Supporting Information

\title{
Stereochemistry of transition metal complexes controlled by the metallo-anomeric effect
}

\author{
Feng Zhu and Maciej A. Walczak* \\ University of Colorado, Department of Chemistry, \\ Boulder, CO 80309, United States \\ E-mail: maciej.walczak@colorado.edu
}




\section{Table of Contents}

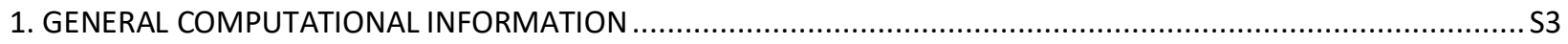

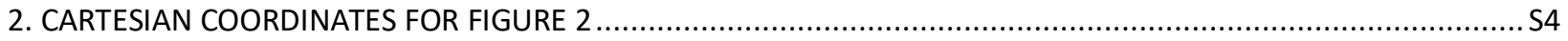

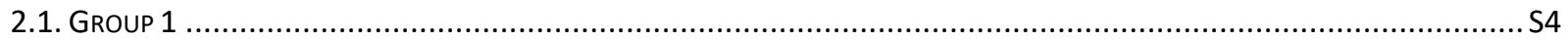

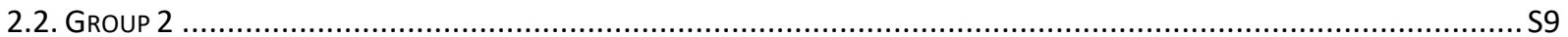

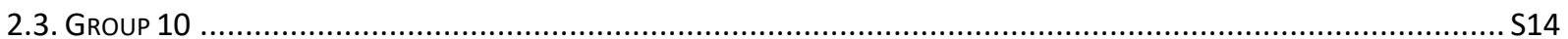

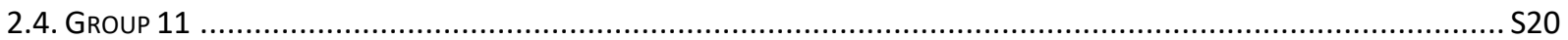

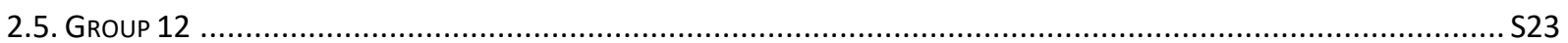

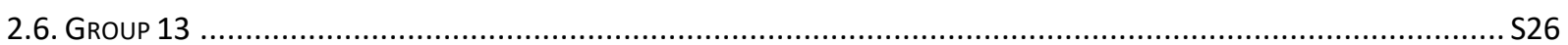

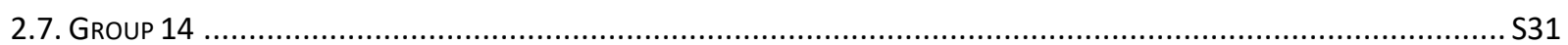

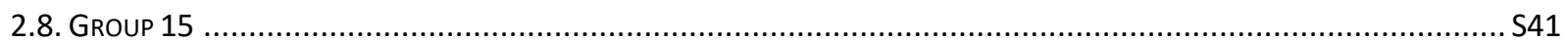

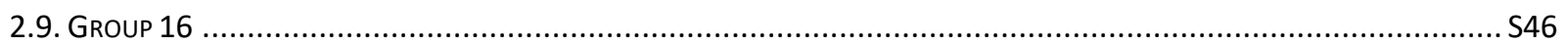

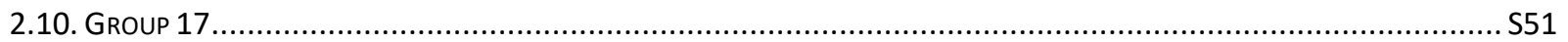

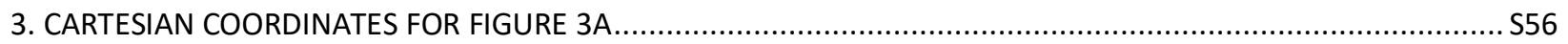

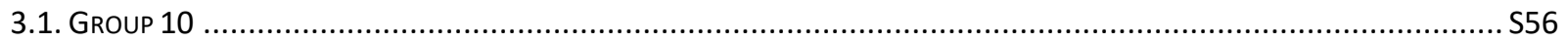

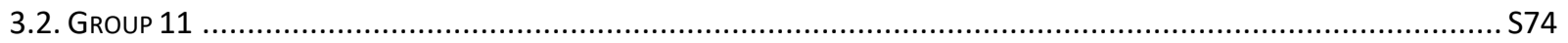

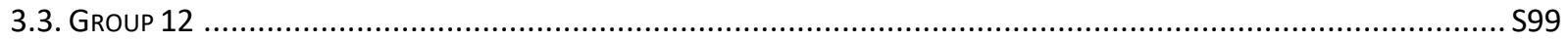

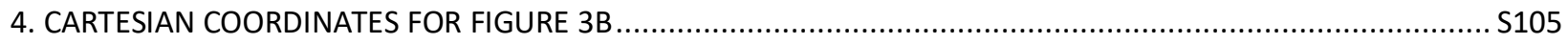

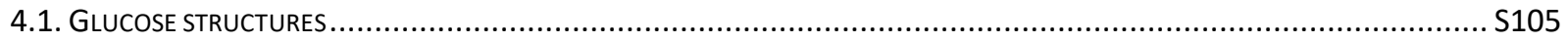

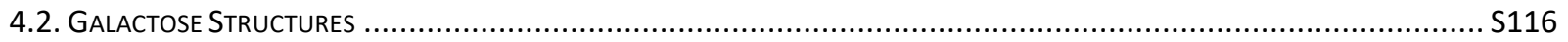

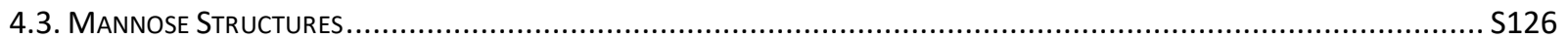

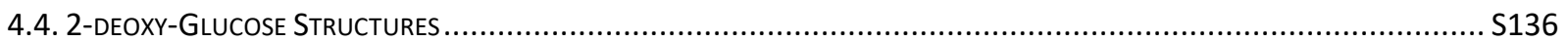

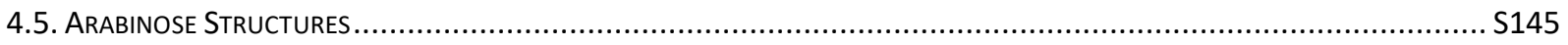

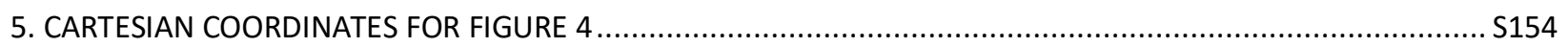

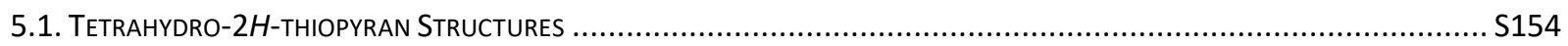

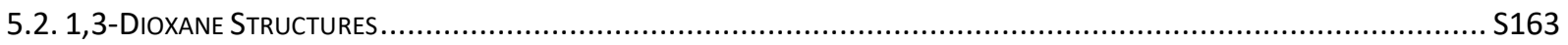

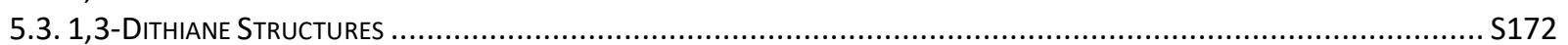

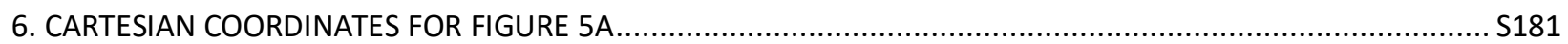

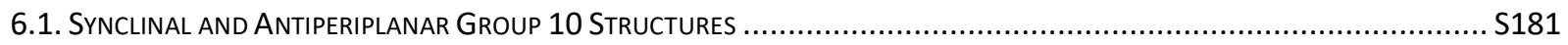

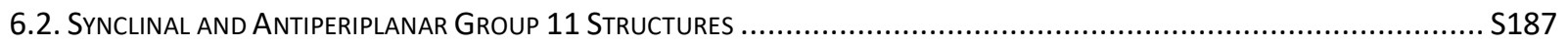

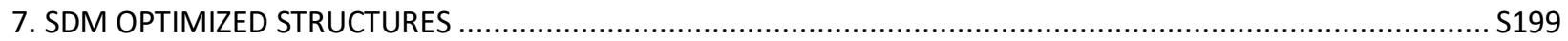

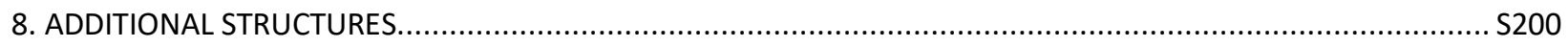

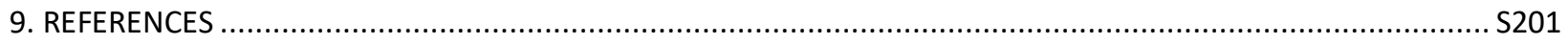




\section{General Computational Information}

All DFT calculations were performed with Gaussian $09^{1}$ or Gaussian 16 software packages. ${ }^{2}$ Geometry optimizations of all the minima were performed at M06-2X using def2TZVP (Figures 2, 3, and 4) or def2QZVPP (Figure 5) basis sets. Vibrational frequencies were computed at the same level to evaluate thermal corrections at $298 \mathrm{~K}$. All structures in Figures 2, 3, and 4 are characterized by NIMAG=0. The single-point energies were computed at the same level of theory with def2QZVP or def2QZVPP basis sets. SMD solvation model for benzene and DMSO was used to optimize selected structures. ${ }^{3}$ To correct the Gibbs free energies under pressure of $1 \mathrm{~atm}$ to the standard state in solution (1 $\mathrm{mol} / \mathrm{L})$, a correction of $R T \ln (\mathrm{cs} / \mathrm{cg})$ (about $1.89 \mathrm{kcal} / \mathrm{mol}$ ) is added to energies of all species. cs is the standard molar concentration in solution ( $1 \mathrm{~mol} / \mathrm{L}), \mathrm{cg}$ is the standard molar concentration in gas phase $(0.0446 \mathrm{~mol} / \mathrm{L})$, and $R$ is the gas constant. Natural Bond Orbital analyses ${ }^{4-6}$ were performed on optimized structures at M06-2X/def2QZVP or def2QZPP levels of theory. def2TZVP, def2QZVP, and def2QZVPP basis sets were defined by Wigenod. ${ }^{7,8}$ Electronic energies and Gibbs free energy corrections reported in Hartrees. 


\subsection{Group 1}

\section{LITHIUM}

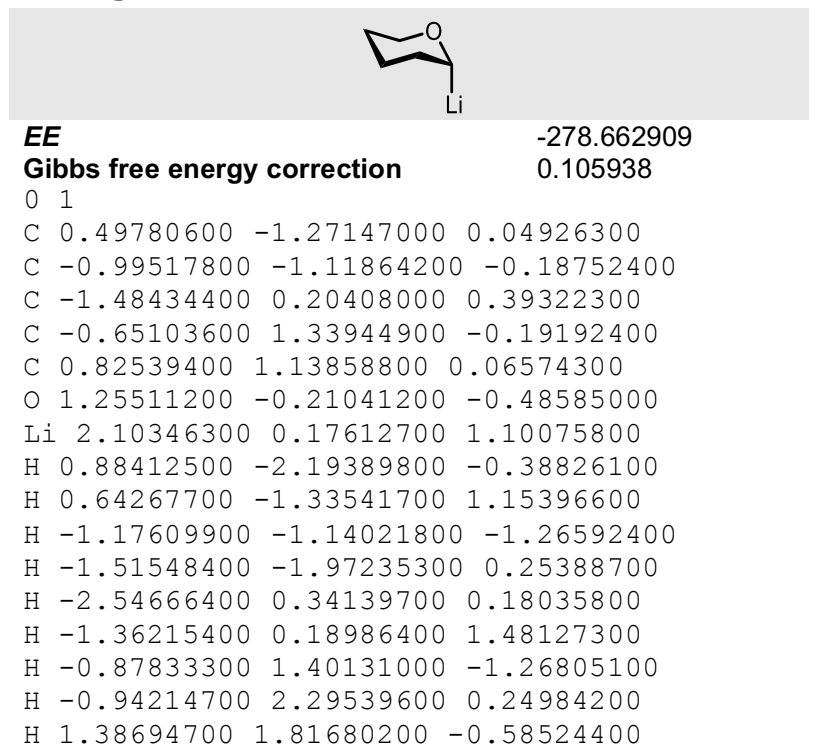

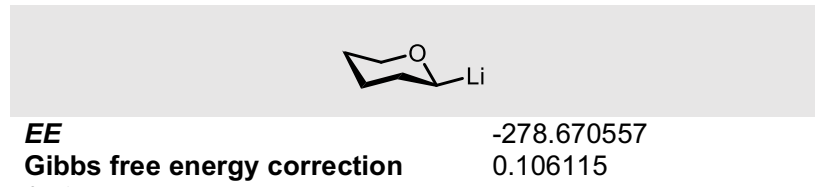

free energy correction $\quad 0.106115$

01

$\begin{array}{llll}\text { C } 0.01910100 & -1.40197700 & 0.24551800\end{array}$

C $-1.26656200-0.80854300-0.30893400$

C $-1.43360900 \quad 0.63645300 \quad 0.15508800$

C $-0.17741600 \quad 1.44261300-0.19511500$

$\begin{array}{llll}\text { C } 1.09333200 & 0.81792600 & 0.34542000\end{array}$

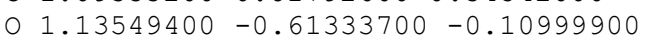

Li $2.65003900 \quad 0.14563800-0.55932600$

$\mathrm{H} \quad 0.18916800 \quad-2.40977600 \quad-0.14219900$

$\mathrm{H}-0.03603700-1.45618600 \quad 1.34119300$

$\mathrm{H}-1.22099500-0.84015300-1.40234200$

$\mathrm{H}-2.11184600-1.42833800 \quad 0.00074500$

$\mathrm{H}-2.33001100 \quad 1.07455600 \quad-0.29254900$

$\mathrm{H}-1.57467300 \quad 0.64970100 \quad 1.24147800$

$\mathrm{H}-0.12068600 \quad 1.54304400-1.28621400$

$\mathrm{H}-0.25396800 \quad 2.45740400 \quad 0.20405700$

H $1.01590800 \quad 0.76070100 \quad 1.44194000$

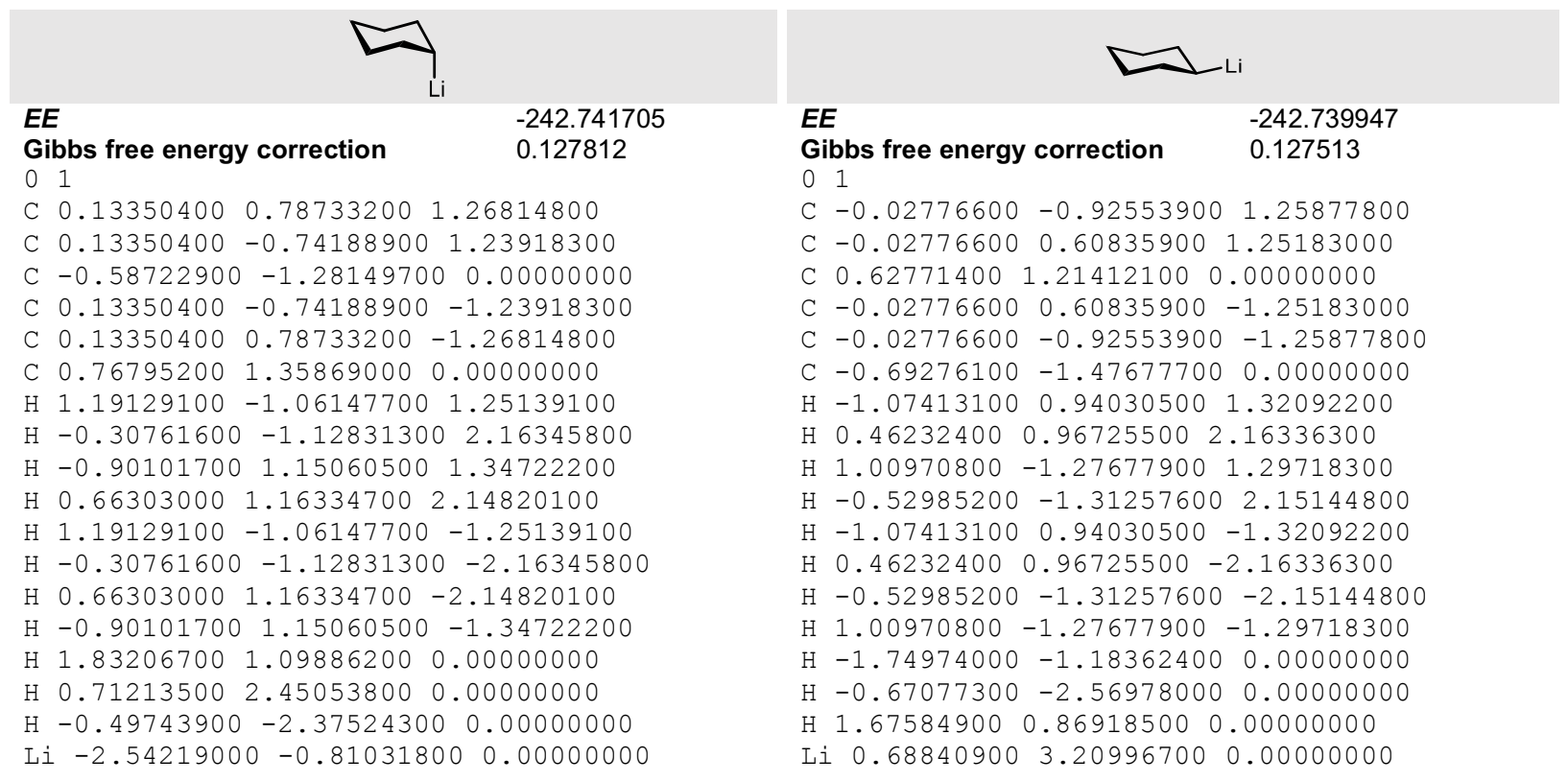




\section{SODIUM}

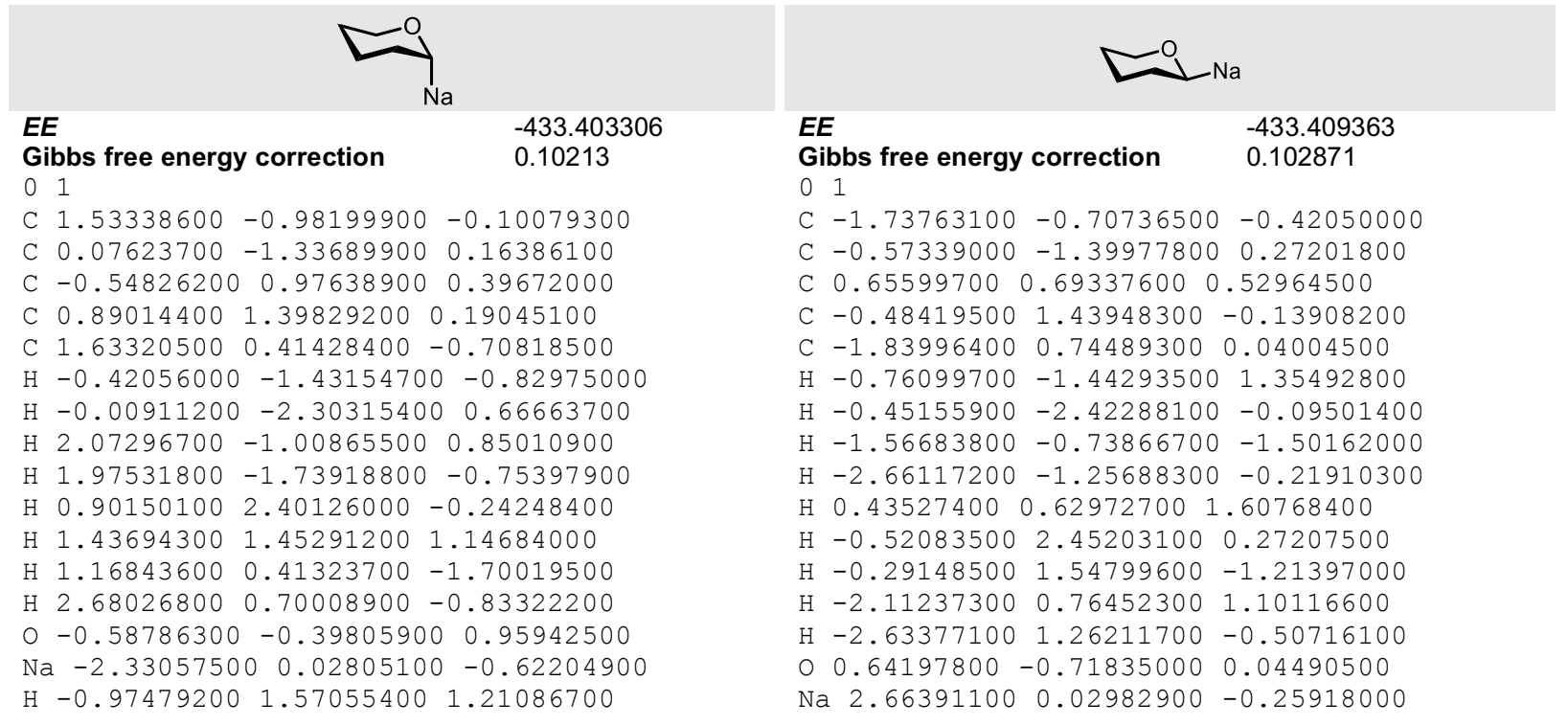

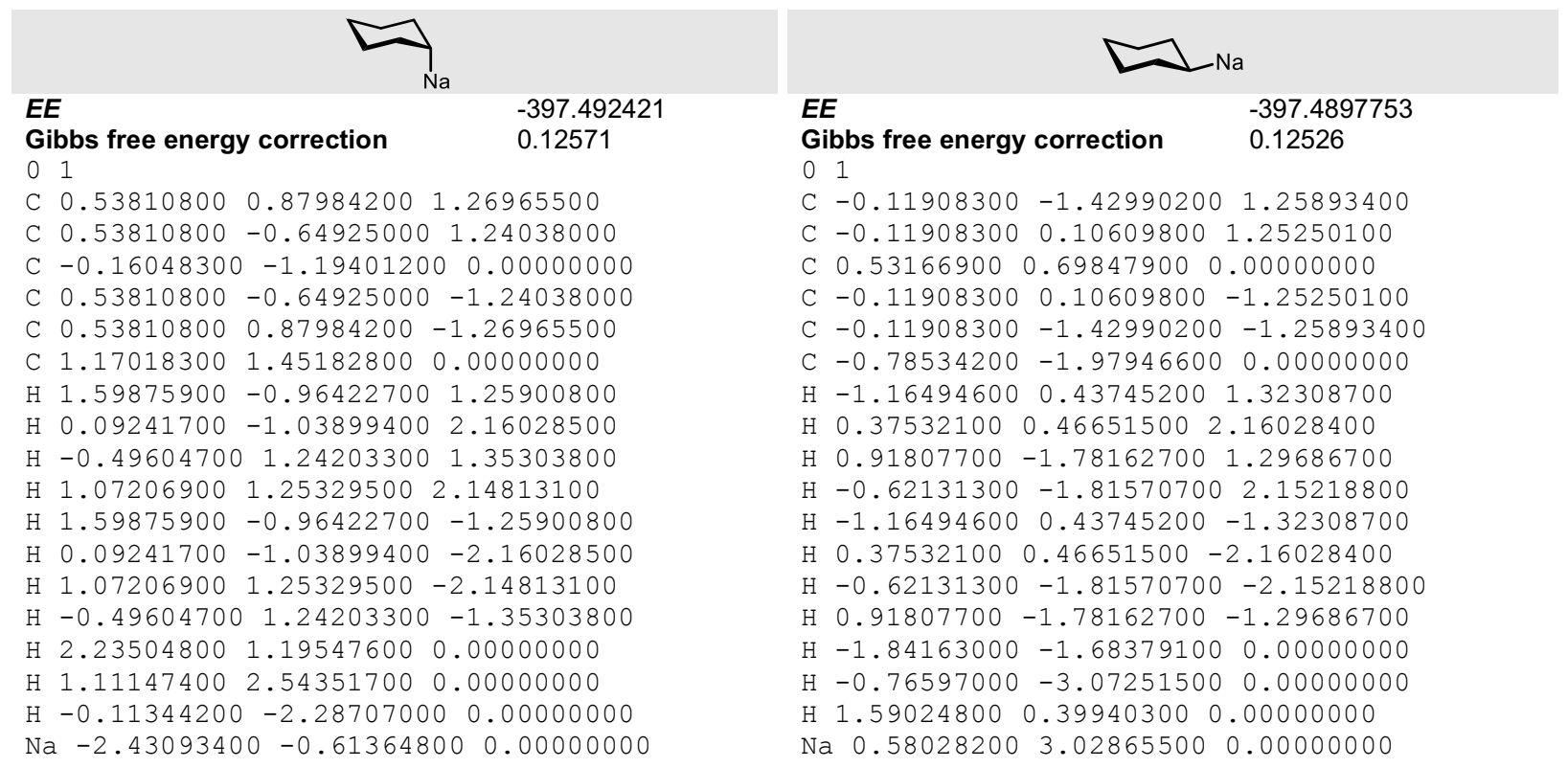




\section{POTASSIUM}

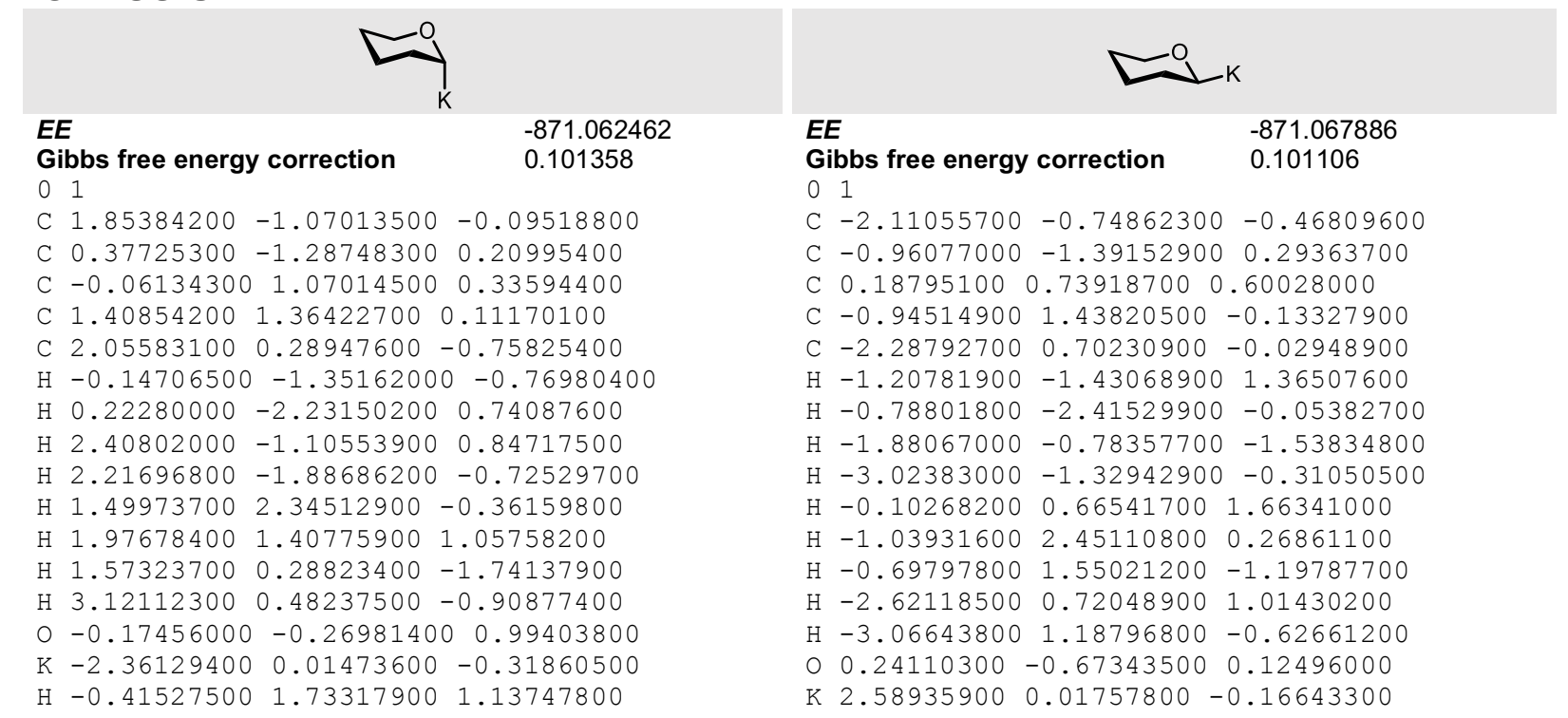

\begin{tabular}{|c|c|c|}
\hline$E E$ & -835.143265 & -835.140450913 \\
\hline $\begin{array}{l}\text { Gibbs free energy correction } \\
0 \quad 1\end{array}$ & 0.123006 & $\begin{array}{l}\text { Gibbs free energy correction } \\
01\end{array}$ \\
\hline C $0.93716500 \quad 0.88753200$ & 1.27028200 & C $-0.11434500-1.91348000 \quad 1.25940600$ \\
\hline C $0.93716500-0.64297000$ & 01.23605800 & 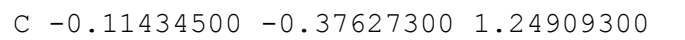 \\
\hline C $0.22417300-1.18127000$ & 00.00000000 & C $0.52777800 \quad 0.23645600 \quad 0.00000000$ \\
\hline C $0.93716500-0.64297000$ & $0-1.23605800$ & $C-0.11434500-0.37627300-1.24909300$ \\
\hline C $0.93716500 \quad 0.88753200$ & -1.27028200 & $C-0.11434500-1.91348000-1.25940600$ \\
\hline C $1.56716900 \quad 1.46071100$ & 0.00000000 & $C-0.78038600-2.46285400 \quad 0.00000000$ \\
\hline $\mathrm{H} \quad 2.00288000-0.94938400$ & 01.25954400 & $\mathrm{H}-1.16511800-0.05371000 \quad 1.32937500$ \\
\hline $\mathrm{H} \quad 0.49578900-1.03149900$ & 02.16001300 & $\mathrm{H} \quad 0.37970800 \quad-0.01732800 \quad 2.15941500$ \\
\hline $\mathrm{H}-0.09630100 \quad 1.25405200$ & 01.35761200 & $\mathrm{H} \quad 0.92368800 \quad-2.26308800 \quad 1.29552300$ \\
\hline $\mathrm{H} \quad 1.47332700 \quad 1.26243600$ & 2.14756500 & $\mathrm{H}-0.61641500 \quad-2.30303700 \quad 2.15220800$ \\
\hline $\mathrm{H} \quad 2.00288000-0.94938400$ & $0-1.25954400$ & $\mathrm{H}-1.16511800-0.05371000-1.32937500$ \\
\hline $\mathrm{H} \quad 0.49578900-1.03149900$ & $0-2.16001300$ & $\mathrm{H} \quad 0.37970800-0.01732800-2.15941500$ \\
\hline $\mathrm{H} \quad 1.47332700 \quad 1.26243600$ & -2.14756500 & $\mathrm{H}-0.61641500-2.30303700-2.15220800$ \\
\hline $\mathrm{H}-0.09630100 \quad 1.25405200$ & $0-1.35761200$ & $\mathrm{H} \quad 0.92368800-2.26308800-1.29552300$ \\
\hline $\mathrm{H} 2.63107900 \quad 1.20003300$ & 0.00000000 & $\mathrm{H}-1.83645300-2.16443200 \quad 0.00000000$ \\
\hline $\mathrm{H} \quad 1.512943002 .55311100$ & 0.00000000 & $\mathrm{H}-0.76474900-3.55628900 \quad 0.00000000$ \\
\hline $\mathrm{H} \quad 0.28429000-2.27563000$ & $0 \quad 0.00000000$ & $\mathrm{H} 1.58941200-0.05873200 \quad 0.00000000$ \\
\hline$K-2.39051100-0.3768480$ & 000.00000000 & K $0.32778900 \quad 2.94153700 \quad 0.00000000$ \\
\hline
\end{tabular}




\section{RUBIDIUM}

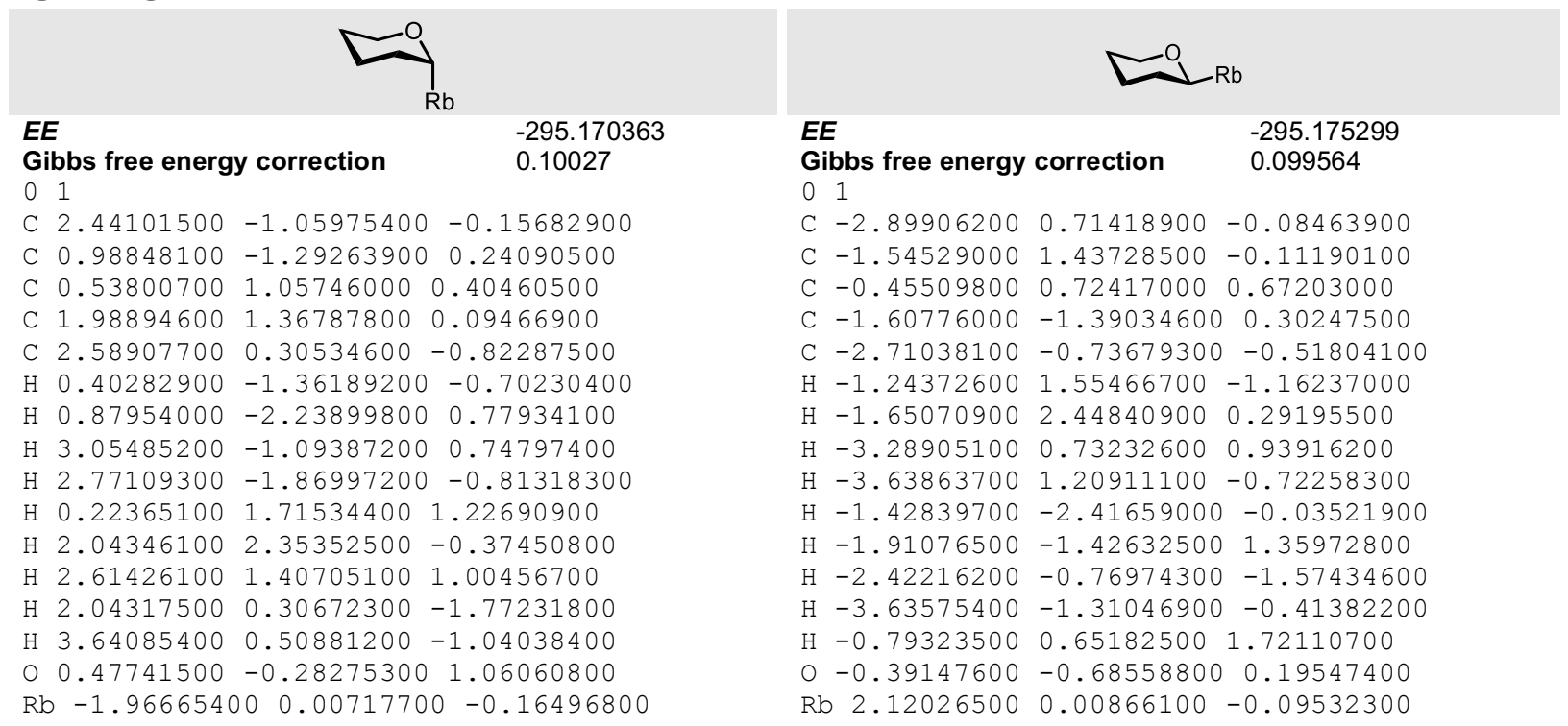

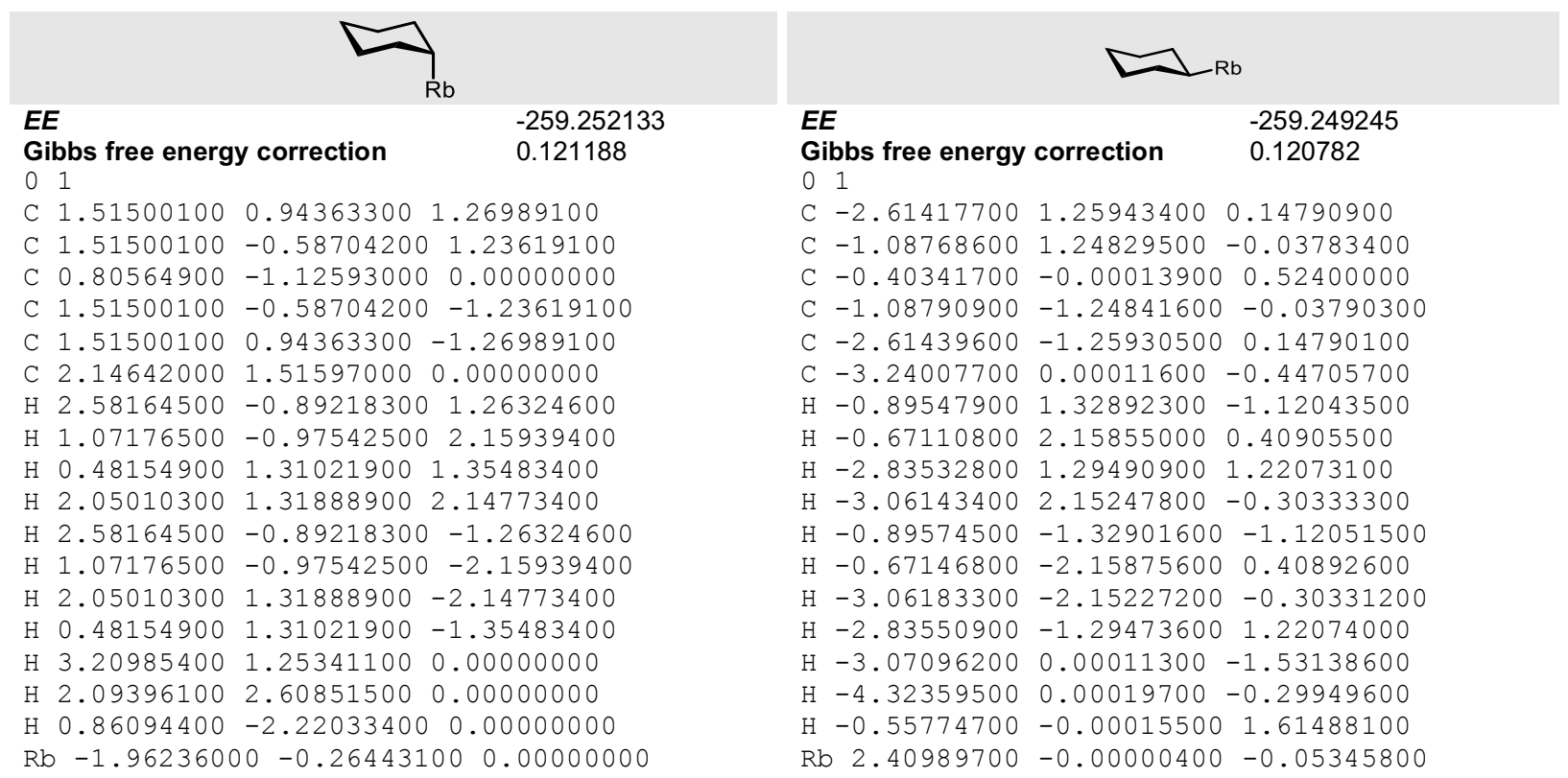




\section{CESIUM}

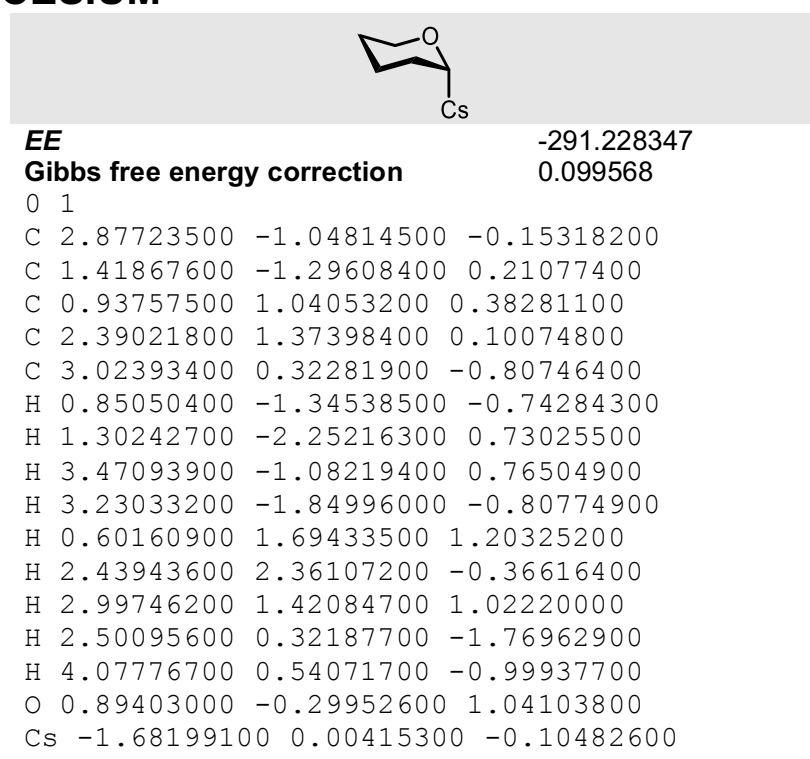

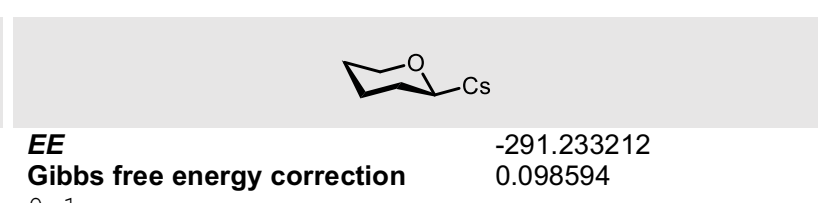

01

C $-3.25747500 \quad 0.78007300-0.14283600$

C $-1.86980000 \quad 1.43094500-0.06134400$

C $-0.87227600 \quad 0.64876000 \quad 0.77704700$

C $-2.09849000-1.39321200 \quad 0.28832200$

C $-3.11311800-0.67235000-0.58807100$

H $-1.49117100 \quad 1.54884800-1.08838000$

$\mathrm{H}-1.95181800 \quad 2.44043000 \quad 0.35201100$

H $-3.71601500 \quad 0.80498100 \quad 0.85194100$

$\mathrm{H}-3.92392200 \quad 1.32306700-0.82105900$

H $-1.94362900-2.41944100-0.06086000$

H $-2.47126000-1.43636200 \quad 1.32247200$

$\mathrm{H}-2.76100300-0.70523100-1.62479700$

H $-4.07016300-1.20035100-0.55173200$

$\mathrm{H}-1.26518700 \quad 0.582341001 .80609200$

$\begin{array}{llll}0 & -0.84662700 & -0.74506600 & 0.27735300\end{array}$

Cs $1.77516600 \quad 0.00467200-0.07351300$

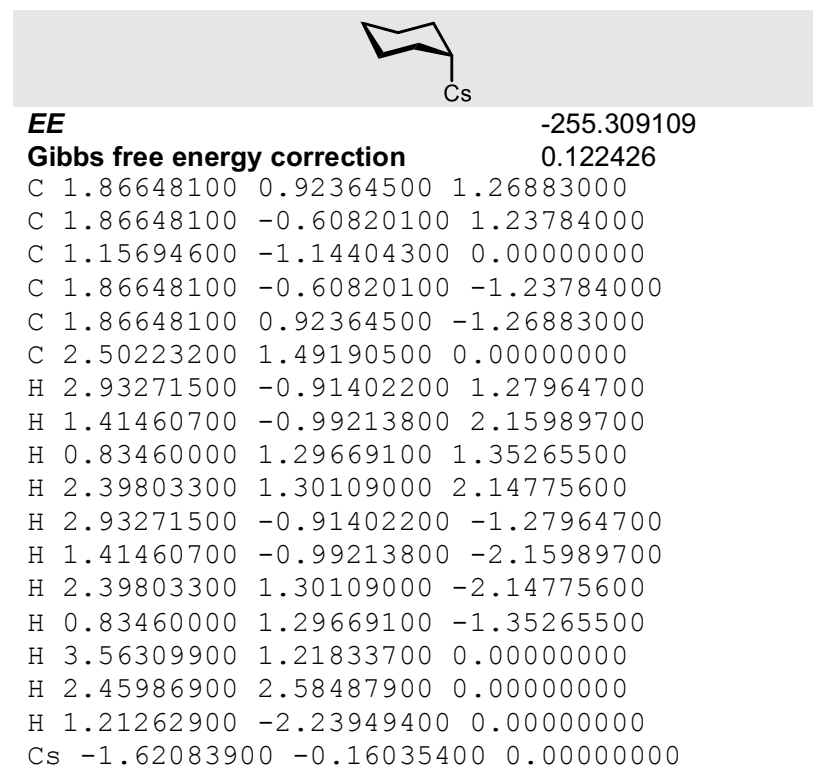

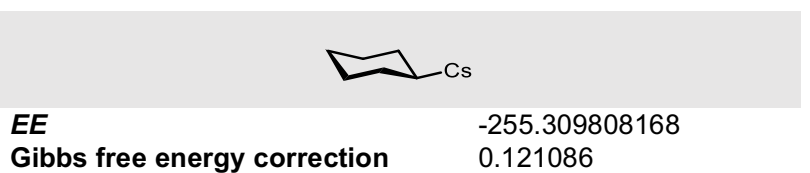

01

C $-0.65500300 \quad 2.71018400 \quad 1.25846800$

C $-0.655003001 .17226100 \quad 1.24898000$

C $-1.23050400 \quad 0.52456600 \quad 0.00000000$

C $-0.65500300 \quad 1.17226100-1.24898000$

C $-0.65500300 \quad 2.71018400-1.25846800$

C $0.01545600 \quad 3.25396000 \quad 0.00000000$

$\mathrm{H} \quad 0.41365800 \quad 0.88486600 \quad 1.39172900$

$\mathrm{H}-1.17442800 \quad 0.80813300 \quad 2.14275900$

$\mathrm{H}-1.69341100 \quad 3.05655900 \quad 1.28901400$

$\mathrm{H}-0.15597400 \quad 3.10120500 \quad 2.15247800$

$\mathrm{H} \quad 0.41365800 \quad 0.88486600-1.39172900$

$\mathrm{H}-1.17442800 \quad 0.80813300-2.14275900$

$\mathrm{H}-0.15597400 \quad 3.10120500-2.15247800$

$\mathrm{H}-1.69341100 \quad 3.05655900-1.28901400$

$\mathrm{H} 1.07014100 \quad 2.94641800 \quad 0.00000000$

$\mathrm{H} \quad 0.01101300 \quad 4.34700800 \quad 0.00000000$

H $-2.32172200 \quad 0.60525700 \quad 0.00000000$

Cs $0.53584100-1.68837600 \quad 0.00000000$ 


\subsection{Group 2}

\section{BERYLIUM}

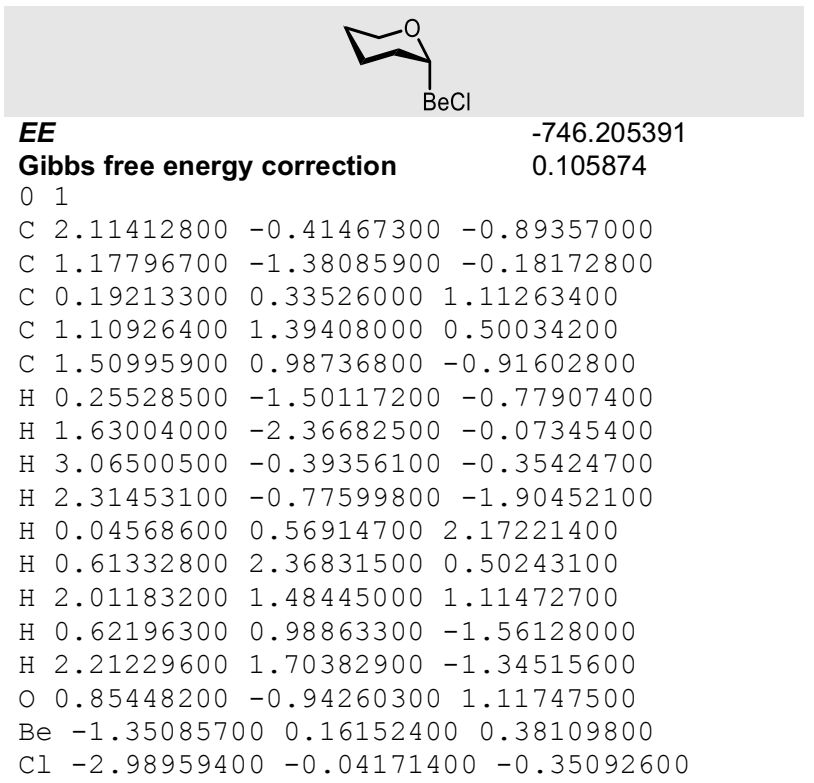

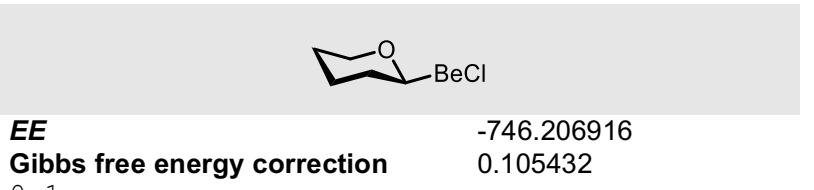

01

C $-2.69917500-0.17406800-0.44785300$

C $-1.87840300-1.30780800 \quad 0.14538200$

$\begin{array}{lllll}\text { C } & 0.01596100 & 0.08186700 & 0.48538200\end{array}$

C $-0.71627500 \quad 1.30228100 \quad-0.08203800$

C $-2.22532200 \quad 1.16453300 \quad 0.11287300$

$\mathrm{H}-2.05384800-1.36766200 \quad 1.22993700$

$\mathrm{H}-2.14456200-2.26795600-0.29780300$

$\mathrm{H}-2.57503900-0.18427000-1.53447500$

$\mathrm{H}-3.75801400-0.33738400-0.23537500$

$\mathrm{H}-0.34567700 \quad 2.21005800 \quad 0.40143500$

$\mathrm{H}-0.49997400 \quad 1.39471700-1.15127700$

H $-2.45247800 \quad 1.20726400 \quad 1.18360500$

$\mathrm{H}-2.75581200 \quad 1.99228700-0.36202400$

$0-0.49524000-1.12805300-0.09170500$

$\mathrm{Be} 1.69143500 \quad 0.01597700 \quad 0.19100500$

$\mathrm{H}-0.18684700 \quad 0.05408600 \quad 1.57098700$

Cl $3.46986600-0.00832100-0.12458100$

\begin{tabular}{|c|c|c|}
\hline \multicolumn{3}{|r|}{-710.301894} \\
\hline & $\begin{array}{l}\text { ibbs free energy correction } \\
1\end{array}$ & 0.129668 \\
\hline C & $-1.34295000-1.26354200$ & -0.62850200 \\
\hline $\mathrm{C}$ & $-0.96713000-1.25451600$ & 0.85275400 \\
\hline $\mathrm{C}$ & $-0.14894100-0.00011900$ & 1.21183100 \\
\hline $\mathrm{C}$ & $\begin{array}{lll}-0.96703400 & 1.25439900 & 0\end{array}$ & 0.85292900 \\
\hline $\mathrm{C}$ & $-1.34285100 \quad 1.26365900$ & -0.62832600 \\
\hline $\mathrm{C}$ & $-2.11184200 \quad 0.00011500$ & -1.01025400 \\
\hline $\mathrm{H}$ & $0.03232600-0.000199002$ & 2.29341800 \\
\hline $\mathrm{H}$ & $-1.89105300-1.26734700$ & 1.44537600 \\
\hline $\mathrm{H}$ & $-0.42339500-2.16763600$ & 1.10738500 \\
\hline $\mathrm{H}$ & $-0.43030700-1.32624800$ & -1.23610200 \\
\hline $\mathrm{H}$ & $-1.93412100-2.15017300$ & -0.86662500 \\
\hline $\mathrm{H}$ & $-1.89095700 \quad 1.267220001$ & 1.44555200 \\
\hline $\mathrm{H}$ & $-0.42322800 \quad 2.167442001$ & 1.10768900 \\
\hline $\mathrm{H}$ & $-1.93395300 \quad 2.15036900$ & -0.86632700 \\
\hline $\mathrm{H}$ & $-0.43020400 \quad 1.32637900$ & -1.23591900 \\
\hline $\mathrm{H}$ & $-3.07258400 \quad 0.00011600$ & -0.48437000 \\
\hline & $-2.33682400 \quad 0.00019900$ & -2.07861200 \\
\hline & $=1.35980200-0.00005800$ & 0.44694100 \\
\hline & L $2.97527000 \quad 0.00000800$ & -0.37187200 \\
\hline
\end{tabular}

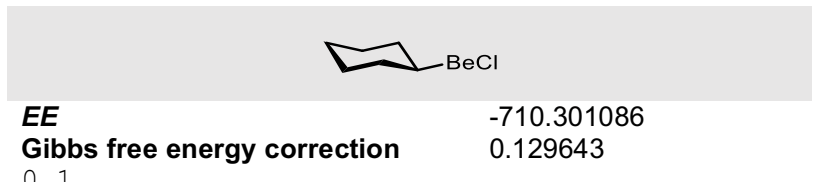

01

C $-2.78570200 \quad 0.00005400 \quad-0.39014300$

C $-2.12386900-1.25906900 \quad 0.16373200$

C $-0.62082100-1.26265300-0.11557200$

C $0.07104800 \quad-0.00004800 \quad 0.43797600$

C $-0.62073500 \quad 1.26261400 \quad-0.11555300$

C $-2.12378600 \quad 1.25912400 \quad 0.16374900$

$\mathrm{H}-0.17097000 \quad-2.16635500 \quad 0.30345600$

$\mathrm{H}-2.28288500-1.30183400 \quad 1.24672200$

$\mathrm{H}-2.58978800-2.15096700-0.26188300$

$\mathrm{H}-2.69633800 \quad 0.00006400-1.48228100$

$\mathrm{H}-3.85345800 \quad 0.00008100-0.16168100$

$\mathrm{H}-0.08350300 \quad-0.00005500 \quad 1.52802700$

$\mathrm{H}-0.17082300 \quad 2.16627800 \quad 0.30348600$

$\mathrm{H}-0.46831700 \quad 1.31585900-1.20026200$

$\mathrm{H}-2.28280300 \quad 1.30187400 \quad 1.24673700$

$\mathrm{H}-2.58965000 \quad 2.15106000-0.26185000$

$\mathrm{H}-0.46840000-1.31589600-1.20028000$

Be $1.73708800-0.00014000 \quad 0.18315600$

Cl $3.52539900 \quad 0.00001900-0.09752700$ 


\section{MAGNESIUM}

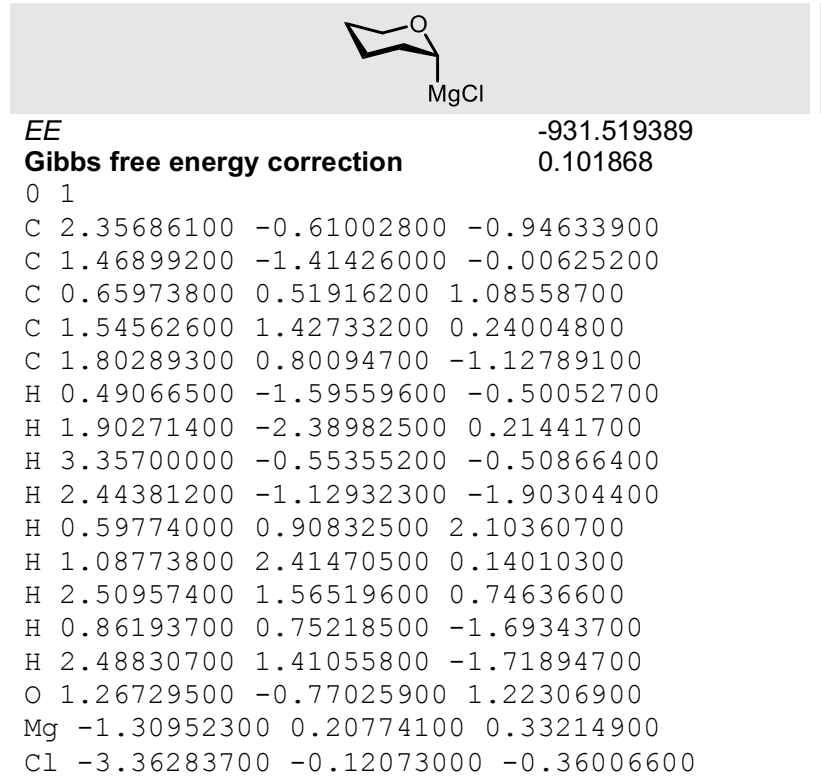

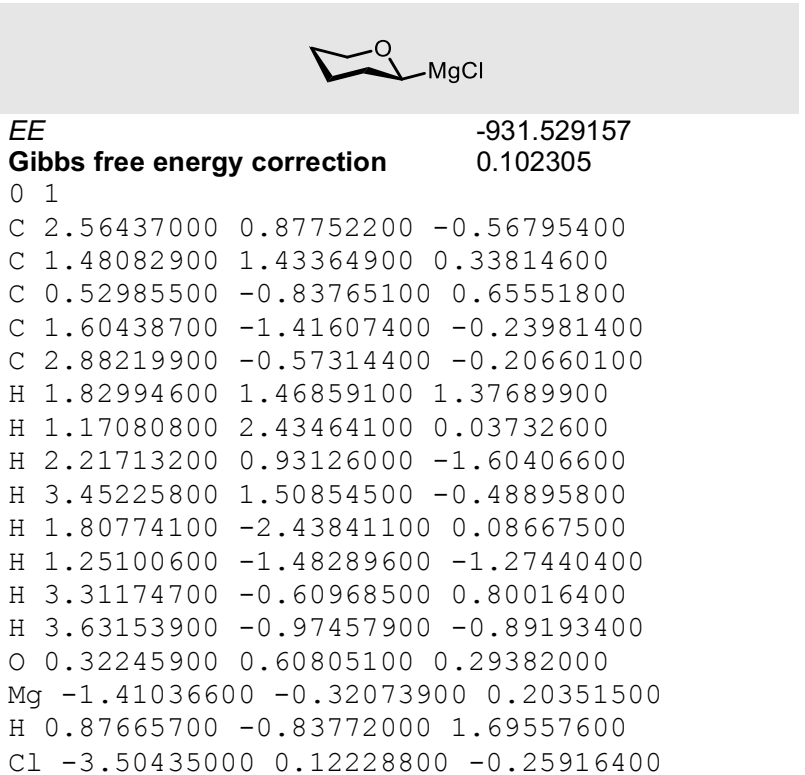

\begin{tabular}{|c|c|}
\hline & $\mathrm{MgCl}$ \\
\hline$E E$ & -895.612415 \\
\hline $\begin{array}{l}\text { Gibbs free energy } \\
01\end{array}$ & correction \\
\hline C -1.58563100 & $-1.26737700-0.70630900$ \\
\hline$C-1.40332700$ & $-1.24941400 \quad 0.81133800$ \\
\hline$C-0.64270200$ & $-0.00026000 \quad 1.27208500$ \\
\hline$C-1.40323100$ & $1.24912600 \quad 0.81179600$ \\
\hline C -1.58553500 & $1.26766100-0.70584300$ \\
\hline$C-2.28640500$ & $0.00025800-1.19397600$ \\
\hline $\mathrm{H}-0.56746100$ & $-0.00046000 \quad 2.36270100$ \\
\hline $\mathrm{H}-2.40402800$ & $-1.25945100 \quad 1.26832000$ \\
\hline $\mathrm{H}-0.90876300$ & $-2.16677400 \quad 1.14130500$ \\
\hline $\mathrm{H}-0.60406500$ & $-1.34925900-1.19761100$ \\
\hline $\mathrm{H}-2.15014100$ & $-2.14930900-1.01584000$ \\
\hline $\mathrm{H}-2.40393200$ & $1.25907900 \quad 1.26878100$ \\
\hline $\mathrm{H}-0.90859600$ & $2.16632700 \quad 1.14210100$ \\
\hline $\mathrm{H}-2.14997300$ & $2.14975100-1.01505100$ \\
\hline $\mathrm{H}-0.60396300$ & $1.34964900-1.19712200$ \\
\hline $\mathrm{H}-3.31146000$ & $0.00022700-0.80941000$ \\
\hline $\mathrm{H}-2.35892700$ & $0.00046000-2.28332400$ \\
\hline $\operatorname{Mg} 1.29259300$ & $-0.00010300 \quad 0.45283000$ \\
\hline Cl 3.31183400 & $0.00006100-0.40196200$ \\
\hline
\end{tabular}

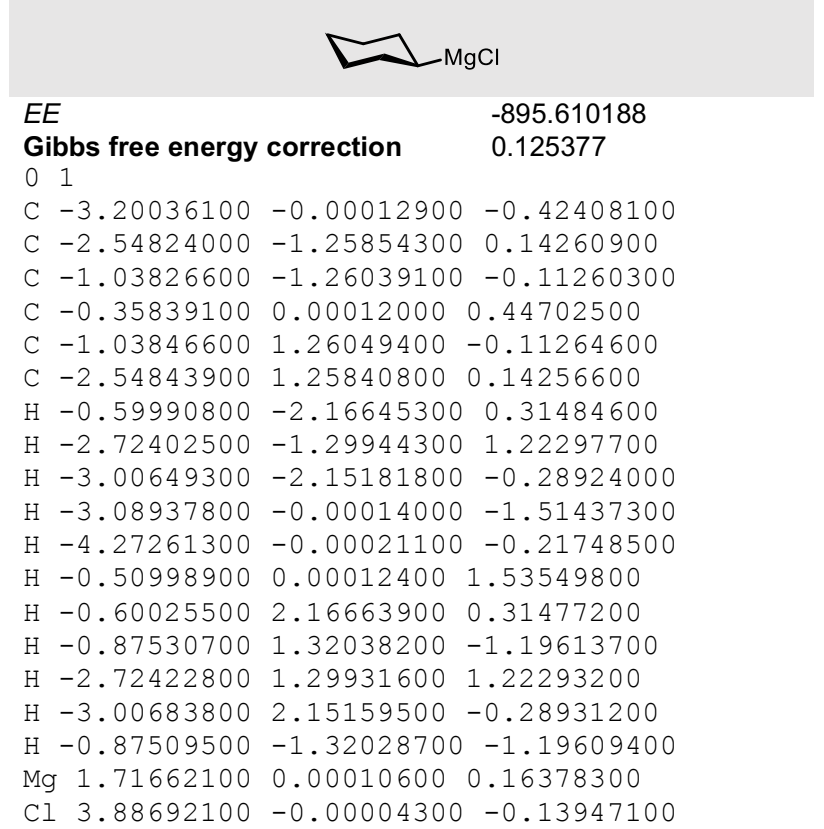




\section{CALCIUM}

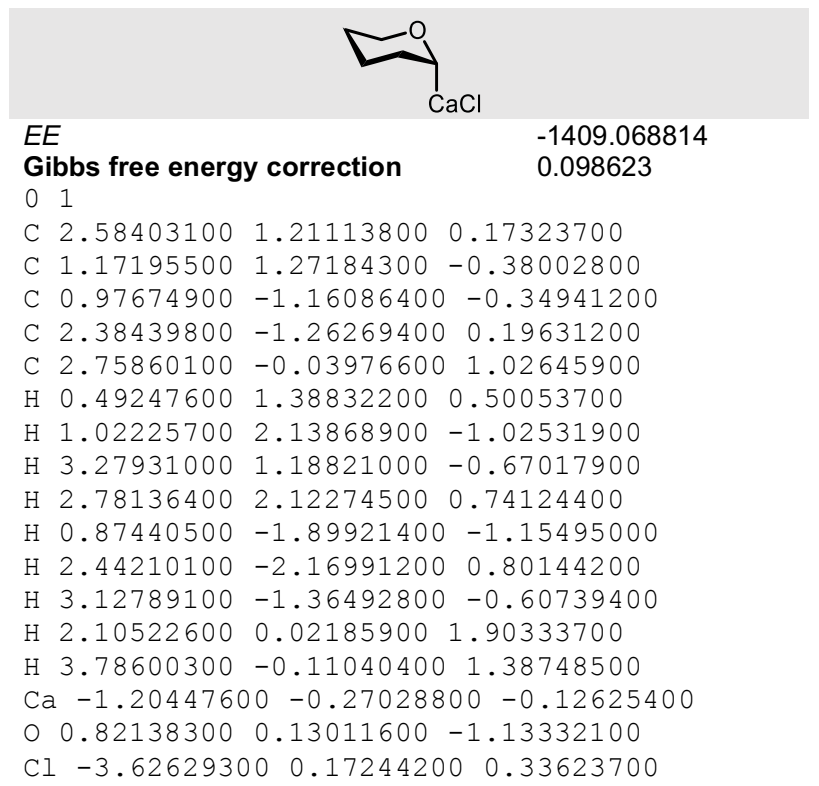

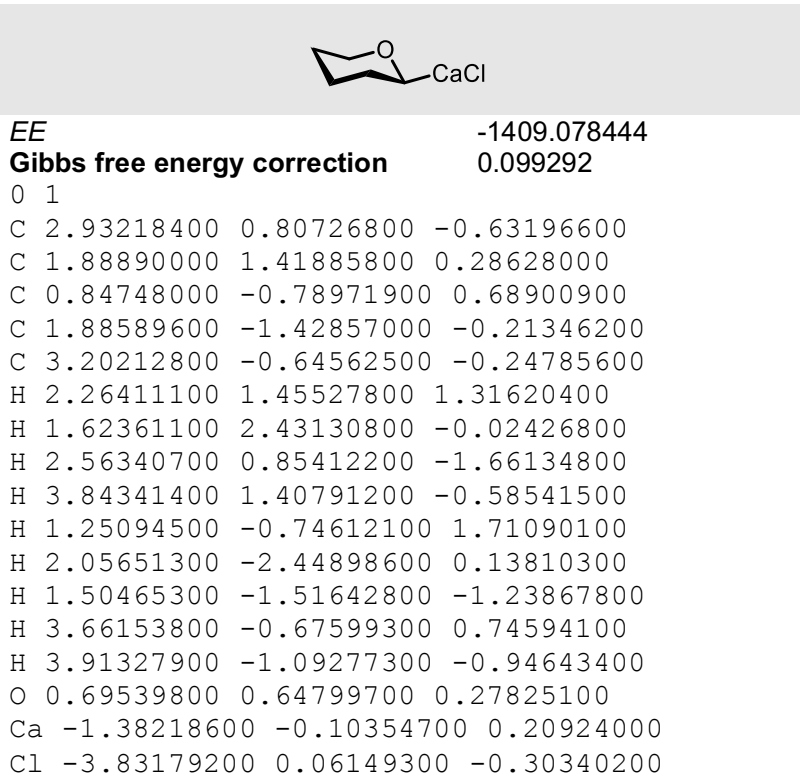

\begin{tabular}{|c|c|}
\hline$E E$ & -1373.140522 \\
\hline $\begin{array}{l}\text { Gibbs free energy } \\
0 \quad 1\end{array}$ & correction \\
\hline$C-1.74184800$ & $-1.26999800-0.68739600$ \\
\hline$C-1.69720300$ & $-1.23964600 \quad 0.84588000$ \\
\hline$C-0.95702400$ & $0.00079600 \quad 1.36441100$ \\
\hline$C-1.69766600$ & $1.24043300 \quad 0.84465200$ \\
\hline$C-1.74227400$ & $1.26923400-0.68865000$ \\
\hline$C-2.38351300$ & $-0.00076500-1.24759600$ \\
\hline $\mathrm{H}-0.97291600$ & $0.00132400 \quad 2.45582400$ \\
\hline $\mathrm{H}-2.74859700$ & $-1.26047200 \quad 1.18178700$ \\
\hline $\mathrm{H}-1.24656800$ & $-2.16410400 \quad 1.21967000$ \\
\hline $\mathrm{H}-0.72582400$ & $-1.38696300-1.11893800$ \\
\hline $\mathrm{H}-2.27742200$ & $-2.14972000-1.05035900$ \\
\hline $\mathrm{H}-2.74907200$ & $1.26119400 \quad 1.18052700$ \\
\hline $\mathrm{H}-1.24736700$ & $2.16542000 \quad 1.21752300$ \\
\hline $\mathrm{H}-2.27812700$ & $2.14841000-1.05252300$ \\
\hline $\mathrm{H}-0.72625600$ & $1.38607800-1.12022000$ \\
\hline $\mathrm{H}-3.43685900$ & $-0.00080200-0.95192500$ \\
\hline $\mathrm{H}-2.36259200$ & $-0.00130500-2.33951200$ \\
\hline $\mathrm{Ca} 1.07586000$ & $0.00003900 \quad 0.05211300$ \\
\hline Cl 3.56303400 & $-0.00001000-0.19129000$ \\
\hline
\end{tabular}

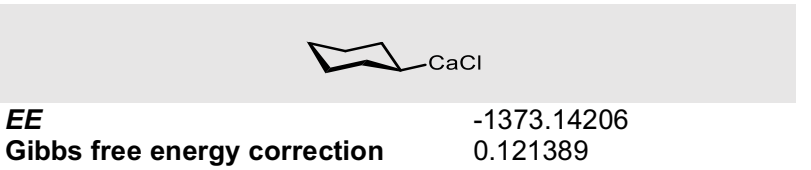

\section{1}

C $-2.85350900 \quad 0.00032600-1.00237700$

C $-2.60230200-1.25582500-0.17570200$

C $-1.18013500-1.25348600 \quad 0.40104200$

C $-0.76922300-0.00019100 \quad 1.16935200$

C $-1.17986500 \quad 1.25339300 \quad 0.40134000$

C $-2.60206900 \quad 1.25615900-0.17530700$

$\mathrm{H}-1.02858900-2.14170000 \quad 1.01931800$

$\mathrm{H}-3.30542100-1.28283900 \quad 0.66198700$

$\mathrm{H}-2.77854600-2.15204000-0.77649400$

$\mathrm{H}-2.17995500 \quad 0.00044000-1.87029000$

$\mathrm{H}-3.86900600 \quad 0.00052600-1.40247100$

$\mathrm{H}-1.18608900-0.00029000 \quad 2.17402400$

$\mathrm{H}-1.02805900 \quad 2.14141000 \quad 1.01982000$

$\mathrm{H}-0.52741800 \quad 1.42549600-0.50247800$

$\mathrm{H}-3.30515000 \quad 1.28296100 \quad 0.66242000$

$\mathrm{H}-2.77821800 \quad 2.15259000-0.77580100$

$\mathrm{H}-0.52762700-1.42553200-0.50273500$

Ca $1.28666100-0.00039700 \quad 0.08112900$

Cl $3.75902800 \quad 0.00027300-0.29646900$ 


\section{STRONTIUM}

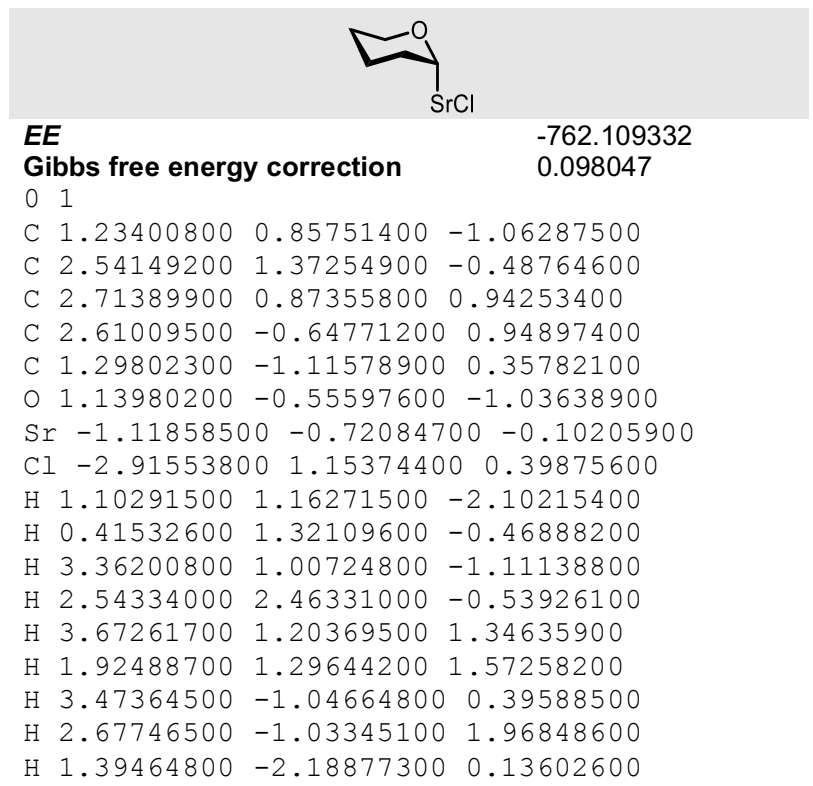

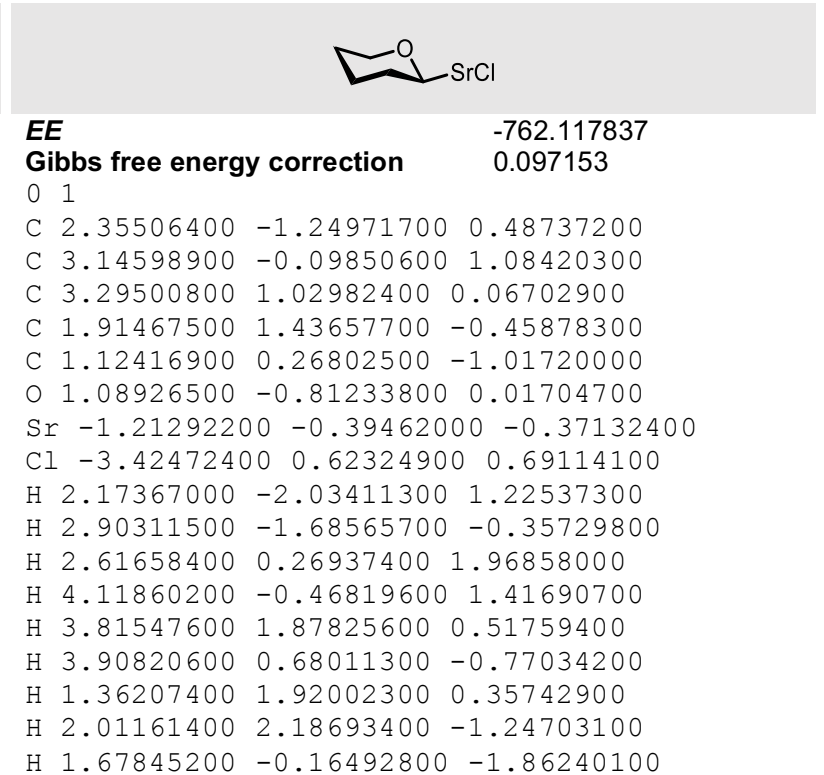

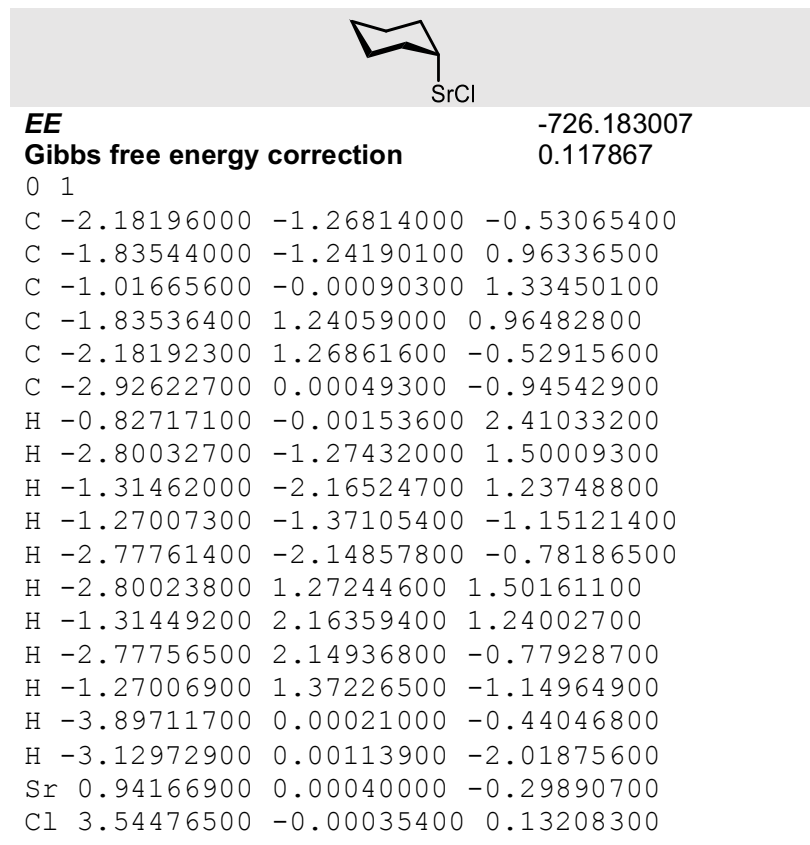

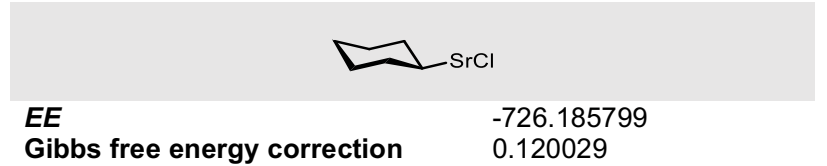

$0 \quad 1$

C $3.34907300-0.00039300 \quad 0.75238200$

C $2.97548800-1.25680300-0.02661800$

C $1.48137900-1.25363600-0.37969200$

C $0.96184800-0.00027200-1.07039600$

C $1.482003001 .25317800-0.38024400$

C $2.97611000 \quad 1.25583200-0.02720800$

$\mathrm{H} 1.24053800-2.14068700-0.97193600$

H $3.54478000-1.28366600-0.96048700$

$\mathrm{H} 3.24134400-2.15274400 \quad 0.54127100$

$\mathrm{H} 2.81306600-0.00003300 \quad 1.71145400$

$\mathrm{H} 4.41339800-0.00059600 \quad 0.99518700$

H $1.21090800-0.00050300-2.13011200$

$\mathrm{H} 1.24153500 \quad 2.14005200-0.97291600$

$\mathrm{H} \quad 0.97283700 \quad 1.42119200 \quad 0.60957500$

H $3.54540300 \quad 1.28199000-0.96109500$

H $3.24239900 \quad 2.15191500 \quad 0.54026100$

$\mathrm{H} \quad 0.97208000-1.42094900 \quad 0.61016900$

Sr $-1.11465400 \quad 0.00090100 \quad 0.25854900$

$\mathrm{Cl}-3.73157900-0.00103800-0.12032800$ 


\section{BARIUM}

\begin{tabular}{|c|c|}
\hline & $\mathrm{BaCl}$ \\
\hline EE & -756.88938 \\
\hline $\begin{array}{l}\text { Gibbs free energy } \\
01\end{array}$ & correction \\
\hline C -2.80612300 & $1.25549600 \quad 0.62699900$ \\
\hline C -1.48767200 & $0.73536900 \quad 1.17165700$ \\
\hline$C-1.41670100$ & $-1.04520300-0.46244100$ \\
\hline$C-2.73293100$ & $-0.57418000-1.04382400$ \\
\hline$C-2.91337400$ & $0.92913800-0.85792300$ \\
\hline $\mathrm{H}-0.68218100$ & $1.29866500 \quad 0.65908800$ \\
\hline $\mathrm{H}-1.39638900$ & $0.91763500 \quad 2.24395600$ \\
\hline $\mathrm{H}-3.62525700$ & $0.77917600 \quad 1.17340300$ \\
\hline $\mathrm{H}-2.86323800$ & $2.33087400 \quad 0.80869100$ \\
\hline $\mathrm{H}-1.44756700$ & $-2.14474200-0.38928700$ \\
\hline $\mathrm{H}-2.75185300$ & $-0.83695500-2.10395800$ \\
\hline $\mathrm{H}-3.59013500$ & $-1.07753700-0.57083600$ \\
\hline $\mathrm{H}-2.12628600$ & $1.46064900-1.40245000$ \\
\hline $\mathrm{H}-3.87443800$ & $1.26201800-1.25437400$ \\
\hline $0-1.33230600$ & $-0.66222500 \quad 0.98153100$ \\
\hline Cl 2.28218100 & $1.82237800-0.33346600$ \\
\hline Ba 1.11356300 & $-0.66921700 \quad 0.03652900$ \\
\hline
\end{tabular}

\begin{tabular}{|c|c|}
\hline$E E$ & -756.896593 \\
\hline $\begin{array}{l}\text { Gibbs free energy } \\
01\end{array}$ & 0.096195 \\
\hline$C-3.25698700$ & $0.52840400-0.98501100$ \\
\hline$C-2.64154100$ & $-0.85746300-0.89824500$ \\
\hline$C-1.27467300$ & $-0.23426500 \quad 1.03869300$ \\
\hline C -1.88906500 & $1.15308300 \quad 1.01128700$ \\
\hline$C-3.29300900$ & $1.17853500 \quad 0.39567300$ \\
\hline $\mathrm{H}-3.27051600$ & $-1.51118900-0.28047000$ \\
\hline $\mathrm{H}-2.53824100$ & $-1.31016800-1.88731800$ \\
\hline $\mathrm{H}-2.65258100$ & $1.13894300-1.66334400$ \\
\hline $\mathrm{H}-4.25710400$ & $0.45065300-1.41805700$ \\
\hline $\mathrm{H}-1.87321300$ & $-0.89705600 \quad 1.67912100$ \\
\hline H -1.91246800 & $1.53831600 \quad 2.03342500$ \\
\hline $\mathrm{H}-1.25073800$ & $1.84155200 \quad 0.43936300$ \\
\hline $\mathrm{H}-3.97657300$ & 0.614967001 .04006900 \\
\hline $\mathrm{H}-3.67949800$ & $2.19817600 \quad 0.32508900$ \\
\hline $0-1.34714700$ & $-0.81069100-0.32168300$ \\
\hline Cl 2.71401900 & $1.60261900-0.44060900$ \\
\hline $\mathrm{Ba} 1.14609700$ & $-0.63273100 \quad 0.11467000$ \\
\hline
\end{tabular}

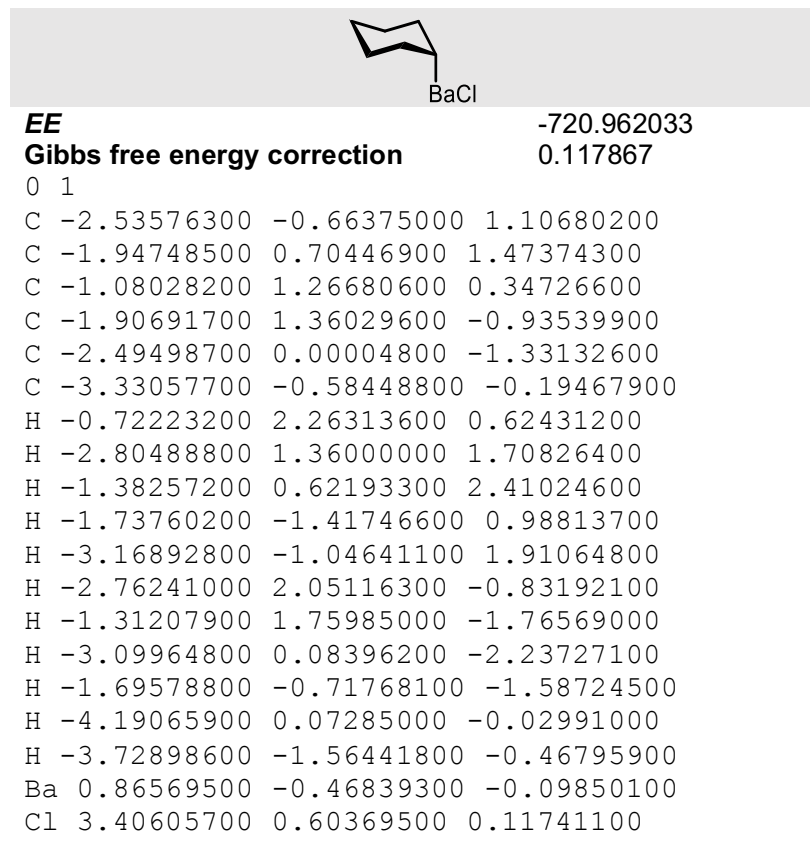

\begin{tabular}{|c|c|}
\hline \multirow{2}{*}{$\begin{array}{l}E E \\
\text { Gibbs free energy correction }\end{array}$} & \multirow{2}{*}{$\begin{array}{l}-720.968106 \\
0.1196\end{array}$} \\
\hline & \\
\hline C 3.69420400 & $0.00080600 \quad 0.51629100$ \\
\hline C 3.24055200 & $-1.25688100-0.21754600$ \\
\hline C 1.71643900 & $-1.25618500-0.40385200$ \\
\hline C 1.13132300 & $-0.00128900-1.02304600$ \\
\hline C 1.71555700 & $1.25474600-0.40536100$ \\
\hline C 3.23966400 & $1.25713300-0.21934100$ \\
\hline H 1.41655900 & $-2.13892300-0.97665800$ \\
\hline $\mathrm{H} 3.70713900$ & $-1.28347600-1.20676300$ \\
\hline $\mathrm{H} \quad 3.56729900$ & $-2.15171800 \quad 0.31965200$ \\
\hline H 3.26354500 & 0.001408001 .52666000 \\
\hline $\mathrm{H} \quad 4.77872900$ & $0.00124900 \quad 0.64229500$ \\
\hline $\mathrm{H} \quad 1.22207100$ & $-0.00180700-2.10844200$ \\
\hline H 1.41449300 & $2.13659300-0.97897100$ \\
\hline H 1.31106400 & $1.42380100 \quad 0.62715500$ \\
\hline $\mathrm{H} 3.70613500$ & $1.28270600-1.20863100$ \\
\hline $\mathrm{H} \quad 3.56569600$ & $2.15298300 \quad 0.31659800$ \\
\hline $\mathrm{H} 1.31168300$ & $-1.42450900 \quad 0.62865500$ \\
\hline $\mathrm{Ba}-1.0138270$ & $00 \quad 0.00042000 \quad 0.42398700$ \\
\hline $\mathrm{Cl}-3.5833240$ & $00-0.00069300-0.63574600$ \\
\hline
\end{tabular}




\section{NICKEL}
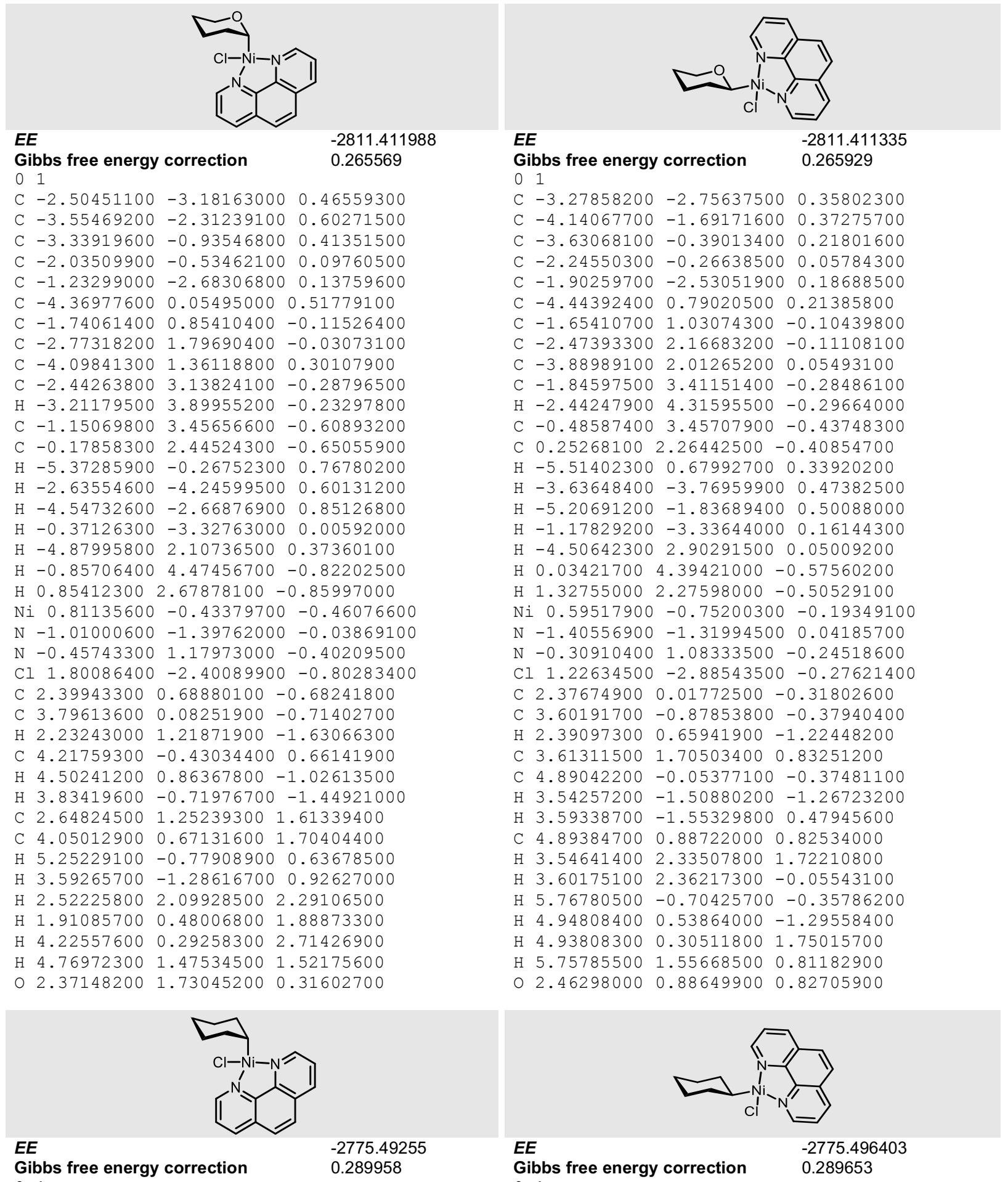

01

$\begin{array}{llll}\text { C }-2.50282500 & -3.21737200 & 0.39861600\end{array}$

$\begin{array}{llll}\text { C }-3.58668500 & -2.38704700 & 0.50670300\end{array}$

C $-3.40996500-1.00054400 \quad 0.34919100$

C $-2.10871700-0.55045400 \quad 0.09264300$

C $-1.23686600-2.67048900 \quad 0.12917600$

$\begin{array}{llll}\text { C }-4.47829000 & -0.04887400 & 0.43028600\end{array}$

C $-1.85537300 \quad 0.85208100-0.08035700$

C $-2.92336400 \quad 1.75828200-0.01074600$

C $-4.24531000 \quad 1.27045800 \quad 0.25464500$

C $-2.63159300 \quad 3.11724300-0.21345800$

$\mathrm{H}-3.42886500 \quad 3.84928500-0.16879700$

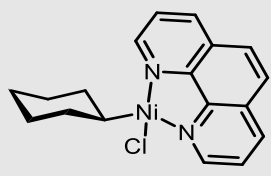

\section{$E E$}

Gibbs free energy correction

$-2775.496403$

0.289653

01

C $-3.25363200 \quad-2.82777400 \quad 0.09437900$

C $-4.15421800-1.79603300 \quad 0.05968000$

C $-3.68306700-0.47150400 \quad 0.00556000$

C $-2.29455600-0.29235200-0.01468800$

C $-1.87696400 \quad-2.54556800 \quad 0.07670600$

C $-4.53766400 \quad 0.67868600-0.02212000$

C $-1.74158900 \quad 1.03140400-0.06489300$

C $-2.60156100 \quad 2.13819900-0.07423600$

C $-4.01978500 \quad 1.92660000-0.05744800$

C $-2.01122300 \quad 3.41314700-0.09325600$

$\mathrm{H}-2.64022800 \quad 4.29511200-0.10011800$ 


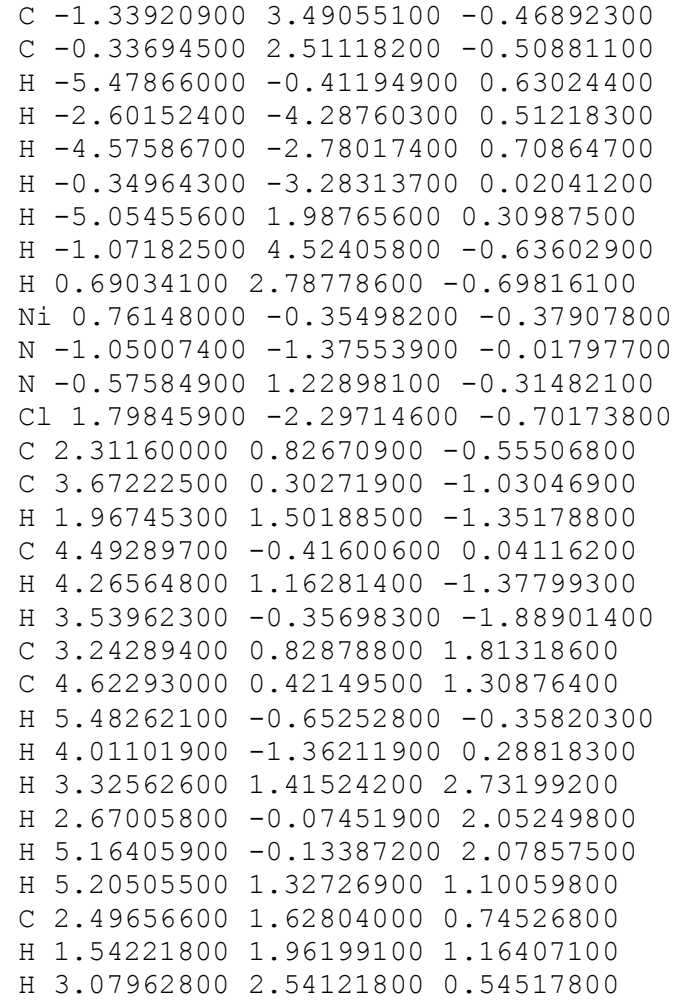

C $-0.64572400 \quad 3.51633700-0.09589000$

$\begin{array}{llll}\text { C } 0.13210800 & 2.34925800 & -0.09589700\end{array}$

$\mathrm{H}-5.60957200 \quad 0.52572400 \quad-0.00858200$

$\mathrm{H}-3.58053300 \quad-3.85697100 \quad 0.13821100$

$\mathrm{H}-5.22117600-1.98344000 \quad 0.07565400$

$\mathrm{H}-1.12107800 \quad-3.32174200 \quad 0.11120000$

$\mathrm{H}-4.66763600 \quad 2.79402600-0.07161400$

$\mathrm{H}-0.15212300 \quad 4.47755900-0.10144000$

$\mathrm{H} \quad 1.21170300 \quad 2.40902800-0.10247000$

$\mathrm{Ni} 0.57993300-0.67231600-0.06868100$

$\mathrm{N}-1.41720900-1.31345700 \quad 0.02332400$

$\mathrm{N}-0.39154000 \quad 1.13948700-0.09023200$

$\begin{array}{lllll}\text { Cl } & 1.30873600 & -2.75616500 & 0.21937900\end{array}$

$\begin{array}{llll}\text { C } 2.33837600 & 0.12706800 & -0.27253600\end{array}$

C $3.44605200-0.69726900-0.92711400$

H $2.17909300 \quad 0.98749600 \quad-0.94321500$

C $4.119800001 .45500200 \quad 0.96640600$

C $4.72900700 \quad 0.12449800-1.08732100$

H $3.11717500-1.07941300-1.89647400$

H $3.65990800-1.57396900-0.31207000$

C $5.21089900 \quad 0.64970300 \quad 0.26364200$

H $4.46357500 \quad 1.78052600 \quad 1.95226900$

H $3.91816400 \quad 2.36351400 \quad 0.38431800$

H $5.51197900-0.47775300-1.55571000$

$\mathrm{H} \quad 4.53956200 \quad 0.97324400 \quad-1.75542900$

$\mathrm{H} \quad 5.48171200 \quad-0.20215500 \quad 0.89681600$

H $6.11297500 \quad 1.25513300 \quad 0.14410400$

C $2.82674500 \quad 0.64060700 \quad 1.08526600$

H $3.00965000 \quad-0.22631100 \quad 1.73064300$

H $2.06341700 \quad 1.23779600 \quad 1.59846600$ 
PALLADIUM
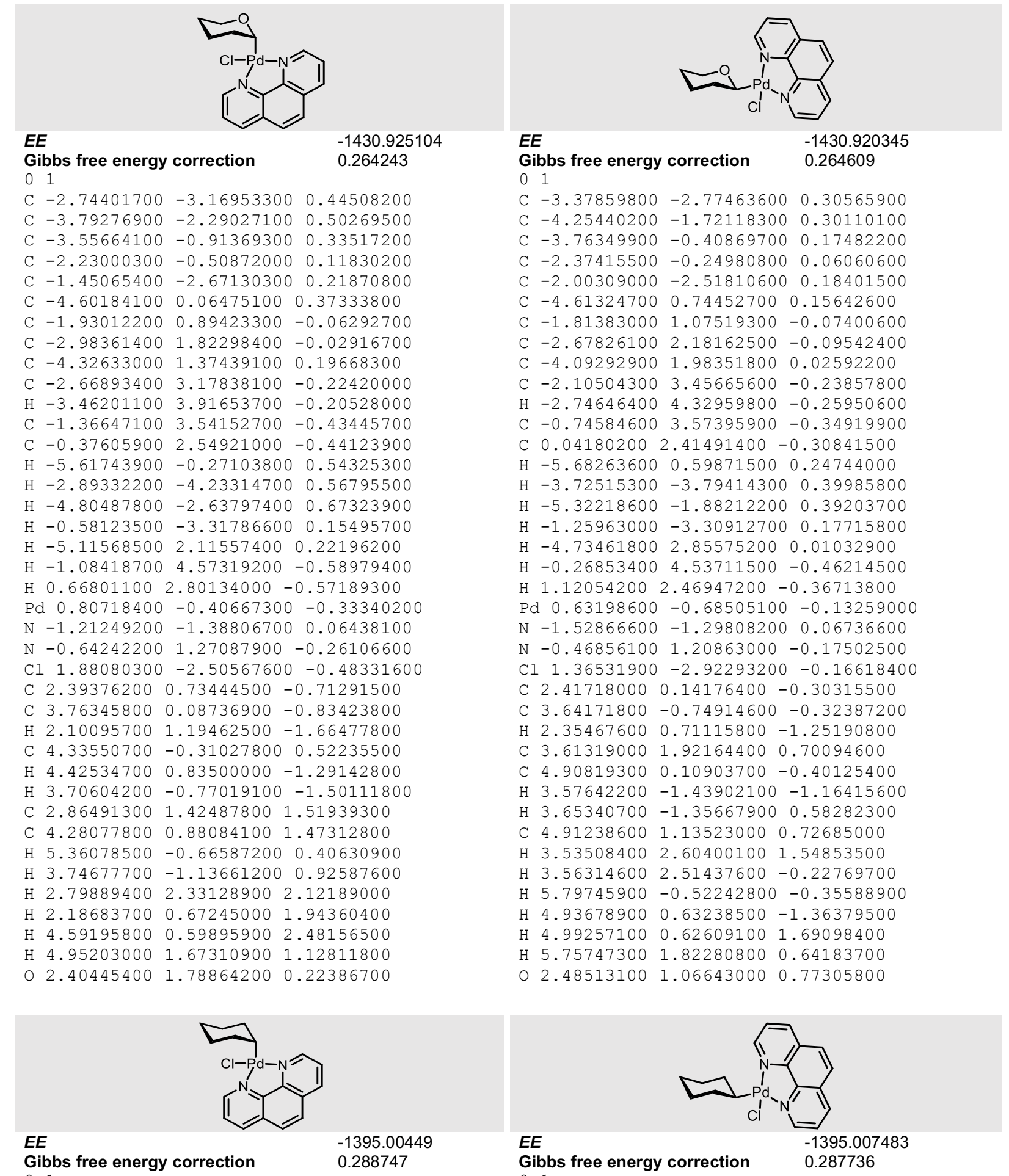

\section{1}

C $-2.70673300-3.21792300 \quad 0.35342500$

C $-3.78216600-2.37342700 \quad 0.43032800$

C $-3.58764100-0.98622600 \quad 0.29861300$

C $-2.27360100-0.53577300 \quad 0.09584700$

C $-1.42807800-2.67507400 \quad 0.14446200$

C $-4.66251300-0.04148000 \quad 0.35808700$

C $-2.01799400 \quad 0.87945300-0.05080700$

C $-3.10008300 \quad 1.77522700 \quad 0.00213900$

C $-4.42851600 \quad 1.28007200 \quad 0.21420400$

C $-2.82790500 \quad 3.14387100-0.16114400$

$\mathrm{H}-3.64289800 \quad 3.85661600-0.12521500$

C $-1.53774900 \quad 3.55275200-0.36642400$

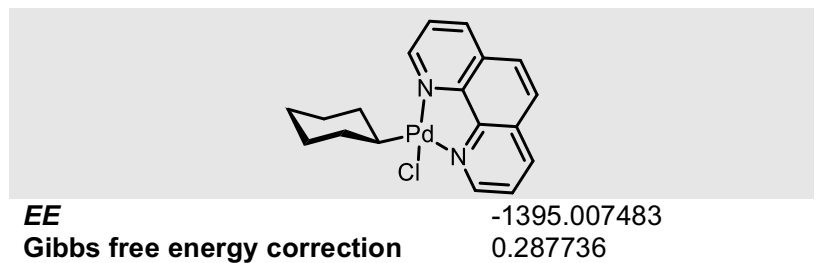

Gibbs
0

C $-3.36347200-2.82571600 \quad 0.16962800$

C $-4.26538300-1.79514600 \quad 0.13805800$

C $-3.80368300-0.46825900 \quad 0.05757200$

C $-2.41490800-0.27186500 \quad 0.00888700$

C $-1.99064800 \quad-2.53123900 \quad 0.12088400$

C $-4.68226000 \quad 0.66270100 \quad 0.03017600$

C $-1.88453300 \quad 1.07042400-0.06991000$

C $-2.77697500 \quad 2.15569400-0.08139800$

C $-4.190033001 .91820100-0.03396300$

C $-2.23408000 \quad 3.45074500-0.13516400$

$\mathrm{H}-2.89834800 \quad 4.30642400-0.14445400$

C $-0.87474500 \quad 3.60923300-0.16962000$ 
$\begin{array}{llll}\text { C } & -0.52124400 & 2.58927200 & -0.39838600\end{array}$ $\mathrm{H}-5.66740000-0.41249500 \quad 0.51731000$ $\mathrm{H}-2.82311900-4.28827900 \quad 0.44862700$ $\mathrm{H}-4.78341600 \quad-2.75571200 \quad 0.58926200$ $\mathrm{H}-0.53800600-3.29166600 \quad 0.06981400$ $\mathrm{H}-5.24049600 \quad 1.99526100 \quad 0.25461200$ $\mathrm{H}-1.28732700 \quad 4.59534000-0.50083800$ $\mathrm{H} \quad 0.50876500 \quad 2.88495400-0.55427100$ $\mathrm{Pd} 0.75866800-0.32949900-0.29220200$ $\mathrm{N}-1.22908900-1.38172000 \quad 0.02309500$ $\mathrm{N}-0.74311000 \quad 1.30102600-0.24193400$ Cl $1.90496000-2.38144400-0.41761600$ C $2.31279100 \quad 0.89888000-0.55409700$ C $3.63236400 \quad 0.35805000-1.10074500$ $\mathrm{H} 1.880013001 .54445700-1.33071100$ C $4.54617400-0.29845100-0.06608500$ H $4.16791400 \quad 1.21819800-1.53048800$ $\mathrm{H} \quad 3.43896200-0.33524500-1.92009500$ C $3.36205700 \quad 0.93002500 \quad 1.76261300$ $\begin{array}{llll}\text { C } 4.72247400 & 0.57267600 & 1.17326800\end{array}$ $\mathrm{H} \quad 5.51557900-0.50357500-0.52657600$ $\begin{array}{lllll}\mathrm{H} & 4.11933600 & -1.25647600 & 0.22903900\end{array}$ $\mathrm{H} \quad 3.47739700 \quad 1.53174300 \quad 2.66714500$ H $2.83921000 \quad 0.00998000 \quad 2.04384000$ $\mathrm{H} 5.33494300 \quad 0.05421500 \quad 1.91398600$ H $5.25214200 \quad 1.49681100 \quad 0.91217600$ C $2.52351400 \quad 1.69048300 \quad 0.73767700$ $\mathrm{H} \quad 1.56955600 \quad 1.98267000 \quad 1.18565000$ H $3.05027200 \quad 2.62513900 \quad 0.49152500$
C $-0.06072100 \quad 2.46865800-0.16224800$ $\mathrm{H}-5.75034000 \quad 0.48761900 \quad 0.06669600$ $\mathrm{H}-3.68678600 \quad-3.85514900 \quad 0.23266100$ $\mathrm{H}-5.33154000-1.98412700 \quad 0.17616400$ $\mathrm{H}-1.22659400 \quad-3.30180500 \quad 0.14703100$ $\mathrm{H}-4.85323900 \quad 2.77399300-0.04929900$ $\mathrm{H}-0.41857000 \quad 4.58825500-0.20452200$ $\mathrm{H} 1.01821400 \quad 2.56384500-0.19074200$ Pd $0.60977000-0.62751300-0.08806400$ $\mathrm{N}-1.54424900-1.29800000 \quad 0.04290500$ $\mathrm{N}-0.53989300 \quad 1.24327700 \quad-0.12154400$ $\begin{array}{lllll}\text { Cl } & 1.44331700 & -2.82011700 & 0.10884300\end{array}$ $\begin{array}{llll}\text { C } 2.38751000 & 0.24817700 & -0.24095300\end{array}$ C $3.52108000-0.55608300-0.85868100$

H $2.16803800 \quad 1.08462100-0.92348100$

C $4.05842400 \quad 1.64936800 \quad 1.02156100$

$\begin{array}{llll}\text { C } 4.77300600 & 0.31888600 & -0.99432800\end{array}$

H $3.22222200-0.95555600-1.82906300$

H $3.74515700-1.41536200-0.22456500$

C $5.19777800 \quad 0.87541700 \quad 0.36271200$

H $4.35661100 \quad 2.00158600 \quad 2.01204000$

$\mathrm{H} 3.83720700 \quad 2.53989800 \quad 0.42060100$

H $5.58617700-0.26043700-1.43747300$

$\mathrm{H} \quad 4.56791100 \quad 1.15144300-1.67733300$

H $5.48313400 \quad 0.04252900 \quad 1.01380800$

H $6.07838200 \quad 1.51368500 \quad 0.25871700$

C $2.79279300 \quad 0.78776600 \quad 1.12519700$

H $2.98531900-0.06030300 \quad 1.79015200$

H $1.98530700 \quad 1.36767700 \quad 1.58371000$ 


\section{PLATINUM}

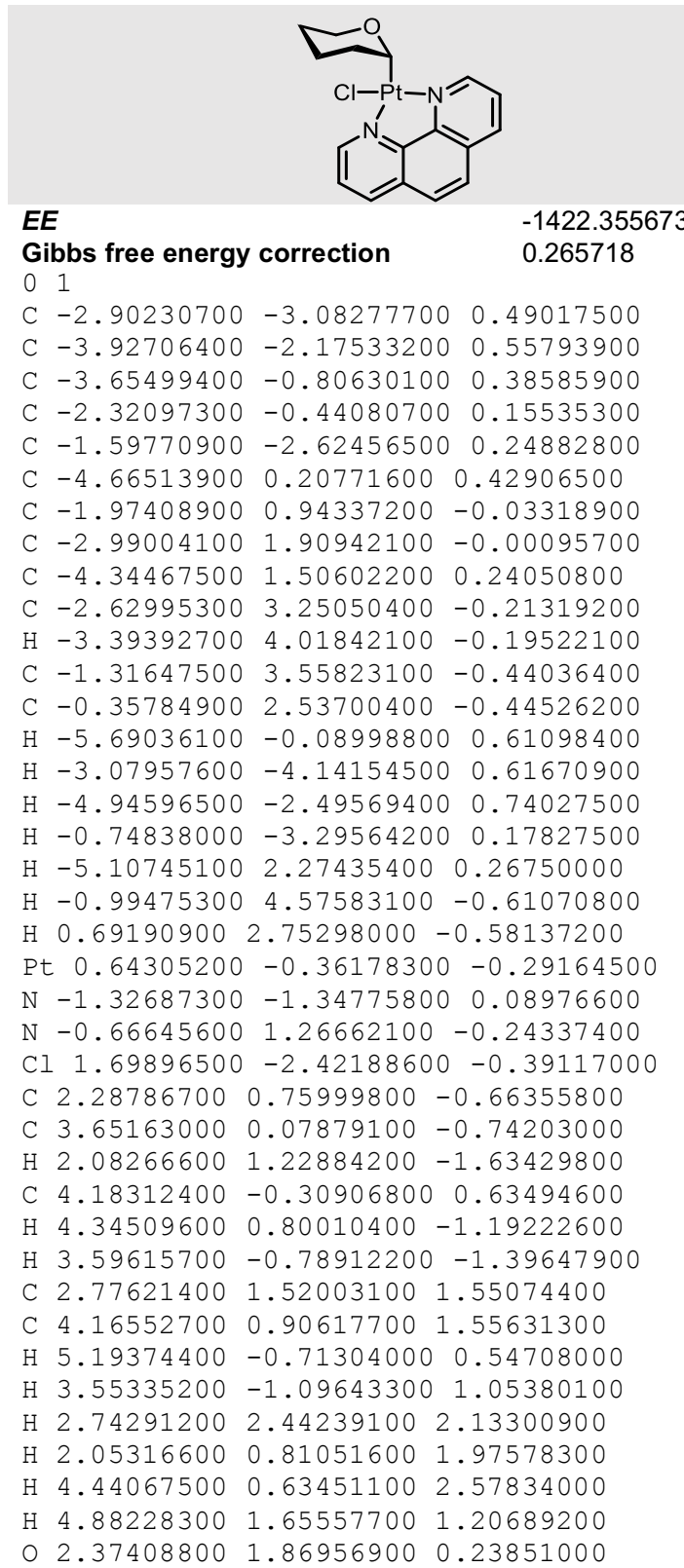

$\begin{array}{llll}0 & 2.37408800 & 1.86956900 & 0.23851000\end{array}$

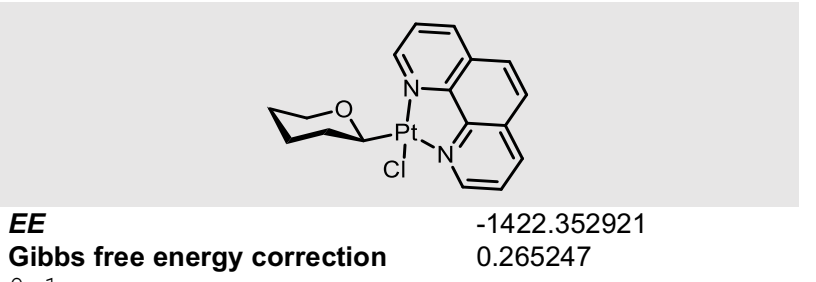

Gibbs free energy correction

0.265247

C $-3.49221600-2.66433500 \quad 0.31071300$

C $-4.35162500-1.59653000 \quad 0.31003500$

$\begin{array}{llll}\text { C }-3.83898400 & -0.29266100 & 0.18455700\end{array}$

C $-2.44882000-0.15936300 \quad 0.06695500$

C $-2.11312700-2.43463400 \quad 0.18485500$

C $-4.66060000 \quad 0.88060900 \quad 0.16986400$

C $-1.85836300 \quad 1.14568300-0.06356600$

C $-2.69028200 \quad 2.27378700-0.08205300$

C $-4.10925500 \quad 2.10681400 \quad 0.04061700$

C $-2.08458600 \quad 3.53355600-0.22201400$

$\mathrm{H}-2.70044300 \quad 4.42442700 \quad-0.24029300$

C $-0.72232800 \quad 3.60905800-0.33251400$

C $0.03941900 \quad 2.43438200-0.29682000$

$\mathrm{H}-5.73310400 \quad 0.76174900 \quad 0.26243800$

$\mathrm{H}-3.85586400-3.67772700 \quad 0.40573800$

$\mathrm{H}-5.42135700-1.74095400 \quad 0.40431200$

$\mathrm{H}-1.38429700 \quad-3.23816300 \quad 0.17787400$

$\mathrm{H}-4.72968000 \quad 2.99425200 \quad 0.02755500$

$\mathrm{H}-0.21653800 \quad 4.55777800-0.44251700$

$\mathrm{H} \quad 1.11711700 \quad 2.46298000 \quad-0.35392300$

Pt $0.49489200-0.58152200-0.11812800$

$\mathrm{N}-1.61891900-1.22177600 \quad 0.06737700$

$\mathrm{N}-0.50287700 \quad 1.23659100-0.16450100$

Cl $1.23413300 \quad-2.77505000 \quad-0.07751000$

$\begin{array}{lllll}\text { C } 2.31963900 & 0.24696500 & -0.26857600\end{array}$

C $3.53466300-0.66778900-0.31611000$

H $2.31573400 \quad 0.84589000-1.20193300$

$\begin{array}{llll}\text { C } 3.59704700 & 1.98964000 & 0.72385100\end{array}$

C $4.82126800 \quad 0.15354600-0.41648300$

H $3.43857800-1.35313600-1.15818000$

H $3.55194100-1.28008500 \quad 0.58765300$

C $4.87818200 \quad 1.17320400 \quad 0.71578300$

H $3.56220600 \quad 2.67369700 \quad 1.57366800$

H $3.54274200 \quad 2.58766700 \quad-0.20248200$

H $5.69563900-0.50008300-0.39421600$

$\mathrm{H} \quad 4.84260800 \quad 0.68237000-1.37645900$

H $4.96674500 \quad 0.65682800 \quad 1.67551200$

H $5.73872500 \quad 1.83945800 \quad 0.61527900$

○ $2.45246900 \quad 1.16779100 \quad 0.82461800$
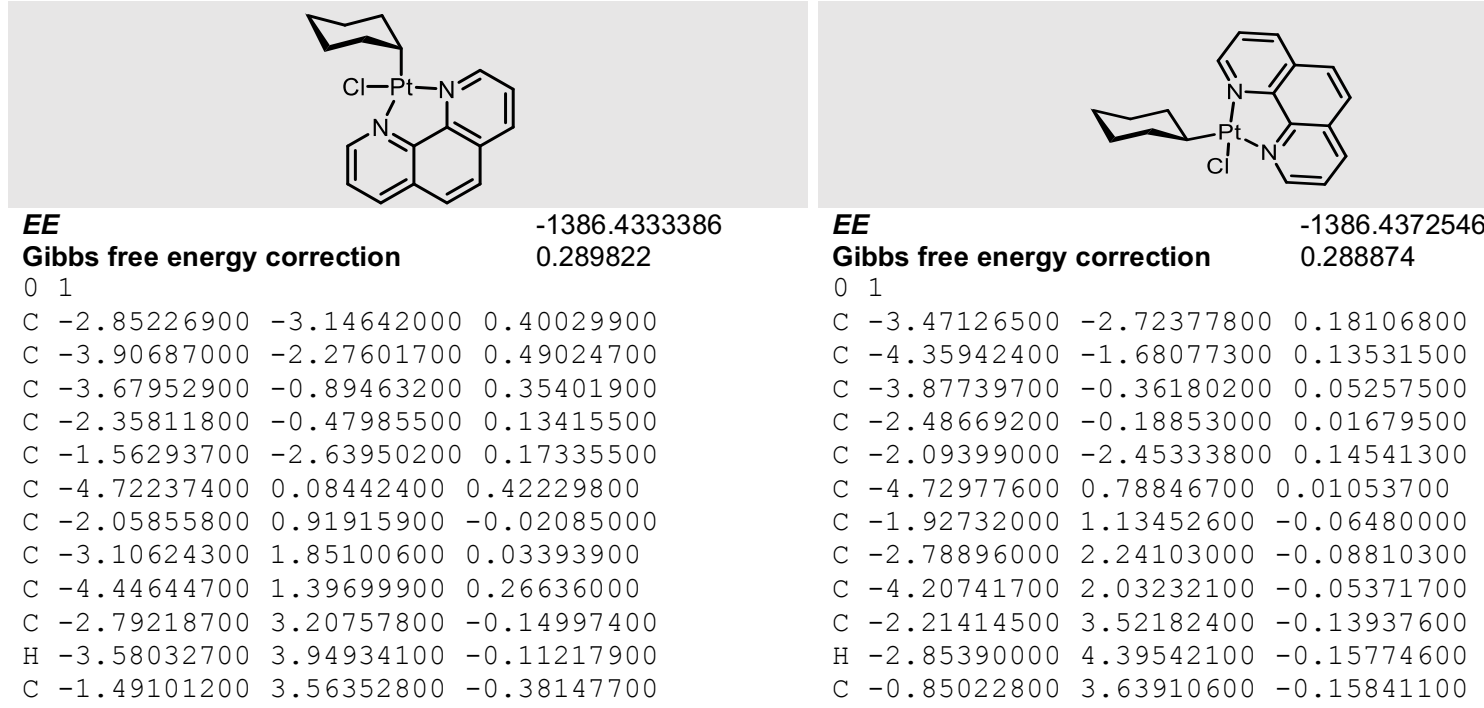


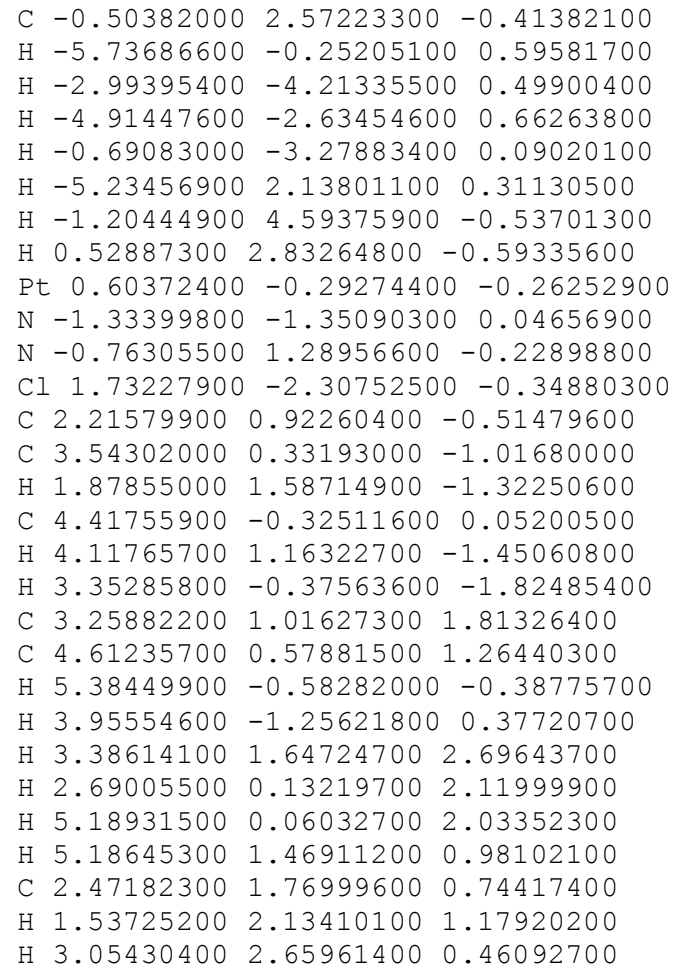

C $-0.06064200 \quad 2.48361100-0.14096200$ $\mathrm{H}-5.80190400 \quad 0.63876900 \quad 0.03671500$ $\mathrm{H}-3.81032700 \quad-3.74799600 \quad 0.24554600$ $\mathrm{H}-5.42816200-1.85544900 \quad 0.16358800$ $\mathrm{H}-1.34352100 \quad-3.23568800 \quad 0.18179400$ $\mathrm{H}-4.85118300 \quad 2.90250100-0.07904800$ $\mathrm{H}-0.36564100 \quad 4.60449700-0.18943100$ $\mathrm{H} 1.01786600 \quad 2.55286000-0.15942800$ Pt $0.47846400-0.52834200-0.05542900$ $\mathrm{N}-1.62860500-1.22624600 \quad 0.06635300$ $\mathrm{N}-0.57081800 \quad 1.26498300 \quad-0.10484600$ $\mathrm{Cl} 1.29731300-2.68404900 \quad 0.10777600$ $\begin{array}{llll}\text { C } 2.30143700 & 0.33618800 & -0.21801900\end{array}$ $\begin{array}{llll}\text { C } 3.42338100 & -0.50647200 & -0.82778900\end{array}$

H $2.15888000 \quad 1.17839400-0.91472400$ C $4.04816700 \quad 1.72162900 \quad 0.98992300$

C $4.69488800 \quad 0.32776500-1.00342900$

H $3.10461300-0.92608300-1.78356200$

H $3.63879200-1.35511800-0.17549300$

C $5.15821700 \quad 0.90596700 \quad 0.33157600$

H $4.37285100 \quad 2.08975900 \quad 1.96658300$

H $3.84117500 \quad 2.60423100 \quad 0.37165000$

H $5.48656600-0.28197900-1.44547700$

H $4.500651001 .14938000-1.70299600$

H $5.43392900 \quad 0.08161200 \quad 0.99788500$

H $6.05288200 \quad 1.51893600 \quad 0.19789400$

C $2.76440200 \quad 0.89758400 \quad 1.13071800$

H $2.94469000 \quad 0.05782600 \quad 1.81006600$

H 1.983732001 .504553001 .60103100 


\section{COPPER}

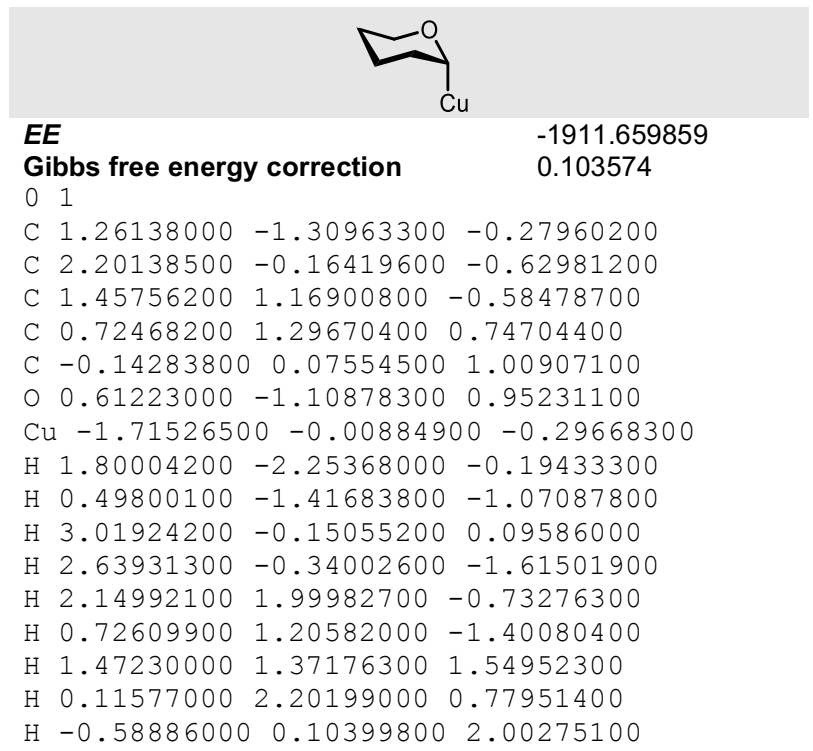

\begin{tabular}{|c|c|c|}
\hline \multicolumn{2}{|r|}{$E E$} & -1911.654874 \\
\hline & $\begin{array}{l}\text { ibbs free energy correction } \\
1\end{array}$ & 0.102243 \\
\hline $\mathrm{C}$ & $-1.79567200-1.29047300$ & 00.18072600 \\
\hline C & $-2.63633700-0.13538700$ & $0-0.34103400$ \\
\hline $\mathrm{C}$ & $-2.08342700 \quad 1.18695800$ & 0.18295200 \\
\hline $\mathrm{C}$ & $-0.58517400 \quad 1.28038000$ & -0.12896800 \\
\hline $\mathrm{C}$ & $0.13962000 \quad 0.03985600 \quad 0$ & 0.37504800 \\
\hline & $-0.43262600-1.13964100$ & $0-0.17260700$ \\
\hline & u $2.10017100-0.00568000$ & $0-0.04820300$ \\
\hline $\mathrm{H}$ & $-2.12088800-2.24297800$ & $0-0.24016900$ \\
\hline $\mathrm{H}$ & $-1.88414400-1.34978500$ & 01.27608000 \\
\hline $\mathrm{H}$ & $-2.59852800-0.14373400$ & $0-1.43423800$ \\
\hline $\mathrm{H}$ & $-3.67917500-0.27396300$ & $0-0.04542800$ \\
\hline $\mathrm{H}$ & $-2.62339000 \quad 2.03297900$ & -0.24911400 \\
\hline $\mathrm{H}$ & $-2.22564800 \quad 1.23265100$ & 1.26801800 \\
\hline $\mathrm{H}$ & $-0.45162400 \quad 1.37173100$ & -1.21085200 \\
\hline $\mathrm{H}$ & $-0.15145200 \quad 2.17212600$ & 0.32961900 \\
\hline & $0.05685600-0.00517200$ & 1.47249700 \\
\hline
\end{tabular}

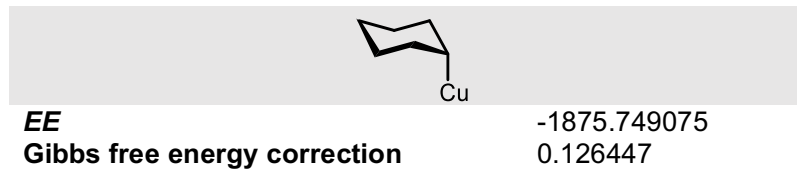

01

0.126447

C $1.39467300 \quad 1.26636700-0.46817400$

C $0.64542000 \quad 1.25108500 \quad 0.86294700$

C $-0.20874600 \quad 0.00000400 \quad 1.02504800$

C $0.64541500-1.25108000 \quad 0.86294900$

C $1.39467500-1.26637200-0.46817000$

C $2.23197300-0.00000100-0.64513900$

$\mathrm{H}-0.71467800 \quad 0.00000500 \quad 1.99258000$

$\mathrm{H} 1.39620100 \quad 1.27339000 \quad 1.66995900$

$\mathrm{H} \quad 0.04801700 \quad 2.15857000 \quad 0.97280600$

H $0.66667100 \quad 1.33668800-1.28597400$

$\mathrm{H} \quad 2.03457300 \quad 2.14951700-0.53488500$

$\mathrm{H} 1.39619800-1.27338700 \quad 1.66996000$

$\mathrm{H} \quad 0.04801000 \quad-2.15856400 \quad 0.97281600$

H $2.03457700-2.14952100-0.53487000$

$\mathrm{H} \quad 0.66667800-1.33669800-1.28597100$

$\mathrm{H} 3.03134000 \quad-0.00000200 \quad 0.10396400$

$\mathrm{H} \quad 2.71896300 \quad 0.00000000-1.62306200$

$\mathrm{Cu}-1.72231100 \quad 0.00000000-0.31496900$

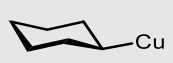

EE $\quad-1875.746877$

Gibbs free energy correction $\quad 0.126674$

01

$\begin{array}{llll}\text { C } 2.01536800 & -1.25694400 & 0.20247600\end{array}$

C $0.52093800-1.25862000-0.14862100$

C $-0.17288100 \quad-0.00000300 \quad 0.36794400$

C $0.52093200 \quad 1.25861700 \quad-0.14861500$

C $2.015366001 .25694800 \quad 0.20247200$

C $2.70154200 \quad 0.00000000-0.32455800$

H $0.42405000-1.31572900-1.23891800$

$\mathrm{H} 0.05084600 \quad-2.15809500 \quad 0.25457400$

H $2.12439800-1.29552700 \quad 1.29186100$

H $2.49694400-2.15280300-0.19866100$

H $0.424041001 .31573700-1.23891100$

H $0.05084300 \quad 2.15809000 \quad 0.25458800$

$\mathrm{H} 2.49693000 \quad 2.15280500-0.19868300$

H $2.12440900 \quad 1.29554300 \quad 1.29185500$

$\mathrm{H} 2.65501400 \quad 0.00000000-1.41970100$

H $3.76024800 \quad 0.00000000-0.05436200$

$\mathrm{H}-0.14208100-0.00000800 \quad 1.46385800$

$\mathrm{Cu}-2.14045600 \quad 0.00000000 \quad-0.03841700$ 


\section{SILVER}

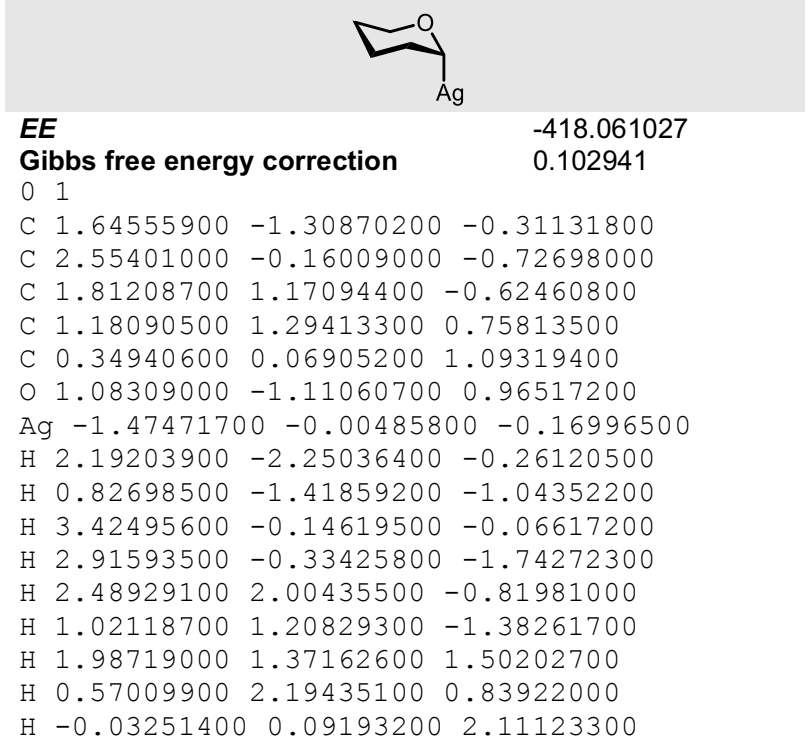

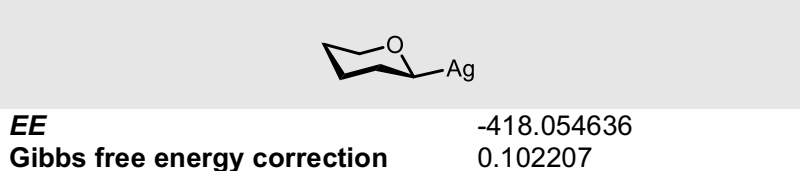

\section{Gibbs free energy correction $\quad 0.102207$}

01

C $-3.11212600-0.13601300-0.35545300$

C $-2.27781600-1.29107100 \quad 0.17598400$

C $-0.35216100 \quad 0.03954300 \quad 0.39546900$

C $-1.06266200 \quad 1.28038400 \quad-0.11940300$

C $-2.56536700 \quad 1.18625800 \quad 0.17514200$

$\mathrm{H}-2.37885000 \quad-1.35229000 \quad 1.26986700$

$\mathrm{H}-2.59426400-2.24362500-0.25078900$

$\mathrm{H}-3.06176600-0.14429700-1.44807100$

$\mathrm{H}-4.15820500-0.27475000-0.07177600$

$\mathrm{H}-0.43419300-0.00750000 \quad 1.49074100$

$\mathrm{H}-0.63305500 \quad 2.17074700 \quad 0.34464600$

$\mathrm{H}-0.91517400 \quad 1.36900000-1.19923700$

$\mathrm{H}-2.72085600 \quad 1.23304300 \quad 1.25824300$

$\mathrm{H}-3.09875600 \quad 2.03256800-0.26417400$

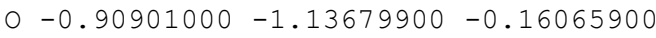

Ag $1.77634000-0.00347000-0.03137500$

\begin{tabular}{|c|c|c|c|}
\hline \multicolumn{3}{|c|}{$\begin{array}{l}\text { EE } \\
\text { Gibbs free energy correction }\end{array}$} & $\begin{array}{l}-382.149474 \\
0.125935\end{array}$ \\
\hline $\mathrm{C}$ & -1.38243300 & 1.19462500 & -1.26708500 \\
\hline $\mathrm{C}$ & -1.38243300 & -0.33258400 & $0-1.25116400$ \\
\hline $\mathrm{C}$ & -0.72918200 & -0.89714200 & 00.00000000 \\
\hline $\mathrm{C}$ & -1.38243300 & -0.33258400 & 01.25116400 \\
\hline $\mathrm{C}$ & -1.38243300 & 1.19462500 & 1.26708500 \\
\hline $\mathrm{C}$ & -2.02290500 & 1.76096500 & 0.00000000 \\
\hline $\mathrm{H}$ & -0.75144400 & -1.98675800 & 0.0 .00000000 \\
\hline $\mathrm{H}$ & -2.43321200 & -0.66654400 & $0-1.27281600$ \\
\hline $\mathrm{H}$ & -0.91509200 & -0.72520900 & $0-2.15606900$ \\
\hline $\mathrm{H}$ & -0.34735200 & 1.54944500 & -1.34113900 \\
\hline $\mathrm{H}$ & -1.91050200 & 1.56433500 & -2.14927800 \\
\hline $\mathrm{H}$ & -2.43321200 & -0.66654400 & 01.27281600 \\
\hline $\mathrm{H}$ & -0.91509200 & -0.72520900 & 02.15606900 \\
\hline $\mathrm{H}$ & -1.91050200 & 1.56433500 & 2.14927800 \\
\hline $\mathrm{H}$ & -0.34735200 & 1.54944500 & 1.34113900 \\
\hline $\mathrm{H}$ & -3.08828600 & 1.50617900 & 0.00000000 \\
\hline & -1.96260800 & 2.85162600 & 0.00000000 \\
\hline & 1.41926800 & -0.454 & 00.00000000 \\
\hline
\end{tabular}

\begin{tabular}{|c|c|c|}
\hline E & & -382.146969 \\
\hline & $\begin{array}{l}\text { ibbs free energy correction } \\
1\end{array}$ & 0.126001 \\
\hline $\mathrm{C}$ & $-0.35380700 \quad 2.48798800$ & -1.25683200 \\
\hline $\mathrm{C}$ & $-0.35380700 \quad 0.95206000$ & -1.25866000 \\
\hline $\mathrm{C}$ & $0.30841400 \quad 0.40444900$ & 0.00000000 \\
\hline $\mathrm{C}$ & $-0.35380700 \quad 0.95206000$ & 1.25866000 \\
\hline $\mathrm{C}$ & $-0.35380700 \quad 2.48798800$ & 1.25683200 \\
\hline $\mathrm{C}$ & $-1.02372900 \quad 3.03570900$ & 0.00000000 \\
\hline $\mathrm{H}$ & $-1.39206600 \quad 0.60677100$ & -1.31362000 \\
\hline $\mathrm{H}$ & $0.14796200 \quad 0.58552100$ & -2.15612100 \\
\hline H & 0.681399002 .84382900 & -1.29678500 \\
\hline H & $-0.85511600 \quad 2.86297000$ & -2.15305400 \\
\hline H & $-1.39206600 \quad 0.60677100$ & 1.31362000 \\
\hline $\mathrm{H}$ & $0.14796200 \quad 0.585521002$ & 2.15612100 \\
\hline & $-0.85511600 \quad 2.86297000$ & 2.15305400 \\
\hline H & 0.681399002 .84382900 & 1.29678500 \\
\hline $\mathrm{H}$ & $-2.07937100 \quad 2.74105200$ & 0.00000000 \\
\hline & $-1.00141500 \quad 4.12804800$ & 0.00000000 \\
\hline & $1.36947400 \quad 0.67114200$ & 0.00000000 \\
\hline & $0.36872800-1.77148800$ & 00.00000000 \\
\hline
\end{tabular}


GOLD

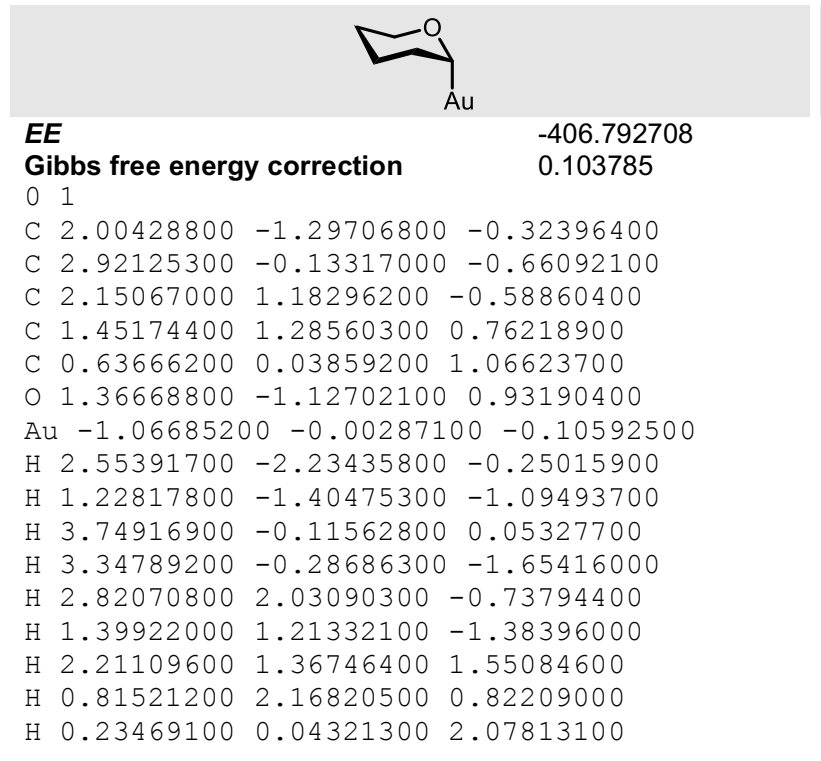

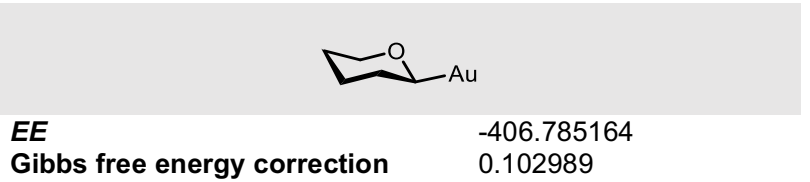

Gibbs free energy correction $\quad 0.102989$

01

C $-3.47887700-0.11444700-0.33704000$

C $-2.65391400-1.28331000 \quad 0.17627600$

C $-0.72008100 \quad 0.01637400 \quad 0.37825300$

C $-1.401739001 .27296900-0.13537800$

C $-2.89962100 \quad 1.19586900 \quad 0.18770200$

$\mathrm{H}-2.73850900-1.35277100 \quad 1.27034300$

$\mathrm{H}-2.97984300-2.22992000-0.25363500$

$\mathrm{H}-3.45103500-0.12034300-1.42993500$

$\mathrm{H}-4.52022200-0.23630200-0.03069000$

$\mathrm{H}-0.78541600-0.03394900 \quad 1.47427500$

$\mathrm{H}-0.94848500 \quad 2.15432700 \quad 0.32013300$

$\mathrm{H}-1.26293400 \quad 1.34392000-1.21621400$

$\mathrm{H}-3.03579100 \quad 1.24361300 \quad 1.27320600$

$\mathrm{H}-3.42228500 \quad 2.05383700-0.23919600$

$0-1.28589800-1.14025200-0.18070500$

Au $1.27034300-0.00285000-0.01698100$

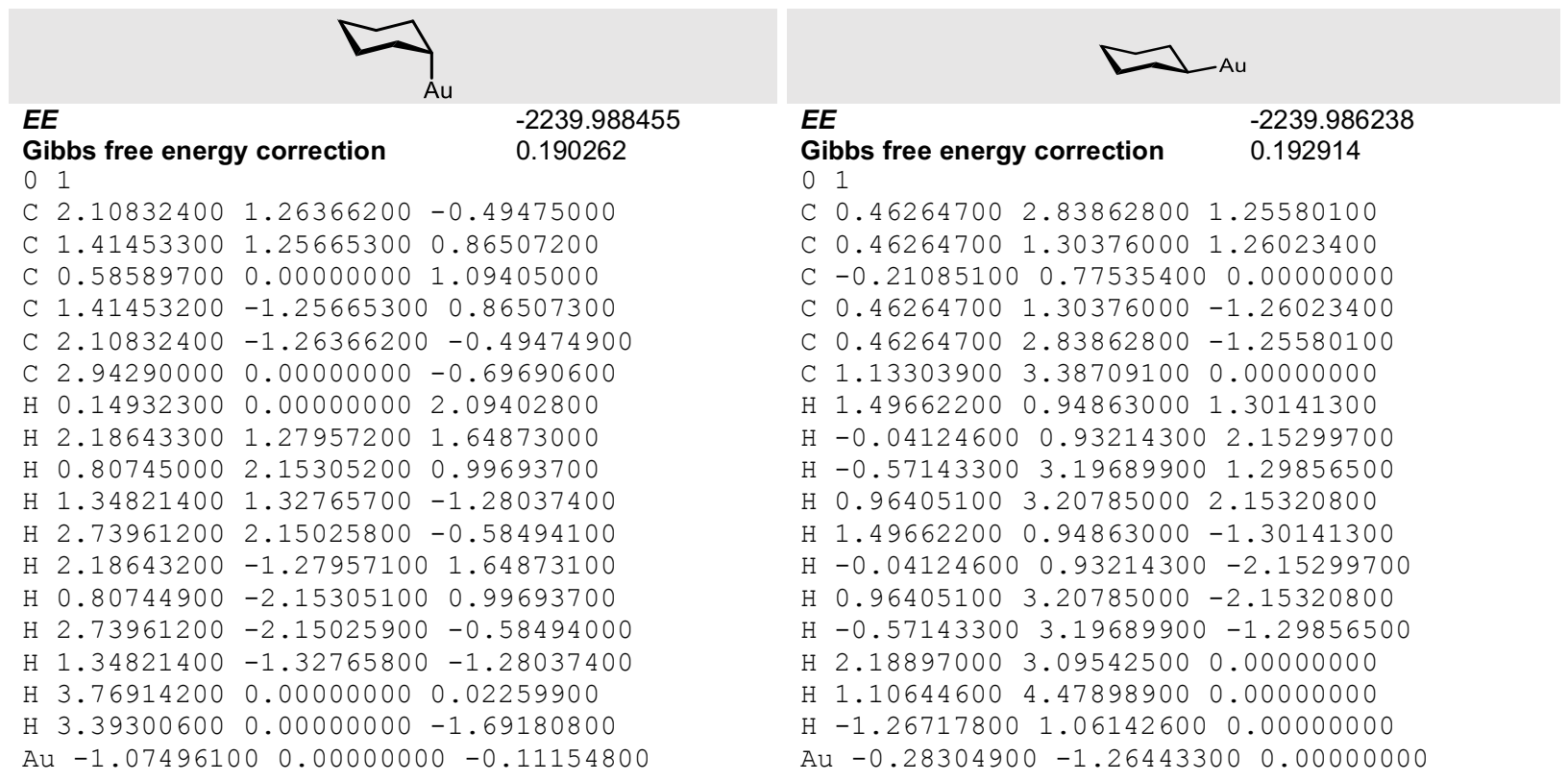




\subsection{Group 12}

\section{ZINC}

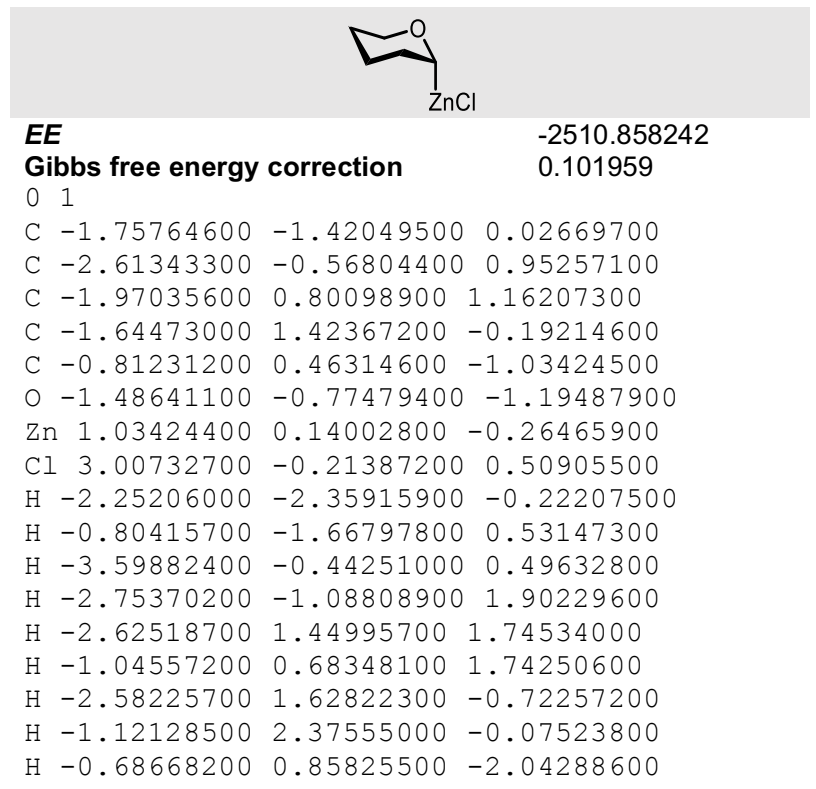
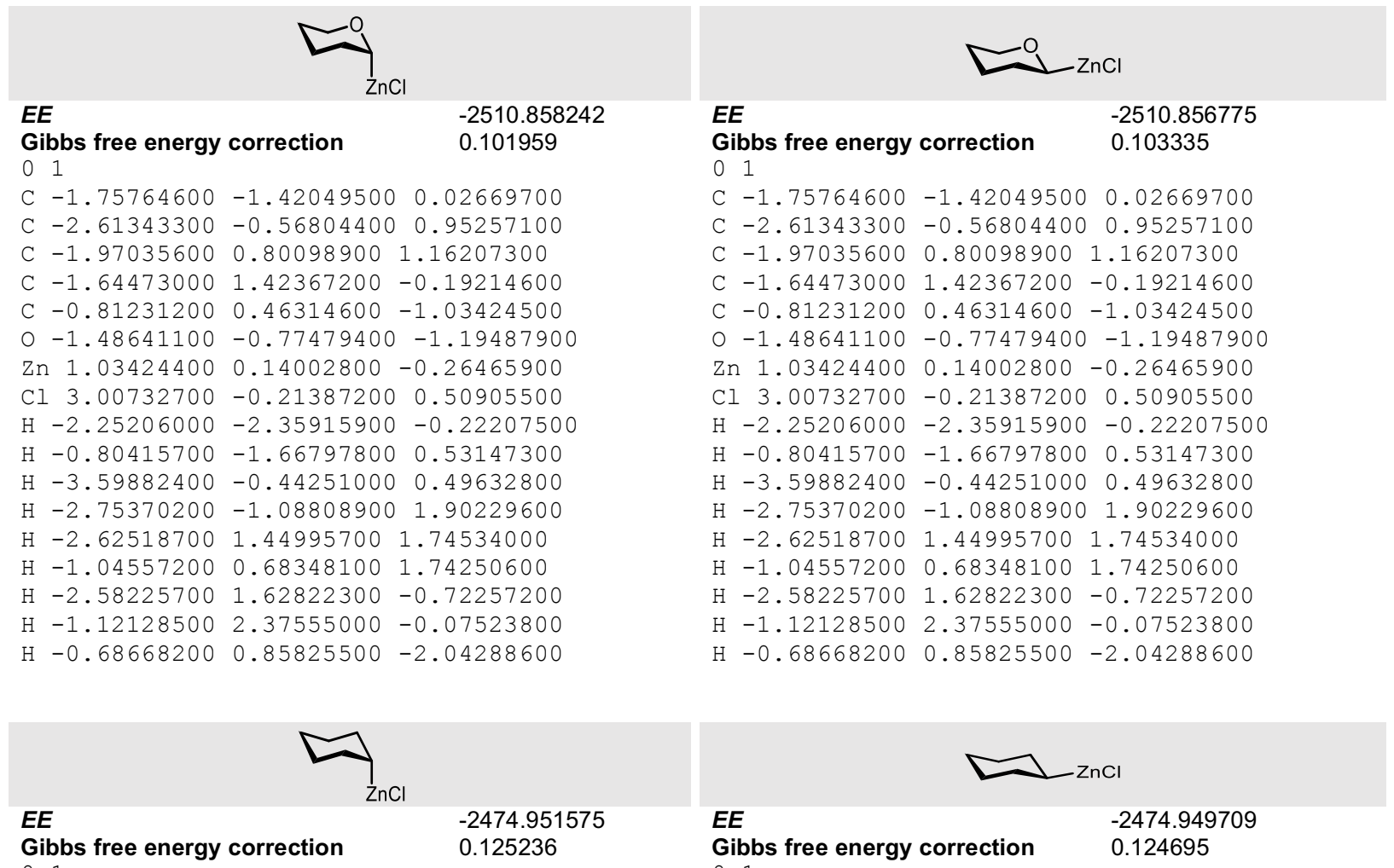

01

C $1.67399700 \quad 1.07436600 \quad 1.26603300$

C $1.67399700-0.45324000 \quad 1.25221800$

C $0.98519500-1.00588600 \quad 0.00000000$

$\begin{array}{llll}\text { C } 1.67399700 & -0.45324000 & -1.25221800\end{array}$

C $1.673997001 .07436600-1.26603300$

C $2.317014001 .63873900 \quad 0.00000000$

$\mathrm{H} 2.71810900 \quad-0.79558000 \quad 1.26053500$

$\begin{array}{lllll}\mathrm{H} & 1.21486400 & -0.84096600 & 2.16453400\end{array}$

$\mathrm{H} 0.64050800 \quad 1.44055800 \quad 1.34085300$

$\begin{array}{llll}\mathrm{H} & 2.19533200 & 1.44715800 & 2.15002700\end{array}$

$\mathrm{H} 2.71810900-0.79558000-1.26053500$

$\mathrm{H} \quad 1.21486400-0.84096600-2.16453400$

$\mathrm{H} \quad 2.19533200 \quad 1.44715800-2.15002700$

$\mathrm{H} 0.64050800 \quad 1.44055800-1.34085300$

$\mathrm{H} \quad 3.38027500 \quad 1.37678700 \quad 0.00000000$

$\mathrm{H} 2.26153800 \quad 2.72906100 \quad 0.00000000$

$\mathrm{H} \quad 1.04365000 \quad-2.09690100 \quad 0.00000000$

$\mathrm{Zn}-0.95337700-0.52543300 \quad 0.00000000$

$\mathrm{Cl}-3.03594000 \quad 0.00006400 \quad 0.00000000$

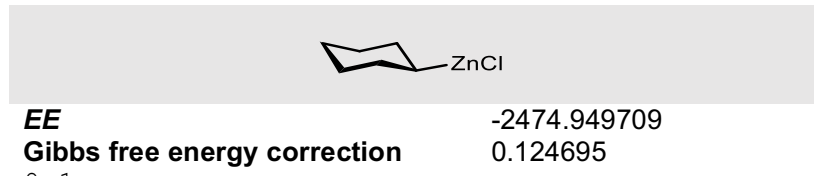

01

C $-0.37714000-2.78510300 \quad 1.25822500$

C $-0.37714000-1.25482700 \quad 1.26180200$

C $0.29290800 \quad-0.69148100 \quad 0.00000000$

C $-0.37714000-1.25482700-1.26180200$

C $-0.37714000-2.78510300-1.25822500$

C $-1.04422900-3.33385200 \quad 0.00000000$

$\mathrm{H}-1.41551200-0.90701000 \quad 1.31502800$

H $0.11902600 \quad-0.88973700 \quad 2.16434500$

$\mathrm{H} \quad 0.65836400 \quad-3.13988300 \quad 1.30062700$

$\mathrm{H}-0.87930900-3.16291200 \quad 2.15196400$

$\mathrm{H}-1.41551200-0.90701000-1.31502800$

H $0.11902600 \quad-0.88973700 \quad-2.16434500$

$\mathrm{H}-0.87930900-3.16291200-2.15196400$

$\mathrm{H} \quad 0.65836400 \quad-3.13988300-1.30062700$

$\mathrm{H}-2.10117700-3.04465400 \quad 0.00000000$

$\mathrm{H}-1.01668500 \quad-4.42541500 \quad 0.00000000$

$\mathrm{H} \quad 1.33927500-1.02165600 \quad 0.00000000$

Zn $0.36291500 \quad 1.29514800 \quad 0.00000000$

$\mathrm{Cl} 0.44031100 \quad 3.43926600 \quad 0.00000000$ 
CADMIUM

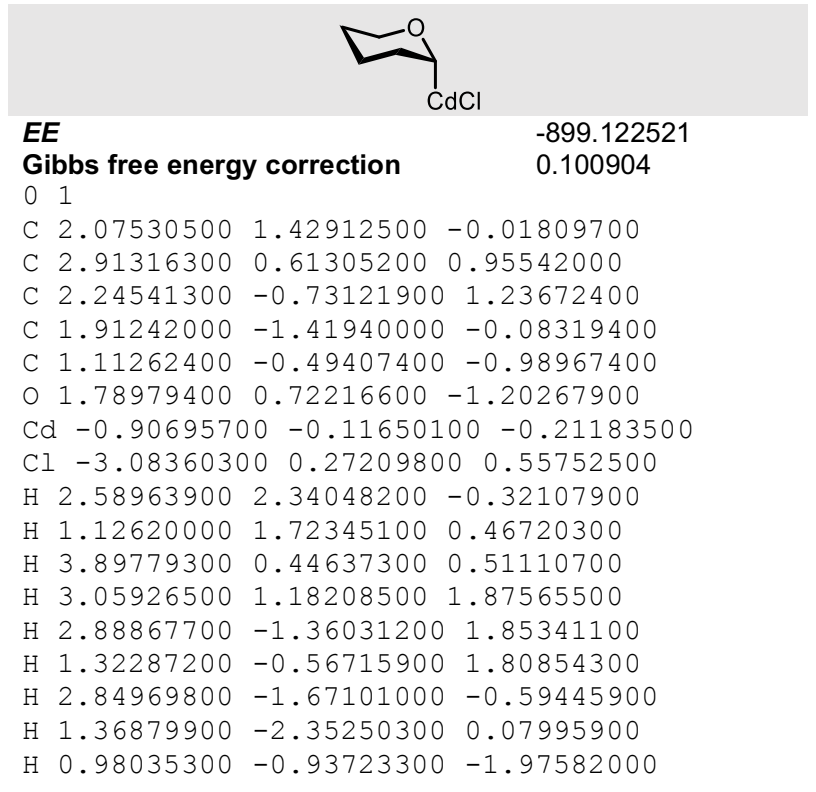

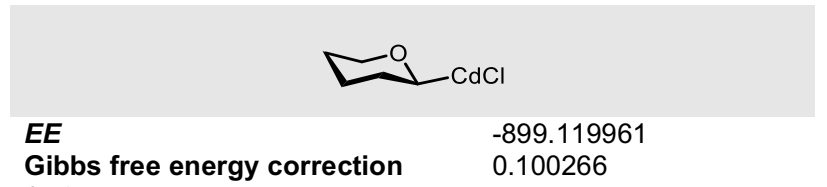

free energy correction $\quad 0.100266$

C $-2.86356200-1.32098000 \quad 0.10994900$

C $-3.68857700-0.19958600-0.50012300$

C $-3.22688800 \quad 1.14694800 \quad 0.05151900$

C $-1.71476100 \quad 1.29234200-0.13554200$

C $-0.99762000 \quad 0.08234200 \quad 0.45173000$

o $-1.47662400-1.12675500-0.12074000$

Cd $1.13000200 \quad 0.02874600 \quad 0.11420500$

Cl $3.42486900-0.05447500-0.27814000$

$\mathrm{H}-3.11224800-2.28815700-0.32687000$

$\mathrm{H}-3.04389000-1.37336400 \quad 1.19352500$

$\mathrm{H}-3.55735400-0.21938400-1.58563100$

$\mathrm{H}-4.74748700-0.36812700-0.29295000$

$\mathrm{H}-3.75574000 \quad 1.96837300-0.43575300$

$\mathrm{H}-3.46210500 \quad 1.20034500 \quad 1.11980900$

$\mathrm{H}-1.49317600 \quad 1.37161500-1.20394000$

$\mathrm{H}-1.35187500 \quad 2.20563800 \quad 0.34237200$

$\mathrm{H}-1.17755000 \quad 0.05699400 \quad 1.53668600$

\begin{tabular}{|c|c|c|}
\hline EE & -863.215072 & -863.213012 \\
\hline $\begin{array}{l}\text { Gibbs free energy } \\
01\end{array}$ & correction & $\begin{array}{l}\text { Gibbs free energy correction } \\
01\end{array}$ \\
\hline C -2.15419100 & $1.26250800 \quad 0.76442800$ & C $3.14356600 \quad 1.25746100-0.11940400$ \\
\hline C -1.83986200 & $1.25626600-0.73044100$ & C $1.63527800 \quad 1.26164600 \quad 0.14648200$ \\
\hline$C-1.06494100$ & $0.00564900-1.14637600$ & C $0.97042600 \quad 0.00036700-0.41542300$ \\
\hline$C-1.83947200$ & $-1.24925500-0.74263600$ & C $1.63468900-1.26136700 \quad 0.14629600$ \\
\hline$C-2.15324200$ & $-1.27008100 \quad 0.75214800$ & C $3.14299300-1.25791700-0.11954300$ \\
\hline$C-2.89913300$ & $-0.00611700 \quad 1.17825000$ & C $3.79918900-0.00040800 \quad 0.44410900$ \\
\hline $\mathrm{H}-0.89938100$ & $0.01099000-2.22493700$ & $\mathrm{H} 1.47367200 \quad 1.31488000 \quad 1.22918900$ \\
\hline $\mathrm{H}-2.79271400$ & $1.27148700-1.27824900$ & $\mathrm{H} 1.18947100 \quad 2.16271100-0.28077200$ \\
\hline $\mathrm{H}-1.30832500$ & $2.16778400-1.01211400$ & $\mathrm{H} \quad 3.31371500 \quad 1.29920300-1.20051200$ \\
\hline $\mathrm{H}-1.21875200$ & $1.33594700 \quad 1.33527600$ & $\mathrm{H} \quad 3.60112800 \quad 2.15188600 \quad 0.30946300$ \\
\hline $\mathrm{H}-2.74244700$ & 2.144537001 .02437900 & $\mathrm{H} 1.47304800-1.31467400 \quad 1.22900000$ \\
\hline $\mathrm{H}-2.79252100$ & $-1.25934300-1.29022400$ & $\mathrm{H} 1.18845900-2.16217600-0.28106800$ \\
\hline $\mathrm{H}-1.30773500$ & $-2.15773900-1.03361100$ & $\mathrm{H} \quad 3.60012300-2.15260800 \quad 0.30923200$ \\
\hline $\mathrm{H}-2.74062200$ & -2.155151001 .00364800 & $\mathrm{H} 3.31313800-1.29962000-1.20065200$ \\
\hline $\mathrm{H}-1.21750000$ & $-1.34808500 \quad 1.32184200$ & $\mathrm{H} \quad 3.69573400-0.00045000 \quad 1.53485100$ \\
\hline $\mathrm{H}-3.88578500$ & $-0.00420700 \quad 0.70359500$ & $\mathrm{H} 4.86958600-0.00063700 \quad 0.22930800$ \\
\hline $\mathrm{H}-3.06886500$ & $-0.01142900 \quad 2.25648300$ & $\mathrm{H} 1.09621500 \quad 0.00042200-1.50348600$ \\
\hline Cl 3.05768900 & $-0.00334900 \quad 0.66457800$ & $\mathrm{Cl}-3.47206100-0.00021400 \quad 0.24048500$ \\
\hline $\mathrm{Cd} 0.91039600$ & $0.00142300-0.26158600$ & $\mathrm{Cd}-1.16137700 \quad 0.00012500-0.10329000$ \\
\hline
\end{tabular}


MERCURY

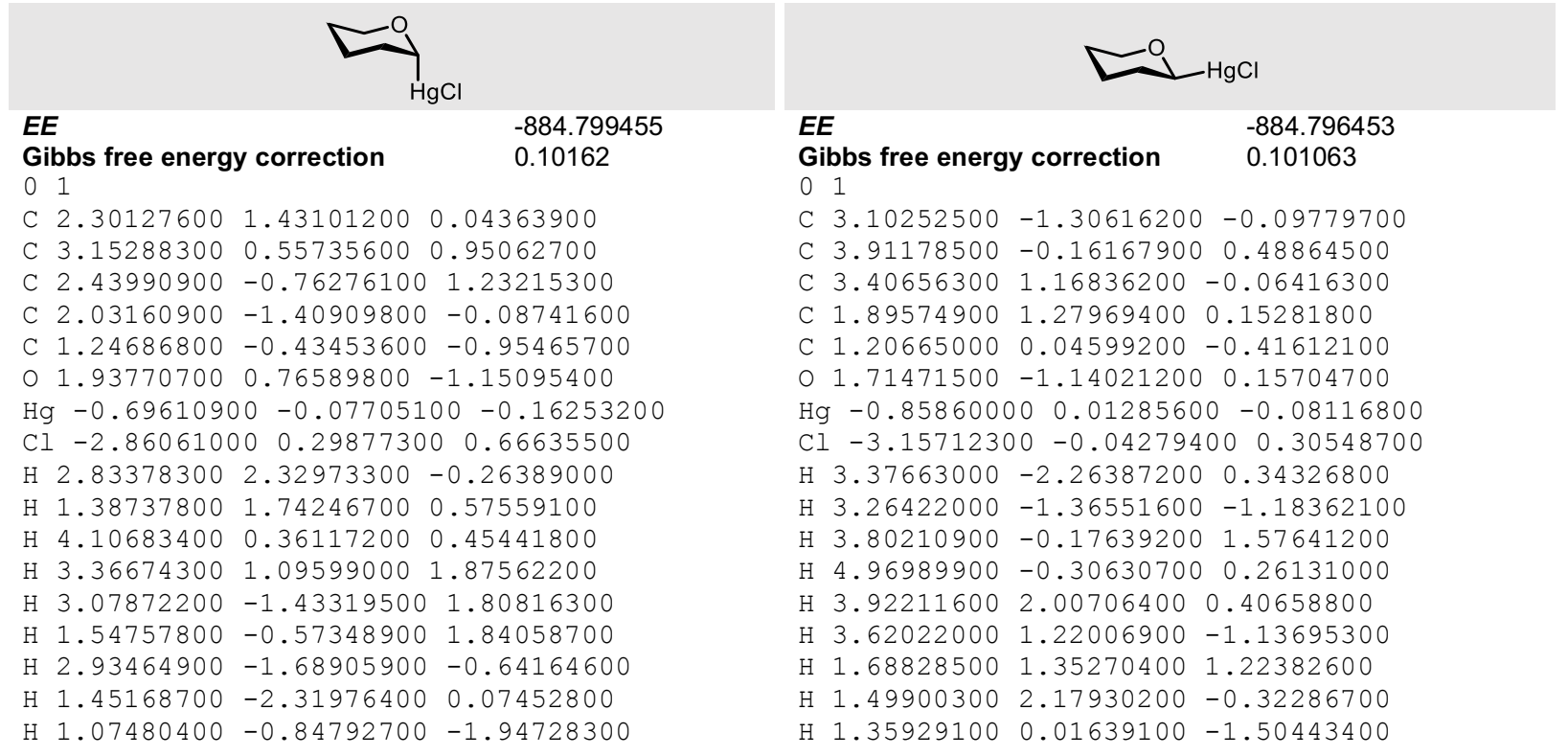

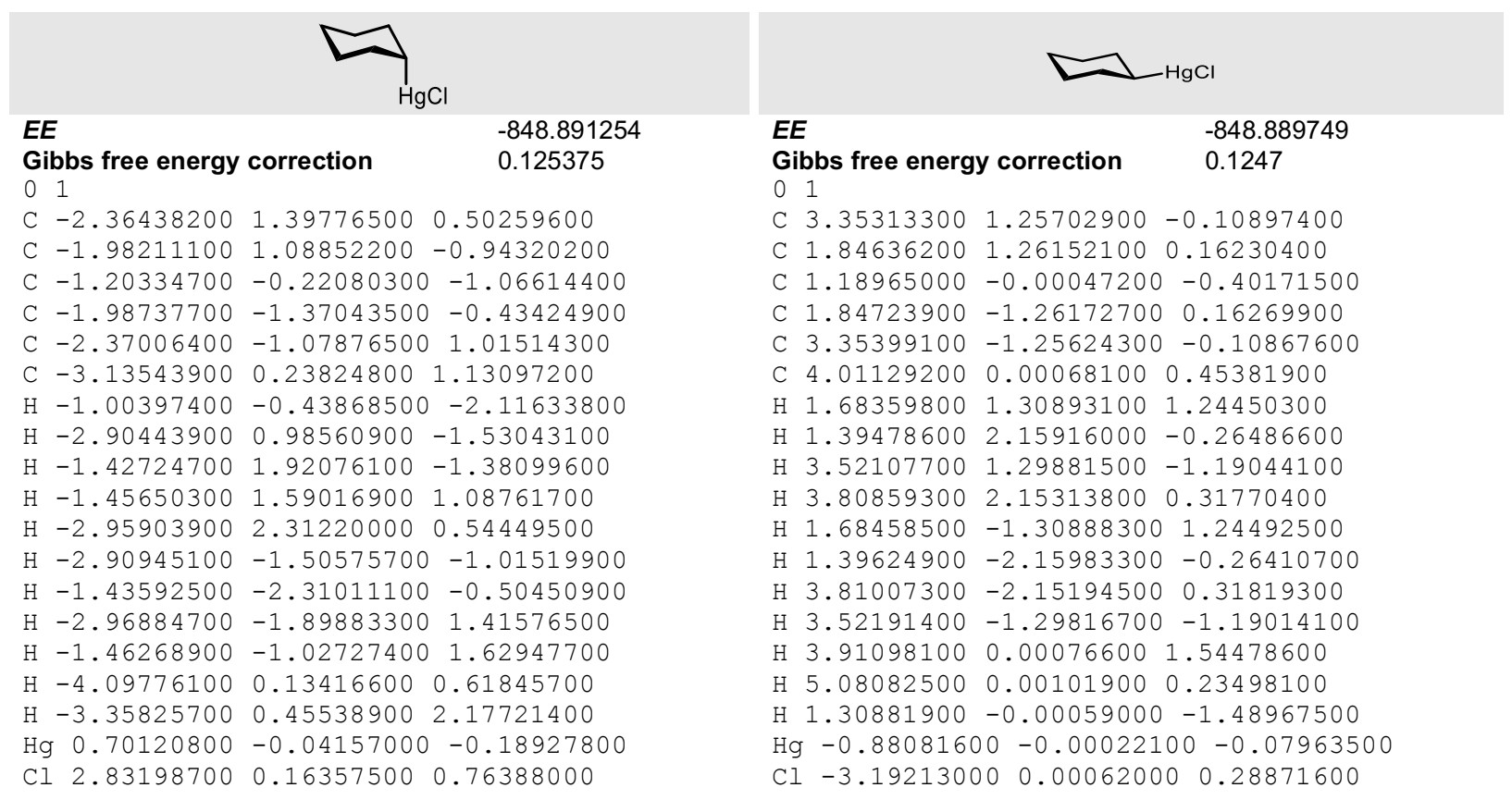




\section{BORON}
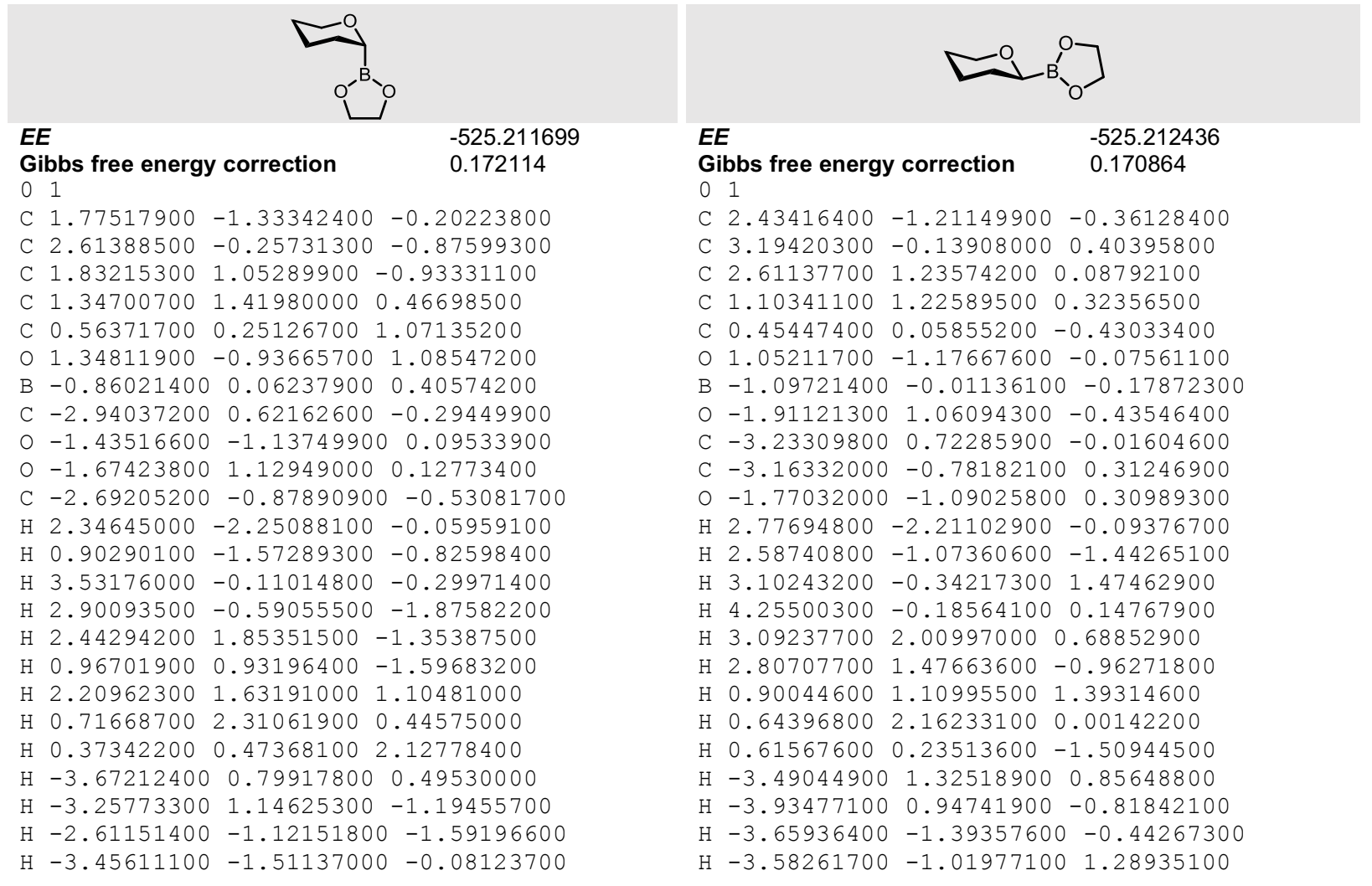

\section{Gibbs free energy correction $\quad 0.170864$}

01

C $2.43416400-1.21149900-0.36128400$

$\begin{array}{llll}\text { C } 3.19420300 & -0.13908000 & 0.40395800\end{array}$

C $2.61137700 \quad 1.23574200 \quad 0.08792100$

C $1.10341100 \quad 1.22589500 \quad 0.32356500$

C $0.45447400 \quad 0.05855200-0.43033400$

$\begin{array}{llll}0 & 1.05211700 & -1.17667600 & -0.07561100\end{array}$

B $-1.09721400-0.01136100-0.17872300$

$\begin{array}{llll}\mathrm{O} & -1.91121300 & 1.06094300 & -0.43546400\end{array}$

C $-3.23309800 \quad 0.72285900-0.01604600$

$\begin{array}{llll}\text { C }-3.16332000 & -0.78182100 & 0.31246900\end{array}$

$\begin{array}{lllll}0 & -1.77032000 & -1.09025800 & 0.30989300\end{array}$

H $2.77694800-2.21102900-0.09376700$

H $2.58740800-1.07360600-1.44265100$

H $3.10243200-0.34217300 \quad 1.47462900$

$\mathrm{H} \quad 4.25500300 \quad-0.18564100 \quad 0.14767900$

$\mathrm{H} \quad 3.09237700 \quad 2.00997000 \quad 0.68852900$

H $2.80707700 \quad 1.47663600-0.96271800$

$\mathrm{H} 0.90044600 \quad 1.10995500 \quad 1.39314600$

$\mathrm{H} \quad 0.64396800 \quad 2.16233100 \quad 0.00142200$

$\mathrm{H} \quad 0.61567600 \quad 0.23513600 \quad-1.50944500$

$\mathrm{H}-3.49044900 \quad 1.32518900 \quad 0.85648800$

$\mathrm{H}-3.93477100 \quad 0.94741900-0.81842100$

$\mathrm{H}-3.65936400-1.39357600-0.44267300$

$\mathrm{H}-3.58261700-1.01977100 \quad 1.28935100$

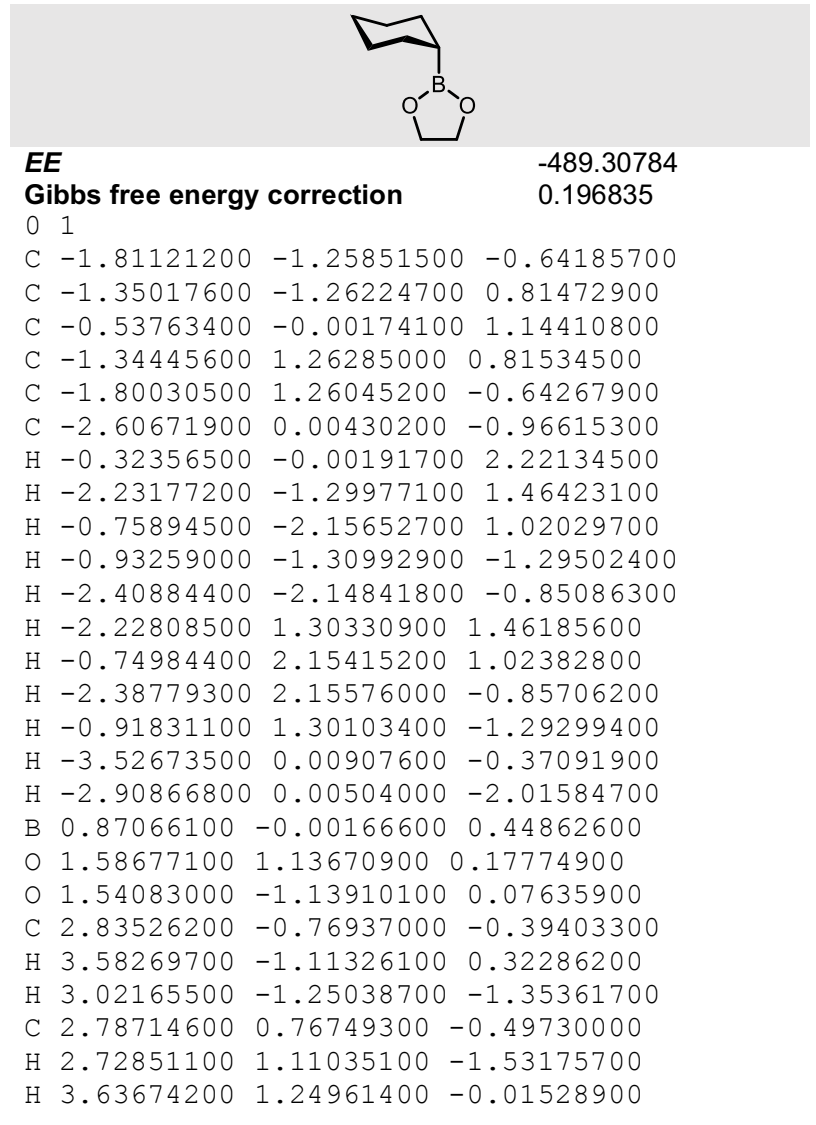

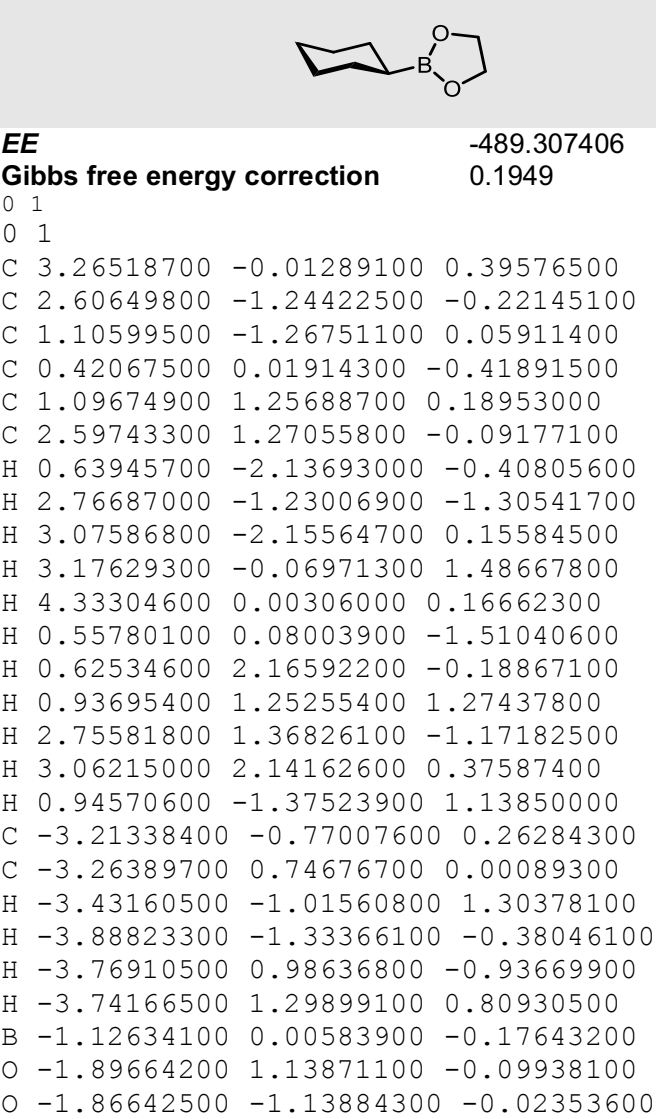


ALUMINUM

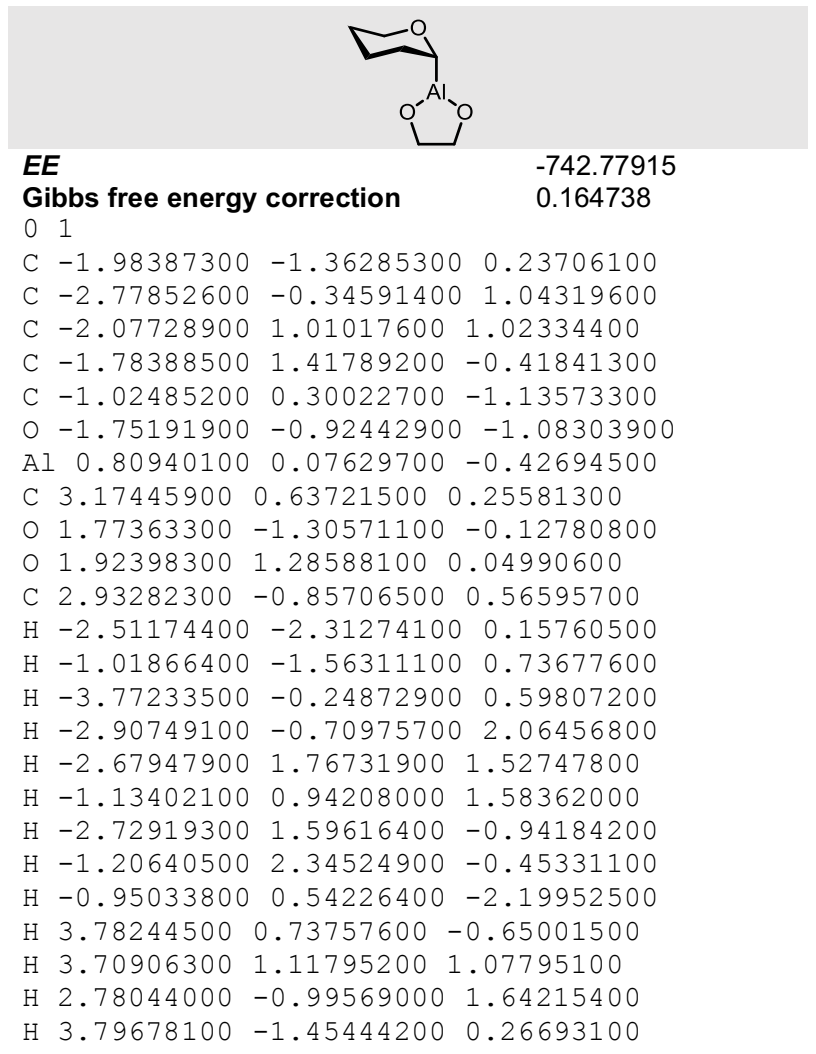
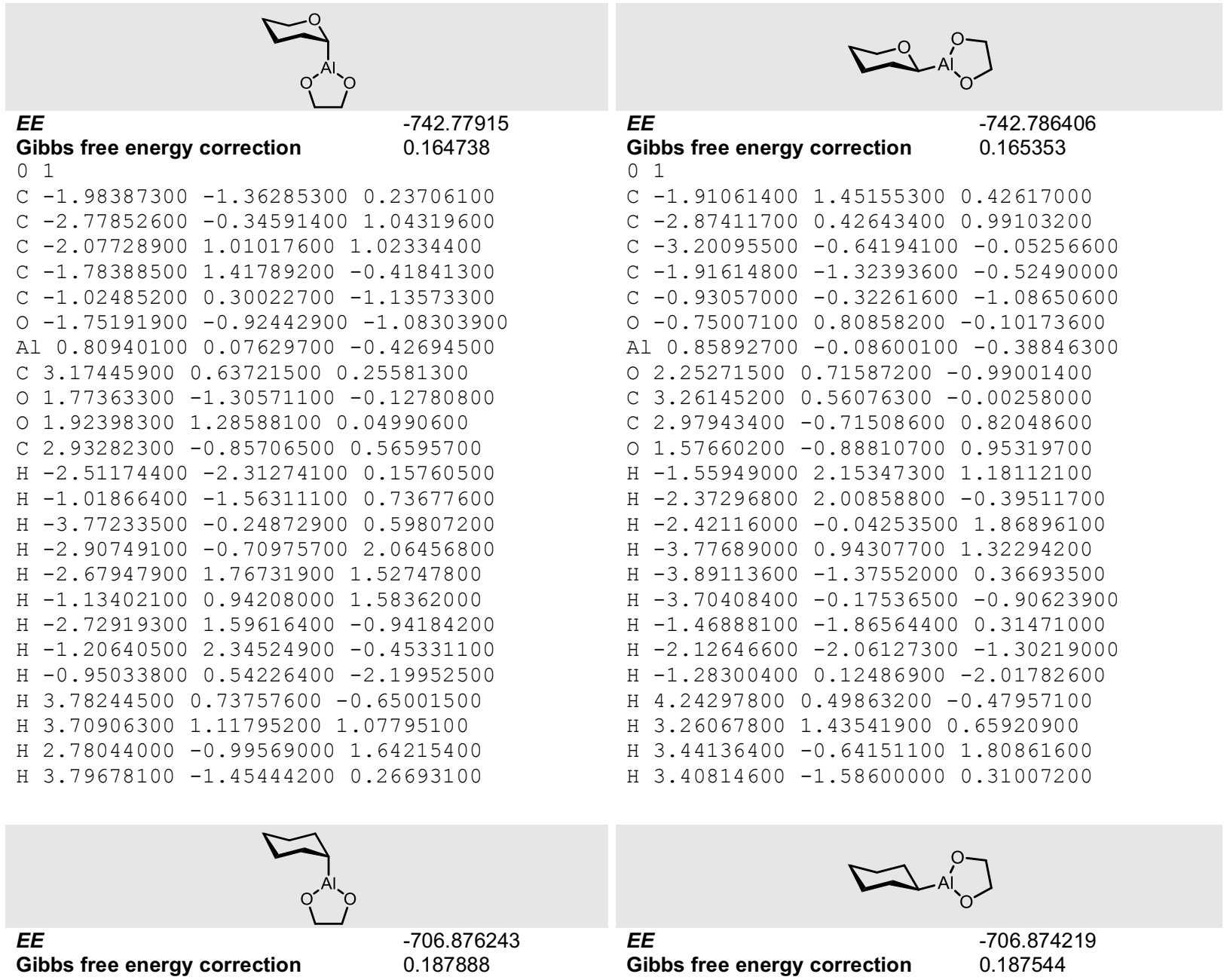

0

$\begin{array}{llll}\text { C } 2.01051700 & -1.25552600 & 0.73272800\end{array}$

C $1.75457700-1.27317100-0.77366500$

C $0.98837000-0.01823400-1.22396300$

C $1.75669700 \quad 1.24787700-0.81019800$

C $2.011526001 .27258500 \quad 0.69609600$

C $2.74625600 \quad 0.01462000 \quad 1.15592400$

$\mathrm{H} 0.88705100-0.03350500-2.31425900$

$\mathrm{H} 2.72086400-1.30768600-1.29223000$

$\mathrm{H} 1.21598500-2.18236900-1.05052500$

$\mathrm{H} 1.05181800-1.31822800 \quad 1.26625700$

$\begin{array}{llll}\mathrm{H} & 2.57937900 & -2.13851600 & 1.02978700\end{array}$

H $2.72372700 \quad 1.26548700-1.32818400$

$\mathrm{H} \quad 1.22015500 \quad 2.14961200-1.11414200$

H $2.57910500 \quad 2.16395700 \quad 0.96967100$

$\mathrm{H} \quad 1.05202200 \quad 1.34954100 \quad 1.22740200$

H $3.74625500 \quad 0.00845100 \quad 0.70952100$

$\begin{array}{llll}\mathrm{H} & 2.88436200 & 0.03025800 & 2.23870000\end{array}$

Al $-0.80688500-0.00514800-0.47089700$

$0-1.87561500 \quad 1.29644900-0.15254500$

$\begin{array}{llll}0 & -1.80327200 & -1.29457600 & 0.06127900\end{array}$

$\begin{array}{llll}C & -3.09521600 & -0.74876700 & 0.30018300\end{array}$

$\mathrm{H}-3.72155700 \quad-0.91083200-0.58390300$

$\mathrm{H}-3.56293300-1.25981200 \quad 1.14455300$

C $-2.96860300 \quad 0.76441900 \quad 0.58648800$

$\mathrm{H}-2.79104000 \quad 0.92862000 \quad 1.65508800$

$\mathrm{H}-3.88934000 \quad 1.28414100 \quad 0.31249500$

\begin{tabular}{|c|c|c|c|}
\hline \multicolumn{3}{|c|}{$E E$} & -706.874219 \\
\hline \multicolumn{3}{|c|}{ Gibbs free energy correction } & 0.187544 \\
\hline $\mathrm{C}$ & 3.64735800 & -0.00904500 & 0.41919800 \\
\hline C & 2.99710700 & -1.24829100 & -0.19162300 \\
\hline $\mathrm{C}$ & 1.49173800 & -1.27129100 & 0.07357500 \\
\hline $\mathrm{C}$ & 0.813244000 & $0.01348600-$ & -0.43516100 \\
\hline $\mathrm{C}$ & 1.480990001 & 1.25859900 & 0.17748000 \\
\hline C & $2.98631100 \quad 1$ & $1.27035500-$ & -0.08858200 \\
\hline $\mathrm{H}$ & $1.04205600-$ & -2.15481000 & -0.38537600 \\
\hline & 3.16618600 & -1.24736400 & -1.27387600 \\
\hline & 3.46248700 & -2.15461500 & 0.20208900 \\
\hline & 3.54786400 & -0.05403400 & 1.50944500 \\
\hline $\mathrm{H}$ & 4.717094000 & 0.004493000 & 0.20135500 \\
\hline $\mathrm{H}$ & 0.971666000 & 0.05969200 & -1.52250600 \\
\hline $\mathrm{H}$ & 1.023382002 & 2.17281300 & -0.20761800 \\
\hline $\mathrm{H}$ & 1.317895001 & 1.26532400 & 1.26184900 \\
\hline $\mathrm{H}$ & 3.154735001 & 1.35928900 & -1.16725400 \\
\hline & 3.444090002 & $2.14547700 \quad 0$ & 0.37796300 \\
\hline $\mathrm{H}$ & $1.32807100-$ & -1.36840700 & 1.15346200 \\
\hline $\mathrm{C}$ & -3.46485600 & -0.75358200 & 0.34107200 \\
\hline $\mathrm{C}$ & -3.52010400 & 0.74119500 & -0.04789900 \\
\hline $\mathrm{H}$ & -3.57173800 & -0.86202700 & 1.42588500 \\
\hline $\mathrm{H}$ & -4.27948400 & -1.30297100 & -0.13578800 \\
\hline $\mathrm{H}$ & -3.89963600 & 0.84769800 & -1.07005100 \\
\hline $\mathrm{H}$ & -4.19076800 & 1.28566200 & 0.62037200 \\
\hline 0 & -2.21261600 & 1.29789400 & 0.02180300 \\
\hline O & -2.21427100 & -1.30164500 & -0.05855900 \\
\hline & -1.10842500 & 00.00194000 & -0.16802100 \\
\hline
\end{tabular}


GALLIUM
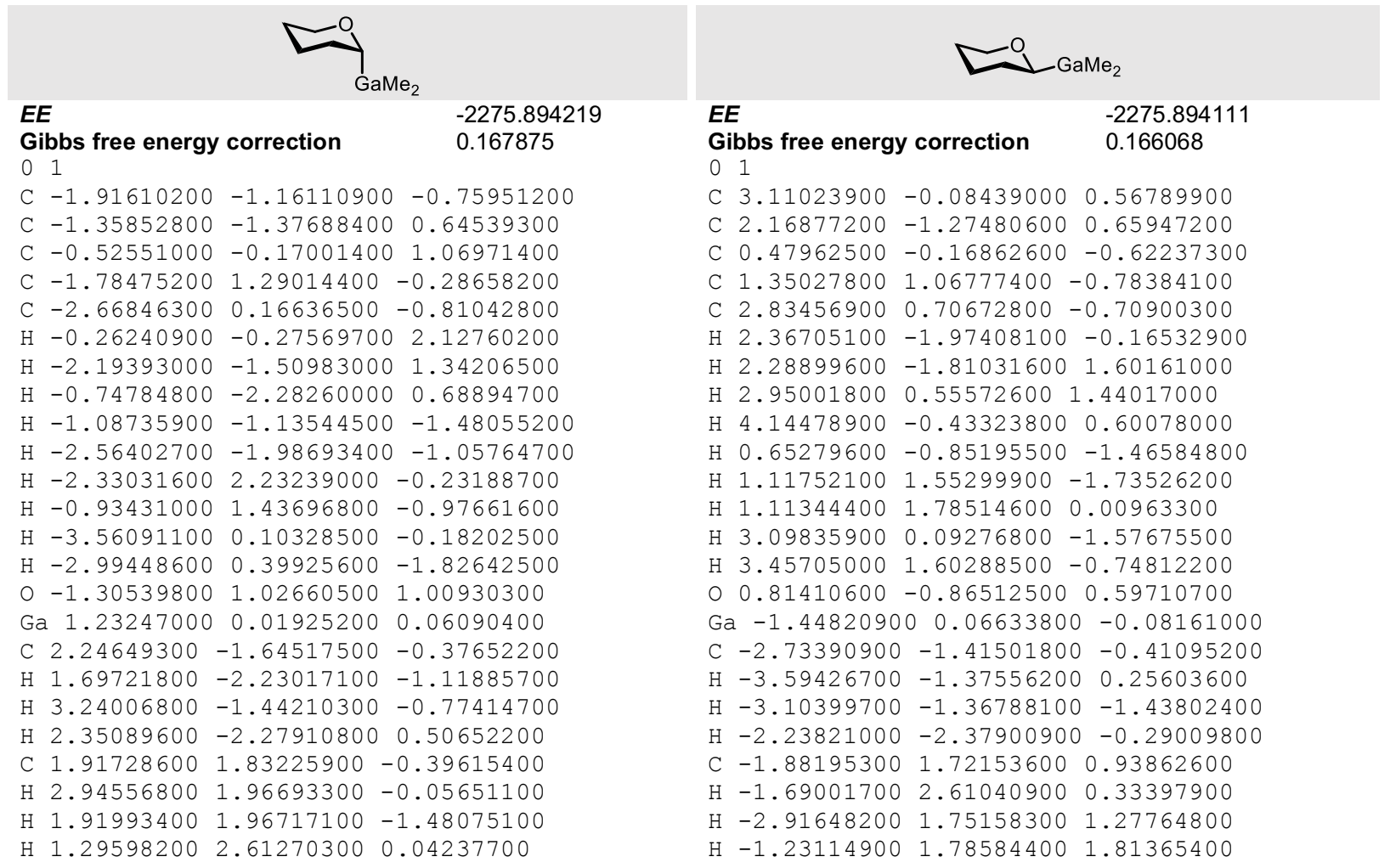

Gibbs free energy correction $\quad 0.166068$

01

$\begin{array}{llll}\text { C } 3.11023900 & -0.08439000 & 0.56789900\end{array}$

C $2.16877200-1.27480600 \quad 0.65947200$

$\begin{array}{llll}\text { C } 0.47962500 & -0.16862600 & -0.62237300\end{array}$

C $1.35027800 \quad 1.06777400-0.78384100$

C $2.83456900 \quad 0.70672800-0.70900300$

H $2.36705100-1.97408100-0.16532900$

H $2.28899600-1.81031600 \quad 1.60161000$

H $2.95001800 \quad 0.55572600 \quad 1.44017000$

$\mathrm{H} \quad 4.14478900-0.43323800 \quad 0.60078000$

$\mathrm{H} \quad 0.65279600-0.85195500-1.46584800$

$\mathrm{H} 1.117521001 .55299900-1.73526200$

H $1.11344400 \quad 1.78514600 \quad 0.00963300$

H $3.09835900 \quad 0.09276800-1.57675500$

$\mathrm{H} 3.45705000 \quad 1.60288500-0.74812200$

$\begin{array}{lllll}0 & 0.81410600 & -0.86512500 & 0.59710700\end{array}$

$\mathrm{Ga}-1.44820900 \quad 0.06633800-0.08161000$

C $-2.73390900-1.41501800-0.41095200$

$\mathrm{H}-3.59426700-1.37556200 \quad 0.25603600$

$\mathrm{H}-3.10399700-1.36788100-1.43802400$

$\mathrm{H}-2.23821000-2.37900900-0.29009800$

C $-1.88195300 \quad 1.72153600 \quad 0.93862600$

H $-1.69001700 \quad 2.61040900 \quad 0.33397900$

$\mathrm{H}-2.91648200 \quad 1.75158300 \quad 1.27764800$

$\mathrm{H}-1.23114900 \quad 1.78584400 \quad 1.81365400$

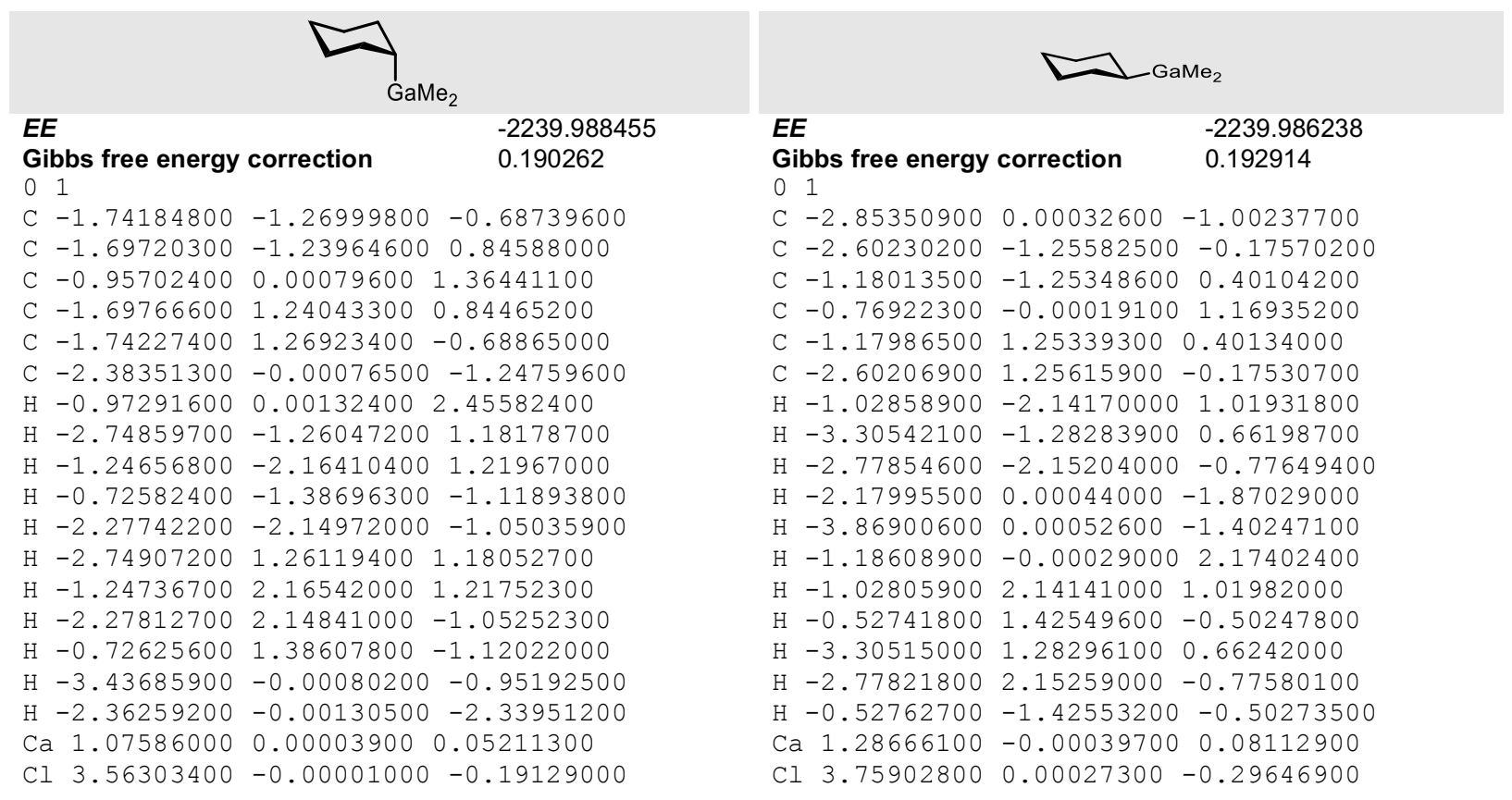


INDIUM

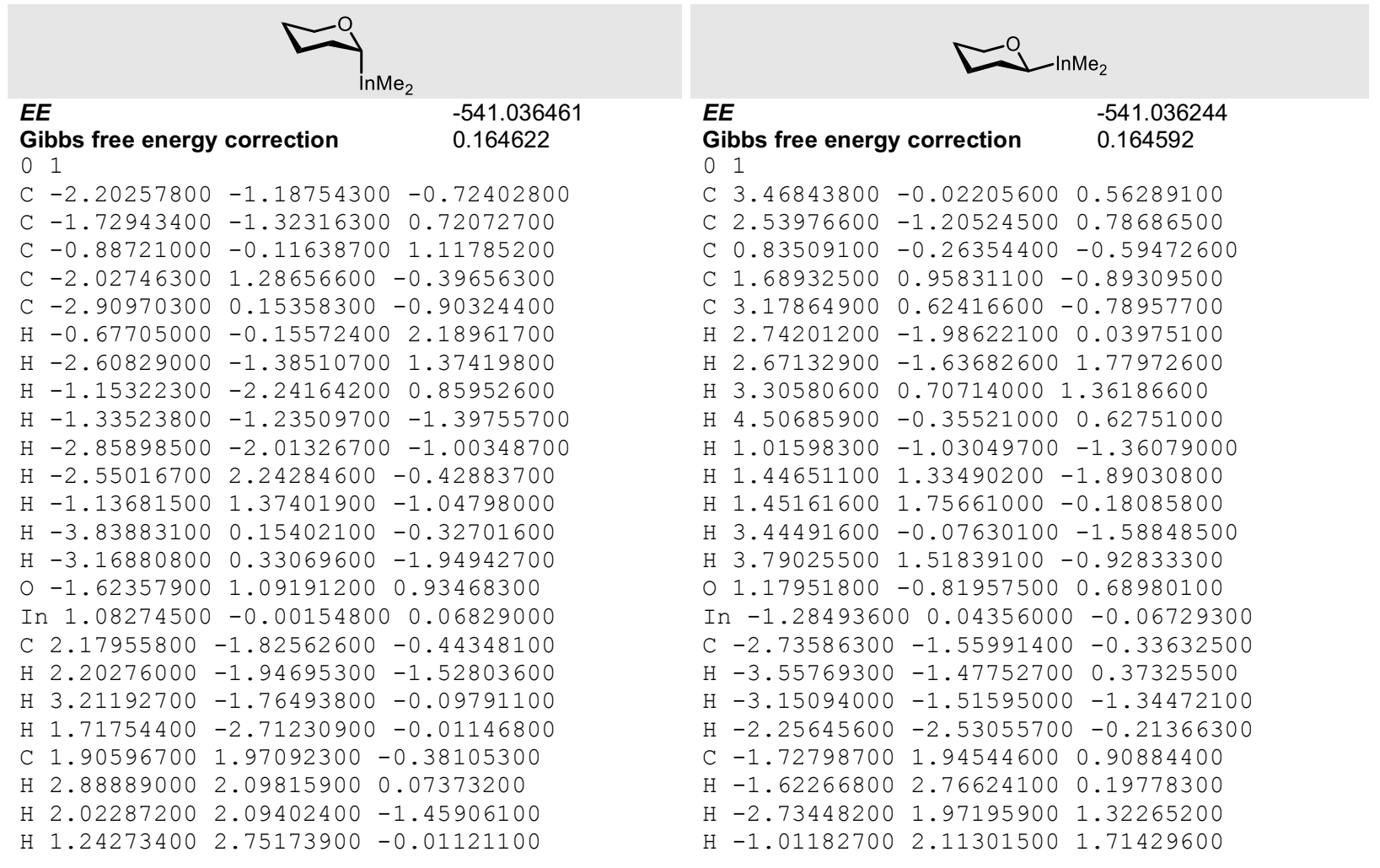

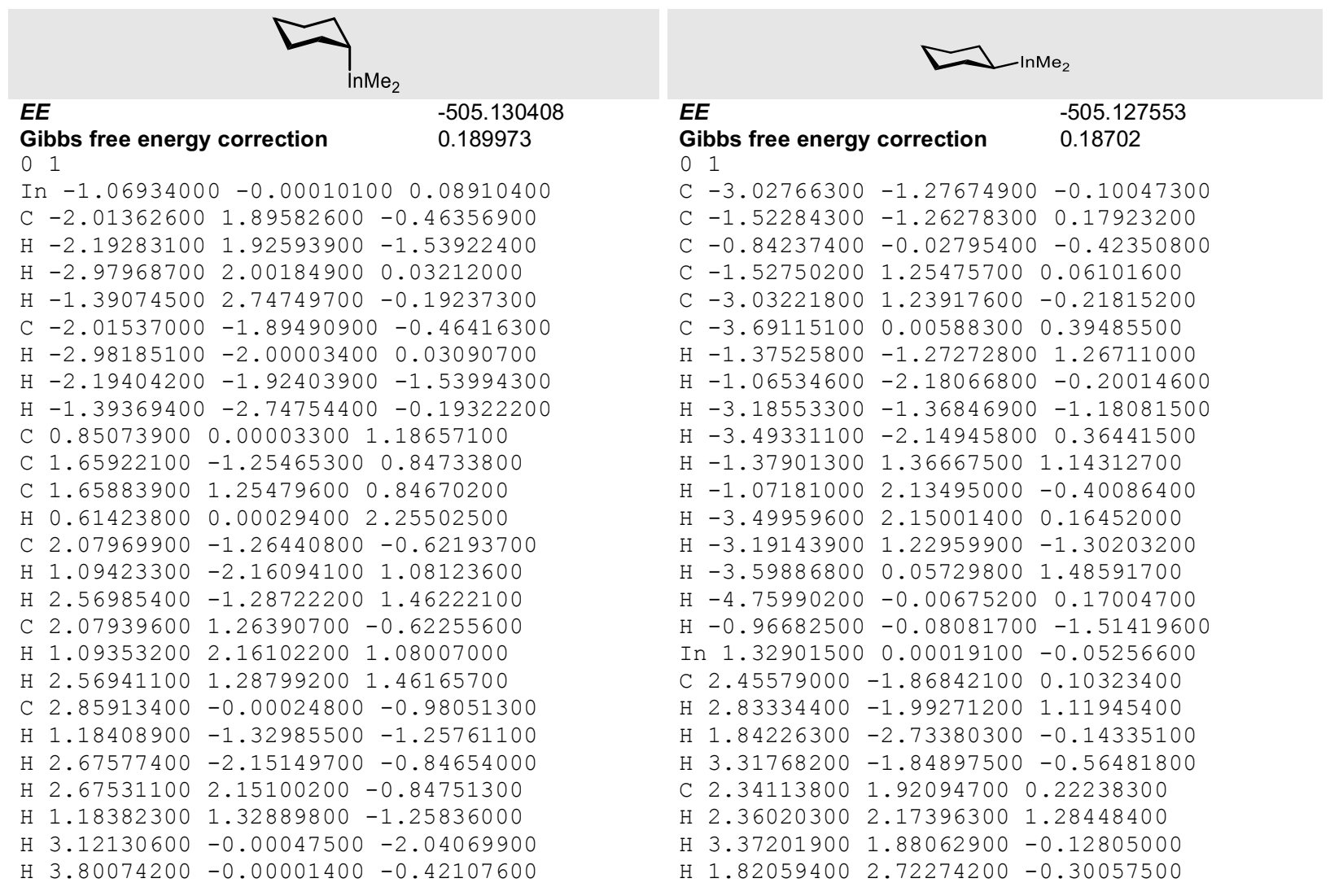




\section{THALLIUM}

\begin{tabular}{|c|c|c|c|}
\hline EE & & -523.325447 & -523.324053 \\
\hline $\begin{array}{l}\text { Gibbs free energy } \\
01\end{array}$ & correction & 0.164134 & $\begin{array}{l}\text { Gibbs free energy correction } \\
01\end{array}$ \\
\hline$C-2.44388300$ & -1.17538500 & -0.80102300 & C $3.83263400 \quad 0.00304700 \quad 0.48395600$ \\
\hline C -1.95580900 & -1.39202800 & 0.62851200 & C $2.95723400-1.21773000 \quad 0.72031900$ \\
\hline C -1.14819800 & -0.19289600 & 01.10308000 & C $1.14935900-0.30149900-0.50718800$ \\
\hline$C-2.32318900$ & 1.27515800 & -0.31870400 & C $1.92979200 \quad 0.96427000 \quad-0.81877200$ \\
\hline$C-3.18452800$ & 0.15650900 & -0.88778900 & C $3.43493600 \quad 0.68690200-0.82183400$ \\
\hline $\mathrm{H}-0.90913100$ & -0.29184100 & 02.16321600 & $\mathrm{H} 3.13731200-1.96504600-0.06615600$ \\
\hline $\mathrm{H}-2.82808500$ & -1.51670800 & 01.28246300 & $\mathrm{H} 3.16501100-1.67935000 \quad 1.68624900$ \\
\hline $\mathrm{H}-1.35420600$ & -2.30068600 & 0.70633600 & $\mathrm{H} 3.69834300 \quad 0.69677000 \quad 1.31889000$ \\
\hline $\mathrm{H}-1.58188800$ & -1.15849300 & $0-1.48149700$ & $\mathrm{H} 4.88332500-0.29586500 \quad 0.46954500$ \\
\hline $\mathrm{H}-3.08335400$ & -1.99770000 & $0-1.12582800$ & $\mathrm{H} 1.29901800-1.03664000-1.30975200$ \\
\hline $\mathrm{H}-2.86939200$ & 2.21757600 & -0.27874500 & $\mathrm{H} 1.60749900 \quad 1.36397700-1.78419200$ \\
\hline $\mathrm{H}-1.44133000$ & 1.42553200 & -0.96634400 & $\mathrm{H} 1.70562200 \quad 1.72470400-0.06257800$ \\
\hline $\mathrm{H}-4.10910000$ & 0.09828200 & -0.30700800 & $\mathrm{H} 3.67675500 \quad 0.02811000-1.66272400$ \\
\hline $\mathrm{H}-3.45628500$ & 0.39349900 & -1.91878600 & $\mathrm{H} 4.00052100 \quad 1.61036000-0.96344800$ \\
\hline $0-1.89840400$ & 1.00366800 & 0.99560100 & $01.58208300 \quad-0.87567500 \quad 0.72699100$ \\
\hline Tl 0.85129400 & 0.00451800 & 0.05039300 & $\mathrm{Tl}-1.02370400 \quad 0.01856000-0.03839500$ \\
\hline C 2.10511000 & -1.76349500 & -0.45055600 & $C-2.55134000-1.55005500-0.32769800$ \\
\hline H 2.21370900 & -1.82185100 & -1.53314000 & $\mathrm{H}-3.34915100-1.44023500 \quad 0.40293400$ \\
\hline H 3.09444900 & -1.64215900 & -0.01193500 & $\mathrm{H}-2.97074100-1.44827000-1.32817000$ \\
\hline $\mathrm{H} \quad 1.65565500$ & -2.68357500 & -0.08448900 & $\mathrm{H}-2.09597800-2.53323100-0.23456100$ \\
\hline C 1.58217800 & $2.04764800-$ & -0.43019700 & C $-1.42859600 \quad 1.99762400 \quad 0.88707400$ \\
\hline H 2.57738500 & 2.19977600 & -0.01568200 & $\mathrm{H}-1.07677100 \quad 2.78702600 \quad 0.22421900$ \\
\hline H 1.63664100 & 2.16212200 & -1.51260800 & $\mathrm{H}-2.48854300 \quad 2.13409400 \quad 1.08649400$ \\
\hline $\mathrm{H} \quad 0.89727200$ & $2.78785900-$ & -0.02253500 & $\mathrm{H}-0.87295600 \quad 2.06031200 \quad 1.82215400$ \\
\hline
\end{tabular}

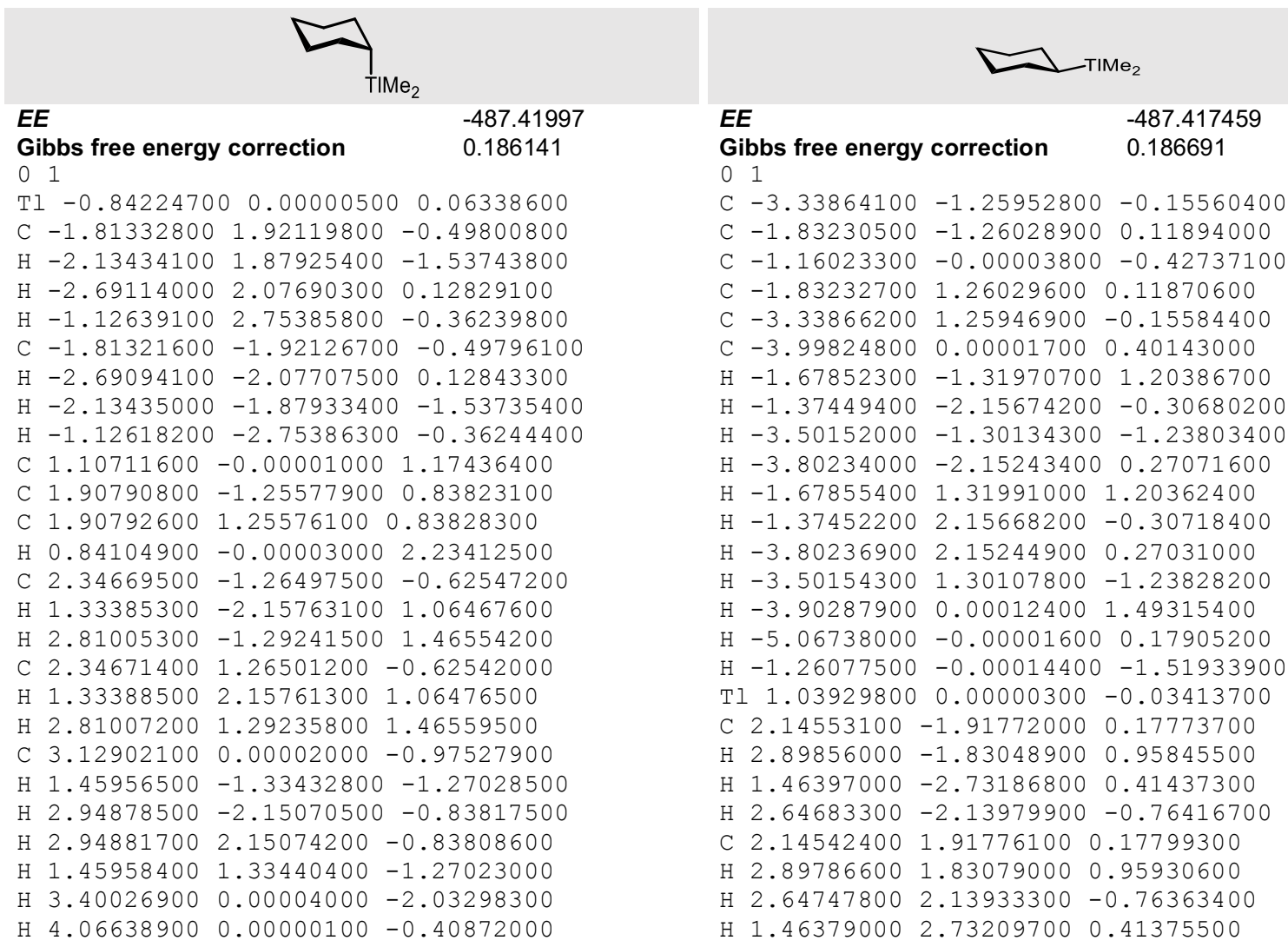




\section{CARBON}

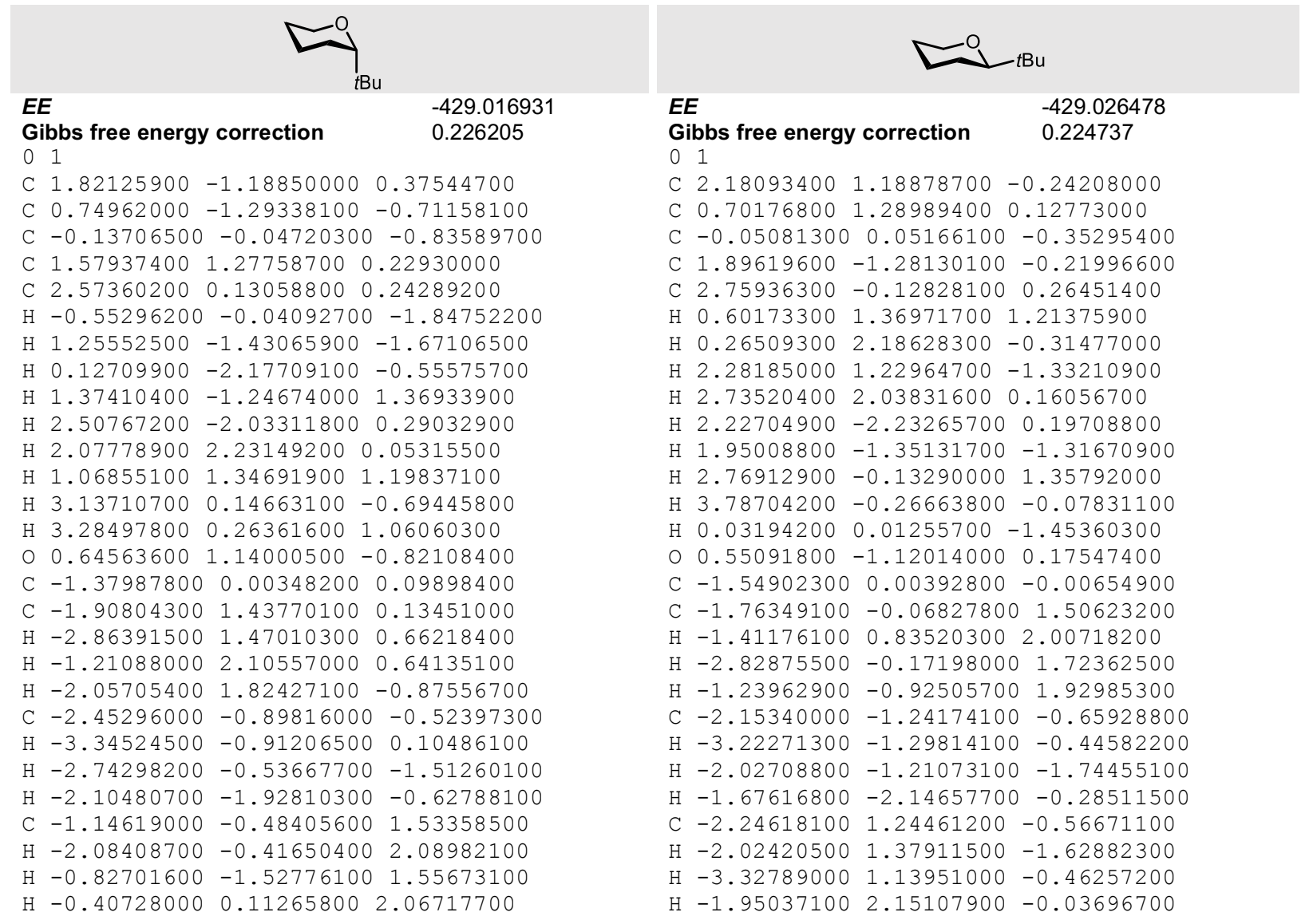

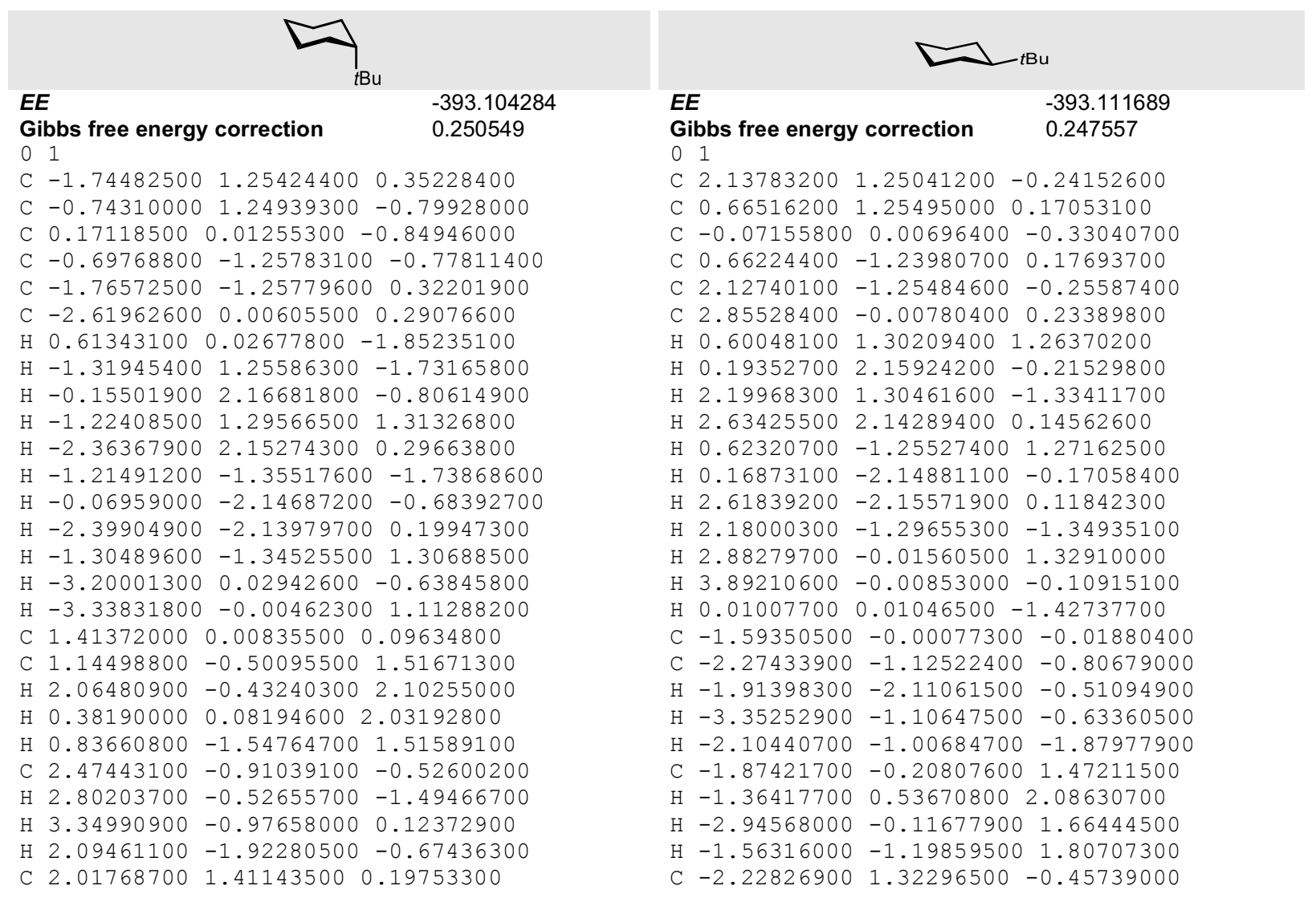


H $2.98569500 \quad 1.36384700 \quad 0.70119900$

H $2.17487600 \quad 1.84816600-0.79192900$

H $1.37885600 \quad 2.08609800 \quad 0.77090400$
$\mathrm{H}-1.95209900 \quad 1.56962400-1.48626800$

$\mathrm{H}-3.31681600 \quad 1.25014500-0.41003400$

$\mathrm{H}-1.92661500 \quad 2.15144800 \quad 0.18407300$ 
SILICON

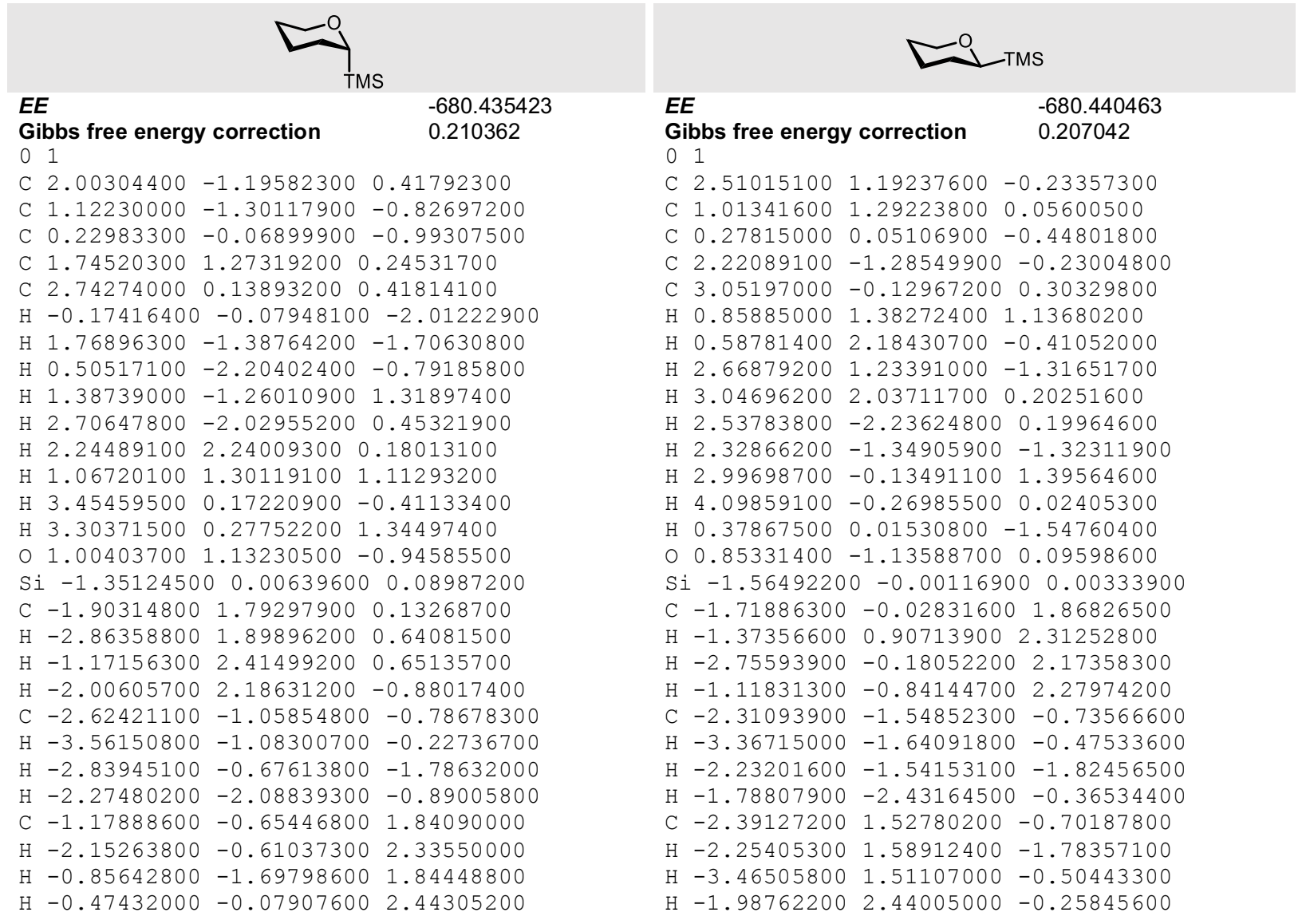

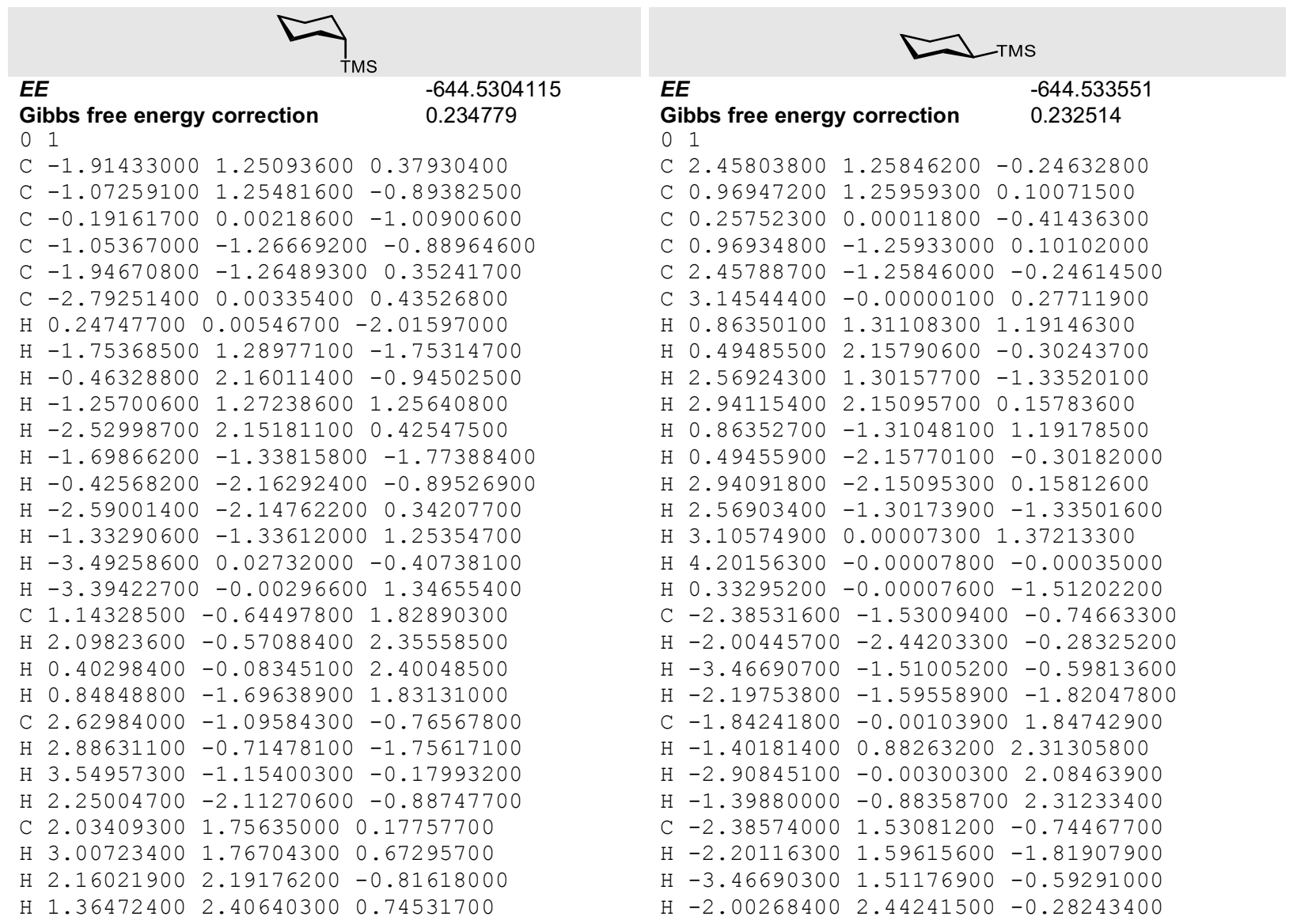


GERMANIUM
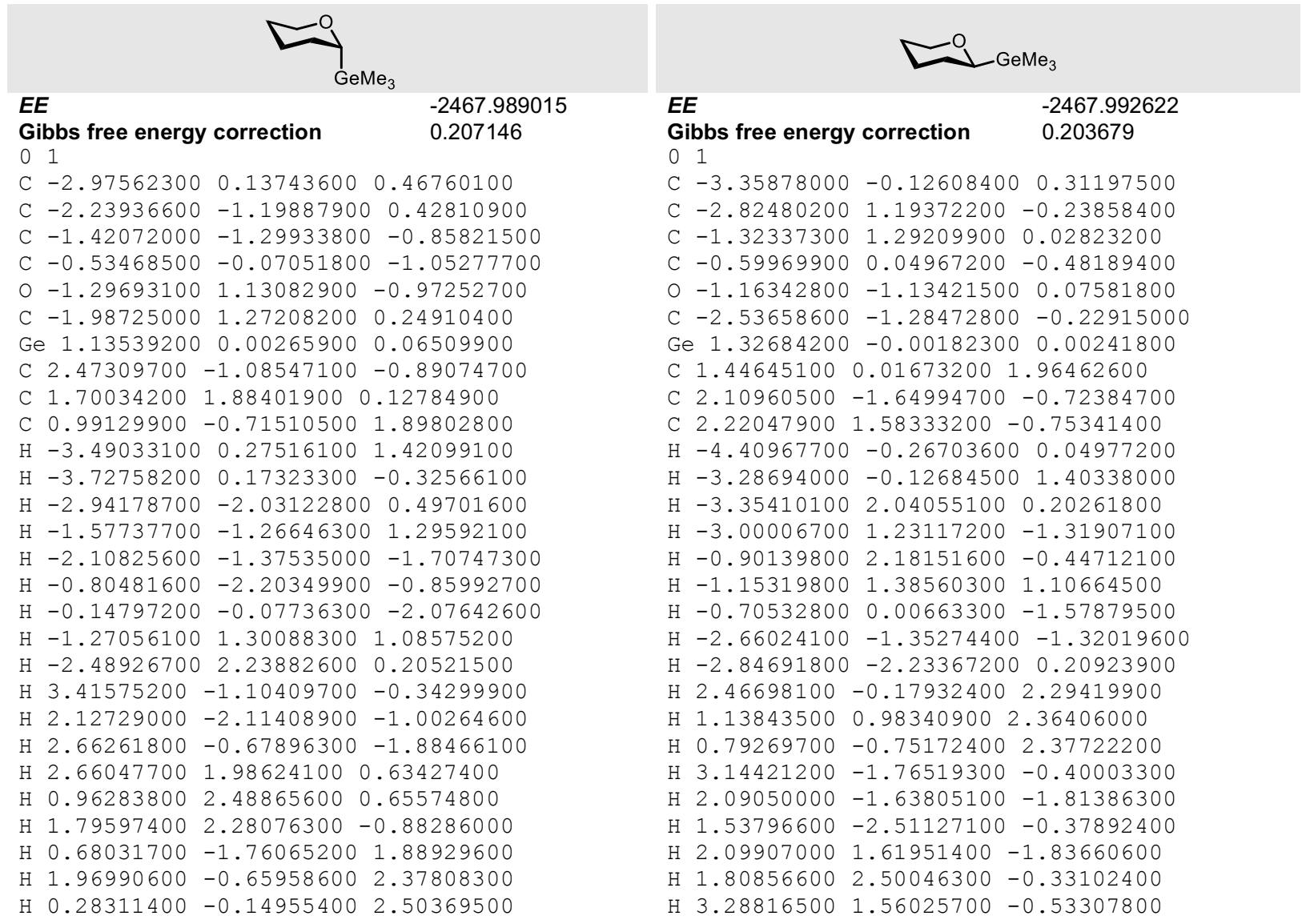

01

$\begin{array}{llll}\text { C } & -3.35878000 & -0.12608400 & 0.31197500\end{array}$

C $-2.82480200 \quad 1.19372200 \quad-0.23858400$

C $-1.32337300 \quad 1.29209900 \quad 0.02823200$

C $-0.59969900 \quad 0.04967200-0.48189400$

$\begin{array}{lllll}0 & -1.16342800 & -1.13421500 & 0.07581800\end{array}$

C $-2.53658600-1.28472800-0.22915000$

Ge $1.32684200-0.00182300 \quad 0.00241800$

C $1.44645100 \quad 0.01673200 \quad 1.96462600$

C $2.10960500-1.64994700-0.72384700$

C $2.22047900 \quad 1.58333200-0.75341400$

$\mathrm{H}-4.40967700 \quad-0.26703600 \quad 0.04977200$

$\mathrm{H}-3.28694000-0.12684500 \quad 1.40338000$

$\mathrm{H}-3.35410100 \quad 2.04055100 \quad 0.20261800$

$\mathrm{H}-3.00006700 \quad 1.23117200-1.31907100$

$\mathrm{H}-0.90139800 \quad 2.18151600-0.44712100$

$\mathrm{H}-1.15319800 \quad 1.38560300 \quad 1.10664500$

$\mathrm{H}-0.70532800 \quad 0.00663300-1.57879500$

$\mathrm{H}-2.66024100-1.35274400-1.32019600$

$\mathrm{H}-2.84691800 \quad-2.23367200 \quad 0.20923900$

$\mathrm{H} 2.46698100 \quad-0.17932400 \quad 2.29419900$

$\mathrm{H} \quad 1.13843500 \quad 0.98340900 \quad 2.36406000$

H $0.79269700 \quad-0.75172400 \quad 2.37722200$

H $3.14421200-1.76519300-0.40003300$

H $2.09050000-1.63805100-1.81386300$

$\mathrm{H} 1.53796600-2.51127100-0.37892400$

$\mathrm{H} 2.09907000 \quad 1.61951400-1.83660600$

$\mathrm{H} 1.80856600 \quad 2.50046300-0.33102400$

H $3.28816500 \quad 1.56025700-0.53307800$

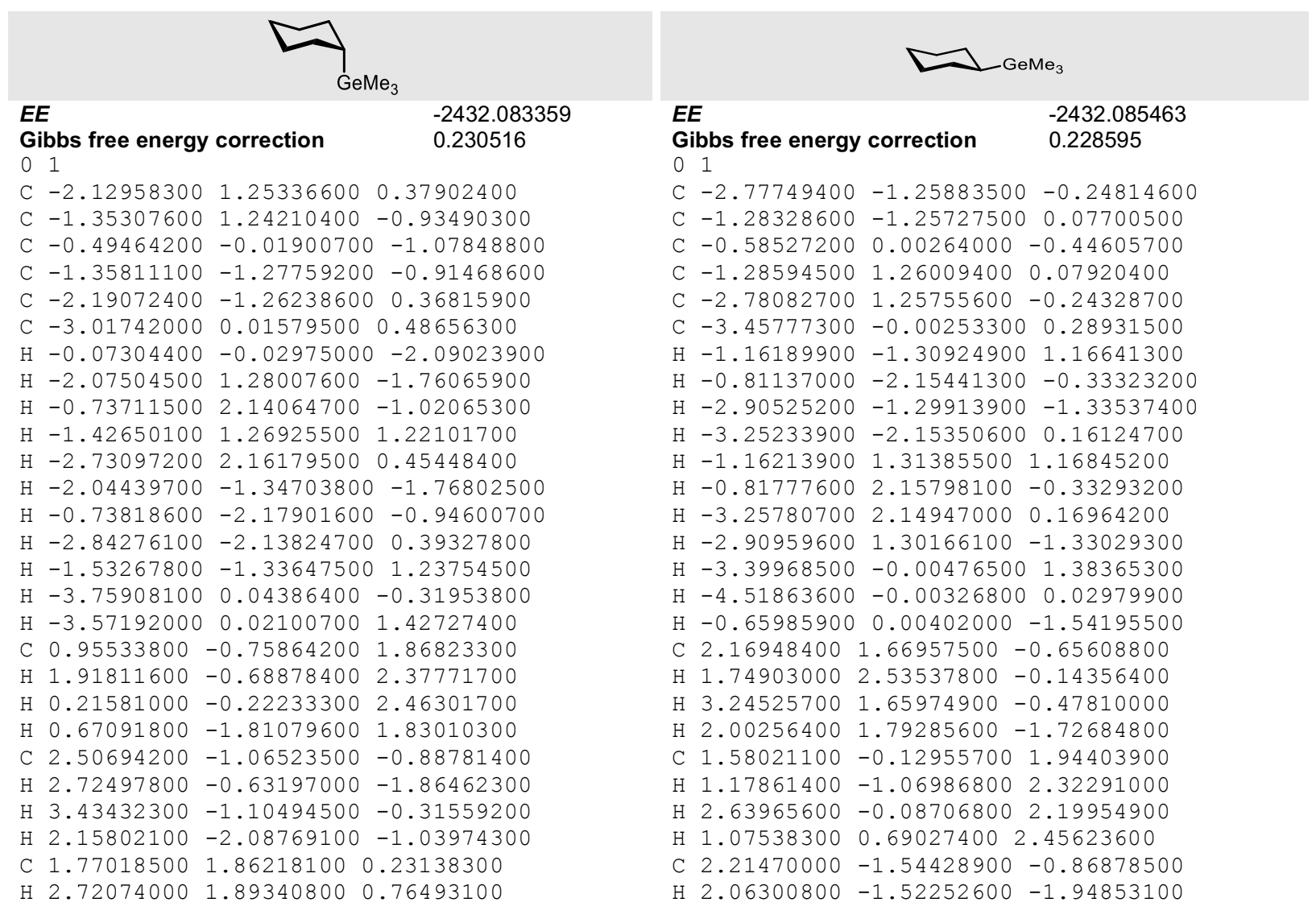


H $1.91388400 \quad 2.31974900-0.74838400$

$\begin{array}{lllll}\mathrm{H} & 1.04871400 & 2.46420600 & 0.78582500\end{array}$

Ge $1.14352300 \quad 0.00123500 \quad 0.05667000$
H $3.28781300-1.53632000-0.67529000$

$\mathrm{H} 1.80910100-2.48118200-0.48532300$

Ge $1.34509800 \quad 0.00099400 \quad-0.00905200$ 


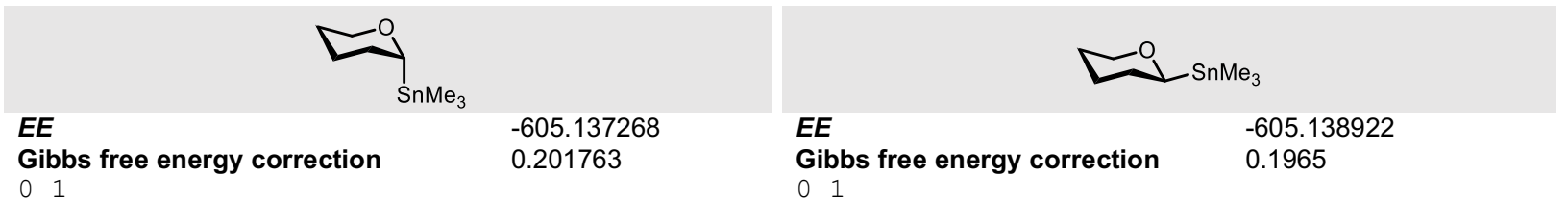

$\begin{array}{llll}\text { C }-3.17507600 & 0.13748000 & 0.53352100\end{array}$

C $-2.44923300-1.20220000 \quad 0.44284700$

C $-1.72960700-1.30415900-0.90042600$

C $-0.84506400-0.08489600-1.14271600$

$0-1.59349900 \quad 1.12078600-1.02424400$

C $-2.20745100 \quad 1.27239500 \quad 0.23523000$

Sn $1.01199700 \quad 0.00178300 \quad 0.05082600$

C $2.59285200-0.71496900-1.23739000$

C $1.35271700 \quad 2.06437700 \quad 0.58992900$

C $0.97920300-1.24261900 \quad 1.82063500$

$\mathrm{H}-3.61618500 \quad 0.28115600 \quad 1.52227600$

$\mathrm{H}-3.98617400 \quad 0.16973600-0.19929000$

$\mathrm{H}-3.14884100 \quad-2.03030100 \quad 0.56765900$

$\mathrm{H}-1.72122400-1.27389300 \quad 1.25748300$

$\mathrm{H}-2.47929200-1.36302100-1.69804700$

$\mathrm{H}-1.12895000-2.21694400-0.95439200$

$\mathrm{H}-0.49771000-0.09419700 \quad-2.17873200$

$\mathrm{H}-1.43739100 \quad 1.31683400 \quad 1.02485900$

$\mathrm{H} \quad-2.71545500 \quad 2.23674000 \quad 0.21418100$

$\mathrm{H} \quad 3.55540400-0.66609600-0.72851100$

H $2.41186700-1.75033100-1.52710200$

H $2.65090200-0.11069200-2.14265900$

H 2.39367000 2.34175100 0.42793100

$\mathrm{H} \quad 1.10852700 \quad 2.23463600 \quad 1.63830600$

$\mathrm{H} \quad 0.71914500 \quad 2.70567600 \quad-0.02306400$

$\mathrm{H} \quad 0.59651600 \quad-2.23694200 \quad 1.58830700$

$\mathrm{H} \quad 1.98704200 \quad-1.34952500 \quad 2.22212900$

$\begin{array}{lllll}\mathrm{H} & 0.34626500 & -0.80245400 & 2.59155900\end{array}$

01

C $-3.66141400 \quad-0.13228300 \quad 0.32377000$

C $-3.14987800 \quad 1.18822500-0.24663800$

C $-1.64346000 \quad 1.29887400-0.01120200$

C $-0.92130800 \quad 0.05974300-0.53007300$

$\begin{array}{llll}0 & -1.46360800 & -1.12707200 & 0.04708600\end{array}$

C $-2.84301400-1.28925600-0.22696500$

Sn $1.19039600 \quad-0.00268700 \quad 0.00242100$

$\begin{array}{lllll}\text { C } 1.27542100 & 0.02958800 & 2.15655000\end{array}$

C $2.05756100-1.81294700-0.77886900$

C $2.18627600 \quad 1.73683400-0.80841100$

$\mathrm{H}-4.71672200 \quad-0.28290100 \quad 0.08572400$

$\mathrm{H}-3.56617300-0.12495600 \quad 1.41339600$

$\mathrm{H}-3.67696500 \quad 2.03358800 \quad 0.20034600$

$\mathrm{H}-3.34725900 \quad 1.21720300-1.32354000$

$\mathrm{H}-1.23691000 \quad 2.18901500-0.49941400$

$\mathrm{H}-1.456040001 .402821001 .06380500$

$\mathrm{H}-1.04815400 \quad 0.00863900-1.62318800$

$\mathrm{H}-2.98868900-1.36544800-1.31468600$

$\mathrm{H}-3.13730600 \quad-2.23719200 \quad 0.22459500$

$\begin{array}{llll}\mathrm{H} & 2.27052400 & -0.23441900 & 2.51282900\end{array}$

$\mathrm{H} 1.02509500 \quad 1.02033900 \quad 2.53521200$

$\mathrm{H} \quad 0.55853900 \quad-0.68699800 \quad 2.55647200$

H $3.06384600-1.96013500-0.38785900$

H $2.11128400-1.77407400-1.86641100$

H $1.44235700-2.66481800-0.49183800$

H $2.07559600 \quad 1.77562400-1.89194800$

$\mathrm{H} \quad 1.76461100 \quad 2.64902700-0.38617300$

H $3.25032100 \quad 1.71295400-0.57402100$

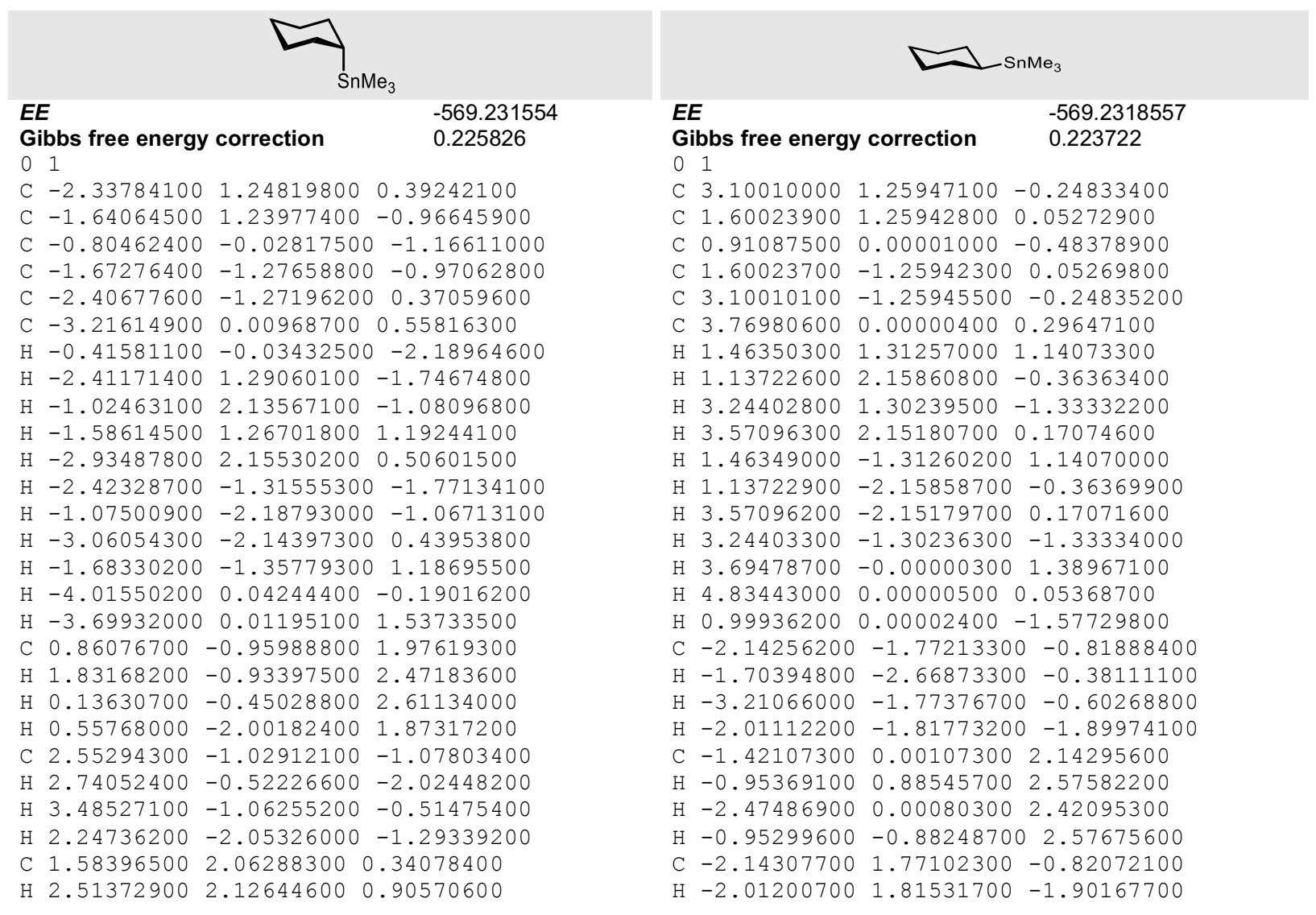


$\mathrm{H} 1.72908000 \quad 2.56455700-0.61604300$

$\begin{array}{lllll}\mathrm{H} & 0.80998500 & 2.59684500 & 0.89288300\end{array}$

Sn $1.01530500-0.00191900 \quad 0.04271800$
$\mathrm{H}-3.21110100 \quad 1.77276900-0.60416600$

$\mathrm{H}-1.70444600 \quad 2.66822100-0.38419300$

Sn $-1.20746100 \quad 0.00000200-0.00887100$ 
LEAD
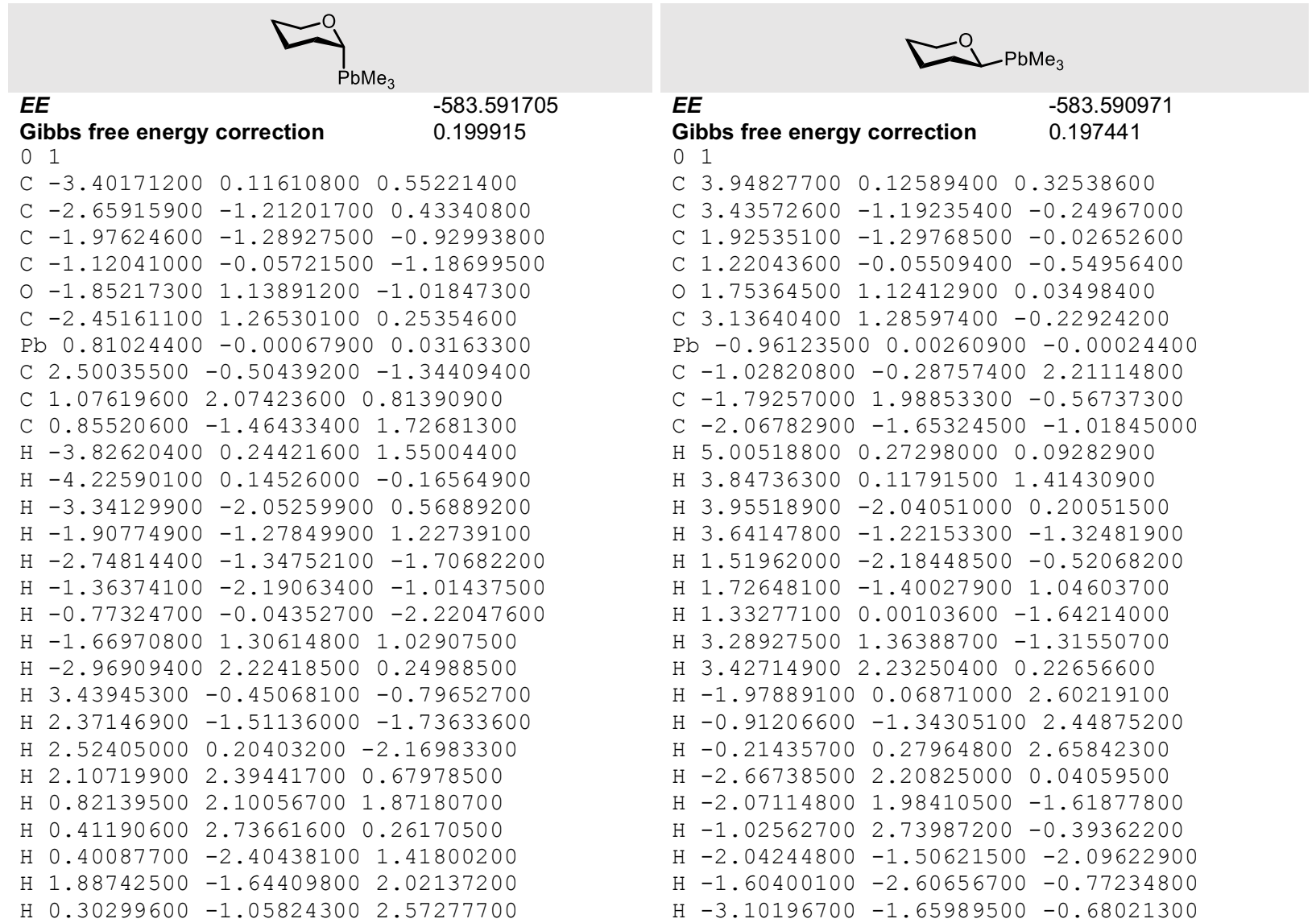

01

$\begin{array}{llll}\text { C } 3.94827700 & 0.12589400 & 0.32538600\end{array}$

C $3.43572600-1.19235400-0.24967000$

C $1.92535100-1.29768500-0.02652600$

C $1.22043600-0.05509400-0.54956400$

$\begin{array}{lllll}0 & 1.75364500 & 1.12412900 & 0.03498400\end{array}$

C $3.136404001 .28597400-0.22924200$

$\mathrm{Pb}-0.96123500 \quad 0.00260900-0.00024400$

$\begin{array}{llll}\text { C }-1.02820800 & -0.28757400 & 2.21114800\end{array}$

C $-1.79257000 \quad 1.98853300 \quad-0.56737300$

C $-2.06782900-1.65324500-1.01845000$

H $5.00518800 \quad 0.27298000 \quad 0.09282900$

H $3.84736300 \quad 0.11791500 \quad 1.41430900$

H $3.95518900-2.04051000 \quad 0.20051500$

H $3.64147800-1.22153300-1.32481900$

$\mathrm{H} \quad 1.51962000-2.18448500-0.52068200$

$\mathrm{H} 1.72648100-1.40027900 \quad 1.04603700$

H $1.33277100 \quad 0.00103600-1.64214000$

$\mathrm{H} 3.28927500 \quad 1.36388700-1.31550700$

$\begin{array}{llll}\mathrm{H} & 3.42714900 & 2.23250400 & 0.22656600\end{array}$

$\mathrm{H}-1.97889100 \quad 0.06871000 \quad 2.60219100$

$\mathrm{H}-0.91206600-1.34305100 \quad 2.44875200$

$\mathrm{H}-0.21435700 \quad 0.27964800 \quad 2.65842300$

$\mathrm{H}-2.66738500 \quad 2.20825000 \quad 0.04059500$

$\mathrm{H}-2.07114800 \quad 1.98410500-1.61877800$

$\mathrm{H}-1.02562700 \quad 2.73987200-0.39362200$

$\mathrm{H}-2.04244800-1.50621500 \quad-2.09622900$

$\mathrm{H}-1.60400100-2.60656700-0.77234800$

$\mathrm{H}-3.10196700-1.65989500 \quad-0.68021300$

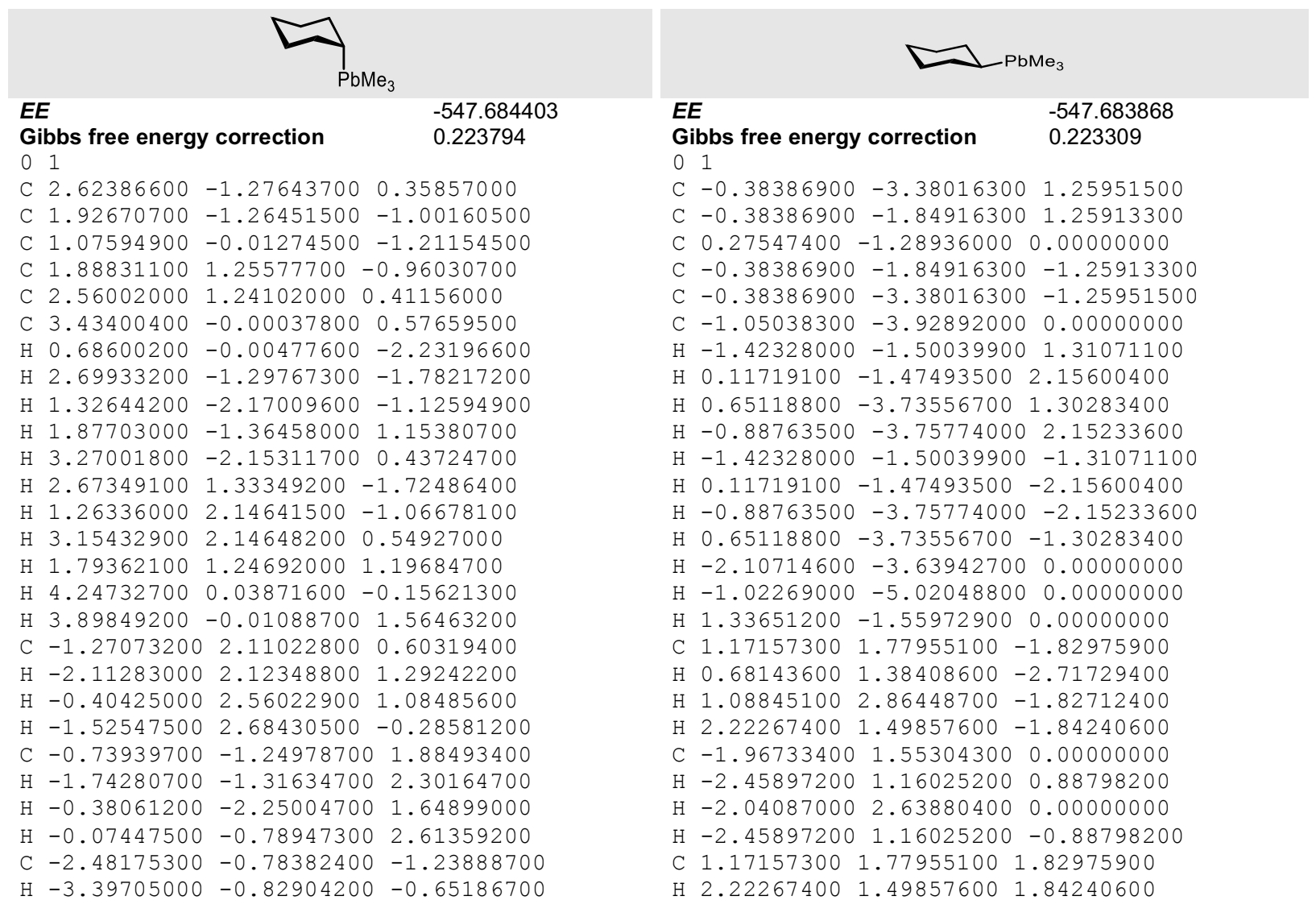


$\mathrm{H}-2.63318300-0.12448800-2.09150400$

$\mathrm{H}-2.23566000-1.78207500-1.59634000$ $\mathrm{Pb}-0.81079200 \quad-0.00370100 \quad 0.02847700$
H $1.08845100 \quad 2.86448700 \quad 1.82712400$

$\mathrm{H} \quad 0.68143600 \quad 1.38408600 \quad 2.71729400$

$\mathrm{Pb} \quad 0.18853100 \quad 0.95234200 \quad 0.00000000$ 


\section{NITROGEN}

\begin{tabular}{|c|c|c|}
\hline$E E$ & & -405.742424 \\
\hline & $\begin{array}{l}\text { bbs free energy correction } \\
1\end{array}$ & 0.185773 \\
\hline $\mathrm{C}$ & $2.26787600-0.13254400$ & -0.47697400 \\
\hline $\mathrm{C}$ & $1.28689700-1.29077200$ & -0.37965800 \\
\hline $\mathrm{C}$ & $-0.31301700 \quad 0.00556800$ & 0.79756200 \\
\hline C & $0.61113500 \quad 1.220217000$ & 0.79171200 \\
\hline $\mathrm{C}$ & $1.516023001 .19689700-$ & -0.43691900 \\
\hline $\mathrm{H}$ & $0.66606000-1.33100300$ & -1.28078600 \\
\hline $\mathrm{H}$ & $1.80578500-2.24269900$ & -0.26971900 \\
\hline $\mathrm{H}$ & $2.96238100-0.18387000$ & 0.36662000 \\
\hline $\mathrm{H}$ & $2.85351600-0.22710800$ & -1.39388200 \\
\hline $\mathrm{H}$ & $-0.85533700-0.04500700$ & 01.75656700 \\
\hline $\mathrm{H}$ & $0.04398300 \quad 2.14755800 \quad 0$ & 0.86603200 \\
\hline $\mathrm{H}$ & 1.220992001 .148332001 & 1.69568500 \\
\hline $\mathrm{H}$ & $0.909910001 .30053900-$ & -1.34140500 \\
\hline $\mathrm{H}$ & 2.214526002 .03488300 & -0.41155000 \\
\hline 0 & $0.45690100-1.17692200$ & 0.76838700 \\
\hline $\mathrm{N}$ & $-1.25955600 \quad 0.00295200$ & -0.31126000 \\
\hline C & $-2.02724800 \quad 1.22939700$ & -0.40655000 \\
\hline $\mathrm{H}$ & $-1.39588100 \quad 2.06731700$ & -0.69849000 \\
\hline $\mathrm{H}$ & $-2.79114800 \quad 1.10724400$ & -1.17410000 \\
\hline $\mathrm{H}$ & $-2.53611600 \quad 1.48570800$ & 0.53840300 \\
\hline C & $-2.14341200-1.14803300$ & $0-0.22361700$ \\
\hline $\mathrm{H}$ & $-2.78855100-1.10637600$ & 00.66964700 \\
\hline $\mathrm{H}$ & $-2.78073600-1.18214700$ & $0-1.10724700$ \\
\hline $\mathrm{H}$ & $-1.55723300-2.06303600$ & -0.17738800 \\
\hline
\end{tabular}

\begin{tabular}{|c|c|c|}
\hline \multicolumn{2}{|r|}{$E E$} & -405.740817 \\
\hline $\begin{array}{l}\text { Gib } \\
0\end{array}$ & $\begin{array}{l}\text { bbs free energy correction } \\
1\end{array}$ & 0.185221 \\
\hline $\mathrm{C}$ & $-1.92324600 \quad 1.18738600$ & 0.27415000 \\
\hline $\mathrm{C}$ & $-0.46912000 \quad 1.28678400$ & -0.18531600 \\
\hline $\mathrm{C}$ & $0.29223200 \quad 0.02195000 \quad 0$ & 0.19288100 \\
\hline $\mathrm{C}$ & $-1.67414200-1.27876500$ & $0 \quad 0.18231700$ \\
\hline $\mathrm{C}$ & $-2.55781700-0.10914800$ & $0-0.21595800$ \\
\hline $\mathrm{H}$ & $-0.41155700 \quad 1.39566300$ & -1.27221800 \\
\hline $\mathrm{H}$ & $-0.00348900 \quad 2.16037200$ & 0.27124000 \\
\hline $\mathrm{H}$ & $-1.95396400 \quad 1.20737200$ & 1.36893700 \\
\hline $\mathrm{H}$ & $-2.48672800 \quad 2.05489800$ & -0.07255300 \\
\hline $\mathrm{H}$ & $-2.04200500-2.22038100$ & $0-0.22433000$ \\
\hline $\mathrm{H}$ & $-1.64192200-1.36830200$ & 01.27830500 \\
\hline $\mathrm{H}$ & $-2.64794700-0.09325700$ & $0-1.30526700$ \\
\hline $\mathrm{H}$ & $-3.55874900-0.23920900$ & 00.20087700 \\
\hline $\mathrm{H}$ & $0.30393100-0.06568500$ & 1.30415700 \\
\hline 0 & $-0.36553500-1.11722500$ & $0-0.32236000$ \\
\hline $\mathrm{N}$ & $1.64028200-0.01624400$ & -0.32628800 \\
\hline $\mathrm{C}$ & $2.406213001 .16683700 \quad 0$ & 0.01765300 \\
\hline $\mathrm{H}$ & 3.432525001 .02759200 & -0.32041900 \\
\hline & 2.010499002 .04960700 & -0.48031700 \\
\hline $\mathrm{H}$ & $2.43119200 \quad 1.355363001$ & 1.10510400 \\
\hline $\mathrm{C}$ & $2.33428400-1.21432500$ & 0.11997900 \\
\hline $\mathrm{H}$ & $1.76763100-2.09614300$ & -0.16455500 \\
\hline & $3.31456700-1.25826300$ & -0.35430300 \\
\hline & $2.47790100-1.22242700$ & 1.21399100 \\
\hline
\end{tabular}

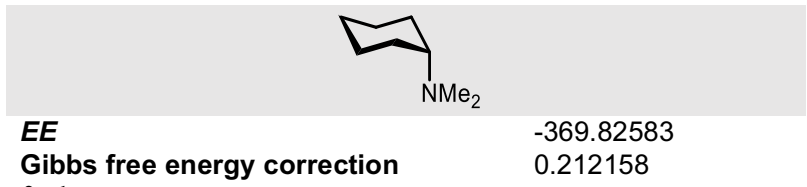

01

C $-2.28688100 \quad 0.00005100-0.52925300$

C $-1.430872001 .26372700-0.44455400$

C $-0.55220400 \quad 1.23793600 \quad 0.80461600$

C $0.35158600 \quad-0.00017200 \quad 0.82147800$

C $-0.55218500-1.23830300 \quad 0.80420700$

C $-1.43089700-1.26366400-0.44493900$

$\mathrm{H} \quad 0.02935500 \quad 2.15544900 \quad 0.89528100$

$\mathrm{H}-0.79270600 \quad 1.32768900-1.33021800$

$\mathrm{H}-2.06783300 \quad 2.15069800-0.43629900$

$\mathrm{H}-3.00072700-0.00006300 \quad 0.30252200$

$\mathrm{H}-2.87457300 \quad 0.00019100-1.44951500$

$\mathrm{H} \quad 0.93307300 \quad-0.00031500 \quad 1.76252000$

$\mathrm{H} \quad 0.02939100 \quad-2.15584100 \quad 0.89447000$

$\mathrm{H}-1.18730000-1.19331400 \quad 1.69416200$

$\mathrm{H}-0.79275100-1.32739100-1.33063000$

$\mathrm{H}-2.06788700-2.15061600 \quad-0.43691600$

$\mathrm{H}-1.18738600 \quad 1.19259400 \quad 1.69450400$

$\mathrm{N} 1.28298200-0.00001300-0.31271200$

C $2.11385100-1.18991500-0.33522200$

$\mathrm{H} 1.52044200-2.07632600-0.55411900$

$\mathrm{H} 2.85998300-1.09233300-1.12324700$

$\begin{array}{llll}\mathrm{H} & 2.64288300 & -1.35277200 & 0.61983500\end{array}$

C $2.113274001 .19027600-0.33547500$

$\begin{array}{lllll}\mathrm{H} & 1.51943800 & 2.07635700 & -0.55456900\end{array}$

H $2.64225200 \quad 1.35361000 \quad 0.61953800$

H $2.85944000 \quad 1.09285000-1.12348600$

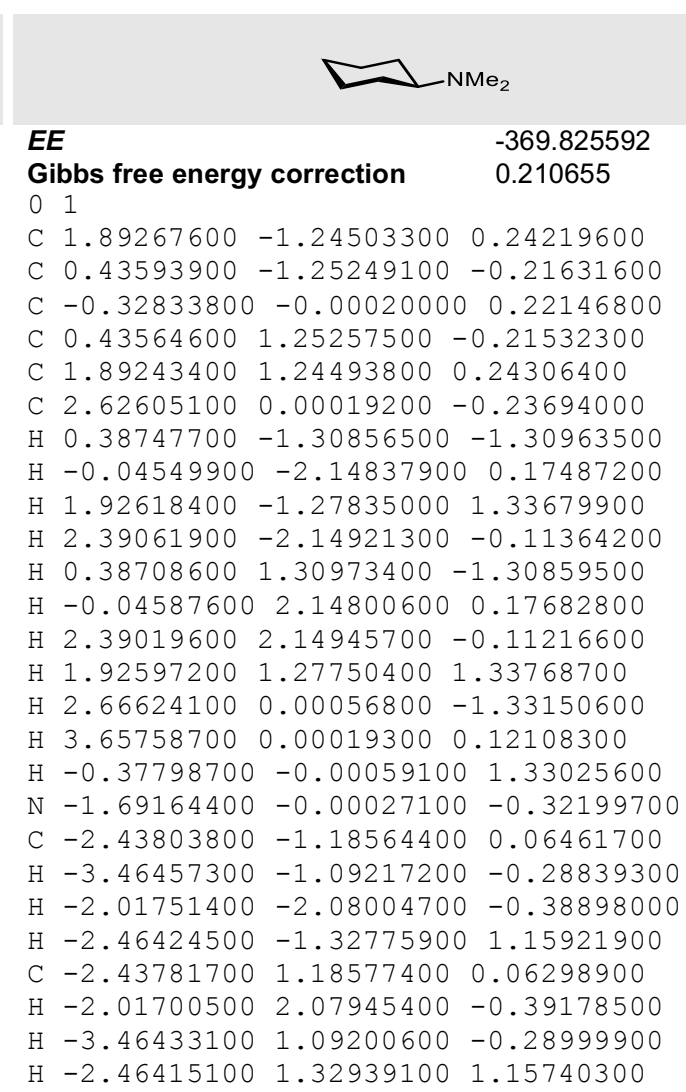


PHOSPHORUS

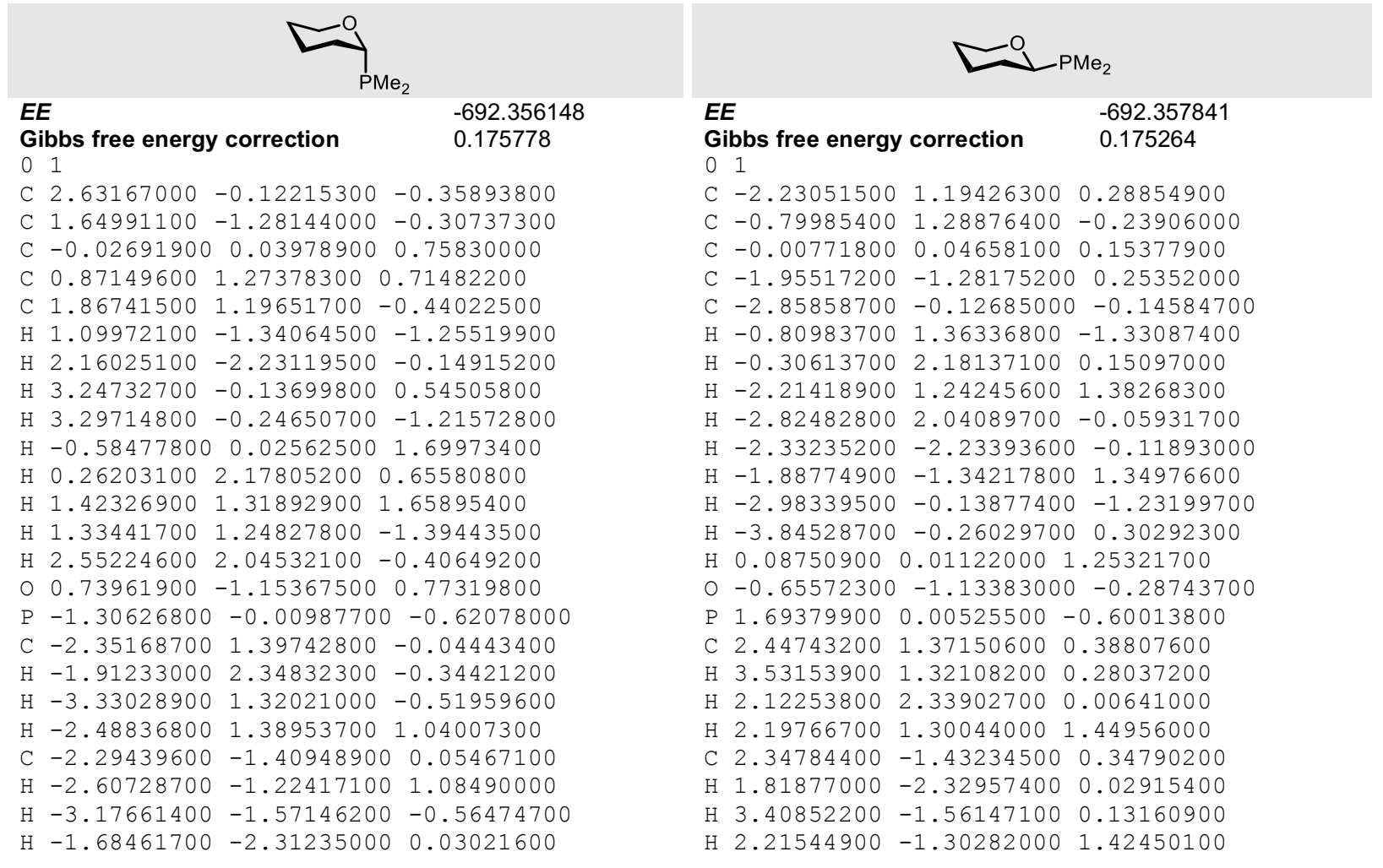

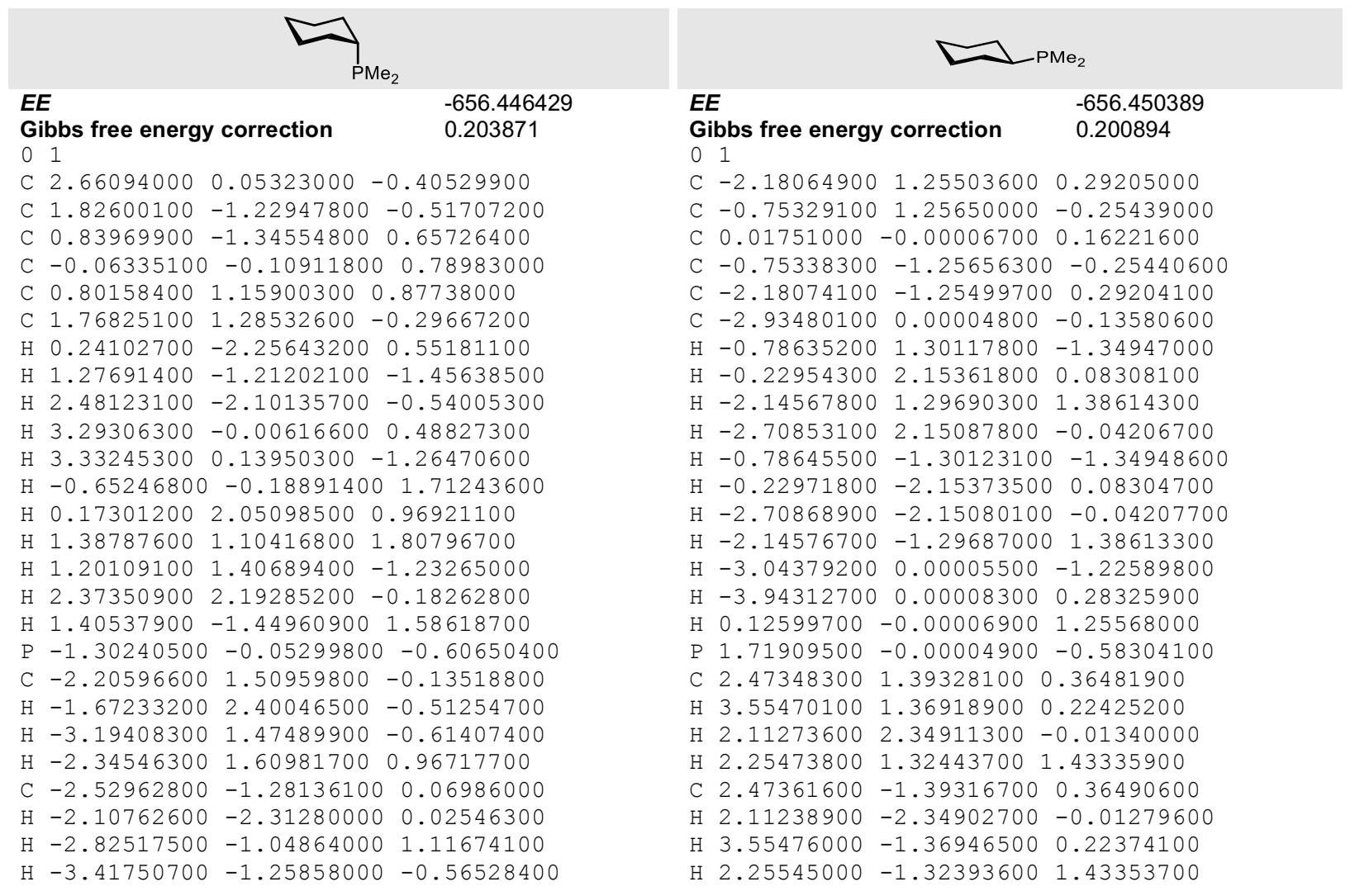


ARSENIC

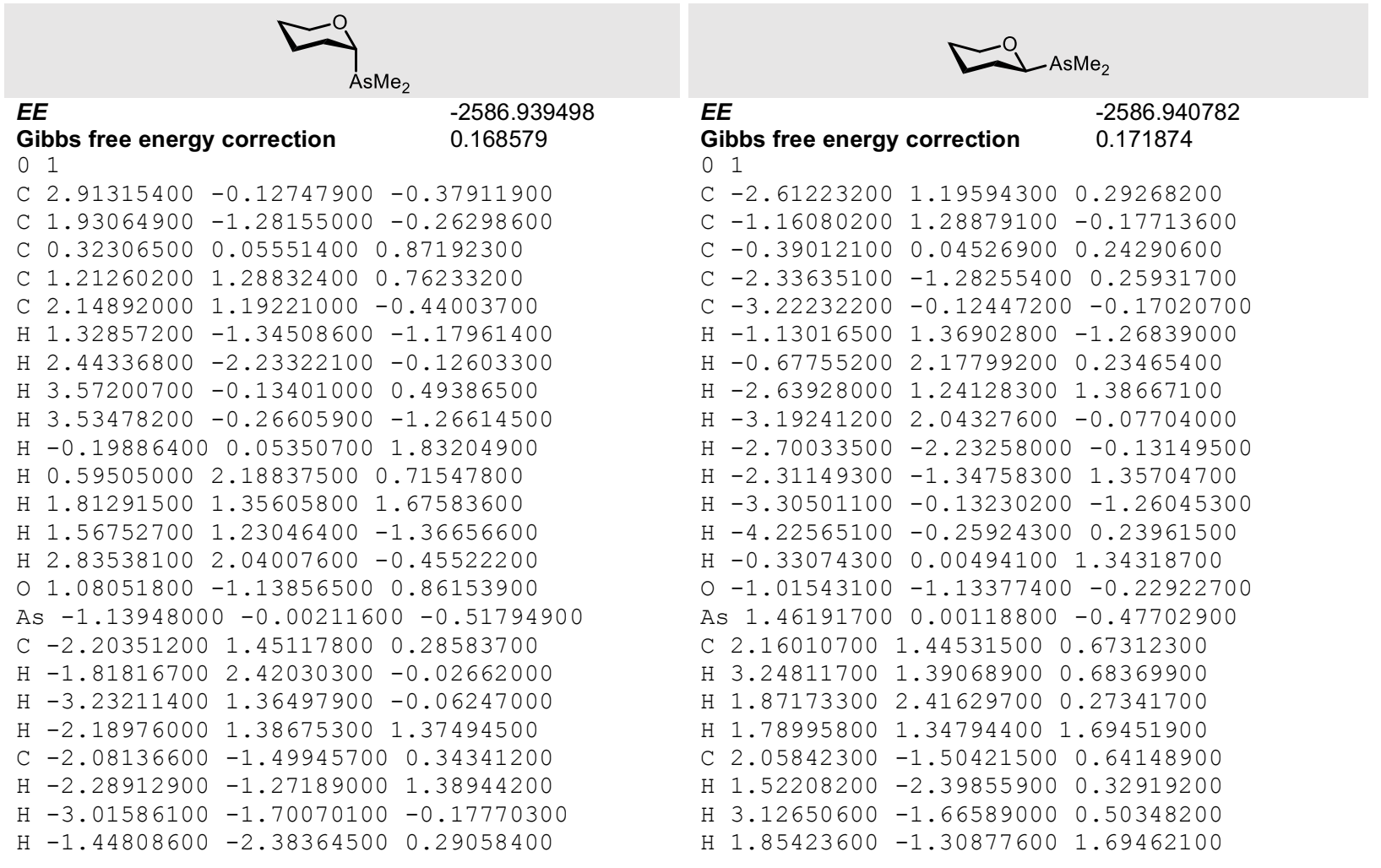

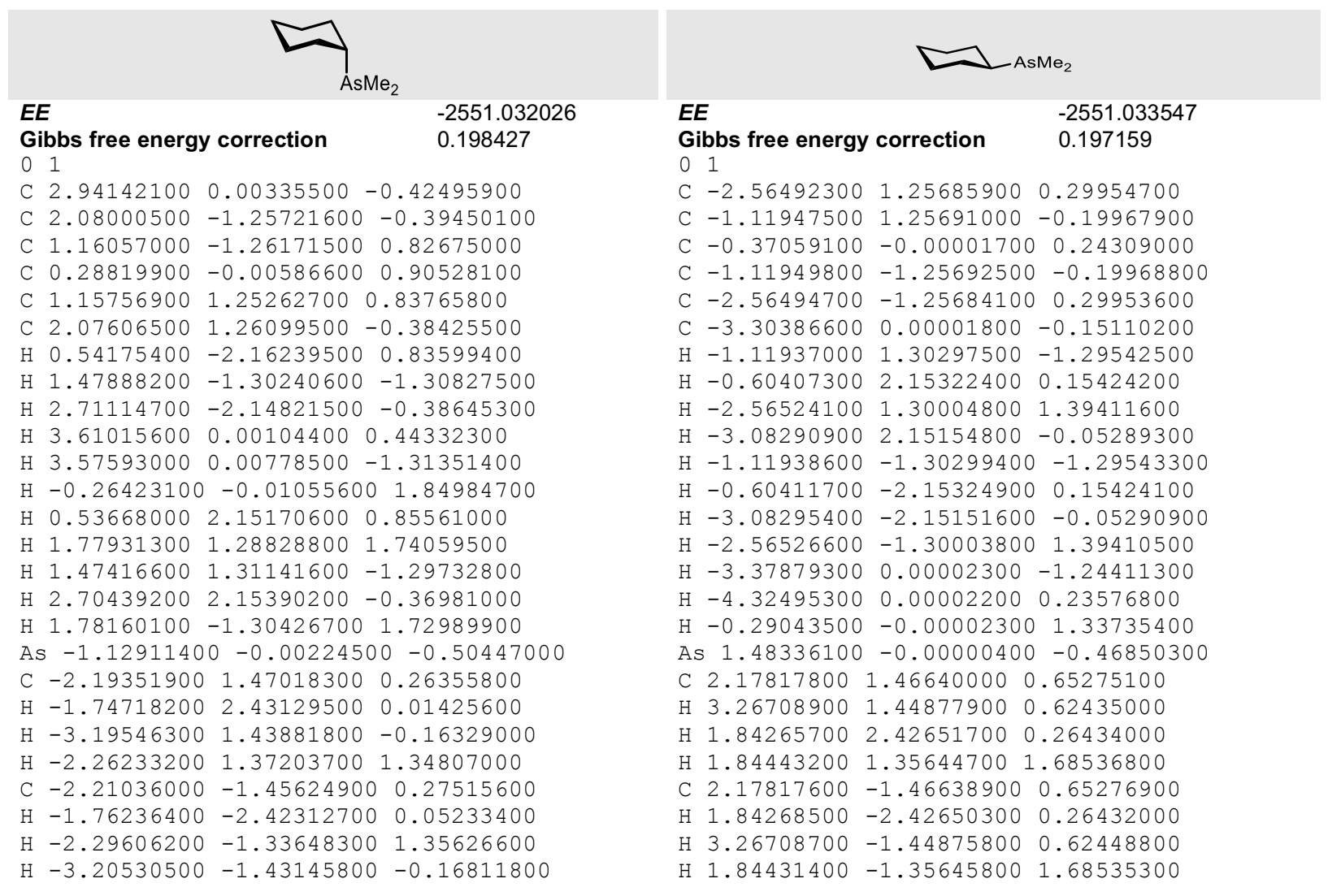


ANTIMONY

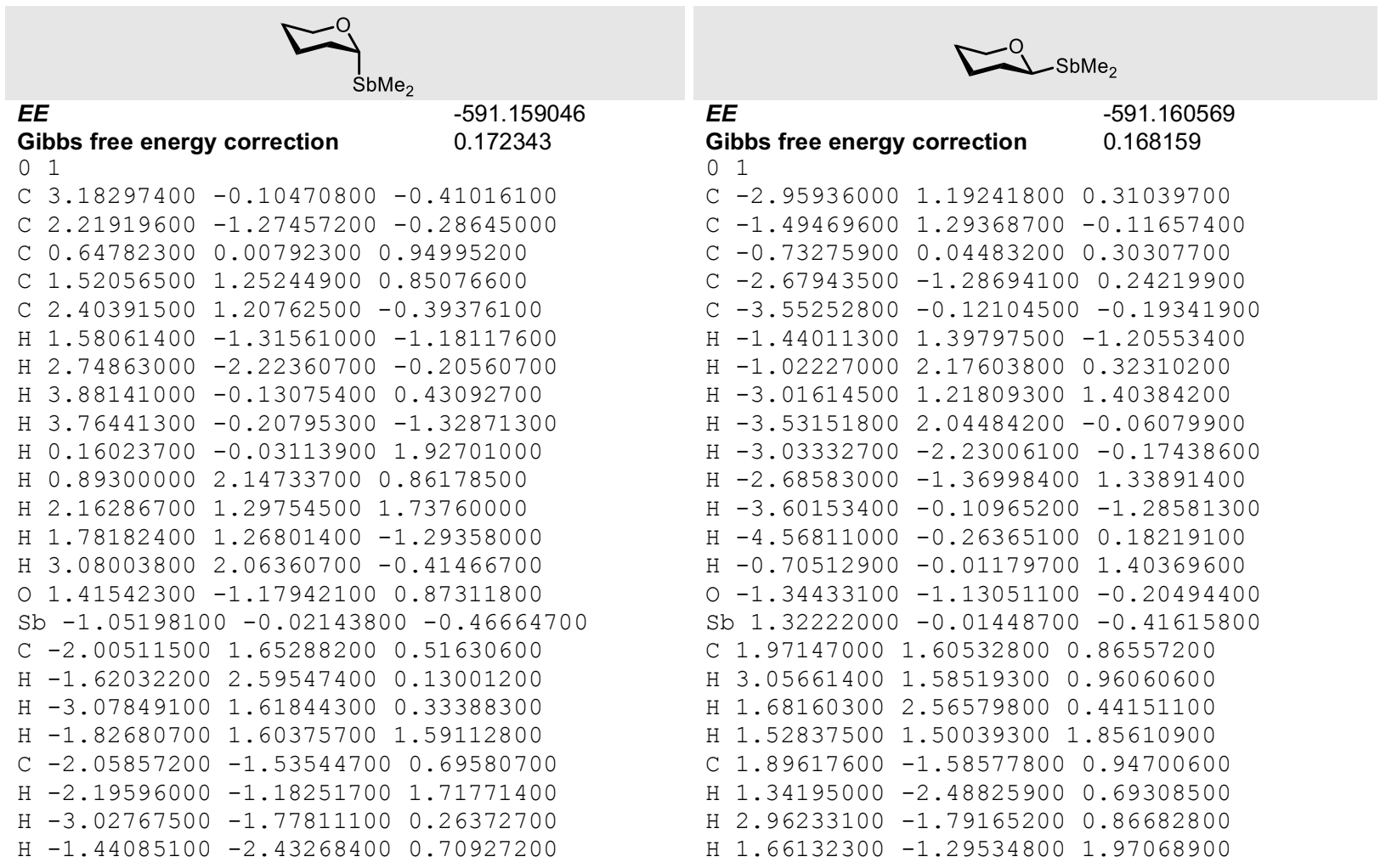

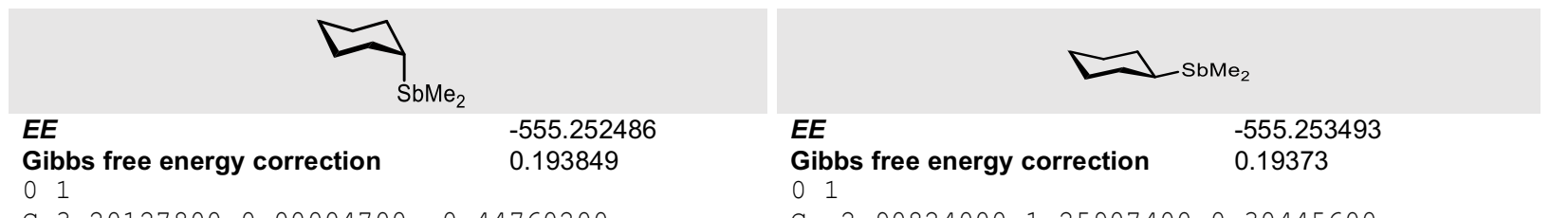

C $3.20127800 \quad 0.00004700-0.44760200$

C $2.34144100-1.26020400-0.37502100$

C $1.47164100-1.25862200 \quad 0.88204100$

C $0.60787300 \quad-0.00008200 \quad 0.98807400$

C $1.47158400 \quad 1.25851100 \quad 0.88215800$

C $2.341379001 .26024800-0.37490700$

H $0.85144600 \quad-2.15868500 \quad 0.91810800$

$\mathrm{H} \quad 1.70494300-1.31055200-1.26518300$

$\begin{array}{llll}\mathrm{H} & 2.97268300 & -2.15091100 & -0.39038700\end{array}$

H $3.90577200 \quad 0.00002600 \quad 0.39155300$

H $3.79812800 \quad 0.00010400-1.36176100$

$\mathrm{H} \quad 0.08750000 \quad-0.00013700 \quad 1.95029700$

$\mathrm{H} \quad 0.85134800 \quad 2.15854200 \quad 0.91831400$

$\mathrm{H} \quad 2.13198800 \quad 1.30519500 \quad 1.75776700$

$\mathrm{H} \quad 1.70487800 \quad 1.31064500-1.26506600$

$\mathrm{H} \quad 2.97257700 \quad 2.15098800 \quad-0.39019400$

H $2.13204200-1.305359001 .75765100$

Sb $-1.03502100-0.00002500-0.45752700$

C $-2.10052300 \quad 1.58717700 \quad 0.55274100$

$\mathrm{H}-1.67714700 \quad 2.55661700 \quad 0.29439100$

$\mathrm{H}-3.14651800 \quad 1.57767400 \quad 0.24856500$

$\mathrm{H}-2.04122200 \quad 1.44493300 \quad 1.63201000$

C $-2.10077500-1.58696300 \quad 0.55289700$

$\mathrm{H}-1.67739400 \quad-2.55647300 \quad 0.29481500$

$\mathrm{H}-2.04164100-1.44450100 \quad 1.63214600$

$\mathrm{H}-3.14672000-1.57748200 \quad 0.24854900$

01

$\begin{array}{llll}\text { C }-2.90824000 & 1.25907400 & 0.30445600\end{array}$

C $-1.45362900 \quad 1.25890300 \quad-0.16930700$

C $-0.71418100 \quad-0.00017700 \quad 0.28436600$

C $-1.45385700-1.25897100 \quad-0.16971300$

C $-2.90846000-1.25904000 \quad 0.30407200$

C $-3.63704300 \quad 0.00015100-0.15954100$

$\mathrm{H}-1.43996200 \quad 1.31053800-1.26517200$

$\mathrm{H}-0.94334400 \quad 2.15517000 \quad 0.19421000$

$\mathrm{H}-2.92651800 \quad 1.30261100 \quad 1.39867000$

$\mathrm{H}-3.42244100 \quad 2.15208200-0.05780600$

$\mathrm{H}-1.44021400-1.31023600-1.26559200$

$\mathrm{H}-0.94373000-2.15546000 \quad 0.19348400$

$\mathrm{H}-3.42282200-2.15184600-0.05846000$

$\mathrm{H}-2.92673600-1.30290800 \quad 1.39827200$

$\mathrm{H}-3.68966200 \quad 0.00032200 \quad-1.25388100$

$\mathrm{H}-4.66590400 \quad 0.00018600 \quad 0.20596800$

$\mathrm{H}-0.65492400-0.00035000 \quad 1.37974100$

$\mathrm{Sb} 1.34476600-0.00017100-0.41186700$

C $1.98101500 \quad 1.58767200 \quad 0.91060500$

H $3.06680800 \quad 1.57996300 \quad 0.99829600$

H $1.67499700 \quad 2.55629500 \quad 0.51846200$

H $1.54268300 \quad 1.44447200 \quad 1.89837300$

C $1.98149600-1.58673200 \quad 0.91190300$

H $1.67390900-2.55556700 \quad 0.52149300$

H $3.06742000-1.58004600 \quad 0.99798600$

$\mathrm{H} 1.54474900-1.441779001 .90011300$ 
BISMUTH

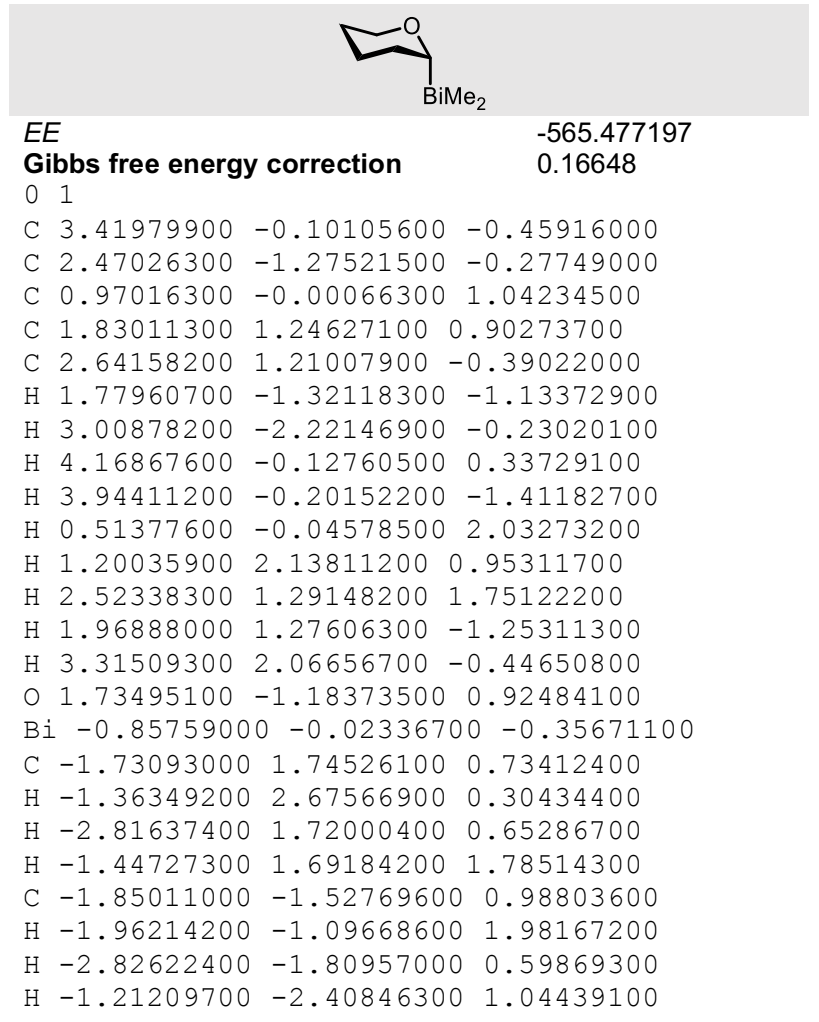

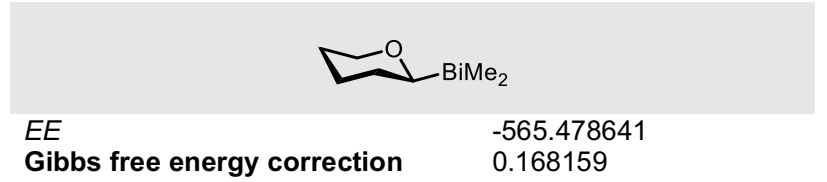

Gibbs free energy correction $\quad 0.168159$

$\begin{array}{llll}\text { C }-3.30689600 & 1.19127200 & 0.30862100\end{array}$

C $-1.82491300 \quad 1.29460600-0.05771100$

$\begin{array}{llll}\text { C }-1.08260000 & 0.04142100 & 0.37640200\end{array}$

$\begin{array}{llll}\text { C }-3.02544800 & -1.28799900 & 0.22343900\end{array}$

C $-3.87936200-0.11601700-0.23383500$

$\mathrm{H}-1.730556001 .41341100-1.14299400$

$\mathrm{H}-1.36820100 \quad 2.17072300 \quad 0.40989800$

$\mathrm{H}-3.40718500 \quad 1.20468800 \quad 1.39902200$

$\mathrm{H}-3.86305400 \quad 2.04843700-0.07618700$

$\mathrm{H}-3.36337500-2.22601400-0.21764300$

$\mathrm{H}-3.07576700-1.38336100 \quad 1.31789800$

$\mathrm{H}-3.88323000-0.09195300-1.32723900$

$\mathrm{H}-4.90988700 \quad-0.26222900 \quad 0.09725900$

$\mathrm{H}-1.08209000-0.02895400 \quad 1.47542800$

$\begin{array}{llll}0 & -1.67277900 & -1.12742000 & -0.16802800\end{array}$

Bi $1.07170900-0.02399100-0.31205800$

C $1.64942500 \quad 1.71187800 \quad 1.00929800$

H $2.72198700 \quad 1.68796400 \quad 1.19548700$

H $1.39451000 \quad 2.65373800 \quad 0.52624900$

$\mathrm{H} 1.11898700 \quad 1.63279500 \quad 1.95753800$

C $1.60663700-1.56335500 \quad 1.23645100$

$\mathrm{H} \quad 1.05057000 \quad-2.47460500 \quad 1.02288400$

H $2.67397400-1.77461900 \quad 1.20968600$

H $1.33261500-1.19021300 \quad 2.22172600$

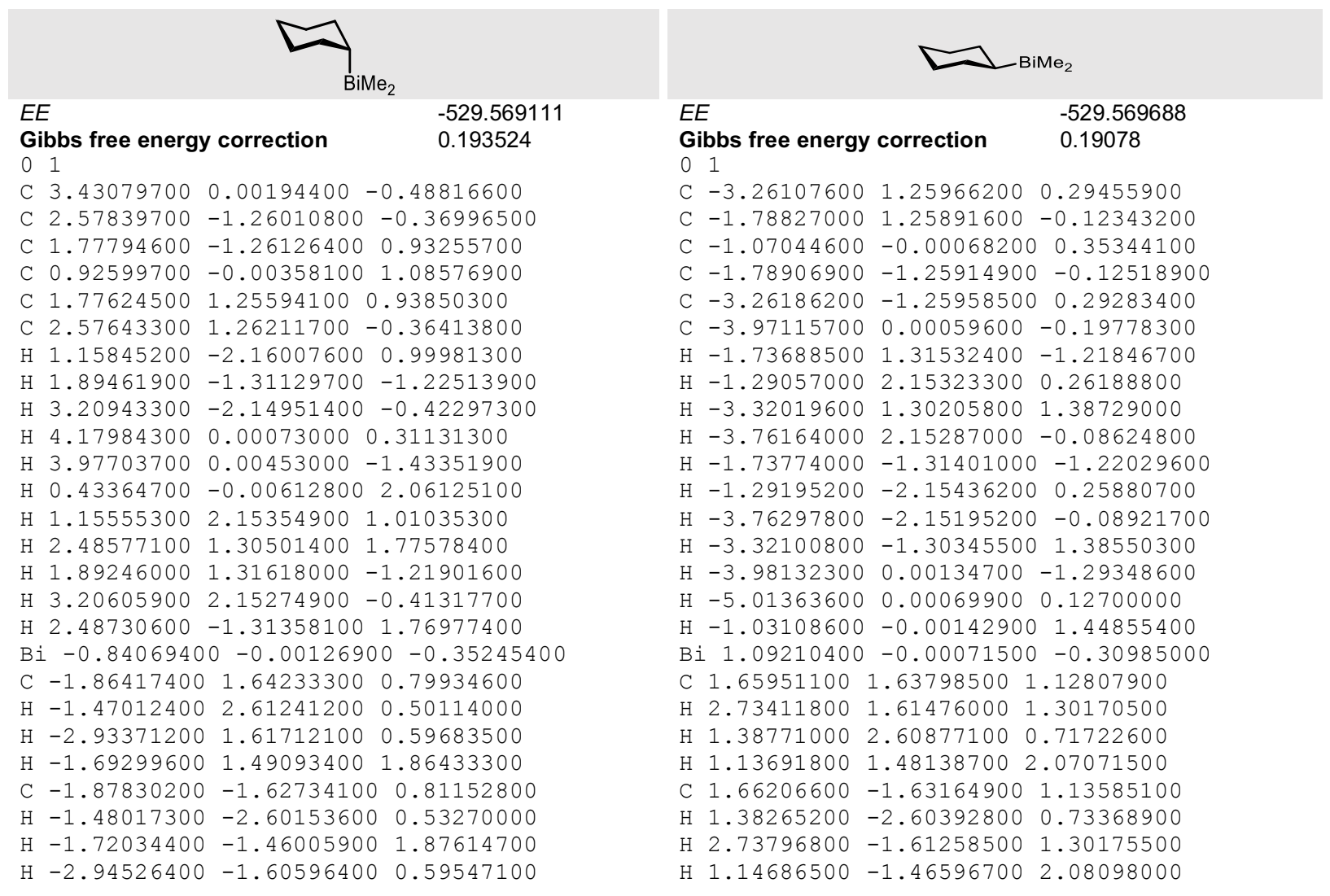


2.9. Group 16

OXYGEN
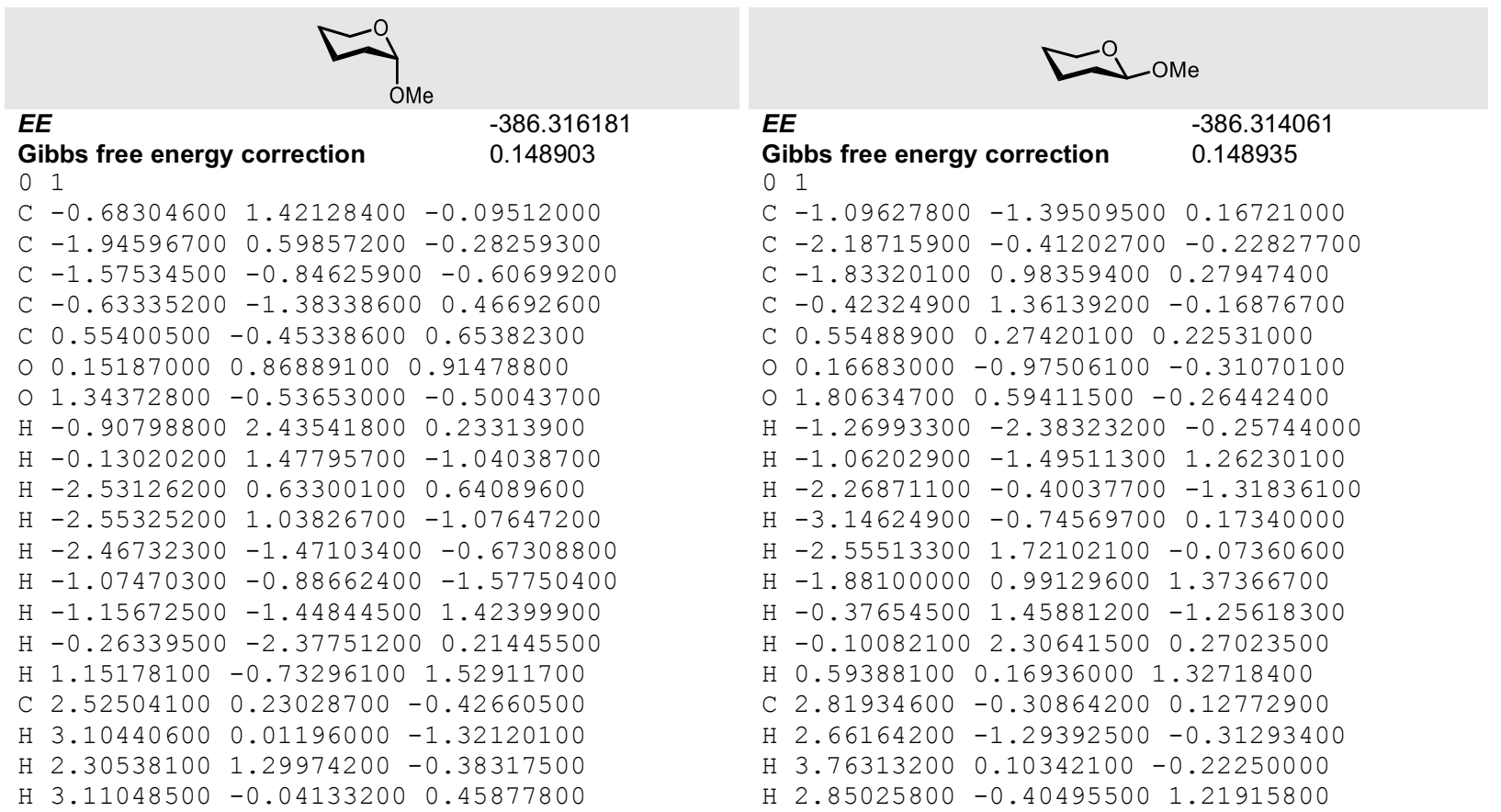

01

C $-1.09627800-1.39509500 \quad 0.16721000$

C $-2.18715900-0.41202700 \quad-0.22827700$

C $-1.83320100 \quad 0.98359400 \quad 0.27947400$

C $-0.42324900 \quad 1.36139200-0.16876700$

C $0.55488900 \quad 0.27420100 \quad 0.22531000$

$\begin{array}{lllll}0 & 0.16683000 & -0.97506100 & -0.31070100\end{array}$

$\begin{array}{llll}0 & 1.80634700 & 0.59411500 & -0.26442400\end{array}$

$\mathrm{H}-1.26993300-2.38323200-0.25744000$

$\mathrm{H}-1.06202900-1.49511300 \quad 1.26230100$

$\mathrm{H}-2.26871100-0.40037700-1.31836100$

$\mathrm{H}-3.14624900-0.74569700 \quad 0.17340000$

$\mathrm{H}-2.55513300 \quad 1.72102100-0.07360600$

$\mathrm{H}-1.88100000 \quad 0.99129600 \quad 1.37366700$

$\mathrm{H}-0.37654500 \quad 1.45881200-1.25618300$

$\mathrm{H}-0.10082100 \quad 2.30641500 \quad 0.27023500$

$\begin{array}{lllll}\mathrm{H} & 0.59388100 & 0.16936000 & 1.32718400\end{array}$

$\begin{array}{llll}\text { C } 2.81934600 & -0.30864200 & 0.12772900\end{array}$

H $2.66164200-1.29392500-0.31293400$

H $3.76313200 \quad 0.10342100-0.22250000$

H $2.85025800 \quad-0.40495500 \quad 1.21915800$

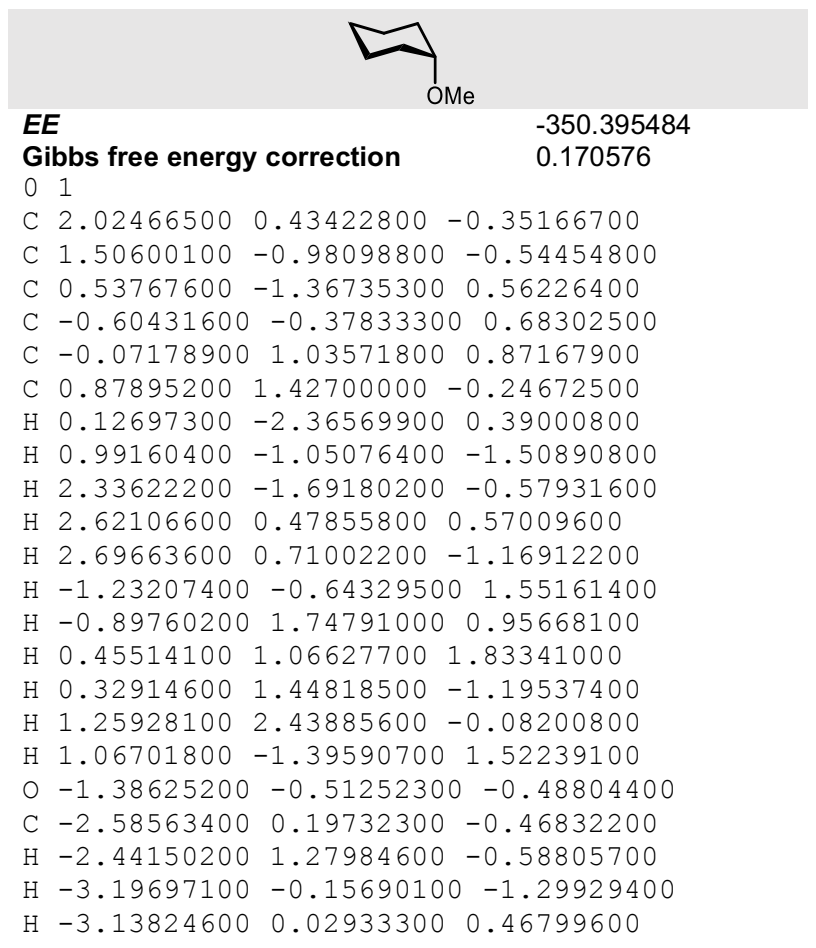

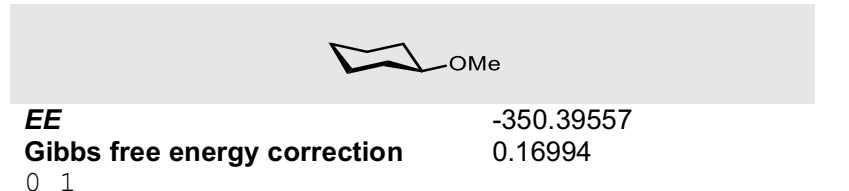

$\begin{array}{llll}\text { C } 2.27357200 & 0.26083300 & -0.21091000\end{array}$

$\begin{array}{llll}\text { C } 1.33564900 & 1.38089400 & 0.20290400\end{array}$

C $-0.08313500 \quad 1.11988700 \quad-0.27755500$

C $-0.59092700 \quad-0.22274300 \quad 0.21946700$

C $0.34642800-1.33758300-0.18764000$

$\begin{array}{llll}\text { C } 1.76441900 & -1.07924000 & 0.29021000\end{array}$

$\mathrm{H}-0.74494100 \quad 1.92649500 \quad 0.05087000$

$\mathrm{H} 1.33585700 \quad 1.46167000 \quad 1.29802300$

H $1.690725002 .34337800-0.17504600$

H $2.34128800 \quad 0.23371900-1.30621300$

H $3.28458800 \quad 0.45077900 \quad 0.15999700$

$\mathrm{H}-0.64642900-0.18545500 \quad 1.32562300$

$\mathrm{H}-0.03928400-2.28647800 \quad 0.19425300$

$\mathrm{H} 0.32603000-1.40595000-1.28287400$

$\mathrm{H} 1.78517900-1.08376200 \quad 1.38793100$

H $2.42378500-1.88906600-0.03295800$

$\mathrm{H}-0.11444700 \quad 1.10507800-1.37508100$

$\begin{array}{lllll}0 & -1.86328900 & -0.55056200 & -0.28974900\end{array}$

C $-2.90157500 \quad 0.26249700 \quad 0.16494400$

$\mathrm{H}-3.84249400 \quad-0.20644700 \quad-0.12535600$

$\mathrm{H}-2.88552700 \quad 0.36304700 \quad 1.26028500$

H $-2.87460200 \quad 1.27020700-0.26997300$ 


\section{SULFUR}

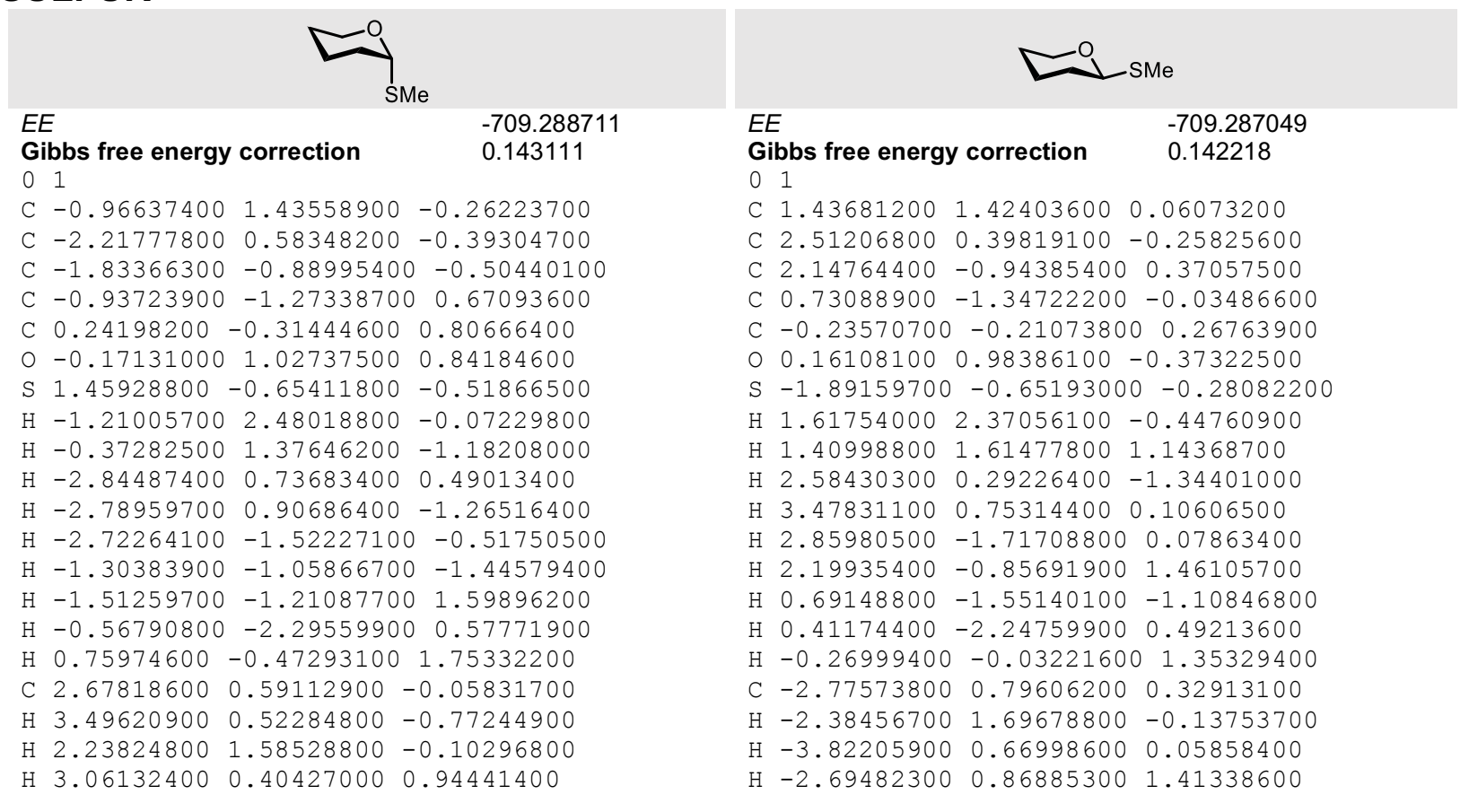

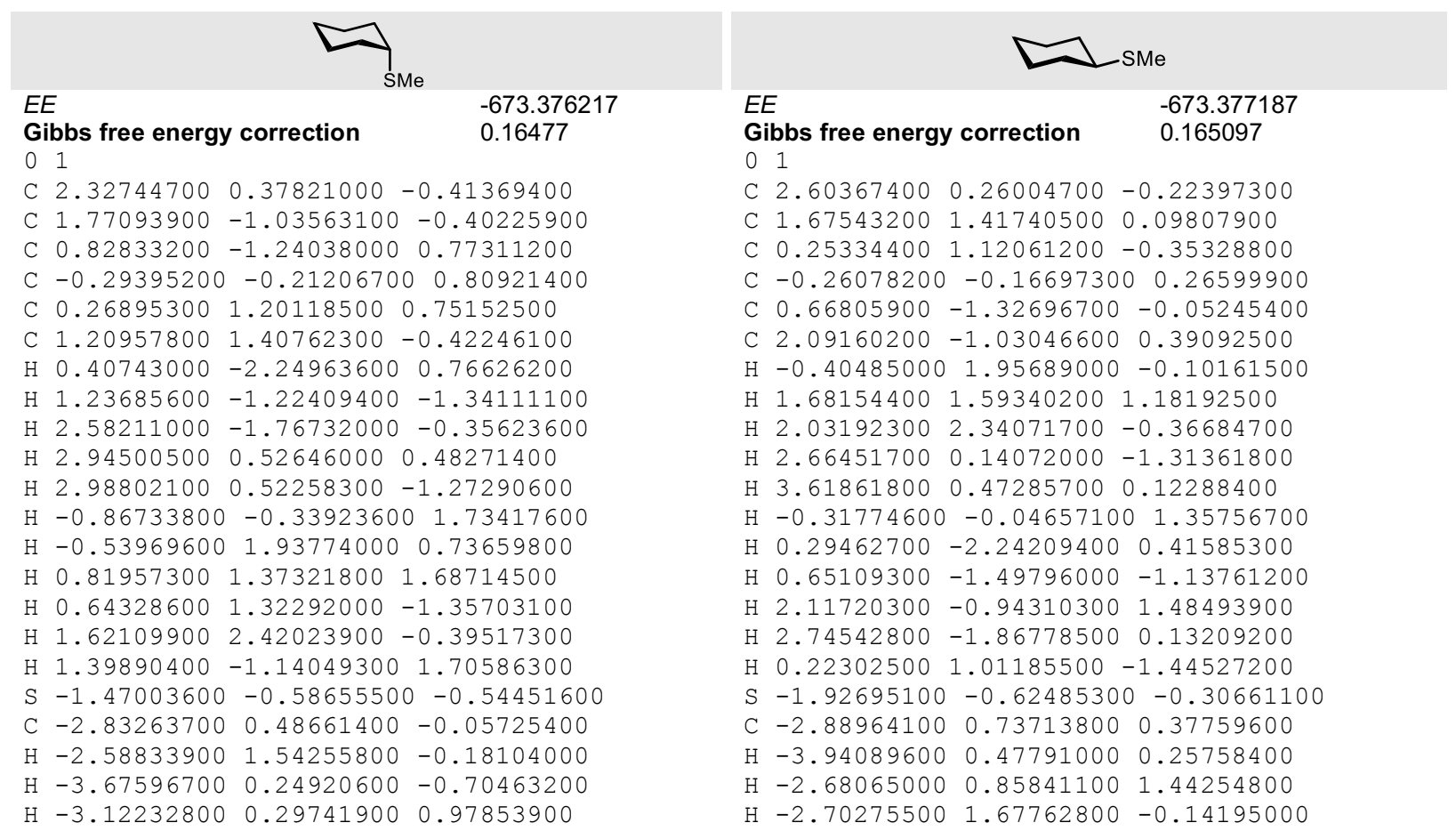




\section{SELENIUM}

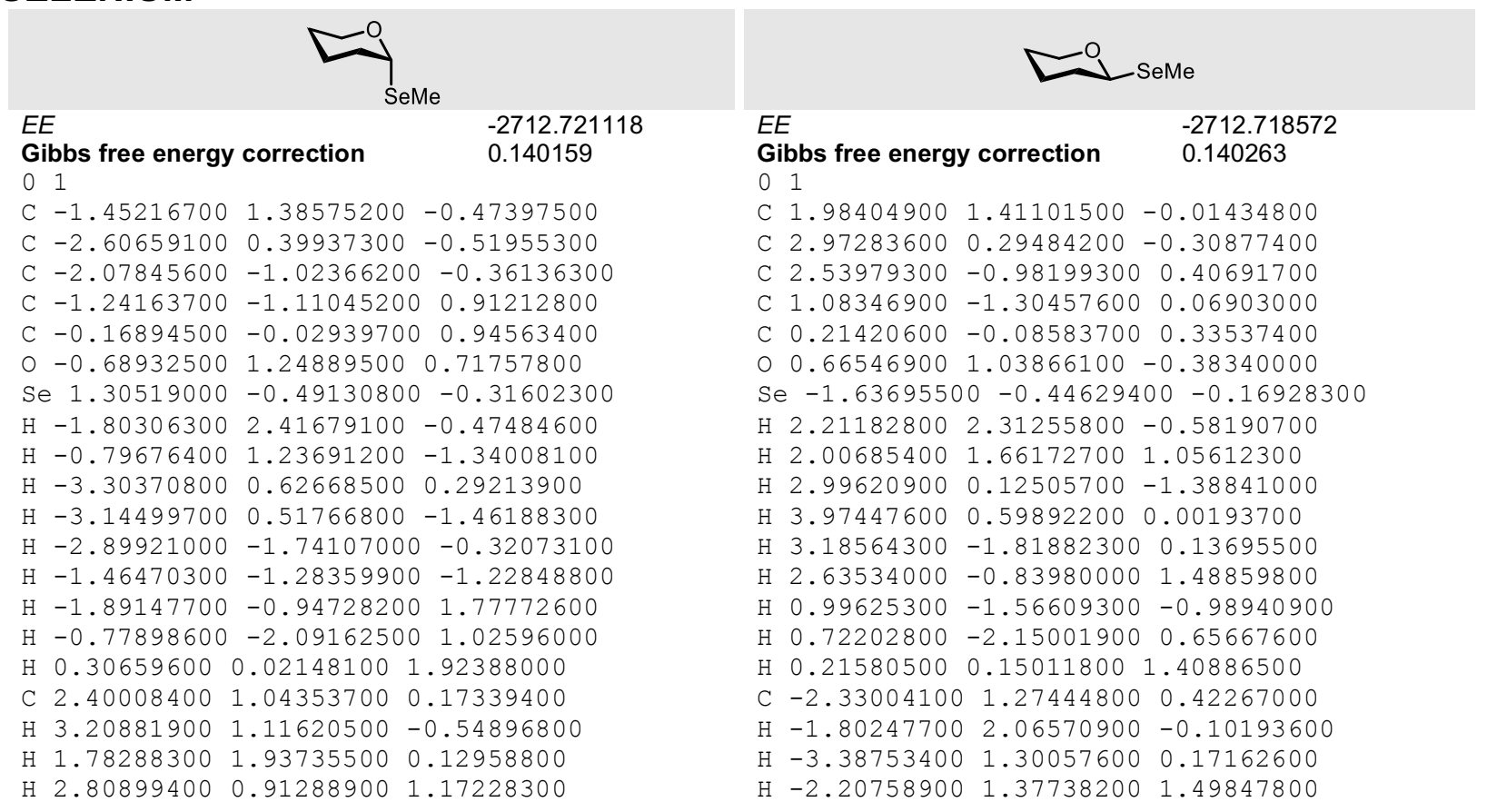

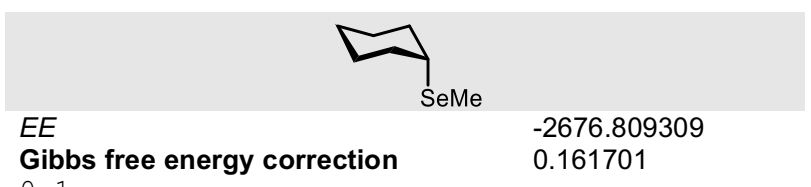

01

$\begin{array}{llll}\text { C } 2.69415900 & 0.20947900 & -0.49274500\end{array}$

C $2.02848500-1.12718400-0.21019200$

C $1.14953800-1.04387300 \quad 1.02706900$

C $0.11714300 \quad 0.06818500 \quad 0.93292400$

C $0.77324500 \quad 1.39733300 \quad 0.59445400$

$\begin{array}{lllll}\text { C } 1.66407200 & 1.31745200 & -0.63342300\end{array}$

$\mathrm{H} 0.65731800 \quad-2.00037000 \quad 1.22395000$

$\mathrm{H} 1.42324000-1.42486700-1.07578600$

$\mathrm{H} 2.78036000-1.91020600-0.07892600$

$\begin{array}{lllll}\text { H } 3.37264300 & 0.45139100 & 0.33666800\end{array}$

$\mathrm{H} 3.31351000 \quad 0.14710800-1.39165000$

$\mathrm{H}-0.41817400 \quad 0.14932200 \quad 1.88373800$

$\mathrm{H} \quad 0.01754300 \quad 2.17918500 \quad 0.47362100$

$\mathrm{H} 1.38510400 \quad 1.69363600 \quad 1.45903800$

$\mathrm{H} 1.04330000 \quad 1.12809800-1.51651500$

$\begin{array}{lllll}\mathrm{H} & 2.15616600 & 2.28011800 & -0.79699300\end{array}$

$\begin{array}{llll}\mathrm{H} & 1.78753400 & -0.83213300 & 1.89629100\end{array}$

Se $-1.29009200-0.44737200-0.35649300$

C $-2.60412000 \quad 0.85740300 \quad 0.23259300$

$\mathrm{H}-2.27714600 \quad 1.87269900 \quad 0.01181700$

$\mathrm{H}-3.52612300 \quad 0.65608200 \quad-0.31001900$

$\mathrm{H}-2.78728500 \quad 0.74781400 \quad 1.30143900$

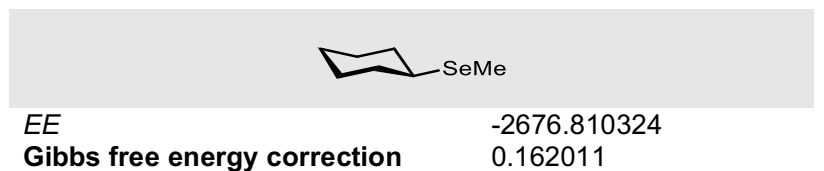

01

C $3.05764400 \quad 0.16740800-0.26720300$

C $2.21709400 \quad 1.40118400 \quad 0.01102300$

C $0.763485001 .17027000-0.37571800$

$\begin{array}{lllll}\text { C } 0.19635500 & -0.04216800 & 0.33765700\end{array}$

C $1.03426200-1.27863300 \quad 0.06470800$

C $2.48904300-1.04809800 \quad 0.44386000$

$\mathrm{H} 0.16680800 \quad 2.06045700-0.15550300$

H $2.27291200 \quad 1.64134800 \quad 1.08087200$

H $2.61455900 \quad 2.26878200-0.52292900$

H $3.07070700-0.02205700-1.34861700$

H $4.09614600 \quad 0.33419100 \quad 0.03179800$

H $0.17056900 \quad 0.14574600 \quad 1.41919900$

H $0.62428000 \quad-2.13902600 \quad 0.60177500$

H $0.97265300-1.51544700-1.00671300$

H $2.55812200-0.89655500 \quad 1.52893500$

H $3.08028500-1.93975600 \quad 0.21747600$

$\mathrm{H} \quad 0.68992000 \quad 1.00244700-1.45823000$ $\mathrm{Se}-1.65255000-0.43487000-0.19153100$

C $-2.46354900 \quad 1.19338900 \quad 0.49398000$

$\mathrm{H}-3.54290900 \quad 1.06564300 \quad 0.43631000$

$\mathrm{H}-2.17791200 \quad 1.33900900 \quad 1.53577900$

$\mathrm{H}-2.17545600 \quad 2.06069100-0.09794000$ 
TELLURIUM
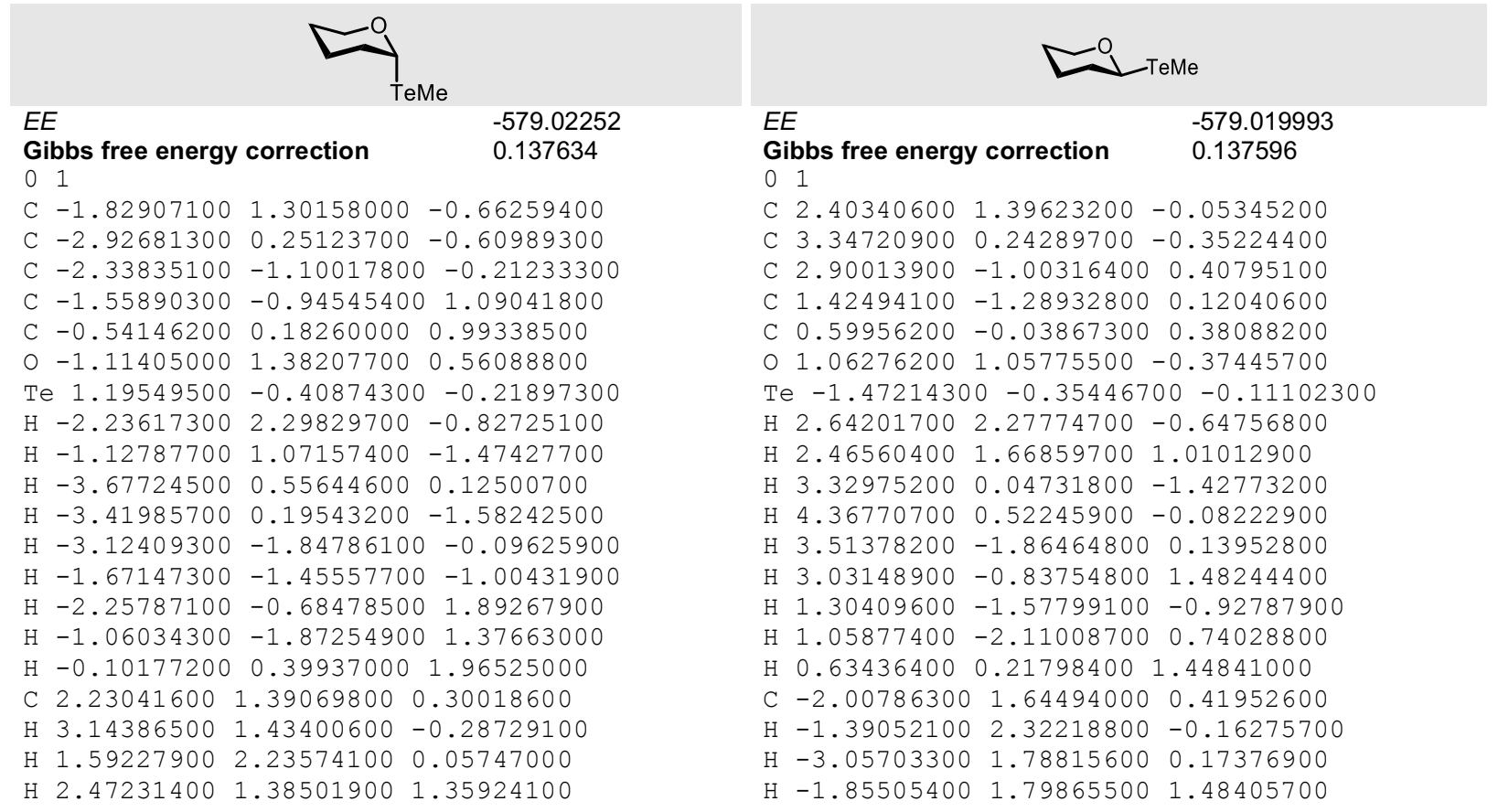

Gibss free energy correction $\quad 0.137596$

01

C $2.403406001 .39623200-0.05345200$

C $3.34720900 \quad 0.24289700-0.35224400$

C $2.90013900-1.00316400 \quad 0.40795100$

C $1.42494100-1.28932800 \quad 0.12040600$

C $0.59956200-0.03867300 \quad 0.38088200$

$\begin{array}{llll}0 & 1.06276200 & 1.05775500 & -0.37445700\end{array}$

$\mathrm{Te}-1.47214300-0.35446700-0.11102300$

H $2.64201700 \quad 2.27774700 \quad-0.64756800$

$\mathrm{H} 2.465604001 .668597001 .01012900$

H $3.32975200 \quad 0.04731800-1.42773200$

$\mathrm{H} \quad 4.36770700 \quad 0.52245900 \quad-0.08222900$

$\mathrm{H} 3.51378200-1.86464800 \quad 0.13952800$

H $3.03148900-0.83754800 \quad 1.48244400$

$\mathrm{H} 1.30409600-1.57799100-0.92787900$

$\mathrm{H} \quad 1.05877400 \quad-2.11008700 \quad 0.74028800$

$\mathrm{H} \quad 0.63436400 \quad 0.21798400 \quad 1.44841000$

C $-2.00786300 \quad 1.64494000 \quad 0.41952600$

$\mathrm{H}-1.39052100 \quad 2.32218800 \quad-0.16275700$

$\mathrm{H}-3.05703300 \quad 1.78815600 \quad 0.17376900$

$\mathrm{H}-1.85505400 \quad 1.79865500 \quad 1.48405700$

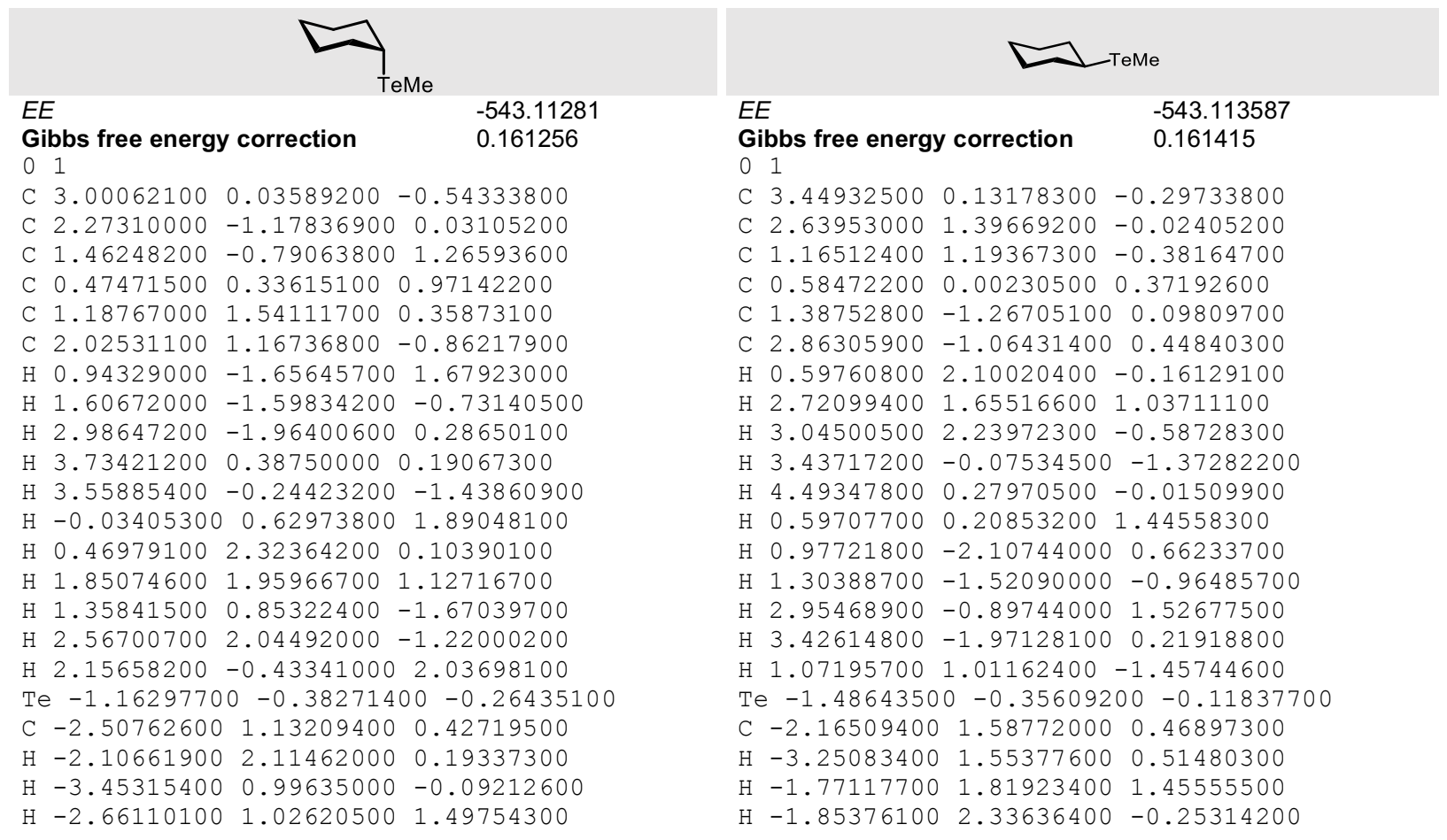


POLONIUM

\begin{tabular}{|c|c|c|}
\hline$E E$ & -548.757682 & -548.754714 \\
\hline $\begin{array}{l}\text { Gibbs free energy } \\
01\end{array}$ & correction & $\begin{array}{l}\text { Gibbs free energy correction } \\
01\end{array}$ \\
\hline$C-2.19989600$ & $1.22281900-0.77718500$ & C $2.842079001 .37387200-0.06951100$ \\
\hline$C-3.23986200$ & $0.12103700-0.65570400$ & C $3.73660900 \quad 0.18430000-0.37804300$ \\
\hline$C-2.59268800$ & $-1.15190800-0.11654900$ & C $3.26096200 \quad-1.03907400 \quad 0.40118100$ \\
\hline$C-1.86961000$ & -0.830535001 .18797500 & C $1.77022100-1.27284000 \quad 0.14407300$ \\
\hline$C-0.91457400$ & $0.33960500 \quad 1.01403800$ & C $0.99882100 \quad 0.00839000 \quad 0.41223500$ \\
\hline $0-1.52899700$ & $1.45795600 \quad 0.45109800$ & $01.48273800 \quad 1.08156600-0.35938300$ \\
\hline Po 0.96620000 & $-0.29054600-0.13386600$ & Po $-1.17644300-0.24194900-0.06859600$ \\
\hline $\mathrm{H}-2.65416900$ & $2.17498100-1.04802600$ & $\mathrm{H} \quad 3.09995200 \quad 2.24178300-0.67549700$ \\
\hline $\mathrm{H}-1.45958900$ & $0.95810200-1.54240900$ & H 2.93608100 $1.65109000 \quad 0.99043500$ \\
\hline $\mathrm{H}-4.02966000$ & $0.44871400 \quad 0.02627700$ & $\mathrm{H} 3.69042000-0.01860600-1.45138500$ \\
\hline $\mathrm{H}-3.69720200$ & $-0.05077500-1.63211000$ & $\mathrm{H} \quad 4.77204100 \quad 0.42855700-0.13121600$ \\
\hline $\mathrm{H}-3.33979200$ & $-1.93043000 \quad 0.04361500$ & $\mathrm{H} \quad 3.83871300 \quad-1.92384700 \quad 0.12840300$ \\
\hline $\mathrm{H}-1.88001400$ & $-1.53820800-0.85271300$ & $\mathrm{H} \quad 3.41822400 \quad-0.86962900 \quad 1.47150900$ \\
\hline $\mathrm{H}-2.61221000$ & -0.537810001 .93942000 & $\mathrm{H} 1.62090100-1.56399400-0.90005100$ \\
\hline $\mathrm{H}-1.33193600$ & $-1.69589200 \quad 1.57808700$ & $\mathrm{H} \quad 1.38779700 \quad-2.07656400 \quad 0.77687700$ \\
\hline $\mathrm{H}-0.50551500$ & $0.66906000 \quad 1.96660200$ & $\mathrm{H} 1.05239600 \quad 0.27015100 \quad 1.47712500$ \\
\hline C 1.896555001 & $1.68015800 \quad 0.33953100$ & $\begin{array}{llll}C-1.58983900 & 1.89193000 & 0.41066300\end{array}$ \\
\hline H 2.70447600 & $1.85035500-0.36619000$ & $\mathrm{H}-0.90814000 \quad 2.49321300-0.18226100$ \\
\hline $\mathrm{H} \quad 1.12344900$ & $2.43338600 \quad 0.21378600$ & $\mathrm{H}-2.62083000 \quad 2.09940100 \quad 0.13683400$ \\
\hline H 2.27382400 & $1.67364200 \quad 1.35700000$ & $\mathrm{H}-1.44136000 \quad 2.06017200 \quad 1.47274300$ \\
\hline
\end{tabular}

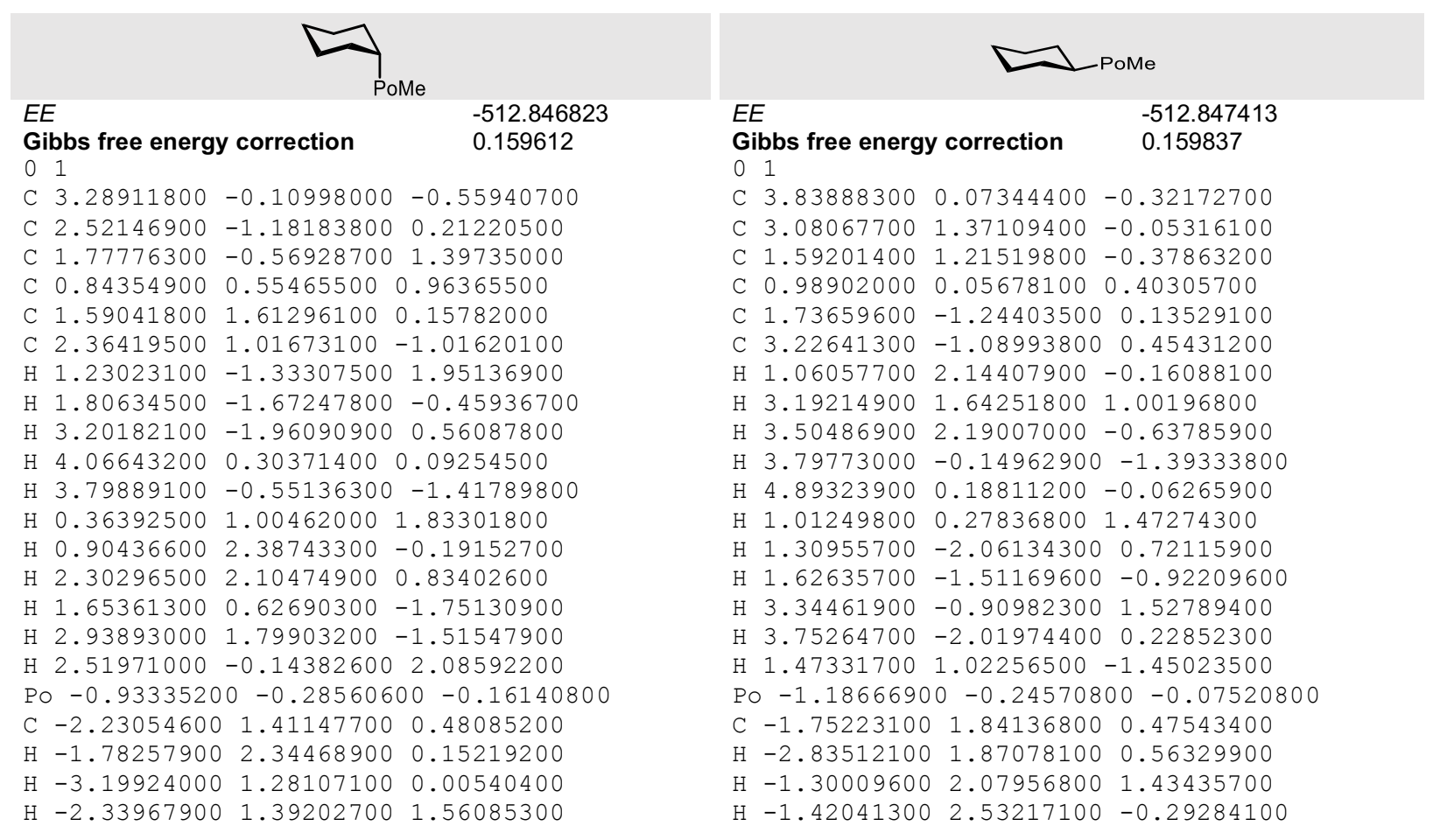




\section{FLUORINE}

EE
Gibbs free energy correction

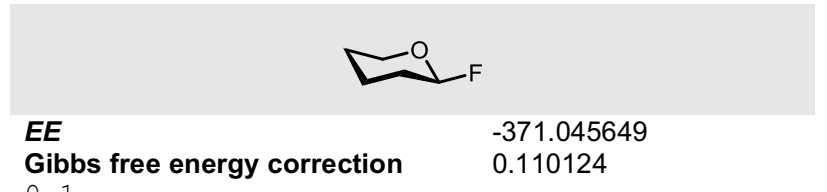

01

C $-1.79674900-0.09135200 \quad-0.28788000$

C $-0.96592800-1.27231200 \quad 0.18976800$

$\begin{array}{lllll}\text { C } & 0.98594200 & -0.00559500 & 0.30779100\end{array}$

C $0.30415100 \quad 1.25867400-0.16692900$

C $-1.17596100 \quad 1.21046900 \quad 0.21268600$

$\mathrm{H}-1.01278200-1.34533500 \quad 1.28606500$

$\mathrm{H}-1.31978200 \quad-2.21331300 \quad-0.22777500$

$\mathrm{H}-1.81870300-0.09609500-1.38063700$

$\mathrm{H}-2.82364700 \quad-0.19857000 \quad 0.06670700$

$\mathrm{H} \quad 0.80432900 \quad 2.12454200 \quad 0.26900300$

$\mathrm{H} \quad 0.42355500 \quad 1.30300700-1.25232800$

$\mathrm{H}-1.27326000 \quad 1.26124000 \quad 1.30242700$

H $-1.699645002 .07673900-0.19220500$

$00.38604100-1.14665900-0.21844100$

F 2.28043300 -0.03006300 - 0.11816900

$\mathrm{H} \quad 0.99897800 \quad-0.06767400 \quad 1.40717500$

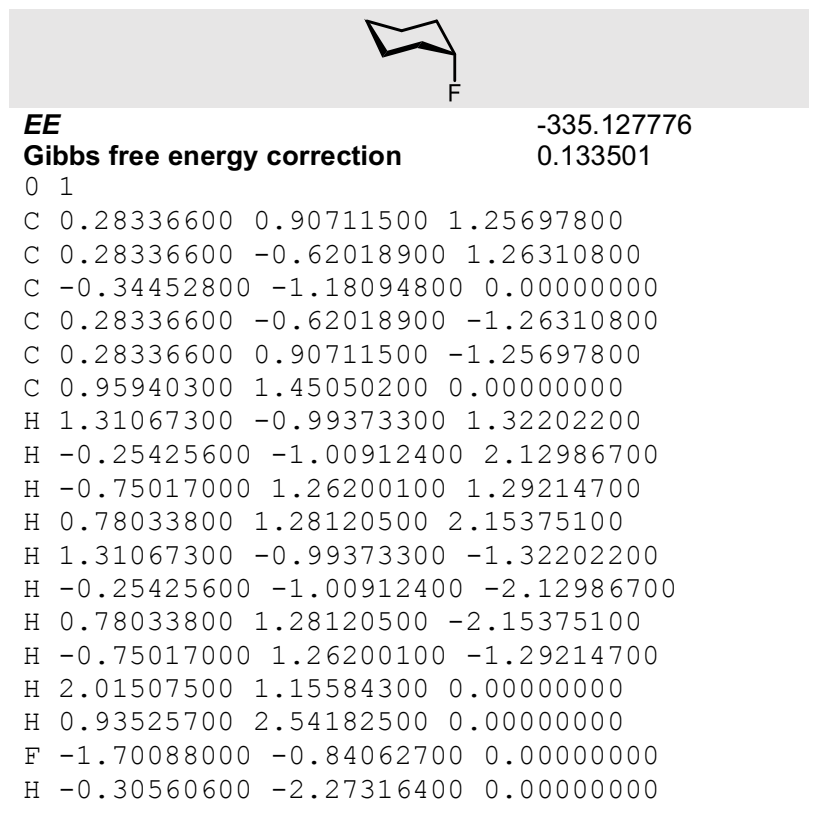

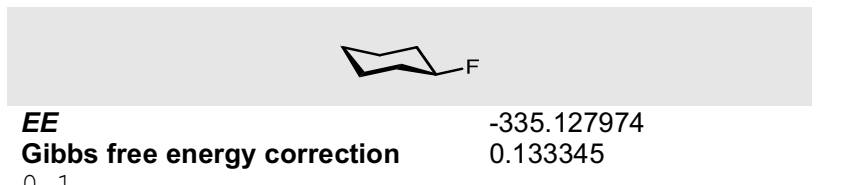

01

C $-0.08807300-1.14949900 \quad 1.25914500$

$\begin{array}{llll}\text { C }-0.08807300 & 0.37895200 & 1.25541800\end{array}$

$\begin{array}{llll}\text { C } & 0.57892700 & 0.90178700 & 0.00000000\end{array}$

C $-0.08807300 \quad 0.37895200-1.25541800$

C $-0.08807300-1.14949900-1.25914500$

C $-0.75337200-1.70179200 \quad 0.00000000$

$\mathrm{H}-1.11628000 \quad 0.75373300 \quad 1.27402700$

H $0.42266000 \quad 0.77756300 \quad 2.13358400$

H $0.94439400-1.51173100 \quad 1.30845100$

$\mathrm{H}-0.59231900-1.51901000 \quad 2.15341500$

$\mathrm{H}-1.11628000 \quad 0.75373300 \quad-1.27402700$

H $0.42266000 \quad 0.77756300 \quad-2.13358400$

$\mathrm{H}-0.59231900-1.51901000-2.15341500$

H $0.94439400-1.51173100-1.30845100$

$\mathrm{H}-1.81145900-1.41971800 \quad 0.00000000$

$\mathrm{H}-0.71536600 \quad-2.79276300 \quad 0.00000000$

$\mathrm{H} 1.64098800 \quad 0.63057000 \quad 0.00000000$

F $0.52548300 \quad 2.29193400 \quad 0.00000000$ 


\section{CHLORINE}

\begin{tabular}{|c|c|}
\hline$E E$ & -731.398678 \\
\hline $\begin{array}{l}\text { Gibbs free energy correction } \\
01\end{array}$ & 0.107894 \\
\hline C $1.96408200-0.10696200$ & $0-0.38756400$ \\
\hline C $1.01227100-1.28493900$ & -0.27754500 \\
\hline$C-0.62846700 \quad 0.01552000$ & 0.82460000 \\
\hline C 0.204491001 .27953200 & 0.69610600 \\
\hline C 1.167411001 .19168600 & -0.48257200 \\
\hline $\mathrm{H} \quad 0.42555600-1.39101100$ & -1.19597500 \\
\hline H $1.53991500-2.21866500$ & -0.09232900 \\
\hline $\mathrm{H} 2.60835800-0.08490000$ & 0.49588400 \\
\hline H $2.60496200-0.24159500$ & -1.26085200 \\
\hline $\mathrm{H}-0.45635600 \quad 2.14216800$ & 0.62112700 \\
\hline H $0.77197300 \quad 1.36858400$ & 1.62808200 \\
\hline $\mathrm{H} \quad 0.60042200 \quad 1.20818800$ & -1.41669100 \\
\hline H 1.830197002 .05753900 & -0.48931500 \\
\hline $0 \quad 0.11890000-1.13795200$ & 0.82381400 \\
\hline $\mathrm{H}-1.22606100 \quad 0.00456400$ & 1.73129600 \\
\hline $\mathrm{Cl}-1.88052300-0.018252$ & $200-0.52058200$ \\
\hline
\end{tabular}

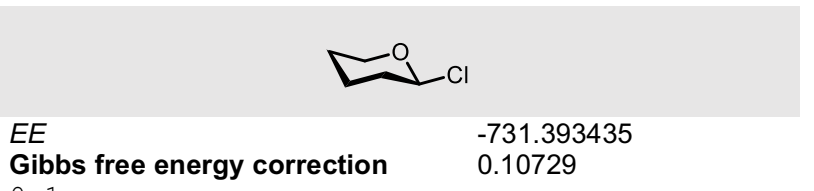

01

C $-2.18312200-0.10234600-0.32302600$

C $-1.36043400-1.27670100 \quad 0.18071700$

$\begin{array}{llll}\text { C } 0.57894000 & 0.00326700 & 0.35934500\end{array}$

C $-0.09543600 \quad 1.26555300-0.14175500$

C $-1.58626900 \quad 1.20340400 \quad 0.19486300$

$\mathrm{H}-1.43564700-1.34898800 \quad 1.27506200$

$\mathrm{H}-1.69341600-2.22084600-0.24707700$

$\mathrm{H}-2.17110400-0.10849500-1.41586400$

$\mathrm{H}-3.21986100-0.21592500 \quad-0.00045900$

H $0.38094900 \quad 2.13706400 \quad 0.30784200$

$\mathrm{H} \quad 0.05160900 \quad 1.31682900-1.22308800$

$\mathrm{H}-1.71556500 \quad 1.25551600 \quad 1.28104900$

$\mathrm{H}-2.10267000 \quad 2.06565600-0.22841500$

$00.00535600-1.14502200-0.18944600$

Cl $2.30557300-0.01358000-0.07697000$

$\mathrm{H} \quad 0.54605100 \quad-0.04884300 \quad 1.45414700$

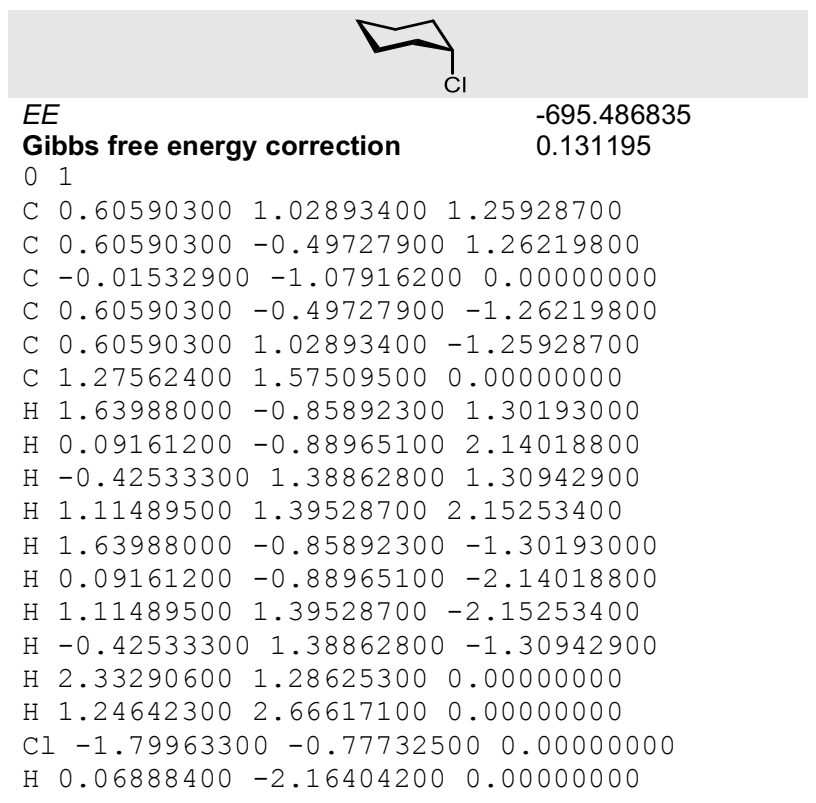

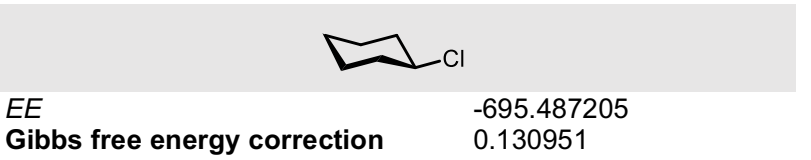

Gibbs free energy correction $\quad 0.130951$

01

C $-0.16535200-1.54541600 \quad 1.25769400$

C $-0.16535200 \quad-0.01609300 \quad 1.25819700$

C $0.50538500 \quad 0.50784100 \quad 0.00000000$

C $-0.16535200-0.01609300-1.25819700$

C $-0.16535200-1.54541600-1.25769400$

C $-0.83206600-2.09701600 \quad 0.00000000$

$\mathrm{H}-1.19386100 \quad 0.35671400 \quad 1.28227000$

$\begin{array}{lllll}\mathrm{H} & 0.34322400 & 0.37600200 & 2.13979400\end{array}$

$\mathrm{H} \quad 0.86715700-1.90738200 \quad 1.30727700$

$\mathrm{H}-0.67013300-1.91229600 \quad 2.15304800$

$\mathrm{H}-1.19386100 \quad 0.35671400-1.28227000$

H $0.34322400 \quad 0.37600200 \quad-2.13979400$

$\mathrm{H}-0.67013300-1.91229600-2.15304800$

$\mathrm{H} \quad 0.86715700-1.90738200-1.30727700$

$\mathrm{H}-1.88959800-1.81258300 \quad 0.00000000$

$\mathrm{H}-0.79672000-3.18798300 \quad 0.00000000$

$\mathrm{H} 1.56151800 \quad 0.23287300 \quad 0.00000000$

Cl $0.49179700 \quad 2.30675100 \quad 0.00000000$ 


\section{BROMINE}

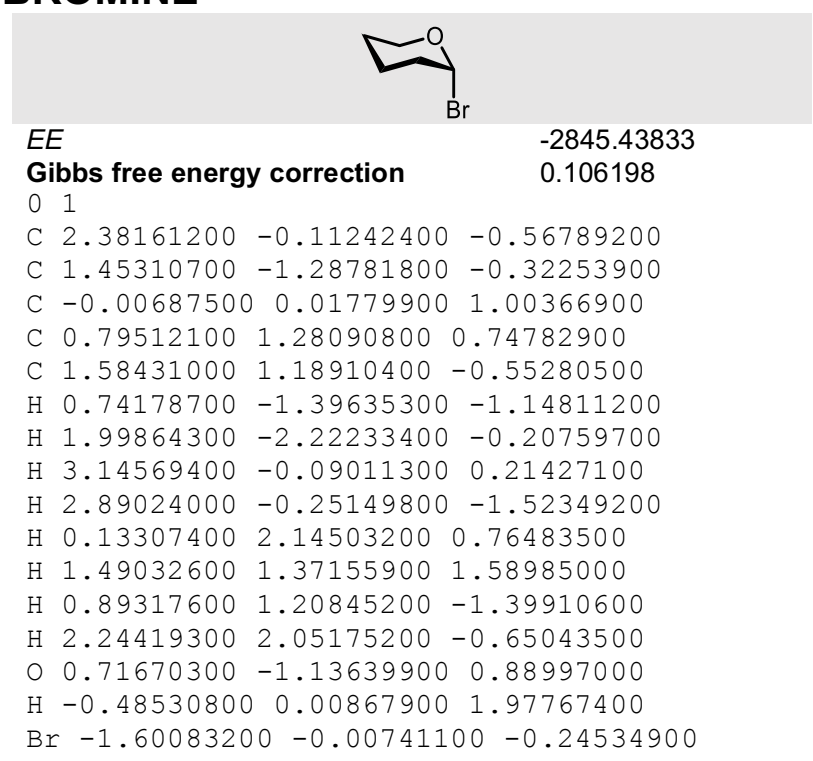

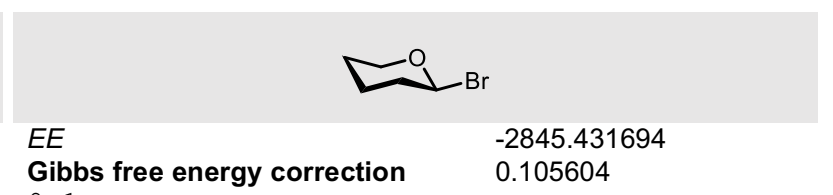

$0 \quad 1$

C $-2.75086000-0.10489000-0.34798700$

C $-1.93890500-1.27819500 \quad 0.17426900$

C $-0.00770700 \quad 0.00548900 \quad 0.39192500$

C $-0.67025700 \quad 1.26730100-0.12271900$

C $-2.16820100 \quad 1.20126000 \quad 0.18436000$

$\mathrm{H}-2.03688900-1.35040500 \quad 1.26658800$

$\mathrm{H}-2.25966600-2.22303000-0.26124100$

$\mathrm{H}-2.71319700-0.11034700-1.44026800$

$\mathrm{H}-3.79480600-0.22037900-0.05022400$

$\mathrm{H}-0.20552400 \quad 2.13975400 \quad 0.33652000$

$\mathrm{H}-0.50519800 \quad 1.32228700-1.20111800$

$\mathrm{H}-2.31971800 \quad 1.25204800 \quad 1.26763400$

$\mathrm{H}-2.67660300 \quad 2.06328100-0.24917100$

$0-0.56339500-1.14448300-0.16622900$

Br $1.89371800-0.00621800-0.04295800$

$\mathrm{H}-0.04577900-0.04550500 \quad 1.48554100$

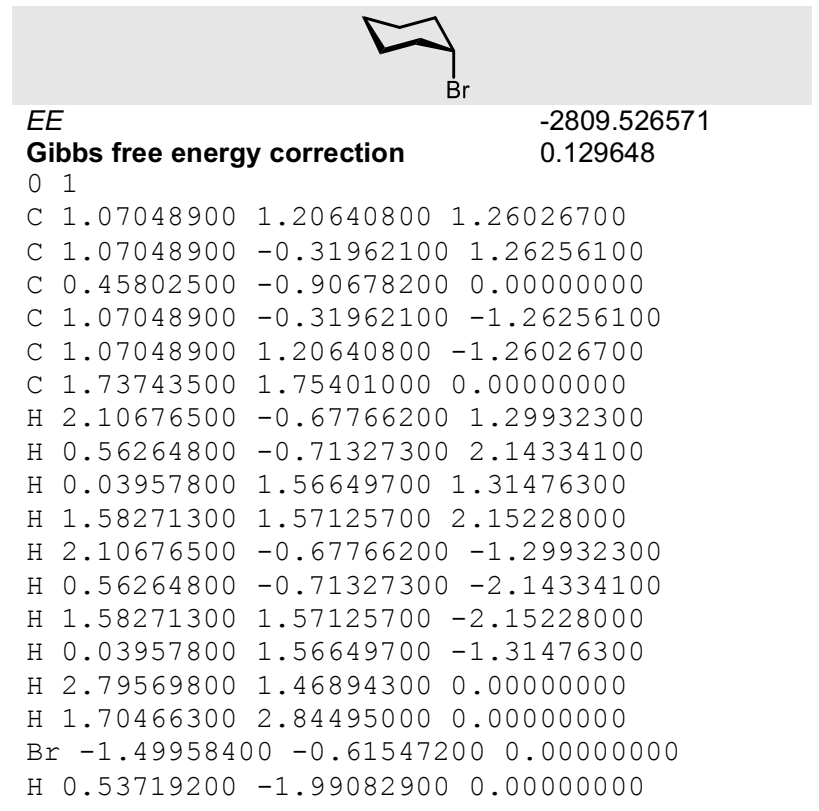

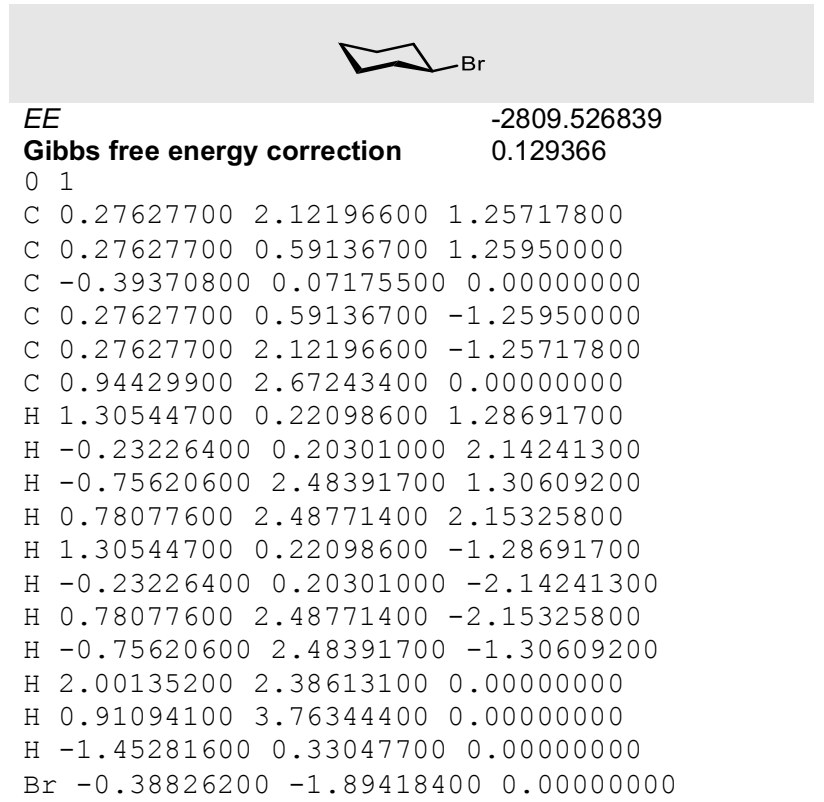


IODINE

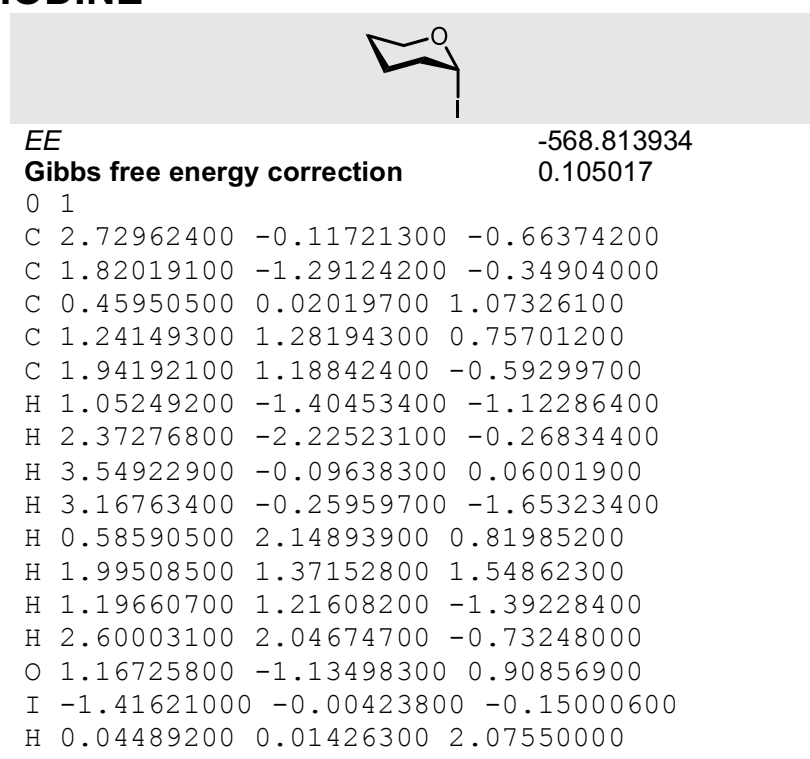

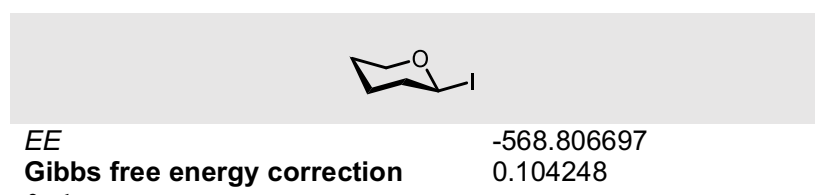

Gibbs
0

C $-3.19709000-0.11141000-0.35584200$

C $-2.38406500-1.28204800 \quad 0.17039000$

C $-0.46137200 \quad 0.01167700 \quad 0.40237700$

C $-1.12222500 \quad 1.27178000-0.12020500$

C $-2.62268400 \quad 1.19758500 \quad 0.17872300$

$\mathrm{H}-2.48711500-1.35560200 \quad 1.26205200$

$\mathrm{H}-2.69778400-2.22784500-0.26838900$

$\mathrm{H}-3.15400200-0.11706300-1.44791600$

$\mathrm{H}-4.24222900-0.23071400-0.06354200$

$\mathrm{H}-0.66752800 \quad 2.14868200 \quad 0.34130100$

$\mathrm{H}-0.95433600 \quad 1.33132800-1.19804900$

$\mathrm{H}-2.77907700 \quad 1.24838000 \quad 1.26126100$

$\mathrm{H}-3.13320800 \quad 2.05683800 \quad-0.25823900$

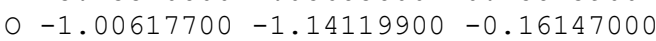

$\mathrm{H}-0.51073400-0.03706500 \quad 1.49549700$

I $1.64905800-0.00401600-0.02801600$

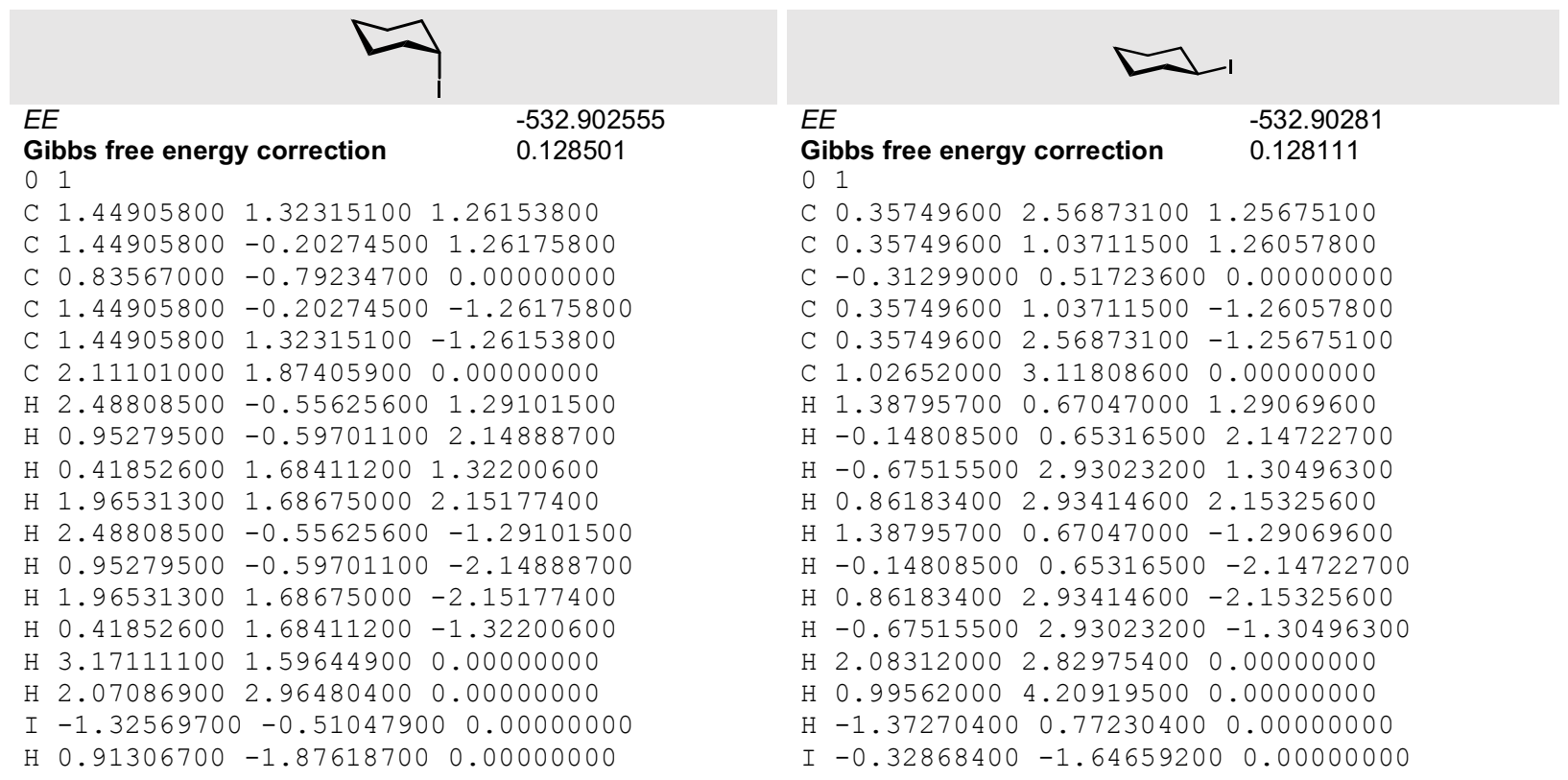




\section{ASTATIUM}

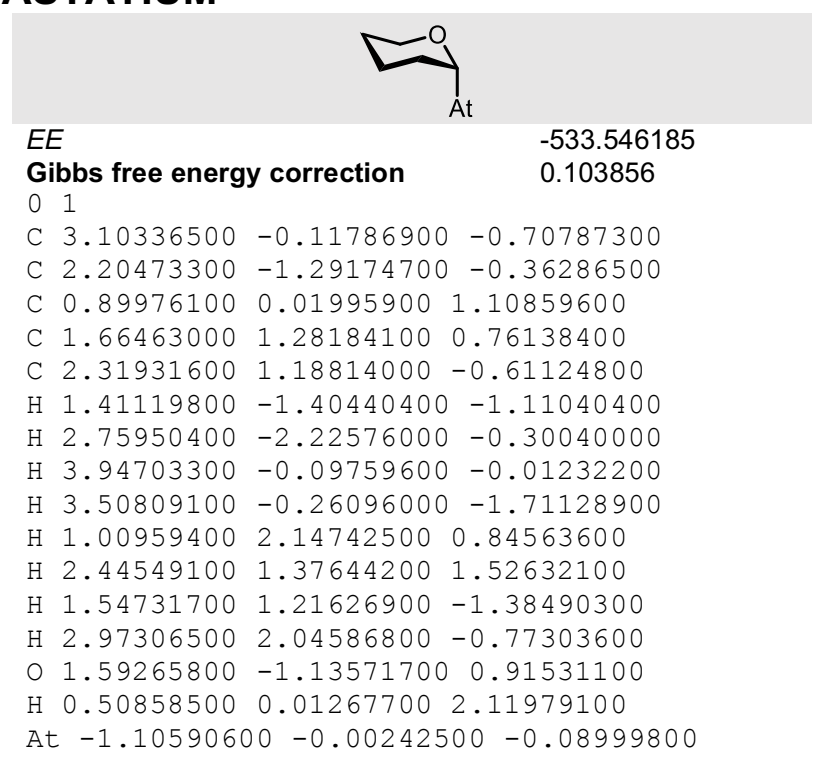

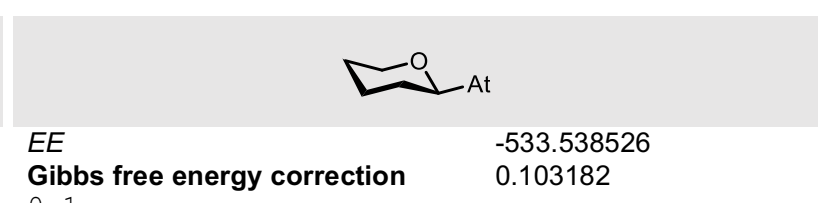

Giblos

C $-3.64219100-0.11461200-0.37616800$

C $-2.83782400-1.28324100 \quad 0.16654800$

C $-0.91980100 \quad 0.01491000 \quad 0.42538500$

C $-1.57539200 \quad 1.27335200-0.10363700$

C $-3.08138300 \quad 1.19536400 \quad 0.16976700$

$\mathrm{H}-2.95929400-1.35555700 \quad 1.25629400$

$\mathrm{H}-3.14112700-2.23023900-0.27707100$

$\mathrm{H}-3.57661700-0.12046400-1.46724100$

$\mathrm{H}-4.69295700-0.23602100-0.10556200$

$\mathrm{H}-1.13168200 \quad 2.15117900 \quad 0.36694400$

$\mathrm{H}-1.39327000 \quad 1.33775100-1.17896400$

$\mathrm{H}-3.25703200 \quad 1.24471500 \quad 1.24934700$

$\mathrm{H}-3.58626100 \quad 2.05365900-0.27594100$

$0-1.45397900-1.13980000-0.14152900$

At $1.27851700-0.00243600-0.01935700$

$\mathrm{H}-0.96428100-0.03422000 \quad 1.51836900$

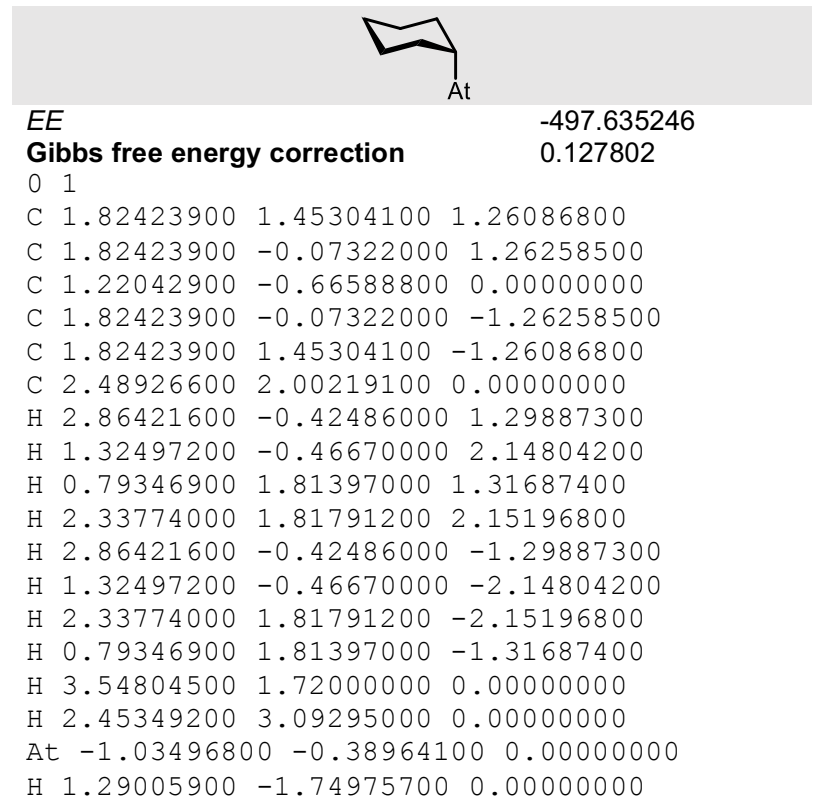

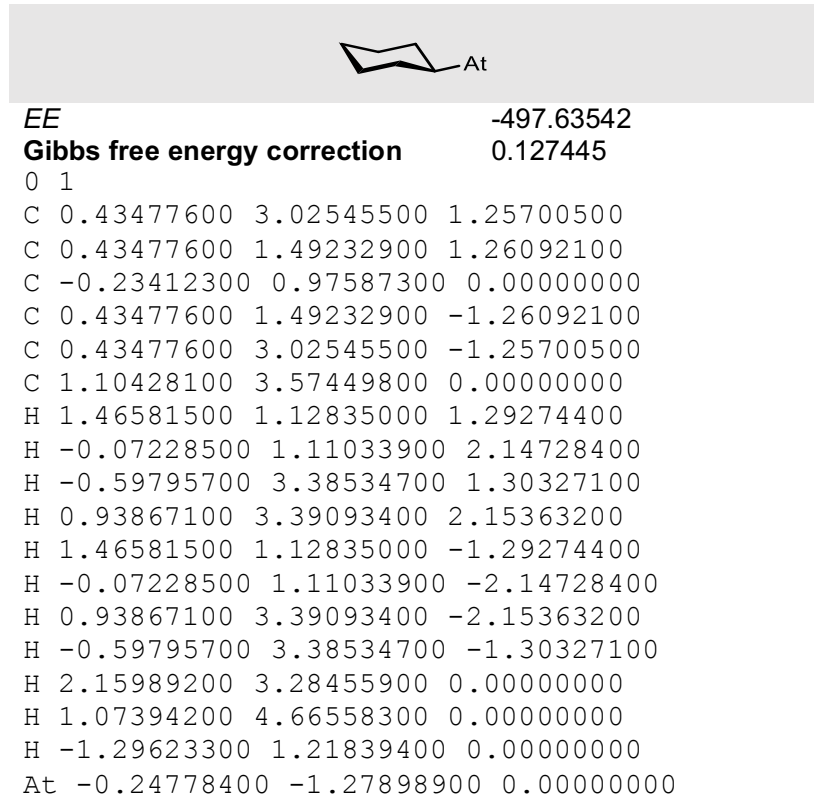




\subsection{Group 10}

\section{NICKEL}
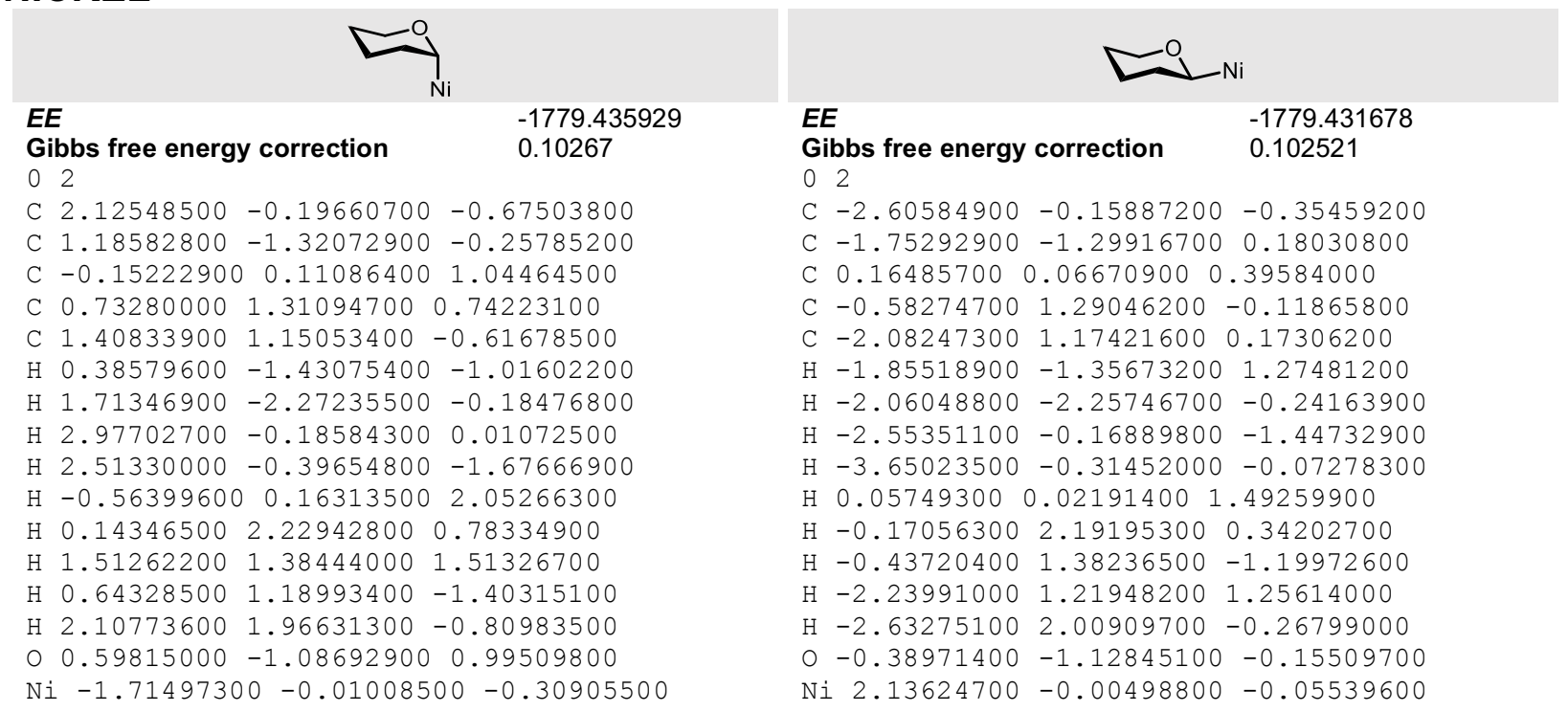

02

C $-2.60584900-0.15887200 \quad-0.35459200$

C $-1.75292900-1.29916700 \quad 0.18030800$

C $0.16485700 \quad 0.06670900 \quad 0.39584000$

C $-0.58274700 \quad 1.29046200-0.11865800$

C $-2.08247300 \quad 1.17421600 \quad 0.17306200$

$\mathrm{H}-1.85518900-1.35673200 \quad 1.27481200$

$\mathrm{H}-2.06048800-2.25746700 \quad-0.24163900$

$\mathrm{H}-2.55351100-0.16889800-1.44732900$

$\mathrm{H}-3.65023500-0.31452000 \quad-0.07278300$

$\mathrm{H} \quad 0.05749300 \quad 0.02191400 \quad 1.49259900$

$\mathrm{H}-0.17056300 \quad 2.19195300 \quad 0.34202700$

$\mathrm{H}-0.43720400 \quad 1.38236500-1.19972600$

$\mathrm{H}-2.23991000 \quad 1.21948200 \quad 1.25614000$

$\mathrm{H}-2.63275100 \quad 2.00909700-0.26799000$

$0-0.38971400-1.12845100-0.15509700$

Ni $2.13624700-0.00498800 \quad-0.05539600$

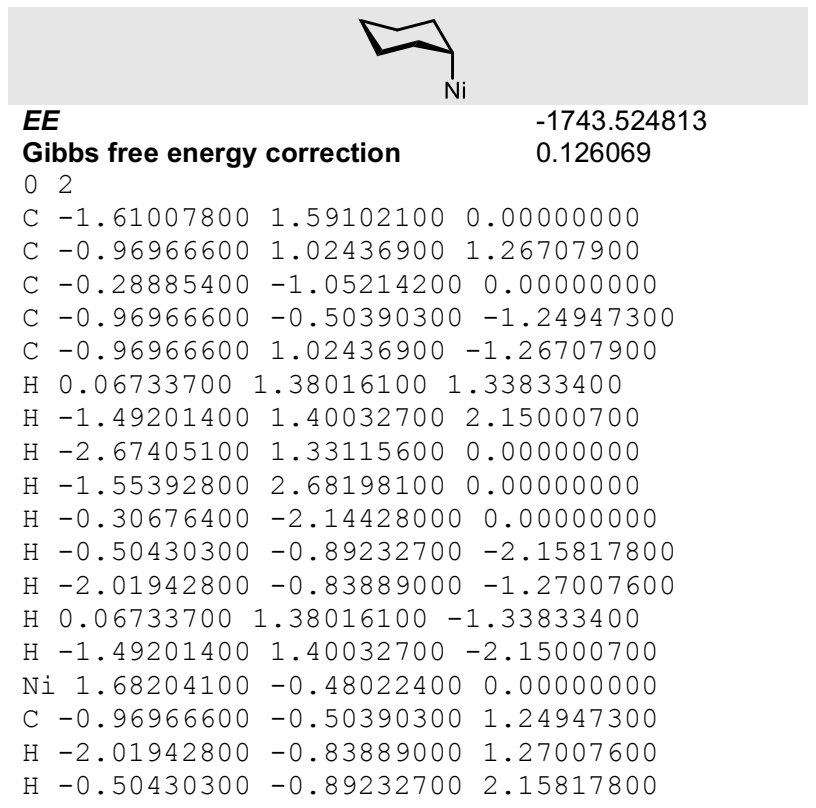

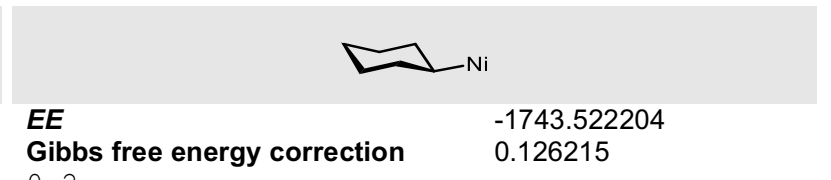

02

C $-0.92664600 \quad 2.54176800 \quad 0.00000000$

C $-0.25899000 \quad 1.99282600 \quad 1.25837600$

C $0.40271800 \quad-0.10566700 \quad 0.00000000$

C $-0.25899000 \quad 0.45859600-1.25754200$

C $-0.25899000 \quad 1.99282600-1.25837600$

$\mathrm{H} \quad 0.77666300 \quad 2.34742200 \quad 1.29854600$

H $-0.76114400 \quad 2.37214900 \quad 2.15245800$

$\mathrm{H}-1.98285000 \quad 2.24908100 \quad 0.00000000$

$\mathrm{H}-0.90274400 \quad 3.63401000 \quad 0.00000000$

$\mathrm{H} 1.46051900 \quad 0.18927300 \quad 0.00000000$

H $0.23916200 \quad 0.09186600 \quad-2.15825500$

$\mathrm{H}-1.29901100 \quad 0.11528500-1.31358000$

$\mathrm{H} \quad 0.77666300 \quad 2.34742200 \quad-1.29854600$

$\mathrm{H}-0.76114400 \quad 2.37214900-2.15245800$

$\mathrm{Ni} 0.45975200 \quad-2.14141000 \quad 0.00000000$

C $-0.25899000 \quad 0.45859600 \quad 1.25754200$

$\begin{array}{lllll}\mathrm{H} & 0.23916200 & 0.09186600 & 2.15825500\end{array}$

$\mathrm{H}-1.29901100 \quad 0.11528500 \quad 1.31358000$ 
NICKEL

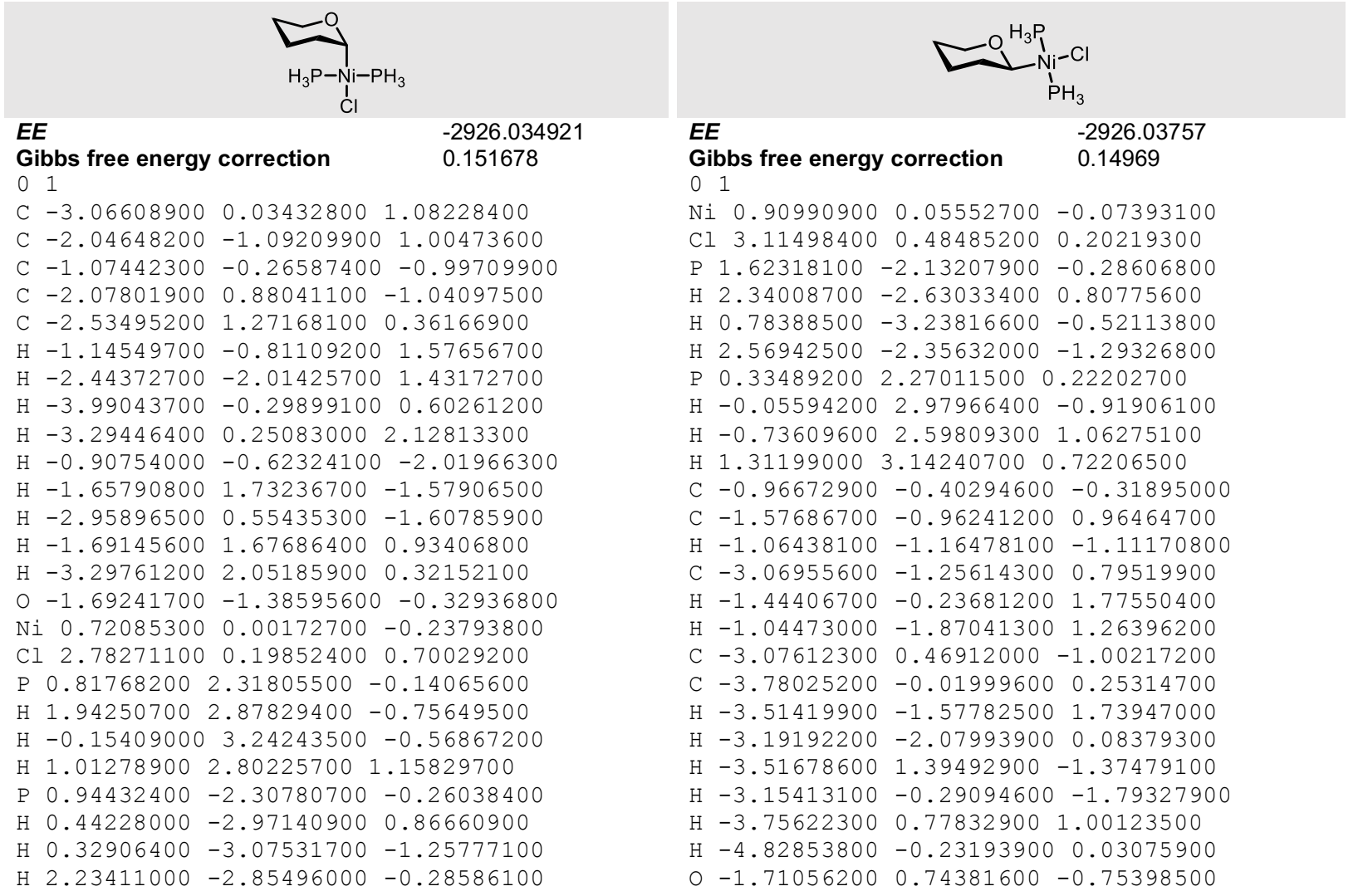

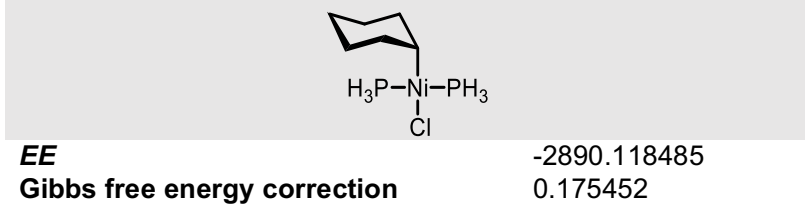

$\begin{array}{ll}0 & 1\end{array}$

C $3.26851400 \quad 0.11796000 \quad 0.96642000$

C $2.18163600 \quad 1.14976800 \quad 1.25588800$

C $1.01075200 \quad 0.60619600-0.93116700$

C $2.10873200-0.42732900-1.20888100$

C $2.73570400-0.99405900 \quad 0.06609200$

$\mathrm{H} 1.37376700 \quad 0.67733900 \quad 1.82867800$

$\mathrm{H} 2.58179000 \quad 1.95491600 \quad 1.87600400$

$\mathrm{H} \quad 4.10417000 \quad 0.61707900 \quad 0.46428100$

$\mathrm{H} \quad 3.66083000-0.300769001 .89549600$

$\mathrm{H} \quad 0.724621001 .05142700-1.89341000$

H $1.74065100-1.23478400-1.84680000$

H 2.91514700 $0.05534600-1.77986400$

$\mathrm{H} 1.99279500-1.55903100 \quad 0.64050000$

$\mathrm{H} 3.53476800-1.69583600-0.18486300$

$\mathrm{Ni}-0.70024000-0.03752800-0.19123500$

$\mathrm{H}-2.98003200 \quad 2.08032600-0.84623200$

$\mathrm{H}-1.95771300 \quad 2.67607900 \quad 0.92219000$

$\mathrm{H}-1.14356900 \quad 3.17201700 \quad-0.98484300$

$\mathrm{H}-1.44977500-3.04773700-0.78517000$

$\mathrm{H} \quad 0.68817500 \quad-3.07594200-0.75277300$

$\mathrm{H}-0.41268200-2.93339500 \quad 1.06811800$

Cl $-2.68134200-0.63402300 \quad 0.78081400$

C $1.61779300 \quad 1.70872500-0.04991900$

$\mathrm{H} \quad 0.90332900 \quad 2.50590700 \quad 0.17327600$

$\mathrm{H} \quad 2.44271900 \quad 2.19454300-0.59132000$

$\mathrm{P}-1.68705400 \quad 2.06382800 \quad-0.30941900$

$\mathrm{P}-0.38416100-2.34888800-0.20478900$

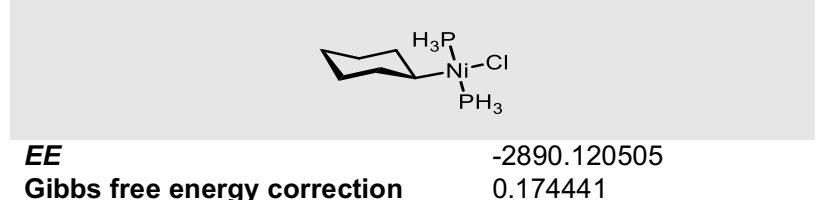

$\begin{array}{ll}\text { Gibbs } \\ 0 & 1\end{array}$

$\mathrm{Ni} 0.88885700-0.03200300-0.00013300$

Cl $3.10976600 \quad 0.48479000 \quad 0.00003100$

$\mathrm{H}-0.62310200 \quad 2.97290300-0.00236100$

H $1.22478800 \quad 2.93360700 \quad 1.06378400$

$\mathrm{H} 1.22844600 \quad 2.93436800-1.06224600$

H $0.81600200 \quad-3.36413300 \quad 0.00037400$

H 2.48267600 -2.55690900 -1.06184700

H 2.48197500 $-2.55661900 \quad 1.06342500$

C $-1.01503500-0.47801000 \quad 0.00030900$

C $-1.73531800 \quad 0.01985800 \quad 1.25684600$

$\mathrm{H}-1.11468700-1.57160200 \quad 0.00131100$

C $-3.21431700-0.38129000 \quad 1.26064500$

$\mathrm{H}-1.68376300 \quad 1.11471000 \quad 1.31553200$

$\mathrm{H}-1.24613200-0.35812800 \quad 2.15936200$

C $-3.21436500-0.38338500-1.26011900$

C $-3.91745300 \quad 0.11668600-0.00014000$

$\mathrm{H}-3.71305700 \quad 0.00665700 \quad 2.15232700$

$\mathrm{H}-3.28679900-1.47328100 \quad 1.30444200$

$\mathrm{H}-3.71313400 \quad 0.00311900-2.15241500$

$\mathrm{H}-3.28691800-1.47544000-1.30211600$

$\mathrm{H}-3.91270300 \quad 1.21298900-0.00103900$

$\mathrm{H}-4.96505800-0.19217900 \quad 0.00013000$

C $-1.73533600 \quad 0.01766200-1.25708500$

$\mathrm{H}-1.24626000-0.36199200-2.15895600$

$\mathrm{H}-1.68362400 \quad 1.11239400-1.31769800$

P $0.59762700 \quad 2.27298000-0.00056600$

P $1.63425700-2.21930900 \quad 0.00046400$ 
NICKEL

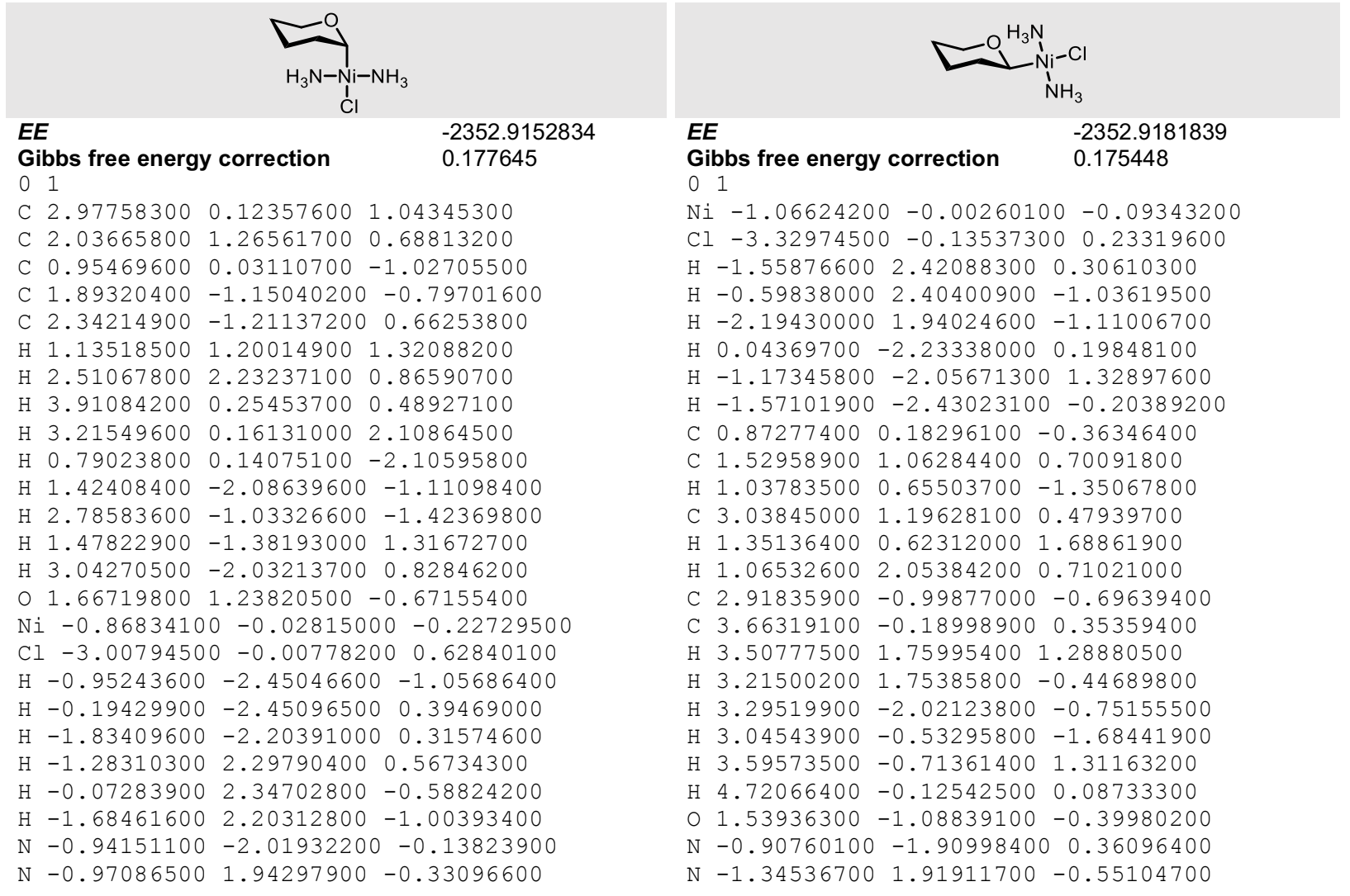

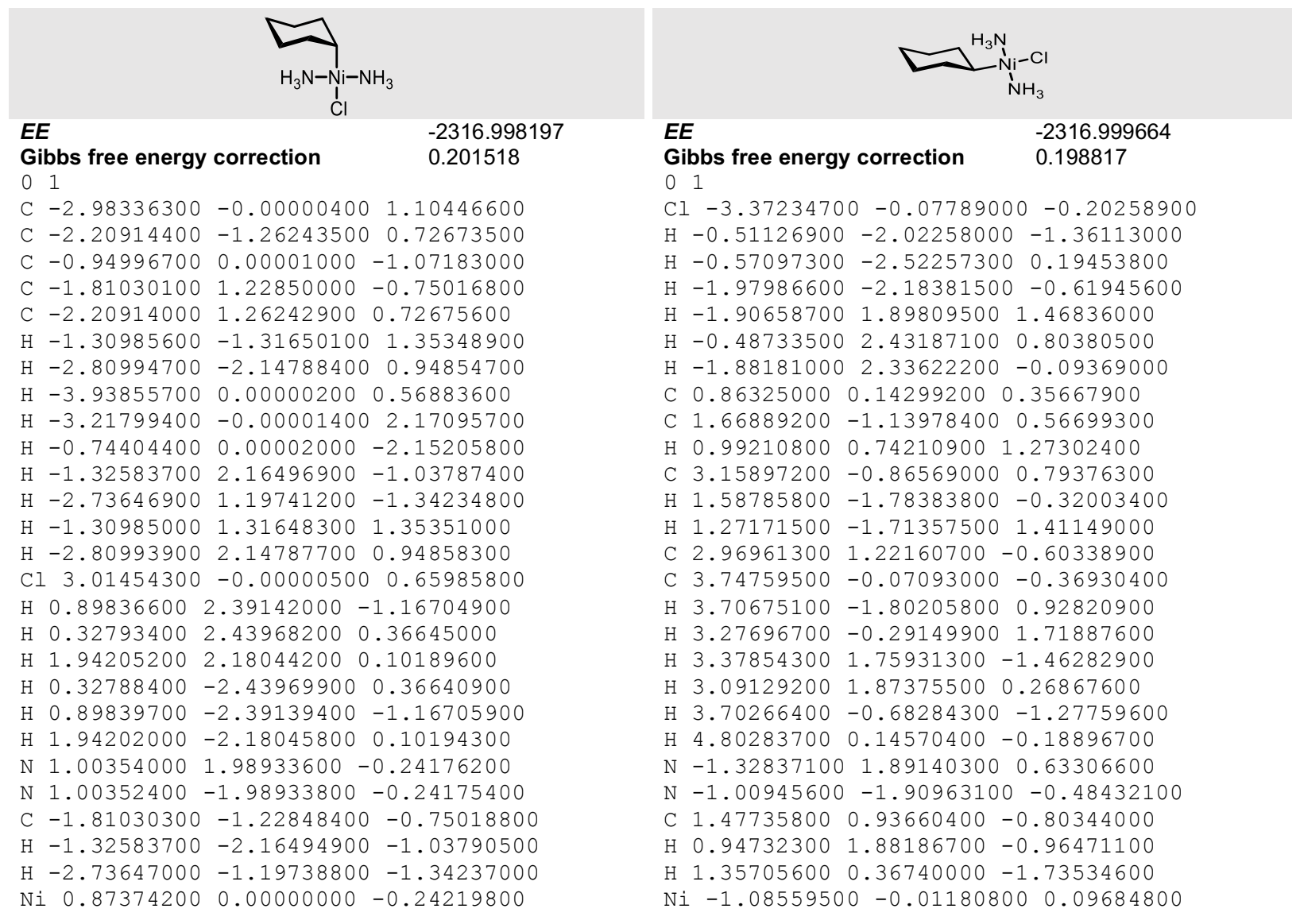


NICKEL

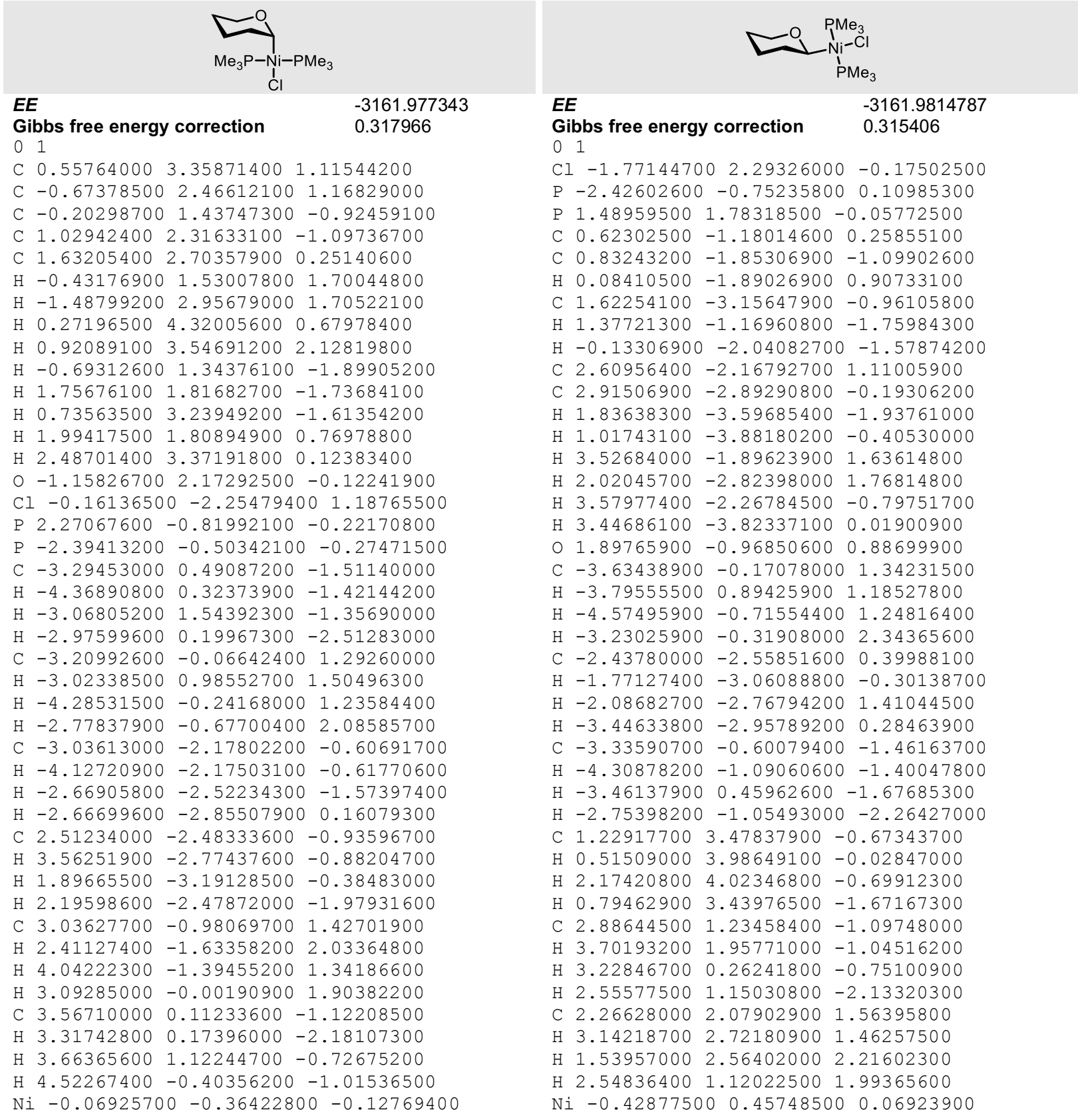

$\begin{array}{lll} & \\ \text { EE flibbs free energy correction } & -3126.058942 \\ & & 0.34111\end{array}$

01

C $1.68483200 \quad 3.24900500 \quad 0.95628900$

C $0.28411600 \quad 2.784392001 .34330100$

C $0.03382900 \quad 1.53356900-0.85919200$

C $1.43995800 \quad 2.01390800-1.22904000$

C $2.328992002 .25788600-0.00867800$

$\mathrm{H} \quad 0.35385500 \quad 1.83937600 \quad 1.89742600$

$\mathrm{H}-0.18357000 \quad 3.51037300 \quad 2.01251100$

$\mathrm{H} \quad 1.61243900 \quad 4.22877000 \quad 0.47163200$

$\mathrm{H} 2.30823400 \quad 3.37821700 \quad 1.84379900$

$\mathrm{H}-0.55835700 \quad 1.52390200-1.78199700$

$\mathrm{H} 1.91777600 \quad 1.31977400-1.92165100$

$\mathrm{H} \quad 1.36313800 \quad 2.96959300-1.76953300$

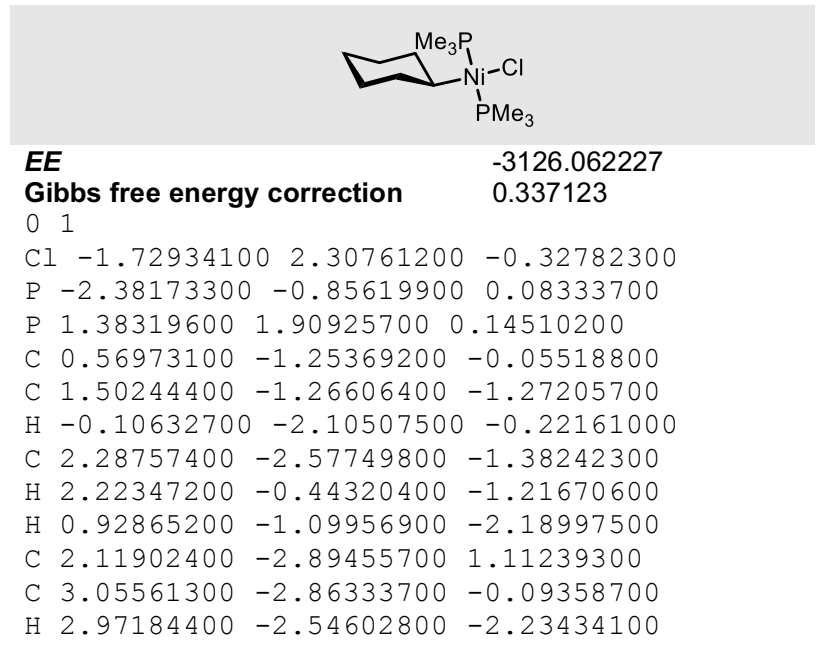


H 2.48512100 $1.31929900 \quad 0.53313300$

$\mathrm{H} \quad 3.31510900 \quad 2.61492400-0.31913600$ $\mathrm{Cl}-0.61936300-2.01043000 \quad 1.40783100$ P $1.97904500-1.25482400-0.24958700$ P $-2.50892000-0.14066200-0.28887700$ C $-3.25736900 \quad 1.01868600-1.48848000$ $\mathrm{H}-4.34467000 \quad 0.93114500-1.47663300$ $\mathrm{H}-2.97570500 \quad 2.04572200-1.26168200$ $\mathrm{H}-2.89327100 \quad 0.77876900-2.48776700$ C $-3.46013500 \quad 0.15729300 \quad 1.23666200$ $\mathrm{H}-3.25061800 \quad 1.15685000 \quad 1.61715900$ $\mathrm{H}-4.53112000 \quad 0.05780000 \quad 1.05425200$ $\mathrm{H}-3.13785600-0.57380500 \quad 1.97766600$ C $-3.18324500-1.73472900-0.86469600$ $\mathrm{H}-4.26843300-1.68234700-0.96529500$ $\mathrm{H}-2.74522300-1.98635300-1.83081100$ $\mathrm{H}-2.90753900-2.50475200-0.14644500$ C $1.72519600-2.95515400-0.87143300$ H $2.67277600-3.49513400-0.90594700$ $\mathrm{H} \quad 1.02377600-3.46702500-0.21597700$ $\mathrm{H} 1.30226300-2.91338500-1.87568900$ C $2.83694600-1.54473500 \quad 1.33491200$ H $2.12730000-1.98869700 \quad 2.03131500$ $\mathrm{H} 3.68959500 \quad-2.21059700 \quad 1.19302300$ $\mathrm{H} \quad 3.18771400 \quad-0.59701500 \quad 1.74372600$ C $3.36788900-0.73397600-1.32690000$ $\mathrm{H} 3.03118400-0.67509600-2.36170500$ $\mathrm{H} \quad 3.75146400 \quad 0.24016600-1.02976500$ $\mathrm{H} \quad 4.17081800-1.46985400-1.25943100$ $\mathrm{Ni}-0.18353700-0.24368400-0.01882100$ C $-0.57570800 \quad 2.57527100 \quad 0.09636500$ $\mathrm{H}-1.58788900 \quad 2.30975000 \quad 0.40957700$ $\mathrm{H}-0.66375300 \quad 3.54388200 \quad-0.41960600$
H $1.58602000-3.39836100-1.56849500$

H $2.68465300-3.08766800 \quad 2.02766200$

H $1.41382500-3.72538800 \quad 0.99333000$

H $3.80447200-2.07647700 \quad 0.05805100$

H $3.60392800-3.80445500-0.17686700$

C $-3.97103900-0.01907100 \quad 0.37215500$

$\mathrm{H}-4.12756800 \quad 0.71628600 \quad-0.41487500$

$\mathrm{H}-4.79091200-0.73857100 \quad 0.39336900$

$\mathrm{H}-3.92456700 \quad 0.52063200 \quad 1.31742100$

C $-2.41668600-2.18447700 \quad 1.33737100$

$\mathrm{H}-1.53654000 \quad-2.81922400 \quad 1.24194800$

$\mathrm{H}-2.40978100-1.73345900 \quad 2.32991700$

$\mathrm{H}-3.31522800 \quad-2.79391200 \quad 1.23112000$

C $-2.68565000-1.74966100-1.47833400$

$\mathrm{H}-3.57633300-2.37549000-1.40683500$

$\mathrm{H}-2.82287300-1.01721100-2.27406100$

$\mathrm{H}-1.82444400-2.37099000-1.72313000$

C $1.07314700 \quad 3.33341900 \quad 1.24325500$

$\mathrm{H} \quad 0.98456100 \quad 2.98297200 \quad 2.27178900$

$\mathrm{H} 1.89343300 \quad 4.05003400 \quad 1.17799900$

$\mathrm{H} \quad 0.13534500 \quad 3.80195700 \quad 0.95337400$

C $1.645686002 .70436600-1.47584500$

H $2.38714700 \quad 3.50179100-1.40704100$

$\mathrm{H} \quad 1.98261700 \quad 1.95860700 \quad-2.19658800$

$\mathrm{H} \quad 0.69069500 \quad 3.10964600-1.80896900$

C $3.097800001 .49617800 \quad 0.64993400$

$\mathrm{H} \quad 3.74105500 \quad 2.36513500 \quad 0.50134900$

H $3.110471001 .22917500 \quad 1.70666200$

H $3.49465100 \quad 0.65774700 \quad 0.08064600$

C $1.33295700-1.58460900 \quad 1.22825600$

H $0.64236400-1.64008100 \quad 2.07623300$

H $2.03746400-0.78101900 \quad 1.46539800$

$\mathrm{Ni}-0.47031600 \quad 0.42110600 \quad 0.00157300$ 
PALLADIUM

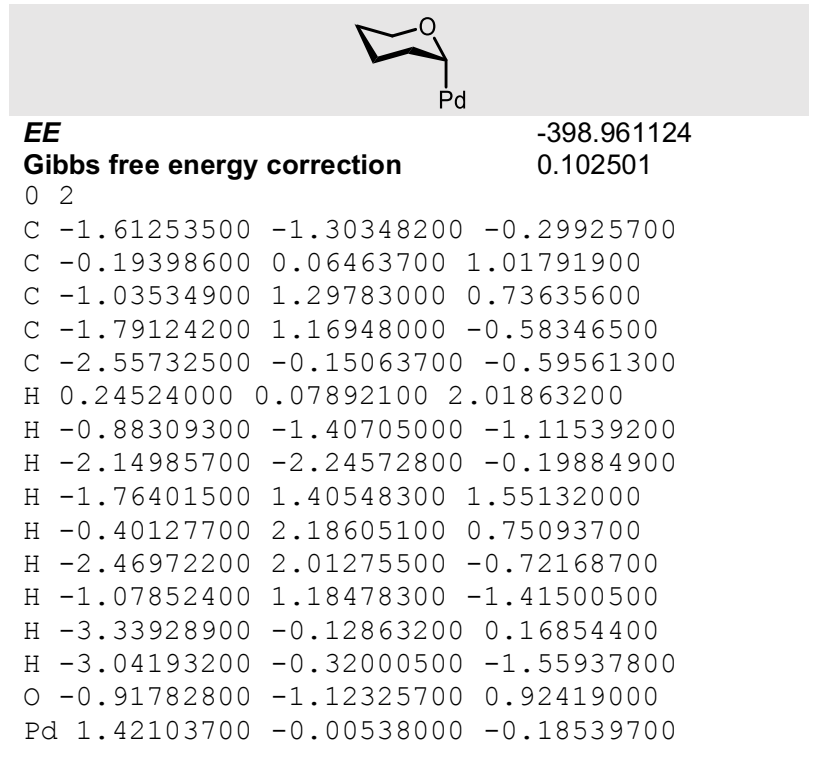

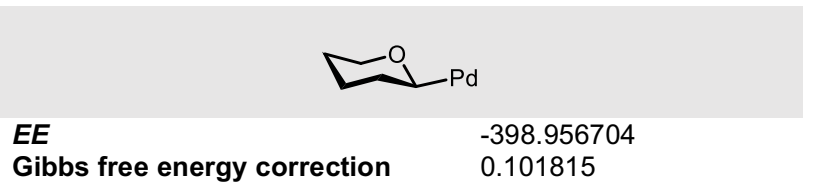

Gibbs free energy correction $\quad 0.101815$

02

C $-2.16835900-1.29335100 \quad 0.17138900$

C $-2.99125400-0.13372900-0.36757700$

C $-2.44871900 \quad 1.18683500 \quad 0.17182100$

C $-0.94108500 \quad 1.28152200-0.10578300$

C $-0.24498000 \quad 0.04030600 \quad 0.41805500$

$\mathrm{H}-2.47160300-2.24252100-0.26969400$

$\mathrm{H}-2.28756800-1.36375600 \quad 1.26210500$

$\mathrm{H}-2.93087700-0.13979600-1.45932800$

$\mathrm{H}-4.03998900-0.26930100-0.09344500$

$\mathrm{H}-2.97470100 \quad 2.03464000-0.27127800$

$\mathrm{H}-2.61593600 \quad 1.23230300 \quad 1.25291100$

$\mathrm{H}-0.77851300 \quad 1.35904400-1.18487900$

$\mathrm{H}-0.51594400 \quad 2.17183400 \quad 0.36164500$

$\mathrm{H}-0.30062800-0.01078900 \quad 1.51846700$

$0-0.79173800-1.13075800-0.13842000$

Pd $1.69600100-0.00467600-0.03775200$

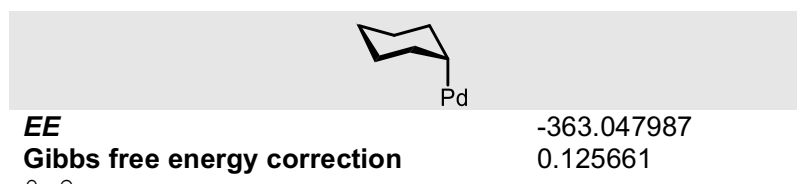

02

0.125661

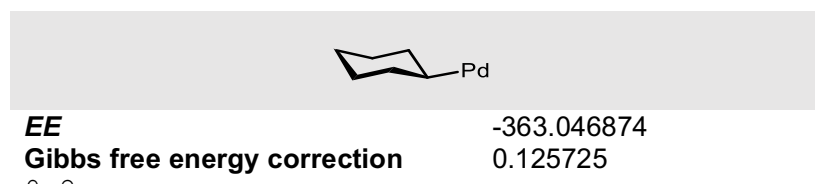

02

C $-1.93307100 \quad 1.77527400 \quad 0.00000000$

C $-1.27403300 \quad 1.22324700 \quad 1.26274900$

C $-0.64090500-0.87638100 \quad 0.00000000$

C $-1.27403300-0.30621900-1.25890000$

C $-1.27403300 \quad 1.22324700 \quad-1.26274900$

$\mathrm{H}-0.24011400 \quad 1.58378300 \quad 1.31658400$

$\mathrm{H}-1.78722600 \quad 1.59744700 \quad 2.15118500$

$\mathrm{H}-2.991414001 .49156700 \quad 0.00000000$

$\mathrm{H}-1.89915300 \quad 2.86671600 \quad 0.00000000$

$\mathrm{H}-0.67212000-1.97053900 \quad 0.00000000$

$\mathrm{H}-0.77756200-0.69356000-2.15156200$

$\mathrm{H}-2.31834600-0.65064400-1.31173300$

$\mathrm{H}-0.240114001 .58378300-1.31658400$

$\mathrm{H}-1.78722600 \quad 1.59744700-2.15118500$

Pd $1.34412700-0.48825000 \quad 0.00000000$

C $-1.27403300-0.30621900 \quad 1.25890000$

$\mathrm{H}-2.31834600-0.65064400 \quad 1.31173300$

$\mathrm{H}-0.77756200-0.69356000 \quad 2.15156200$

C $-0.97440900 \quad 2.93001400 \quad 0.00000000$

C $-0.30764500 \quad 2.38280700 \quad 1.25930900$

C $0.35408100 \quad 0.30748900 \quad 0.00000000$

C $-0.30764500 \quad 0.84580900-1.25800200$

C $-0.30764500 \quad 2.38280700-1.25930900$

$\mathrm{H} \quad 0.72715300 \quad 2.73808000 \quad 1.30325900$

$\mathrm{H}-0.81193800 \quad 2.75641500 \quad 2.15344700$

$\mathrm{H}-2.03104700 \quad 2.64051800 \quad 0.00000000$

$\mathrm{H}-0.94640500 \quad 4.02194500 \quad 0.00000000$

$\mathrm{H} 1.42403100 \quad 0.55396600 \quad 0.00000000$

$\mathrm{H} \quad 0.19648500 \quad 0.47308600-2.15224100$

$\mathrm{H}-1.34619300 \quad 0.49881900-1.30434800$

$\mathrm{H} \quad 0.72715300 \quad 2.73808000-1.30325900$

$\mathrm{H}-0.81193800 \quad 2.75641500-2.15344700$

$\mathrm{Pd} 0.32886700-1.70255700 \quad 0.00000000$

C $-0.30764500 \quad 0.84580900 \quad 1.25800200$

$\begin{array}{lllll}\mathrm{H} & 0.19648500 & 0.47308600 & 2.15224100\end{array}$

$\mathrm{H}-1.34619300 \quad 0.49881900 \quad 1.30434800$ 
PALLADIUM

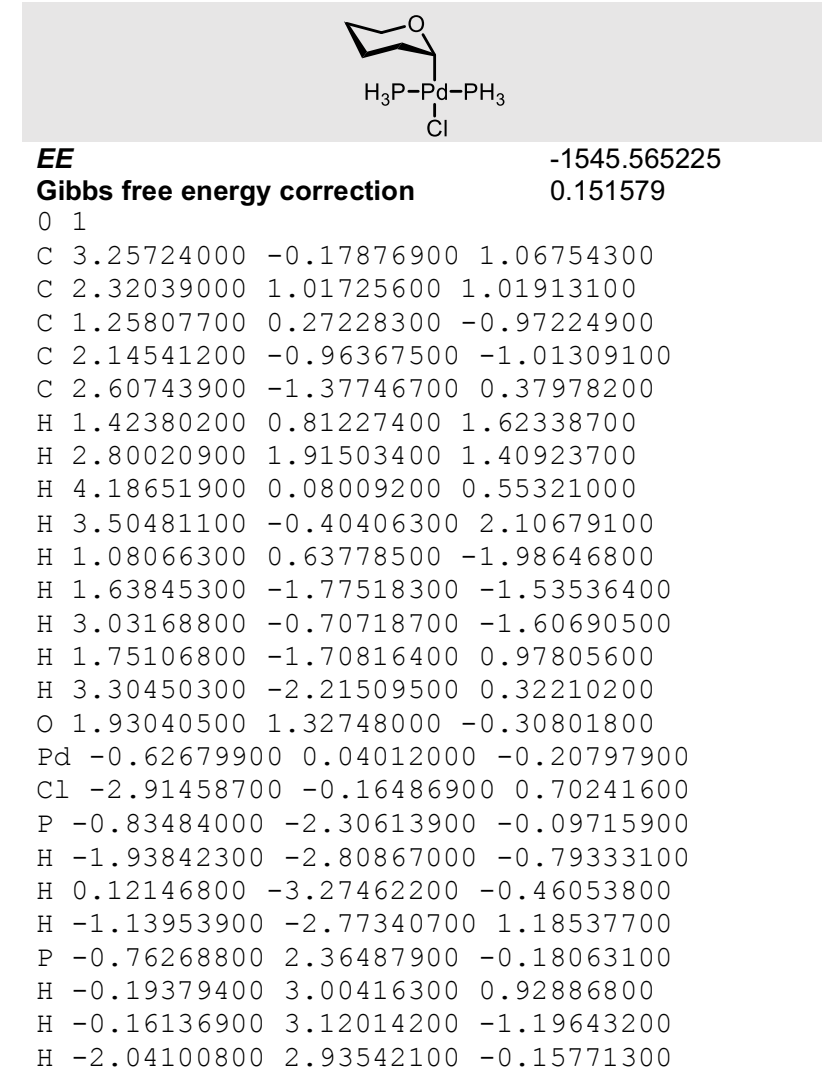

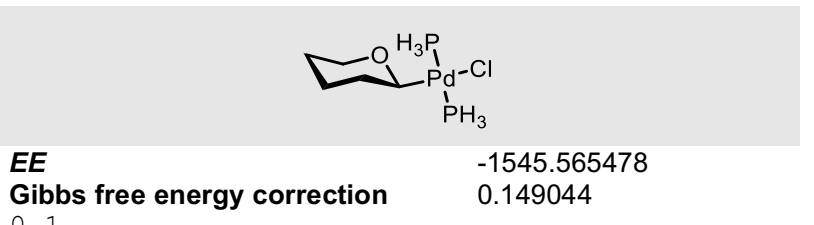

$\begin{array}{ll}0 & 1\end{array}$

Pd $0.78781100 \quad 0.04513600 \quad-0.06040200$

Cl $3.18511900 \quad 0.47100600 \quad 0.22547300$

P $1.55057800-2.16248900-0.28925400$

H 2.26442700 $-2.64721400 \quad 0.81147100$

$\mathrm{H} \quad 0.73391000-3.28293200-0.53798500$

$\mathrm{H} 2.50832300-2.35409400-1.29120800$

P $0.22622400 \quad 2.28099400 \quad 0.21259300$

$\mathrm{H}-0.16798000 \quad 2.97766300-0.93494500$

$\mathrm{H}-0.83079000 \quad 2.63025800 \quad 1.06228700$

H $1.22861600 \quad 3.13420400 \quad 0.69263500$

C $-1.16621000-0.41232300-0.28889700$

C $-1.77168000-0.93830400 \quad 1.00490000$

$\mathrm{H}-1.23787400-1.18386100-1.07071200$

C $-3.26253800-1.23830500 \quad 0.81580700$

$\mathrm{H}-1.64860700-0.18919900 \quad 1.79310100$

$\mathrm{H}-1.23911100-1.83615900 \quad 1.32730300$

C $-3.24985100 \quad 0.45047700-1.01368700$

C $-3.96998700-0.01586900 \quad 0.24079300$

$\mathrm{H}-3.71533100-1.54227900 \quad 1.76149700$

$\mathrm{H}-3.37362800-2.07782100 \quad 0.12146900$

$\mathrm{H}-3.67709200 \quad 1.37272200-1.40778100$

$\mathrm{H}-3.31537200-0.32093100-1.79410500$

$\mathrm{H}-3.96020900 \quad 0.79692800 \quad 0.97290100$

$\mathrm{H}-5.01367700-0.23693600 \quad 0.00690500$

O $-1.88404700 \quad 0.72808300-0.74637300$

\begin{tabular}{|c|c|c|}
\hline$E$ & -1509.64807 & -1509.650829 \\
\hline & $\begin{array}{l}\text { ibbs free energy correction } \\
1\end{array}$ & $\begin{array}{l}\text { Gibbs free energy correction } \\
01\end{array}$ \\
\hline & $3.41010000-0.00259000 \quad 0.99490300$ & $\mathrm{Pd}-0.77406800-0.04353200-0.00012000$ \\
\hline & $2.39048700 \quad 1.10351800 \quad 1.25404900$ & $\mathrm{Cl}-3.19218200 \quad 0.45488100 \quad 0.00020800$ \\
\hline & $1.21173000 \quad 0.58715300-0.93761900$ & $\mathrm{H}-2.29898900-2.60291700-1.06656600$ \\
\hline & $2.22222600-0.53612800-1.16862100$ & $\mathrm{H}-0.61467600-3.37987200 \quad 0.00039600$ \\
\hline & $2.80982000-1.10369100 \quad 0.12344200$ & $\mathrm{H}-2.29900500-2.60221400 \quad 1.06689400$ \\
\hline & $1.55352700 \quad 0.70248300 \quad 1.83701000$ & $\mathrm{H}-1.21439100 \quad 2.93617600 \quad 1.06571000$ \\
\hline & $2.84150800 \quad 1.90086500 \quad 1.84810700$ & $\mathrm{H} \quad 0.63145700 \quad 3.06285300-0.00135900$ \\
\hline & $4.27833300 \quad 0.42768900 \quad 0.48441400$ & $\mathrm{H}-1.21677900 \quad 2.93622100-1.06453000$ \\
\hline & $3.77101300-0.420848001 .93644300$ & $\begin{array}{llll}C & 1.21878400 & -0.47279400 & -0.00006600\end{array}$ \\
\hline & $0.93522900 \quad 1.01565400-1.90564900$ & $\begin{array}{llll}C & 1.90682400 & 0.04755100 & -1.25747700\end{array}$ \\
\hline & $1.79620700-1.32826900-1.78699400$ & $\mathrm{H} 1.31491700-1.56345600 \quad 0.00006900$ \\
\hline & $3.05442500-0.11633200-1.75200500$ & $\begin{array}{llll}\text { C } 3.39160400 & -0.33848700 & -1.25974300\end{array}$ \\
\hline & $2.03156400-1.60698300 \quad 0.70632500$ & $\mathrm{H} 1.83480000 \quad 1.14026300-1.30016500$ \\
\hline & $3.56422900-1.85814800-0.11061700$ & $\mathrm{H} 1.41684800-0.33564100-2.15541800$ \\
\hline & $d-0.60887200 \quad 0.00026700-0.18168200$ & C $3.39136800-0.33797500 \quad 1.26010600$ \\
\hline & $1-2.82786000-0.57962500 \quad 0.74970800$ & $\begin{array}{llll}C & 4.08955800 & 0.16827700 & 0.00014100\end{array}$ \\
\hline & $-1.46851000-2.98778000-0.77581100$ & H $3.88007400 \quad 0.05710200 \quad-2.15305000$ \\
\hline & $0.66399100-3.13663400-0.63032700$ & $\mathrm{H} 3.47780200-1.42909900-1.30635600$ \\
\hline & $\begin{array}{llll}-0.52114600 & -2.91356800 & 1.13201800\end{array}$ & $\begin{array}{llll}\mathrm{H} & 3.87957600 & 0.05814900 & 2.15332100\end{array}$ \\
\hline & $-1.75411000 \quad 2.71913500 \quad 1.03460000$ & $\mathrm{H} \quad 3.47771400-1.42854900 \quad 1.30730200$ \\
\hline & $\begin{array}{llll}-0.90455300 & 3.27164000 & -0.84407100\end{array}$ & $\mathrm{H} 4.07498900 \quad 1.26403700-0.00008800$ \\
\hline $\mathrm{H}$ & $\begin{array}{llll}-2.77766800 & 2.22679100 & -0.77341400\end{array}$ & $\mathrm{H} 5.13938000-0.13204200 \quad 0.00032000$ \\
\hline $\mathrm{P}$ & $-0.39617000-2.34886200-0.14358200$ & $P-0.55770000 \quad 2.30888800-0.00016900$ \\
\hline $\mathrm{P}$ & $\begin{array}{llll}-1.49423600 & 2.15560900 & -0.22171700\end{array}$ & $\mathrm{P}-1.46152100-2.25578100 \quad 0.00005600$ \\
\hline $\mathrm{C}$ & $1.86525700 \quad 1.66264800-0.06687800$ & $\begin{array}{llll}\text { C } 1.90653400 & 0.04785400 & 1.25737400\end{array}$ \\
\hline & $1.19635300 \quad 2.50455000 \quad 0.11951900$ & $\mathrm{H} 1.41642200-0.33525500 \quad 2.15528200$ \\
\hline & $2.71969800 \quad 2.07444400-0.62338800$ & $\mathrm{H} \quad 1.83434400 \quad 1.14056600 \quad 1.29990900$ \\
\hline
\end{tabular}


PALLADIUM

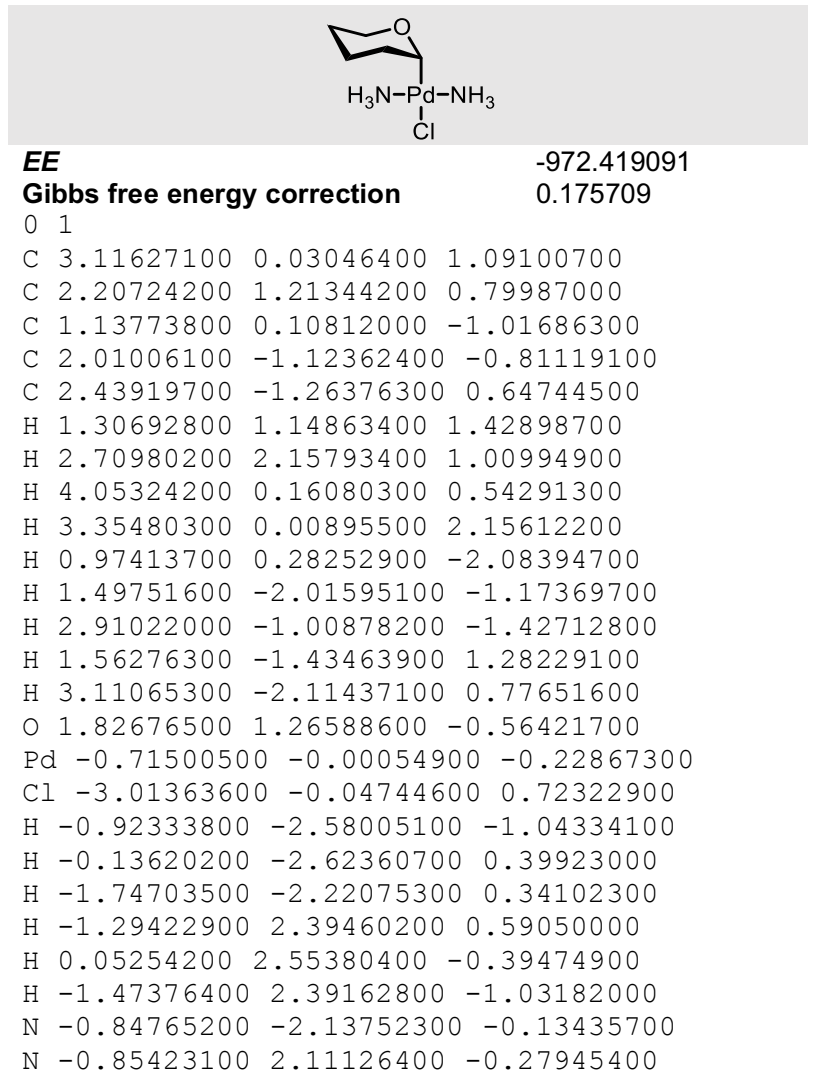

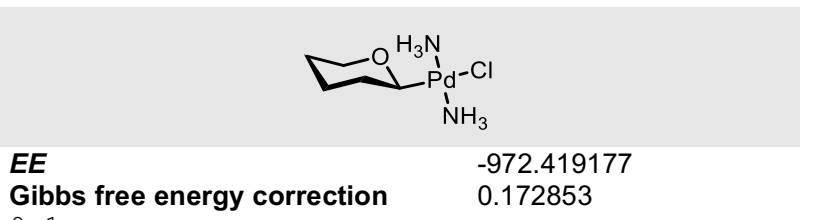
Gibbs free energy correction $\quad 0.172853$
01

$\mathrm{Pd}-0.89114800 \quad 0.01922500-0.06291900$

Cl $-3.33250200-0.23851400 \quad 0.22531700$

$\mathrm{H}-2.33895400 \quad 2.03619600-0.48614400$

$\mathrm{H}-0.96320100 \quad 2.75101300 \quad 0.09012700$

$\mathrm{H}-1.06276200 \quad 2.33477700-1.49781500$

$\mathrm{H} \quad 0.12735200-2.40477100-0.11733400$

$\mathrm{H}-0.45665300-2.14813900 \quad 1.40306300$

$\mathrm{H}-1.52544600-2.47155600 \quad 0.19591600$

C $1.06876200 \quad 0.26796800 \quad-0.31842200$

C $1.72355500 \quad 0.95328700 \quad 0.87314000$

H $1.20053200 \quad 0.88949700 \quad-1.22044500$

C $3.23177300 \quad 1.10747800 \quad 0.64894500$

H $1.54857400 \quad 0.35282000 \quad 1.77122200$

$\mathrm{H} 1.26209900 \quad 1.92899200 \quad 1.04331600$

C $3.07371800-0.86299600-0.86958600$

C $3.84162100-0.24336800 \quad 0.28662000$

H $3.71397900 \quad 1.52693300 \quad 1.53395600$

H $3.40125600 \quad 1.81051200-0.17342600$

H $3.43108600-1.86768400-1.09663200$

$\mathrm{H} 3.18926400-0.24533900-1.77140000$

$\mathrm{H} \quad 3.78508200-0.91736800 \quad 1.14599000$

H $4.89490000 \quad-0.14161600 \quad 0.01629500$

$01.69514400-0.98628900-0.56287400$

$\mathrm{N}-0.64420400-2.01703600 \quad 0.41563400$

$N-1.32143900 \quad 2.05878000-0.55763300$

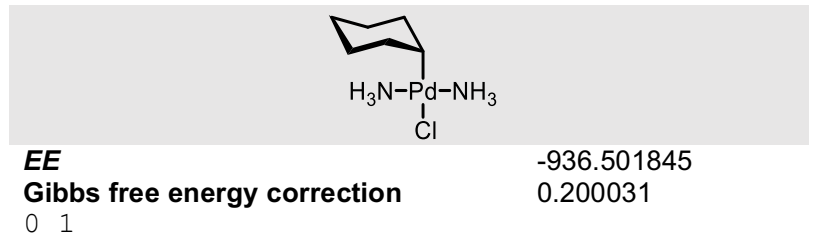

0

C $3.10691300 \quad 0.00066900 \quad 1.15266200$

C $2.33639300 \quad 1.26340200 \quad 0.76775200$

C $1.13770700-0.00091300-1.07232000$

C $1.95980300-1.23782700 \quad-0.71237500$

C $2.33600700-1.26248200 \quad 0.76987500$

H $1.42737600 \quad 1.31739200 \quad 1.37797700$

$\mathrm{H} 2.93371400 \quad 2.14924600 \quad 0.99532800$

$\mathrm{H} 4.06885200 \quad 0.00006700 \quad 0.62894900$

$\mathrm{H} \quad 3.32808500 \quad 0.00154200 \quad 2.22166700$

$\mathrm{H} \quad 0.92581800-0.00179400-2.14746000$

$\mathrm{H} 1.45339400-2.15974000-1.00408300$

H 2.88982100 -1.21861400 -1.29777300

$\mathrm{H} 1.42689900-1.31521500 \quad 1.38009500$

$\mathrm{H} \quad 2.93305100-2.14811600 \quad 0.99897600$

$\mathrm{Pd}-0.71449100-0.00007400-0.23505100$

Cl $-3.02246700 \quad 0.00049900 \quad 0.72526100$

$\mathrm{H}-0.87982600-2.57173000-1.09461100$

$\mathrm{H}-0.23724300-2.60085800 \quad 0.41868300$

$\mathrm{H}-1.83687800-2.21606500 \quad 0.20866400$

$\mathrm{H}-0.23630700 \quad 2.60151100 \quad 0.41827400$

$\mathrm{H}-0.88035400 \quad 2.57178200-1.09428900$

$\mathrm{H}-1.83591100 \quad 2.21578900 \quad 0.20987700$

$\mathrm{N}-0.90011100-2.12663700-0.18423200$

$\mathrm{N}-0.89945100 \quad 2.12668700-0.18387700$

C $1.960042001 .23639900-0.71444300$

$\mathrm{H} \quad 1.45375700 \quad 2.15787300-1.00767200$

$\mathrm{H} 2.890030001 .21603400-1.29984300$

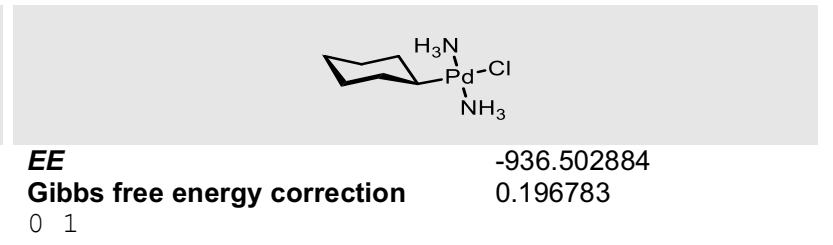

01

$\mathrm{Pd}-0.90707900 \quad 0.03030300 \quad 0.07639300$

$\mathrm{Cl}-3.37045500-0.20527000-0.21231800$

$\mathrm{H}-0.22218200-2.21968900-1.30872300$

$\mathrm{H}-0.48078400-2.63752700 \quad 0.26008800$

$\mathrm{H}-1.76437300-2.22089400-0.69890500$

$\mathrm{H}-1.09881800 \quad 2.27888300 \quad 1.56886100$

$\mathrm{H}-0.80172200 \quad 2.75436700 \quad 0.02295500$

$\mathrm{H}-2.28373500 \quad 2.12790800 \quad 0.42735900$

$\begin{array}{llll}\text { C } 1.07358600 & 0.28281500 & 0.28068900\end{array}$

C $1.80923800-0.92645900 \quad 0.84713000$

$\mathrm{H} 1.18798100 \quad 1.10701000 \quad 0.99848200$

C $3.30385500-0.63355300 \quad 1.02288000$

H $1.71573900-1.77433100 \quad 0.15786200$

H $1.36995200-1.23512800 \quad 1.79932100$

$\begin{array}{llll}\text { C } 3.19521000 & 0.99636700 & -0.89490800\end{array}$

C $3.92997100-0.20301800-0.30139500$

H $3.81754700-1.51206900 \quad 1.42009900$

$\mathrm{H} \quad 3.42753600 \quad 0.16734500 \quad 1.75923700$

$\mathrm{H} 3.627078001 .26843800-1.86073700$

H $3.326346001 .85938000-0.23337000$

H $3.87935300-1.03886100-1.00823000$

$\begin{array}{lllll}\mathrm{H} & 4.98824300 & 0.02923600 & -0.16508400\end{array}$

$\mathrm{N}-1.28014300 \quad 2.06738700 \quad 0.59437600$

$\mathrm{N}-0.78593300 \quad-2.02643800-0.48887900$

C $1.69585200 \quad 0.70960300-1.04554100$

$\mathrm{H} 1.188989001 .58982700-1.45015500$

$\mathrm{H} 1.56246100-0.08939300-1.78531900$ 
PALLADIUM

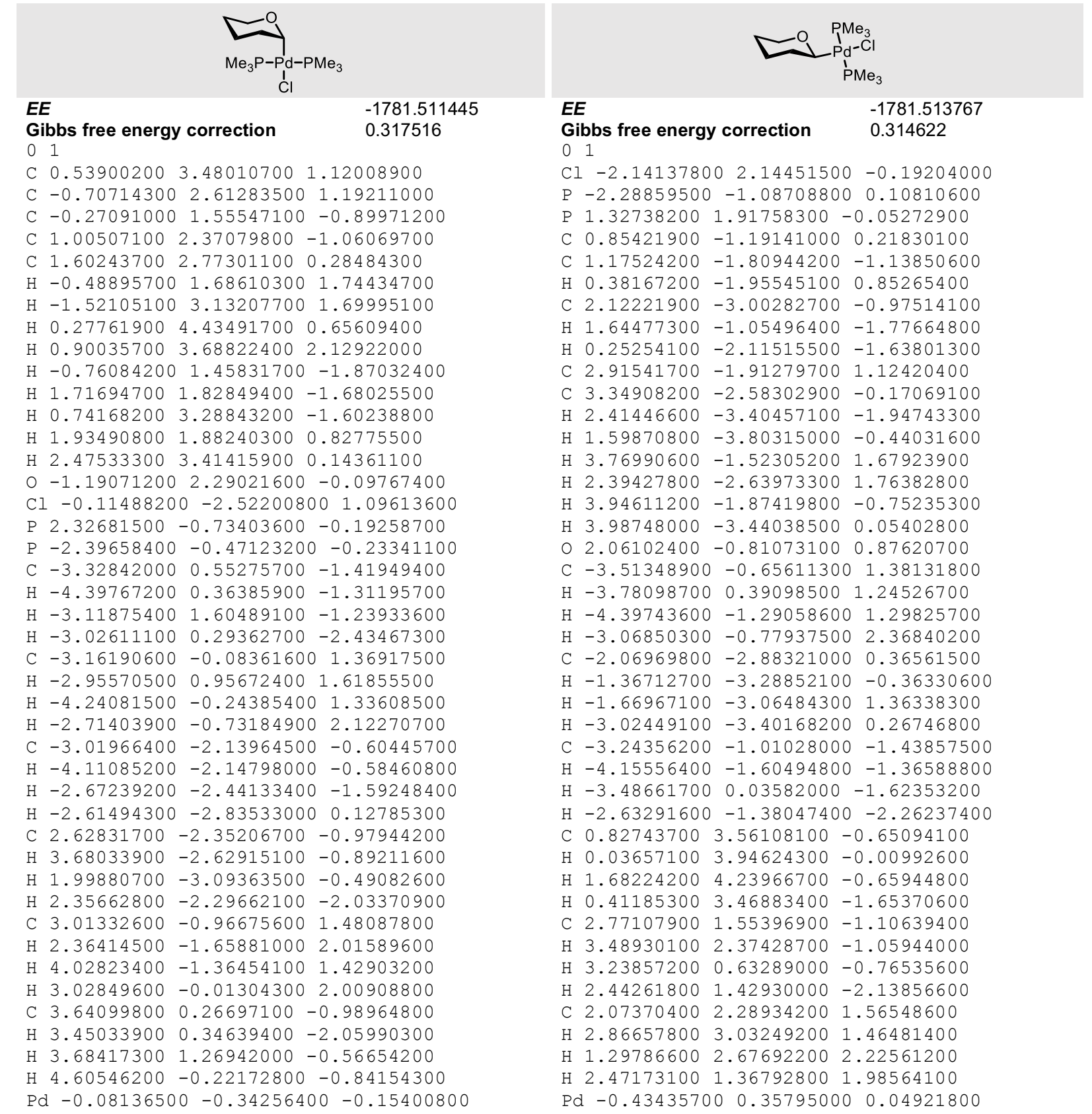

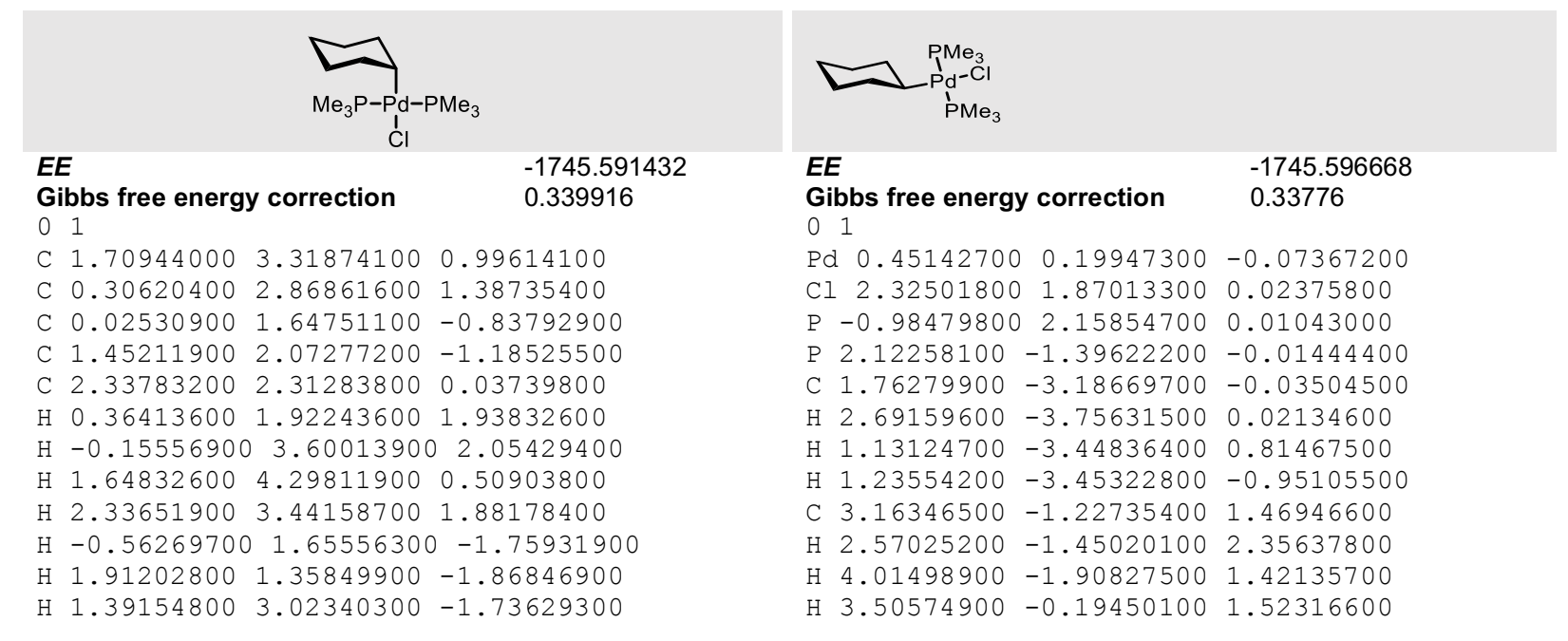


$\mathrm{H} 2.48017000 \quad 1.37594500 \quad 0.58392300$ $\mathrm{H} \quad 3.32733200 \quad 2.65348000-0.27921200$ $\mathrm{Pd}-0.19222700 \quad-0.24965500-0.06764600$ $\mathrm{Cl}-0.67708900-2.32423800 \quad 1.27212100$ P 2.05368400 -1.20796700 -0.23070900 P $-2.49431100-0.06060400-0.26182100$ C $-3.24676300 \quad 1.16462700-1.39174200$ $\mathrm{H}-4.33453200 \quad 1.09442900-1.34440200$ $\mathrm{H}-2.94241700 \quad 2.17971100-1.14467600$ $\mathrm{H}-2.92342200 \quad 0.94585700 \quad-2.40996800$ C $-3.36920000 \quad 0.18647200 \quad 1.31473500$ $\mathrm{H}-3.09560900 \quad 1.14794200 \quad 1.74907400$ $\mathrm{H}-4.45046300 \quad 0.14483000 \quad 1.17387100$ $\mathrm{H}-3.04787900-0.60784700 \quad 1.98914400$ C $-3.21833700-1.60744600-0.89454400$ $\mathrm{H}-4.30471900-1.52064500-0.95570200$ $\mathrm{H}-2.81456400-1.81213700-1.88609200$ H $-2.93535100 \quad-2.41949200 \quad-0.22729400$ C $1.86360100-2.87710600-0.94761600$ $\mathrm{H} 2.81945800-3.40395400-0.94406800$ $\mathrm{H} \quad 1.12572200-3.42485200-0.36525500$ $\mathrm{H} \quad 1.50697900-2.78647300-1.97396200$ C $2.80751500-1.55383300 \quad 1.39434800$ $\begin{array}{llll}\mathrm{H} & 2.05376000 & -2.04056200 & 2.01311200\end{array}$ $\mathrm{H} \quad 3.68133200 \quad-2.19938700 \quad 1.28982400$ H $3.10574000 \quad-0.618912001 .86951300$ C $3.49392200-0.62248500-1.20553500$ $\begin{array}{llll}\mathrm{H} & 3.21596400 & -0.54727900 & -2.25696900\end{array}$ $\mathrm{H} \quad 3.84146500 \quad 0.35087200 \quad-0.86585200$ H $4.30672400-1.34451300-1.10934400$ C $-0.55885800 \quad 2.67272400 \quad 0.14407500$ $\mathrm{H}-1.57177300 \quad 2.41575300 \quad 0.45330800$ $\mathrm{H} \quad-0.63253900 \quad 3.64167300 \quad-0.37331100$
C $3.31080100-1.21213800-1.38007400$

$\mathrm{H} 4.14754700-1.90224900-1.25905500$

H $2.81034100-1.41024800-2.32767300$

H $3.66457100-0.18169600-1.37693600$

C $-0.52552900 \quad 3.40923400-1.23434100$

$\mathrm{H}-1.05334200 \quad 4.34682200-1.05059300$

$\mathrm{H} \quad 0.55131600 \quad 3.56245700-1.18230400$

$\mathrm{H}-0.78142500 \quad 3.03934900 \quad-2.22740700$

C $-0.66619000 \quad 3.01422300 \quad 1.58930400$

$\mathrm{H} \quad 0.41158500 \quad 3.14156300 \quad 1.68921600$

$\mathrm{H}-1.16553400 \quad 3.98422400 \quad 1.61715200$

$\mathrm{H}-1.02676900 \quad 2.39767700 \quad 2.41354000$

C $-2.81531000 \quad 2.23527000-0.08781600$

$\mathrm{H}-3.14472200 \quad 2.00164200 \quad-1.09983800$

$\mathrm{H}-3.27669800 \quad 1.52486600 \quad 0.59730500$

$\mathrm{H}-3.15131700 \quad 3.24257700 \quad 0.16454000$

C $-1.02152700-1.21402500-0.08724900$

C $-1.76844900-1.22460400 \quad 1.24368700$

C $-1.97718400-1.05657600-1.26518000$

$\mathrm{H}-0.55633500-2.19679400 \quad-0.19784900$

C $-2.86319900-2.29910200 \quad 1.25805300$

$\mathrm{H}-2.23271900-0.24864800 \quad 1.42209700$

H $-1.07169200-1.38926800 \quad 2.07018400$

C $-3.05967900-2.14263500-1.24557400$

$\mathrm{H}-1.42739100-1.08882000-2.20893800$

$\mathrm{H}-2.47100300-0.08324100-1.22205300$

C $-3.81747500 \quad-2.12565900 \quad 0.07954000$

$\mathrm{H}-3.41079500 \quad-2.26787100 \quad 2.20316900$

$\mathrm{H}-2.39430000-3.28738300 \quad 1.19466400$

$\mathrm{H}-3.74938800-2.00383800-2.08170800$

$\mathrm{H}-2.59118900-3.12383900-1.37915200$

$\mathrm{H}-4.33820100-1.16585100 \quad 0.17932000$

$\mathrm{H}-4.58475300 \quad-2.90307600 \quad 0.09307300$ 
PALLADIUM
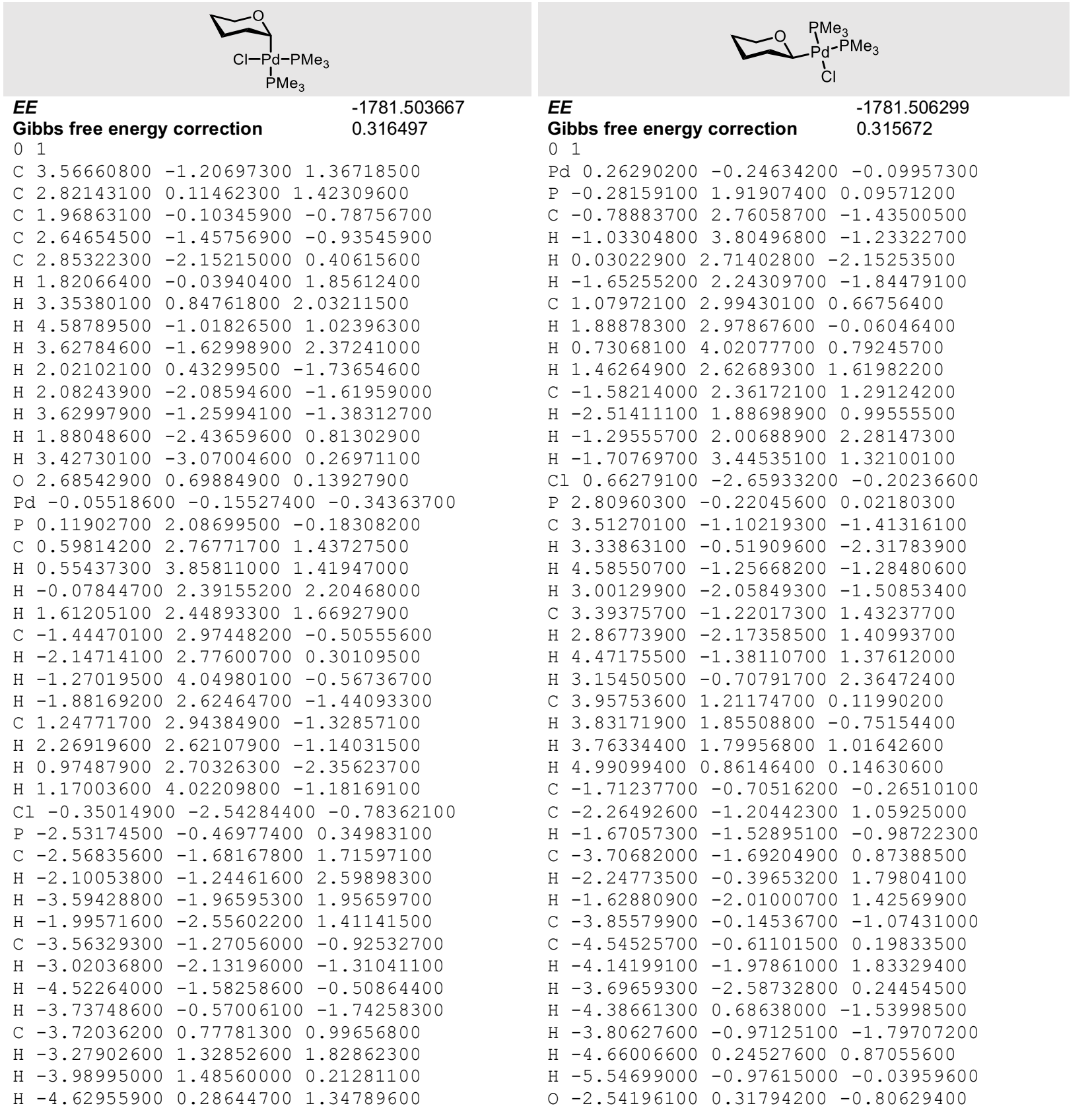

\section{1}

Pd $0.26290200-0.24634200-0.09957300$

P $-0.28159100 \quad 1.91907400 \quad 0.09571200$

C $-0.78883700 \quad 2.76058700-1.43500500$

$\mathrm{H}-1.03304800 \quad 3.80496800-1.23322700$

$\mathrm{H} \quad 0.03022900 \quad 2.71402800 \quad-2.15253500$

H $-1.65255200 \quad 2.24309700 \quad-1.84479100$

C $1.07972100 \quad 2.99430100 \quad 0.66756400$

H $1.88878300 \quad 2.97867600-0.06046400$

$\mathrm{H} \quad 0.73068100 \quad 4.02077700 \quad 0.79245700$

$\mathrm{H} \quad 1.46264900 \quad 2.62689300 \quad 1.61982200$

$\begin{array}{llll}\text { C }-1.58214000 & 2.36172100 & 1.29124200\end{array}$

$\mathrm{H}-2.51411100 \quad 1.88698900 \quad 0.99555500$

$\mathrm{H}-1.29555700 \quad 2.00688900 \quad 2.28147300$

H $-1.70769700 \quad 3.44535100 \quad 1.32100100$

Cl $0.66279100 \quad-2.65933200 \quad-0.20236600$

P $2.80960300-0.22045600 \quad 0.02180300$

C $3.51270100-1.10219300-1.41316100$

H $3.33863100-0.51909600-2.31783900$

$\mathrm{H} \quad 4.58550700-1.25668200-1.28480600$

H $3.00129900-2.05849300-1.50853400$

C $3.39375700-1.22017300 \quad 1.43237700$

H $2.86773900-2.17358500 \quad 1.40993700$

$\mathrm{H} 4.47175500-1.38110700 \quad 1.37612000$

H $3.15450500-0.707917002 .36472400$

$\begin{array}{llll}\text { C } 3.95753600 & 1.21174700 & 0.11990200\end{array}$

$\mathrm{H} 3.831719001 .85508800-0.75154400$

$\mathrm{H} \quad 3.76334400 \quad 1.79956800 \quad 1.01642600$

H $4.99099400 \quad 0.86146400 \quad 0.14630600$

C $-1.71237700-0.70516200 \quad-0.26510100$

C $-2.26492600-1.20442300 \quad 1.05925000$

$\mathrm{H}-1.67057300-1.52895100 \quad-0.98722300$

C $-3.70682000-1.69204900 \quad 0.87388500$

$\mathrm{H}-2.24773500-0.39653200 \quad 1.79804100$

$\mathrm{H}-1.62880900-2.01000700 \quad 1.42569900$

C $-3.85579900-0.14536700-1.07431000$

C $-4.54525700-0.61101500 \quad 0.19833500$

$\mathrm{H}-4.14199100-1.97861000 \quad 1.83329400$

$\mathrm{H}-3.69659300 \quad-2.58732800 \quad 0.24454500$

$\mathrm{H}-4.38661300 \quad 0.68638000-1.53998500$

$\mathrm{H}-3.80627600-0.97125100-1.79707200$

$\mathrm{H}-4.66006600 \quad 0.24527600 \quad 0.87055600$

$\mathrm{H}-5.54699000-0.97615000-0.03959600$

$\begin{array}{llll}0 & -2.54196100 & 0.31794200 & -0.80629400\end{array}$

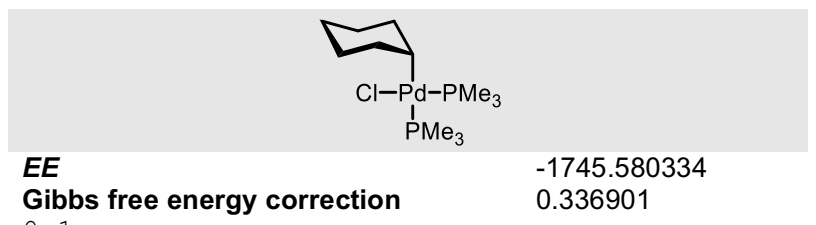

$0 \quad 1$

C $3.86919800-1.01250400 \quad 1.21332700$

C $2.91347500 \quad 0.06765100 \quad 1.70857000$

C $1.99885000 \quad 0.41733800-0.64529200$

C $2.88454700-0.74280900-1.10495800$

C $3.27953500-1.72312200-0.00034300$

$\mathrm{H} \quad 1.97284900 \quad-0.39691500 \quad 2.02764100$

$\mathrm{H} \quad 3.33160900 \quad 0.58486600 \quad 2.57563200$

$\mathrm{H} \quad 4.82333000 \quad-0.54292600 \quad 0.94718700$

H $4.08012700 \quad-1.73140300 \quad 2.00796900$

$\mathrm{H} 1.960497001 .14101500-1.46218500$

$\mathrm{H} 2.41823300-1.28033100-1.92895300$

$\mathrm{H} 3.80626100-0.28651200-1.49839100$

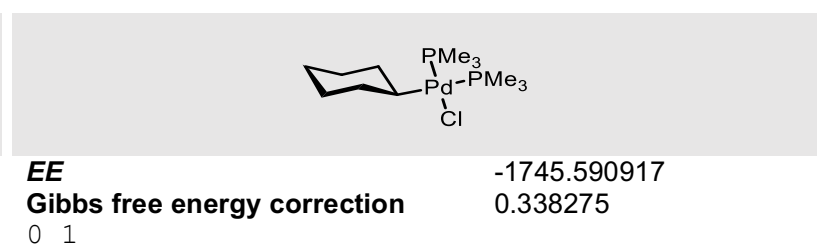

01

$\mathrm{Pd}-0.21785100-0.10485700-0.00080500$

P $-0.14632600 \quad 2.10311800 \quad-0.00138800$

C $0.63855300 \quad 2.87740200 \quad 1.45136800$

$\mathrm{H} \quad 0.67791000 \quad 3.96176700 \quad 1.33658600$

$\mathrm{H} \quad 0.05070600 \quad 2.63142900 \quad 2.33577500$

H $1.64629200 \quad 2.48995400 \quad 1.59204100$

C $-1.74484200 \quad 2.98262900-0.04307200$

$\mathrm{H}-2.33922400 \quad 2.70095100 \quad 0.82499500$

$\mathrm{H}-1.59325300 \quad 4.06339800 \quad-0.04113900$

$\mathrm{H}-2.29067700 \quad 2.69831600-0.94199300$

C $0.70089100 \quad 2.87080000-1.42280000$

H $1.71636800 \quad 2.48955300-1.51558400$ 
$\mathrm{H} \quad 2.39861600 \quad-2.28687600 \quad 0.30639300$ $\mathrm{H} 3.99406800-2.44564600-0.40129900$ $\mathrm{Pd} 0.00088300-0.08041700-0.32400500$ $\mathrm{P}-0.43235300 \quad 2.11437400-0.16965600$ C $-0.66054100 \quad 2.80198400 \quad 1.50614600$ $\mathrm{H}-0.95264900 \quad 3.85239500 \quad 1.46035800$ $\mathrm{H}-1.43837400 \quad 2.23376000 \quad 2.01586900$ $\mathrm{H} \quad 0.26069900 \quad 2.70741600 \quad 2.07935100$ C $-2.01846300 \quad 2.54146800-0.96666900$ $\mathrm{H}-2.83369900 \quad 2.04533400-0.44545600$ $\mathrm{H}-2.18341000 \quad 3.61995900-0.94715900$ $\mathrm{H}-2.00574300 \quad 2.19526900-1.99992500$ C $0.65971700 \quad 3.33984900-0.96978300$ $\mathrm{H} 1.67516600 \quad 3.28377300-0.58493000$ $\mathrm{H} \quad 0.68520700 \quad 3.13910800-2.04102800$ $\mathrm{H} \quad 0.26452400 \quad 4.34303400-0.80450000$ Cl $0.15624000-2.44193100-0.95274000$ $\mathrm{P}-2.33602000-0.92536000 \quad 0.36515000$ C $-2.05065300-2.15823400 \quad 1.68157000$ $\mathrm{H}-1.63688800-1.65948400 \quad 2.55897900$ $\mathrm{H}-2.98162500-2.65705200 \quad 1.95649800$ $\mathrm{H}-1.32613400-2.88381200 \quad 1.31609800$ C $-3.24622800-1.87706100-0.89719400$ $\mathrm{H}-2.56009300-2.59795600-1.33818300$ $\mathrm{H}-4.10277100-2.38871300-0.45462700$ $\mathrm{H}-3.59555600-1.20029200-1.67767100$ C $-3.72162800 \quad 0.02916100 \quad 1.11700900$ $\mathrm{H}-3.35638600 \quad 0.68548300 \quad 1.90757600$ $\mathrm{H}-4.22353000 \quad 0.63588600 \quad 0.36351800$ $\mathrm{H}-4.45762100-0.65314200 \quad 1.54636200$ C $2.626184001 .06878900 \quad 0.59190700$ $\mathrm{H} 2.02328600 \quad 1.88763000 \quad 0.98721700$ H $3.58492800 \quad 1.51788700 \quad 0.29031100$
H $0.15500400 \quad 2.61526100 \quad-2.33112100$ H $0.72787700 \quad 3.95615100-1.31385100$ $\mathrm{Cl}-0.06439400-2.56246500-0.04945400$ P $-2.68581200 \quad-0.67457600 \quad 0.00959200$ C $-3.09535400-1.77129000 \quad 1.40929400$ $\mathrm{H}-2.99788100-1.21865400 \quad 2.34409900$ $\mathrm{H}-4.11492500-2.15032700 \quad 1.31931100$ $\mathrm{H}-2.38303500-2.59491800 \quad 1.41223000$ C $-3.13400800-1.68905200-1.43932500$ $\mathrm{H}-2.41935000-2.50758200-1.51113400$ $\mathrm{H}-4.14872400-2.07906300-1.34190800$ $\mathrm{H}-3.06646800-1.08275100-2.34297600$

C $-4.11131200 \quad 0.48478600 \quad 0.06702900$ $\mathrm{H}-4.07330500 \quad 1.08621700 \quad 0.97528200$

$\mathrm{H}-4.09542900 \quad 1.15429500 \quad-0.79257000$ $\mathrm{H}-5.04996600 \quad-0.07238800 \quad 0.05817000$

C $1.84511400 \quad 0.07578600 \quad 0.01307000$

$\begin{array}{llll}\text { C } 2.39572000 & -0.59252100 & 1.26777700\end{array}$

H $2.18318900 \quad 1.11759100 \quad 0.03914100$

C $3.92853900-0.52722100 \quad 1.27695800$

$\mathrm{H} 2.07585800-1.63584700 \quad 1.29366400$

$\mathrm{H} \quad 1.99630800 \quad-0.11179600 \quad 2.16620200$

C $3.94900600-0.47680300-1.24030200$

C $4.50781400-1.15215800 \quad 0.00996900$

$\mathrm{H} \quad 4.31751400-1.03275600 \quad 2.16395200$

H $4.25022700 \quad 0.51934300 \quad 1.34061200$

$\mathrm{H} \quad 4.35233400 \quad-0.94680400-2.14033300$

$\mathrm{H} \quad 4.27186400 \quad 0.57126100-1.25684700$

$\mathrm{H} \quad 4.24404000-2.21454100-0.01349800$

H $5.59865700-1.09309200 \quad 0.01998700$

C $2.41632800-0.54088500-1.25831200$

H $2.03136600-0.02461300-2.14325200$

$\mathrm{H} 2.09596600-1.58200000-1.33107900$ 
PALLADIUM
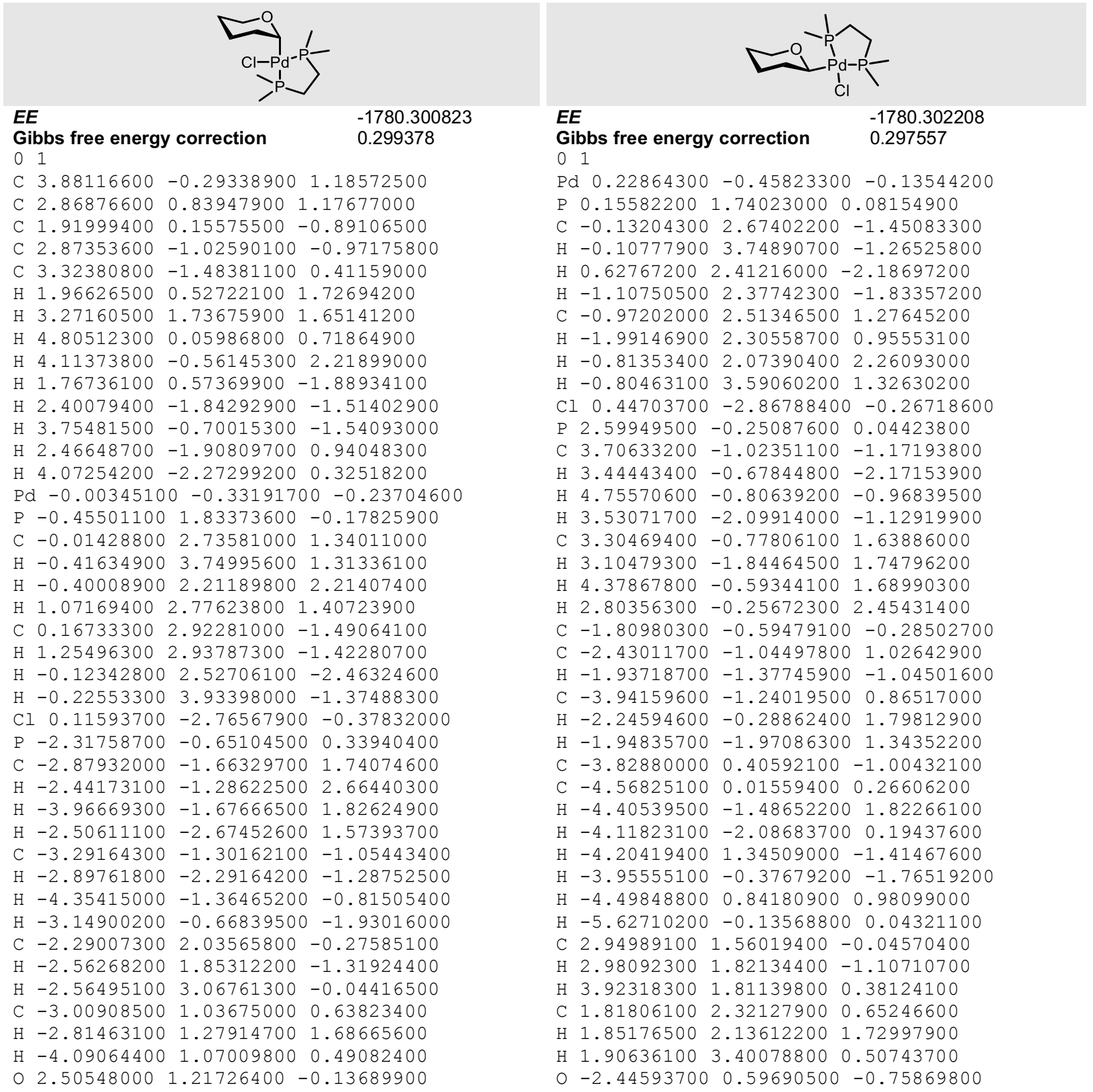

01

$\mathrm{Pd} 0.22864300-0.45823300-0.13544200$

P $0.15582200 \quad 1.74023000 \quad 0.08154900$

C $-0.13204300 \quad 2.67402200-1.45083300$

$\mathrm{H}-0.10777900 \quad 3.74890700-1.26525800$

$\mathrm{H} \quad 0.62767200 \quad 2.41216000-2.18697200$

$\mathrm{H}-1.10750500 \quad 2.37742300-1.83357200$

C $-0.97202000 \quad 2.51346500 \quad 1.27645200$

$\mathrm{H}-1.99146900 \quad 2.30558700 \quad 0.95553100$

$\mathrm{H}-0.81353400 \quad 2.07390400 \quad 2.26093000$

$\mathrm{H}-0.80463100 \quad 3.59060200 \quad 1.32630200$

$\mathrm{Cl} \quad 0.44703700-2.86788400-0.26718600$

$\begin{array}{llll}\text { P } 2.59949500 & -0.25087600 & 0.04423800\end{array}$

C $3.70633200-1.02351100-1.17193800$

H $3.44443400-0.67844800-2.17153900$

H $4.75570600-0.80639200-0.96839500$

H $3.53071700-2.09914000-1.12919900$

C $3.30469400-0.77806100 \quad 1.63886000$

H $3.10479300-1.84464500 \quad 1.74796200$

H $4.37867800 \quad-0.59344100 \quad 1.68990300$

$\mathrm{H} 2.80356300 \quad-0.25672300 \quad 2.45431400$

C $-1.80980300-0.59479100 \quad-0.28502700$

C $-2.43011700-1.04497800 \quad 1.02642900$

H $-1.93718700-1.37745900-1.04501600$

C $-3.94159600-1.24019500 \quad 0.86517000$

$\mathrm{H}-2.24594600-0.28862400 \quad 1.79812900$

$\mathrm{H}-1.94835700-1.97086300 \quad 1.34352200$

C $-3.82880000 \quad 0.40592100-1.00432100$

C $-4.56825100 \quad 0.01559400 \quad 0.26606200$

$\mathrm{H}-4.40539500-1.48652200 \quad 1.82266100$

$\mathrm{H}-4.11823100 \quad-2.08683700 \quad 0.19437600$

$\mathrm{H}-4.20419400 \quad 1.34509000-1.41467600$

$\mathrm{H}-3.95555100 \quad-0.37679200 \quad-1.76519200$

$\mathrm{H}-4.49848800 \quad 0.84180900 \quad 0.98099000$

$\mathrm{H}-5.62710200 \quad-0.13568800 \quad 0.04321100$

C $2.949891001 .56019400-0.04570400$

$\mathrm{H} 2.980923001 .82134400-1.10710700$

H $3.92318300 \quad 1.81139800 \quad 0.38124100$

$\begin{array}{llll}\text { C } 1.81806100 & 2.32127900 & 0.65246600\end{array}$

H $1.85176500 \quad 2.13612200 \quad 1.72997900$

H $1.90636100 \quad 3.40078800 \quad 0.50743700$

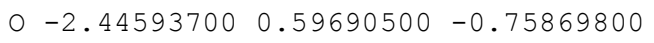

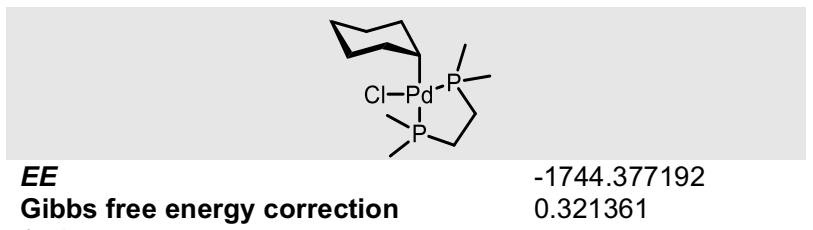

01 0.321361

C $4.29688400-0.47731300-0.38053600$

$\begin{array}{llll}\text { C } 3.62162700 & -0.70749100 & 0.96931200\end{array}$

C $1.80185800 \quad 1.00094400 \quad 0.29705700$

C $2.502238001 .13327900-1.05614200$

C $3.26746000-0.12701600-1.45250200$

H $3.00546700 \quad-1.60287500 \quad 0.90874800$

$\mathrm{H} 4.37611100-0.88082200 \quad 1.74105000$

$\begin{array}{lllll}\mathrm{H} & 5.01951900 & 0.34412400 & -0.29836500\end{array}$

H $4.85887900-1.36719200-0.67158700$

$\mathrm{H} 1.51406700 \quad 2.00684300 \quad 0.61954900$

$\mathrm{H} 1.78914600 \quad 1.40572500-1.84023300$

$\mathrm{H} \quad 3.22600900 \quad 1.96152600-0.99652100$

H $2.57009200-0.96135100-1.56571900$

H $3.75989100 \quad 0.02766500 \quad-2.41569300$

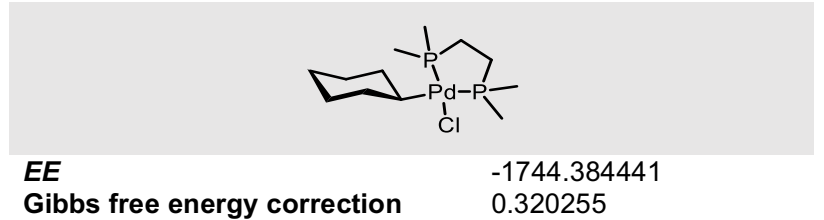

Gibbs free energy correction $\quad 0.320255$

\section{1}

$\mathrm{Pd}-0.13231800 \quad-0.27681200 \quad 0.00052900$

P $-0.69046800 \quad 1.83698000 \quad 0.00639500$

C $-0.42847200 \quad 2.72455800 \quad 1.57471400$

$\mathrm{H}-0.76747900 \quad 3.75938100 \quad 1.50781000$

H $-0.96533300 \quad 2.21378000 \quad 2.37379000$

$\mathrm{H} \quad 0.63497500 \quad 2.70291700 \quad 1.81451200$

C $0.00642100 \quad 2.97070500-1.23417000$

H $1.08419400 \quad 3.04484900-1.09280900$

$\mathrm{H}-0.18124600 \quad 2.56983000-2.22975400$

$\mathrm{H}-0.43890900 \quad 3.96319900-1.14958500$

$\mathrm{Cl} 0.32214700-2.67588300-0.08908600$

P $-2.46732100-0.76625500 \quad 0.01836900$

C $-3.16767300-1.94975100 \quad 1.20562100$

$\mathrm{H}-2.88849900-1.65613900 \quad 2.21694700$ 
$\begin{array}{lllll}\mathrm{Pd} & 0.02354000 & -0.12860300 & 0.12812300\end{array}$ $\begin{array}{llll}\mathrm{P} & -1.03190400 & 1.78881800 & 0.02879500\end{array}$ C $-1.23446000 \quad 2.65268600 \quad 1.61952100$ $\mathrm{H}-1.81586100 \quad 3.56807900 \quad 1.50012000$ $\mathrm{H}-1.73261200 \quad 1.99236000 \quad 2.32893200$ $\mathrm{H}-0.24941600 \quad 2.89542700 \quad 2.01782100$ C $-0.46437200 \quad 3.10467100-1.09124800$ $\mathrm{H} \quad 0.54385700 \quad 3.41189200 \quad-0.81551400$ $\mathrm{H}-0.43818000 \quad 2.72187200-2.11101100$ $\mathrm{H}-1.13317800 \quad 3.96521300-1.04248000$ Cl $0.80188100-2.46848200 \quad 0.06134300$ P $-2.13188500-1.13730200-0.06179200$ C $-2.68771100-2.42033000 \quad 1.09685000$ $\mathrm{H}-2.64199300 \quad-2.03716200 \quad 2.11566800$ $\mathrm{H}-3.69990700 \quad-2.76019200 \quad 0.87404500$ $\mathrm{H}-1.98386100-3.24912700 \quad 1.01201000$ C $-2.46677000-1.87454400-1.69180300$ $\mathrm{H}-1.74019300-2.67550800-1.83426300$ $\mathrm{H}-3.48102200-2.27060000-1.75852400$ $\mathrm{H}-2.30991200-1.13167800 \quad-2.47389500$ C $2.75814400 \quad 0.48487400 \quad 1.37887300$ H $2.20788200 \quad 0.24939300 \quad 2.29411900$ $\mathrm{H} \quad 3.43290500 \quad 1.31697500 \quad 1.63408100$ C $-2.76806300 \quad 1.49963400-0.53789700$ $\mathrm{H}-2.714164001 .38339800-1.62416400$ $\mathrm{H}-3.37904200 \quad 2.38300900-0.33662900$ C $-3.35934300 \quad 0.23644700 \quad 0.09541100$ $\mathrm{H}-3.52361200 \quad 0.39164700 \quad 1.16526400$ $\mathrm{H}-4.32382300-0.01649500-0.34972100$
$\mathrm{H}-4.25322500 \quad-2.02012200 \quad 1.12585700$

H $-2.71103900-2.91846900 \quad 0.99888600$

C $-3.20065600-1.28834700-1.56594900$

$\mathrm{H}-2.73858800-2.23643700-1.84343700$

$\mathrm{H}-4.28246500-1.40984900-1.49419000$

$\mathrm{H}-2.96403000-0.55955000-2.34109800$

C $1.86713000 \quad 0.30556300 \quad 0.04036300$

$\begin{array}{llll}\text { C } 2.53524600 & -0.33395300 & 1.25324400\end{array}$

H $1.98535200 \quad 1.39350700 \quad 0.13733700$

C $4.03631900-0.01902600 \quad 1.27891200$

H $2.39445400-1.41689300 \quad 1.21126700$

H $2.06621700 \quad 0.01283000 \quad 2.17915500$

C $4.04125100 \quad 0.18188100-1.23002400$

$\begin{array}{llll}\text { C } 4.70725500 & -0.46515100 & -0.01804700\end{array}$

H $4.50597400 \quad-0.50534800 \quad 2.13748900$

H $4.18147000 \quad 1.06042800 \quad 1.40522400$

$\mathrm{H} \quad 4.51240100-0.16352300-2.15360400$

H $4.189404001 .26740400-1.18349000$

H $4.62153500-1.55329400-0.10519800$

H $5.77383900-0.22885000 \quad 0.00253700$

C $2.53871700-0.12521600-1.25850100$

H $2.07425600 \quad 0.37076000-2.11651000$

H 2.39160900 $-1.20062500-1.39368500$

C $-3.28419700 \quad 0.84909700 \quad 0.39601200$

$\mathrm{H}-3.24250200 \quad 0.97251600 \quad 1.48179700$

$\mathrm{H}-4.33719000 \quad 0.85577100 \quad 0.10645200$

C $-2.51105200 \quad 1.97795300-0.29420000$

$\mathrm{H}-2.64182000 \quad 1.91069900-1.37814300$

$\mathrm{H}-2.86813600 \quad 2.96385700 \quad 0.01325800$ 


\section{PLATINUM}

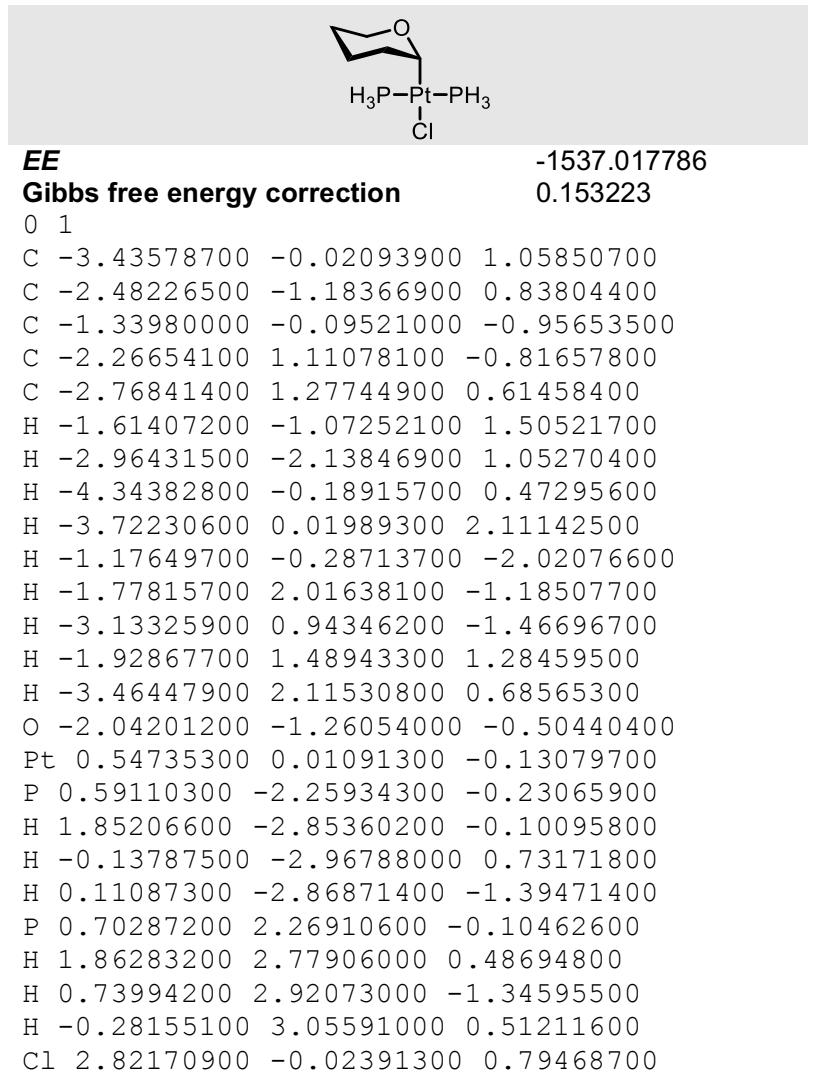

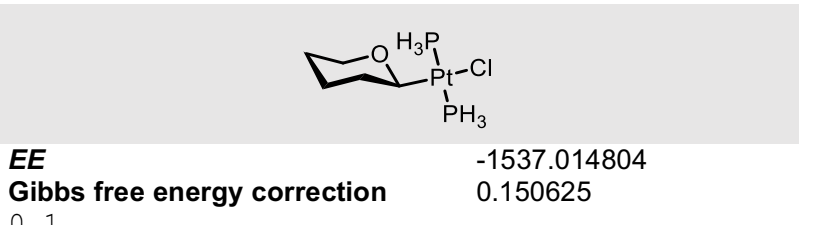

01

Pt $-0.65815400-0.02209300-0.04550700$

$\mathrm{Cl}-3.06325900-0.41149300 \quad 0.24539800$

$\mathrm{P}-1.31204600 \quad 2.13414800 \quad-0.28953200$

$\mathrm{H}-1.99328600 \quad 2.68472800 \quad 0.80069000$

$\mathrm{H}-0.38605500 \quad 3.16114500-0.54614200$

$\mathrm{H}-2.23660300 \quad 2.37627300-1.31084400$

P $-0.17731500-2.21527700 \quad 0.22292900$

H $0.23865800-2.92167400-0.90900100$

$\mathrm{H} \quad 0.83149400 \quad-2.57151400 \quad 1.12314100$

$\mathrm{H}-1.23540300 \quad-3.01723500 \quad 0.66961900$

C $1.32030800 \quad 0.38825600-0.28693100$

C $1.96410900 \quad 0.97675500 \quad 0.96690900$

H $1.441076001 .10910200-1.11240500$

C $3.45824500 \quad 1.22710500 \quad 0.75183000$

H $1.82605900 \quad 0.280142001 .79940100$

H $1.45764800 \quad 1.90571500 \quad 1.24133400$

C $3.38927200-0.57624600-0.96026800$

$\begin{array}{llll}\text { C } 4.13125600 & -0.04849500 & 0.25633900\end{array}$

H $3.92586000 \quad 1.58214700 \quad 1.67231500$

H $3.58681000 \quad 2.01502000 \quad 0.00186500$

H $3.79288800-1.53169300-1.29707900$

$\mathrm{H} \quad 3.47181200 \quad 0.14159500-1.78907500$

H $4.10428500-0.80996900 \quad 1.04141400$

H $5.17893100 \quad 0.12863900 \quad 0.00336900$

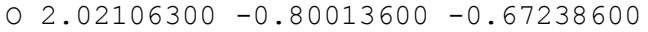

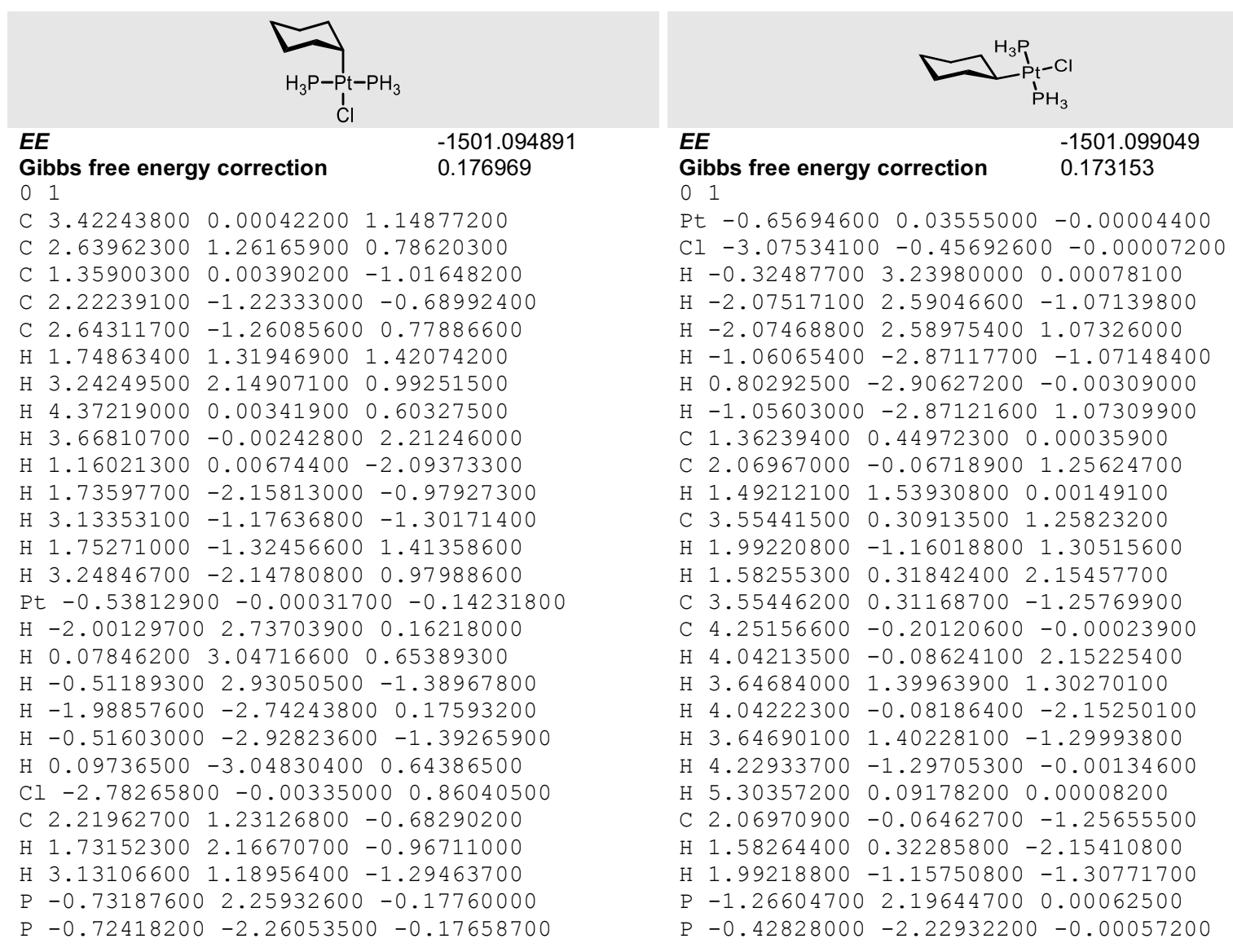




\section{PLATINUM}

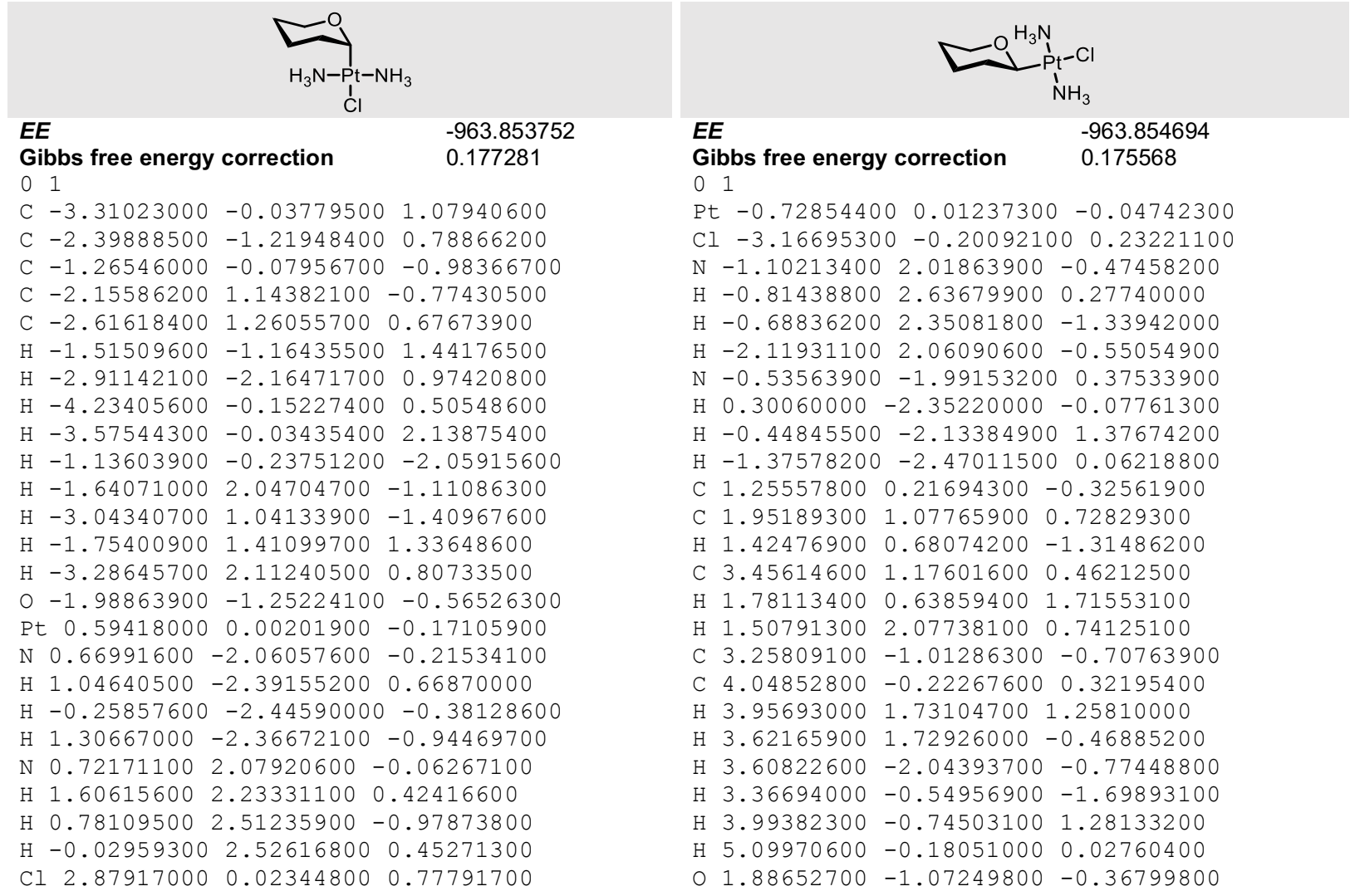

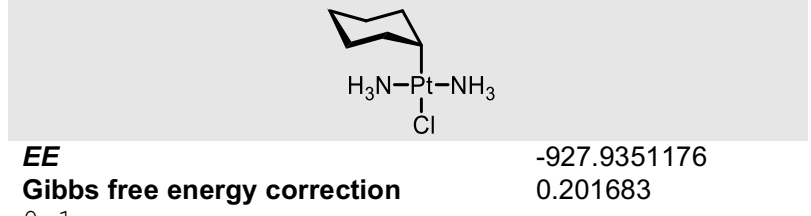

$\begin{array}{ll}0 & 1\end{array}$

C $-3.31822200 \quad 0.00007900 \quad 1.13517700$

C $-2.53216900-1.26122700 \quad 0.77832900$

C $-1.26706700-0.00004400-1.03057400$

C $-2.11413500 \quad 1.23453700 \quad-0.69242800$

C $-2.53210300 \quad 1.26132500 \quad 0.77824300$

$\mathrm{H}-1.64157100-1.30356400 \quad 1.41610200$

$\mathrm{H}-3.13232200-2.14879100 \quad 0.99334600$

$\mathrm{H}-4.25988000 \quad 0.00009600 \quad 0.57552000$

$\mathrm{H}-3.57833200 \quad 0.00012600 \quad 2.19564900$

$\mathrm{H}-1.09121500-0.00009400-2.11364600$

$\mathrm{H}-1.60692400 \quad 2.16190800 \quad-0.97108900$

$\mathrm{H}-3.02746200 \quad 1.21465300-1.30251100$

$\mathrm{H}-1.64152400 \quad 1.30369500 \quad 1.41604700$

$\mathrm{H}-3.13225600 \quad 2.14891400 \quad 0.99317400$

Pt $0.59811800-0.00001700-0.17565500$

$\mathrm{N} 0.74564700 \quad-2.07068600-0.12056800$

$\mathrm{H} \quad 1.67164400 \quad-2.23579500 \quad 0.27544700$

$\mathrm{H} \quad 0.04772800 \quad-2.51223500 \quad 0.46973100$

$\mathrm{H} \quad 0.70096700 \quad-2.49837300-1.03973900$

$\mathrm{N} 0.74569700 \quad 2.07065700-0.12061100$

$\mathrm{H} \quad 1.67166400 \quad 2.23573500 \quad 0.27549200$

$\mathrm{H} \quad 0.70113900 \quad 2.49843100-1.03975100$

$\mathrm{H} \quad 0.04772200 \quad 2.51218900 \quad 0.46964100$

Cl 2.88707300 $0.00003500 \quad 0.78769700$

C $-2.11422900-1.23455900-0.69234600$

$\mathrm{H}-1.60711300-2.16199000-0.97097300$

$\mathrm{H}-3.02757200-1.21460700-1.30240000$

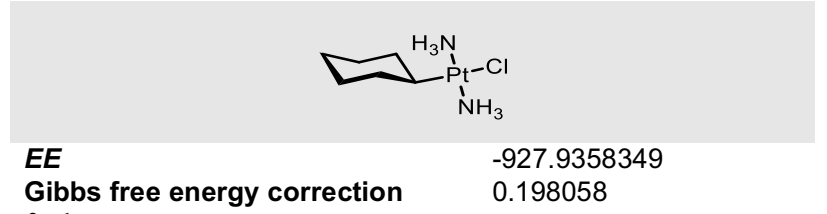

01

Pt $-0.74614400 \quad 0.02413500-0.05004800$

Cl $-3.20031700-0.19974600 \quad 0.21888800$

$\mathrm{N}-1.07734500 \quad 1.99266600-0.60589900$

$\mathrm{H}-0.61449000 \quad 2.66239000 \quad 0.00039700$

$\mathrm{H}-0.79365700 \quad 2.17909100-1.56243600$

$\mathrm{H}-2.08682400 \quad 2.11778100-0.53081900$

$\mathrm{N}-0.62898400-1.96679900 \quad 0.52424000$

$\mathrm{H}-0.25488900-2.56148700-0.20831200$

$\mathrm{H}-0.07852800-2.11393300 \quad 1.36421200$

$\mathrm{H}-1.59640200-2.23292800 \quad 0.70983000$

C $1.26082600 \quad 0.26286400-0.27108700$

C $1.93022800 \quad 0.77578700 \quad 1.00903300$

H $1.41079400 \quad 1.03451600-1.04392900$

C $3.42597700 \quad 1.04056600 \quad 0.81302400$

$\mathrm{H} 1.80356800 \quad 0.03239900 \quad 1.80602500$

$\mathrm{H} 1.43744400 \quad 1.684720001 .36596200$

C $3.49291000-0.72080900-0.98096000$

C $4.14427000-0.20426100 \quad 0.29865800$

$\mathrm{H} \quad 3.87723100 \quad 1.38247900 \quad 1.74777500$

H $3.55181700 \quad 1.85009600 \quad 0.08540600$

$\mathrm{H} 3.99436000-1.62921200-1.32393300$

H $3.61244900 \quad 0.02595800-1.77320300$

$\mathrm{H} \quad 4.09515400 \quad-0.98664300 \quad 1.06482200$

$\mathrm{H} \quad 5.20279800 \quad 0.00653400 \quad 0.13129300$

C $2.00057800-0.98405500-0.76453400$

H $1.54720000-1.35685900-1.68736300$

$\mathrm{H} \quad 1.91217700-1.78339200-0.01624400$ 


\section{PLATINUM}

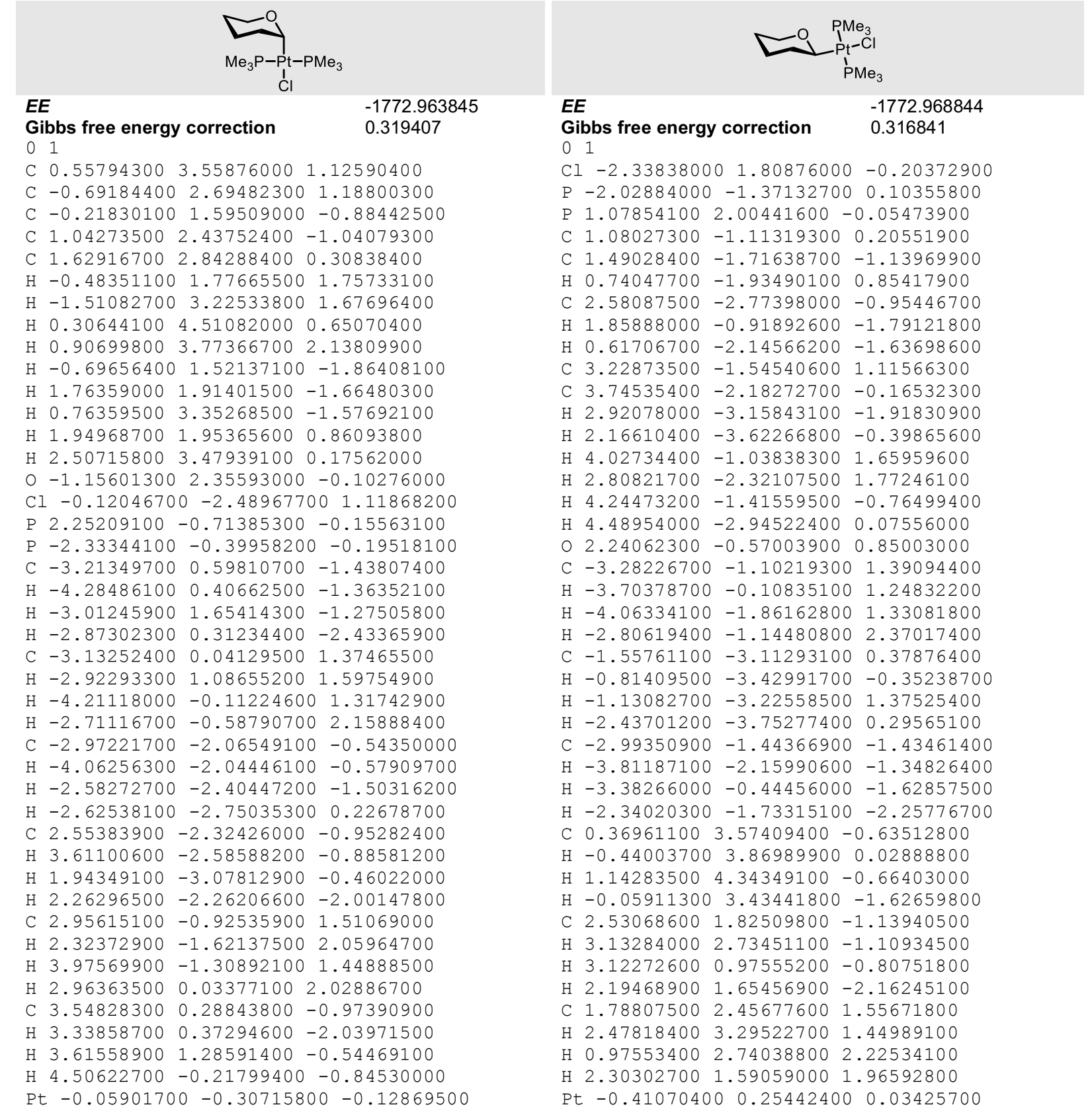

\begin{tabular}{|c|c|}
\hline $\begin{array}{l}E E \\
\text { Gibbs free energy correction }\end{array}$ & $\begin{array}{l}-1737.0428369 \\
0.339673\end{array}$ \\
\hline
\end{tabular}

01

C $1.97615200 \quad 3.24884700 \quad 1.01001700$

C $0.54316100 \quad 2.89668100 \quad 1.39328600$

C $0.19215800 \quad 1.66617100-0.82770100$

C $1.64203300 \quad 2.02542300-1.16998300$

$\begin{array}{llll}\text { C } 2.53645500 & 2.19748700 & 0.05802700\end{array}$

H $0.53425600 \quad 1.95434500 \quad 1.95267400$

H $0.12629100 \quad 3.66457000 \quad 2.04902800$

$\begin{array}{lllll}\mathrm{H} & 1.98642100 & 4.22747800 & 0.51786800\end{array}$

H $2.60506300 \quad 3.33229400 \quad 1.89886400$

$\mathrm{H}-0.37857900 \quad 1.74801200-1.75771200$

H $2.06178900 \quad 1.29971700-1.86539100$

$\mathrm{H} \quad 1.63469600 \quad 2.98607900-1.70442900$

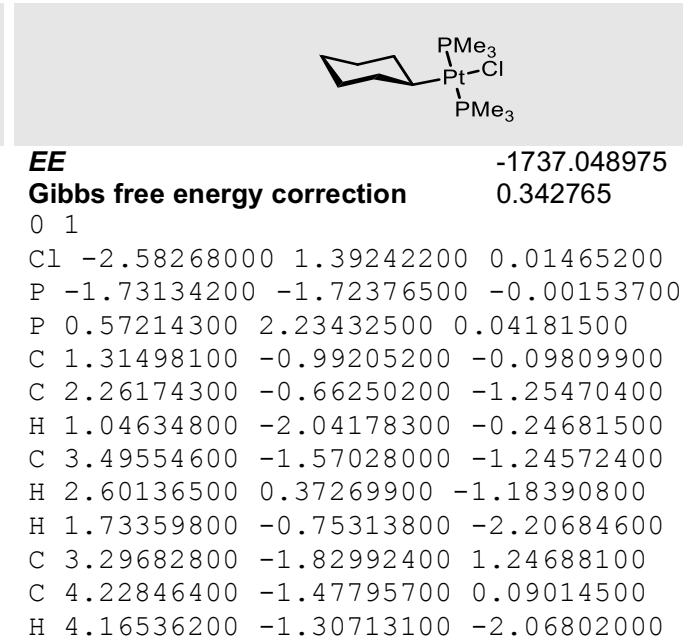


H 2.60243400 $1.25439700 \quad 0.60870600$ $\mathrm{H} 3.552118002 .46176600-0.24933600$ $\mathrm{Cl}-0.81462200 \quad-2.24973300 \quad 1.28849300$ P $1.91132400-1.29523100-0.20539800$ $\begin{array}{lllll}\mathrm{P} & -2.40592300 & 0.13831200 & -0.23493500\end{array}$ C $-3.041016001 .40068100-1.39111900$ $\mathrm{H}-4.13138800 \quad 1.40376500-1.36016900$ $\mathrm{H}-2.67203300 \quad 2.39575300-1.15500000$ $\mathrm{H}-2.71717000 \quad 1.14156500-2.39941900$ C $-3.26766700 \quad 0.47608000 \quad 1.32997700$ $\mathrm{H}-2.92295000 \quad 1.41893800 \quad 1.75328200$ $\mathrm{H}-4.34756900 \quad 0.51580800 \quad 1.18039300$ $\mathrm{H}-3.01472000-0.33120700 \quad 2.01781400$ C $-3.25275500-1.34544400-0.85893600$ $\mathrm{H}-4.32505500-1.15925800 \quad-0.93770000$ $\mathrm{H}-2.85444900-1.59682700-1.84159200$ $\mathrm{H}-3.05649400-2.17228600-0.17969500$ C $1.61938000-2.94705300-0.92157200$ H $2.54892800-3.51839800-0.93584800$ $\mathrm{H} \quad 0.86523500-3.46188100-0.33082200$ $\mathrm{H} \quad 1.25226900-2.83112000-1.94143200$ C $2.66815900-1.672002001 .40812700$ $\mathrm{H} \quad 1.90065700 \quad-2.11586400 \quad 2.04135300$ $\begin{array}{lllll}\mathrm{H} & 3.50343000 & -2.36383600 & 1.28940500\end{array}$ $\mathrm{H} \quad 3.02449200 \quad-0.75270100 \quad 1.87288500$ C $3.36150500-0.79733500-1.20774500$ $\mathrm{H} 3.06718800-0.70142300-2.25250400$ $\mathrm{H} \quad 3.78962300 \quad 0.14372200-0.87267000$ $\mathrm{H} \quad 4.11702700-1.58071400-1.12826300$ Pt $-0.17577000-0.22307700-0.06995700$ C $-0.32535300 \quad 2.74493000 \quad 0.14610700$ $\mathrm{H}-1.35326800 \quad 2.55963100 \quad 0.45755200$ $\mathrm{H}-0.33387300 \quad 3.71014000 \quad-0.38147000$
H $3.18312800-2.60730500-1.41136700$

H $3.82210500-1.74502700 \quad 2.20135100$

H $2.98779600-2.87674200 \quad 1.14799800$

H $4.59624400 \quad-0.45378500 \quad 0.22507400$

H $5.10508200 \quad-2.12958400 \quad 0.09350600$

C $-2.76508000-1.75439200 \quad 1.49340900$

$\mathrm{H}-3.31965200-0.81836600 \quad 1.54092700$

$\mathrm{H}-3.45141900-2.60219200 \quad 1.46894000$

$\mathrm{H}-2.12400500-1.83003100 \quad 2.37158400$

C $-1.01137200-3.39897600-0.00881900$

$\mathrm{H}-0.46639400-3.57261600 \quad-0.93627900$

$\mathrm{H}-0.31977600-3.51269600 \quad 0.82632800$

$\mathrm{H}-1.80770600-4.13876700 \quad 0.08167600$

C $-2.93486100-1.80110600-1.36100700$

$\mathrm{H}-3.61340000-2.64480000-1.22620500$

$\mathrm{H}-3.49150700-0.86495100-1.37125500$

$\mathrm{H}-2.40459000-1.90704300-2.30698200$

C $-0.07788900 \quad 3.38129900-1.21320300$

$\mathrm{H}-1.16521900 \quad 3.36318500-1.17007100$

H $0.29453300 \quad 4.39108500-1.03329200$

$\mathrm{H} \quad 0.24230300 \quad 3.04823700-2.20046200$

C $2.35850700 \quad 2.62932100-0.03717600$

H $2.49896700 \quad 3.67143000 \quad 0.25478400$

H $2.94021500 \quad 1.99190500 \quad 0.62610800$

$\mathrm{H} 2.724911002 .50098300-1.05456600$

C $0.09685700 \quad 3.00621600 \quad 1.62086400$

$\mathrm{H} \quad 0.41134800 \quad 4.05052000 \quad 1.65102400$

$\mathrm{H}-0.98526800 \quad 2.93420400 \quad 1.72121400$

$\mathrm{H} \quad 0.56629500 \quad 2.46169300 \quad 2.44066000$

Pt $-0.41134300 \quad 0.11931400-0.07021400$

$\begin{array}{llll}\text { C } 2.04985200 & -0.94015400 & 1.24502500\end{array}$

H $1.37600400-1.24186400 \quad 2.05164900$

H $2.35015400 \quad 0.08883400 \quad 1.47114100$ 


\section{COPPER}

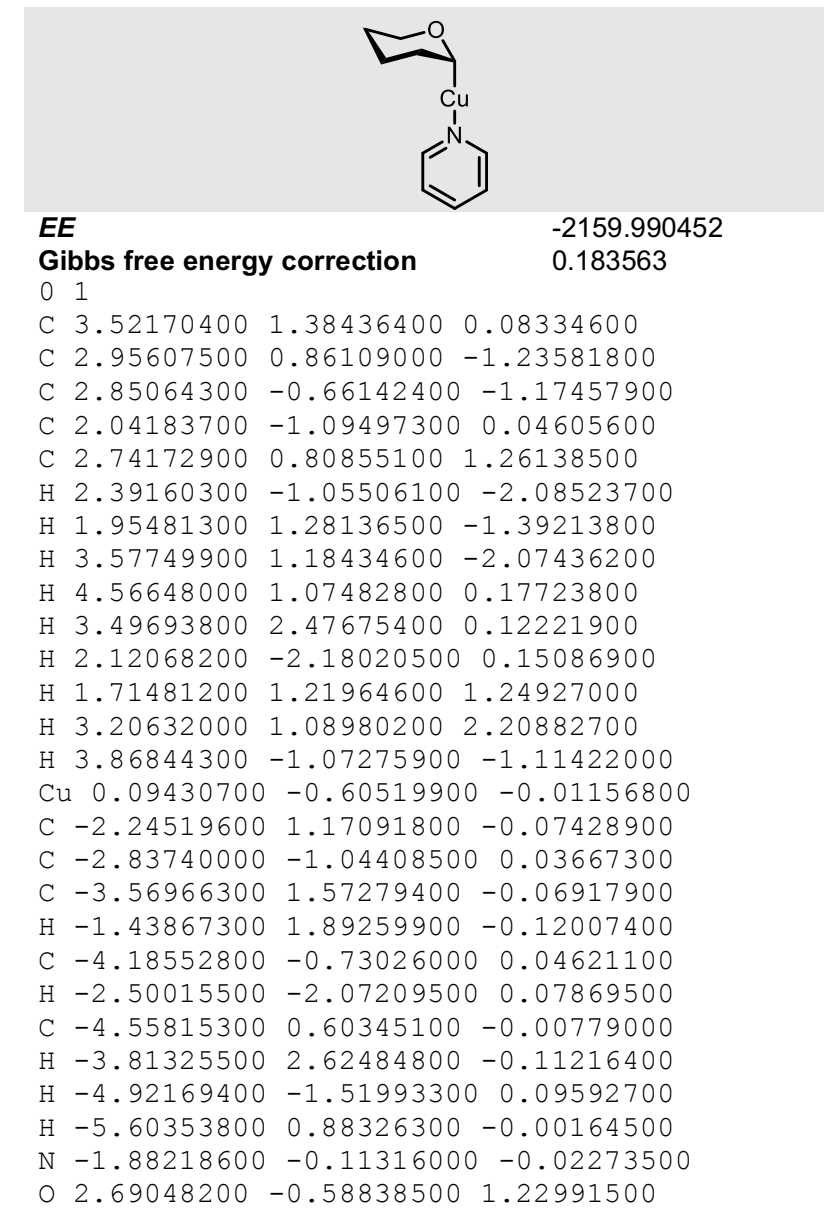

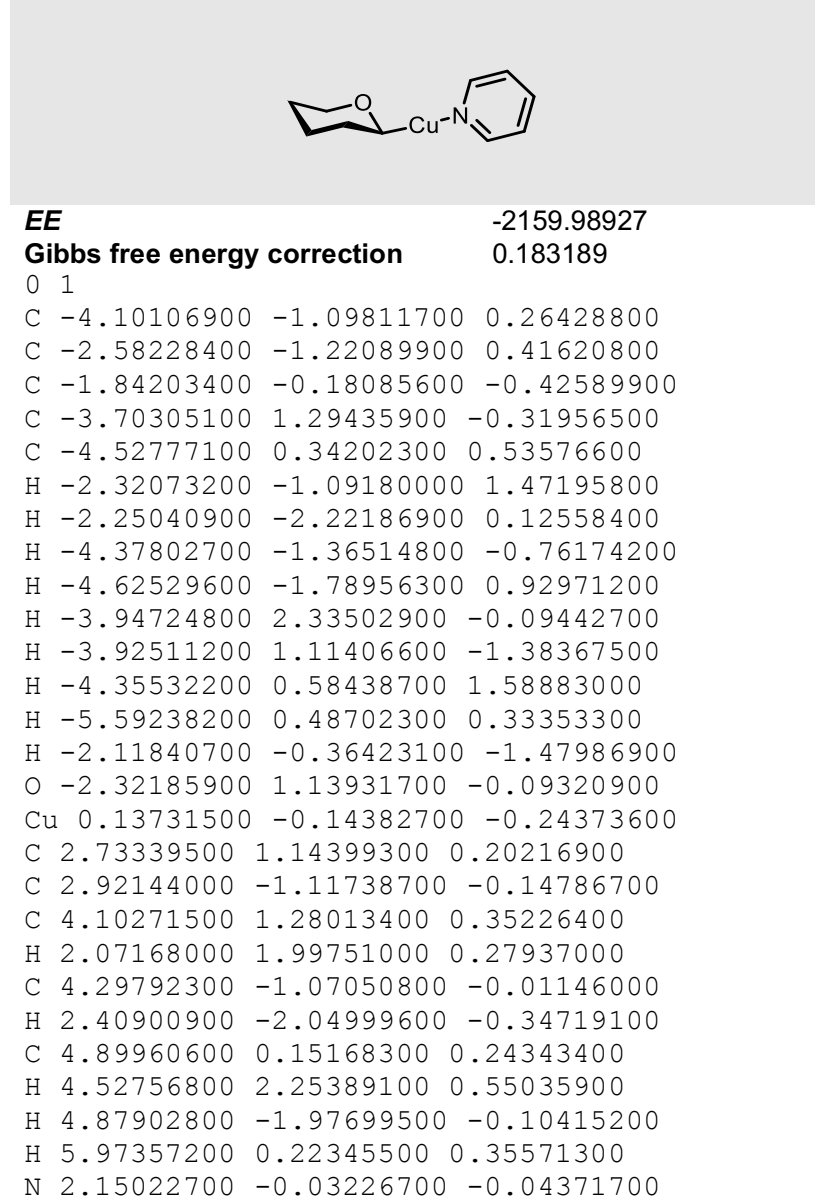

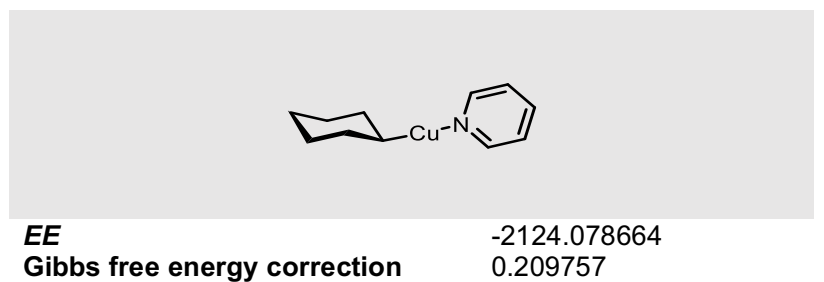

01

C $-4.00735500-0.77391500 \quad 0.99900900$

C $-2.48914300-0.59560500 \quad 1.11319700$

C $-1.81621300-0.35577300-0.24657300$

C $-4.01220400 \quad 0.65584200-1.07166200$

C $-4.64836100 \quad 0.42045800 \quad 0.29623500$

$\mathrm{H}-2.29583400 \quad 0.25822400 \quad 1.77589700$

$\mathrm{H}-2.05736000-1.46913400 \quad 1.60960200$

$\mathrm{H}-4.21407800 \quad-1.68099700 \quad 0.41967900$

$\mathrm{H}-4.45593700-0.91730700 \quad 1.98676900$

$\mathrm{H}-4.46438300 \quad 1.52770900-1.55423300$

$\mathrm{H}-4.21900400-0.20768900-1.71410400$

$\mathrm{H}-4.50404200 \quad 1.31506300 \quad 0.91364800$

$\mathrm{H}-5.72758600 \quad 0.27626100 \quad 0.19916000$

$\mathrm{H}-2.01377600-1.24707000-0.86167000$

$\mathrm{Cu} \quad 0.16239500-0.18456500-0.13091800$

C $2.75777300 \quad 1.01647800 \quad 0.59852400$

C $2.97711700-0.95339500-0.55839900$

$\begin{array}{llll}\text { C } 4.13052200 & 1.16385700 & 0.69833400\end{array}$

H $2.08247200 \quad 1.74862500 \quad 1.02305900$

C $4.35853100-0.88450700-0.50460500$

H $2.47501500-1.77808300-1.04841300$

C $4.94607900 \quad 0.19475100 \quad 0.13607000$

H $4.54393400 \quad 2.02272800 \quad 1.20768700$ 
$\mathrm{H}-1.97904300-2.05494900 \quad-0.10324800$

C $-4.57928600 \quad 0.00327700 \quad 0.53204700$

$\mathrm{H}-4.38461700 \quad 2.15066500 \quad 0.48308500$

$\mathrm{H}-4.38958100 \quad-2.14466000 \quad 0.48756700$

$\mathrm{H}-5.63031300 \quad 0.00476800 \quad 0.78996500$

$\mathrm{N}-1.88792400-0.00052200-0.12822100$
$\mathrm{H} \quad 4.95428900-1.66423700 \quad-0.95747800$

H $6.02327900 \quad 0.27938300 \quad 0.19628100$

N $2.18765600 \quad-0.02186500-0.01782500$

C $-2.49394600 \quad 0.82931800 \quad-0.95052100$

$\mathrm{H}-2.06590500 \quad 0.98497700-1.94472400$

$\mathrm{H}-2.30093800 \quad 1.75193400-0.38741400$ 


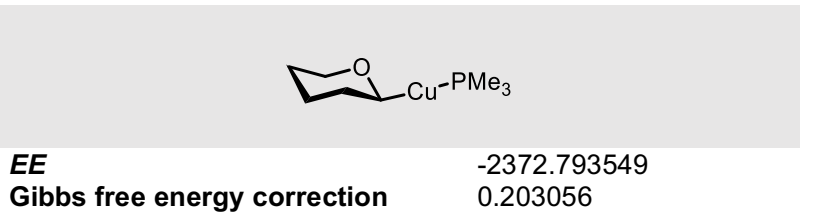

\section{Gibbs free energy correction} 0.203056

$\begin{array}{llll}\text { C } & -4.35716000 & 0.23483200 & 0.52676100\end{array}$

C $-3.51540300 \quad 1.33158100-0.11148300$

C $-1.65383300-0.10447000-0.47156600$

C $-2.41263900-1.28467000 \quad 0.13738500$

C $-3.92839100-1.12681800-0.01335700$

$\mathrm{H}-3.71876500 \quad 1.36351500-1.19402800$

$\mathrm{H}-3.76238300 \quad 2.30946100 \quad 0.30828500$

$\mathrm{H}-4.20361200 \quad 0.26596800 \quad 1.60981200$

$\begin{array}{lllll}\mathrm{H} & -5.41768200 & 0.42225600 & 0.33830500\end{array}$

$\mathrm{H}-1.91327100-0.07757400 \quad-1.54557900$

$\mathrm{H}-2.07769400 \quad-2.21156200-0.33737400$

$\mathrm{H}-2.17096400-1.36651400 \quad 1.20283200$

$\mathrm{H}-4.18746900-1.18681400-1.07638100$

$\mathrm{H}-4.46605800-1.93197300 \quad 0.49505200$

$\begin{array}{llll}0 & -2.13884300 & 1.12853100 & 0.10373900\end{array}$

$\mathrm{Cu} 0.34176600 \quad-0.10029400 \quad-0.24311200$

P $2.65663200 \quad 0.01179300 \quad 0.06631700$

C $3.23211000-0.13100600 \quad 1.79356800$

$\mathrm{H} 4.31842600-0.04782100 \quad 1.85319000$

$\mathrm{H} 2.77626000 \quad 0.65642200 \quad 2.39308200$

H 2.92106500 -1.09207200 2.20206200

C $3.441751001 .56934400-0.47418600$

$\mathrm{H} \quad 4.51571700 \quad 1.55456300-0.28205700$

$\mathrm{H} 3.267829001 .71277100-1.54022600$

H $2.99063100 \quad 2.40589000 \quad 0.05864800$

C $3.67159700-1.24689200-0.78331100$

H $4.73249700-1.10031400-0.57457000$

$\mathrm{H} 3.37310700-2.24046600-0.45017900$

H $3.50421000-1.18375300-1.85815000$

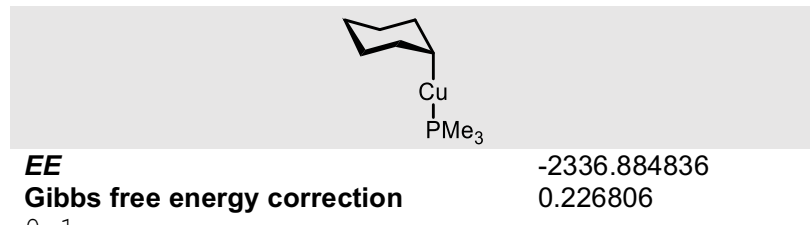

\section{Gibbs free energy correction $\quad 0.226806$}

01

C $3.381141001 .32116100-0.00555000$

C $2.69124200 \quad 0.80828000-1.26984000$

C $1.79610300-1.18892400 \quad 0.00525100$

$\begin{array}{llll}\text { C } 2.55199800 & -0.70291100 & 1.24650800\end{array}$

C $2.69028500 \quad 0.81982800 \quad 1.26283400$

$\mathrm{H} 1.691352001 .25559000-1.34002000$

H $3.248418001 .13126000-2.15365900$

$\mathrm{H} \quad 4.41631500 \quad 0.96233300-0.00350400$

H $3.42779600 \quad 2.41336700-0.01050500$

$\mathrm{H} \quad 1.79314000 \quad-2.28478700 \quad 0.01022400$

$\mathrm{H} 2.06434300-1.04038200 \quad 2.16524900$

H $3.57095000-1.12543600 \quad 1.26387400$

$\mathrm{H} 1.690327001 .267750001 .32817700$

H $3.24680100 \quad 1.15086200 \quad 2.14408300$

$\mathrm{Cu}-0.13303900-0.59371200 \quad 0.00210600$

P $-2.36181900 \quad 0.13893700 \quad-0.00072300$

C $-3.40393300-0.38258600 \quad 1.40610900$

$\mathrm{H}-2.95134800 \quad-0.04446700 \quad 2.33785900$

$\mathrm{H}-4.41042300 \quad 0.03057500 \quad 1.32332300$

$\mathrm{H}-3.46214800-1.47036900 \quad 1.43157200$

C $-2.56357400 \quad 1.95426300 \quad 0.02431800$

$\mathrm{H}-2.07410000 \quad 2.38498500 \quad-0.84891300$

$\mathrm{H}-3.61829300 \quad 2.23386500 \quad 0.02215200$

$\mathrm{H}-2.08443100 \quad 2.35959700 \quad 0.91524100$

C $-3.38631000-0.34139100-1.43492900$

$\mathrm{H}-3.44372800-1.42796100-1.49288700$

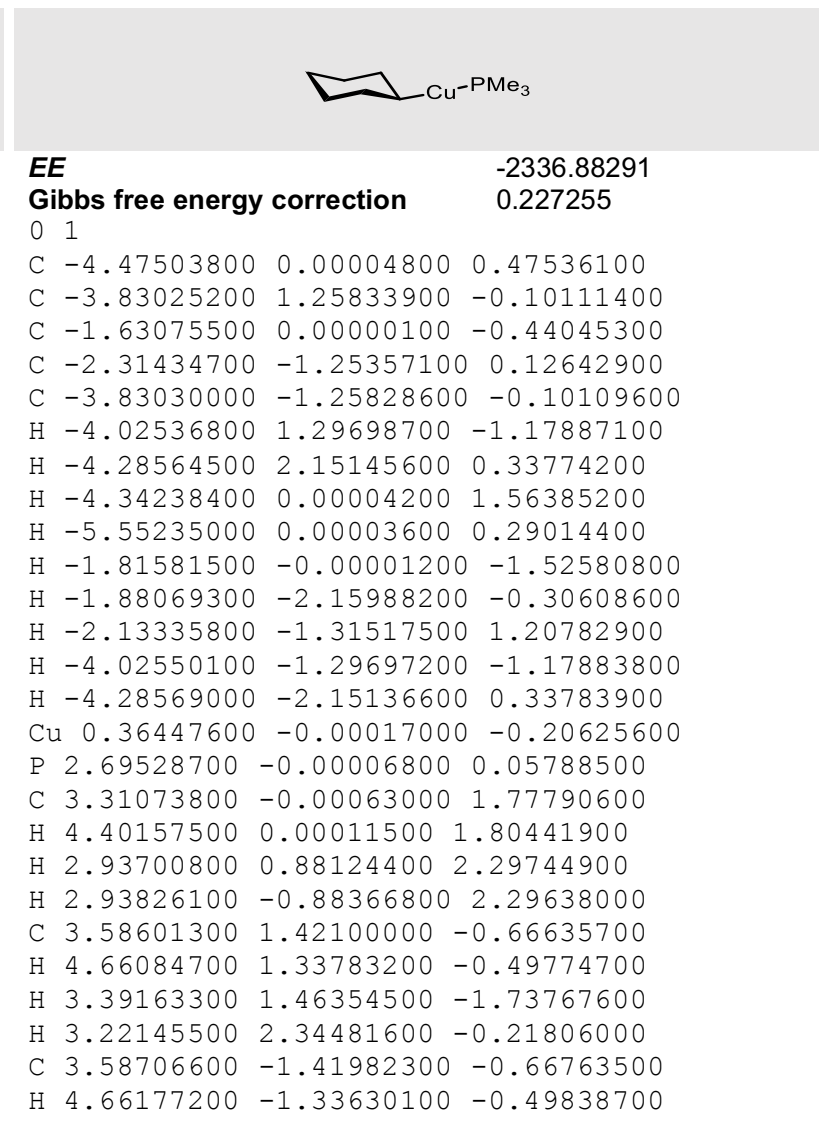


$\mathrm{H}-4.39392000 \quad 0.06911200-1.35256900$

H $-2.92238500 \quad 0.02382600-2.35074100$

C $2.55289200-0.71424900-1.23982200$

H $2.06584100-1.05998700-2.15579700$

H $3.57183400-1.13695800-1.25266200$
H $3.22276200-2.34438100-0.22067100$

H $3.39325900-1.46113700-1.73910800$

C $-2.31427900 \quad 1.25356000 \quad 0.12644800$

$\mathrm{H}-1.88058300 \quad 2.15985900-0.30604800$

$\mathrm{H}-2.13335900 \quad 1.31509600 \quad 1.20785400$ 
$\begin{array}{llll}\text { C } 3.71206000 & -0.14680600 & 1.10990400\end{array}$

$\begin{array}{llll}\text { C } 4.50664700 & -0.54827400 & -0.12494600\end{array}$

C $3.89065200-1.79523600-0.75294300$

C $2.39134200-1.57271300-0.97439100$

$\begin{array}{llll}\text { C } 1.67429000 & -1.10611900 & 0.29364900\end{array}$

$\begin{array}{llll}0 & 2.35368100 & 0.07441900 & 0.80810600\end{array}$

$\mathrm{H} \quad 4.09990700 \quad 0.78002300 \quad 1.54130100$

H $3.79473200-0.93770700 \quad 1.87147300$

$\mathrm{H} \quad 4.47643000 \quad 0.27858400 \quad-0.84238300$

H $5.55401800 \quad-0.71103800 \quad 0.14303200$

H $4.40072600-2.04967000-1.68638200$

H $4.02586700-2.64136400-0.07009600$

H $2.26073900-0.82657400-1.76830200$

$\mathrm{H} \quad 1.92008800-2.49349900-1.32972200$

$\mathrm{H} 1.82020100-1.89417900 \quad 1.05254600$

$\mathrm{Cu}-0.31035400-0.65177300 \quad 0.06872300$

P $-2.65084600-1.03646600 \quad 0.03922200$

P $-0.29230100 \quad 1.93441500-0.17548800$

C $0.30099800 \quad 2.54378500 \quad 1.44446700$

$\mathrm{H} \quad 0.65267100 \quad 3.57548100 \quad 1.37773600$

$\mathrm{H}-0.50310700 \quad 2.48654900 \quad 2.17929600$

$\mathrm{H} 1.11570300 \quad 1.88928300 \quad 1.75652300$

C $-1.47121600 \quad 3.27092500 \quad-0.64258800$

$\mathrm{H}-2.34928200 \quad 3.23845200 \quad 0.00417300$

$\mathrm{H}-1.00796000 \quad 4.25620800 \quad-0.55580200$

$\mathrm{H}-1.80154600 \quad 3.12875700-1.67235200$

C $1.132444002 .34066200-1.24750700$

H $1.40618700 \quad 3.39450700-1.16348000$

$\mathrm{H} 1.964074001 .71138000-0.92815500$

$\mathrm{H} \quad 0.89250000 \quad 2.11236500 \quad-2.28624900$

C $-3.48107400 \quad-0.15760600 \quad 1.41469100$

$\mathrm{H}-3.18709000 \quad 0.89255300 \quad 1.39298100$

$\mathrm{H}-4.56739300 \quad-0.22909300 \quad 1.33751500$

$\mathrm{H}-3.15943100 \quad-0.58088600 \quad 2.36605100$

C $-3.50636400-0.30827400-1.40577800$

$\mathrm{H}-3.19472000-0.82263300-2.31468700$

$\mathrm{H}-4.59086400-0.37970000-1.30621400$

$\mathrm{H}-3.21895800 \quad 0.74031200-1.49154100$

C $-3.43790400-2.68600000 \quad 0.13675700$

$\mathrm{H}-4.52673400-2.61082600 \quad 0.14187300$

$\mathrm{H}-3.12289500-3.28724600-0.71580500$

$\mathrm{H}-3.10710000 \quad-3.18927600 \quad 1.04496100$

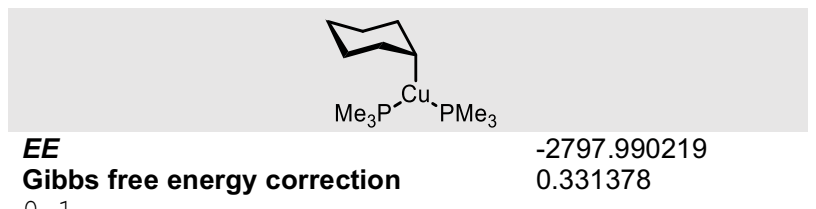

0

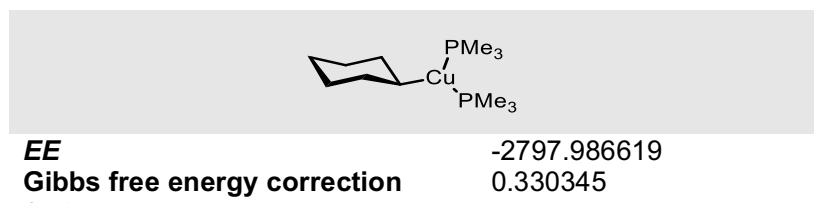

$0 \quad 1$

$\mathrm{Cu} 0.07130200-0.14225500-0.47997100$

P 2.44304200 $-0.41924200 \quad 0.06059500$

P $-1.43535100-1.95098500 \quad 0.04888000$

C $3.44795000-1.90285100 \quad 0.46617200$

$\mathrm{H} 3.35776800-2.63492200-0.33657700$

$\mathrm{H} \quad 4.50146000-1.64898300 \quad 0.60027500$

$\mathrm{H} \quad 3.07259100 \quad-2.35674700 \quad 1.38372600$

C $2.93269000 \quad 0.70627500 \quad 1.42059100$

H 2.51509900 $0.34817700 \quad 2.36190400$

$\mathrm{H} 4.01882600 \quad 0.77039100 \quad 1.51071300$

$\mathrm{H} 2.52409600 \quad 1.69820600 \quad 1.22602800$

C $3.40214000 \quad 0.27949900-1.33351700$

H 2.96677600 $1.24057100-1.60827500$

$\mathrm{H} 4.45390300 \quad 0.41226600-1.07288700$

C $4.05728700-0.45998300 \quad 1.26406400$

C $4.71881900-0.35997600-0.10838600$

C $4.01774200-1.26386700-1.11952800$

C $2.52028800-0.94288400-1.19945900$

C $1.81061100-1.01275500 \quad 0.16132300$

H $4.55791400 \quad 0.20466400 \quad 1.97527400$

H $4.17425900-1.48138100 \quad 1.64308900$

$\mathrm{H} \quad 4.65646600 \quad 0.67855700-0.45721600$

H $5.78213000-0.60504800-0.04326400$

H $4.48988100-1.16908600-2.10246800$

H $4.13791300-2.30713500-0.80633600$

H $2.41462200 \quad 0.06481700-1.62629000$

H $2.04427800-1.61983700-1.91503400$

H $1.91516300 \quad-2.04978600 \quad 0.51533800$ 
$\begin{array}{llll}\mathrm{H} & 3.32613600 & -0.38329400 & -2.19567100\end{array}$ C $-1.81076300-2.06447600 \quad 1.83895000$ $\mathrm{H}-2.14420700-1.09077900 \quad 2.19880800$ $\mathrm{H}-2.58735100 \quad-2.80554800 \quad 2.03715200$ $\mathrm{H}-0.90655500 \quad-2.33757800 \quad 2.38339200$ C $-3.11194500-1.72543700-0.64827900$ $\mathrm{H}-3.80171600-2.49503700-0.29655500$ $\mathrm{H}-3.48459500-0.74146800-0.36115400$ $\mathrm{H}-3.05749800-1.75785100-1.73648100$ C $-1.11510400-3.71150300-0.35165500$ $\mathrm{H}-1.93084400-4.35347200 \quad-0.01368100$ $\mathrm{H}-0.99656200-3.82348300-1.42934200$ $\mathrm{H}-0.18782300 \quad-4.02779200 \quad 0.12670200$ C $-0.432666001 .74552800-1.18890500$ C $-1.94309800 \quad 1.99030500-1.11853700$ C $0.26642300 \quad 2.86883100-0.41908300$ $\mathrm{H}-0.12503300 \quad 1.80645000 \quad-2.24036700$ C $-2.43927100 \quad 2.01530600 \quad 0.32824800$ $\mathrm{H}-2.49029800 \quad 1.22926800-1.68461000$ $\mathrm{H}-2.21405800 \quad 2.96204300-1.56910800$ C $-0.17823800 \quad 2.89985700 \quad 1.04435800$

$\begin{array}{lllll}\mathrm{H} & 1.35686400 & 2.76970200 & -0.47397100\end{array}$ $\mathrm{H} \quad 0.03338000 \quad 3.85884600-0.85038500$ C $-1.69444500 \quad 3.07228500 \quad 1.14452400$ $\mathrm{H}-2.26157200 \quad 1.03105600 \quad 0.78284400$ $\mathrm{H}-3.51667900 \quad 2.20194300 \quad 0.37340200$ $\mathrm{H} \quad 0.32311200 \quad 3.70223400 \quad 1.59436900$ $\mathrm{H} \quad 0.10395100 \quad 1.95265400 \quad 1.52391100$ $\mathrm{H}-2.01569300 \quad 3.04821000 \quad 2.18932700$ $\mathrm{H}-1.95864000 \quad 4.06203200 \quad 0.75635600$
$\mathrm{Cu}-0.20288300 \quad-0.68893900 \quad 0.01953800$ P $-2.52476100-1.21604700 \quad 0.02069200$ P $-0.623044001 .96369100-0.16600500$ C $-0.23265900 \quad 2.45531900 \quad 1.55845000$ $\mathrm{H}-0.00895500 \quad 3.52154400 \quad 1.63198100$ $\mathrm{H}-1.08123000 \quad 2.22483800 \quad 2.20443600$ $\mathrm{H} \quad 0.62527500 \quad 1.87921200 \quad 1.90628300$ C $-1.93166500 \quad 3.20012700-0.56319300$ $\mathrm{H}-2.83626700 \quad 2.98315000 \quad 0.00691200$ $\mathrm{H}-1.60575400 \quad 4.21634100-0.33081700$ $\mathrm{H}-2.17880800 \quad 3.14386500-1.62399800$ C $0.79343600 \quad 2.69988000-1.06478400$ $\mathrm{H} \quad 0.91715200 \quad 3.75665000-0.81952000$ $\mathrm{H} 1.70028700 \quad 2.15561500 \quad-0.80207700$ H $0.63762800 \quad 2.59524500-2.13872800$ C $-3.43873700 \quad-0.35833600 \quad 1.35649300$ $\mathrm{H}-3.24701900 \quad 0.71327000 \quad 1.28672800$ $\mathrm{H}-4.51327800 \quad-0.53493100 \quad 1.28323500$ $\mathrm{H}-3.08277400-0.70586600 \quad 2.32623400$ C $-3.42648100-0.61879600-1.45568600$ $\mathrm{H}-3.06999100-1.14594200-2.34043300$ $\mathrm{H}-4.50254000-0.77097500-1.35554400$ $\mathrm{H}-3.22120700 \quad 0.44431000-1.58643000$ C $-3.17078300 \quad-2.92097100 \quad 0.17970400$ $\mathrm{H}-4.26200700-2.93882900 \quad 0.17592700$

$\mathrm{H}-2.79797000-3.52572400-0.64669000$

$\mathrm{H}-2.80622900-3.35886600 \quad 1.10864900$

C $2.56153300-0.13573800 \quad 1.17223000$

$\begin{array}{llll}\mathrm{H} & 2.11027600 & -0.22033800 & 2.16643700\end{array}$

H $2.47511400 \quad 0.92412200 \quad 0.89002700$ 
COPPER

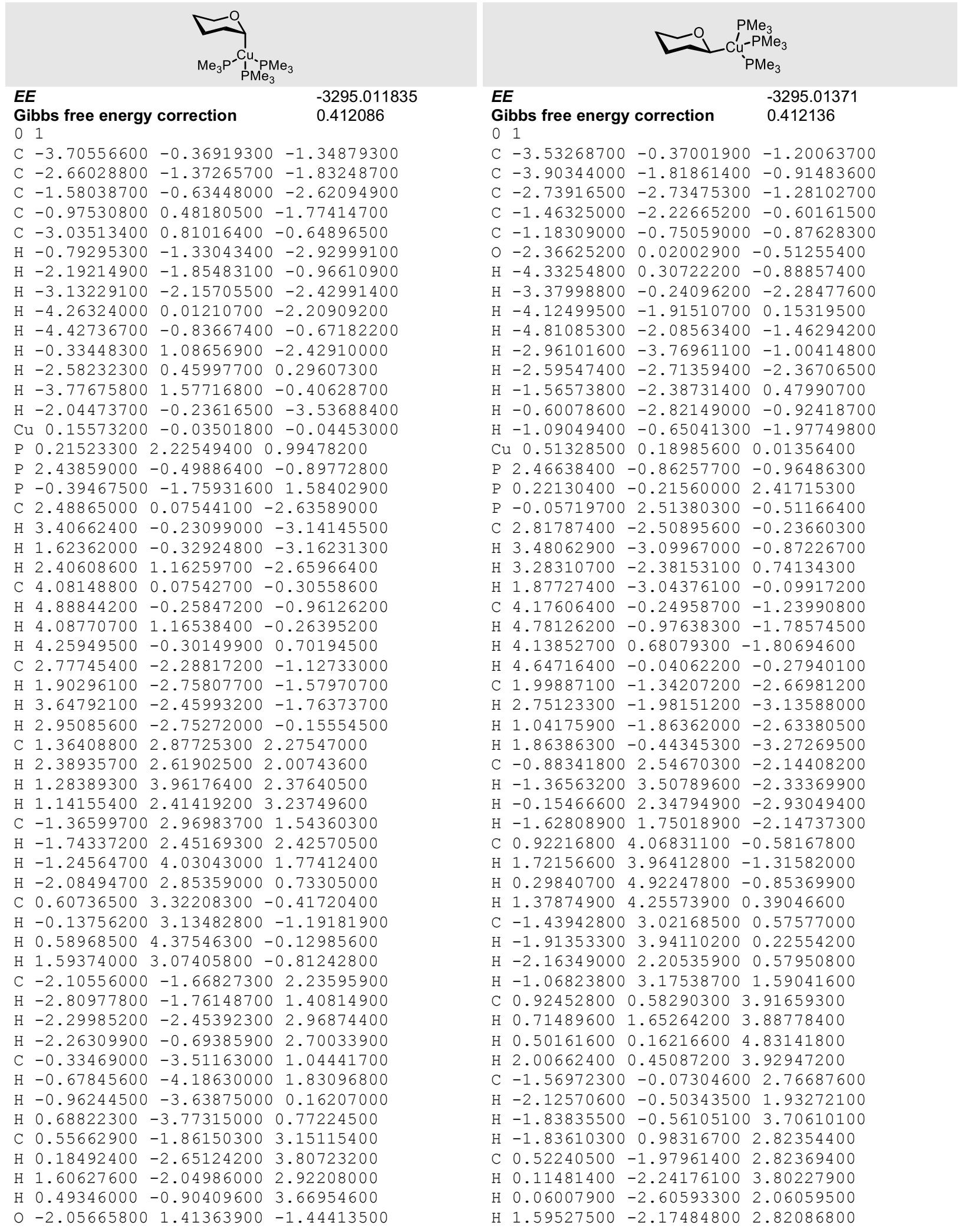

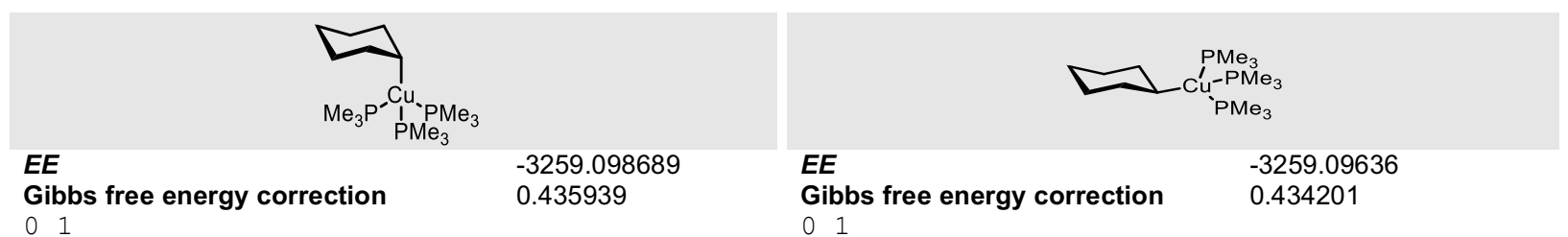


$\begin{array}{llll}\text { C } & -3.18791400 & -2.10881600 & -0.79204800\end{array}$ C $-1.77515600-2.69759200-0.76906000$ C $-0.94017200-2.15122100-1.92865300$ C $-0.87194800-0.62187500-1.89278300$ C $-3.18646100-0.58105500-0.88638500$ $\mathrm{H} \quad 0.05888400 \quad-2.60039500-1.90365500$ $\mathrm{H}-1.28079300 \quad-2.43058400 \quad 0.17383200$ H $-1.83367700-3.78971900-0.80475400$ $\mathrm{H}-3.71034300 \quad-2.50686800 \quad-1.66901100$ $\mathrm{H}-3.75745300 \quad-2.43918100 \quad 0.08232600$ $\mathrm{H}-0.30120700 \quad-0.28689800 \quad-2.77142200$ $\mathrm{H}-2.79301700-0.15844800 \quad 0.04497900$

$\mathrm{H}-4.21410900-0.22153600 \quad-0.99814800$ $\mathrm{H}-1.40827100-2.51163500-2.86311100$ $\begin{array}{ccccc}\mathrm{Cu} & 0.15576300 & 0.10059000 & -0.16739800\end{array}$ P $0.05548000 \quad 2.56319500-0.38343900$

$\begin{array}{llll}\text { P } 2.41950500 & -0.90332400 & -0.37974800\end{array}$ $\begin{array}{llll}\mathrm{P} & -0.45077500 & -0.28322900 & 2.20197200\end{array}$ C $2.74021900-0.92026600-2.18270100$ $\mathrm{H} 3.63844600-1.48906800-2.43133800$ H $1.87486600-1.35205300-2.68595100$ $\begin{array}{lllll}\mathrm{H} & 2.85195700 & 0.10569400 & -2.53554600\end{array}$ C $4.06628700-0.37788900 \quad 0.24962300$ $\mathrm{H} \quad 4.86982000-1.00061400-0.14957400$ $\mathrm{H} \quad 4.24647000 \quad 0.65993600-0.03200500$ $\begin{array}{lllll}\mathrm{H} & 4.07725600 & -0.43831300 & 1.33857900\end{array}$ C $2.49574800-2.69851400-0.00000300$ $\begin{array}{llll}\mathrm{H} & 1.58445800 & -3.18237000 & -0.35193500\end{array}$ $\mathrm{H} 3.36078400-3.16950300-0.47152400$ H $2.56066400-2.84108100 \quad 1.07905800$ C $0.99817100 \quad 3.98115400 \quad 0.31455100$ $\mathrm{H} 2.04241100 \quad 3.90991500 \quad 0.00915000$ $\mathrm{H} \quad 0.59418900 \quad 4.93926600 \quad-0.01967700$ $\mathrm{H} \quad 0.96185900 \quad 3.94023300 \quad 1.40384300$ C $-1.64955800 \quad 3.05267100 \quad 0.08764100$ $\mathrm{H}-1.74687400 \quad 3.01469600 \quad 1.17394500$ $\mathrm{H}-1.89315700 \quad 4.06072600-0.25435400$ $\mathrm{H}-2.35672800 \quad 2.34147900-0.34005400$ $\begin{array}{llll}\text { C } 0.07113800 & 2.99592600 & -2.16301900\end{array}$ $\mathrm{H}-0.53907000 \quad 2.27201800 \quad-2.70336900$ $\mathrm{H}-0.30345600 \quad 4.00578200-2.34178800$ $\mathrm{H} \quad 1.09224600 \quad 2.92126700 \quad-2.53909600$ $\begin{array}{llll}\text { C }-2.17758800 & -0.64489700 & 2.70261700\end{array}$ $\mathrm{H}-2.54940300-1.49191500 \quad 2.12393300$ $\mathrm{H}-2.24780200-0.87155000 \quad 3.76825700$ $\begin{array}{lllll}\mathrm{H} & -2.80906000 & 0.21483900 & 2.47500500\end{array}$ C $0.41756600-1.72257900 \quad 2.93751900$ $\mathrm{H} 0.12825500 \quad-1.88440600 \quad 3.97777700$ $\mathrm{H} \quad 0.18994000 \quad-2.61752900 \quad 2.35678300$ H $1.49358600-1.54879500 \quad 2.88876600$ C $-0.01183100 \quad 1.01683700 \quad 3.42302300$ $\mathrm{H}-0.22876900 \quad 0.70923500 \quad 4.44802200$ $\mathrm{H} \quad 1.05075000 \quad 1.24562400 \quad 3.33208600$ $\mathrm{H}-0.57181700 \quad 1.92595700 \quad 3.19972600$ C $-2.30595000-0.10739000-2.04269600$ $\mathrm{H}-2.34000700 \quad 0.98590700-2.10489400$ $\mathrm{H}-2.76739500-0.47380800 \quad-2.97785000$
C $-3.75632000 \quad 1.52638400-1.06144100$ C $-4.57428800 \quad 0.32316400 \quad-0.59810600$ C $-3.86186100-0.98579200-0.93143900$ C $-2.44933900-0.99570800-0.33395000$ C $-1.57888900 \quad 0.19193600-0.76413300$ $\mathrm{H}-4.26969900 \quad 2.45601700-0.79466800$ $\mathrm{H}-3.68143800 \quad 1.50454100 \quad-2.15473900$ $\mathrm{H}-4.70960100 \quad 0.38399700 \quad 0.48904400$ $\mathrm{H}-5.57420800 \quad 0.34292000-1.03936100$ $\mathrm{H}-4.44932700-1.83748900-0.57286200$ $\mathrm{H}-3.78509500-1.08408800 \quad-2.02055900$ $\mathrm{H}-2.55798700 \quad-0.99186100 \quad 0.76199600$ $\mathrm{H}-1.96016800-1.94841600-0.57659300$ $\mathrm{H}-1.48350700 \quad 0.13112200-1.86277300$ $\begin{array}{cllll}\mathrm{Cu} & 0.39564700 & 0.02029000 & 0.01273000\end{array}$ P $1.32602500-1.91268700-1.20872100$ $\begin{array}{llll}\mathrm{P} & 0.27766500 & -0.45887400 & 2.40973600\end{array}$ P $1.55224700 \quad 2.10853400-0.47746400$ C $0.98045900-3.57715300-0.51626000$ $\mathrm{H} 1.16404600-4.36494100-1.24981100$ H $1.61431000 \quad-3.74834100 \quad 0.35453500$ $\mathrm{H}-0.06069100-3.62155600-0.19441100$ C $3.04598800-2.15956900-1.80821200$ $\mathrm{H} 3.13275100-3.04854600-2.43655300$ $\mathrm{H} 3.35364800-1.28603000-2.38424300$ H $3.71936200-2.25580000-0.95626200$ C $0.40385200-2.00984500-2.78761000$ H $0.67198000-2.89818400-3.36299600$ $\mathrm{H}-0.66458200-2.01753000-2.56925600$ $\mathrm{H} \quad 0.61088200-1.11671900-3.37827500$ C $1.07469200 \quad 2.62127500-2.17036100$ $\mathrm{H} \quad 1.32356500 \quad 3.66567800-2.36905500$ $\mathrm{H} \quad 1.59174400 \quad 1.98738700-2.89230500$ H $0.00278600 \quad 2.46522100 \quad-2.29376300$ C $3.326036002 .59291700-0.47122100$ $\mathrm{H} 3.88304000 \quad 1.93848800-1.14239300$ $\mathrm{H} \quad 3.46296300 \quad 3.62899300 \quad-0.78830600$ H $3.73258300 \quad 2.47031500 \quad 0.53299100$ C $0.83882500 \quad 3.46773300 \quad 0.52539300$ H $1.11378500 \quad 4.44980400 \quad 0.13517700$ $\mathrm{H}-0.24694200 \quad 3.36907000 \quad 0.53411100$ $\mathrm{H} \quad 1.19785700 \quad 3.37982900 \quad 1.55198100$ $\begin{array}{llll}\text { C } 1.59070600 & -0.28687200 & 3.68422300\end{array}$ H $1.95295400 \quad 0.74180100 \quad 3.69239800$ $\mathrm{H} \quad 1.22454800 \quad-0.54360400 \quad 4.68057900$ H $2.42886700 \quad-0.93694600 \quad 3.43222300$ C $-1.06039300 \quad 0.53212000 \quad 3.17418200$ $\mathrm{H}-1.97748300 \quad 0.38114300 \quad 2.60402500$ $\mathrm{H}-1.22738100 \quad 0.25369200 \quad 4.21676300$ $\mathrm{H}-0.80475000 \quad 1.59096000 \quad 3.11893300$ C $-0.31288000 \quad-2.15723200 \quad 2.77187400$ H $-0.58879900-2.27320000 \quad 3.82207800$ $\mathrm{H}-1.17811600 \quad-2.37264900 \quad 2.14389100$ $\mathrm{H} \quad 0.47170100 \quad-2.87405100 \quad 2.52784600$ C $-2.33977800 \quad 1.49016300-0.47173000$ $\mathrm{H}-2.42343000 \quad 1.63688400 \quad 0.61590500$ $\mathrm{H}-1.78386700 \quad 2.35795000 \quad-0.84498100$ 


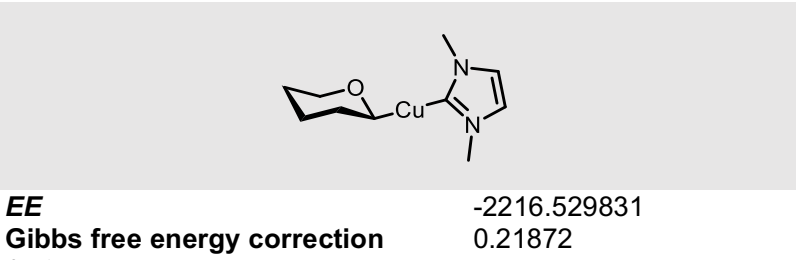

Gibbs free energy correction $\quad 0.21872$

01

C $-4.54330000 \quad 0.48686900 \quad 0.49531000$

C $-3.63456100 \quad 1.28772900-0.42778200$

C $-1.92916700-0.38492500-0.43169500$

C $-2.76984200-1.27162500 \quad 0.48557100$

C $-4.26917600-1.00525700 \quad 0.32752200$

$\mathrm{H}-3.88623400 \quad 1.05794300-1.47575300$

$\mathrm{H}-3.77157900 \quad 2.36142300 \quad-0.27499000$

$\mathrm{H}-4.33660800 \quad 0.78402900 \quad 1.52843400$

$\mathrm{H}-5.58967600 \quad 0.72648300 \quad 0.28619900$

$\mathrm{H}-2.23945700-0.61200400-1.46791000$

$\mathrm{H}-2.54466100 \quad-2.32067900 \quad 0.27182500$

$\mathrm{H}-2.48804400-1.09239500 \quad 1.52942200$

$\mathrm{H}-4.58095000-1.31443200-0.67668500$

$\mathrm{H}-4.85787600-1.58878200 \quad 1.04130100$

$0-2.274199001 .00736800-0.19783000$

$\mathrm{Cu} 0.06446800-0.40033000-0.23650100$

C $2.03403400-0.03517900-0.01169300$

N 2.55629000 $1.20249300 \quad 0.13273100$

C $4.27963400-0.13800200 \quad 0.19990800$

$\begin{array}{llll}\text { C } 3.92683300 & 1.16379600 & 0.26300700\end{array}$

H $5.24318800 \quad-0.61238400 \quad 0.26134400$

$\begin{array}{lllll}\mathrm{H} & 4.52175600 & 2.05129600 & 0.38811600\end{array}$

C $3.04262300-2.29319700-0.08139400$

$\mathrm{H} 1.99846000-2.57139800-0.19931800$

H $3.60845600-2.62865600-0.94980600$

H $3.44350600 \quad-2.76018500 \quad 0.81746500$

C $1.76854300 \quad 2.42497400 \quad 0.14271500$

H $2.03498900 \quad 3.04729700-0.71065600$

H $0.71450000 \quad 2.15945700 \quad 0.08122400$

$\mathrm{H} \quad 1.95012300 \quad 2.97387100 \quad 1.06579200$

N $3.11091200 \quad-0.84840100 \quad 0.03246400$
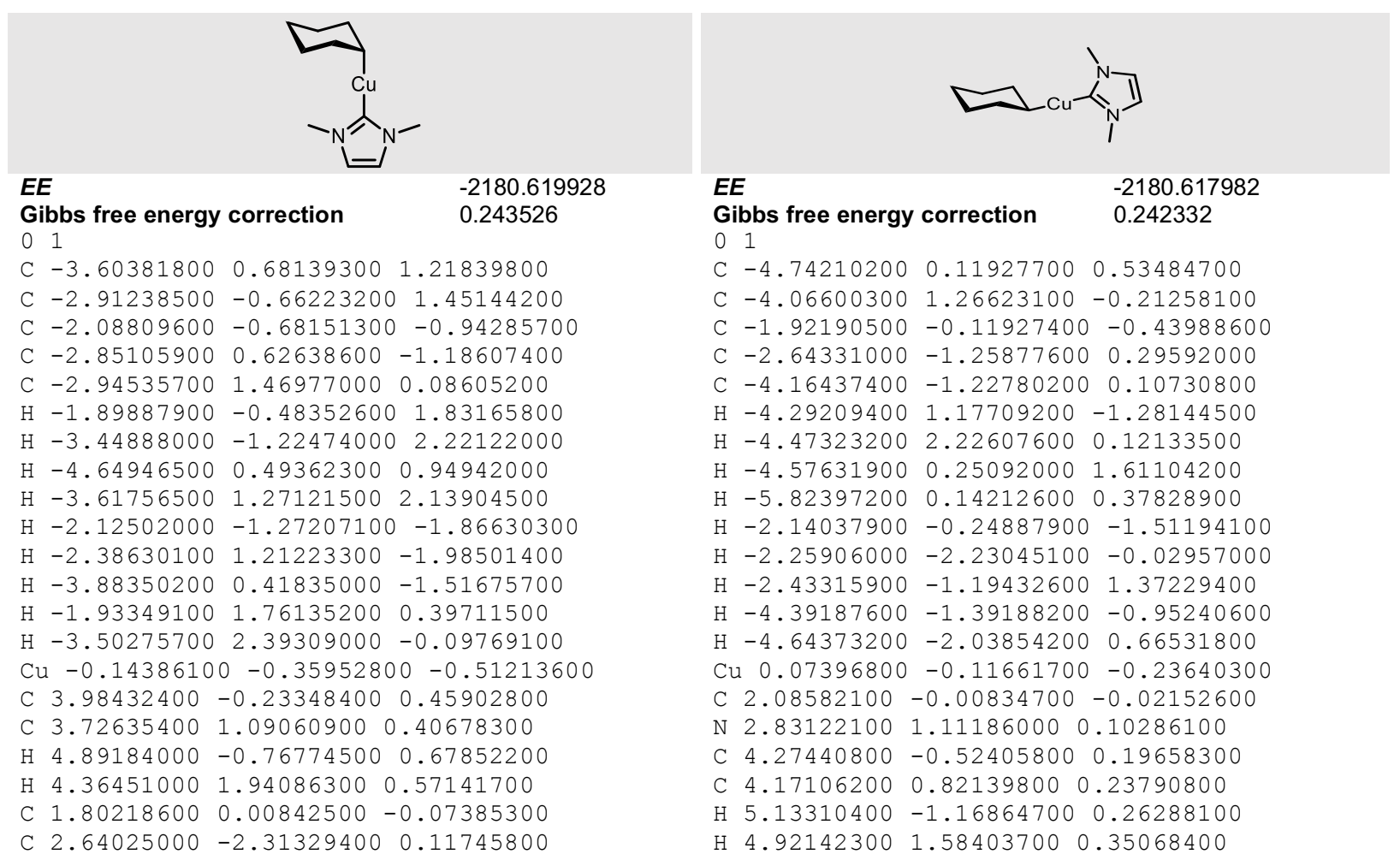

H $2.83940800 \quad-2.74487200 \quad 1.09777700$
$\mathrm{H} \quad 1.61467600-2.53035300-0.17106500$
$\mathrm{H} 3.32281100-2.74240900-0.61501400$
C $1.70775100 \quad 2.48387800-0.07617600$
$\mathrm{H} \quad 0.66983500 \quad 2.27978000-0.32895000$
$\mathrm{H} 1.74868500 \quad 3.04984400 \quad 0.85363500$
$\mathrm{H} 2.16932600 \quad 3.06173300-0.87596800$
$\begin{array}{llll}\mathrm{N} & 2.79972600 & -0.87132400 & 0.16268600\end{array}$
$\begin{array}{llll}\mathrm{N} & 2.39344700 & 1.21366300 & 0.08127100\end{array}$
C $-2.82132400-1.46397000 \quad 0.15267900$
$\mathrm{H}-2.33496700 \quad-2.42477600 \quad 0.34561800$
$\mathrm{H}-3.85346000-1.69746900-0.16170800$

C $2.65909600-2.41599600-0.05387000$

$\mathrm{H} 1.58025000-2.50004700-0.15988700$

H $3.14453100-2.86163100-0.92135800$

H $2.97641300 \quad-2.93678900 \quad 0.84875600$

C $2.28537700 \quad 2.45744100 \quad 0.10359500$

H $2.73449900 \quad 3.04311900-0.69754200$

$\mathrm{H} \quad 1.21175700 \quad 2.38782100 \quad-0.05587400$

H $2.47769800 \quad 2.94070200 \quad 1.06084900$

$\begin{array}{llll}\mathrm{N} & 2.99369800 & -1.00664600 & 0.03870700\end{array}$

C $-2.54546900 \quad 1.22316600-0.02391300$

$\mathrm{H}-2.08981700 \quad 2.04951500-0.57959500$

$\mathrm{H}-2.33113800 \quad 1.41561900 \quad 1.03655700$ 


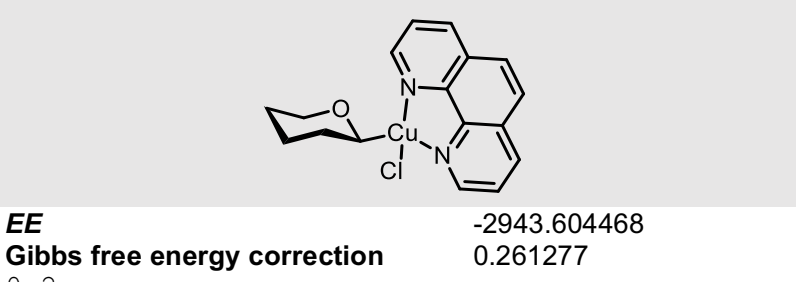

$\begin{array}{ll}0 & \text { Gibbs } \\ 0 & 2\end{array}$

C $3.52003600-2.55284900 \quad 0.42897200$

C $4.30178700-1.42915400 \quad 0.36005400$

C $3.69529900-0.17227200 \quad 0.18010800$

C $2.29744700-0.14961100 \quad 0.09004900$

$\begin{array}{llll}\text { C } 2.12465900 & -2.42674200 & 0.32203900\end{array}$

C $4.42519400 \quad 1.05777400 \quad 0.08147000$

C $1.61284900 \quad 1.10148400-0.10713900$

C $2.35391100 \quad 2.28634100-0.20604000$

C $3.78335700 \quad 2.23316500-0.10292100$

C $1.63318700 \quad 3.47708200-0.40953700$

H $2.16721300 \quad 4.41617600-0.49285200$

C $0.26699700 \quad 3.43094400-0.49843800$

C $-0.38826600 \quad 2.19396500-0.38065900$

H $5.50501900 \quad 1.02403300 \quad 0.15552800$

H $3.95573100-3.53313300 \quad 0.56007000$

$\mathrm{H} \quad 5.38059500-1.49436300 \quad 0.43685300$

$\mathrm{H} 1.45920400 \quad-3.28266500 \quad 0.34596500$

$\mathrm{H} \quad 4.33884700 \quad 3.15958800 \quad-0.17929000$

$\mathrm{H}-0.31717600 \quad 4.32695300-0.65265400$

$\mathrm{H}-1.46742400 \quad 2.11311200-0.40749700$

$\mathrm{Cu}-0.60960900-0.90293500-0.01415500$

N $1.54043700-1.25864800 \quad 0.16615500$

$\mathrm{N} \quad 0.26747600 \quad 1.06762200-0.19373100$

Cl $-0.98325000-3.08962900-0.57365700$

C $-2.51769800 \quad-0.39444800 \quad 0.34617900$

C $-3.38401400-0.41170400-0.90416300$

$\mathrm{H}-2.86788500-1.17802700 \quad 1.03544600$

C $-3.948029001 .16042700 \quad 1.41800900$

C $-4.83769000-0.06475400-0.56870200$

$\mathrm{H}-3.31191500-1.39583300-1.36974200$

$\mathrm{H}-3.00254700 \quad 0.31957800 \quad-1.62868000$

C $-4.89106900 \quad 1.23762600 \quad 0.22631300$

$\mathrm{H}-3.91101000 \quad 2.10826900 \quad 1.95874500$

H $-4.29280700 \quad 0.38208000 \quad 2.11390900$

$\mathrm{H}-5.44479500 \quad 0.00978600-1.47399100$

$\mathrm{H}-5.26068900-0.87241500 \quad 0.03758900$

$\mathrm{H}-4.58068400 \quad 2.07206700-0.41150200$

$\mathrm{H}-5.90534900 \quad 1.45149600 \quad 0.57254700$

$\begin{array}{llll}0 & -2.62419400 & 0.86945700 & 1.01270900\end{array}$
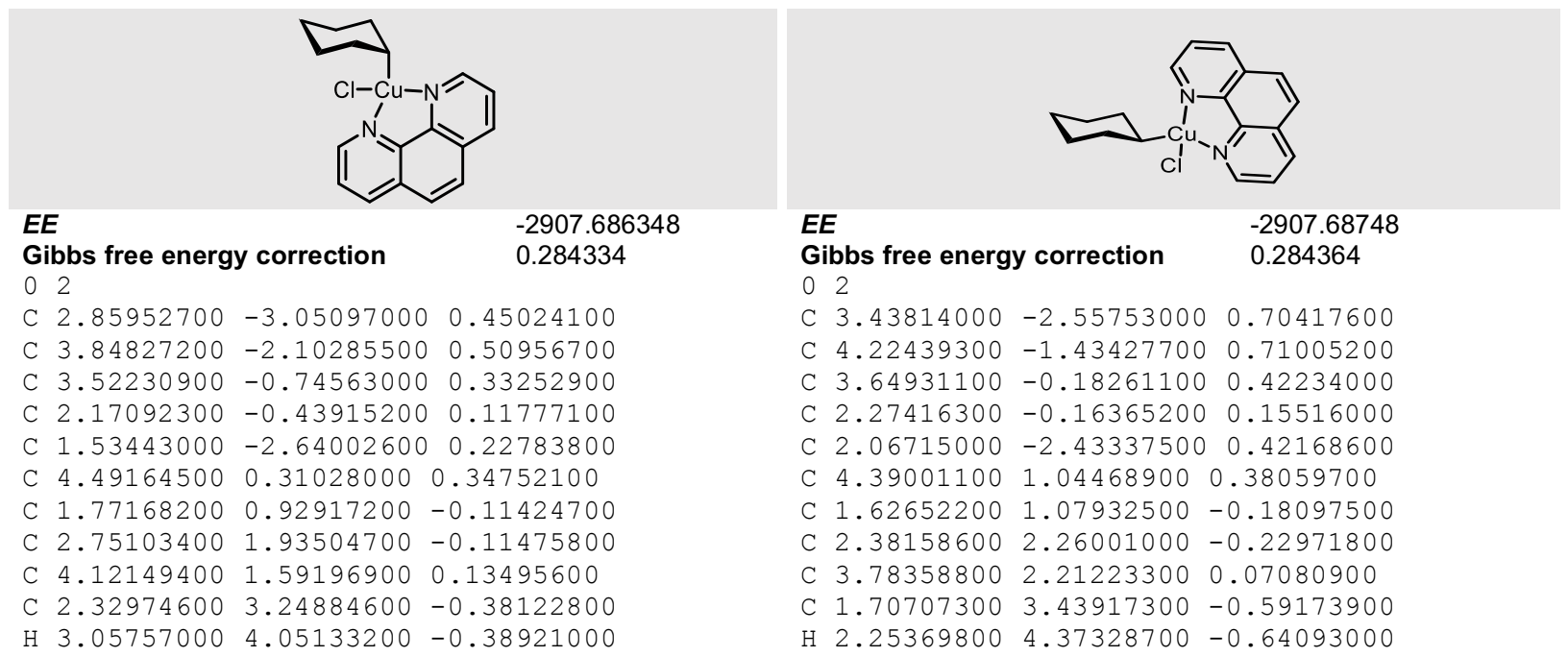


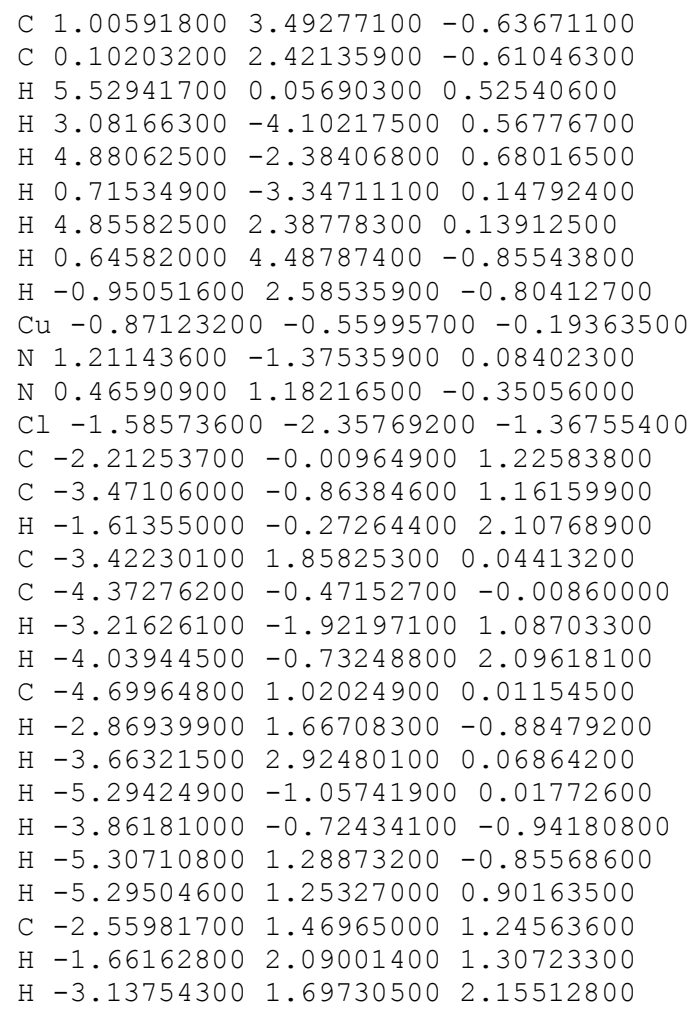

C $0.36894300 \quad 3.38867900-0.88561400$

C $-0.29840100 \quad 2.15736700-0.80604000$

H $5.45050500 \quad 1.01464100 \quad 0.59790500$

H $3.85316800-3.53406900 \quad 0.90963900$

H $5.28456000-1.49711700 \quad 0.92460500$

$\mathrm{H} \quad 1.40087000 \quad-3.28861600 \quad 0.38072100$

H $4.34820200 \quad 3.13552500 \quad 0.03513700$

$\mathrm{H}-0.17870100 \quad 4.27410500-1.17521700$

$\mathrm{H}-1.35543300 \quad 2.07928700-1.02835200$

$\mathrm{Cu}-0.63119400-0.91202600-0.21532000$

$\mathrm{N} 1.51148100-1.26909900 \quad 0.16966000$

N $0.30791900 \quad 1.04189800-0.46034400$

Cl $-0.95195300-3.02953200-0.99139500$

C $-2.48904300-0.40363600 \quad 0.36429500$

C $-3.39942400-0.13939200-0.82963700$

$\mathrm{H}-2.79730600-1.35074500 \quad 0.82319000$

C $-4.04274500 \quad 0.94393800 \quad 1.83949400$

C $-4.84930100 \quad 0.10843300-0.39653100$

$\mathrm{H}-3.35083000-0.97995100-1.52512200$

$\mathrm{H}-3.06052800 \quad 0.74612100-1.38653100$

C $-4.92961900 \quad 1.23513500 \quad 0.63092600$

$\mathrm{H}-4.09782200 \quad 1.76471400 \quad 2.56011400$

$\mathrm{H}-4.41321800 \quad 0.04685900 \quad 2.34629600$

$\mathrm{H}-5.47503200 \quad 0.33769500 \quad-1.26322400$

$\mathrm{H}-5.24371200-0.81092900 \quad 0.04840600$

$\mathrm{H}-4.59846800 \quad 2.17051700 \quad 0.16265100$

H $-5.96360700 \quad 1.39277200 \quad 0.94686000$

C $-2.58964100 \quad 0.70370900 \quad 1.40751000$

$\mathrm{H}-2.20060700 \quad 1.65047300 \quad 1.01137500$

$\mathrm{H}-1.97995000 \quad 0.46732300 \quad 2.28452500$ 
COPPER

\begin{tabular}{|c|c|}
\hline$E E$ & -2832.288903 \\
\hline $\begin{array}{l}\text { Gibbs free energy } \\
-1 \quad 2\end{array}$ & correction \\
\hline$C-0.84160200$ & $-1.25547700 \quad 0.71898100$ \\
\hline$C-2.56819900$ & $0.32749100 \quad 0.82344400$ \\
\hline$C-3.02897600$ & $-0.03292900-0.57965800$ \\
\hline$C-1.82806900$ & $-0.45381000-1.42330800$ \\
\hline C -1.09418500 & $-1.59264600-0.71412900$ \\
\hline $\mathrm{H}-3.41266300$ & $0.55255900 \quad 1.47583300$ \\
\hline $\mathrm{H}-0.17396600$ & $-1.86789600 \quad 1.30958900$ \\
\hline $\mathrm{H}-3.75499500$ & $-0.85190200-0.52463200$ \\
\hline $\mathrm{H}-3.53340500$ & $0.83237300-1.01667900$ \\
\hline $\mathrm{H}-2.14275400$ & $-0.76330100-2.42253300$ \\
\hline $\mathrm{H}-1.15380900$ & $0.40067700-1.52890200$ \\
\hline $\mathrm{H}-1.71590300$ & $-2.50276500-0.77259000$ \\
\hline $\mathrm{H}-0.13749900$ & $-1.82122400-1.18644400$ \\
\hline $\mathrm{H}-1.89605200$ & $1.19050300 \quad 0.78199000$ \\
\hline $0-1.87745000$ & $-0.75166400 \quad 1.45117200$ \\
\hline $\mathrm{Cu} 1.34787500$ & $0.42239100 \quad 0.04504600$ \\
\hline Cl 2.55882800 & $-1.45108100-0.02646100$ \\
\hline Cl 0.38343300 & $2.42985700-0.09020600$ \\
\hline
\end{tabular}

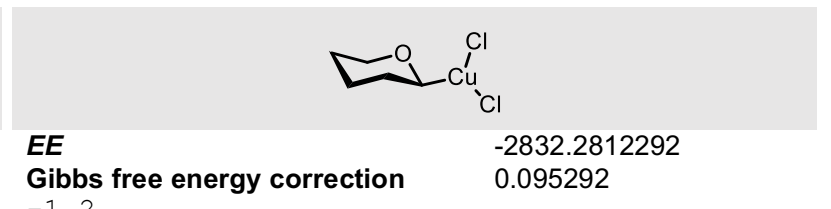

$-12$

C $-3.51389200-0.03309800-0.29064400$

C $-2.62325700-1.08610400-0.93916800$

C $-0.71825400-0.41624400 \quad 0.27816900$

C $-1.49480100 \quad 0.66734900 \quad 1.00362600$

C $-2.98254000 \quad 0.30955300 \quad 1.09930000$

$\mathrm{H}-2.68626700-2.02345800-0.36265700$

$\mathrm{H}-2.94321900-1.29488900-1.96290200$

$\mathrm{H}-3.50087000 \quad 0.86311400-0.91803100$

$\mathrm{H}-4.54565300-0.39541200-0.24664400$

$\mathrm{H}-0.72902700-1.35718000 \quad 0.85090600$

$\mathrm{H}-1.06720900 \quad 0.81694500 \quad 1.99842800$

$\mathrm{H}-1.37357900 \quad 1.60902000 \quad 0.46066800$

$\mathrm{H}-3.10234900-0.56306700 \quad 1.75192100$

$\mathrm{H}-3.55907600 \quad 1.12665100 \quad 1.54310900$

$0-1.28427800-0.66199900-1.00800600$

$\mathrm{Cu} 1.21884000 \quad 0.05548400-0.00732200$

Cl $1.42255900 \quad 2.27019100 \quad-0.35598400$

Cl 2.48518100 $-1.78451500 \quad 0.25327100$

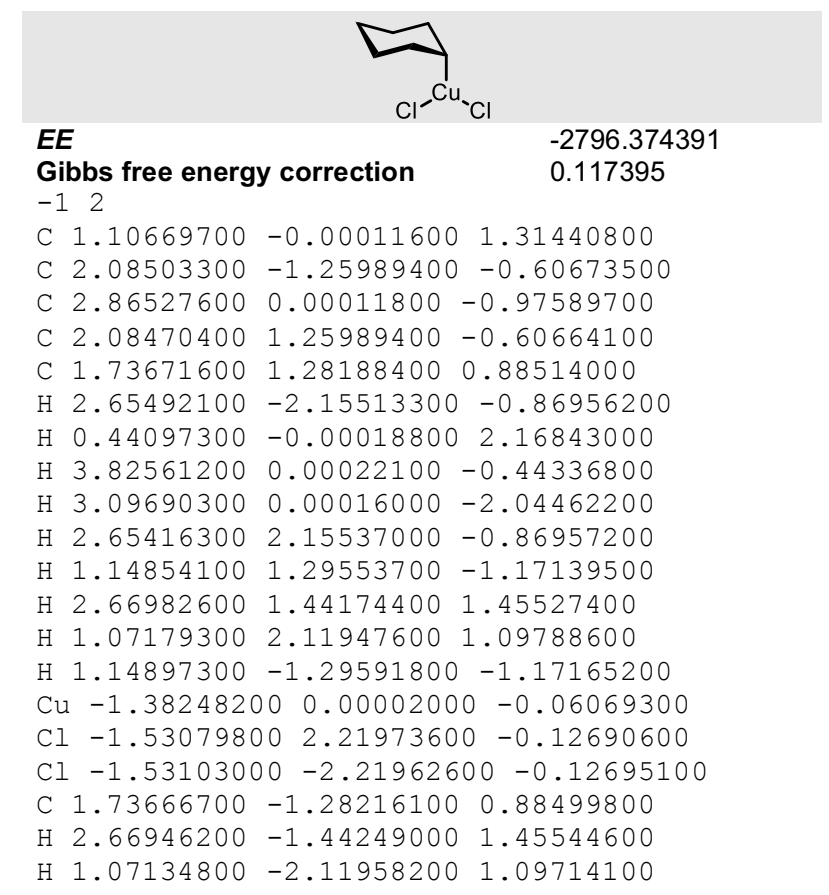

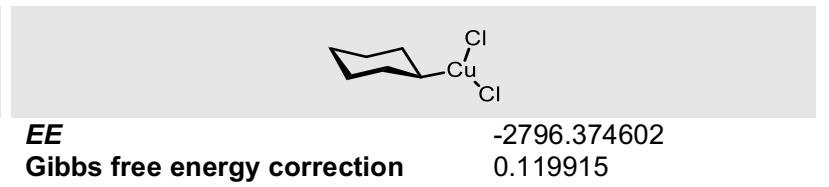

Gibbs

C $-3.56748900 \quad 0.00579000-0.36239500$

C $-2.82769800-1.33119000-0.37307000$

C $-0.72481500-0.16796000 \quad 0.41605900$

C $-1.46847400 \quad 1.15704500 \quad 0.43791100$

C $-2.97209600 \quad 0.95865500 \quad 0.67342500$

$\mathrm{H}-2.97235000-1.82699400 \quad 0.59375700$

$\mathrm{H}-3.24953500-1.99186300-1.13750500$

$\mathrm{H}-3.48112700 \quad 0.46518300-1.35424700$

$\mathrm{H}-4.63502100-0.14706200-0.17604200$

$\mathrm{H}-0.74091900-0.63519700 \quad 1.40944800$

$\mathrm{H}-1.04815000 \quad 1.82312500 \quad 1.19436800$

$\mathrm{H}-1.32372900 \quad 1.66854300-0.52071100$

$\mathrm{H}-3.12495600 \quad 0.53607400 \quad 1.67357700$

$\mathrm{H}-3.49682400 \quad 1.91925000 \quad 0.64864200$

$\mathrm{Cu} 1.25372900 \quad 0.03995900 \quad 0.05296200$

Cl $1.78427200 \quad 2.19189900-0.34566500$

Cl 2.15734800 $-2.02404700 \quad 0.18055500$

C $-1.32421600-1.12615400-0.60361400$

$\mathrm{H}-0.79776600-2.08223300-0.57630200$

$\mathrm{H}-1.18657400-0.71825800-1.61391300$ 


\section{SILVER}

\begin{tabular}{|c|c|c|c|c|c|}
\hline \multicolumn{2}{|c|}{$E E$} & -722.919381 & $E E$ & \\
\hline \multicolumn{2}{|c|}{ Gibbs free energy correction } & correction & $\begin{array}{l}\text { Gibbs free energy } \\
0 \quad 1\end{array}$ & correction & 0.218446 \\
\hline $\mathrm{C}$ & -3.80558400 & $0.30017100 \quad 1.39523400$ & C -4.79792000 & 0.46016000 & 0.44438200 \\
\hline C & -3.01719600 & $-0.99059700 \quad 1.19416200$ & $C-3.97124800$ & 1.15641500 & -0.62825600 \\
\hline $\mathrm{C}$ & -2.26821500 & $-0.36020600-0.95858700$ & C -2.11705200 & -0.30443100 & $0-0.35621100$ \\
\hline C & -3.08140500 & $0.93012200-0.90447700$ & $C-2.85540500$ & -1.08550900 & 00.72938600 \\
\hline $\mathrm{C}$ & -3.22297800 & $1.42004300 \quad 0.53472500$ & $C-4.37481600$ & -1.00194300 & 00.55503500 \\
\hline $\mathrm{H}$ & -2.00198200 & $-0.86307400 \quad 1.61065400$ & H -4.19516700 & 0.70862700 & -1.60993500 \\
\hline $\mathrm{H}$ & -3.49745400 & $-1.82144200 \quad 1.71584500$ & $\mathrm{H}-4.21162400$ & 2.22112000 & -0.67902400 \\
\hline $\mathrm{H}$ & -4.84385200 & $0.12046400 \quad 1.10113700$ & $\mathrm{H}-4.62476100$ & 0.96658000 & 1.39906200 \\
\hline $\mathrm{H}$ & -3.80539300 & $0.57079900 \quad 2.45474500$ & $\mathrm{H}-5.86250600$ & 0.55099700 & 0.21139600 \\
\hline $\mathrm{H}$ & -2.32133200 & $-0.76665400-1.97084000$ & $\mathrm{H}-2.39854500$ & -0.74952800 & $0-1.32615400$ \\
\hline $\mathrm{H}$ & -2.61093500 & $1.69268200-1.53103700$ & $\mathrm{H}-2.52626800$ & -2.12845800 & 00.70918500 \\
\hline & -4.09057600 & $0.75156700-1.30448900$ & $\mathrm{H}-2.58888200$ & -0.68548300 & 01.71359500 \\
\hline $\mathrm{H}$ & -2.23174400 & $1.69134500 \quad 0.91810200$ & $\mathrm{H}-4.65553100$ & -1.52574200 & $0-0.36574800$ \\
\hline $\mathrm{H}$ & -3.85076900 & $2.31294200 \quad 0.59090000$ & $\mathrm{H}-4.89830300$ & -1.49588100 & 01.37858700 \\
\hline & $9-0.16289100$ & $0-0.13513900-0.45319300$ & $0-2.58982800$ & 1.05939000 & -0.37119400 \\
\hline $\mathrm{C}$ & $4.15364000-$ & $-0.47382500 \quad 0.48032700$ & Ag 0.02654100 & -0.21284400 & $0-0.18120300$ \\
\hline $\mathrm{C}$ & $4.05323400 \quad 0$ & $0.87204500 \quad 0.46739200$ & C $2.21293700 \quad 0$ & 0.028609000 & 0.00984400 \\
\hline $\mathrm{H}$ & $4.99602000-$ & $\begin{array}{lll}-1.11772700 & 0.66212800\end{array}$ & N 2.87672000 1 & $1.18779700 \quad 0$ & 0.20641800 \\
\hline $\mathrm{H}$ & 4.791343001 & $1.63696400 \quad 0.63321700$ & C $4.43599900-$ & -0.34009900 & 0.15602400 \\
\hline $\mathrm{C}$ & 2.003813000 & $0.03776100 \quad 0.01266500$ & C $4.23668100 \quad 0$ & 0.987113000 & 0.29949700 \\
\hline $\mathrm{C}$ & $2.56859300-$ & $\begin{array}{lll}-2.37195000 & 0.12159700\end{array}$ & H $5.33987800-$ & -0.92345900 & 0.16096500 \\
\hline $\mathrm{H}$ & $2.78216400-$ & $-2.85977800 \quad 1.07188200$ & H $4.93293300 \quad 1$ & 1.792113000 & 0.45535500 \\
\hline $\mathrm{H}$ & $1.50831100-$ & $-2.46339000-0.10066200$ & C $2.95876300-$ & -2.32234100 & -0.21108100 \\
\hline & $3.14699100-$ & $-2.84660100-0.67014100$ & H 1.89183200 & -2.47395700 & -0.35398600 \\
\hline C & 2.203663002 & $2.50626200 \quad 0.07809500$ & Н $3.49660600-$ & -2.67305000 & -1.09111600 \\
\hline $\mathrm{H}$ & 1.141631002 & $2.43388500-0.14306500$ & Н 3.28979900 & -2.87906600 & 0.66490400 \\
\hline $\mathrm{H}$ & 2.342288003 & 3.036031001 .01978400 & C 2.238578002 & $2.48807300 \quad 0$ & 0.31576200 \\
\hline $\mathrm{H}$ & 2.705369003 & $3.04795000-0.72284200$ & H 2.642043003 & $3.16537100-$ & -0.43571400 \\
\hline N & $2.89440100-$ & $\begin{array}{lll}-0.95937100 & 0.20121700\end{array}$ & H $1.17131300 \quad 2$ & $2.35978200 \quad 0$ & 0.15141700 \\
\hline $\mathrm{N}$ & $2.73595200 \quad 1$ & $1.15968800 \quad 0.18091600$ & H 2.403661002 & 2.905059001 & 1.30853500 \\
\hline O & -2.93025800 & $-1.35320200-0.15461700$ & N 3.18979800 & -0.90247600 & -0.01949200 \\
\hline
\end{tabular}

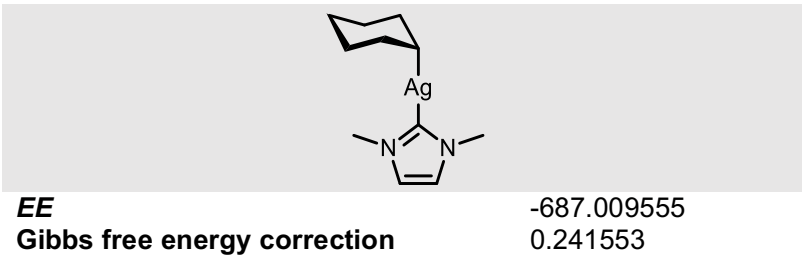

\section{Gibbs free energy correction}

01

C $-3.78967200 \quad 1.35863700 \quad 0.09140800$

$\begin{array}{llll}C & -3.09760500 & 0.77546400 & 1.32390700\end{array}$

C $-2.21862300-1.15734300-0.06120100$

C $-2.97198700-0.59794000-1.27081300$

C $-3.10670100 \quad 0.92363400-1.20567400$

$\mathrm{H}-2.09615100 \quad 1.21400200 \quad 1.41783400$

$\mathrm{H}-3.65283500 \quad 1.04968000 \quad 2.22534400$

$\mathrm{H}-4.82665000 \quad 1.00536900 \quad 0.07429300$

$\mathrm{H}-3.83174200 \quad 2.44936600 \quad 0.15543100$

$\mathrm{H}-2.22408700-2.24982900-0.12539300$

$\mathrm{H}-2.48776900-0.88805300-2.20684300$

$\mathrm{H}-3.99328100-1.01461400-1.30604300$

$\mathrm{H}-2.10594200 \quad 1.37104900-1.25527200$

$\mathrm{H}-3.66848100 \quad 1.30046900-2.06501000$

Ag $-0.13631800-0.54972400-0.03136400$

C $4.24368700 \quad 0.12110400 \quad 0.03517200$

C $3.828556001 .40515300 \quad 0.02615900$

H $5.23287100 \quad-0.30121900 \quad 0.05160500$

$\begin{array}{lllll}\mathrm{H} & 4.38340600 & 2.32669200 & 0.03279300\end{array}$

C $1.98477200 \quad 0.09985700 \quad 0.00034000$ 

C $3.11092900-2.10607100 \quad 0.02352800$
H $3.60066500-2.47627000 \quad 0.92328500$
$\mathrm{H} 2.07943000 \quad-2.44818800 \quad 0.00596300$
$\begin{array}{llll}\mathrm{H} & 3.63272400 & -2.48128700 & -0.85587700\end{array}$
C $1.60777800 \quad 2.54948300-0.01141800$
H $0.56953200 \quad 2.22631100-0.01083500$
$\mathrm{H} 1.79825600 \quad 3.15735900 \quad 0.87204500$
$\mathrm{H} \quad 1.80352900 \quad 3.13703600-0.90744700$
N $3.10450800 \quad-0.65471900 \quad 0.01922000$
N 2.45097200 $1.36748800 \quad 0.00531700$
C $-2.96382100-0.74334000 \quad 1.21026400$
$\mathrm{H}-2.47364000-1.14054700 \quad 2.10286600$
$\mathrm{H}-3.98512600-1.16149200 \quad 1.20360300$

C $2.63944400-2.44601000 \quad 0.05503200$

H $1.55768500-2.45538800-0.05108000$

H $3.09173000-2.95544500-0.79486200$

H $2.91787200 \quad-2.95624300 \quad 0.97620500$

C $2.64014400 \quad 2.44611700 \quad 0.05615800$

H $3.08901900 \quad 2.95448000 \quad-0.79619600$

$\mathrm{H} \quad 1.55794700 \quad 2.45584100 \quad-0.04540500$

H $2.92270000 \quad 2.95721300 \quad 0.97558600$

N $3.07685900 \quad-1.06256100 \quad 0.09869400$

C $-2.77315500 \quad 1.25305900 \quad 0.16537000$

$\mathrm{H}-2.34947700 \quad 2.15755300 \quad-0.28039500$

$\mathrm{H}-2.56407600 \quad 1.31547100 \quad 1.24131300$

Ag $0.04099100 \quad 0.00003300-0.20397900$ 
SILVER

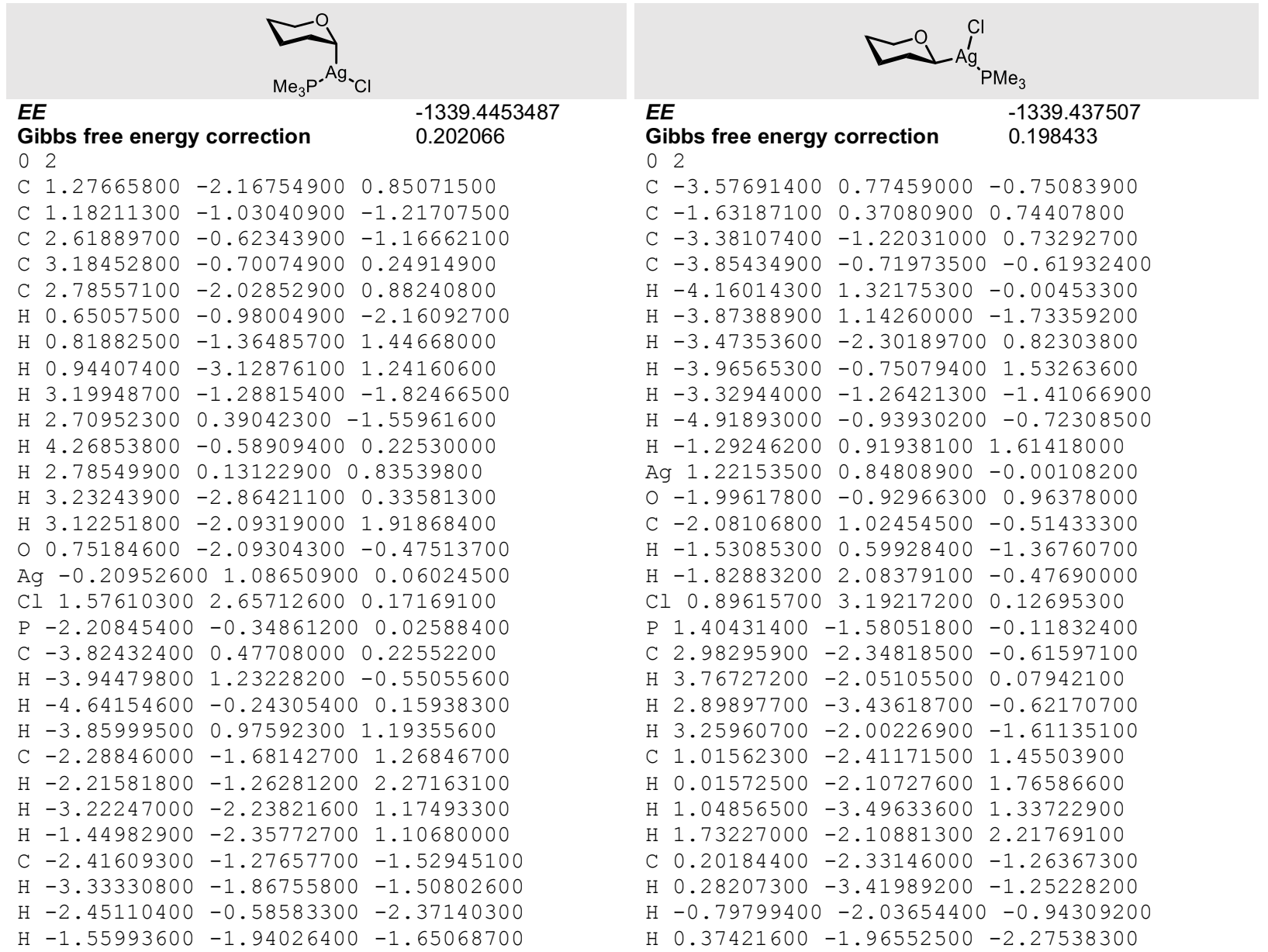

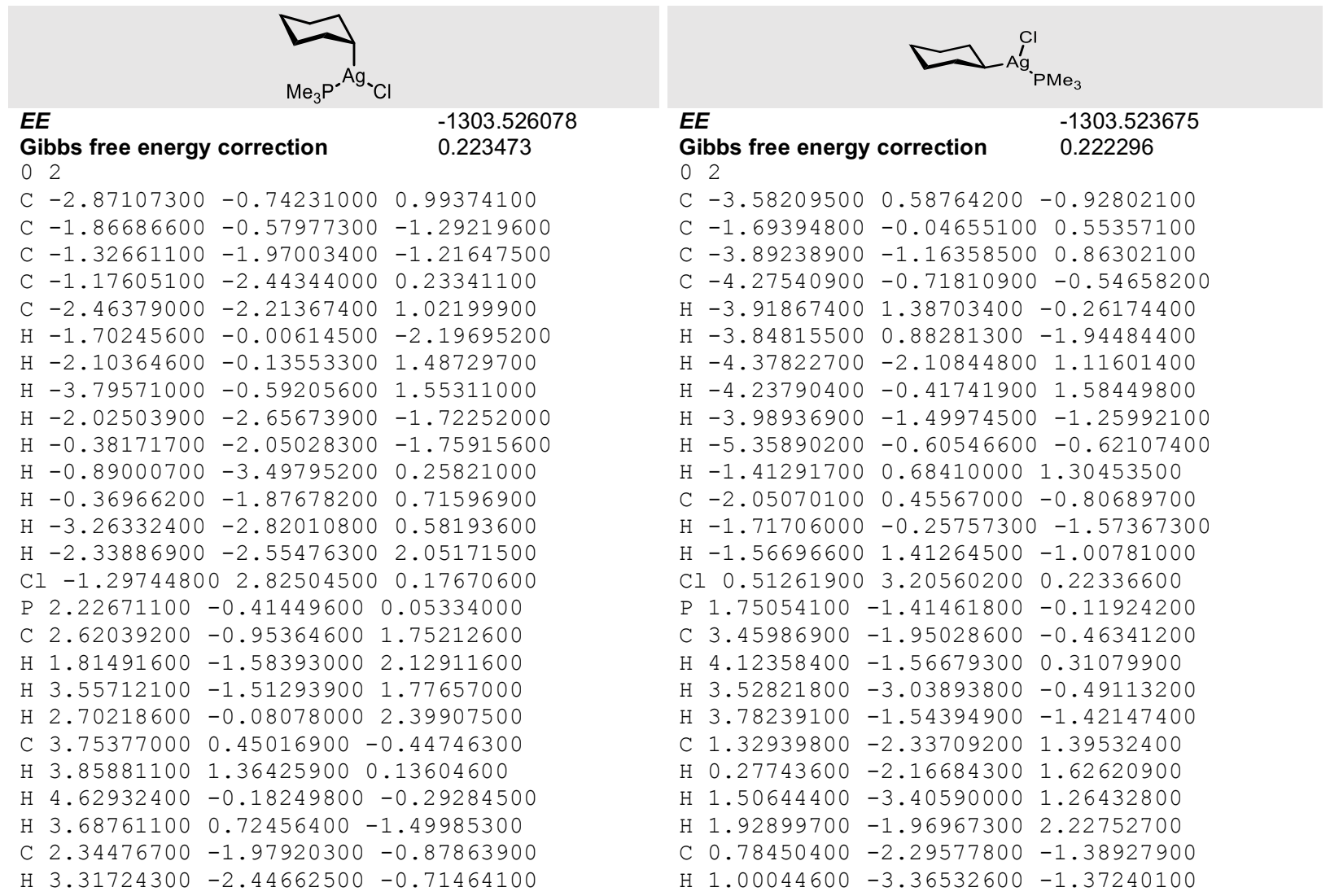


H $1.55923600-2.66004100-0.55092800$

H $2.21365700-1.78587200-1.94294100$

C $-3.04307100-0.23704700-0.44100000$

$\mathrm{H}-3.93938900-0.71338500-0.87177200$

$\mathrm{H}-3.21198900 \quad 0.83955800-0.44744900$

Ag $0.20231600 \quad 0.98794600 \quad-0.06908700$
H $-0.27627800-2.13646300-1.19453600$

$\mathrm{H} \quad 1.01705400-1.89392100-2.37492900$

C $-2.36251400-1.30825600 \quad 0.99210400$

$\mathrm{H}-2.05643900 \quad-2.14511100 \quad 0.34863600$

$\mathrm{H}-2.09384700 \quad-1.57085300 \quad 2.01692000$

Ag $1.20036700 \quad 0.95123200 \quad 0.03609600$ 
GOLD

\begin{tabular}{|c|c|c|}
\hline \multicolumn{2}{|l|}{$E E$} & \\
\hline \multicolumn{2}{|c|}{$\begin{array}{l}\text { Gibbs free energy correction } \\
02\end{array}$} & 0.208569 \\
\hline C 1.27665800 & -2.16754900 & 0.85071500 \\
\hline C 1.18211300 & -1.03040900 & -1.21707500 \\
\hline C 2.61889700 & -0.62343900 & -1.16662100 \\
\hline C 3.18452800 & -0.70074900 & 0.24914900 \\
\hline C 2.78557100 & -2.02852900 & 0.88240800 \\
\hline $\mathrm{H} 0.65057500$ & -0.98004900 & -2.16092700 \\
\hline $\mathrm{H} 0.81882500$ & -1.36485700 & 1.44668000 \\
\hline $\mathrm{H} \quad 0.94407400$ & -3.12876100 & 1.24160600 \\
\hline H 3.19948700 & -1.28815400 & -1.82466500 \\
\hline H 2.70952300 & $0.39042300-$ & -1.55961600 \\
\hline $\mathrm{H} 4.26853800$ & -0.58909400 & 0.22530000 \\
\hline $\mathrm{H} \quad 2.78549900$ & 0.131229000 & 0.83539800 \\
\hline $\mathrm{H} 3.23243900$ & -2.86421100 & 0.33581300 \\
\hline H 3.12251800 & -2.09319000 & 1.91868400 \\
\hline O 0.75184600 & -2.09304300 & -0.47513700 \\
\hline $\mathrm{Ag}-0.2095260$ & 01.08650900 & $0 \quad 0.06024500$ \\
\hline Cl 1.57610300 & 2.65712600 & 0.17169100 \\
\hline$P-2.20845400$ & -0.34861200 & $0 \quad 0.02588400$ \\
\hline$C-3.82432400$ & 0.47708000 & 0.22552200 \\
\hline $\mathrm{H}-3.94479800$ & 1.23228200 & -0.55055600 \\
\hline $\mathrm{H}-4.64154600$ & -0.24305400 & $0 \quad 0.15938300$ \\
\hline $\mathrm{H}-3.85999500$ & 0.97592300 & 1.19355600 \\
\hline$C-2.28846000$ & -1.68142700 & 01.26846700 \\
\hline $\mathrm{H}-2.21581800$ & -1.26281200 & $0 \quad 2.27163100$ \\
\hline $\mathrm{H}-3.22247000$ & -2.23821600 & 1.17493300 \\
\hline H -1.44982900 & -2.35772700 & 1.10680000 \\
\hline C -2.41609300 & -1.27657700 & -1.52945100 \\
\hline $\mathrm{H}-3.33330800$ & -1.86755800 & -1.50802600 \\
\hline-2.45110400 & -0.58583300 & -2.37140300 \\
\hline $\mathrm{H}-1.55993600$ & -1.94026400 & $0-1.65068700$ \\
\hline
\end{tabular}

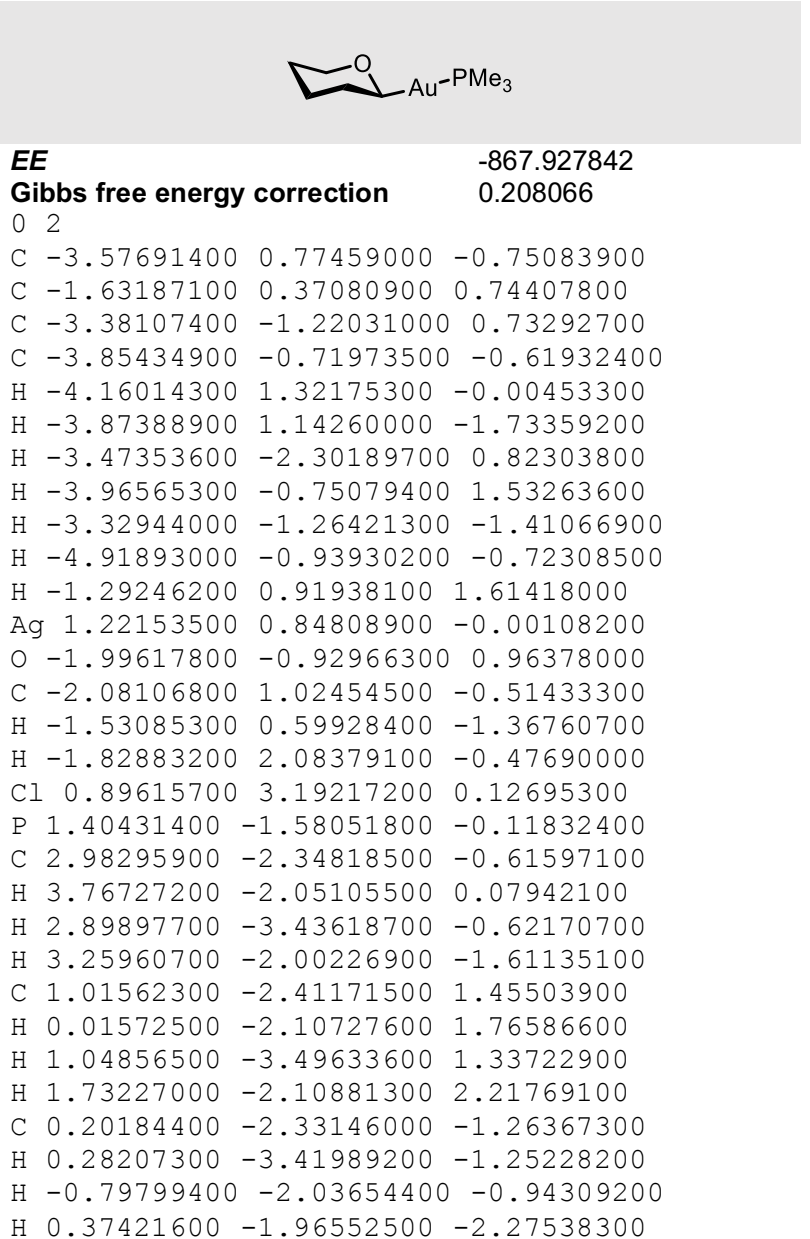

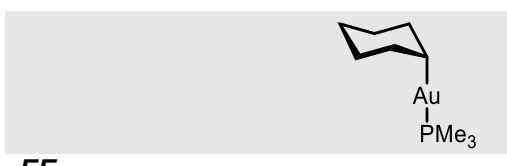

$E E$

Gibbs free energy correction

$\begin{array}{ll}0 & 1 \\ 0 & 3\end{array}$

C $3.601109001 .38400800 \quad 0.00562300$

C $2.87140300 \quad 0.93586700-1.26034100$

C $1.86308900-1.02604900-0.00511600$

C $2.63477100-0.58624400 \quad 1.24422800$

C $2.87345500 \quad 0.92307000 \quad 1.26819700$

H $1.904449001 .44877800-1.31950500$

H $3.44426300 \quad 1.22773200-2.14438800$

H $4.60832600 \quad 0.95256300 \quad 0.00260700$

$\mathrm{H} \quad 3.72453800 \quad 2.46985600 \quad 0.01102800$

$\mathrm{H} 1.81444500-2.11973400-0.01063300$

$\mathrm{H} 2.11598500-0.89810900 \quad 2.15384000$

H $3.61736200-1.082465001 .25846800$

$\mathrm{H} \quad 1.90670800 \quad 1.43543000 \quad 1.33406400$

$\mathrm{H} \quad 3.44783400 \quad 1.20595800 \quad 2.15417600$

$\mathrm{Au}-0.13771300-0.37092300-0.00112600$

$\mathrm{P}-2.34916700 \quad 0.31830600 \quad 0.00121200$

C $-3.02249100 \quad 0.80563100 \quad 1.62377200$

$\mathrm{H}-2.42602500 \quad 1.62208500 \quad 2.02986500$

$\mathrm{H}-4.062958001 .12318300 \quad 1.53907500$

$\mathrm{H}-2.95556000-0.03839500 \quad 2.30957500$

C $-2.71663800 \quad 1.77340500-1.03351100$

$\mathrm{H}-2.45365300 \quad 1.55485600-2.06808800$

$\mathrm{H}-3.77274800 \quad 2.04156700-0.97605000$

$\mathrm{H}-2.10990900 \quad 2.61437800-0.69888400$

C $-3.56943800-0.90311800-0.58413200$
H $0.37421600-1.96552500-2.27538300$ 
$\mathrm{H}-3.51757200-1.79265300 \quad 0.04294500$

$\mathrm{H}-4.58106700-0.49540400-0.55307500$

$\mathrm{H}-3.32652100-1.19270000-1.60606400$

C $2.63307200-0.57365300-1.25102000$

$\mathrm{H} 2.11317100-0.87669500-2.16296400$

H $3.61579400-1.06940500-1.27141200$
H $4.63531000-0.00010800-1.19700300$

$\mathrm{H} 3.31203200-0.88198300-1.99738800$

$\mathrm{H} \quad 3.31255700 \quad 0.88309500 \quad-1.99678100$

C $-2.46659000 \quad 1.25526100 \quad 0.18016100$

$\mathrm{H}-2.02661500 \quad 2.15737800-0.25200500$

$\mathrm{H}-2.28183600 \quad 1.30794300 \quad 1.25999000$ 
GOLD
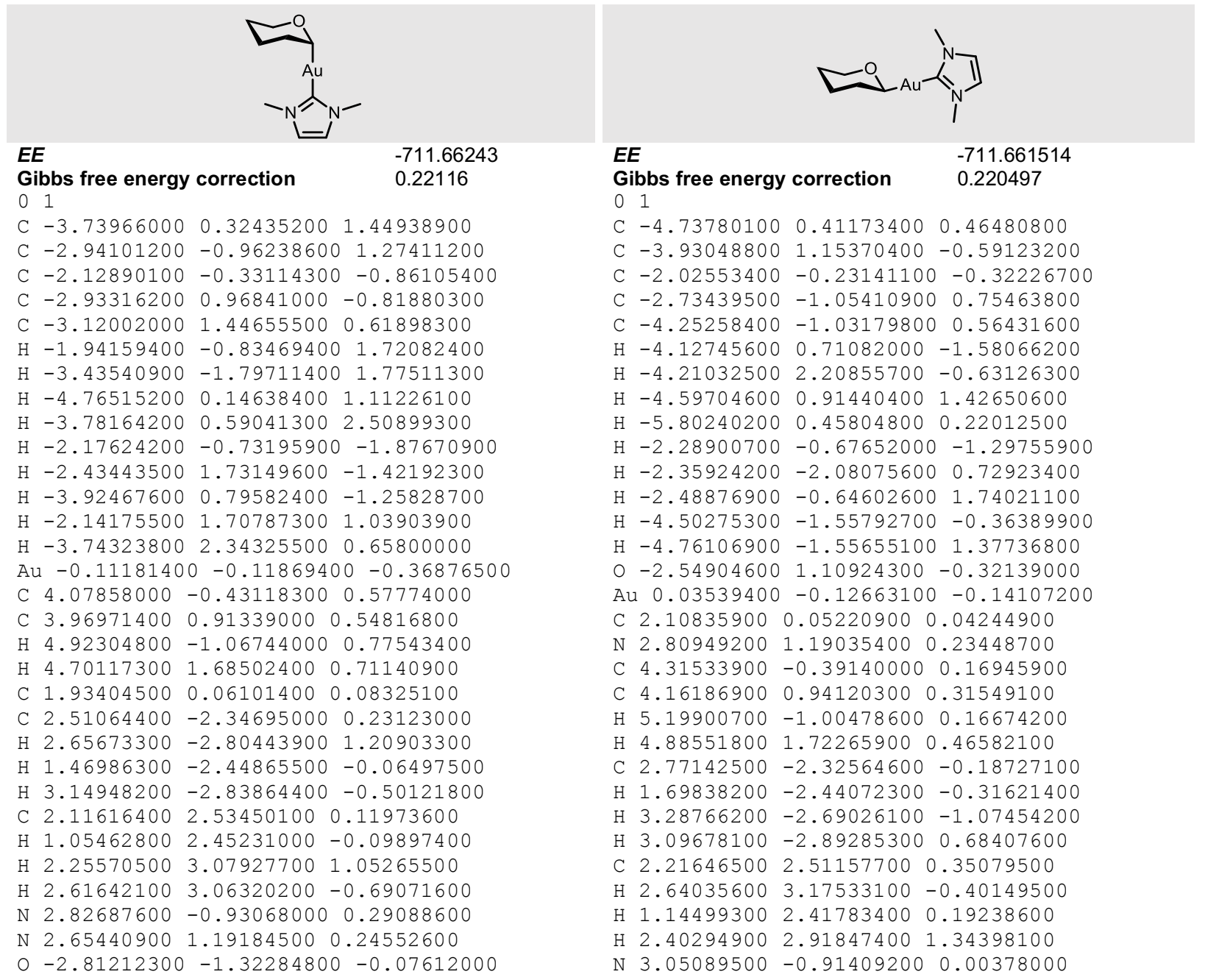

\section{Gibbs free energy correction}

01

$\begin{array}{llll}\text { C } & -4.73780100 & 0.41173400 & 0.46480800\end{array}$

C $-3.93048800 \quad 1.15370400 \quad-0.59123200$

C $-2.02553400-0.23141100 \quad-0.32226700$

C $-2.73439500-1.05410900 \quad 0.75463800$

C $-4.25258400-1.03179800 \quad 0.56431600$

$\mathrm{H}-4.12745600 \quad 0.71082000-1.58066200$

$\mathrm{H}-4.21032500 \quad 2.20855700 \quad-0.63126300$

$\mathrm{H}-4.59704600 \quad 0.91440400 \quad 1.42650600$

$\mathrm{H}-5.80240200 \quad 0.45804800 \quad 0.22012500$

$\mathrm{H}-2.28900700-0.67652000-1.29755900$

$\mathrm{H}-2.35924200 \quad-2.08075600 \quad 0.72923400$

$\mathrm{H}-2.48876900-0.64602600 \quad 1.74021100$

$\mathrm{H}-4.50275300-1.55792700 \quad-0.36389900$

$\mathrm{H}-4.76106900-1.55655100 \quad 1.37736800$

$0-2.54904600 \quad 1.10924300-0.32139000$

$\mathrm{Au} \quad 0.03539400 \quad-0.12663100 \quad-0.14107200$

C $2.10835900 \quad 0.05220900 \quad 0.04244900$

N 2.80949200 $1.19035400 \quad 0.23448700$

$\begin{array}{llll}\text { C } 4.31533900 & -0.39140000 & 0.16945900\end{array}$

C $4.16186900 \quad 0.94120300 \quad 0.31549100$

H $5.19900700-1.00478600 \quad 0.16674200$

H $4.88551800 \quad 1.72265900 \quad 0.46582100$

C $2.77142500-2.32564600-0.18727100$

H $1.69838200-2.44072300-0.31621400$

H $3.28766200-2.69026100-1.07454200$

H $3.09678100 \quad-2.89285300 \quad 0.68407600$

$\begin{array}{llll}\text { C } 2.21646500 & 2.51157700 & 0.35079500\end{array}$

H $2.64035600 \quad 3.17533100-0.40149500$

H $1.14499300 \quad 2.41783400 \quad 0.19238600$

H $2.40294900 \quad 2.91847400 \quad 1.34398100$

N $3.05089500 \quad-0.91409200 \quad 0.00378000$

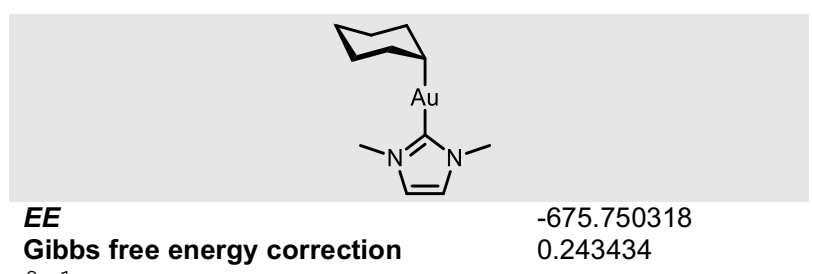

01 0.243434

C $3.779297001 .38853000-0.00366200$

C $3.05939900 \quad 0.91714600-1.26691300$

C $2.07624500-1.05084800 \quad 0.00214900$

$\begin{array}{llll}\text { C } 2.84669800 & -0.59054300 & 1.24692500\end{array}$

C $3.05972700 \quad 0.92297700 \quad 1.26192000$

$\mathrm{H} \quad 2.08305600 \quad 1.41170700-1.33247500$

H $3.62851100 \quad 1.21250900-2.15253500$

$\mathrm{H} \quad 4.79396200 \quad 0.97471300 \quad-0.00282700$

$\mathrm{H} 3.88452600 \quad 2.47667000-0.00617100$

H $2.06039200 \quad-2.14626800 \quad 0.00467800$

$\mathrm{H} \quad 2.33423300 \quad-0.90379200 \quad 2.15972200$

$\mathrm{H} 3.83882600-1.06801700 \quad 1.26391800$

H 2.083407001 .417853001 .32545300

$\mathrm{H} \quad 3.62906800 \quad 1.222403002 .14602600$

$\mathrm{Au} 0.08413900-0.42691500 \quad 0.00094800$

C $-4.17404400 \quad 0.20410900-0.00163600$

C $-3.764353001 .48950700-0.00122800$

$\mathrm{H}-5.16133000-0.22263300-0.00235000$

$\mathrm{H}-4.32273600 \quad 2.40878400-0.00147600$

C $-1.91793700 \quad 0.19307800-0.00017000$

C $-3.03543600-2.02087100-0.00119400$

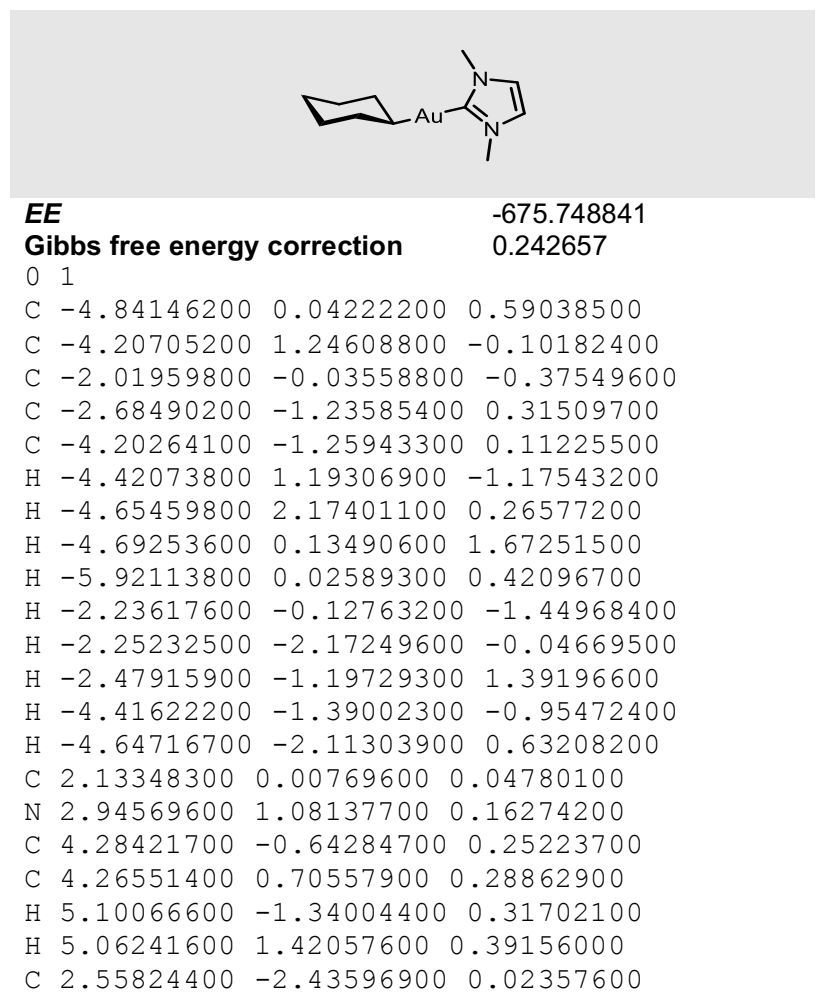




\begin{abstract}
H $-3.54006300-2.39290700-0.89189900$
$\mathrm{H}-2.00249100-2.35814600 \quad 0.00019700$

$\mathrm{H}-3.54256000 \quad-2.39312600 \quad 0.88799600$

C $-1.54937100 \quad 2.64696700 \quad 0.00043200$

$\mathrm{H}-0.51049200 \quad 2.32701400 \quad 0.00015600$

$\mathrm{H}-1.74754500 \quad 3.24340500-0.88914500$

$\mathrm{H}-1.74741900 \quad 3.24220800 \quad 0.89084200$

$\mathrm{N}-3.03338500-0.56917500-0.00096900$

$\mathrm{N}-2.38666300 \quad 1.46045100-0.00035800$

C $2.84640400-0.59628900-1.24490500$

$\mathrm{H} \quad 2.33373700 \quad-0.91376300-2.15612900$

H $3.83853700-1.07382200-1.25991400$
\end{abstract}

$\mathrm{H} 1.47740400-2.45631200-0.08731500$

H $3.02287500-2.91476700-0.83741800$

H $2.84215400 \quad-2.96452100 \quad 0.93267400$

$\begin{array}{llll}\text { C } 2.49054400 & 2.46002500 & 0.15529500\end{array}$

H $2.93808800 \quad 2.99592500 \quad-0.68072200$

H $1.40903700 \quad 2.45649800 \quad 0.04889600$

H $2.764018002 .94773900 \quad 1.09003900$

N 2.97518500 $-1.04769500 \quad 0.10525200$

C $-2.68916900 \quad 1.26246200 \quad 0.10138600$

$\mathrm{H}-2.25967700 \quad 2.12527300-0.41482000$

$\mathrm{H}-2.48363600 \quad 1.40805100 \quad 1.16917300$

$\mathrm{Au} \quad 0.04908200-0.01427700-0.16478300$ 
GOLD

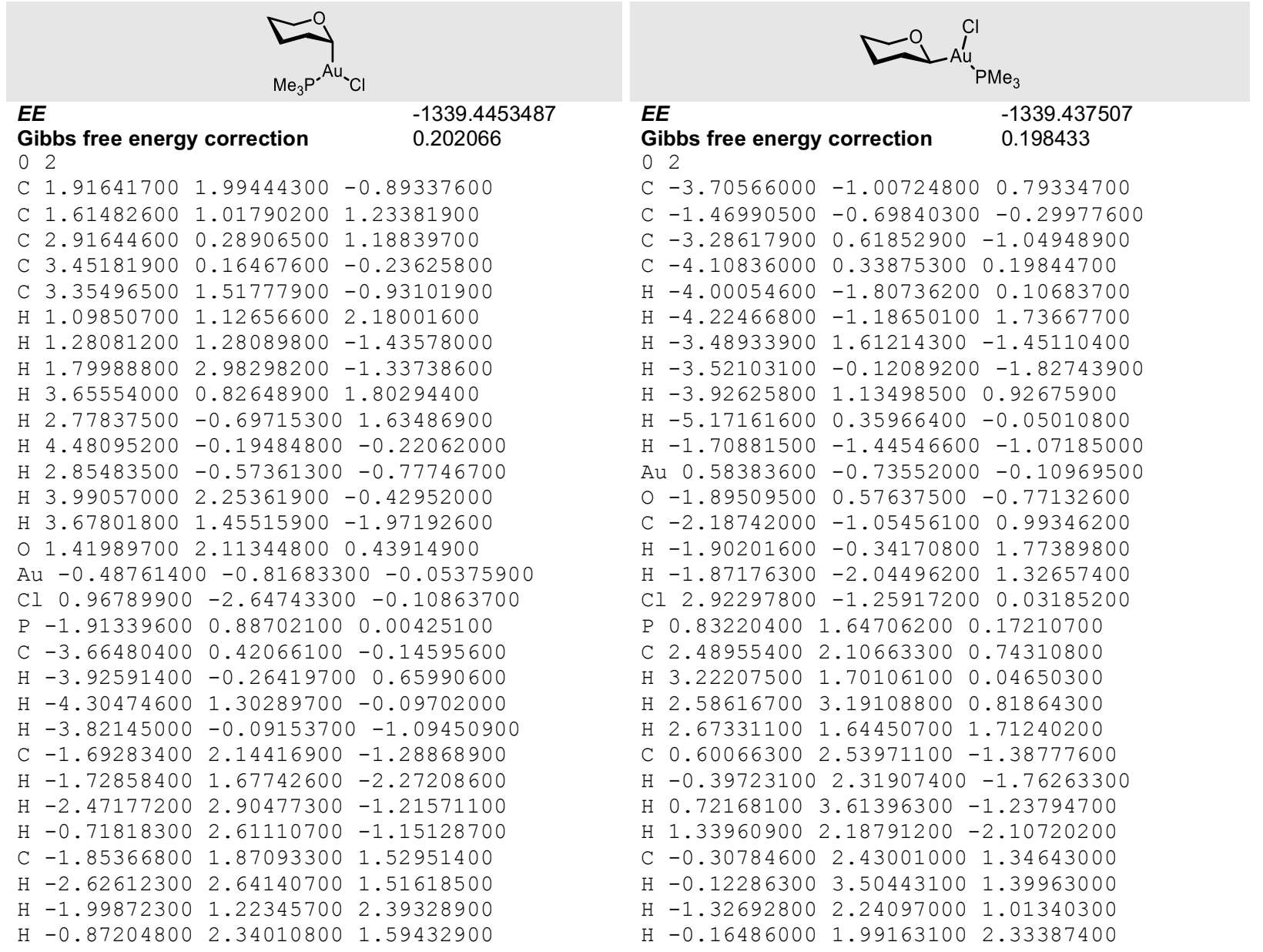

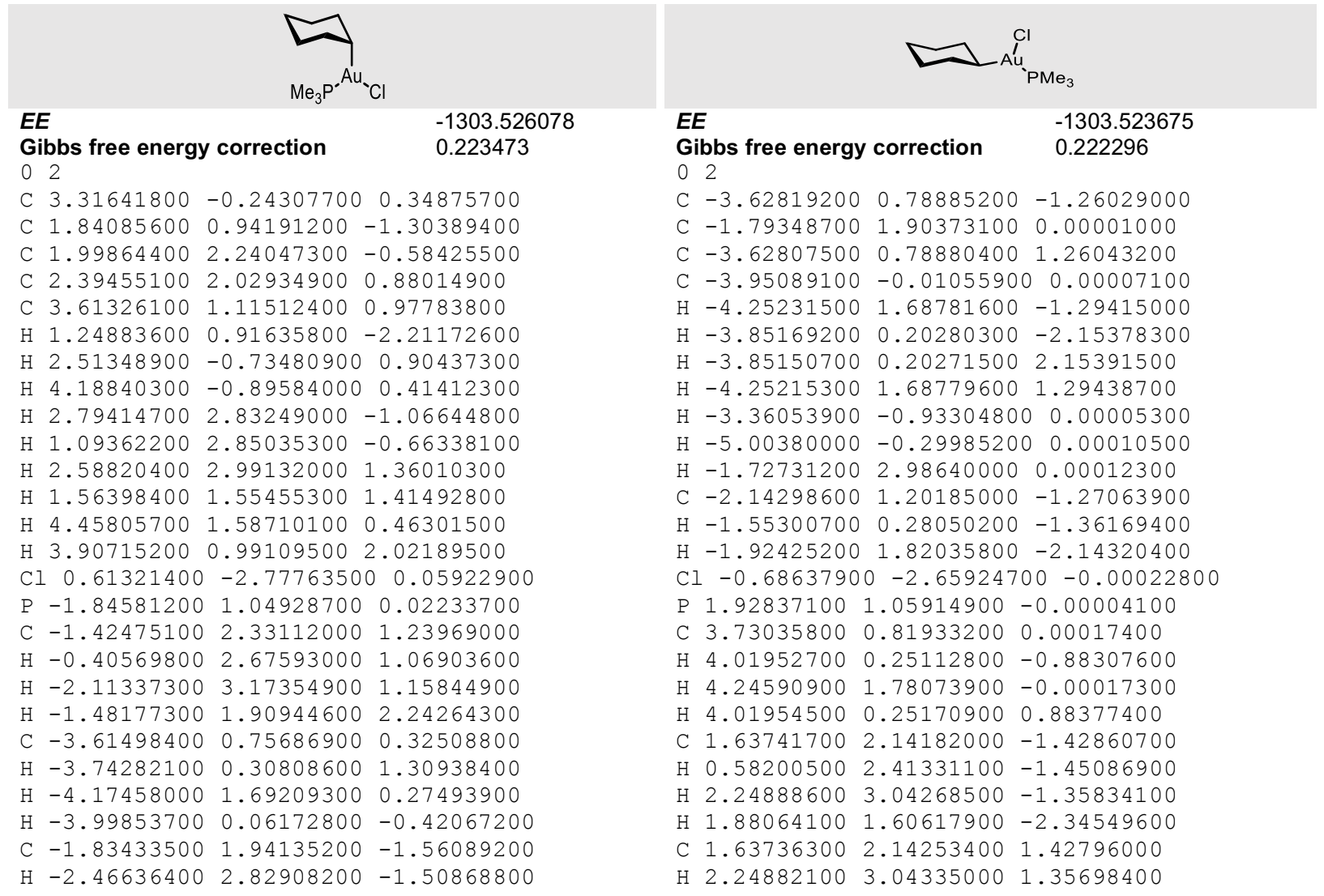


$\mathrm{H}-0.81277700 \quad 2.23225300-1.80257000$

$\mathrm{H}-2.19864100 \quad 1.28053900-2.34684600$

C $2.88590400-0.10644000-1.11433300$

H $3.77046400 \quad 0.16485300-1.71463200$

$\mathrm{H} 2.53427600-1.06892400-1.48937900$

$\mathrm{Au}-0.59565700-0.78368200 \quad 0.05064100$
H $0.58197800 \quad 2.41403200 \quad 1.45062600$

$\mathrm{H} \quad 1.88105600 \quad 1.60742200 \quad 2.34505700$

C $-2.14283900 \quad 1.20164800 \quad 1.27059900$

$\mathrm{H}-1.55301600 \quad 0.28019400 \quad 1.36137100$

$\mathrm{H}-1.92375700 \quad 1.81993400 \quad 2.14322800$

Au $0.70855500-0.79359800 \quad 0.00009300$ 
GOLD

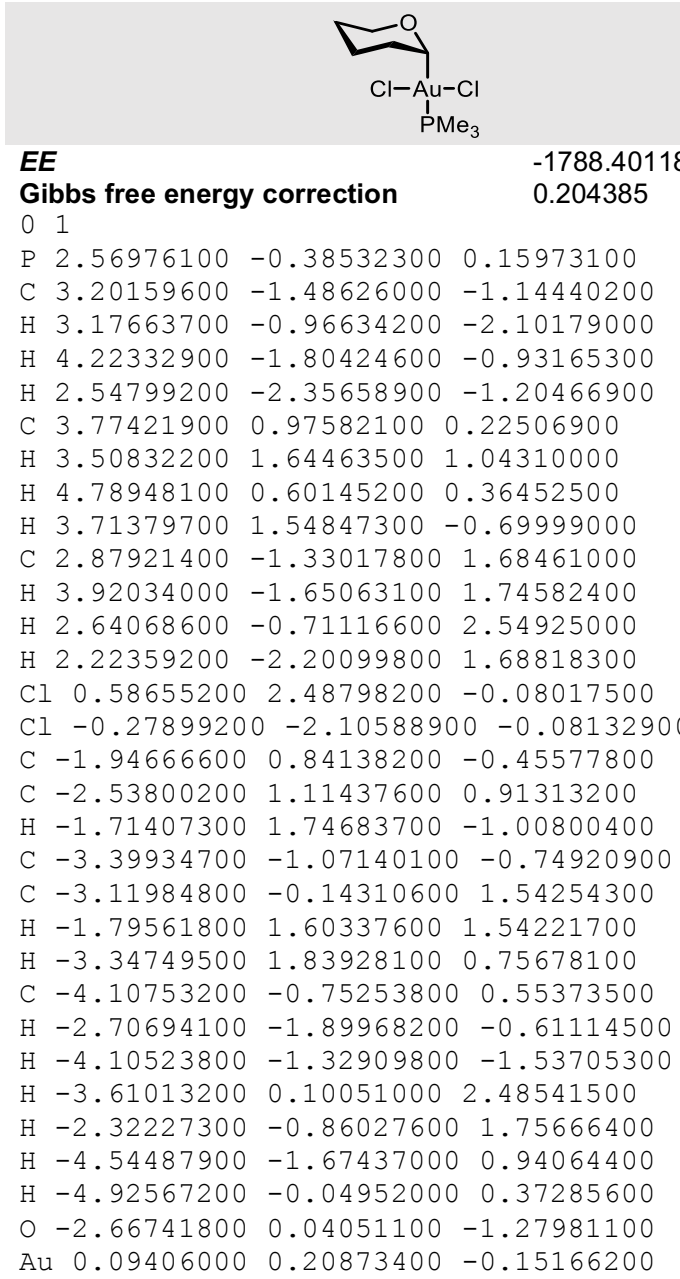

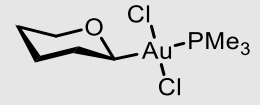

\section{EE \\ Gibbs free energy correction}

01

$\begin{array}{llll}\text { P } 2.63049100 & -0.24581400 & -0.01592900\end{array}$

C $3.14751000-1.27423200-1.42070800$

H $2.93900500-0.74719200-2.35135200$

$\mathrm{H} 4.21205500-1.50526800-1.36463100$

H $2.56465100-2.19503700-1.40350800$

C $3.702001001 .21696400-0.08369700$

H $3.493199001 .85124900 \quad 0.77714400$

$\mathrm{H} \quad 4.75440700 \quad 0.93038200 \quad-0.09093400$

H $3.46513700 \quad 1.78994700-0.97956700$

C $3.19478800-1.17417800 \quad 1.43980400$

H $4.25727200-1.40945700 \quad 1.36507600$

$\begin{array}{llll}\mathrm{H} & 3.01699700 & -0.58294800 & 2.33773100\end{array}$

H $2.61341000-2.09368100 \quad 1.50567400$

C $-1.87756500 \quad 0.43030400 \quad-0.01147600$

C $-2.51617700 \quad-0.04917100 \quad 1.27409600$

$\mathrm{H}-2.00108000 \quad 1.51137200-0.13867200$

C $-3.74894600-0.04248900-1.32057100$

C $-4.03624200 \quad 0.13031600 \quad 1.15074200$

H $-2.28052100-1.10370600 \quad 1.42677700$

$\mathrm{H}-2.12395000 \quad 0.52125600 \quad 2.11823600$

C $-4.53495000-0.54872900-0.12206000$

$\mathrm{H}-4.00870500-0.57609000-2.23352900$

$\mathrm{H}-3.93990800 \quad 1.02865600-1.47504900$

$\mathrm{H}-4.53406700-0.27754800 \quad 2.03192100$

$\mathrm{H}-4.27363800 \quad 1.19817700 \quad 1.11064500$

$\mathrm{H}-5.59949500-0.36002800-0.27672600$

$\mathrm{H}-4.39447900-1.62980700-0.04401500$

$\begin{array}{lllll}0 & -2.35343700 & -0.24739800 & -1.13275800\end{array}$

$\mathrm{Cl} 0.46197400 \quad 2.45448800 \quad-0.00939900$

$\mathrm{Cl}-0.07577100 \quad-2.15625300 \quad 0.09146500$

$\mathrm{Au} \quad 0.18559300 \quad 0.15333400 \quad 0.00194800$

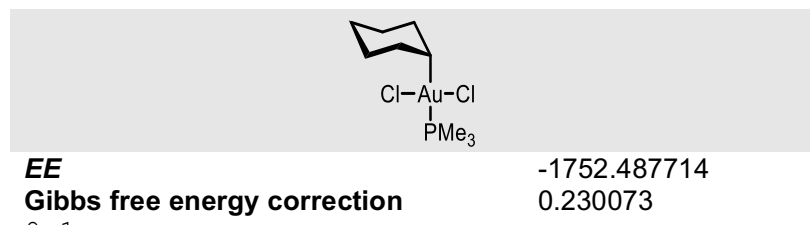

01 0.230073

$\begin{array}{llll}\text { P } & -2.50389000 & -0.28938500 & 0.32192300\end{array}$

C $-2.71865500-1.31172300 \quad 1.80926100$

$\mathrm{H}-2.34141500-0.77182400 \quad 2.67744500$

$\mathrm{H}-3.77022500-1.55668700 \quad 1.96481400$

$\mathrm{H}-2.13813300 \quad-2.22564200 \quad 1.68650700$

C $-3.57606900 \quad 1.15001800 \quad 0.59571100$

$\mathrm{H}-3.56838600 \quad 1.77428400 \quad-0.29692900$

$\mathrm{H} \quad-4.59556700 \quad 0.83711000 \quad 0.82536700$

$\mathrm{H}-3.17520200 \quad 1.74419100 \quad 1.41619600$

C $-3.33719100-1.23881000-0.98424600$

$\mathrm{H}-4.35907400-1.48706300-0.69427000$

$\mathrm{H}-3.35406600-0.65144700-1.90200500$

$\mathrm{H}-2.76938100-2.15151200-1.16305600$

Cl $-0.52181600 \quad 2.43904300 \quad-0.27383000$

Cl $0.10377600-2.16413600-0.28549600$

C $1.91024300 \quad 0.60893900-0.72174800$

C $2.77221700-0.56516100-1.16274200$

$\mathrm{H} 1.70053800 \quad 1.26145000-1.56961400$

C $3.04958500 \quad 0.52527400 \quad 1.55055500$

C $3.36901400-1.39379100-0.02427800$

$\mathrm{H} 2.23550300-1.20799700-1.86046600$

H $3.60024900-0.11454800-1.72914300$

C $4.03259800-0.52157200 \quad 1.03645600$

H $2.19574300 \quad 0.02235600 \quad 2.01915700$

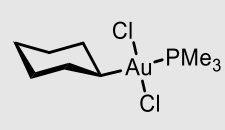

\section{EE $\quad-1752.493853$ \\ Gibbs free energy correction $\quad 0.22908$}

01

P 2.66492100 $-0.22039300 \quad 0.00018300$

C $3.22717600-1.19141200-1.42965300$

H $3.02996000-0.63488200-2.34552900$

$\mathrm{H} 4.29382600-1.40910800-1.36054700$

H $2.65933200-2.12135100-1.45743000$

C $3.71808200 \quad 1.25825200 \quad 0.00043800$

H $3.486863001 .85920400 \quad 0.87933400$

H $4.77468900 \quad 0.98732900 \quad 0.00045900$

H $3.48694600 \quad 1.85941600-0.87833600$

C $3.22754400-1.19216000 \quad 1.42935100$

$\mathrm{H} \quad 4.29472300-1.40749900 \quad 1.36104500$

H $3.02813300 \quad-0.63744100 \quad 2.34584000$

$\mathrm{H} 2.66164600-2.12334400 \quad 1.45518100$

C $-1.88282800 \quad 0.40641100 \quad-0.00029600$

C $-2.48961900-0.17352500 \quad 1.26277800$

$\mathrm{H}-1.98006700 \quad 1.49225200-0.00068500$

C $-4.00498300 \quad 0.07989100-1.25757500$

C $-4.00475000 \quad 0.08086900 \quad 1.25783800$

$\mathrm{H}-2.30548500 \quad-1.24945100 \quad 1.30487100$

$\mathrm{H}-2.03924700 \quad 0.27672300 \quad 2.14993600$

C $-4.65093800-0.49400700 \quad 0.00040700$

$\mathrm{H}-4.45035700-0.35753700-2.15344100$

$\mathrm{H}-4.19071900 \quad 1.15784900-1.30119800$

$\mathrm{H}-4.44988500-0.35594800 \quad 2.15412000$ 
$\begin{array}{llll}\mathrm{H} & 3.51323200 & 1.14816400 & 2.31778800\end{array}$

$\mathrm{H} \quad 4.08881700-2.10121200-0.44156100$

$\mathrm{H} \quad 2.58303700-1.98371200 \quad 0.44668900$

$\mathrm{H} 4.39864300-1.14300700 \quad 1.85604600$

$\mathrm{H} \quad 4.90348500 \quad-0.01107400 \quad 0.61016700$

$\mathrm{Au}-0.10386400 \quad 0.15511100 \quad-0.21776500$

C $2.56164000 \quad 1.40276200 \quad 0.40228300$

$\mathrm{H} 1.90524400 \quad 2.19639200 \quad 0.75298400$

$\mathrm{H} \quad 3.43692200 \quad 1.89975500-0.04046100$
$\mathrm{H}-4.19047300 \quad 1.15885000 \quad 1.30073100$

$\mathrm{H}-5.72417100-0.29233100 \quad 0.00038800$

$\mathrm{H}-4.53030700-1.58234700 \quad 0.00084200$

Cl $0.44491400 \quad 2.44843400 \quad-0.00026800$

$\mathrm{Cl}-0.03115500-2.17899200 \quad 0.00008100$

$\mathrm{Au} \quad 0.19725500 \quad 0.14532300-0.00004600$

C $-2.48983500-0.17442000-1.26276800$

$\mathrm{H}-2.30554400-1.25033100-1.30430100$

$\mathrm{H}-2.03984800 \quad 0.27541200 \quad-2.15033300$ 
3.3. Group 12

\section{ZINC}

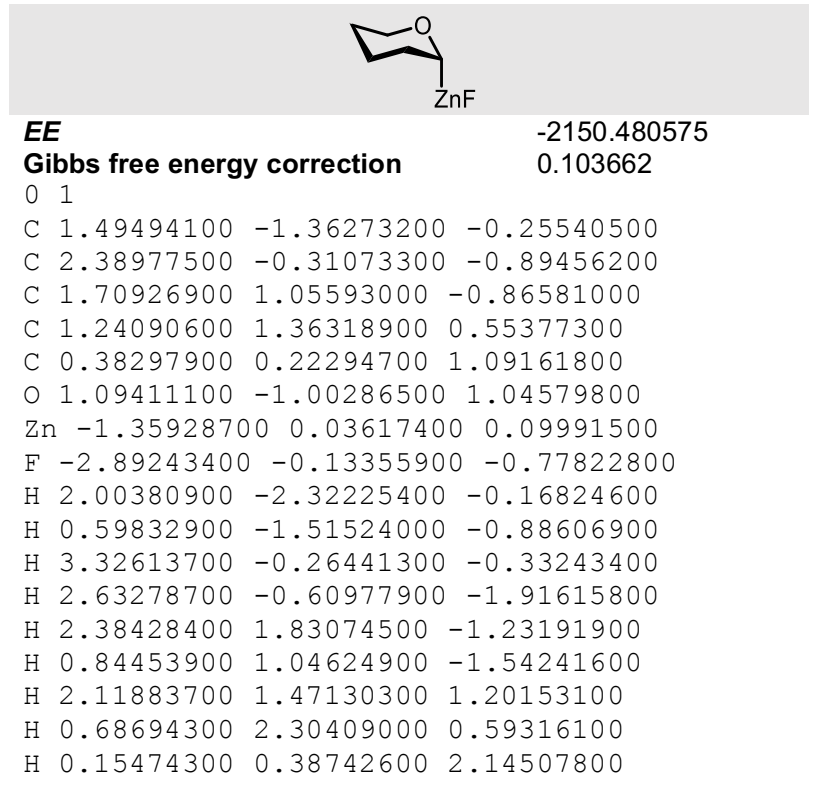

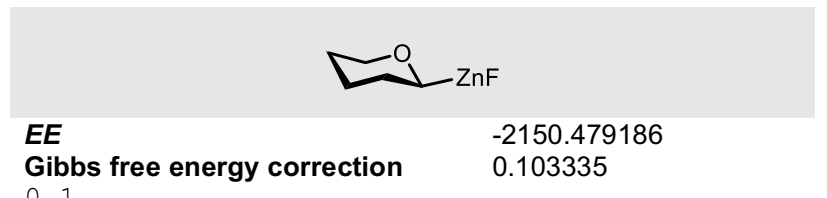

Gibbs free energy correction

0.103335

C $-2.18130600-1.30853700 \quad 0.14227700$

C $-3.00890500-0.17631500-0.44344300$

C $-2.52607600 \quad 1.16187200 \quad 0.11016500$

C $-1.01837400 \quad 1.29786500 \quad-0.10749200$

C $-0.29023600 \quad 0.07733000 \quad 0.45168100$

$0-0.79917700-1.12701200-0.11649100$

Zn $1.65294500 \quad 0.01255800 \quad 0.07114100$

F $3.37956900-0.03873700-0.31807300$

H $-2.44972300-2.27059800 \quad-0.29441900$

$\mathrm{H}-2.33989100-1.36555800 \quad 1.22917500$

$\mathrm{H}-2.89758200-0.18850600-1.53136500$

$\mathrm{H}-4.06514100-0.33845100-0.21832700$

$\mathrm{H}-3.05932700 \quad 1.99110000-0.35874900$

$\mathrm{H}-2.73875900 \quad 1.20697100 \quad 1.18356600$

$\mathrm{H}-0.81942500 \quad 1.38802400-1.17970900$

$\mathrm{H}-0.63872800 \quad 2.20435900 \quad 0.37072500$

$\mathrm{H}-0.45309800 \quad 0.04734400 \quad 1.54032000$

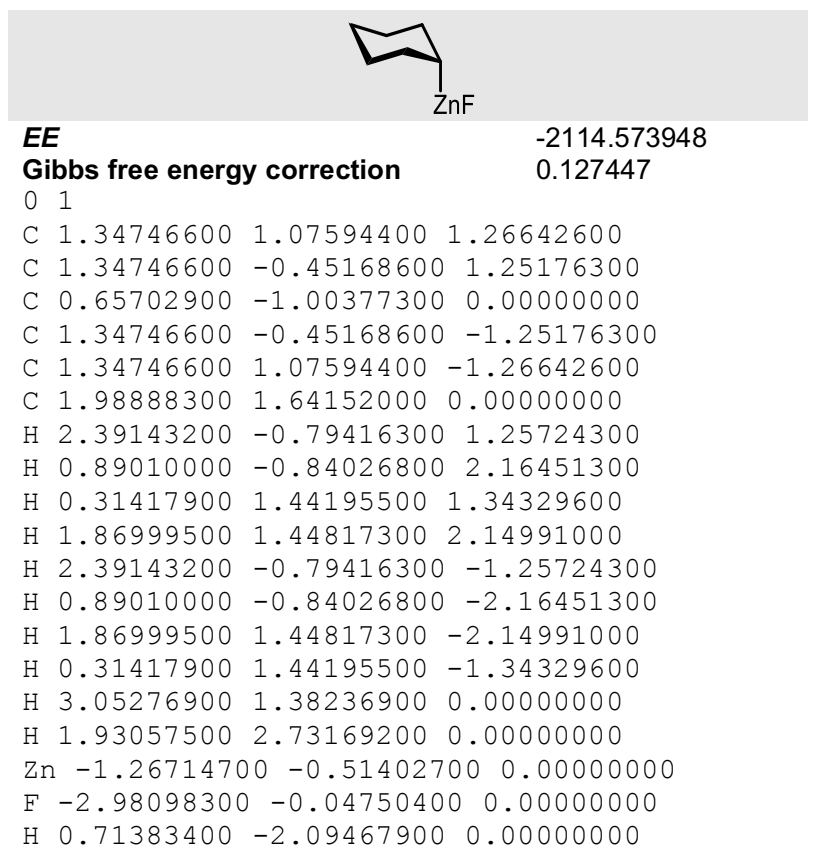

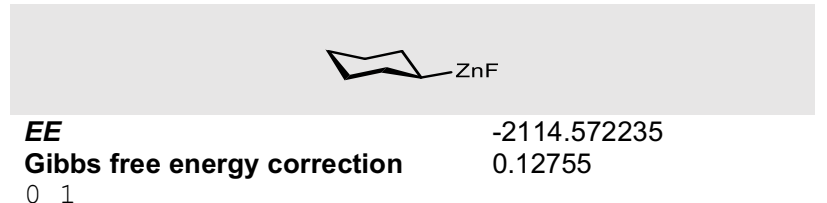

01

C $0.32063100 \quad 2.42216000 \quad 1.25774800$

C $0.32063100 \quad 0.89186300 \quad 1.26202600$

C $-0.34745700 \quad 0.32702600 \quad 0.00000000$

C $0.32063100 \quad 0.89186300-1.26202600$

C $0.32063100 \quad 2.42216000-1.25774800$

C $0.98981100 \quad 2.96964400 \quad 0.00000000$

$\mathrm{H} \quad 1.35905100 \quad 0.54474600 \quad 1.31795300$

$\mathrm{H}-0.17752200 \quad 0.52756200 \quad 2.16379100$

$\mathrm{H}-0.71471300 \quad 2.77756000 \quad 1.29867800$

$\mathrm{H} \quad 0.82202900 \quad 2.79945100 \quad 2.15205300$

H $1.35905100 \quad 0.54474600 \quad-1.31795300$

$\mathrm{H}-0.17752200 \quad 0.52756200 \quad-2.16379100$

$\mathrm{H} \quad 0.82202900 \quad 2.79945100 \quad-2.15205300$

$\mathrm{H}-0.71471300 \quad 2.77756000-1.29867800$

$\mathrm{H} 2.04593500 \quad 2.67729900 \quad 0.00000000$

$\mathrm{H} \quad 0.96524600 \quad 4.06120000 \quad 0.00000000$

$\mathrm{H}-1.39461600 \quad 0.65403200 \quad 0.00000000$ Zn $-0.39795500-1.64792100 \quad 0.00000000$ F $-0.42276600-3.42242500 \quad 0.00000000$ 
ZINC

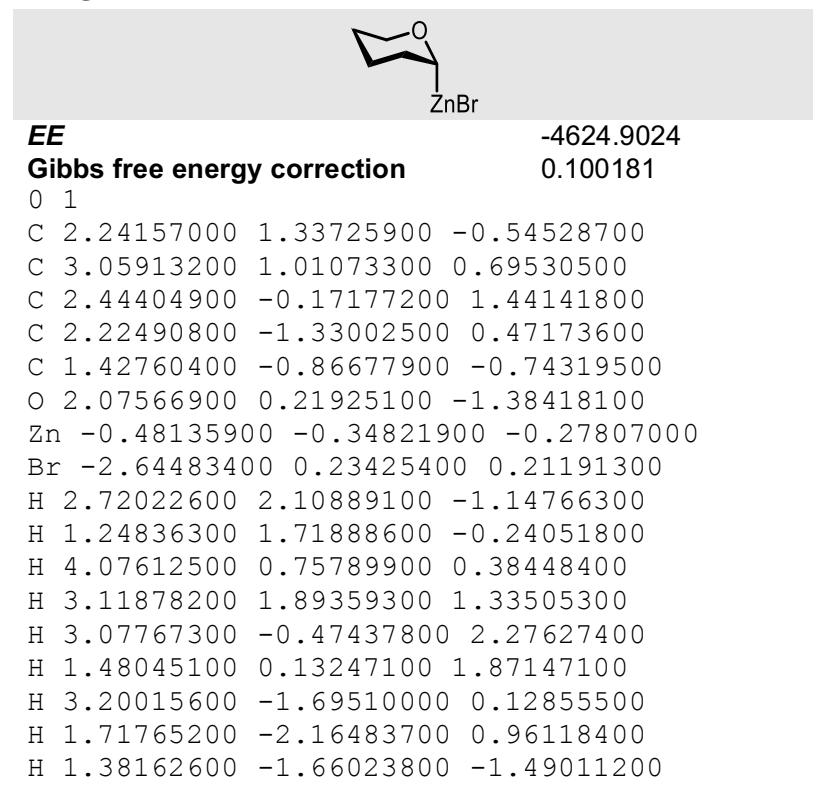

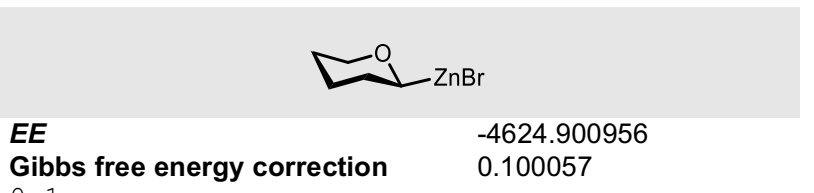

01

$\begin{array}{llll}C & -3.13190800 & -1.31941100 & 0.09877900\end{array}$

C $-3.94860600-0.19780800-0.52234200$

C $-3.49837200 \quad 1.14865300 \quad 0.03907700$

C $-1.98441500 \quad 1.29715000-0.12194900$

C $-1.26973200 \quad 0.08753000 \quad 0.47489600$

$0-1.74274000-1.12432600-0.10759900$

Zn $0.70853200 \quad 0.03409700 \quad 0.19460300$

Br 2.96652800 $-0.02007900-0.14478000$

$\mathrm{H}-3.37378300-2.28580400-0.34391400$

$\mathrm{H}-3.33085900-1.37383500 \quad 1.17916100$

$\mathrm{H}-3.79998600-0.21528500-1.60566600$

$\mathrm{H}-5.01048300-0.36838600-0.33243200$

$\mathrm{H}-4.02204200 \quad 1.96957900-0.45464600$

$\mathrm{H}-3.75087700 \quad 1.19859100 \quad 1.10369800$

$\mathrm{H}-1.74458500 \quad 1.38032500-1.18638300$

$\mathrm{H}-1.63208800 \quad 2.21083900 \quad 0.36350500$

$\mathrm{H}-1.47960900 \quad 0.06173000 \quad 1.55591500$

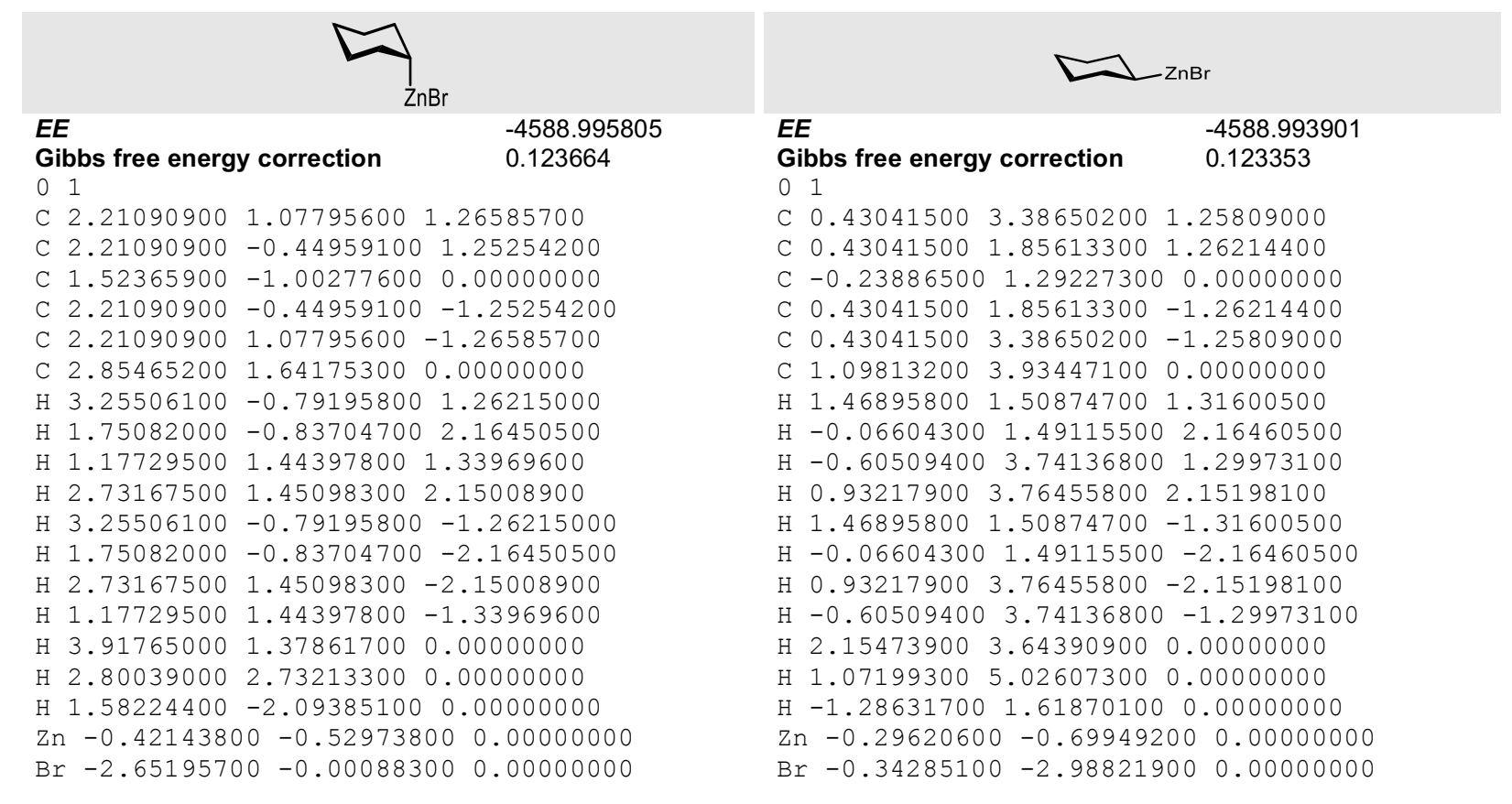




\section{CADMIUM}

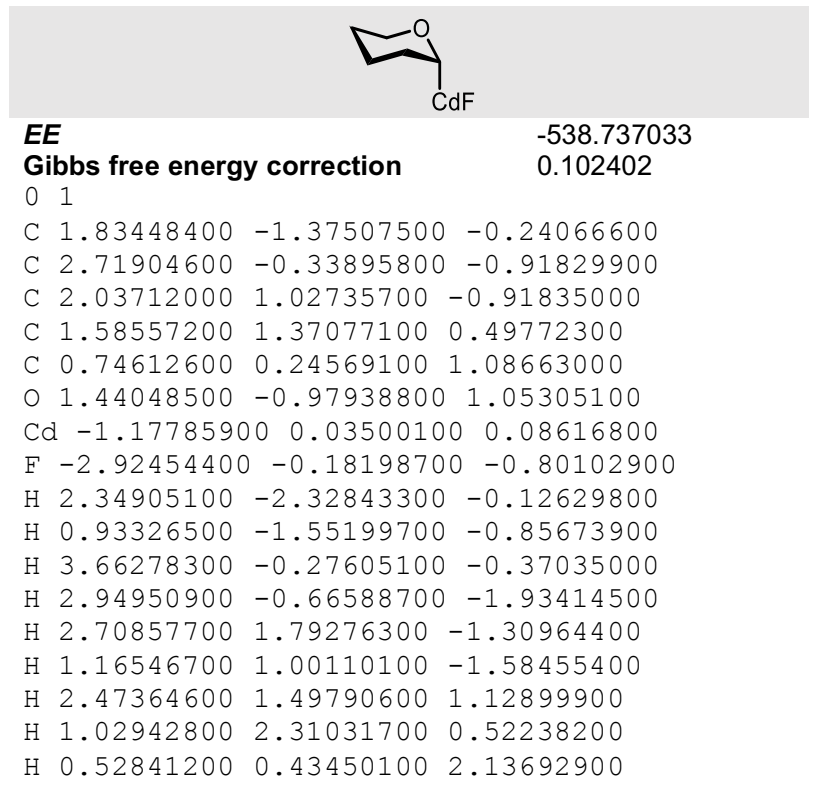

\begin{tabular}{|c|c|c|}
\hline \multicolumn{2}{|c|}{$E E$} & -538.734586 \\
\hline & $\begin{array}{l}\text { bbs free energy correction } \\
1\end{array}$ & 0.102441 \\
\hline $\mathrm{C}$ & $-2.57337900-1.30551900$ & 0.12776500 \\
\hline $\mathrm{C}$ & $-3.38668200-0.16924300$ & -0.46979000 \\
\hline $\mathrm{C}$ & -2.904173001 .16568600 & 0.09151800 \\
\hline $\mathrm{C}$ & $-1.39198000 \quad 1.29276500$ & -0.10556400 \\
\hline $\mathrm{C}$ & $-0.68306300 \quad 0.06779000$ & 0.46243200 \\
\hline 0 & $-1.18563500-1.13008100$ & -0.11301700 \\
\hline $\mathrm{Cd}$ & $1.41737300 \quad 0.00831300$ & 0.06571800 \\
\hline $\mathrm{F}$ & $3.33027500-0.03952400$ & -0.38917400 \\
\hline $\mathrm{H}$ & $-2.83909200-2.26657100$ & -0.31242400 \\
\hline $\mathrm{H}$ & $-2.74652400-1.36142200$ & 1.21232400 \\
\hline $\mathrm{H}$ & $-3.25986300-0.18181000$ & -1.55596600 \\
\hline $\mathrm{H}$ & $-4.44684200-0.32549200$ & -0.25974300 \\
\hline $\mathrm{H}$ & $-3.42450400 \quad 1.99880600$ & -0.38487500 \\
\hline $\mathrm{H}$ & $-3.13201800 \quad 1.21216600$ & 1.16166500 \\
\hline $\mathrm{H}$ & -1.178800001 .38088200 & -1.17500900 \\
\hline $\mathrm{H}$ & $-1.01279200 \quad 2.19615400$ & 0.37847400 \\
\hline & $-0.84521100 \quad 0.03576000$ & 1.54961200 \\
\hline
\end{tabular}

$\begin{array}{ll} & \\ \text { GE } & -489.30784 \\ \text { Gibbs free energy correction } & 0.196835\end{array}$

$0 \quad 1$

C $-1.95142900 \quad 1.26670400 \quad 0.64686300$

C $-1.49877700 \quad 1.25230100-0.81204400$

C $-0.68871800-0.00064100-1.14730100$

C $-1.49928100-1.25293900-0.81112200$

C $-1.95190300-1.26607100 \quad 0.64780600$

C $-2.73279200 \quad 0.00058800 \quad 0.99641200$

$\mathrm{H}-0.42464900-0.00107800-2.20608600$

$\mathrm{H}-2.39583300 \quad 1.26350300-1.44686400$

$\mathrm{H}-0.94295000 \quad 2.16218600-1.04824300$

$\mathrm{H}-1.07322900 \quad 1.34303900 \quad 1.30171200$

$\mathrm{H}-2.56075300 \quad 2.15028700 \quad 0.84583400$

$\mathrm{H}-2.39634600-1.26420000-1.44592400$

$\mathrm{H}-0.94384400-2.16323700-1.04663700$

$\mathrm{H}-2.56153300-2.14928500 \quad 0.84747900$

$\mathrm{H}-1.07368200-1.34221000 \quad 1.30262700$

$\mathrm{H}-3.67138100 \quad 0.00054400 \quad 0.43267600$

$\mathrm{H}-3.00144000 \quad 0.00105300 \quad 2.05435600$

F 2.91217100 $0.00014000 \quad 0.85547800$

$\mathrm{Cd} 1.18278100-0.00003100-0.09195700$

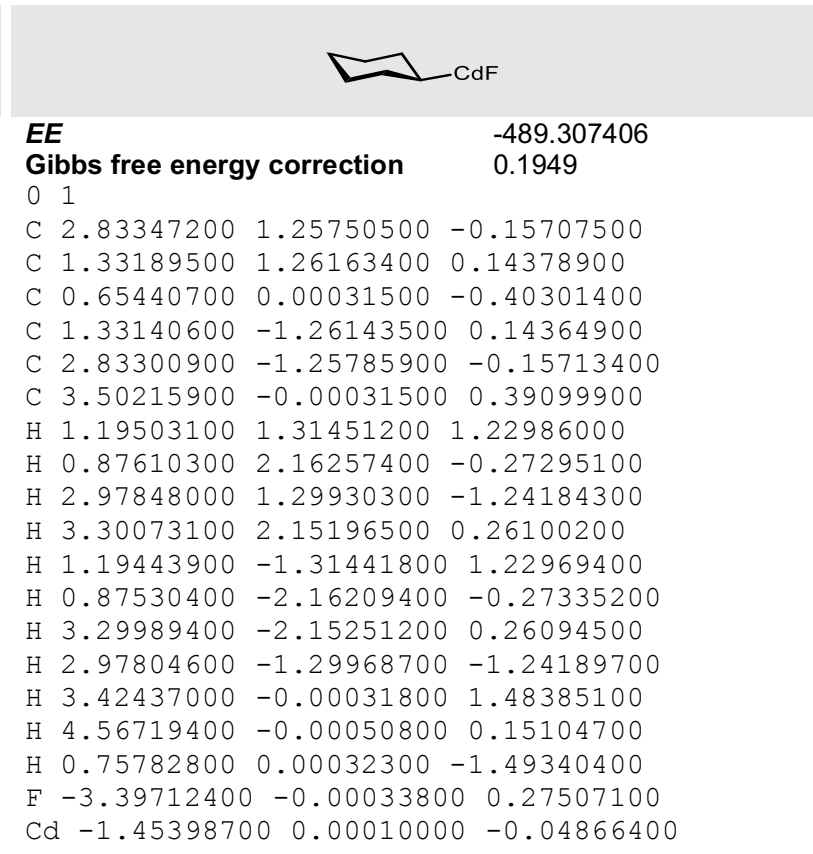




\section{CADMIUM}

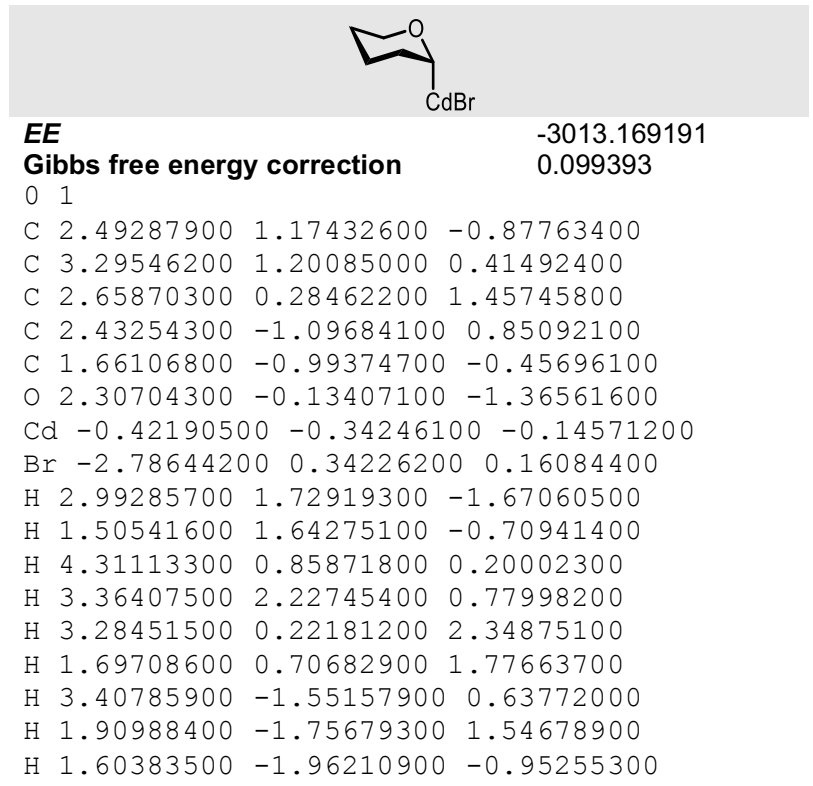
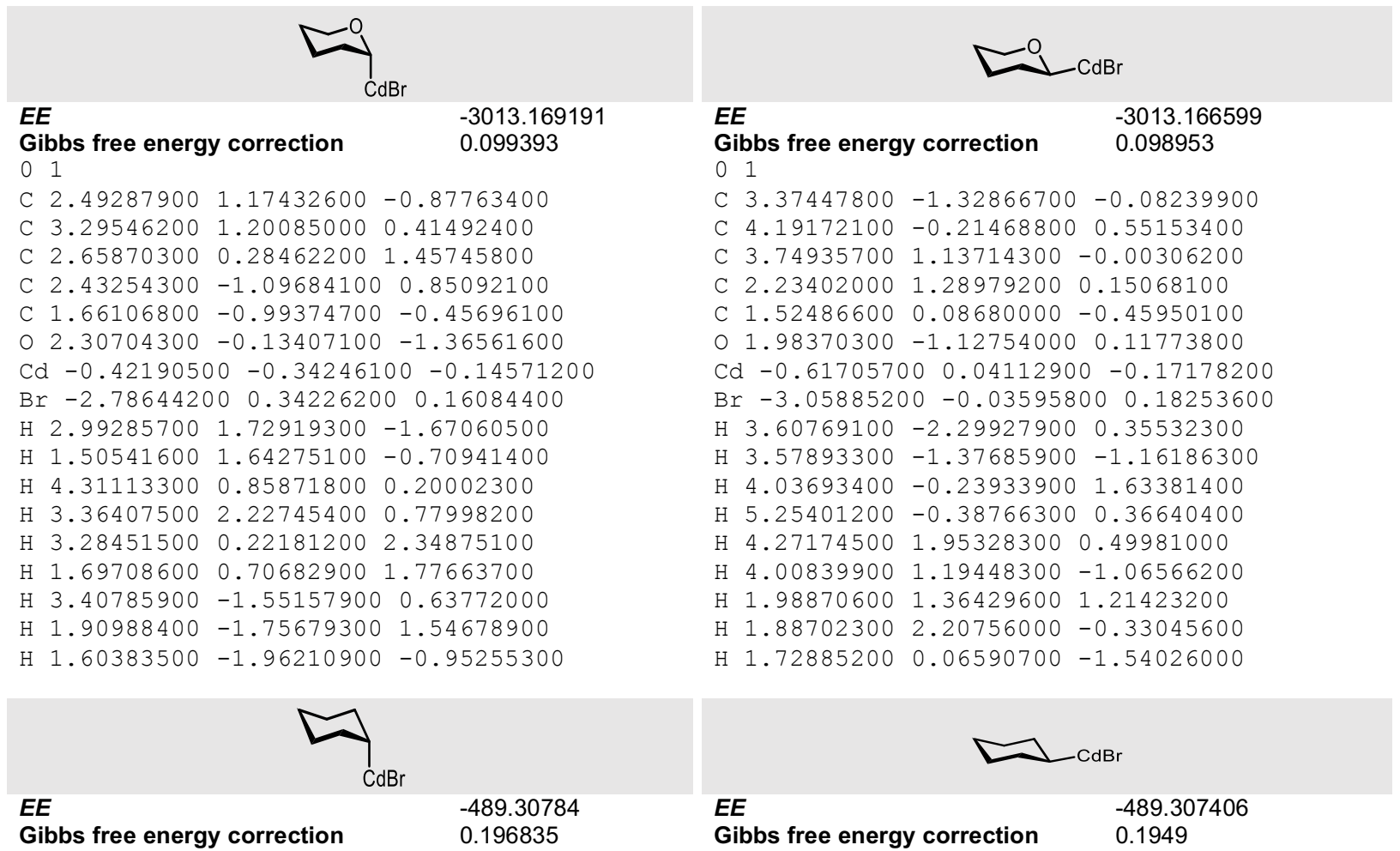

01

C $-2.55168400 \quad 0.85103300 \quad-1.26588400$

C $-2.35832700-0.66422000-1.25317100$

C $-1.61962600-1.13562600-0.00050600$

C $-2.35844100-0.66538500 \quad 1.25253300$

C $-2.55185500 \quad 0.84985600 \quad 1.26660500$

C $-3.26121800 \quad 1.32879400 \quad 0.00053300$

$\mathrm{H}-1.53874100-2.22377400-0.00103200$

$\mathrm{H}-3.35228900-1.13361400-1.26674500$

$\mathrm{H}-1.85099400-0.99157300-2.16320400$

H $-1.57348200 \quad 1.34472900-1.34140100$

$\mathrm{H}-3.11708400 \quad 1.15334000-2.14937200$

$\mathrm{H}-3.35238800-1.13482700 \quad 1.26560700$

$\mathrm{H}-1.85119400-0.99352200 \quad 2.16232700$

$\mathrm{H}-3.11735700 \quad 1.15142500 \quad 2.15028400$

$\mathrm{H}-1.57365700 \quad 1.34348000 \quad 1.34266800$

$\mathrm{H}-4.28269500 \quad 0.93460400 \quad 0.00029700$

$\mathrm{H}-3.34408400 \quad 2.41718800 \quad 0.00103500$

Br 2.76470600 $0.40571000 \quad 0.00017200$

Cd $0.42492000-0.40529200-0.00014900$

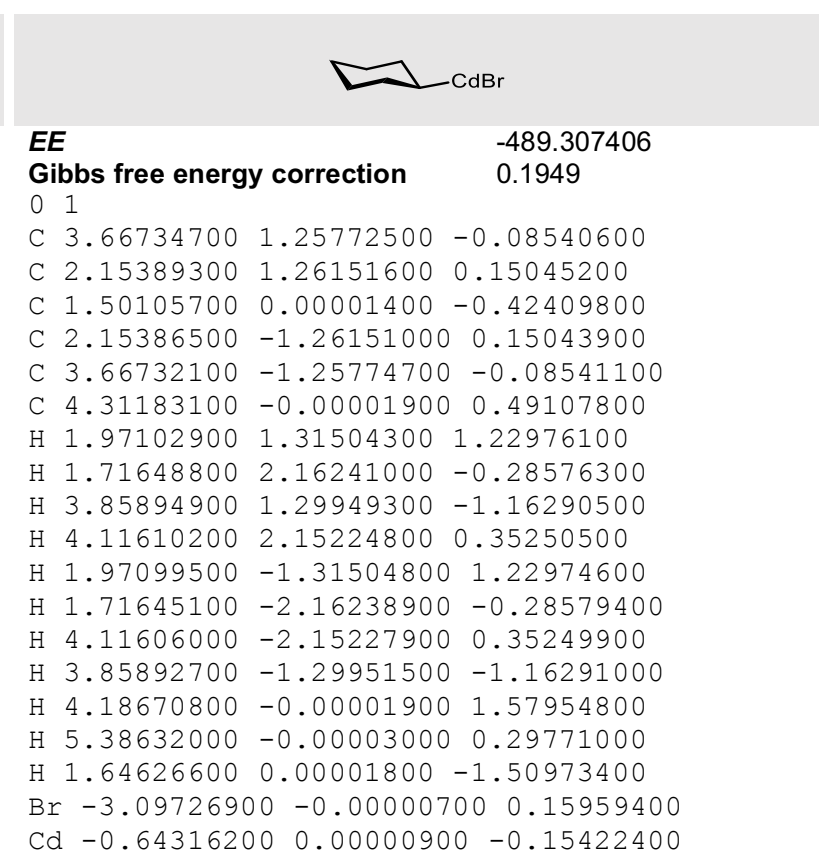




\section{MERCURY}

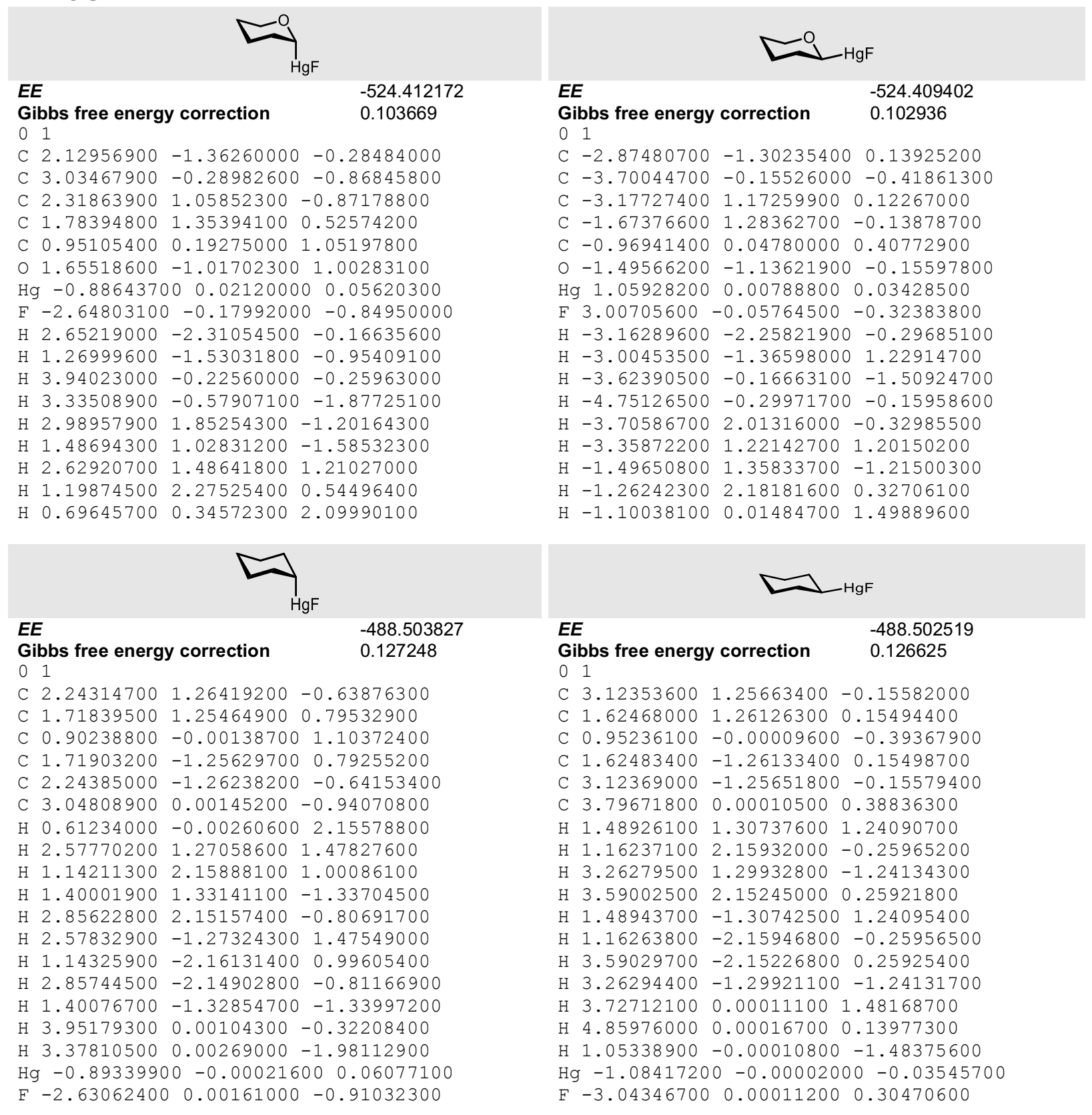




\section{MERCURY}

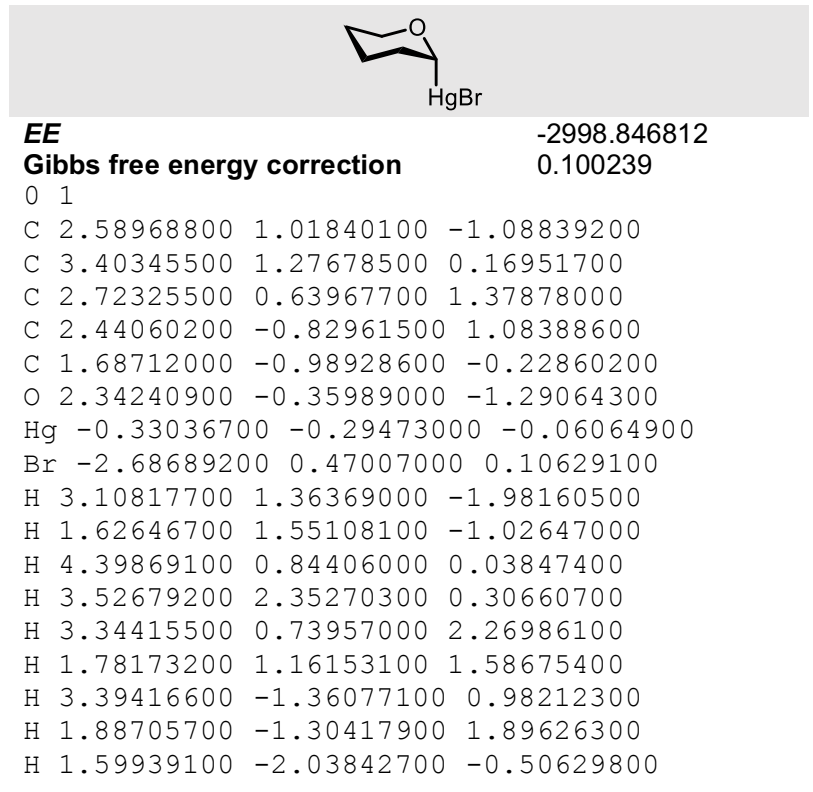

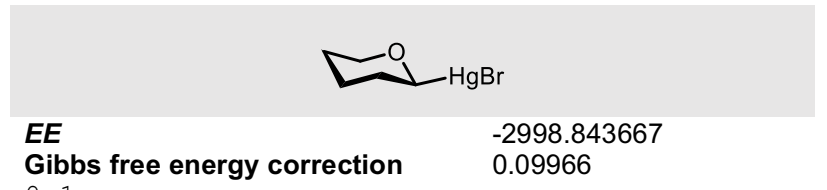

01

C $3.48784500-1.31051700-0.05354400$

$\begin{array}{llll}\text { C } 4.28432200 & -0.16924000 & 0.55644200\end{array}$

C $3.79578800 \quad 1.16343800-0.00503300$

$\begin{array}{llll}\text { C } 2.27997000 & 1.27731800 & 0.17371300\end{array}$

C $1.60424600 \quad 0.04694300 \quad-0.41678200$

$\begin{array}{llll}0 & 2.09413200 & -1.14188400 & 0.16585000\end{array}$

$\mathrm{Hg}-0.47856800 \quad 0.01736500 \quad-0.13228100$

$\mathrm{Br}-2.91898300 \quad-0.02646000 \quad 0.21817500$

H $3.74833000 \quad-2.27001900 \quad 0.39188200$

H $3.67723400-1.36720300-1.13502100$

$\mathrm{H} 4.14778000-0.18680500 \quad 1.64109400$

H $5.34745500 \quad-0.31522000 \quad 0.35484300$

H $4.30107800 \quad 1.99977400 \quad 0.48082100$

$\mathrm{H} 4.036500001 .21758600-1.07199400$

H $2.04548800 \quad 1.34698100 \quad 1.23937400$

H $1.89740200 \quad 2.17928800-0.30902700$

H $1.78254500 \quad 0.01996300-1.50116300$

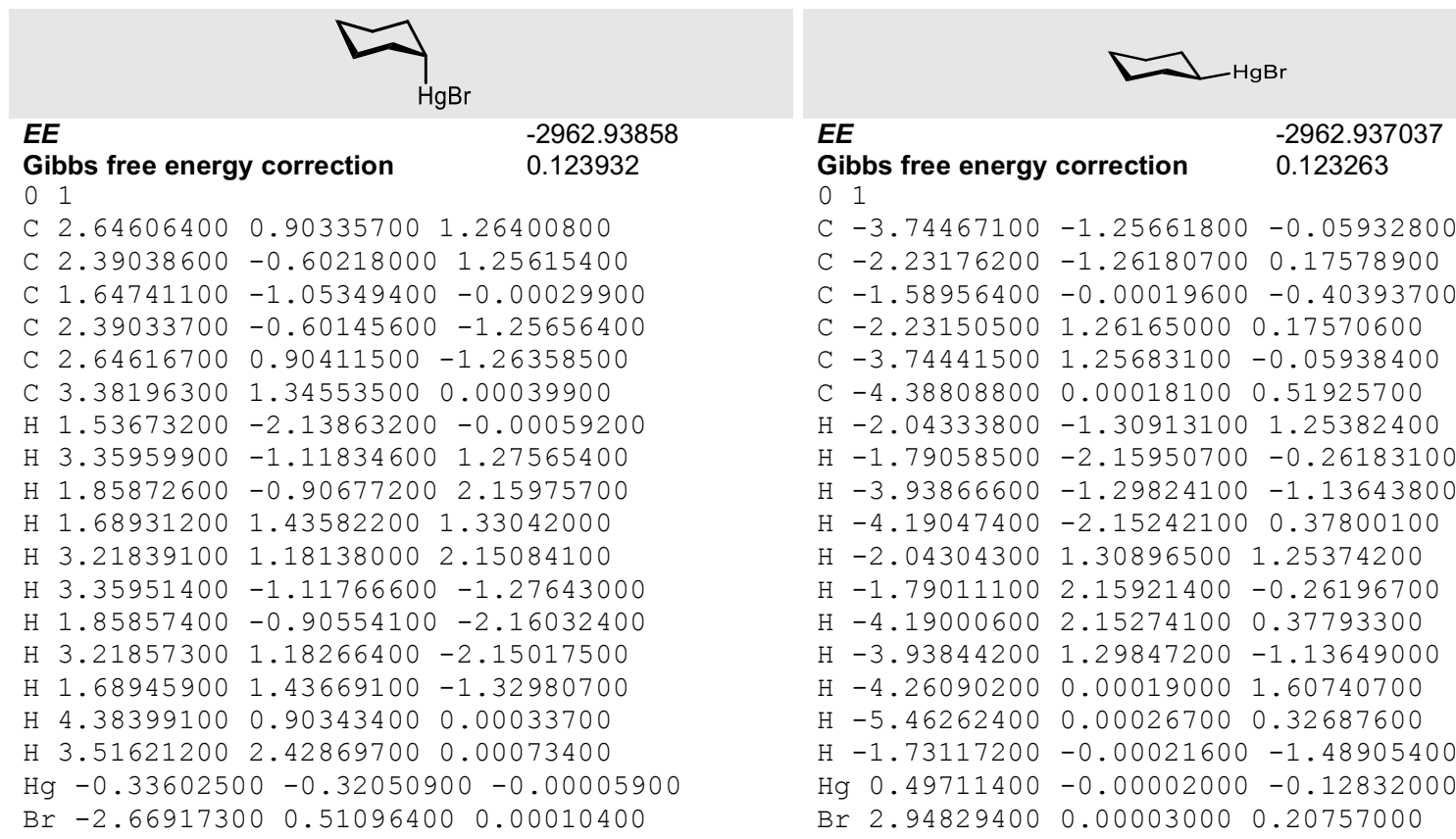




\section{Cartesian Coordinates for Figure 3B}

\subsection{Glucose structures}

\section{NICKEL}

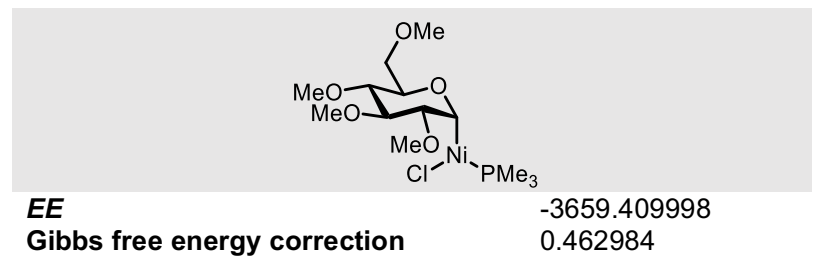

01 0.462984

C $-1.46035000 \quad 0.38515900 \quad 1.58335900$

○ $0.57980300 \quad 1.46740700 \quad 1.06344100$

C $-2.10540600 \quad 0.94411400 \quad 0.31533700$

C $0.12228700 \quad 2.00388300-0.14760800$

$\begin{array}{lllll}\text { C }-1.39118600 & 2.23288100 & -0.07990500\end{array}$

$\begin{array}{lllll}0 & -3.48470800 & 1.14850800 & 0.54037400\end{array}$

$\mathrm{H}-1.97380200 \quad 0.23020500-0.50782000$

C $0.85787900 \quad 3.30031000-0.39031100$

$\mathrm{H} \quad 0.33486700 \quad 1.31540000-0.98300100$

$\begin{array}{lllll}0 & -1.86966900 & 2.67980500 & -1.33546400\end{array}$

$\mathrm{H}-1.59820400 \quad 2.98356700 \quad 0.69447900$

C $-4.31333000 \quad 1.13285700-0.60190800$

$\begin{array}{llll}0 & 2.22531000 & 3.02619000 & -0.56784900\end{array}$

H $0.71451500 \quad 3.96268800 \quad 0.47567000$

H $0.45092100 \quad 3.79053400-1.28355700$

C $-2.42256400 \quad 3.97388800-1.32126500$

$\mathrm{H}-3.98715400 \quad 0.36948300 \quad-1.31624100$

$\mathrm{H}-4.32568100 \quad 2.09454500-1.11719300$

$\mathrm{H}-5.32116600 \quad 0.89426700 \quad-0.26266700$

C $2.98696700 \quad 4.18753600-0.77468100$

$\mathrm{H}-2.77270700 \quad 4.18832000 \quad-2.32958400$

$\mathrm{H}-3.26539900 \quad 4.03865000-0.62442500$

$\mathrm{H}-1.67913700 \quad 4.72647200 \quad-1.03562300$

$\mathrm{H} \quad 4.02338000 \quad 3.88161700-0.90693700$

H 2.91912900 4.86326300 0.08563600

H 2.65492700 4.72534600 -1.67025400

C $0.03007100 \quad 0.16552200 \quad 1.34908100$

$\mathrm{H} \quad 0.45216300 \quad-0.10440400 \quad 2.32223500$

$\mathrm{Ni} 0.72154100-1.15918500 \quad 0.04128400$

$\begin{array}{lllll}\text { Cl } 1.77518300 & -2.43119200 & -1.56188300\end{array}$

P 2.90564300 $-0.50466000 \quad 0.61187400$

P $-1.24011500-2.40350400-0.68128400$

C $-1.51605500-2.08134400-2.46088500$

$\mathrm{H}-0.57091800-2.19049400-2.98908200$

$\mathrm{H}-2.25443600-2.77514900 \quad-2.86587300$

$\mathrm{H}-1.87757000-1.05999600-2.59492300$

C $-2.97028200-2.43639800-0.07384000$

$\mathrm{H}-3.40122800-1.44054300-0.00207100$

$\mathrm{H}-3.56416000-3.05085400-0.75326200$

H $-2.98668200-2.87337700 \quad 0.92248900$

C $-0.89939800-4.19917300-0.64176400$

$\mathrm{H}-1.71838200-4.74521400-1.11276800$

$\mathrm{H} \quad 0.03592300-4.40324900-1.15599500$

$\mathrm{H}-0.81049600-4.51902700 \quad 0.39683700$

C $3.90260600 \quad 0.14262600-0.76542400$

H $4.92738500 \quad 0.32187300-0.43489400$

H $3.89040600 \quad-0.58271200-1.57656400$

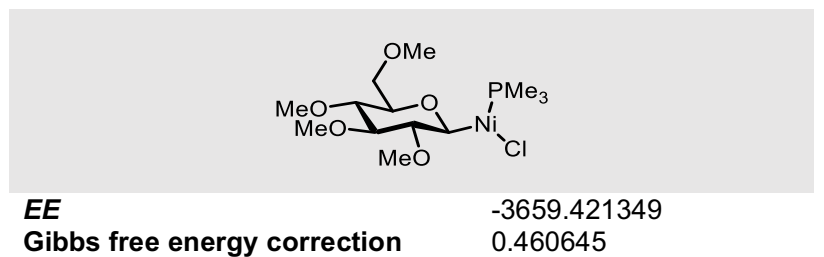

01

C $0.64724200-1.23958300-0.69156300$

o $1.11885500 \quad 0.81080000 \quad 0.41857800$

C $2.01952800-1.78178100-0.31937300$

C $2.39035700 \quad 0.41083200 \quad 0.87483600$

C $2.99486300-0.64073300-0.05802500$

○ $2.55825600-2.58471300-1.35152300$

$\mathrm{H} \quad 1.92781900-2.36832900 \quad 0.60531800$

C $3.26307500 \quad 1.64210900 \quad 0.93129200$

H 2.31196300 $-0.01913700 \quad 1.88354600$

○ $4.17913200-1.13090800 \quad 0.53314300$

$\mathrm{H} \quad 3.22179100-0.16555100-1.02413800$

C $2.45520500-3.97285700-1.12655500$

o $2.78324600 \quad 2.50506900 \quad 1.92987700$

H $3.23234800 \quad 2.14309800-0.04897500$

H $4.29928700 \quad 1.34682100 \quad 1.13877700$

C $5.25881700-1.30222500-0.35823100$

H $1.41368500-4.27839500-1.00332600$

$\mathrm{H} 3.02471400-4.26465800-0.23715600$

H $2.87825400 \quad-4.47101800 \quad-1.99750800$

C $3.510676003 .70315500 \quad 1.99276600$

H $6.09131900 \quad-1.69576800 \quad 0.22230400$

H $5.00619600-1.99937200-1.15950100$

H $5.55619700-0.34336600-0.79925100$

$\mathrm{H} \quad 3.08757100 \quad 4.30436200 \quad 2.79493600$

H $3.43832000 \quad 4.26012500 \quad 1.05035800$

H $4.56987300 \quad 3.51486500 \quad 2.20496900$

C $0.15849100-0.25118500 \quad 0.36933800$

$\mathrm{H} \quad 0.20340000 \quad-0.76130600 \quad 1.34276200$

$\mathrm{Ni}-1.66483300 \quad 0.37977700 \quad 0.01723000$

Cl $-3.82350300 \quad 0.96527900-0.42407900$

$\mathrm{P}-0.86601000 \quad 2.24396900-1.16399200$

P $-2.58258600-1.34331200 \quad 1.29868400$

C $-3.68767200-0.70253000 \quad 2.59977800$

$\mathrm{H}-4.45354500-0.08925200 \quad 2.12784400$

$\mathrm{H}-4.14735700-1.52169800 \quad 3.15477400$

$\mathrm{H}-3.11558800-0.07892800 \quad 3.28686800$

C $-3.66540100-2.47230700 \quad 0.36656700$

$\mathrm{H}-3.05358300-3.09231000-0.28724500$

$\mathrm{H}-4.22450800-3.11559600 \quad 1.04757400$

$\mathrm{H}-4.35171600-1.87787900-0.23500200$

C $-1.52513800-2.50604400 \quad 2.22771800$

$\mathrm{H}-2.13685800-3.25650100 \quad 2.73053000$

$\mathrm{H}-0.84038500-2.99387700 \quad 1.53399000$

$\mathrm{H}-0.94453900-1.96506200 \quad 2.97520500$

C $0.47098100 \quad 2.02727400 \quad-2.39077500$

$\mathrm{H} \quad 0.70795000 \quad 2.98300000-2.86114200$

$\mathrm{H} 1.354428001 .63182600-1.89471600$ 

H $3.45676200 \quad 1.07763900-1.10332000$
C $3.26336100 \quad 0.69294000 \quad 1.94066500$
H $2.69195900 \quad 0.44885100 \quad 2.83590300$
$\begin{array}{lllll}\mathrm{H} & 4.32854600 & 0.67329900 & 2.17679100\end{array}$
$\mathrm{H} 2.96727800 \quad 1.68321500 \quad 1.60180700$
C $3.85363600-1.968031001 .15517400$
$\mathrm{H} \quad 3.79830500 \quad-2.72903900 \quad 0.37890600$
$\mathrm{H} \quad 4.89446000-1.700879001 .34484600$
$\mathrm{H} \quad 3.41535300 \quad-2.36565300 \quad 2.07108900$
$\mathrm{H}-1.58413800 \quad 1.14497800 \quad 2.37172700$
$\begin{array}{llll}0 & -2.01991000 & -0.82743300 & 2.02507700\end{array}$
$\begin{array}{llll}C & -3.09680100 & -0.70345700 & 2.92542700\end{array}$
$\mathrm{H}-2.80593100-0.11118100 \quad 3.80015200$
$\mathrm{H}-3.96547000 \quad-0.23816200 \quad 2.45629700$
$\mathrm{H}-3.35302600-1.71007600 \quad 3.25505400$

$\mathrm{H} \quad 0.14873800 \quad 1.32584300 \quad-3.16146200$

C $-0.22451100 \quad 3.55785400 \quad-0.07504500$

$\mathrm{H} \quad 0.58722000 \quad 3.15746200 \quad 0.53155500$

$\mathrm{H} \quad 0.12283100 \quad 4.40740600 \quad-0.66599400$

$\mathrm{H}-1.02977100 \quad 3.88815000 \quad 0.58178800$

C $-2.11148700 \quad 3.13323500-2.15260200$

$\mathrm{H}-2.92027600 \quad 3.45656300-1.50028000$

$\mathrm{H}-1.65929200 \quad 3.99161700-2.65212000$

$\mathrm{H}-2.54134300 \quad 2.45611600 \quad-2.88989400$

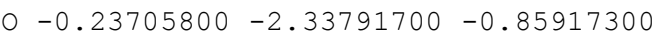

C $-1.05143200-2.23526000-2.00383800$

$\mathrm{H}-1.75001000-1.38982300-1.93406800$

$\mathrm{H}-0.44057000-2.12061100-2.90494300$

$\mathrm{H}-1.62529000-3.15724900-2.08503500$

H $0.75354300-0.71329700-1.64978100$ 


\section{PALLADIUM}

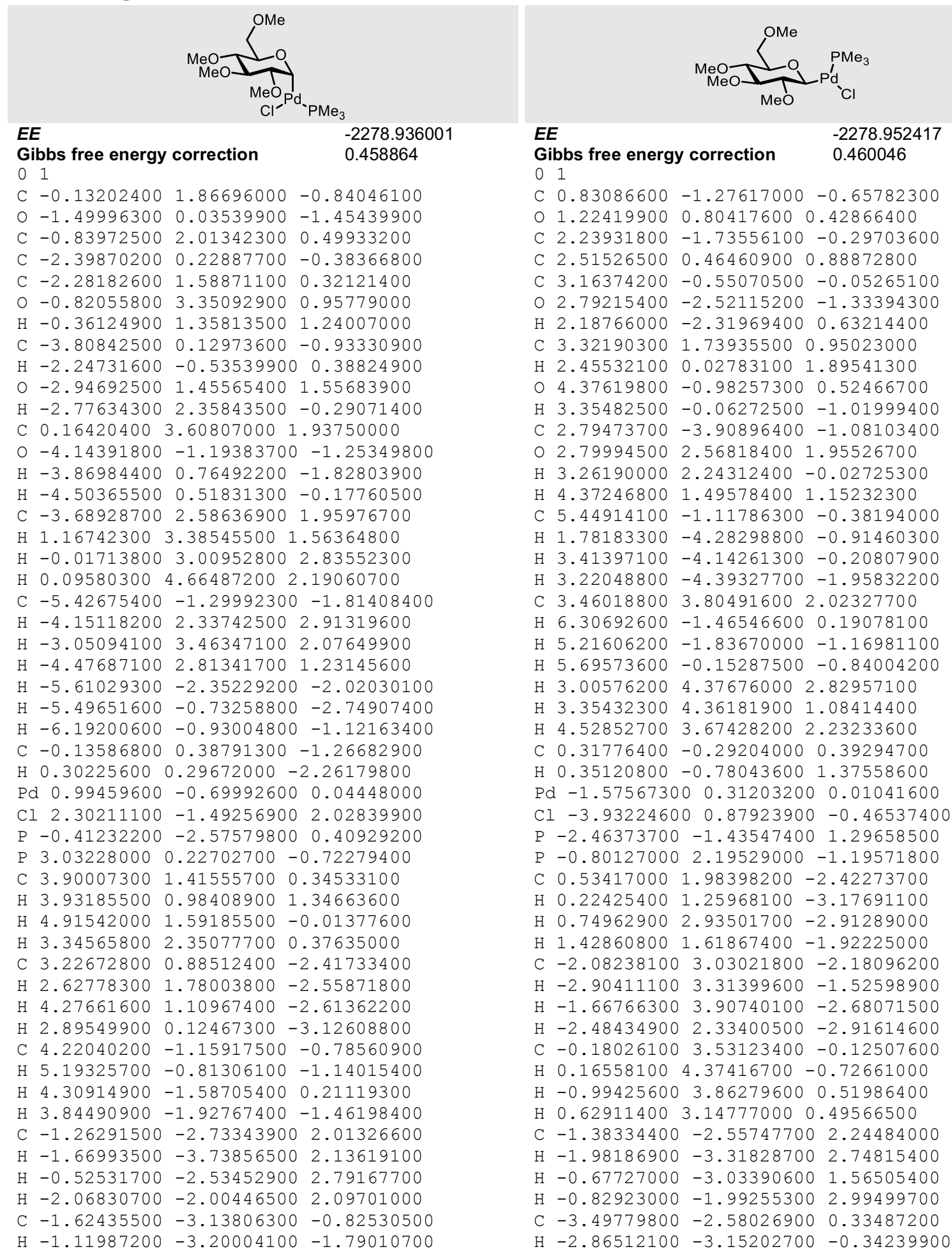


$\mathrm{H}-1.99494300-4.12751400 \quad-0.54969600$

$\mathrm{H}-2.45910200-2.45060700 \quad-0.92469700$

C $0.71440900-4.01230300 \quad 0.44123600$

$\mathrm{H} 1.43524300 \quad-3.88303300 \quad 1.24546300$

$\mathrm{H} \quad 0.14074800 \quad-4.93052500 \quad 0.58125300$

$\mathrm{H} \quad 1.25295900-4.06704100-0.50525700$

$\mathrm{H}-0.71247800 \quad 2.41695300-1.59683700$

$\begin{array}{llll}0 & 1.16765700 & 2.41307300 & -0.78788300\end{array}$

C $1.27147200 \quad 3.72915200-1.28647100$

$\mathrm{H} \quad 0.98470400 \quad 3.76951400-2.34345500$

$\mathrm{H} 0.64517600 \quad 4.42282400 \quad-0.72179800$

$\mathrm{H} \quad 2.31737100 \quad 4.02352600-1.19424200$
$\mathrm{H}-4.02979500-3.26553700 \quad 0.99679000$

$\mathrm{H}-4.20655200-1.98873300-0.24406000$

C $-3.60636800-0.81164400 \quad 2.56859000$

$\mathrm{H}-4.36539900-0.20768900 \quad 2.07323700$

$\mathrm{H}-4.06952400-1.63896400 \quad 3.10892900$

$\mathrm{H}-3.05901800-0.18169000 \quad 3.26949300$

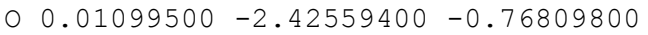

C $-0.71757200-2.49295800-1.97412400$

$\mathrm{H}-1.44954600-1.67841400-2.04399400$

$\mathrm{H}-0.04342400-2.45210500-2.83531000$

$\mathrm{H}-1.24354500-3.44658300-1.98544500$

$\mathrm{H} \quad 0.88658500-0.76592600-1.62749900$

\section{PLATINUM}

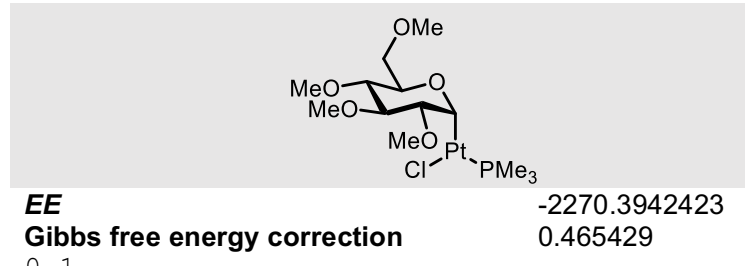

0

C $-1.65044300-0.71775600 \quad 1.38610400$

O $-0.87535700 \quad 1.48265900 \quad 1.09894700$

$\begin{array}{llll}\text { C } & -2.47646200 & -0.62023300 & 0.10409500\end{array}$

C $-1.571546001 .69703600-0.10406900$

C $-2.83946700 \quad 0.84020100 \quad-0.13276600$

$0-3.61554600-1.44236500 \quad 0.24346500$

$\mathrm{H}-1.87493700-0.95596000-0.74958300$

C $-1.91065100 \quad 3.16589600 \quad-0.18671500$

$\mathrm{H}-0.94178700 \quad 1.42529500-0.96433100$

$\begin{array}{lllll}0 & -3.49205800 & 0.98083400 & -1.38025900\end{array}$

$\mathrm{H}-3.50377200 \quad 1.16183500 \quad 0.68073700$

C $-4.22326700-1.87949100-0.95403500$

$\begin{array}{lllll}0 & -0.72376500 & 3.90869300 & -0.31625700\end{array}$

$\mathrm{H}-2.44714100 \quad 3.46583100 \quad 0.72514800$

$\mathrm{H}-2.56008800 \quad 3.33954300-1.05370000$

C $-4.778984001 .54503200-1.30424900$

$\mathrm{H}-3.47291900-2.10628400-1.71611800$

$\mathrm{H}-4.90994100-1.13595500-1.36139600$

$\mathrm{H}-4.77748800-2.78749700-0.71740600$

C $-0.95639800 \quad 5.29189200-0.38820700$

$\mathrm{H}-5.18115800 \quad 1.57233600-2.31551300$

$\mathrm{H}-5.43989200 \quad 0.94539500-0.66874500$

$\mathrm{H}-4.74907700 \quad 2.56538600-0.90580000$

$\mathrm{H} \quad 0.00938000 \quad 5.78244300-0.49596000$

$\mathrm{H}-1.44623700 \quad 5.65821000 \quad 0.52127900$

$\mathrm{H}-1.58452300 \quad 5.54307300-1.25057500$

C $-0.38969000 \quad 0.14311700 \quad 1.28199900$

$\mathrm{H} \quad 0.05256800 \quad 0.15376800 \quad 2.28187900$

Pt $1.11609200-0.31762400-0.03971900$

Cl 2.97231200 $-0.46634900-1.69235900$

P 2.17255300 1.63279100 0.53092100

$\mathrm{P} \quad 0.67734200-2.60727300-0.46701000$

C $-0.91676900-3.36170200-0.96115500$

$\mathrm{H}-1.19618200-2.96568500-1.93871200$

$\mathrm{H}-0.77417400-4.43958400-1.05550900$

$\mathrm{H}-1.70430000-3.15897200-0.24186800$

C $1.22411900-3.61004200 \quad 0.95453100$

$\mathrm{H} \quad 0.64465600 \quad-3.34944500 \quad 1.83524400$

H $1.12120300-4.67346800 \quad 0.73140100$

H 2.27491900 $-3.38402300 \quad 1.13742400$

C $1.71723000-3.31674200-1.78800400$

$\mathrm{H} 1.49900900-4.38271600-1.87375600$

H $1.51430000-2.81514800-2.73262300$

$\mathrm{H} \quad 2.76797800 \quad-3.16192500-1.55812400$

C $2.390421002 .82345400-0.82083900$

$\mathrm{H} 3.00853900 \quad 3.65808900-0.48446100$

$\mathrm{H} 2.86541800 \quad 2.31251100-1.65630600$

H $1.40890800 \quad 3.19177400-1.11715200$

C $1.59474400 \quad 2.67886300 \quad 1.90562700$

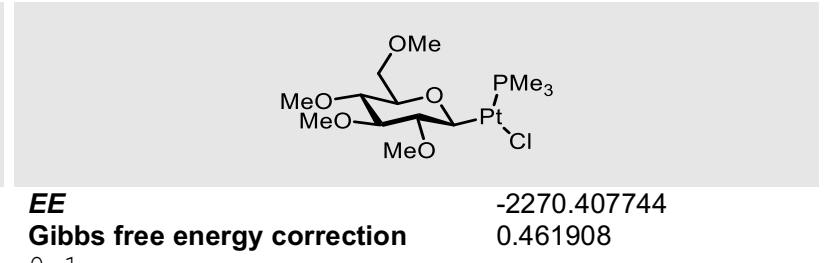

$0 \quad 1$

C $1.06130500-1.29500000-0.63459800$

$\begin{array}{lllll}0 & 1.38209000 & 0.82659400 & 0.38827800\end{array}$

C $2.48499300-1.69112800-0.26377800$

C $2.68046200 \quad 0.54464300 \quad 0.85848900$

C $3.36790100-0.46943800-0.05559000$

○ $3.06266700-2.48888200-1.27900500$

H 2.45655800 $-2.24942200 \quad 0.68224600$

C $3.44190700 \quad 1.84821900 \quad 0.89137600$

H $2.63462300 \quad 0.13070000 \quad 1.87579200$

$04.59616100 \quad-0.84143200 \quad 0.53064700$

H $3.54020400-0.00071400-1.03589200$

C $3.12162600-3.86605200-0.98095000$

$\begin{array}{lllll}0 & 2.88873200 & 2.68166400 & 1.87621600\end{array}$

H $3.36591000 \quad 2.32673500-0.09761300$

$\mathrm{H} 4.50002500 \quad 1.64743000 \quad 1.10068000$

C $5.67090200-0.96490900-0.37513500$

H 2.12512100 $-4.27411000-0.79544200$

$\mathrm{H} 3.75426700-4.04681100-0.10498600$

H $3.56129900-4.36219200-1.84476000$

C $3.50300300 \quad 3.94283100 \quad 1.91477300$

H $6.54190000-1.26598100 \quad 0.20404200$

H $5.46136400-1.71344600-1.14178000$

$\mathrm{H} 5.88259200-0.00532000-0.86129900$

$\mathrm{H} \quad 3.02680400 \quad 4.51696200 \quad 2.70682700$

$\mathrm{H} \quad 3.37827400 \quad 4.47286100 \quad 0.96245400$

$\mathrm{H} \quad 4.57516700 \quad 3.85678200 \quad 2.12749200$

C $0.49991500-0.29885500 \quad 0.38708500$

$\mathrm{H} \quad 0.57476700 \quad-0.75867400 \quad 1.38212600$

Pt $-1.42025800 \quad 0.23418900 \quad 0.00798500$

$\mathrm{Cl}-3.80252300 \quad 0.71883700-0.45794100$

P $-2.24029000-1.49492400 \quad 1.28087200$

$\mathrm{P}-0.75605100 \quad 2.09443800-1.18047200$

C $0.55603900 \quad 1.89363000-2.43073300$

H $0.24690800 \quad 1.14579000-3.16177700$

$\mathrm{H} \quad 0.72905300 \quad 2.84099200-2.94384900$

$\mathrm{H} \quad 1.47185700 \quad 1.56557400-1.94462600$

C $-2.07047500 \quad 2.88675500-2.15460900$

$\mathrm{H}-2.88272800 \quad 3.17806200-1.49173100$

$\mathrm{H}-1.67193700 \quad 3.75756100 \quad-2.67762600$

$\mathrm{H}-2.47654900 \quad 2.17183200 \quad-2.86897200$

C $-0.15952500 \quad 3.45570800-0.13247500$

$\mathrm{H} \quad 0.14488100 \quad 4.30251400 \quad-0.75052200$

$\mathrm{H}-0.97375000 \quad 3.76557800 \quad 0.52275000$

$\mathrm{H} \quad 0.67345100 \quad 3.10657400 \quad 0.47637700$

C $-1.10740700 \quad-2.54442400 \quad 2.24627900$

$\mathrm{H}-1.67754800-3.31780100 \quad 2.76293700$

$\mathrm{H}-0.38154200-3.00496000 \quad 1.57745600$

$\mathrm{H}-0.58220200-1.94077800 \quad 2.98666300$

C $-3.21077100-2.69962600 \quad 0.32987100$ 
$\mathrm{H} 1.33595900 \quad 2.06407600 \quad 2.76716200$

H $2.39767900 \quad 3.36443400 \quad 2.18158300$

$\mathrm{H} \quad 0.71371600 \quad 3.23164600 \quad 1.59190400$

C 3.870815001 .245673001 .06330700

$\mathrm{H} \quad 4.37074900 \quad 0.68500000 \quad 0.27689600$

$\mathrm{H} \quad 4.41222600 \quad 2.16885300 \quad 1.27634900$

$\mathrm{H} \quad 3.83028000 \quad 0.63638700 \quad 1.96645500$

$\mathrm{H}-2.26015100 \quad-0.29607900 \quad 2.19904800$

$\begin{array}{llll}0 & -1.29634000 & -2.03574900 & 1.72507400\end{array}$

$\begin{array}{llll}\text { C }-2.17435500 & -2.68414100 & 2.61972200\end{array}$

$\mathrm{H}-2.23953500 \quad-2.13351000 \quad 3.56424600$

$\mathrm{H}-3.17357100 \quad-2.78792800 \quad 2.19456400$

$\mathrm{H}-1.75476300 \quad-3.67077300 \quad 2.81599900$
$\mathrm{H}-2.54686900-3.23827700 \quad-0.34453500$

$\mathrm{H}-3.70274400-3.40860100 \quad 0.99743600$

$\mathrm{H}-3.95206900-2.15275700-0.25197300$

C $-3.40630700-0.90925200 \quad 2.54730700$

$\mathrm{H}-4.19954800-0.35272900 \quad 2.05093100$

$\mathrm{H}-3.82048400-1.75194300 \quad 3.10306000$

$\mathrm{H}-2.88543700-0.24186100 \quad 3.23346100$

$\begin{array}{llll}0 & 0.28727100 & -2.47949100 & -0.71380900\end{array}$

C $-0.38011200-2.64263500-1.94529300$

$\mathrm{H}-1.13073300-1.85675900-2.09343300$

H $0.33254200-2.63040300 \quad-2.77589100$

$\mathrm{H}-0.87552900-3.61266800-1.92307800$

$\mathrm{H} 1.09416500-0.80988000-1.61751000$ 
COPPER

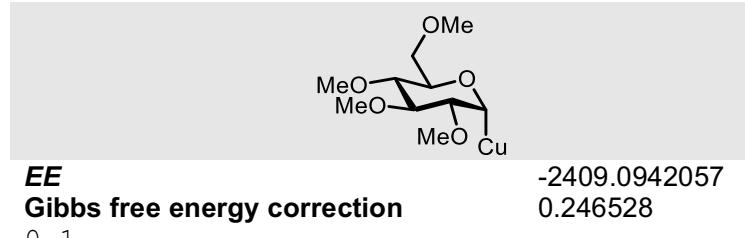

0

$\begin{array}{llll}C & -1.39754100 & -0.30267300 & 0.89267100\end{array}$

$\begin{array}{lllll}0 & 0.92316900 & -0.95281800 & 1.01403500\end{array}$

C $-1.00149100 \quad 0.85254200 \quad-0.01280700$

$\begin{array}{lllll}0 & -2.66681200 & -0.81065700 & 0.53080900\end{array}$

$\mathrm{H}-1.43120500 \quad 0.06621700 \quad 1.92958400$

C $1.35981000 \quad 0.01799900 \quad 0.09881700$

C $0.45467700 \quad 1.24873200 \quad 0.17902700$

$\begin{array}{lllll}0 & -1.83671300 & 1.97354200 & 0.22047200\end{array}$

$\mathrm{H}-1.12207800 \quad 0.51019600-1.04955400$

C $-3.75108600-0.27959700 \quad 1.26107300$

C $2.79243400 \quad 0.36567100 \quad 0.42329900$

$\mathrm{H} 1.31573600-0.38650600-0.92627300$

$\begin{array}{lllll}0 & 0.82880200 & 2.17476200 & -0.82214500\end{array}$

$\mathrm{H} \quad 0.56832500 \quad 1.69915700 \quad 1.17463600$

C $-2.43434300 \quad 2.50184400 \quad-0.94132700$

$\mathrm{H}-3.84307800 \quad 0.79927100 \quad 1.12006700$

$\mathrm{H}-3.64011400-0.48827100 \quad 2.33058600$

$\mathrm{H}-4.65011900-0.77458200 \quad 0.89729600$

$\begin{array}{lllll}0 & 3.62735600 & -0.68883500 & 0.02003800\end{array}$

$\mathrm{H} 2.87884700 \quad 0.53180500 \quad 1.50648600$

$\mathrm{H} \quad 3.06712400 \quad 1.29150600-0.09850000$

C $0.90057900 \quad 3.51203400-0.38242500$

$\mathrm{H}-3.09851800 \quad 1.76664900-1.40860600$

$\mathrm{H}-1.67956500 \quad 2.81237100-1.67054600$

$\mathrm{H}-3.02016700 \quad 3.36834100-0.63783800$

C $4.96851600-0.46414500 \quad 0.36097000$

H $1.16047000 \quad 4.12068500-1.24687600$

$\mathrm{H}-0.05516200 \quad 3.84846900 \quad 0.02861300$

$\mathrm{H} \quad 1.67614300 \quad 3.63214500 \quad 0.38282600$

$\mathrm{H} 5.54658300-1.31456000 \quad 0.00487700$

H $5.09274900-0.37605800 \quad 1.44687300$

H $5.35095200 \quad 0.45094700-0.10810200$

C $-0.38030300-1.43126100 \quad 0.77722200$

$\mathrm{H}-0.57656900-2.17963000 \quad 1.54490100$

$\mathrm{Cu}-0.67635400-2.38182700-0.99535300$

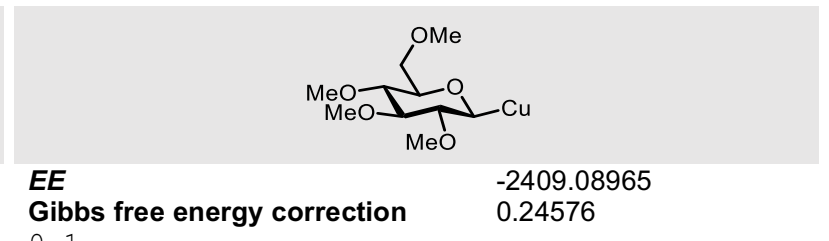

$0 \quad 1$

C $-1.37654400 \quad 0.23822900 \quad 0.15974800$

O $0.52063100-1.23760700 \quad 0.17157000$

C $-0.46194800 \quad 1.39156700-0.23213000$

o $-2.65566900 \quad 0.41639000-0.41415100$

$\mathrm{H}-1.44816300 \quad 0.21255900 \quad 1.25475000$

C $1.43195800-0.25391200-0.28078600$

C $0.97559800 \quad 1.12702600 \quad 0.19128800$

$\begin{array}{lllll}0 & -0.91301200 & 2.60351100 & 0.35016200\end{array}$

$\mathrm{H}-0.48461800 \quad 1.47352700-1.32628000$

C $-3.61365200 \quad 0.97155900 \quad 0.45683800$

C $2.79838400 \quad-0.59345600 \quad 0.26236000$

$\mathrm{H} \quad 1.47630700-0.25658400-1.37930900$

O $1.82348400 \quad 2.11441300-0.36357900$

$\mathrm{H} \quad 1.02776700 \quad 1.15538600 \quad 1.28864700$

C $-1.10718900 \quad 3.65043000-0.57294500$

$\mathrm{H}-3.30725500 \quad 1.95773400 \quad 0.81314000$

$\mathrm{H}-3.77902900 \quad 0.31883800 \quad 1.32272500$

$\mathrm{H}-4.54455000 \quad 1.05754800-0.10159300$

$03.31634700-1.69808100-0.43268200$

H $2.70314400 \quad-0.82377100 \quad 1.33323100$

H $3.45970900 \quad 0.27507600 \quad 0.14591000$

C $2.22406600 \quad 3.11688200 \quad 0.54227400$

$\mathrm{H}-1.87904800 \quad 3.38908400-1.30458100$

$\mathrm{H}-0.17961400 \quad 3.88727000-1.10391800$

$\mathrm{H}-1.43177200 \quad 4.52356000-0.00849600$

C $4.56366200-2.09958500 \quad 0.06464900$

$\mathrm{H} 2.83857600 \quad 3.82380200-0.01272300$

H $1.36226700 \quad 3.63676600 \quad 0.96925800$

$\mathrm{H} 2.82052400 \quad 2.69249300 \quad 1.35835900$

$\mathrm{H} \quad 4.90001400-2.94604300-0.53089000$

H $4.49265100-2.40627500 \quad 1.11525300$

H $5.30214300-1.29175900-0.01181600$

C $-0.78341400-1.06816900-0.35231200$

$\mathrm{Cu}-1.97345300-2.62534000 \quad 0.05878900$

$\mathrm{H}-0.73869100-1.01679700-1.45101800$ 
SILVER

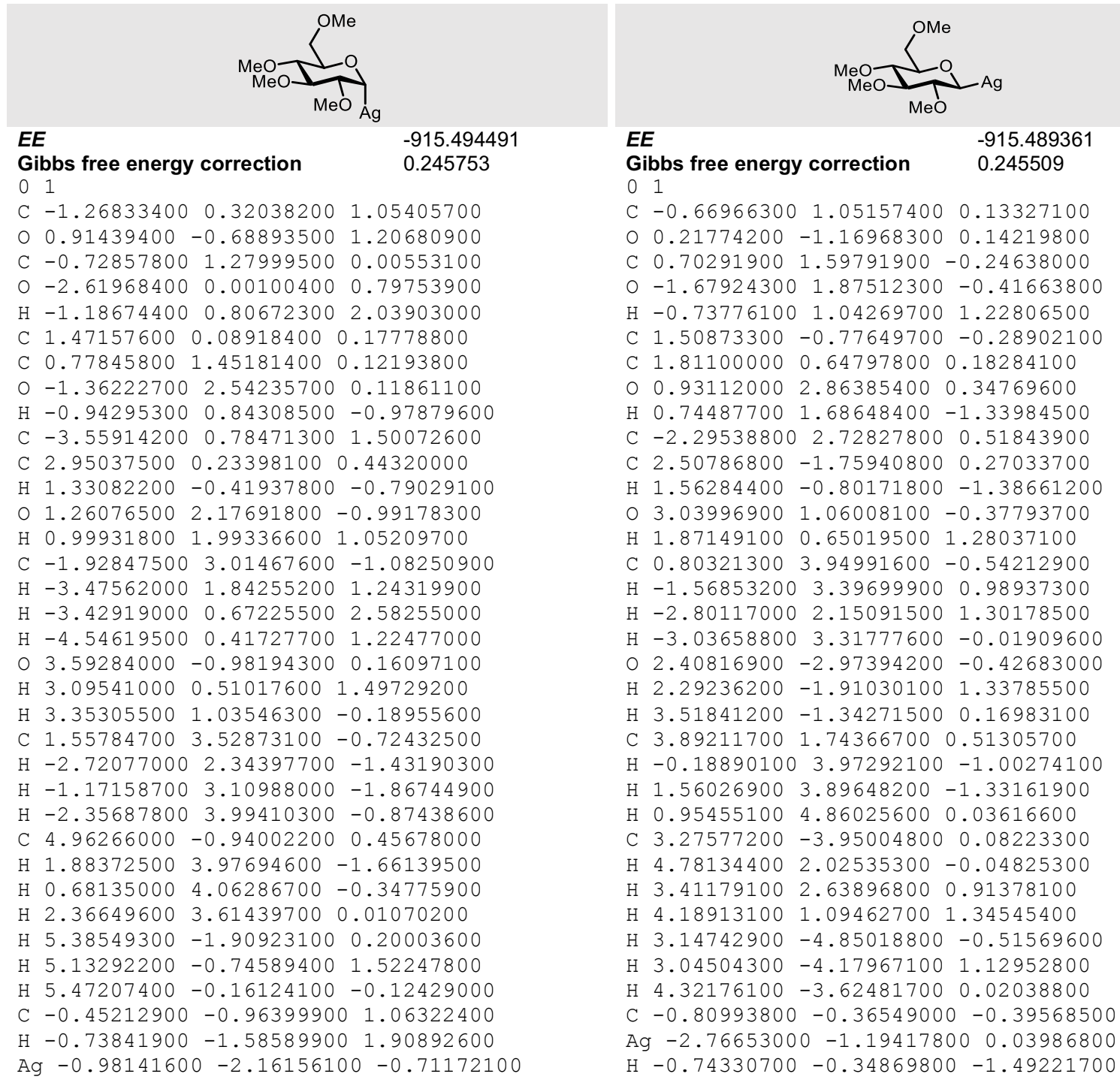


GOLD

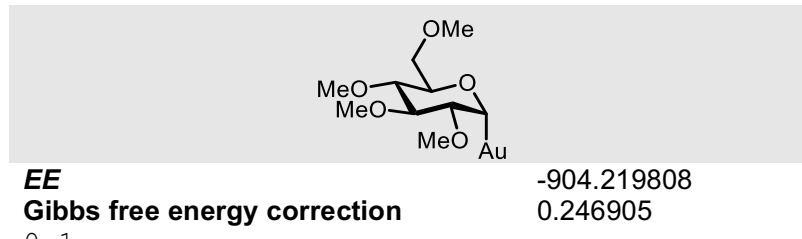

$0 \quad 1$

C $-0.83002000 \quad 1.01962100 \quad 1.06924600$

$\begin{array}{lllll}0 & 0.91775100 & -0.61138700 & 1.23731300\end{array}$

C $-0.07758500 \quad 1.71384900-0.05526800$

$\begin{array}{llllll}0 & -2.22208000 & 1.16665200 & 0.92692200\end{array}$

$\mathrm{H}-0.50478400 \quad 1.46730300 \quad 2.02059400$

C $1.68084600-0.05930900 \quad 0.18040900$

C $1.41574800 \quad 1.44212000 \quad 0.04334600$

o $-0.292685003 .11094000-0.01502400$

$\mathrm{H}-0.43167700 \quad 1.30548200-1.01033900$

C $-2.78808300 \quad 2.17633900 \quad 1.73432300$

C $3.13508800-0.33291400 \quad 0.47607200$

$\mathrm{H} 1.40579300-0.55334200-0.76214000$

o $2.06894300 \quad 1.90200000-1.11810700$

H $1.80863300 \quad 1.95591300 \quad 0.93240900$

C $-1.18342900 \quad 3.58388300-1.00245100$

$\mathrm{H}-2.35246000 \quad 3.15558300 \quad 1.51896400$

$\mathrm{H}-2.64680000 \quad 1.95250700 \quad 2.79702000$

$\mathrm{H}-3.85419500 \quad 2.19548000 \quad 1.51604900$

$\begin{array}{lllll}0 & 3.40098800 & -1.69463100 & 0.27230000\end{array}$

$\mathrm{H} 3.34512000-0.05039400 \quad 1.51776500$

$\mathrm{H} \quad 3.75494900 \quad 0.28417100-0.18699100$

C $2.72893800 \quad 3.14030900-0.97132800$

$\mathrm{H}-2.15785400 \quad 3.09241500-0.92728300$

$\mathrm{H}-0.77619700 \quad 3.41519100-2.00453900$

$\mathrm{H}-1.30392300 \quad 4.65387700-0.84068500$

C $4.72605500-2.03064600 \quad 0.58616100$

H $3.16644000 \quad 3.38371400-1.93770400$

$\mathrm{H} 2.03360000 \quad 3.92774700-0.67318900$

$\mathrm{H} 3.53012400 \quad 3.06925700-0.22642600$

$\mathrm{H} \quad 4.85099300-3.09358800 \quad 0.39065300$

$\mathrm{H} \quad 4.94644000-1.83179500 \quad 1.64171000$

$\mathrm{H} 5.43624600-1.46614900-0.03042700$

C $-0.45692300-0.45731000 \quad 1.13325500$

$\mathrm{H}-0.90154900-0.92845600 \quad 2.00849800$

$\mathrm{Au}-1.27134200-1.49492600-0.44726300$

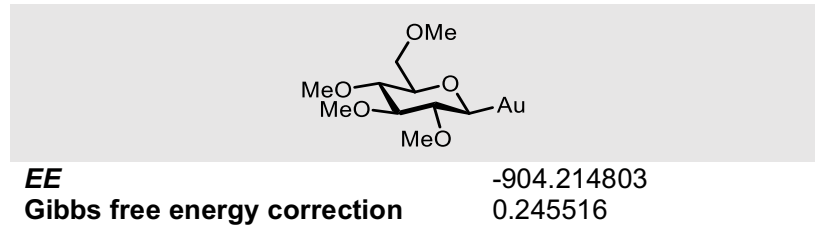

$\begin{array}{ll}0 & 1\end{array}$

C $0.01028800-1.29646700 \quad 0.11347800$

O $-0.39966000 \quad 1.06371700 \quad 0.14279800$

C $-1.45669000-1.53317100-0.23831100$

o $0.78096600-2.31478700-0.48108500$

$\mathrm{H} \quad 0.10193800-1.32083400 \quad 1.20481700$

C $-1.74731300 \quad 0.95229000-0.29171800$

C $-2.33811900-0.37131300 \quad 0.19481300$

o $-1.91961000-2.71500800 \quad 0.38044900$

$\mathrm{H}-1.53784000-1.62224400-1.32979400$

C $1.55171500-3.06324800 \quad 0.43033300$

C $-2.50639500 \quad 2.13437100 \quad 0.25783500$

$\mathrm{H}-1.79180100 \quad 0.98083000-1.38924300$

$0-3.62884000-0.51677700-0.35476600$

$\mathrm{H}-2.38617200-0.35389700 \quad 1.29260000$

C $-2.03700900-3.82229600-0.48720300$

H $0.90930000 \quad-3.60207300 \quad 1.13432600$

H 2.23768200 $-2.41774600 \quad 0.98978100$

$\mathrm{H} 2.13280000-3.77819200-0.14917300$

o $-2.14667300 \quad 3.28681600-0.45618500$

$\mathrm{H}-2.25873700 \quad 2.24634300 \quad 1.32297800$

$\mathrm{H}-3.58300000 \quad 1.94297500 \quad 0.16379900$

C $-4.59485100-1.02796200 \quad 0.53772100$

$\mathrm{H}-1.07805700-4.06467400-0.95154600$

$\mathrm{H}-2.77621300-3.62363200-1.27040500$

$\mathrm{H}-2.37320900-4.66505900 \quad 0.11444300$

C $-2.76492800 \quad 4.44012400 \quad 0.04892700$

$\mathrm{H}-5.52802000-1.10948300-0.01651900$

$\mathrm{H}-4.30474200-2.01009700 \quad 0.91665000$

$\mathrm{H}-4.74251000-0.34709000 \quad 1.38403800$

$\mathrm{H}-2.44473600 \quad 5.28024000-0.56378700$

$\mathrm{H}-2.47257100 \quad 4.62317300 \quad 1.08965200$

$\mathrm{H}-3.85780000 \quad 4.35816200 \quad 0.00310100$

C $0.42774000 \quad 0.07399400-0.40377400$

$\mathrm{H} \quad 0.38039300 \quad 0.08486200-1.50067700$

Au 2.36224300 $0.50694700 \quad 0.03459100$ 
ZINC

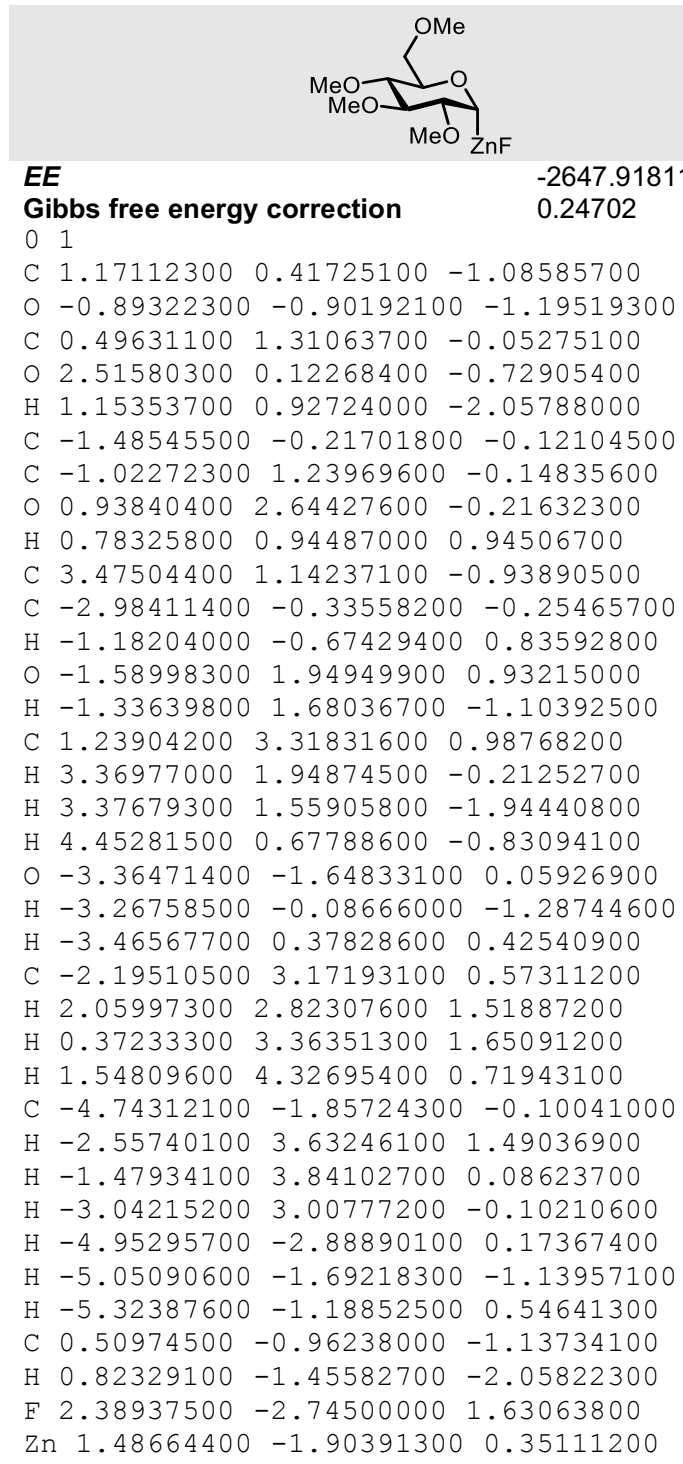

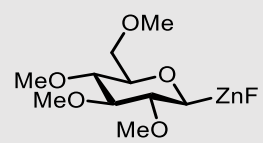

$E E$ $-2647.915085$

Gibbs free energy correction $\quad 0.247318$

C $-0.81470300 \quad 0.94065800 \quad 0.17527100$

$00.12880500-1.28510500 \quad 0.13830100$

C $0.52588600 \quad 1.53223600-0.22971400$

$\begin{array}{lllll}0 & -1.93798500 & 1.61470700 & -0.34828500\end{array}$

$\mathrm{H}-0.86271300 \quad 0.93086100 \quad 1.27414200$

$\begin{array}{llll}\text { C } 1.40293500 & -0.84518400 & -0.29846000\end{array}$

C $1.65172300 \quad 0.58533400 \quad 0.18464900$

$\begin{array}{llllll}0 & 0.72799800 & 2.79033400 & 0.38751600\end{array}$

$\mathrm{H} \quad 0.53922300 \quad 1.63984500 \quad-1.32258700$

C $-2.23143500 \quad 2.88049000 \quad 0.21147000$

C $2.43935900-1.79748100 \quad 0.24543900$

$\mathrm{H} 1.44779200-0.86404200-1.39652800$

O $2.87479100 \quad 1.05272200-0.34594400$

H $1.69319500 \quad 0.57630500 \quad 1.28250500$

$\begin{array}{llll}\text { C } 1.23987100 & 3.78622000 & -0.47066200\end{array}$

$\mathrm{H}-1.58199700 \quad 3.65836300 \quad-0.19174100$

$\mathrm{H}-2.11677600 \quad 2.86127000 \quad 1.29889100$

$\mathrm{H}-3.26625700 \quad 3.10360600-0.04149400$

$\begin{array}{llll}0 & 2.35675900 & -3.01270600 & -0.44962500\end{array}$

H $2.24994700-1.95158600 \quad 1.31756100$

H $3.43580000-1.35293000 \quad 0.12454600$

C $3.739416001 .63516600 \quad 0.60406700$

$\mathrm{H} 0.53840300 \quad 4.00100200-1.28500300$

H $2.19796200 \quad 3.48692800-0.90370000$

H $1.37492600 \quad 4.68627300 \quad 0.12674900$

$\begin{array}{llll}\text { C } 3.25555500 & -3.97061800 & 0.04385000\end{array}$

$\mathrm{H} 4.62137400 \quad 1.97900100 \quad 0.06670000$

$\mathrm{H} 3.26397500 \quad 2.48177300 \quad 1.10755900$

H $4.04566700 \quad 0.90179000 \quad 1.35848300$

H $3.13637400-4.87201800-0.55351300$

H $3.04679400 \quad-4.20597900 \quad 1.09406400$

$\mathrm{H} \quad 4.29215900-3.62122400-0.03612900$

C $-0.91030300-0.48161400-0.37432900$

$\mathrm{H}-0.81273000-0.40889200-1.46892600$

$\mathrm{Zn}-2.75118900-1.10866500-0.01139900$

F $-4.41113500-1.62680400 \quad 0.32057700$ 
CADMIUM

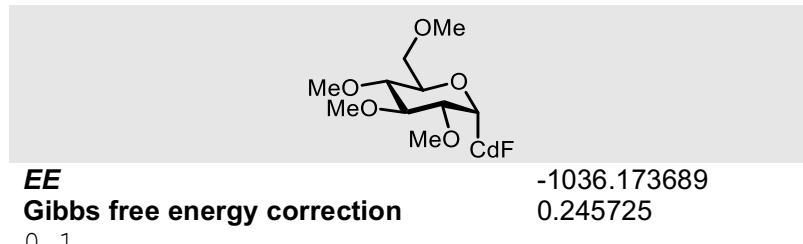

01

C $-0.74186800 \quad 1.00648700 \quad 1.14315600$

$\begin{array}{lllll}0 & 0.84316200 & -0.83465600 & 1.29767800\end{array}$

C $0.09914200 \quad 1.58832600 \quad 0.01647800$

$\begin{array}{llllll}0 & -2.12473800 & 1.17704100 & 0.88960900\end{array}$

$\mathrm{H}-0.47143400 \quad 1.51087100 \quad 2.08097400$

C $1.58359200-0.41819200 \quad 0.17669100$

C $1.542412001 .10696500 \quad 0.07798800$

$\begin{array}{lllll}0 & 0.05133500 & 3.00047000 & 0.05773000\end{array}$

$\mathrm{H}-0.32737900 \quad 1.23310400-0.93319900$

C $-2.69966400 \quad 2.37471700 \quad 1.37745900$

C $2.99262500-0.93757500 \quad 0.32530000$

$\mathrm{H} \quad 1.14977400-0.84644500-0.74305100$

o $2.22575200 \quad 1.52500300-1.08384000$

$\mathrm{H} \quad 2.01845200 \quad 1.52537800 \quad 0.97501500$

C $-0.21513400 \quad 3.61207000-1.18729200$

$\mathrm{H}-2.26779300 \quad 3.25212400 \quad 0.89494200$

$\mathrm{H}-2.55616200 \quad 2.45637300 \quad 2.45904500$

$\mathrm{H}-3.76464600 \quad 2.32057000 \quad 1.16183200$

$\begin{array}{llll}0 & 2.98890300 & -2.32323900 & 0.10927700\end{array}$

$\mathrm{H} 3.35123800-0.70423700 \quad 1.33797100$

$\mathrm{H} 3.64085000-0.43465100-0.40326000$

C $3.105701002 .61026400-0.88606200$

$\mathrm{H}-1.20672500 \quad 3.32836200-1.55620100$

$\mathrm{H} \quad 0.53329800 \quad 3.33569300-1.93476600$

$\mathrm{H}-0.18913500 \quad 4.68849600-1.02721500$

C $4.25833600-2.89503800 \quad 0.28737600$

H $3.53567000 \quad 2.85422300-1.85564500$

H 2.57853200 3.48209900 -0.48889700

$\mathrm{H} \quad 3.91256600 \quad 2.33908300-0.19626300$

$\mathrm{H} \quad 4.16918700-3.96094400 \quad 0.08920700$

$\mathrm{H} \quad 4.61784700-2.74915000 \quad 1.31264500$

$\mathrm{H} \quad 4.98977900-2.46081900-0.40476000$

C $-0.51782700-0.49929100 \quad 1.25847300$

$\mathrm{H}-0.94216000-0.84755600 \quad 2.19959900$

F $-2.95892900-2.17390900-1.64190700$

$\mathrm{Cd}-1.75959600-1.38347400-0.28941400$

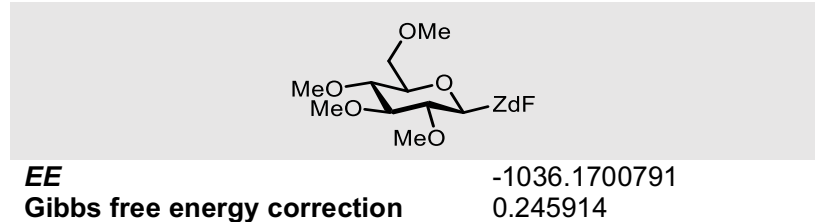

Gibbs
0

C $0.25216100-1.18453400 \quad 0.15662400$

O $-0.20815300 \quad 1.18684700 \quad 0.12437500$

C $-1.19234300-1.47499900-0.22873800$

O $1.18517000-2.09237400-0.38269600$

$\mathrm{H} \quad 0.31626800-1.18559000 \quad 1.25454500$

C $-1.55095000 \quad 1.02830000-0.30311300$

C $-2.09115200-0.31362100 \quad 0.19194800$

$\begin{array}{lllll}0 & -1.64679200 & -2.66126800 & 0.39587200\end{array}$

$\mathrm{H}-1.24068100-1.57977100-1.32089800$

C $1.21347700-3.38376000 \quad 0.19389200$

C $-2.35466100 \quad 2.18324600 \quad 0.24133200$

$\mathrm{H}-1.59768000 \quad 1.05114700-1.40091700$

$0-3.39019400-0.51555800-0.32488300$

$\mathrm{H}-2.11842800-0.29105400 \quad 1.29001800$

C $-2.36074800-3.53203700-0.45415600$

$\mathrm{H} \quad 0.40473700-4.01151600-0.18193700$

$\mathrm{H} \quad 1.13031100 \quad-3.32644700 \quad 1.28296700$

$\mathrm{H} \quad 2.17094400-3.82668900-0.07445700$

o $-2.01490500 \quad 3.34959500-0.45911200$

$\mathrm{H}-2.13119300 \quad 2.29607700 \quad 1.31202100$

$\mathrm{H}-3.42398600 \quad 1.96315800 \quad 0.12649200$

C $-4.34762400-0.90389600 \quad 0.63542300$

$\mathrm{H}-1.72519600-3.89076200-1.27188200$

$\mathrm{H}-3.23877300-3.04140200-0.88242800$

$\mathrm{H}-2.67577600-4.38214600 \quad 0.14858600$

C $-2.68538100 \quad 4.48044700 \quad 0.03188500$

H $-5.28712000-1.05733300 \quad 0.10761400$

$\mathrm{H}-4.05383800-1.83014400 \quad 1.13740600$

$\mathrm{H}-4.48686100-0.12159000 \quad 1.38996300$

$\mathrm{H}-2.37604600 \quad 5.33283700-0.56915000$

$\mathrm{H}-2.42866800 \quad 4.66898900 \quad 1.08088100$

$\mathrm{H}-3.77298200 \quad 4.36212400-0.04550600$

C $0.63488800 \quad 0.18570300-0.39608800$

$\mathrm{H} \quad 0.52773600 \quad 0.14172200-1.48971200$

F $4.62057400 \quad 0.78364700 \quad 0.35338800$

Cd 2.70656400 $0.52142800-0.00659600$ 
MERCURY

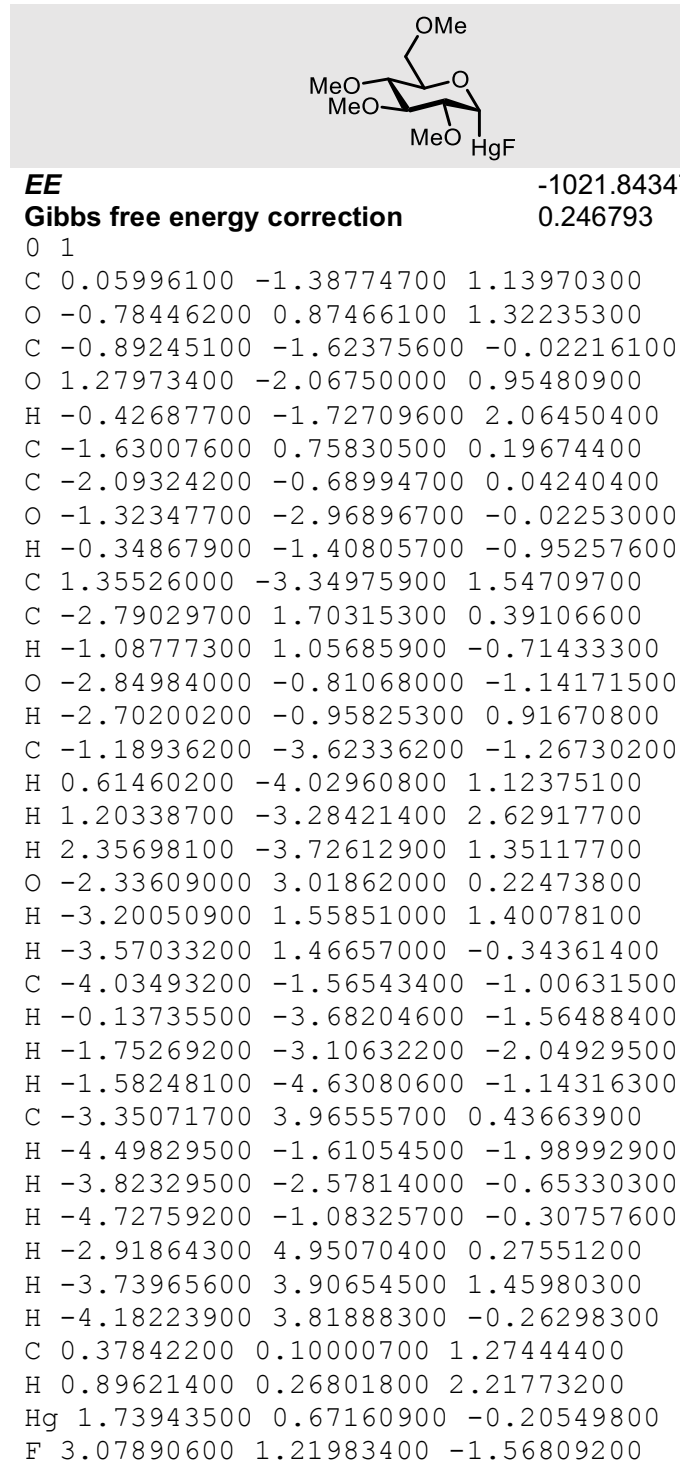

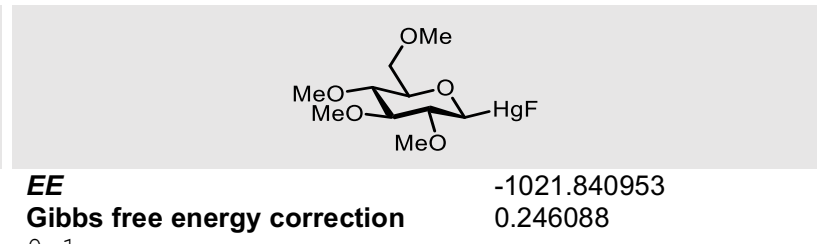

$\begin{array}{ll}0 & 1\end{array}$

C $-0.27741700-1.30886700 \quad 0.11021700$

$\begin{array}{lllll}0 & -0.47656700 & 1.09357300 & 0.10648300\end{array}$

C $-1.75732600-1.41628800-0.23047800$

$\begin{array}{lllll}0 & 0.45912900 & -2.34749300 & -0.49027800\end{array}$

$\mathrm{H}-0.16720900-1.33480900 \quad 1.20207500$

C $-1.838421001 .09115400-0.29524700$

C $-2.52161300-0.17672400 \quad 0.21775800$

$\begin{array}{lllll}0 & -2.30899900 & -2.56698500 & 0.37664000\end{array}$

$\mathrm{H}-1.84195500-1.49103300-1.32258400$

$\begin{array}{llll}\text { C } 0.71843400 & -3.45397100 & 0.35127900\end{array}$

C $-2.48471000 \quad 2.33727400 \quad 0.25658900$

$\mathrm{H}-1.90319400 \quad 1.11432700-1.39196100$

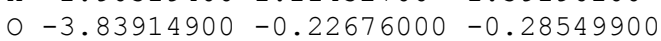

$\mathrm{H}-2.53147900-0.14990300 \quad 1.31610900$

C $-3.01265500-3.41165600-0.50918200$

$\mathrm{H}-0.20833000 \quad-3.91713000 \quad 0.69421600$

$\mathrm{H} \quad 1.30951400 \quad-3.14863800 \quad 1.22179800$

$\mathrm{H} 1.29429300-4.16800700-0.23350900$

$\begin{array}{llll}0 & -2.02697100 & 3.44829400 & -0.46546300\end{array}$

$\mathrm{H}-2.22143100 \quad 2.43063000 \quad 1.31996200$

$\mathrm{H}-3.57482900 \quad 2.24246800 \quad 0.17093400$

C $-4.81599000 \quad-0.59491800 \quad 0.66434500$

$\mathrm{H}-2.34573700-3.80913800-1.28101500$

$\mathrm{H}-3.83881600-2.87967200-0.99014300$

$\mathrm{H}-3.40849400 \quad-4.23656700 \quad 0.08068800$

C $-2.53996100 \quad 4.65648100 \quad 0.03243400$

$\mathrm{H}-5.77119000-0.62687500 \quad 0.14381300$

$\mathrm{H}-4.60066400-1.57536300 \quad 1.09704300$

$\mathrm{H}-4.87462800 \quad 0.14395800 \quad 1.47132600$

$\mathrm{H}-2.14796200 \quad 5.45986000-0.58745800$

$\mathrm{H}-2.22961400 \quad 4.82018400 \quad 1.07091900$

H $-3.63563000 \quad 4.67041100-0.01141100$

C $0.25005500 \quad 0.01634900-0.43216100$

$\mathrm{H} \quad 0.15260600 \quad 0.00046600-1.52625500$

F $4.17708700 \quad 0.47618300 \quad 0.39494400$

Hg $2.25211300 \quad 0.25663900 \quad-0.01159300$ 


\section{NICKEL}

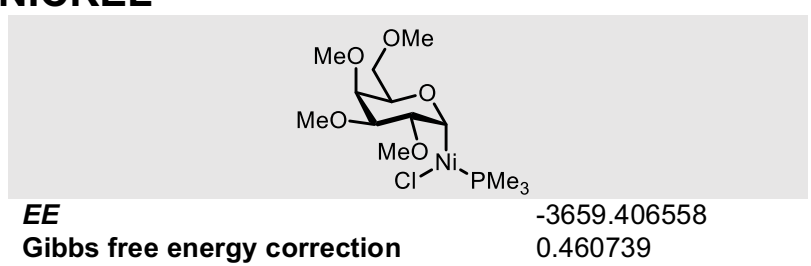

01

C $0.07782900 \quad 1.83930100-0.81965500$

$\begin{array}{llllll}0 & -1.52837500 & 0.11837000 & -1.10140300\end{array}$

C $-0.46655800 \quad 2.15876300 \quad 0.56709500$

$\mathrm{H}-0.51010900 \quad 2.40810400-1.55092800$

C $-2.16736200 \quad 0.39130500 \quad 0.12348600$

C $-1.95537200 \quad 1.83307100 \quad 0.58873400$

$\begin{array}{llll}0 & -0.28873400 & 3.51589100 & 0.92484600\end{array}$

$\mathrm{H} \quad 0.04391600 \quad 1.51553900 \quad 1.30136300$

C $-3.64586800 \quad 0.12201900-0.05526600$

$\mathrm{H}-1.77677700 \quad-0.27209100 \quad 0.91287600$

C $0.84315800 \quad 3.76934400 \quad 1.72556800$

O $-3.87129700-1.26849900-0.06050300$

$\mathrm{H}-3.97240800 \quad 0.57134600 \quad-0.99838100$

$\mathrm{H}-4.21162900 \quad 0.58932200 \quad 0.76274900$

H $1.77193800 \quad 3.55279800 \quad 1.19162000$

$\mathrm{H} \quad 0.81903500 \quad 3.16970500 \quad 2.64150600$

H $0.81797300 \quad 4.82489400 \quad 1.99171300$

C $-5.20671300-1.59547500-0.34649900$

$\mathrm{H}-5.29301000-2.68042000-0.32258900$

$\mathrm{H}-5.49805900-1.23205900-1.33821700$

$\mathrm{H}-5.88746900-1.16521800 \quad 0.39731200$

C $-0.10302900 \quad 0.33765800-1.10367300$

$\begin{array}{lllll}0 & 1.43857200 & 2.22060500 & -0.91958400\end{array}$

C $1.64621900 \quad 3.46694200-1.54503200$

$\mathrm{H} 1.30920800 \quad 3.44183100-2.58694700$

H $1.11979300 \quad 4.27030400-1.02381200$

$\mathrm{H} 2.71926100 \quad 3.66344600-1.52514500$

$\begin{array}{llllll}0 & -2.68319100 & 2.69075300 & -0.26550100\end{array}$

H $-2.33103000 \quad 1.92791700 \quad 1.61598200$

C $-3.24699400 \quad 3.82110800 \quad 0.36265700$

$\mathrm{H}-3.83063300 \quad 4.34307700 \quad-0.39377200$

$\mathrm{H}-3.91513200 \quad 3.51811900 \quad 1.17772500$

$\mathrm{H}-2.47844500 \quad 4.48608100 \quad 0.75787700$

$\mathrm{H} \quad 0.19865200 \quad 0.15286800 \quad-2.13922300$

$\mathrm{Ni} 0.84416300-0.83301700 \quad 0.15916000$

Cl $1.59295100-1.75156100 \quad 2.12102600$

P $-0.51553000-2.72751000-0.15873200$

P $3.05437800-0.14125000-0.32744600$

C $-1.53902800-2.94325000-1.65035500$

$\mathrm{H}-2.38795300-2.26638400-1.59736900$

$\mathrm{H}-0.94373900-2.68992100-2.52813900$

$\mathrm{H}-1.88111400-3.97677900-1.73081500$

C $0.67638400-4.10595600-0.32003000$

$\mathrm{H} \quad 0.15429500-5.05607500-0.44637000$

$\mathrm{H} \quad 1.31569100-3.93516000-1.18726300$

H $1.29801800 \quad-4.13572200 \quad 0.57398000$

C $-1.57729400-3.30478400 \quad 1.20197600$

$\mathrm{H}-1.90775300-4.32899900 \quad 1.01982300$

$\mathrm{H}-0.99635300-3.26042500 \quad 2.12324200$

$\mathrm{H}-2.44698700-2.65409600 \quad 1.27958000$

C $4.13155600-1.59970700-0.07857700$

H $3.77524100-2.42642400-0.69443100$

$\mathrm{H} 5.16191000-1.36688700-0.35361100$

H $4.07689100 \quad-1.90655700 \quad 0.96378800$

C $3.874589001 .09290700 \quad 0.73107200$

$\mathrm{H} \quad 3.44474800 \quad 2.07310000 \quad 0.53802200$

H $3.69225100 \quad 0.81803100 \quad 1.77066000$

H $4.94919300 \quad 1.11282400 \quad 0.54287300$

C $3.54767800 \quad 0.32111700 \quad-2.02630300$

H $4.62795700 \quad 0.46592500-2.08290600$

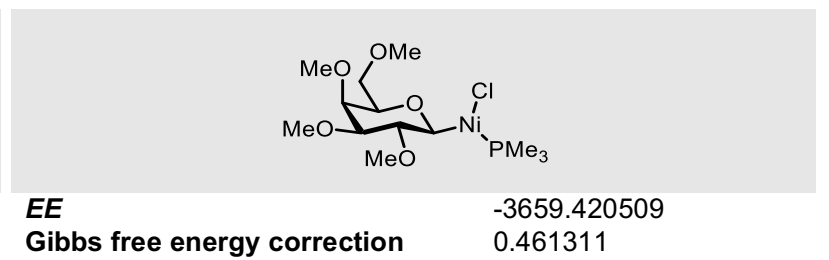

0

C $-0.55481500 \quad 1.42230700-0.46143300$

O $-1.21495800-0.59271700 \quad 0.65042700$

C $-1.79045600 \quad 2.14039000 \quad 0.06633000$

$\mathrm{H}-0.83164100 \quad 0.97139800-1.42156900$

C $-2.37978500-0.01788900 \quad 1.19444000$

C $-2.89967100 \quad 1.11384100 \quad 0.30823100$

o $-2.27537200 \quad 3.11926900-0.82886300$

$\mathrm{H}-1.53437300 \quad 2.60781500 \quad 1.02890700$

C $-3.40355000-1.12089000 \quad 1.34164300$

$\mathrm{H}-2.15572700 \quad 0.39233800 \quad 2.19084400$

C $-1.81514800 \quad 4.42883200-0.57766500$

o $-2.99663600-1.98232400 \quad 2.37436100$

$\mathrm{H}-3.47936700-1.66326600 \quad 0.39148800$

$\mathrm{H}-4.39042300-0.69039300 \quad 1.56719300$

$\mathrm{H}-0.72824600 \quad 4.49101700-0.65032900$

$\mathrm{H}-2.12733400 \quad 4.76712800 \quad 0.41711300$

$\mathrm{H}-2.26964400 \quad 5.07550500-1.32636800$

C $-3.82599000-3.10843500 \quad 2.48517000$

$\mathrm{H}-3.44925700-3.71622800 \quad 3.30533900$

$\mathrm{H}-3.81327600-3.70147900 \quad 1.56273700$

$\mathrm{H}-4.86263500-2.82049300 \quad 2.69872000$

C $-0.12436900 \quad 0.32369200 \quad 0.51614400$

$\mathrm{H} \quad 0.01235000 \quad 0.79764900 \quad 1.50054600$

$\begin{array}{llllll}0 & 0.47989800 & 2.37518300 & -0.66399000\end{array}$

C $1.14706700 \quad 2.22535800-1.89558600$

H $1.70037900 \quad 1.27748400-1.94692300$

$\mathrm{H} \quad 0.43861000 \quad 2.27001300-2.72883800$

H $1.85388100 \quad 3.04850000-1.99145700$

$\begin{array}{llllll}0 & -3.34273700 & 0.54337500 & -0.90755100\end{array}$

$\mathrm{H}-3.74044100 \quad 1.61049900 \quad 0.80997600$

C $-4.471039001 .16186800-1.48863700$

$\mathrm{H}-4.73081500 \quad 0.57766000-2.36986800$

$\mathrm{H}-5.31859500 \quad 1.14888900-0.79353700$

$\mathrm{H}-4.25740100 \quad 2.19072700-1.77820100$

$\mathrm{Ni} 1.53215500-0.56036900-0.05275000$

P 2.84141100 0.91742300 1.19564200

P $0.32354100-2.19124500-1.22949300$

C $3.99650800 \quad 1.91138200 \quad 0.19827700$

H $4.51721800 \quad 1.24898600-0.49191500$

H $3.43140100 \quad 2.65149700-0.36674700$

$\mathrm{H} \quad 4.71466300 \quad 2.42404100 \quad 0.83992200$

C $3.95078800 \quad 0.04064000 \quad 2.34687100$

H $4.59198700 \quad 0.74473300 \quad 2.87928900$

$\mathrm{H} \quad 3.35567100-0.52025400 \quad 3.06764700$

$\mathrm{H} \quad 4.55428100-0.66046300 \quad 1.77274900$

C $2.08600100 \quad 2.18117600 \quad 2.27620600$

H $1.42579200 \quad 2.80923500 \quad 1.67792100$

$\mathrm{H} 1.50043900 \quad 1.69823200 \quad 3.05880000$

$\mathrm{H} 2.85855200 \quad 2.79573600 \quad 2.74066300$

C $1.30531000-3.20087900-2.38647400$

H 2.10931200 $-3.69197800-1.84195900$

$\mathrm{H} \quad 0.67064200 \quad-3.94102000-2.87677800$

$\mathrm{H} 1.76605600-2.55387100 \quad-3.13212100$

C $-0.39543100-3.46018100-0.13412300$

$\mathrm{H} \quad 0.41448300 \quad-3.95489800 \quad 0.40279100$

$\mathrm{H}-1.05720800-2.97931400 \quad 0.58483200$

$\mathrm{H}-0.94140100-4.20330000-0.71833200$

C $-1.07650900-1.69218000-2.28801900$

$\mathrm{H}-1.82417800-1.15892900-1.70323500$

$\mathrm{H}-0.71012100-1.03408400-3.07772700$ 
$\mathrm{H} 3.26844700-0.48876600-2.70174800$

H $3.03377000 \quad 1.22602600-2.33628900$
$\mathrm{H}-1.52412300-2.57444200-2.74944100$

Cl $3.51534400-1.45491100-0.73827500$ 
PALLADIUM

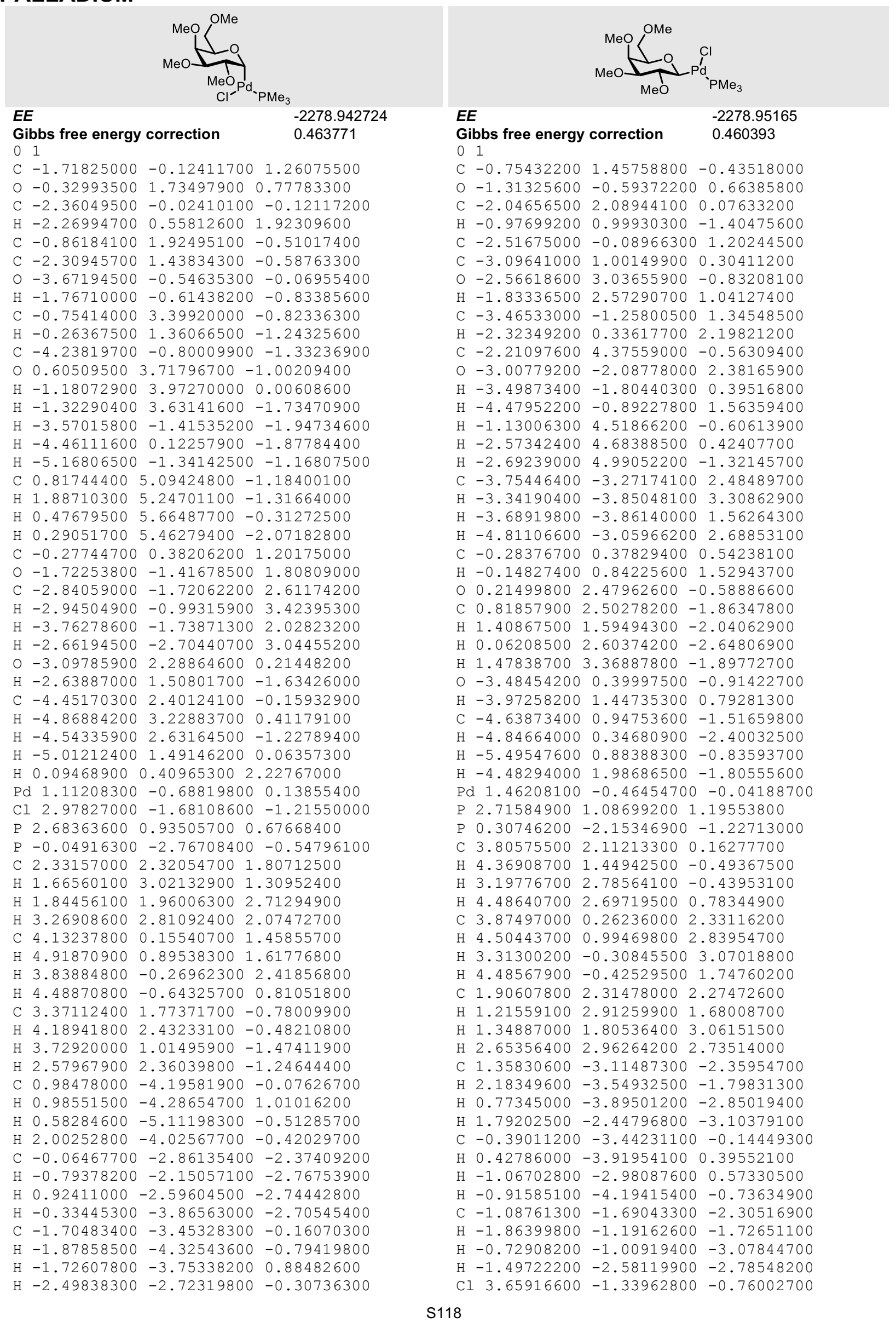




\section{PLATINUM}

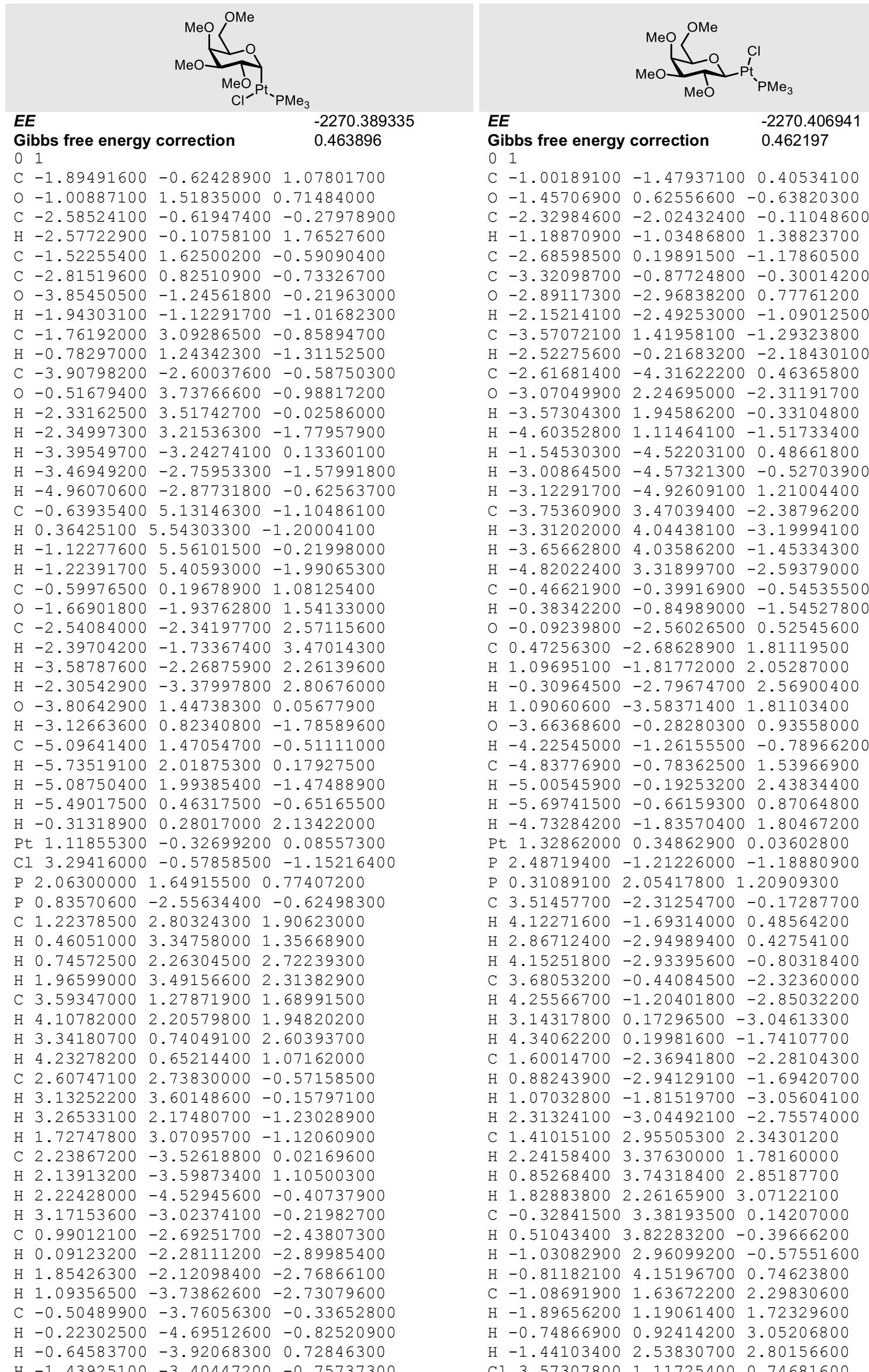

$\mathrm{H}-1.43925100 \quad-3.40447200-0.75737300$ 
COPPER

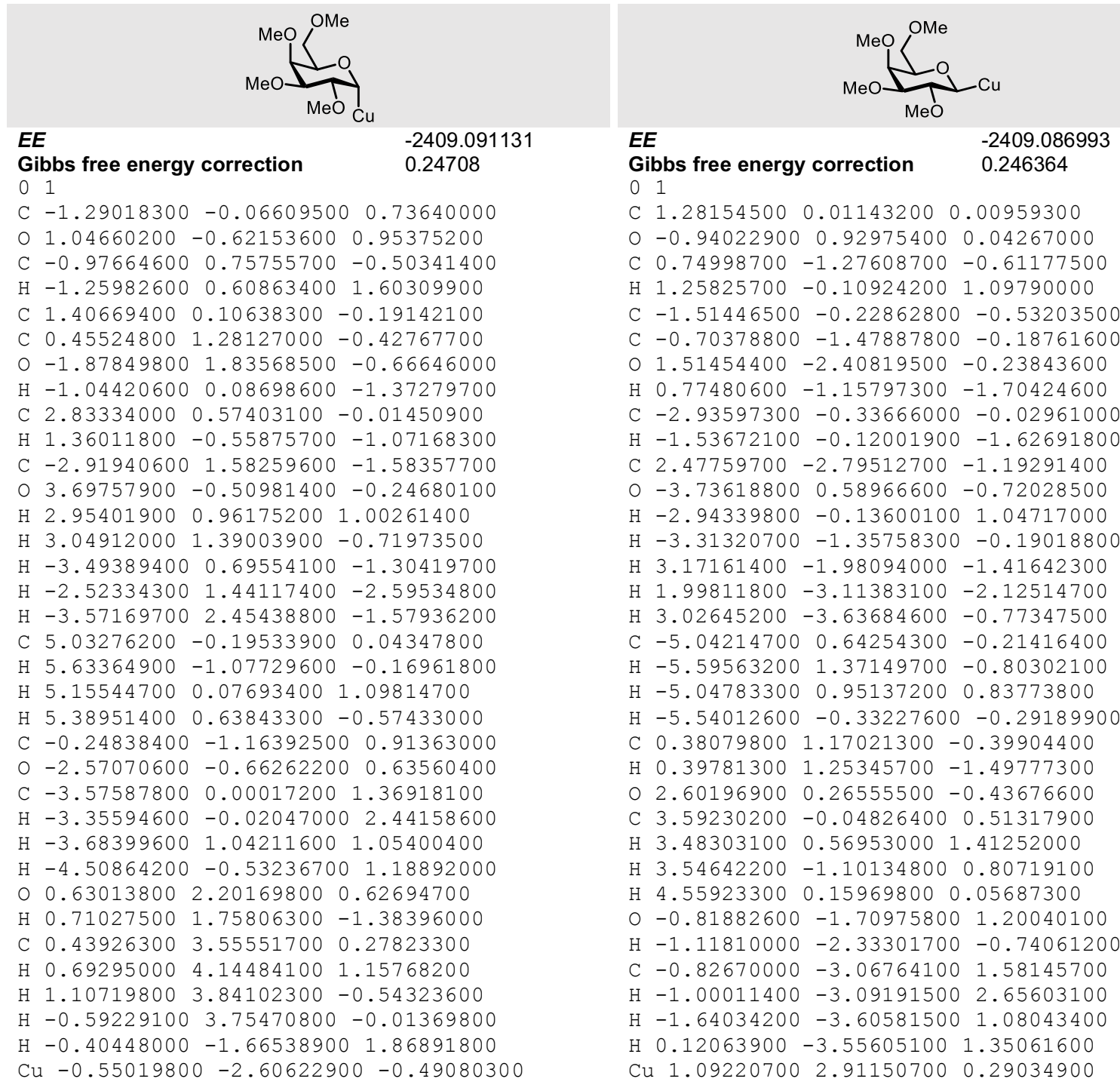


SILVER

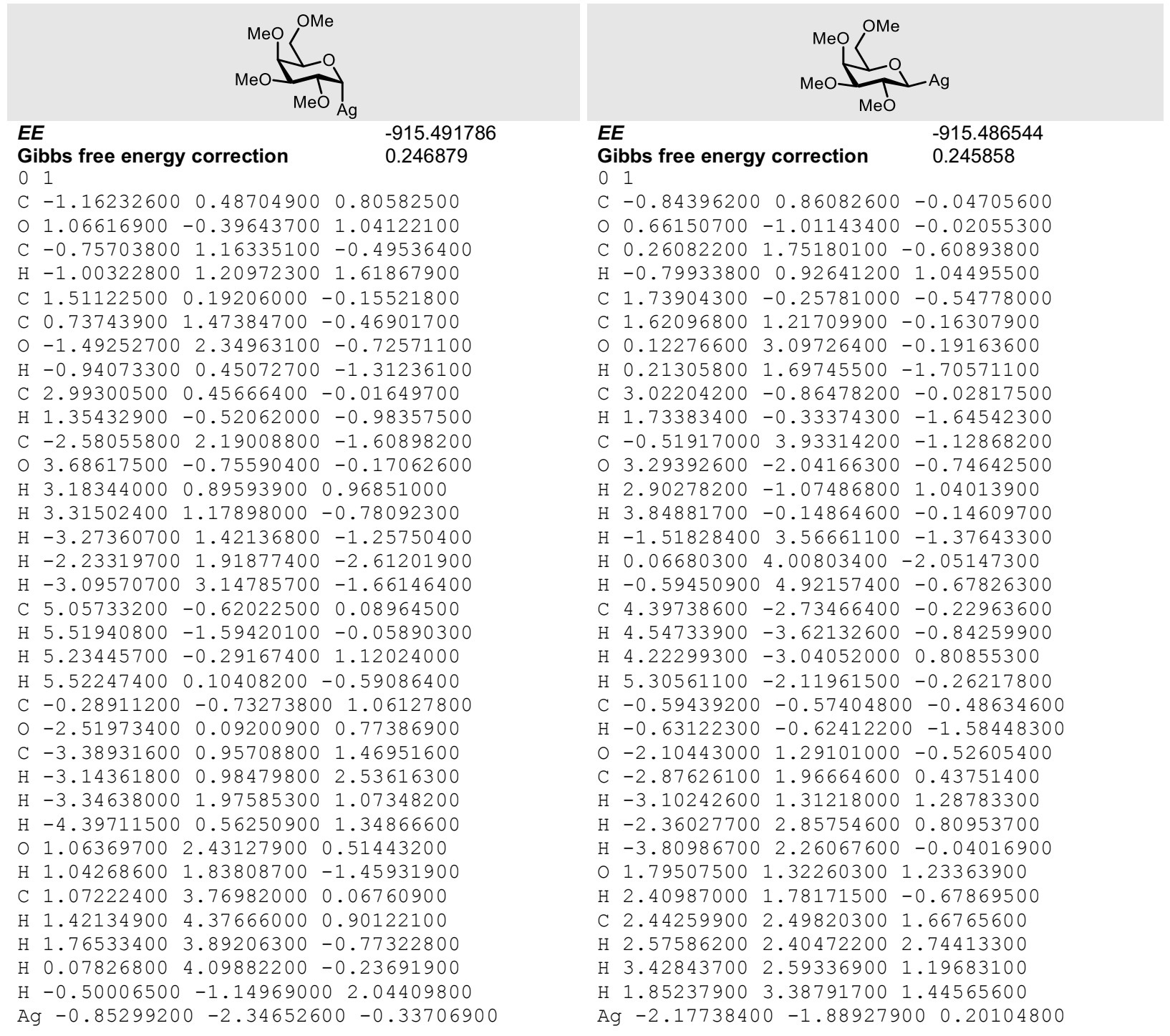


GOLD

\begin{tabular}{|c|c|c|}
\hline \multicolumn{2}{|r|}{$\begin{array}{l}\text { Gibbs free energy correction } \\
01\end{array}$} & $\begin{array}{l}-904.218141 \\
0.247833\end{array}$ \\
\hline & $-0.60059700 \quad 1.20220900$ & 0.80199900 \\
\hline & $1.03368000-0.54631300$ & 1.02801300 \\
\hline & 0.026911001 .63092300 & -0.51678300 \\
\hline & $-0.10239400 \quad 1.76498400$ & 1.60329900 \\
\hline & $1.69543100-0.18975300$ & -0.17228500 \\
\hline & 1.516461001 .29342900 & -0.49293700 \\
\hline & -0.74943900 \\
\hline & -0.445930001 .05331200 & -1.32269400 \\
\hline \multicolumn{2}{|r|}{$3.15294400-0.55284200$} & -0.00743000 \\
\hline & $1.27041100-0.78431100$ & -0.99478700 \\
\hline & -1.233569003 .32234000 & -1.59603600 \\
\hline & $3.28981900-1.94360900$ & -0.13832900 \\
\hline & $3.49500900-0.21328700$ & 0.97616100 \\
\hline & $3.74966300-0.03541900$ & -0.77297500 \\
\hline & $-2.17194200 \quad 2.92076000$ & -1.20594100 \\
\hline & $-1.06959700 \quad 2.92162600$ & -2.60201900 \\
\hline & $-1.29629200 \quad 4.40762000$ & -1.65339500 \\
\hline & $4.59384800-2.37836200$ & 0.1414260 \\
\hline & $4.61615900-3.45809900$ & 0.009233 \\
\hline & $4.87964200-2.13587000$ & 1.17142000 \\
\hline & $5.32132900-1.91879600$ & -0.53923900 \\
\hline & \multicolumn{2}{|c|}{$-0.32166900-0.27417700 \quad 1.06790000$} \\
\hline & & 0.82902500 \\
\hline & $\begin{array}{ll}-1.98726900 & 1.44241300 \\
-2.35743100 & 2.60323300\end{array}$ & 1.54083600 \\
\hline & $-2.07958000 \quad 2.52030200$ & 2.59665400 \\
\hline & $-1.88759100 \quad 3.49784100$ & 1.12240200 \\
\hline & $\begin{array}{ll}-3.43981900 & 2.69079500\end{array}$ & 1.46512800 \\
\hline & $2.20899100 \quad 2.03957300 \quad 0$ & 0.48350900 \\
\hline & 1.942038001 .49039200 & -1.48574300 \\
\hline & $2.78275600 \quad 3.24422100$ & 0.02132600 \\
\hline & $3.34334300 \quad 3.66434500$ & 0.85435500 \\
\hline & $3.47326100 \quad 3.04915400$ & -0.80748400 \\
\hline & $2.02065400 \quad 3.95230300$ & -0.30497000 \\
\hline & $-0.68349800-0.56184300$ & 02.05381900 \\
\hline & & \\
\hline
\end{tabular}

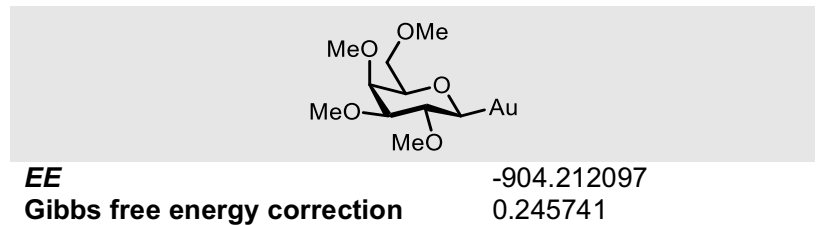

\section{Gibbs}

C $-0.04361700 \quad 1.29376300-0.08162900$

$00.60245700-1.02583700-0.04180100$

C $1.34323500 \quad 1.66592400-0.60555400$

$\mathrm{H}-0.01191000 \quad 1.35193300 \quad 1.01019100$

$\begin{array}{llll}\text { C } 1.89788300 & -0.76111200 & -0.55963900\end{array}$

C $2.36980100 \quad 0.63171100-0.14814600$

$\begin{array}{lllll}0 & 1.73173700 & 2.94483500 & -0.15480800\end{array}$

$\mathrm{H} 1.30568600 \quad 1.65132300-1.70433900$

C $2.81936600-1.84211800-0.04488400$

H $1.86947300-0.81652300-1.65761100$

C $1.48150000 \quad 3.99533200-1.06449200$

$\begin{array}{llll}0 & 2.59686100 & -3.01544300 & -0.78296700\end{array}$

H $2.61521400-1.99949600 \quad 1.01954700$

$\mathrm{H} 3.86528500-1.51767800-0.14898100$

$\mathrm{H} \quad 0.41770700 \quad 4.08302300-1.29235300$

$\mathrm{H} 2.03344900 \quad 3.84130100-1.99822000$

$\mathrm{H} \quad 1.83321800 \quad 4.91214400-0.59480200$

C $3.30906300-4.10961400-0.26970000$

H $3.09026600-4.96947100-0.89952500$

H $3.00598300-4.33121600 \quad 0.75995100$

H $4.39002500-3.92227900-0.28201400$

C $-0.36382900-0.13267400-0.51613800$

$\mathrm{H}-0.43284500-0.17378500-1.61235500$

$\begin{array}{llll}0 & -0.99206800 & 2.18792800 & -0.61889000\end{array}$

C $-1.70992600 \quad 2.91430200 \quad 0.35127000$

$\mathrm{H}-2.24123000 \quad 2.24124400 \quad 1.03296700$

$\mathrm{H}-1.04154300 \quad 3.55847200 \quad 0.93233200$

$\mathrm{H}-2.43674000 \quad 3.52897600-0.17688100$

$\begin{array}{llll}0 & 2.53914100 & 0.64043600 & 1.25094000\end{array}$

H $3.32761800 \quad 0.84213500 \quad-0.64276200$

C $3.589762001 .45686600 \quad 1.72248000$

H $3.650771001 .29896500 \quad 2.79769800$

$\mathrm{H} \quad 4.54240800 \quad 1.16180800 \quad 1.26699300$

H $3.403351002 .51079700 \quad 1.51475800$

Au $-2.19203300 \quad-0.73140900 \quad 0.13612900$ 
ZINC

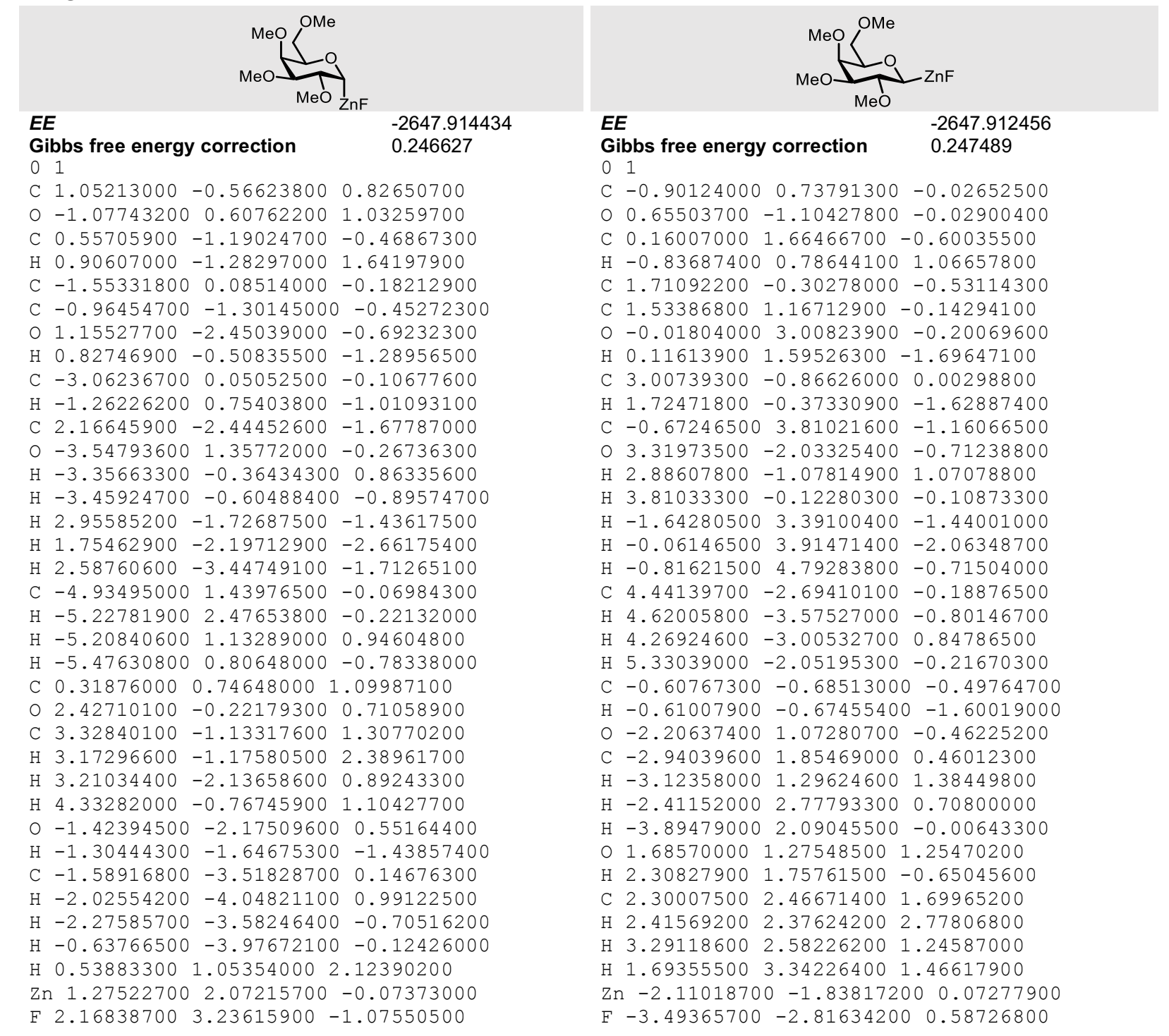


CADMIUM

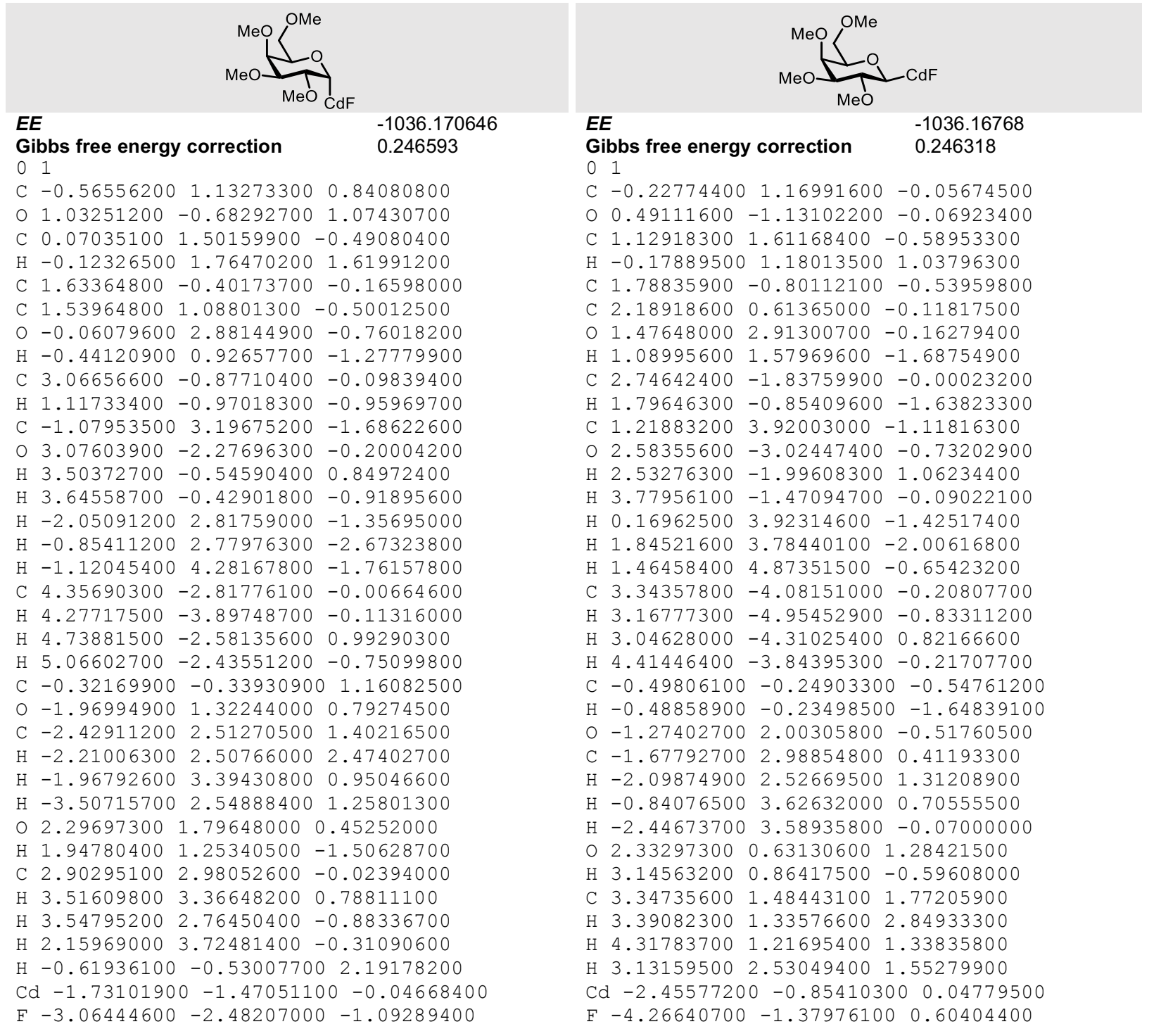


MERCURY

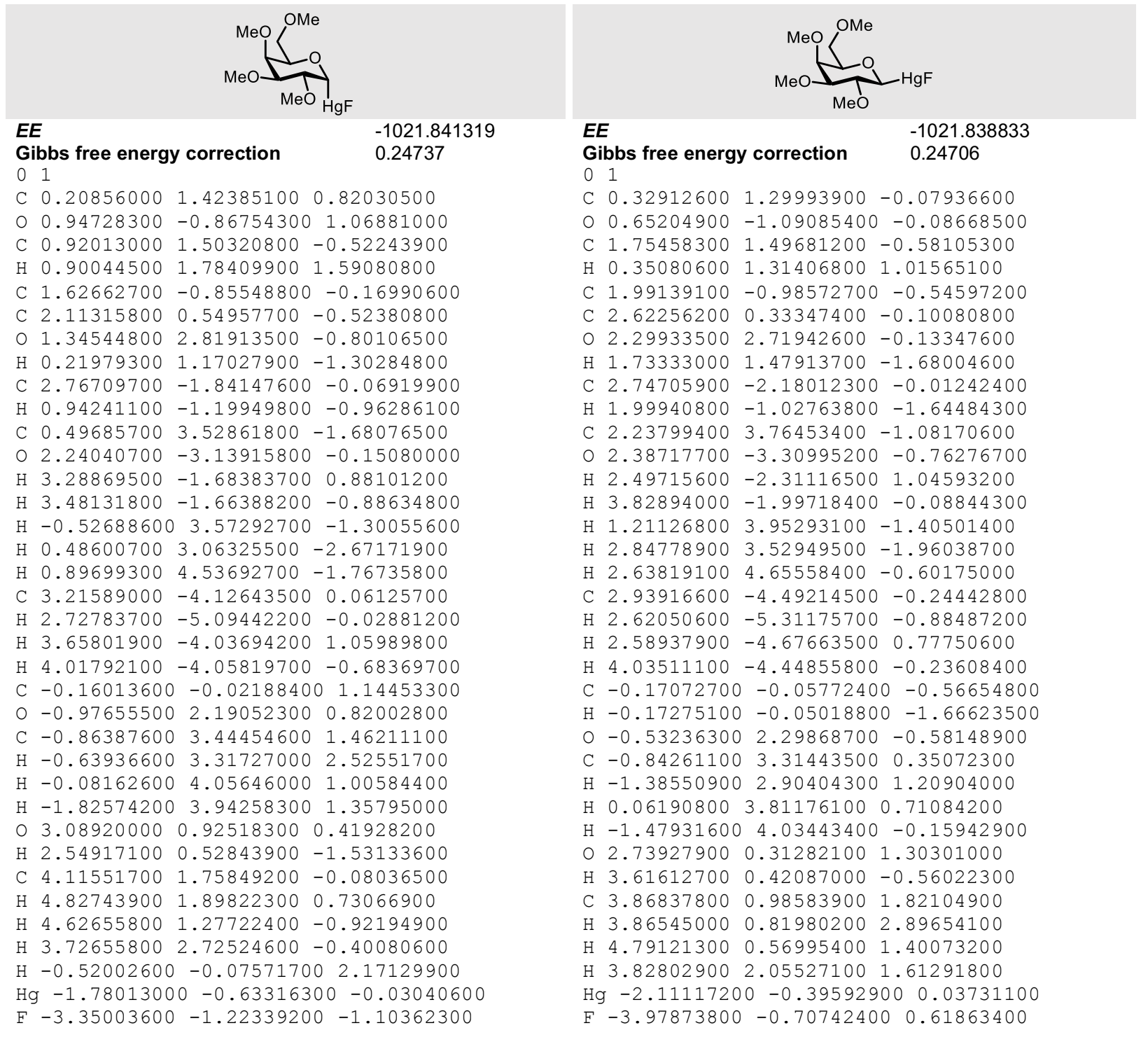




\section{NICKEL}

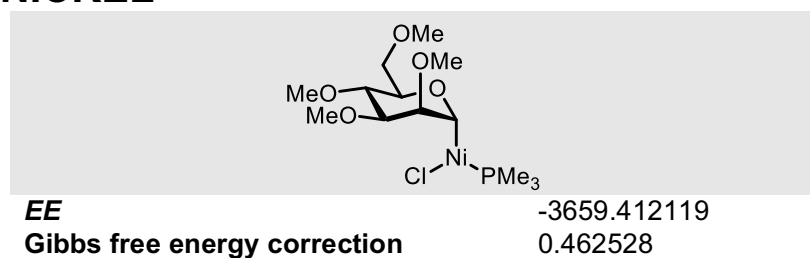

01

C $-1.50811200-0.38526800 \quad 1.44549300$

o $-0.20791800 \quad 1.52867100 \quad 0.89175100$

C $-2.24792700-0.30049000 \quad 0.11536200$

C $-0.85056300 \quad 1.72879900-0.34008300$

C $-2.27475800 \quad 1.15862800-0.32468000$

$0-3.54738200-0.84351700 \quad 0.23492500$

$\mathrm{H}-1.68477500-0.86521100-0.63994000$

C $-0.88165300 \quad 3.21523900-0.60399700$

$\mathrm{H}-0.28922500 \quad 1.23354000-1.15201500$

o $-2.827080001 .28083100-1.62296400$

H $-2.87385400 \quad 1.71671600 \quad 0.40242500$

C $-4.02907100-1.47833800-0.92798600$

$\begin{array}{lllll}0 & 0.43206800 & 3.67671200 & -0.81336500\end{array}$

$\mathrm{H}-1.32427800 \quad 3.71830500 \quad 0.26694100$

$\mathrm{H}-1.50044300 \quad 3.41781500-1.48641200$

C $-4.10988500 \quad 1.86287500-1.64538900$

$\mathrm{H}-3.39467900-2.32944500-1.20615300$

$\mathrm{H}-4.07797200-0.79840500-1.78074600$

$\mathrm{H}-5.02677400-1.84856400-0.69775700$

C $0.48789900 \quad 5.06259100-1.03109000$

$\mathrm{H}-4.45170100 \quad 1.85440500-2.67929500$

$\mathrm{H}-4.81363500 \quad 1.30065100-1.02355500$

$\mathrm{H}-4.07948200 \quad 2.89857000-1.28841200$

H $1.53130600 \quad 5.32939900-1.19042600$

$\mathrm{H} 0.10401600 \quad 5.61457800-0.16548500$

$\mathrm{H}-0.09427400 \quad 5.34848400-1.91473800$

C $-0.09187100 \quad 0.14313700 \quad 1.26171400$

$\mathrm{H} \quad 0.39415400 \quad 0.16504500 \quad 2.24240700$

$\mathrm{H}-1.46835600-1.41943300 \quad 1.80066000$

P $0.13033100-2.95351300-0.19944000$

P 2.74835000 $0.90973600 \quad 0.36795200$

Cl 2.64295800 $-1.62058300-1.50364100$

C $-0.32725300-3.26786300-1.93862500$

$\mathrm{H} \quad 0.54426000-3.07961600-2.56274400$

$\mathrm{H}-0.66554500-4.29773200-2.06300400$

$\mathrm{H}-1.12578300-2.58965800-2.24115500$

C $-1.32895400-3.63145600 \quad 0.68144200$

$\mathrm{H}-2.21255500-3.00995500 \quad 0.54222400$

$\mathrm{H}-1.53430900-4.63678000 \quad 0.30966700$

$\mathrm{H}-1.11977600-3.69038600 \quad 1.74986800$

C $1.34185200-4.27583200 \quad 0.14365600$

H $0.91778500-5.25062700-0.10216900$

$\mathrm{H} 2.23802500-4.09507000-0.44541200$

$\mathrm{H} \quad 1.60444300-4.25827600 \quad 1.20169900$

C $3.187864001 .81798300-1.14494100$

$\mathrm{H} \quad 4.01985600 \quad 2.49695100-0.94897000$

H $3.46486200 \quad 1.09831400-1.91355100$

$\mathrm{H} 2.31953900 \quad 2.39076100-1.46971200$

C 2.530832002 .245904001 .58982600

H 2.18407100 1.83563000 2.53842600

H $3.48010500 \quad 2.76144700 \quad 1.74457800$

H $1.77813500 \quad 2.93683600 \quad 1.21714300$

C $4.33992000 \quad 0.17845100 \quad 0.88635400$

$\mathrm{H} \quad 4.63198100 \quad-0.58029100 \quad 0.16285200$

$\mathrm{H} \quad 5.10918000 \quad 0.94947900 \quad 0.95269800$

$\mathrm{H} \quad 4.22354600-0.29211600 \quad 1.86321000$

$\mathrm{Ni} 1.17062800-0.79904000 \quad 0.05715600$

$\begin{array}{llllll}0 & -2.29211600 & 0.36805500 & 2.36032500\end{array}$

C $-1.77290100 \quad 0.43961000 \quad 3.65959800$

$\mathrm{H}-0.93002900 \quad 1.13476100 \quad 3.72453300$

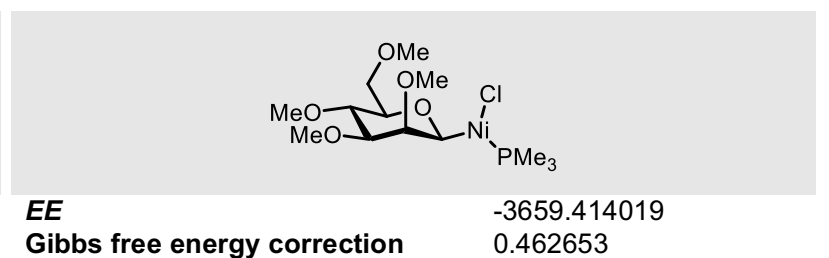

01

C $0.87375800-1.49464400 \quad 0.45008200$

$\begin{array}{lllll}0 & 0.81252000 & 0.83973000 & -0.13118100\end{array}$

C $2.37128100-1.33321400 \quad 0.73783200$

C $2.19181500 \quad 1.03517300 \quad 0.07290800$

C $2.96462200-0.26238400-0.16231500$

$\begin{array}{lllll}0 & 3.07476800 & -2.55954800 & 0.63848500\end{array}$

H 2.44879700 $-0.97106400 \quad 1.77169100$

C $2.641685002 .13049600-0.86410700$

H 2.38633500 1.35561400 1.10966400

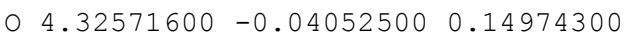

H 2.85483100 $-0.55867700-1.21349800$

C $4.00852900-2.77460300 \quad 1.67372700$

○ $2.14005800 \quad 3.36085100-0.39777000$

H 2.24899200 1.91431400 - 1.86763800

H $3.73699500 \quad 2.15759200-0.90638600$

C $5.22972900-0.67642100-0.72504400$

H $3.50769100 \quad-2.82464000 \quad 2.64665300$

$\mathrm{H} \quad 4.76175600-1.98313700 \quad 1.70573400$

$\mathrm{H} \quad 4.49168800 \quad-3.73019600 \quad 1.47658100$

C $2.41042700 \quad 4.42052300-1.27758800$

$\mathrm{H} \quad 6.23368600-0.46069800-0.36316900$

H $5.07336900-1.75876900-0.74495800$

$\mathrm{H} 5.12711600-0.28612200-1.74397300$

H 2.00124900 5.32768800 -0.83688400

H $1.94103700 \quad 4.25314000-2.25438000$

H $3.48922300 \quad 4.54776800-1.42554500$

C $0.17445900 \quad-0.15977600 \quad 0.68726000$

$\mathrm{H} \quad 0.33399100 \quad 0.10395000 \quad 1.74617000$

Cl $-3.66799200 \quad 0.59739700-0.91683900$

P $-1.55583800 \quad 2.33966500 \quad 0.68579500$

$\mathrm{P}-2.42121600-2.17610000-0.08493800$

C $-2.57901500-2.62901400-1.84087900$

$\mathrm{H}-3.22089000-1.89315600-2.32348000$

$\mathrm{H}-1.59178300-2.59069700-2.29920200$

$\mathrm{H}-3.00383400-3.62792100-1.95162300$

C $-4.12107000-2.39960700 \quad 0.54933800$

$\mathrm{H}-4.11953800-2.28206300 \quad 1.63360000$

$\mathrm{H}-4.76570800-1.63854000 \quad 0.11623500$

$\mathrm{H}-4.49219200-3.39524200 \quad 0.30075200$

C $-1.23519900 \quad 3.35882600-0.78438400$

$\mathrm{H}-2.01820600 \quad 3.15781300-1.51520600$

$\mathrm{H}-1.22312500 \quad 4.42004800-0.52962300$

$\mathrm{H}-0.26709500 \quad 3.06709400-1.18879600$

C $-0.32132400 \quad 2.92930900 \quad 1.89198800$

$\mathrm{H}-0.43104600 \quad 2.38407100 \quad 2.82985400$

$\mathrm{H} \quad 0.67800100 \quad 2.77696100 \quad 1.49122300$

$\mathrm{H}-0.46411000 \quad 3.99413400 \quad 2.08331500$

C $-3.09423500 \quad 3.05305300 \quad 1.36060400$

$\mathrm{H}-2.98250000 \quad 4.12759700 \quad 1.51420400$

$\mathrm{H}-3.90924600 \quad 2.85395000 \quad 0.66772200$

$\mathrm{H}-3.32705400 \quad 2.57869300 \quad 2.31440800$

C $-1.63757000-3.66819800 \quad 0.63012700$

$\mathrm{H}-0.66716300-3.84470400 \quad 0.17523400$

$\mathrm{H}-1.50950800-3.54800800 \quad 1.70598100$

$\mathrm{H}-2.27620600-4.53400900 \quad 0.44800200$

H $0.49065700 \quad-2.24320400 \quad 1.15715600$

○ $0.58275600-1.89598300-0.87908900$

C $1.24143500-3.03990500-1.37709800$

H 2.26927100 $-2.82059800-1.67519500$

H $1.28151200-3.84573800-0.63890700$ 
$\mathrm{H}-2.57351200 \quad 0.79582900 \quad 4.30616700$

$\mathrm{H}-1.44549300 \quad-0.54610600 \quad 4.01471100$
$\mathrm{H} \quad 0.67787100 \quad-3.37014300-2.25022700$

$\mathrm{Ni}-1.71270300 \quad 0.07156400 \quad 0.15113300$ 
PALLADIUM

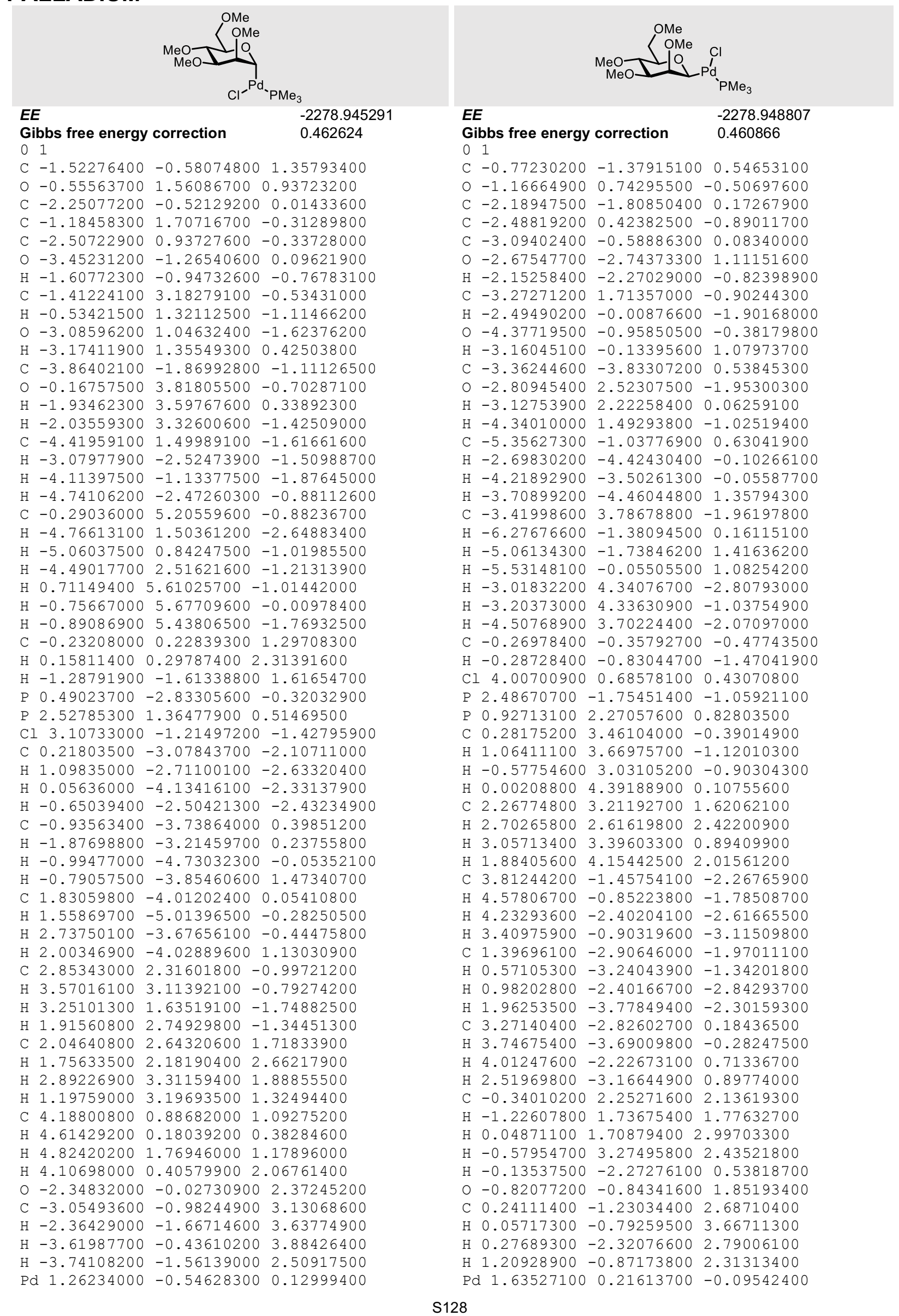




\section{PLATINUM}

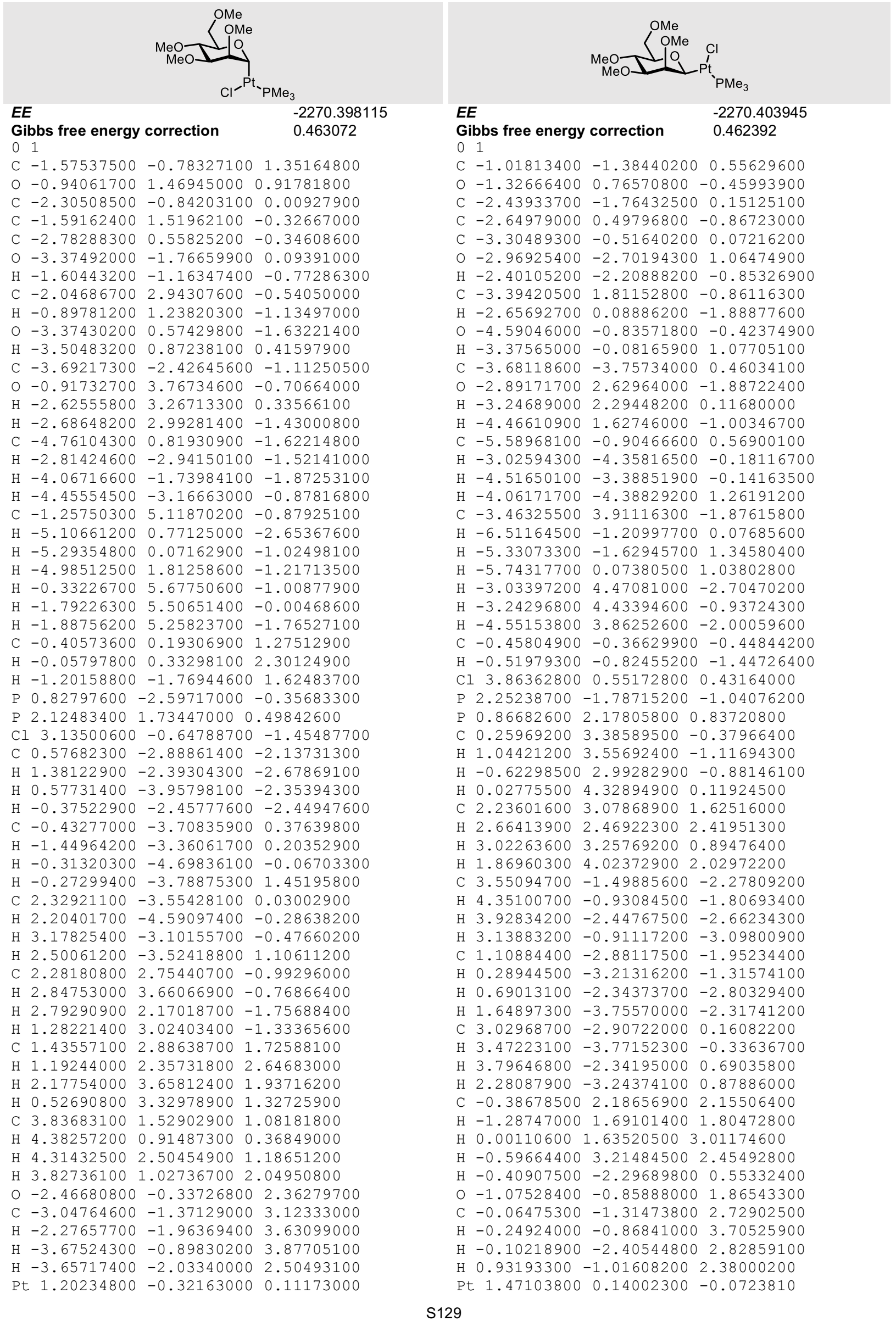


COPPER

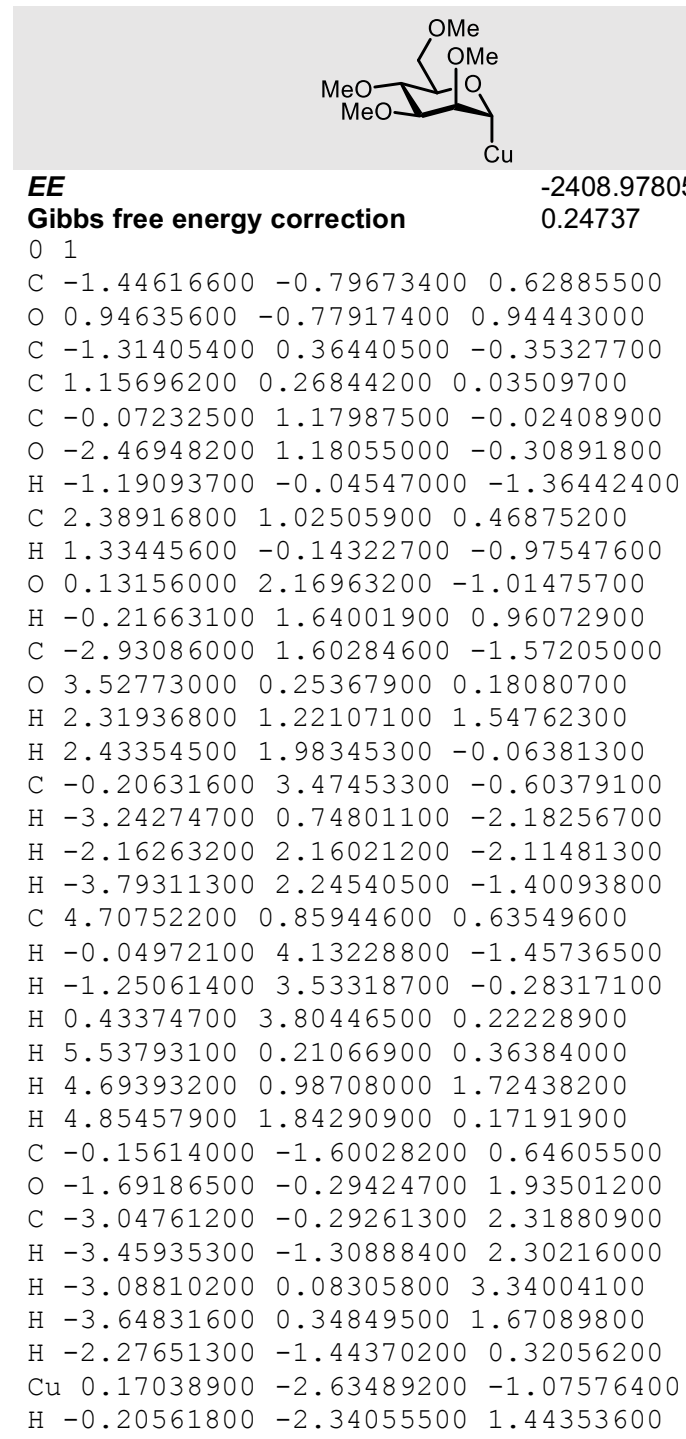

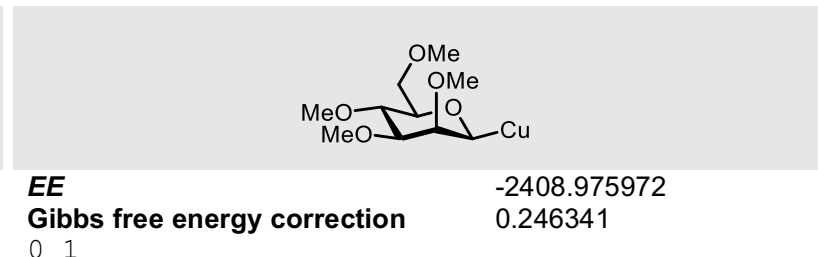

01

C $-1.42251000 \quad 0.50995200-0.36401200$

O $0.17629800-1.29485000-0.09767200$

C $-0.25099400 \quad 1.47151400-0.58543400$

C $1.29117100-0.47167700-0.37107600$

C $1.00175600 \quad 0.95370000 \quad 0.10220600$

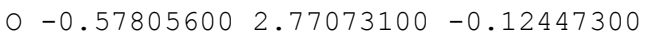

$\mathrm{H}-0.04673700 \quad 1.50811600-1.66406200$

C $2.48755600-1.05792900 \quad 0.33861300$

H $1.49509700-0.45381100-1.45266500$

○ $2.10295100 \quad 1.78616400-0.21351400$

$\mathrm{H} \quad 0.82710000 \quad 0.93728800 \quad 1.18491900$

C $-0.17969600 \quad 3.81142800 \quad-0.98841900$

o $2.90273600-2.22067000-0.33193600$

H 2.19888200 $-1.29352100 \quad 1.37283100$

H $3.29839700-0.31877500 \quad 0.35693600$

$\begin{array}{lllll}\text { C } 2.48785100 & 2.65209700 & 0.82889300\end{array}$

$\mathrm{H}-0.69342200 \quad 3.73876400-1.95346200$

$\mathrm{H} \quad 0.89929700 \quad 3.79722500 \quad-1.16320400$

$\mathrm{H}-0.45865600 \quad 4.74977200-0.51136000$

C $3.97540400-2.84965300 \quad 0.31397700$

H $3.30666700 \quad 3.26455000 \quad 0.45417400$

$\mathrm{H} \quad 1.65994700 \quad 3.29724300 \quad 1.13753900$

H 2.83653400 2.08655300 1.70054900

$\mathrm{H} \quad 4.24314500-3.72866200-0.26925000$

$\mathrm{H} 3.70025400-3.16303200 \quad 1.32837300$

H $4.84604200-2.18517900 \quad 0.38063300$

C $-1.01656100-0.89935600-0.75748800$

$\mathrm{Cu}-2.43252100-2.16087000-0.09670000$

$\mathrm{H}-0.87453900-0.94636200-1.84559400$

$\begin{array}{lllll}0 & -1.82827500 & 0.52536200 & 0.99682900\end{array}$

C $-2.89244600 \quad 1.40387500 \quad 1.28290700$

$\mathrm{H}-3.78412000 \quad 1.13549100 \quad 0.70158500$

$\mathrm{H}-3.12003600 \quad 1.29518300 \quad 2.34232600$

$\mathrm{H}-2.62730700 \quad 2.44056700 \quad 1.07150600$

$\mathrm{H}-2.25786600 \quad 0.82619500-1.00234800$ 
SILVER

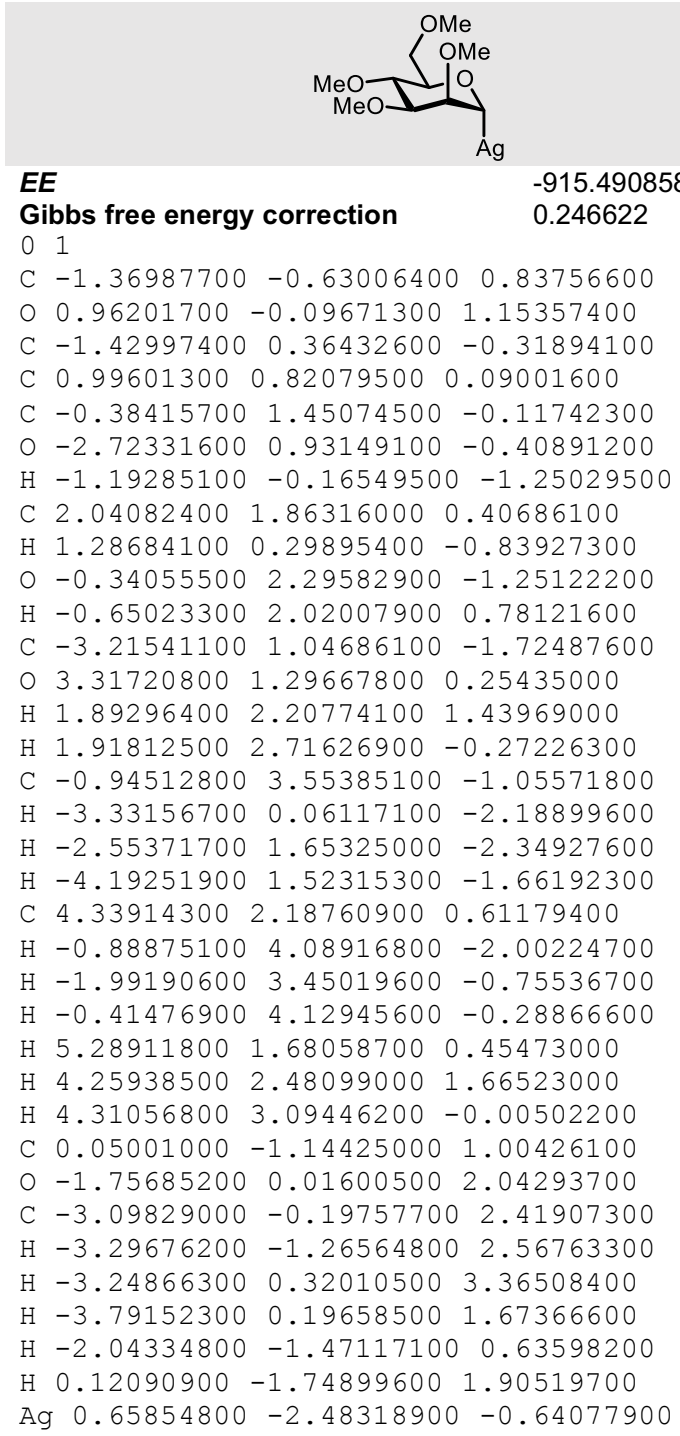

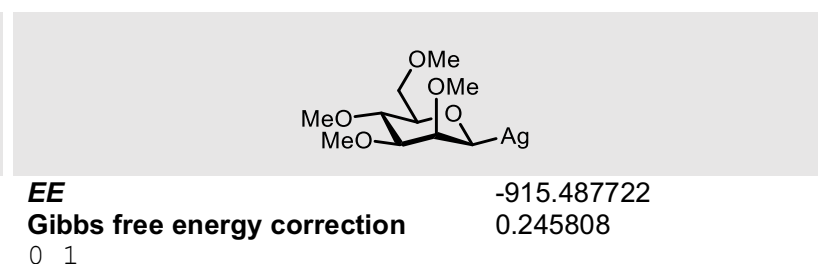

01

C $0.50330800-1.27718500-0.34246800$

$\begin{array}{lllll}0 & 0.17831600 & 1.11035900 & -0.08593900\end{array}$

C $-1.00254200-1.42641700-0.58411900$

C $-1.206272001 .04675600-0.36865100$

C $-1.76252000-0.29980200 \quad 0.09693100$

$0-1.45265900-2.68841400-0.12528200$

$\mathrm{H}-1.18226500-1.34470600 \quad-1.66459800$

C $-1.87740700 \quad 2.19780800 \quad 0.34056800$

$\mathrm{H}-1.37777600 \quad 1.14908700-1.45095200$

$\begin{array}{llll}0 & -3.13739300 & -0.37783200 & -0.23259400\end{array}$

$\mathrm{H}-1.61880100 \quad-0.38578400 \quad 1.18080400$

C $-2.35739900-3.33334400-0.99350900$

$\begin{array}{llll}0 & -1.57131700 & 3.39656100 & -0.32482200\end{array}$

$\mathrm{H}-1.51249100 \quad 2.22938400 \quad 1.37695400$

H $-2.96250800 \quad 2.03461300 \quad 0.35182200$

C $-3.94974400 \quad-0.88154700 \quad 0.80242700$

$\mathrm{H}-1.88479400 \quad-3.55940100-1.95582700$

$\mathrm{H}-3.24585800-2.72242200-1.17381600$

H $-2.64910900-4.26798800 \quad-0.51687700$

C $-2.11593300 \quad 4.51452100 \quad 0.32123400$

$\mathrm{H}-4.96673800 \quad-0.93642700 \quad 0.41695600$

$\mathrm{H}-3.62322400 \quad-1.87728900 \quad 1.11660600$

$\mathrm{H}-3.93448200 \quad-0.21579400 \quad 1.67290800$

$\mathrm{H}-1.84658700 \quad 5.39523800-0.25860900$

$\mathrm{H}-1.71790200 \quad 4.61937500 \quad 1.33777700$

$\mathrm{H}-3.20934600 \quad 4.44646300 \quad 0.38229500$

C $0.94607600 \quad 0.11807600-0.73836700$

$\mathrm{H} \quad 0.86963200 \quad 0.23511800-1.82660300$

$\begin{array}{lllll}0 & 0.80091000 & -1.51425000 & 1.02366700\end{array}$

C $1.25071200-2.81955900 \quad 1.30287100$

H $2.18075400 \quad-3.03399700 \quad 0.76047900$

$\mathrm{H} \quad 1.45059800 \quad-2.86763500 \quad 2.37219800$

H $0.50095200-3.56728400 \quad 1.03877600$

$\mathrm{H} 1.03167500-2.00452000-0.97160400$

Ag $2.99118600 \quad 0.43035600-0.06915800$ 
GOLD

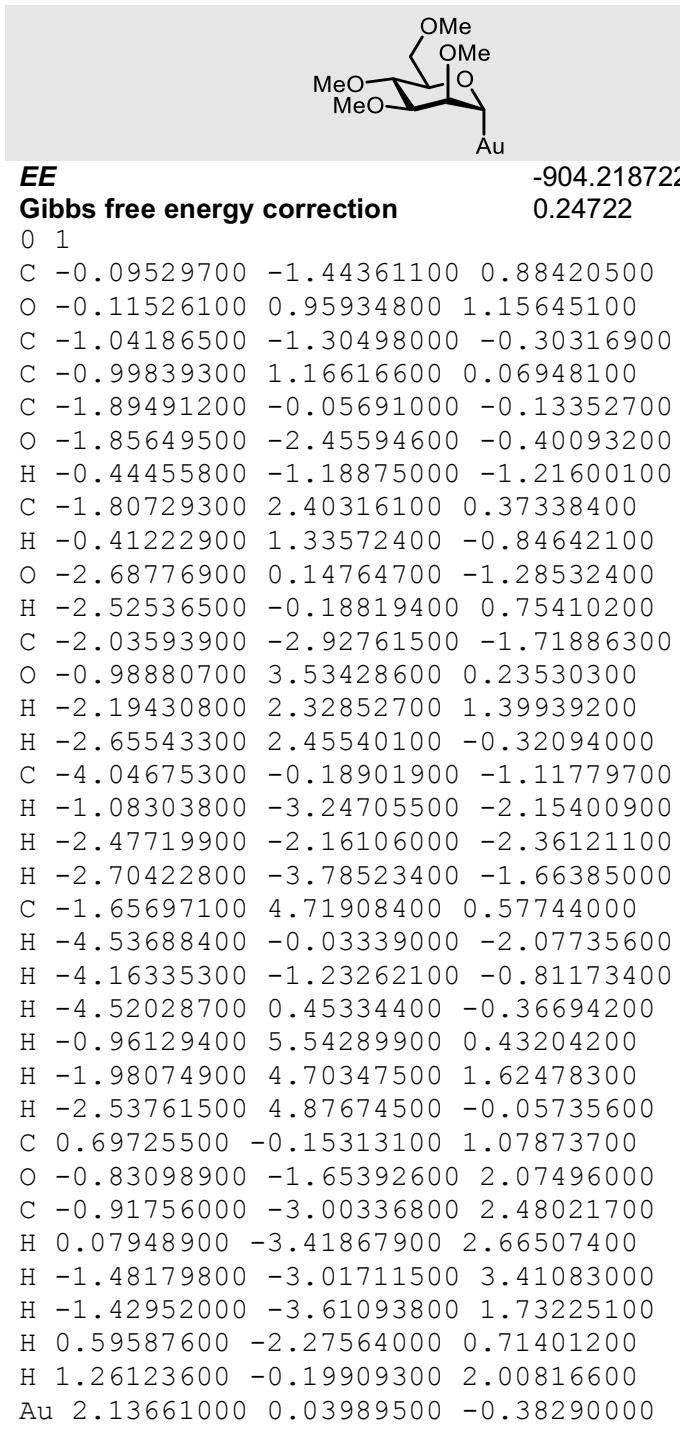

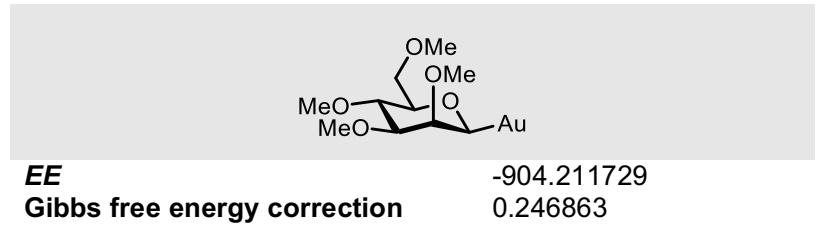

Gibbs

01

C $-0.04357600-1.35459600-0.19464000$

$\begin{array}{lllll}0 & -0.18708500 & 1.04439700 & 0.03145800\end{array}$

C $-1.53032100-1.38975100-0.55991500$

C $-1.55912800 \quad 1.09350200-0.32912000$

C $-2.24962400-0.21020200 \quad 0.07691000$

$\begin{array}{lllll}0 & -2.08844200 & -2.61639800 & -0.13661300\end{array}$

$\mathrm{H}-1.62676600-1.29447800-1.65010900$

C $-2.16876900 \quad 2.28695400 \quad 0.36395500$

$\mathrm{H}-1.65770900 \quad 1.22690000-1.41655700$

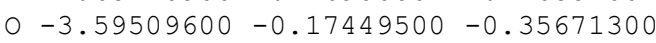

$\mathrm{H}-2.19354000-0.31730900 \quad 1.16655600$

C $-2.97506800-3.20247700-1.06462700$

$\begin{array}{llll}0 & -1.73549800 & 3.46064200 & -0.27179900\end{array}$

$\mathrm{H}-1.85371400 \quad 2.27779600 \quad 1.41665800$

$\mathrm{H}-3.26255500 \quad 2.20859700 \quad 0.32038100$

C $-4.52316700-0.61571500 \quad 0.60878900$

$\mathrm{H}-2.45502700-3.46525800-1.99232000$

$\mathrm{H}-3.80512300-2.53267000-1.30403000$

$\mathrm{H}-3.36127100 \quad-4.11193000 \quad-0.60762600$

C $-2.20056400 \quad 4.61637600 \quad 0.37224600$

$\mathrm{H}-5.50771300-0.58850700 \quad 0.14479700$

$\mathrm{H}-4.30107500-1.63505500 \quad 0.93755800$

$\mathrm{H}-4.52255300 \quad 0.04420300 \quad 1.48348500$

$\mathrm{H}-1.83316200 \quad 5.47494400-0.18614500$

$\mathrm{H}-1.82936000 \quad 4.67282200 \quad 1.40230300$

$\mathrm{H}-3.29697800 \quad 4.64554100 \quad 0.39485200$

C $0.51145800 \quad 0.00483800-0.59442900$

$\mathrm{H} \quad 0.46735200 \quad 0.11644600-1.68611500$

$\begin{array}{lllll}0 & 0.10363300 & -1.55810300 & 1.19143600\end{array}$

C $0.58172300-2.83706600 \quad 1.53762400$

$\mathrm{H} 1.58141400-3.00391100 \quad 1.11868000$

$\mathrm{H} \quad 0.64534500 \quad-2.87115100 \quad 2.62355000$

$\mathrm{H}-0.09556300 \quad-3.61998700 \quad 1.18794300$

$\mathrm{H} \quad 0.47854300-2.13541000-0.75882300$

Au 2.47331100 $0.16846700 \quad-0.09567000$ 
ZINC

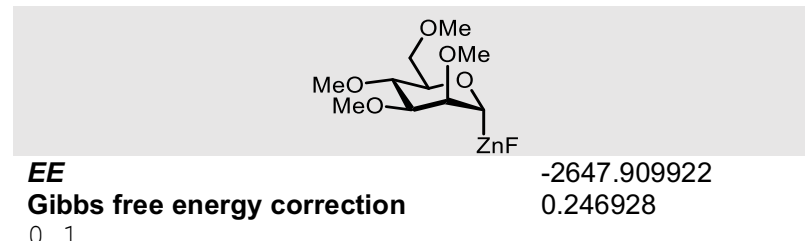

C $1.28969200 \quad 0.86798300 \quad 0.84598200$

$\begin{array}{lllll}0 & -0.94293200 & 0.00389900 & 1.20418600\end{array}$

C $1.44308200-0.06980100-0.35084300$

C $-0.88628600-0.85983400 \quad 0.09657900$

C $0.55753000-1.29505500-0.16586000$

O $2.79467800-0.44515400-0.50558700$

H $1.10367300 \quad 0.45470200-1.25672100$

C $-1.79202700-2.03458600 \quad 0.37459000$

$\mathrm{H}-1.26319700-0.34556500-0.80862100$

○ $0.59535600-2.09561500-1.32866100$

H $0.92009500-1.85195300 \quad 0.70614400$

C $3.24795300-0.46332100-1.84298000$

$0-3.12525400-1.60584600 \quad 0.28550800$

$\mathrm{H}-1.57332900-2.41588300 \quad 1.38167700$

$\mathrm{H}-1.59235900-2.82746700-0.35677300$

C $1.36061400-3.27325800-1.19597300$

H $3.21060000 \quad 0.53938000 \quad-2.28205500$

H 2.65160900 -1.14087200 - 2.45889000

$\mathrm{H} \quad 4.28222600-0.80172400-1.82474800$

C $-4.03628100-2.62656700 \quad 0.59885300$

$\mathrm{H} \quad 1.34696100 \quad-3.77563100 \quad-2.16155100$

$\mathrm{H} 2.39321100-3.04639700-0.91544400$

$\mathrm{H} \quad 0.92798800-3.93871400-0.44089700$

$\mathrm{H}-5.03843000-2.21713800 \quad 0.49146100$

$\mathrm{H}-3.89946800-2.97663400 \quad 1.62853100$

$\mathrm{H}-3.92301700-3.48118900-0.07897600$

C $-0.18674700 \quad 1.18884700 \quad 1.05130000$

$\begin{array}{lllll}0 & 1.77934500 & 0.25395500 & 2.02060500\end{array}$

C $3.11219500 \quad 0.58099000 \quad 2.35217700$

H $3.22365500 \quad 1.66284000 \quad 2.48897400$

$\mathrm{H} \quad 3.33190300 \quad 0.08473300 \quad 3.29553300$

H $3.81132800 \quad 0.24078100 \quad 1.58720500$

H $1.84579800 \quad 1.79534600 \quad 0.65216700$

$\mathrm{H}-0.28281400 \quad 1.72009700 \quad 1.99883300$

Zn $-0.99133100 \quad 2.34903300 \quad-0.37611600$

F $-1.68937900 \quad 3.42977800 \quad-1.59485800$

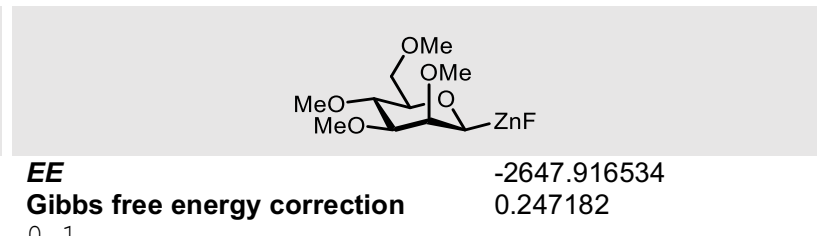

01

C $0.84375500-1.01330900-0.65797900$

○ $0.00952700 \quad 1.24954700-0.30426100$

C $-0.59918500-1.50562200-0.62582200$

C $-1.34193300 \quad 0.85337500-0.41520600$

C $-1.49595300-0.55744600 \quad 0.15469600$

$0-0.66721000-2.82200900-0.11548100$

$\mathrm{H}-0.95357300-1.49890500-1.66617200$

C $-2.18290500 \quad 1.85804600 \quad 0.33431100$

$\mathrm{H}-1.65084400 \quad 0.85118800-1.47127700$

$0-2.84104000-0.97238000 \quad 0.03435600$

$\mathrm{H}-1.18522300-0.54750900 \quad 1.20711100$

C $-1.51801100-3.68761400-0.83827100$

$\begin{array}{lllll}0 & -2.23282100 & 3.05179100 & -0.40128300\end{array}$

$\mathrm{H}-1.72762100 \quad 2.03054000 \quad 1.32016000$

$\mathrm{H}-3.19213100 \quad 1.45246900 \quad 0.47807100$

C $-3.35961000-1.60054100 \quad 1.18544900$

$\mathrm{H}-1.15906100-3.81856300-1.86448700$

$\mathrm{H}-2.54232500-3.30881400-0.86741700$

$\mathrm{H}-1.49823600-4.65087100-0.33189500$

C $-2.94244900 \quad 4.05999200 \quad 0.26816600$

$\mathrm{H}-4.38054700-1.90041800 \quad 0.95572100$

$\mathrm{H}-2.77196100-2.48260500 \quad 1.45652500$

$\mathrm{H}-3.37365600-0.91007600 \quad 2.03588200$

$\mathrm{H}-2.94514200 \quad 4.94042900-0.37103700$

$\mathrm{H}-2.46712700 \quad 4.31182200 \quad 1.22345100$

$\mathrm{H}-3.97776300 \quad 3.75489200 \quad 0.46354500$

C $0.93432600 \quad 0.45800900-1.04459400$

$\mathrm{H} \quad 0.78257100 \quad 0.59159300 \quad-2.11950300$

o $1.51159200-1.09348200 \quad 0.60893400$

C $1.92112100-2.37556300 \quad 1.06344000$

H 2.29687000 $-2.97471300 \quad 0.22941800$

H 2.72290900 $-2.20825700 \quad 1.78010700$

$\mathrm{H} 1.09675400-2.90568900 \quad 1.53507800$

$\mathrm{H} 1.40247100-1.61989700-1.38310900$

F $4.20282900 \quad 1.21114700 \quad 0.68400000$

Zn $2.66342800 \quad 0.85831200-0.12964800$ 
CADMIUM

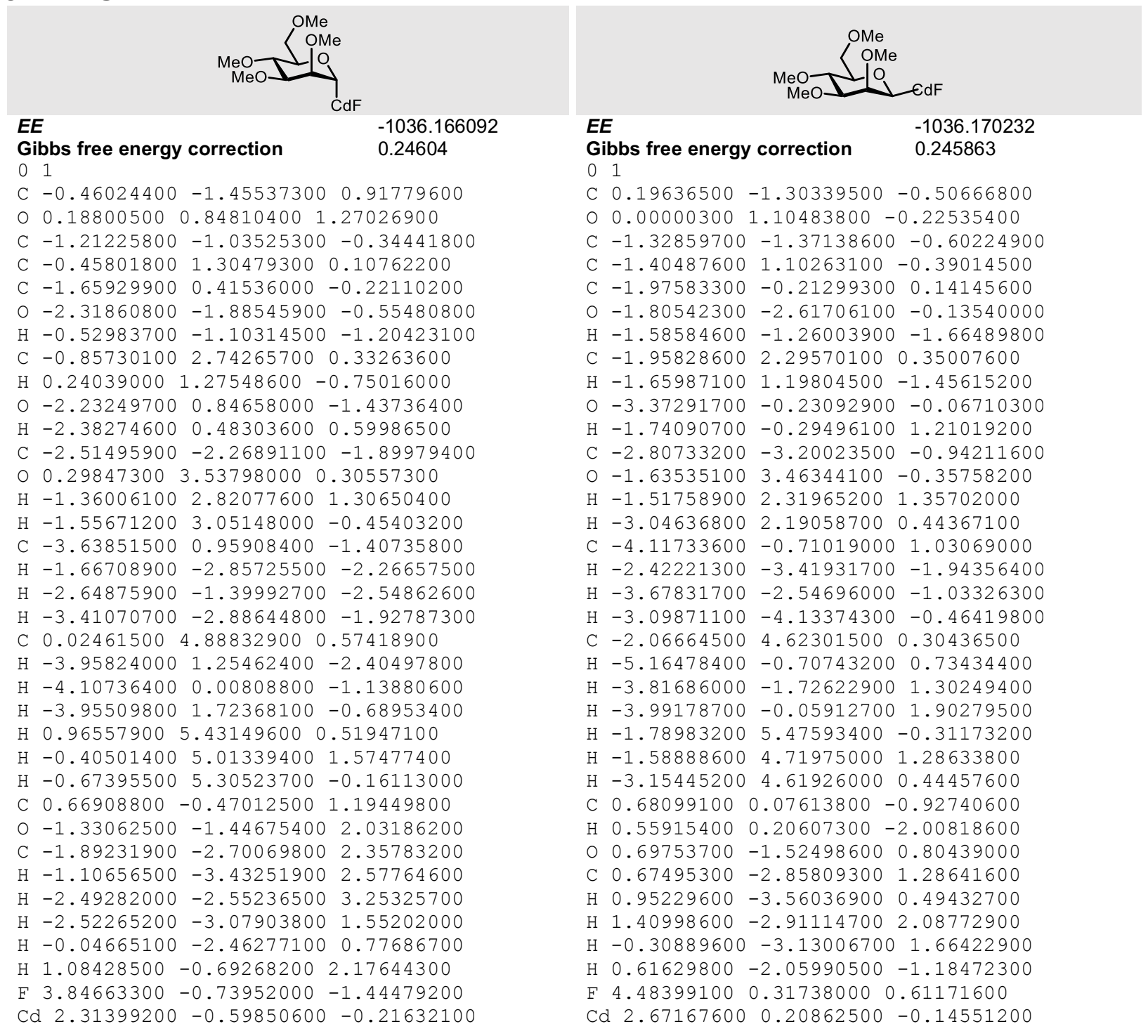


MERCURY

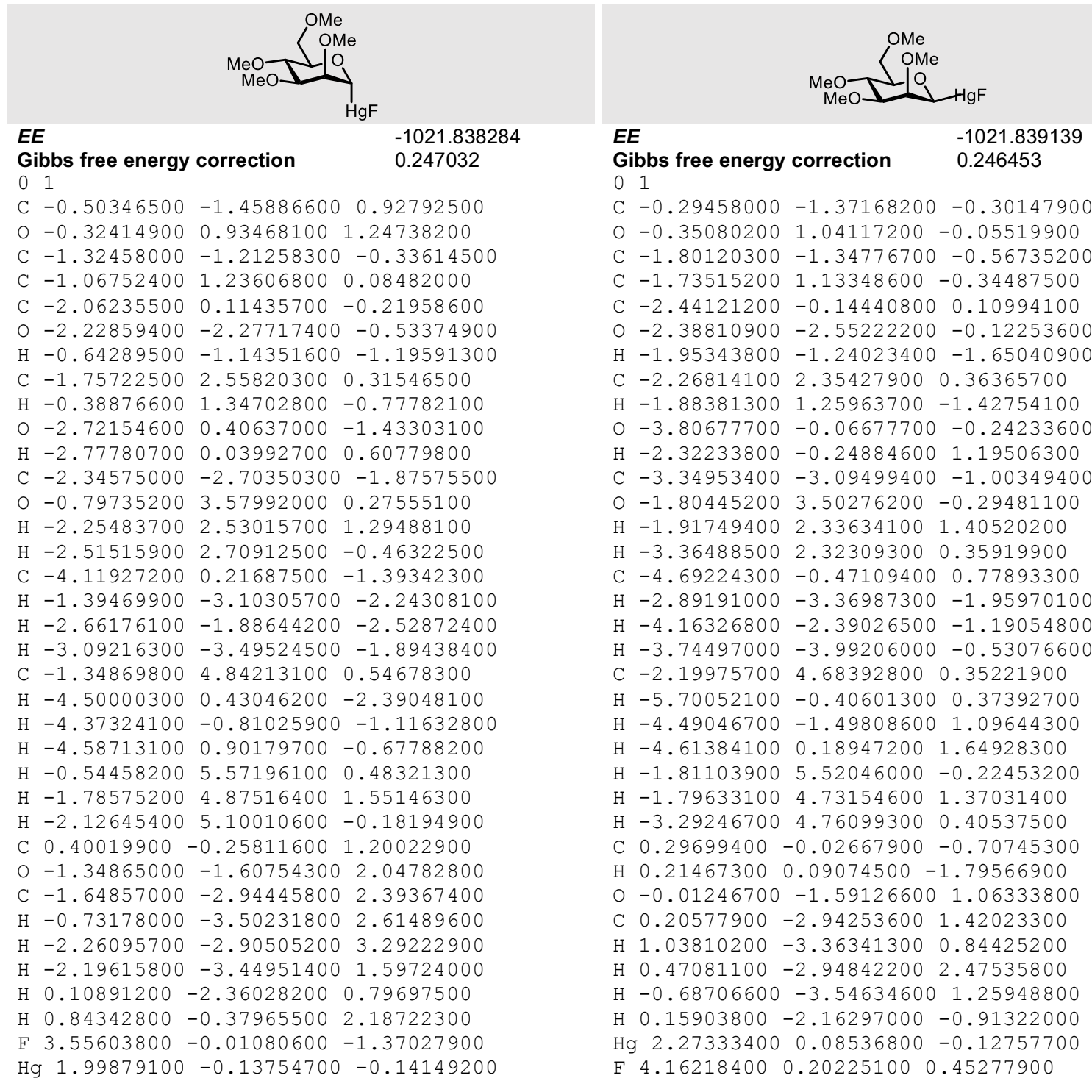




\section{NICKEL}

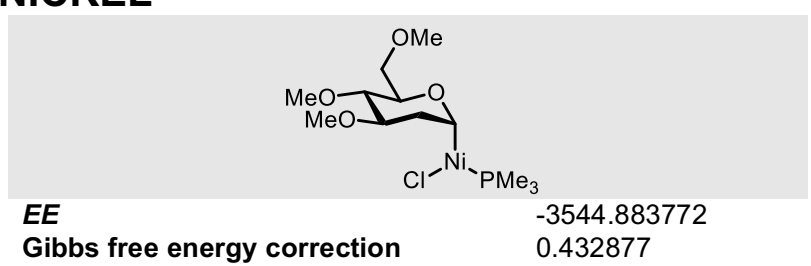

$0 \quad 1$

C $-1.51799000 \quad 0.17577100 \quad 1.80360300$

O $0.36430400 \quad 1.48549200 \quad 1.17347300$

C $-2.29191600 \quad 0.64464000 \quad 0.58333900$

$\mathrm{H}-1.71466900 \quad 0.88749900 \quad 2.61459300$

C $-0.24649400 \quad 1.97641400 \quad 0.01080100$

C $-1.77125600 \quad 2.01501200 \quad 0.17213100$

$\begin{array}{lllll}0 & -3.67677200 & 0.67970000 & 0.88274800\end{array}$

$\mathrm{H}-2.12042500-0.03849600-0.26194200$

C $0.29387500 \quad 3.36362200-0.24528900$

$\mathrm{H}-0.00513400 \quad 1.33647200-0.85550600$

$\begin{array}{lllll}0 & -2.35750700 & 2.43824800 & -1.04518900\end{array}$

$\mathrm{H}-2.01803200 \quad 2.72023200 \quad 0.97704500$

C $-4.53020400 \quad 0.40979100-0.20668500$

$\begin{array}{lllll}0 & 1.65875500 & 3.27506400 & -0.57607800\end{array}$

H $0.16490500 \quad 3.96772400 \quad 0.66404100$

$\mathrm{H}-0.26561800 \quad 3.82997900 \quad-1.06521300$

C $-3.23445500 \quad 3.53187200-0.91284800$

$\mathrm{H}-4.32152700-0.57852500-0.63486000$

$\mathrm{H}-4.43440700 \quad 1.15050300-1.00261900$

$\mathrm{H}-5.54888100 \quad 0.41596900 \quad 0.17783600$

C $2.24354500 \quad 4.53244900-0.79546400$

$\mathrm{H}-3.65438100 \quad 3.73230900-1.89731900$

$\mathrm{H}-4.04484500 \quad 3.31056400-0.21050500$

$\mathrm{H}-2.70427800 \quad 4.42543400-0.56369500$

H $3.28859000 \quad 4.36903800-1.05321600$

H 2.18874300 5.15628400 0.10410100

H $1.74947800 \quad 5.06099000-1.61892100$

C $-0.02472400 \quad 0.13504000 \quad 1.50255300$

H $0.50504700 \quad-0.06999700 \quad 2.43857900$

$\mathrm{H}-1.88084400-0.79337900 \quad 2.13685400$

$\mathrm{P}-1.13897700-2.67482200-0.09433700$

P 2.82038900 $-0.18693000 \quad 0.37066400$

Cl $1.60042500-2.34444300-1.57606500$

C $-1.81600000-2.65985500-1.78984700$

$\mathrm{H}-0.98829700-2.74573400-2.49150000$

$\mathrm{H}-2.51501900-3.48611400-1.92868600$

$\mathrm{H}-2.33456000-1.71799000-1.97278100$

C $-2.67702300-2.79672500 \quad 0.89853400$

$\mathrm{H}-3.25546200-1.87451300 \quad 0.86548800$

$\mathrm{H}-3.28589500-3.61618400 \quad 0.51289700$

$\mathrm{H}-2.42936100-3.00621600 \quad 1.93941700$

C $-0.52649700-4.38681200 \quad 0.07775700$

$\mathrm{H}-1.31918500-5.09847700-0.15827300$

$\mathrm{H} \quad 0.31877200-4.52800300-0.59181500$

$\mathrm{H}-0.19532900-4.54947600 \quad 1.10372300$

C $3.49263700 \quad 0.55001300-1.15062700$

$\mathrm{H} \quad 4.53461000 \quad 0.83917200-1.00136100$

$\mathrm{H} \quad 3.41784000-0.18252900-1.95254700$

H 2.90114200 1.43052600 -1.39969800

C $3.22777400 \quad 1.06257200 \quad 1.63427700$

H 2.82821400 $0.76147000 \quad 2.60257700$

H $4.31083400 \quad 1.17376100 \quad 1.70857500$

$\mathrm{H} 2.76626900 \quad 2.00516900 \quad 1.34916000$

C $4.02025700-1.51215900 \quad 0.74627600$

H $3.93794700-2.28603300-0.01475900$

H $5.03570200-1.11319100 \quad 0.76707800$

$\mathrm{H} \quad 3.78950100-1.94786200 \quad 1.71893800$

$\mathrm{Ni} 0.68252600-1.12273100 \quad 0.14970800$

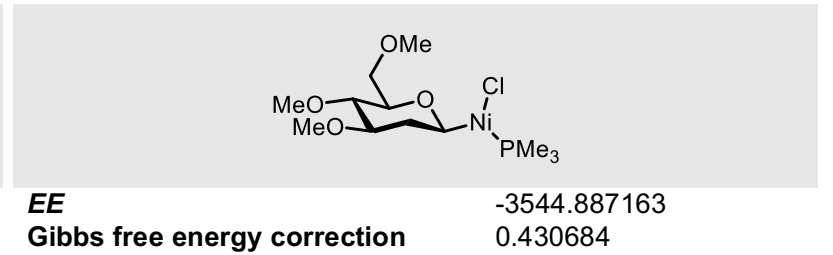

01

C $0.56329800-1.36287100-0.78165000$

O $1.02270200 \quad 0.65650500 \quad 0.43695400$

C $1.96392300-1.87157900-0.47424800$

$\mathrm{H} \quad 0.58519200 \quad-0.84342600-1.74551900$

C $2.31974200 \quad 0.25485400 \quad 0.80877500$

C $2.90328400-0.69902300-0.23637300$

o $2.44179400-2.67223800-1.54203200$

H $1.93671000 \quad-2.46826600 \quad 0.44890800$

C $3.16344800 \quad 1.50155000 \quad 0.93540900$

H 2.29419400 $-0.25942800 \quad 1.78093900$

○ $4.16761300-1.15400000 \quad 0.20754500$

$\mathrm{H} 3.01060400-0.15476500-1.18591000$

C $3.10838000-3.84545400-1.13750400$

$\begin{array}{llll}0 & 2.70401100 & 2.26284700 & 2.02293400\end{array}$

H $3.07744700 \quad 2.08260500 \quad 0.00373800$

$\mathrm{H} \quad 4.21500300 \quad 1.22227200 \quad 1.07596800$

C $5.17408800-1.12142900-0.77834500$

H 2.43086800 $-4.51467200-0.59490100$

$\mathrm{H} \quad 3.96636700-3.62347500-0.49640900$

H $3.45045400-4.34948400-2.04010500$

C $3.39341400 \quad 3.47791900 \quad 2.15163000$

$\mathrm{H} \quad 6.07454100-1.54259900-0.33420800$

$\mathrm{H} \quad 4.89126100-1.70781100-1.65731000$

H $5.38142500-0.09223100-1.09370700$

H 2.98886600 3.99660200 3.01840200

H $3.25794300 \quad 4.10583700 \quad 1.26223800$

H $4.46722200 \quad 3.31261400 \quad 2.30033200$

C $0.07793200-0.41534500 \quad 0.31595400$

$\mathrm{H} \quad 0.10102900 \quad-0.95545500 \quad 1.27573500$

$\mathrm{H}-0.10826200-2.21709000-0.90293600$

$\mathrm{Ni}-1.74336800 \quad 0.20944500-0.03950100$

Cl $-3.91315100 \quad 0.77281500-0.47283100$

P $-2.63943000-1.76369700 \quad 0.83239300$

$\mathrm{P}-0.97322200 \quad 2.22736700-0.94417900$

C $-0.31042100 \quad 3.41668300 \quad 0.26831900$

$\mathrm{H}-1.10437700 \quad 3.67608300 \quad 0.96929800$

$\mathrm{H} \quad 0.51089500 \quad 2.95705300 \quad 0.81652600$

$\mathrm{H} \quad 0.02608800 \quad 4.32428200-0.23651300$

C $-2.23610300 \quad 3.22301400-1.80085800$

$\mathrm{H}-2.67436000 \quad 2.63744900-2.60792700$

$\mathrm{H}-3.03701200 \quad 3.46247800-1.10384100$

$\mathrm{H}-1.79351600 \quad 4.13784800-2.19859400$

C $-3.92377000-1.50200300 \quad 2.09664800$

$\mathrm{H}-4.68918900-0.85239300 \quad 1.67629300$

$\mathrm{H}-4.35968800-2.45300400 \quad 2.40611000$

$\mathrm{H}-3.48346400-1.00803100 \quad 2.96275800$

C $-1.57508900-3.01265900 \quad 1.64195600$

$\mathrm{H}-0.76561100-3.31513600 \quad 0.97758700$

$\mathrm{H}-1.13432200-2.58659600 \quad 2.54340300$

$\mathrm{H}-2.15955300-3.89226000 \quad 1.91515700$

C $-3.50198300-2.74195400-0.43945200$

$\mathrm{H}-3.99639600-3.60896200 \quad 0.00093100$

$\mathrm{H}-4.23470900-2.09526800-0.92066400$

$\mathrm{H}-2.78496700-3.07815700-1.18905900$

C $0.33346100 \quad 2.13981800 \quad-2.21742200$

$\mathrm{H} \quad 1.21246400 \quad 1.65719300-1.79699900$

$\mathrm{H}-0.02642100 \quad 1.55314200-3.06320500$

H $0.59032800 \quad 3.14162300 \quad-2.56578900$ 
PALLADIUM

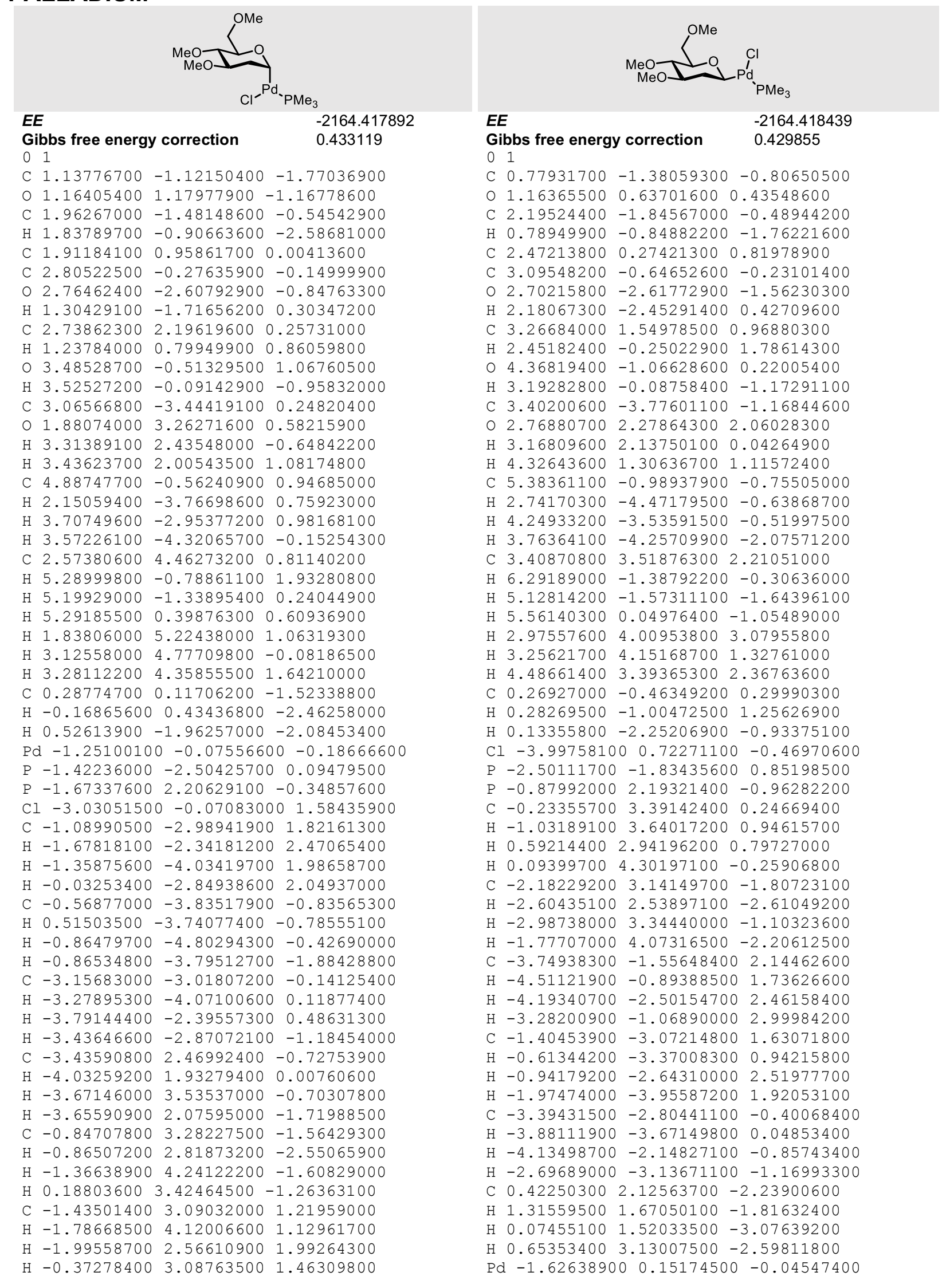




\section{PLATINUM}

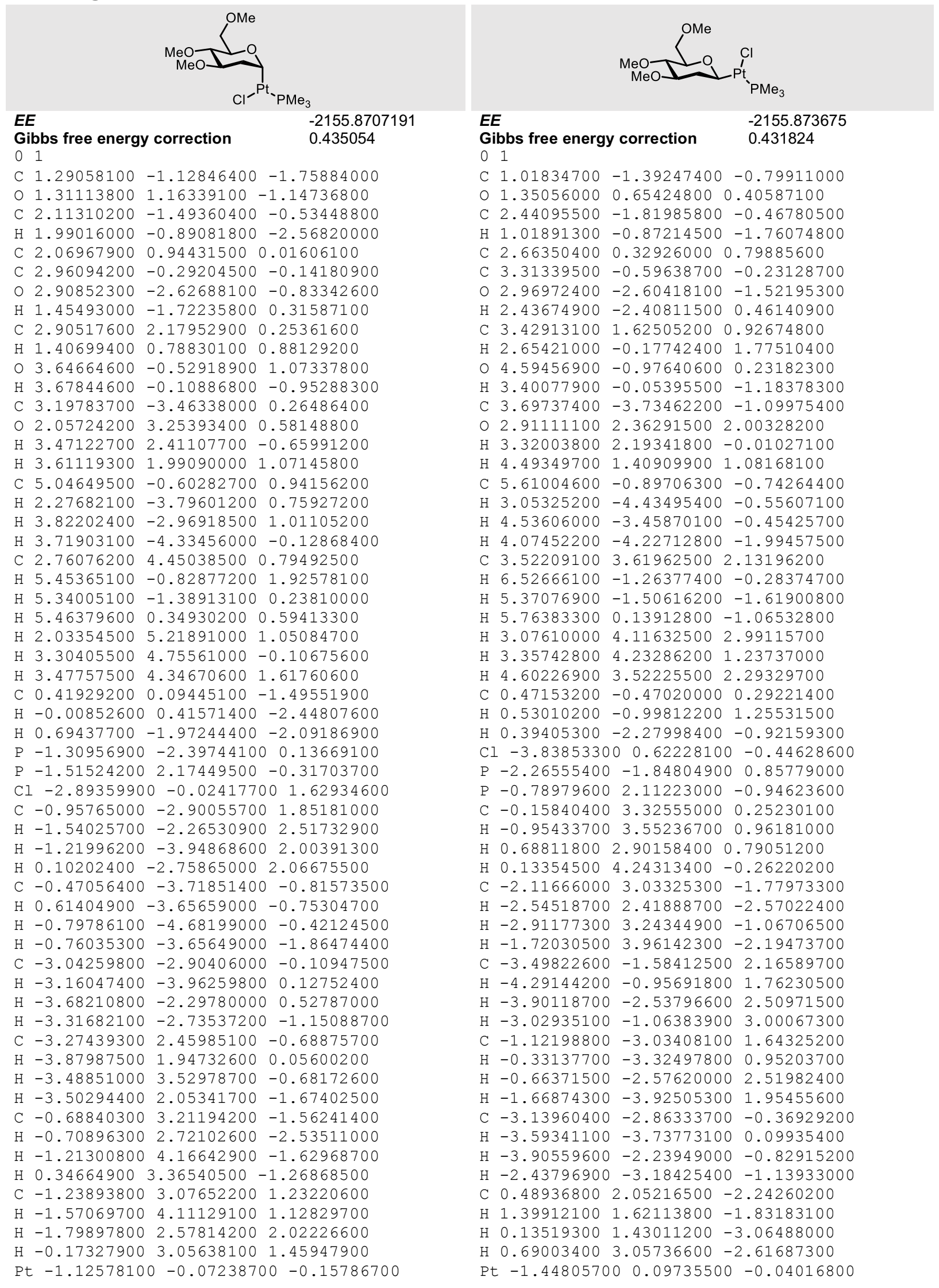


COPPER

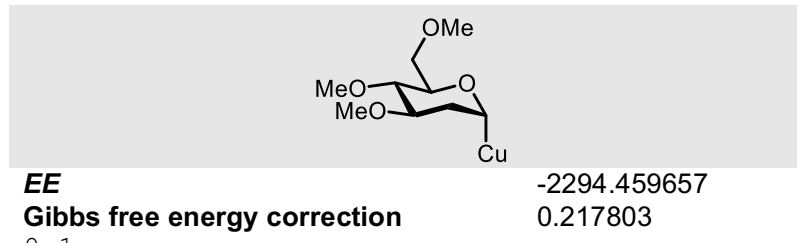

01

C $1.41730600 \quad 0.93657300 \quad 1.28867600$

$\begin{array}{llll}0 & -0.96600400 & 0.68567900 & 1.07998600\end{array}$

C $1.63200700-0.16426900 \quad 0.26667900$

$\mathrm{H} \quad 1.42229700 \quad 0.47576300 \quad 2.28523200$

C $-0.87184400-0.31820100 \quad 0.10267700$

C $0.43455600-1.10438300 \quad 0.25628200$

$\begin{array}{lllll}0 & 2.81468500 & -0.88406700 & 0.56502000\end{array}$

$\begin{array}{lllll}\mathrm{H} & 1.71264400 & 0.28158500 & -0.73546000\end{array}$

C $-2.07659100-1.21878100 \quad 0.23504200$

$\mathrm{H}-0.88480800 \quad 0.13807800-0.90274500$

$0 \quad 0.54415300-2.02811100-0.80962400$

$\mathrm{H} \quad 0.41087300-1.63498900 \quad 1.21787900$

C $3.61245400-1.18326500-0.55675500$

$\begin{array}{lllll}0 & -3.21152600 & -0.54515300 & -0.24538900\end{array}$

$\mathrm{H}-2.20573700-1.48239400 \quad 1.29424600$

$\mathrm{H}-1.90702800-2.13813400-0.33972300$

C $0.91620800-3.32788500-0.41243500$

$\mathrm{H} \quad 3.98201700-0.26739100-1.03138800$

$\mathrm{H} 3.06153800-1.76506300-1.30140300$

$\mathrm{H} \quad 4.46332300-1.76225600-0.20114000$

C $-4.38369700-1.29685100-0.08221900$

$\mathrm{H} 1.01770300-3.92513800-1.31714700$

$\mathrm{H} \quad 1.86574400 \quad-3.32257600 \quad 0.13068200$

$\mathrm{H} \quad 0.14812400 \quad-3.77836600 \quad 0.22680000$

$\mathrm{H}-5.20849100-0.71499800 \quad-0.48889800$

$\mathrm{H}-4.57887000-1.50312200 \quad 0.97686400$

$\mathrm{H}-4.32141200-2.25282600-0.61691600$

C 0.083622001 .626980001 .05008900

$\mathrm{H}-0.13454200 \quad 2.34303300 \quad 1.84085100$

$\mathrm{Cu} \quad 0.03030300 \quad 2.69428200 \quad-0.68594400$

H 2.243256001 .648320001 .24648500

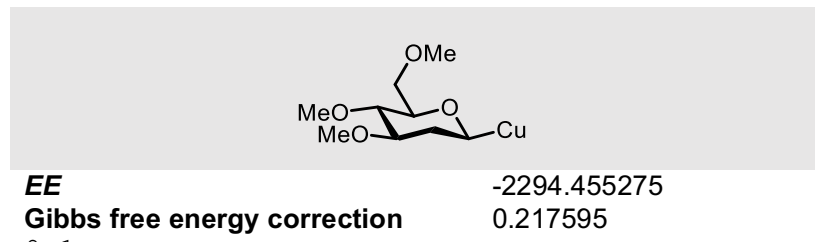

Gibbs
$0 \quad 1$

C $0.49864700-1.77851200 \quad 0.13896000$

$\begin{array}{lllll}0 & 0.98286000 & 0.58574800 & 0.12594200\end{array}$

C $-0.94590900-1.48129800-0.24823300$

H $0.55045500-1.85365400 \quad 1.22809600$

C $-0.31518300 \quad 0.94227300-0.30783900$

C $-1.33350300-0.08082500 \quad 0.20084100$

$\begin{array}{llll}0 & -1.80818800 & -2.44249300 & 0.33663100\end{array}$

$\mathrm{H}-1.04343100-1.51661400 \quad-1.34204100$

C $-0.61823900 \quad 2.32613400 \quad 0.21380400$

$\mathrm{H}-0.35324600 \quad 0.96389900-1.40699200$

$\begin{array}{lllll}0 & -2.61845600 & 0.25325700 & -0.29136900\end{array}$

$\mathrm{H}-1.33006000 \quad-0.05809000 \quad 1.29932500$

C $-2.82067900-2.91544100-0.52093100$

$\begin{array}{lllll}0 & 0.12900400 & 3.27099000 & -0.50868100\end{array}$

$\mathrm{H}-0.35540600 \quad 2.36413800 \quad 1.28076600$

$\mathrm{H}-1.69176800 \quad 2.52744500 \quad 0.10716100$

C $-3.63881900 \quad 0.20561100 \quad 0.67849900$

$\mathrm{H}-2.39217900-3.43689100-1.38418500$

$\mathrm{H}-3.45581800-2.10242500-0.88418300$

$\mathrm{H}-3.42373800 \quad-3.61947800 \quad 0.05057700$

C $-0.06993000 \quad 4.57572700 \quad-0.03764400$

$\mathrm{H}-4.57712400 \quad 0.42576400 \quad 0.17167500$

$\mathrm{H}-3.69918100-0.78160300 \quad 1.14610900$

$\mathrm{H}-3.47310800 \quad 0.95557400 \quad 1.46073700$

H $0.52961800 \quad 5.24425400-0.65250800$

$\mathrm{H} \quad 0.24431200 \quad 4.67314500 \quad 1.00866700$

$\mathrm{H}-1.12434000 \quad 4.87006100-0.11175700$

C $1.41305800-0.67433000-0.37059500$

$\mathrm{Cu} 3.34218000-0.87288300 \quad 0.13759400$

$\mathrm{H} 1.38279400-0.65120200-1.46997000$

$\mathrm{H} \quad 0.79057800-2.74676000-0.27304800$ 


\section{SILVER}

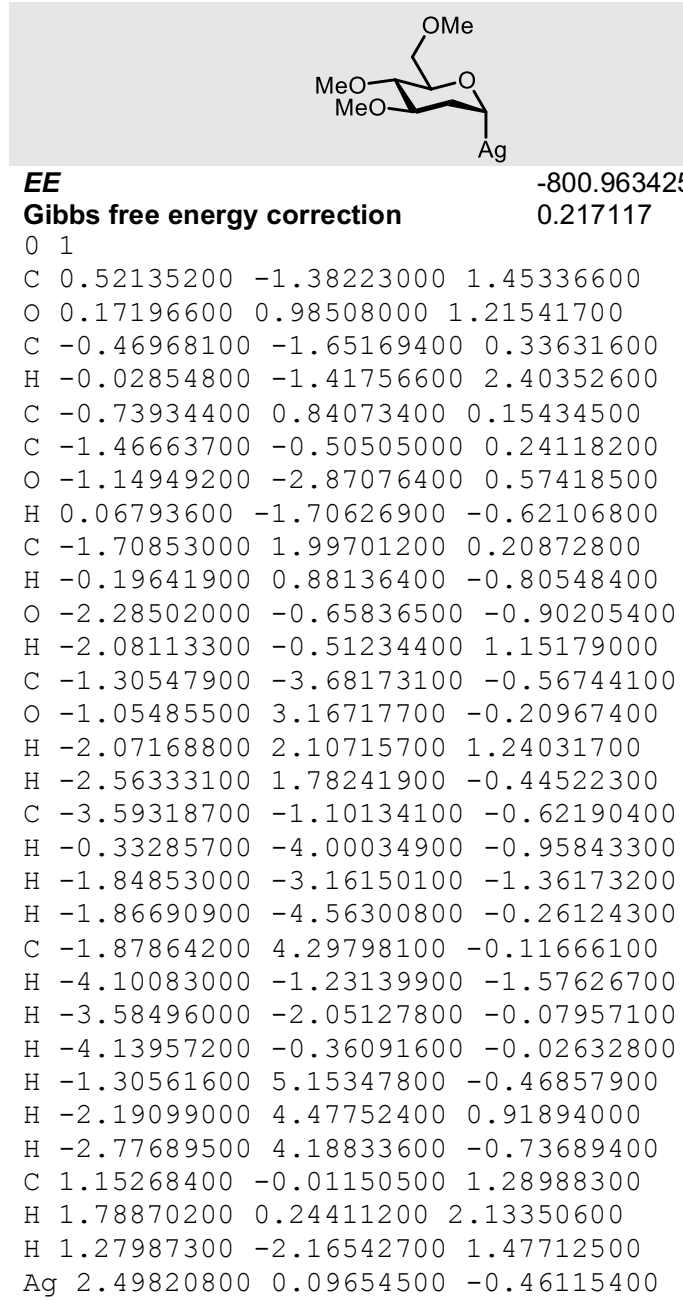

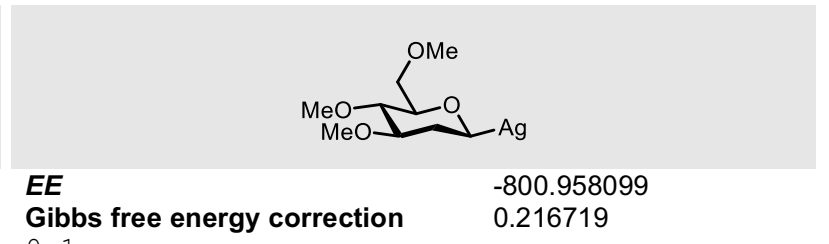

$\begin{array}{ll}0 & 1\end{array}$

C $-0.31679100-1.63818700-0.09794300$

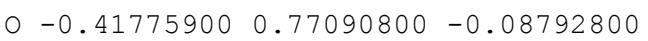

$\begin{array}{llll}\text { C } 1.16447700 & -1.57436500 & 0.26294800\end{array}$

$\mathrm{H}-0.39986500-1.70537000 \quad-1.18529700$

$\begin{array}{llll}\text { C } & 0.92931500 & 0.91902900 & 0.32218600\end{array}$

$\begin{array}{llll}\text { C } 1.76240700 & -0.25416700 & -0.19888700\end{array}$

$\begin{array}{llll}0 & 1.85057100 & -2.66112800 & -0.33396000\end{array}$

H $1.27527400-1.62369600 \quad 1.35490100$

C $1.43660000 \quad 2.23611200-0.21300900$

$\mathrm{H} \quad 0.98924400 \quad 0.93761400 \quad 1.42024600$

$\begin{array}{llll}0 & 3.09209300 & -0.12796800 & 0.27041800\end{array}$

$\mathrm{H} \quad 1.74352200-0.23330300-1.29722100$

$\begin{array}{llll}\text { C } 2.78949500 & -3.29041300 & 0.50699500\end{array}$

$\begin{array}{lllll}0 & 0.85994700 & 3.28860400 & 0.51645700\end{array}$

$\mathrm{H} 1.16422500 \quad 2.31115600 \quad-1.27556300$

H $2.53016800 \quad 2.26584900-0.12563100$

C $4.07539300-0.33786100-0.71633800$

$\mathrm{H} 2.29825800-3.73827400 \quad 1.37804200$

H $3.55190400 \quad-2.58913100 \quad 0.85793500$

H $3.26295500 \quad-4.08051100 \quad-0.07392700$

C $1.24810300 \quad 4.54473700 \quad 0.03094300$

H $5.04515200-0.26827900-0.22602200$

$\mathrm{H} 3.97058500-1.32285600-1.18071800$

$\mathrm{H} \quad 4.01682700 \quad 0.42783800-1.49864500$

$\mathrm{H} \quad 0.77205100 \quad 5.30070300 \quad 0.65241100$

$\mathrm{H} 0.93043300 \quad 4.68429900-1.00947100$

$\mathrm{H} \quad 2.33660000 \quad 4.67282700 \quad 0.08144600$

C $-1.02717500-0.40079400 \quad 0.42249800$

$\mathrm{H}-0.99123000 \quad-0.37796000 \quad 1.51957700$

$\mathrm{H}-0.75366700 \quad-2.54488100 \quad 0.32482600$

Ag $-3.13174900-0.28731700-0.09687600$ 
GOLD

\begin{tabular}{|c|c|c|c|}
\hline \multicolumn{2}{|c|}{$E E$} & \multicolumn{2}{|r|}{-789.69187} \\
\hline \multicolumn{3}{|c|}{$\begin{array}{l}\text { Gibbs free energy correction } \\
01\end{array}$} & \\
\hline C & 0.09680500 & -1.37678800 & 1.43918500 \\
\hline O & -0.24567500 & 0.99544400 & 1.18616300 \\
\hline C & -0.89734700 & -1.64746500 & $0 \quad 0.32497200$ \\
\hline H & -0.44312000 & -1.41174000 & 2.39313300 \\
\hline $\mathrm{C}$ & -1.17555700 & 0.83856300 & 0.12947100 \\
\hline $\mathrm{C}$ & -1.89952700 & -0.50522600 & 0.24122200 \\
\hline 0 & -1.56537300 & -2.86850400 & 0.57552300 \\
\hline $\mathrm{H}$ & -0.36559400 & -1.69935400 & -0.63448700 \\
\hline C & -2.14242700 & 1.99523500 & 0.19887800 \\
\hline $\mathrm{H}$ & -0.64116900 & 0.87611500 & -0.83085200 \\
\hline 0 & -2.73501200 & -0.66481700 & $0-0.88653200$ \\
\hline $\mathrm{H}$ & -2.49875600 & -0.50774800 & 1.16233700 \\
\hline $\mathrm{C}$ & -1.72917700 & -3.68520600 & -0.56265500 \\
\hline 0 & -1.49267600 & 3.16593000 & -0.21860300 \\
\hline $\mathrm{H}$ & -2.49824700 & 2.09939700 & 1.23401800 \\
\hline $\mathrm{H}$ & -3.00220900 & 1.78089600 & -0.44855700 \\
\hline $\mathrm{C}$ & -4.03525500 & -1.11927400 & $0-0.58473800$ \\
\hline $\mathrm{H}$ & -0.75895600 & -3.99759100 & -0.96320800 \\
\hline $\mathrm{H}$ & -2.28520300 & -3.17042500 & -1.35119500 \\
\hline $\mathrm{H}$ & -2.28115000 & -4.56849400 & -0.24595100 \\
\hline C & -2.31845000 & 4.29571400 & -0.12317900 \\
\hline $\mathrm{H}$ & -4.55627900 & -1.25372900 & $0-1.53101700$ \\
\hline $\mathrm{H}$ & -4.00961400 & -2.06886000 & -0.04267600 \\
\hline $\mathrm{H}$ & -4.57772300 & -0.38289800 & 0.01910400 \\
\hline $\mathrm{H}$ & -1.74635800 & 5.15189000 & -0.47439300 \\
\hline $\mathrm{H}$ & -2.62987600 & 4.47316000 & 0.91302400 \\
\hline $\mathrm{H}$ & -3.21669300 & 4.18528200 & -0.74302300 \\
\hline C & 0.71477900 & 0.00424000 & 1.29919200 \\
\hline $\mathrm{H}$ & 1.33114100 & 0.26405500 & 2.15721900 \\
\hline & 0.86476100 & -2.14933200 & 1.45880100 \\
\hline & u 2.04989700 & & \\
\hline
\end{tabular}

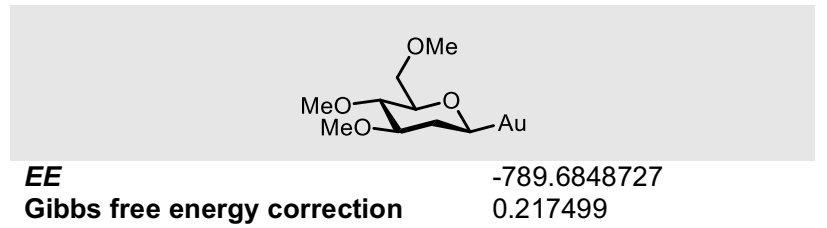

Gibbs free energy correction $\quad 0.217499$

01

C $0.07699200-1.56513600-0.11450100$

$\begin{array}{llll}0 & 0.12495600 & 0.84177500 & -0.10011200\end{array}$

$\begin{array}{llll}\text { C } 1.55689600 & -1.59070200 & 0.25753800\end{array}$

$\mathrm{H}-0.01072900-1.61059400-1.20140300$

$\begin{array}{llll}\text { C } 1.48033800 & 0.91048000 & 0.31565800\end{array}$

C $2.24110400-0.31161700-0.20250400$

$\begin{array}{llll}0 & 2.16927200 & -2.72026600 & -0.33214700\end{array}$

H $1.65814600 \quad-1.64318300 \quad 1.35047800$

C $2.05943400 \quad 2.19707200-0.21924600$

H $1.53661300 \quad 0.92775900 \quad 1.41349600$

$\begin{array}{llll}0 & 3.56940500 & -0.26874300 & 0.27894300\end{array}$

H $2.23064200-0.29006600-1.30072400$

C $3.06000100-3.41112900 \quad 0.51515800$

$\begin{array}{llll}0 & 1.55094300 & 3.27501100 & 0.52073700\end{array}$

$\mathrm{H} \quad 1.78408900 \quad 2.29175200-1.27922200$

H $3.15343300 \quad 2.15935500-0.14017600$

$\begin{array}{llll}\text { C } 4.54847500 & -0.54308400 & -0.69783800\end{array}$

H $2.53395400-3.82534200 \quad 1.38208300$

H $3.86479500 \quad-2.76149300 \quad 0.87025800$

H $3.48255100 \quad-4.23011400 \quad-0.06440300$

C $1.99106300 \quad 4.51244300 \quad 0.02857500$

H $5.51484400-0.53272800-0.19669700$

$\mathrm{H} \quad 4.38674900-1.52098700-1.16042700$

$\mathrm{H} 4.54564000 \quad 0.22260200-1.48187000$

H $1.56587600 \quad 5.28853500 \quad 0.66157800$

$\mathrm{H} 1.65804900 \quad 4.66950000-1.00414300$

$\mathrm{H} 3.08523200 \quad 4.58559300 \quad 0.05689400$

C $-0.54138200-0.28214000 \quad 0.41353700$

$\mathrm{H}-0.51468200-0.25956900 \quad 1.51072300$

H $-0.42197200 \quad-2.44027500 \quad 0.30289100$

$\mathrm{Au}-2.50895400-0.12088700-0.05616400$ 
ZINC
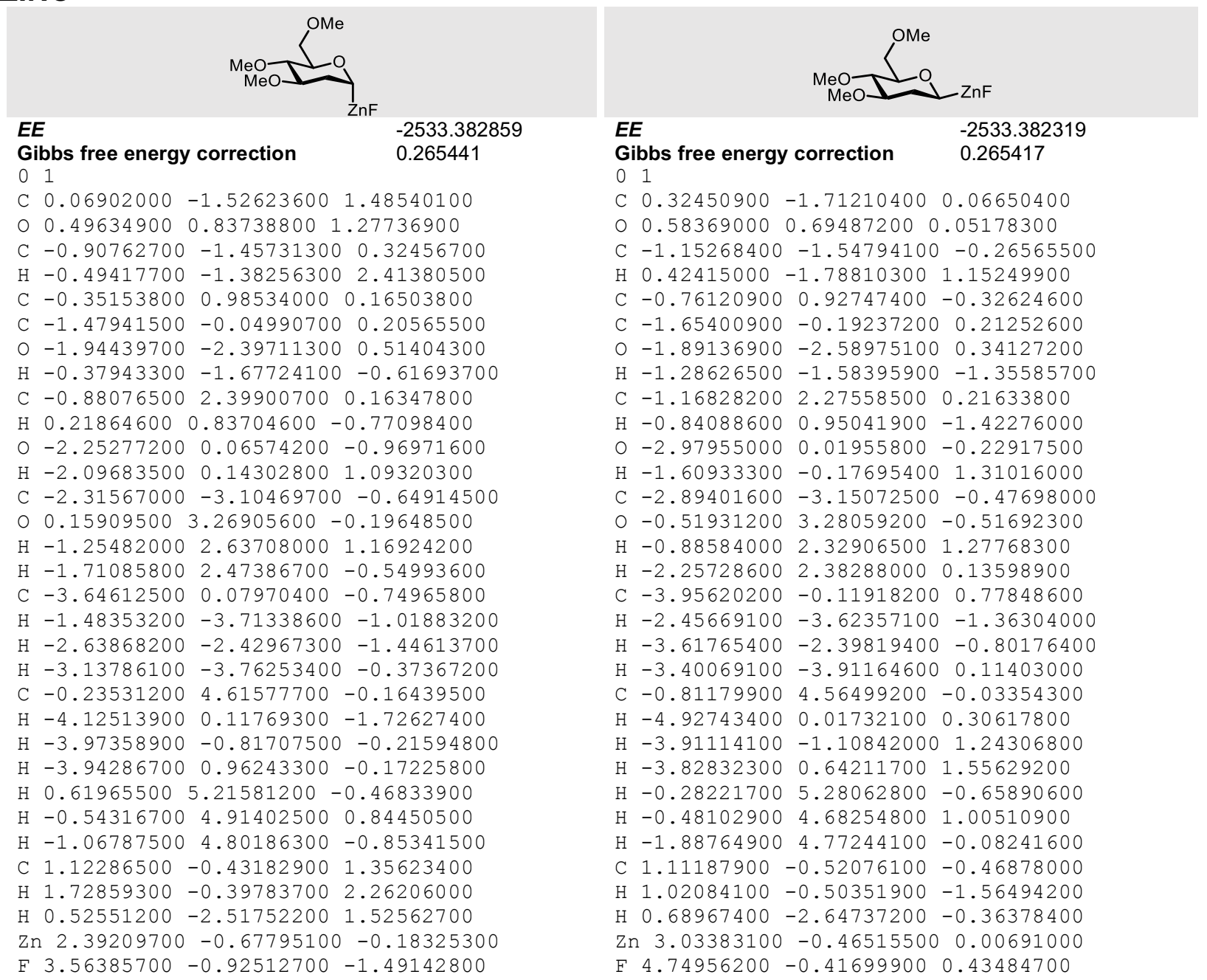

01

C $0.32450900-1.71210400 \quad 0.06650400$

$\begin{array}{llllll}0 & 0.58369000 & 0.69487200 & 0.05178300\end{array}$

C $-1.15268400-1.54794100-0.26565500$

$\mathrm{H} \quad 0.42415000-1.78810300 \quad 1.15249900$

C $-0.76120900 \quad 0.92747400-0.32624600$

C $-1.65400900 \quad-0.19237200 \quad 0.21252600$

$\begin{array}{lllll}0 & -1.89136900 & -2.58975100 & 0.34127200\end{array}$

$\mathrm{H}-1.28626500-1.58395900-1.35585700$

C $-1.16828200 \quad 2.27558500 \quad 0.21633800$

$\mathrm{H}-0.84088600 \quad 0.95041900-1.42276000$

$\begin{array}{lllll}0 & -2.97955000 & 0.01955800 & -0.22917500\end{array}$

$\mathrm{H}-1.60933300 \quad-0.17695400 \quad 1.31016000$

C $-2.89401600-3.15072500-0.47698000$

$\begin{array}{llll}0 & -0.51931200 & 3.28059200 & -0.51692300\end{array}$

$\mathrm{H}-0.88584000 \quad 2.32906500 \quad 1.27768300$

H $-2.25728600 \quad 2.38288000 \quad 0.13598900$

C $-3.95620200-0.11918200 \quad 0.77848600$

$\mathrm{H}-2.45669100-3.62357100-1.36304000$

$\mathrm{H}-3.61765400-2.39819400 \quad-0.80176400$

$\mathrm{H}-3.40069100-3.91164600 \quad 0.11403000$

C $-0.81179900 \quad 4.56499200 \quad-0.03354300$

$\mathrm{H}-4.92743400 \quad 0.01732100 \quad 0.30617800$

$\mathrm{H}-3.91114100-1.10842000 \quad 1.24306800$

$\mathrm{H}-3.82832300 \quad 0.64211700 \quad 1.55629200$

$\mathrm{H}-0.28221700 \quad 5.28062800-0.65890600$

$\mathrm{H}-0.48102900 \quad 4.68254800 \quad 1.00510900$

H $-1.88764900 \quad 4.77244100-0.08241600$

C $1.11187900-0.52076100-0.46878000$

$\mathrm{H} 1.02084100-0.50351900-1.56494200$

$\mathrm{H} \quad 0.68967400 \quad-2.64737200 \quad-0.36378400$

Zn $3.03383100-0.46515500 \quad 0.00691000$

F $4.74956200-0.41699900 \quad 0.43484700$ 
CADMIUM
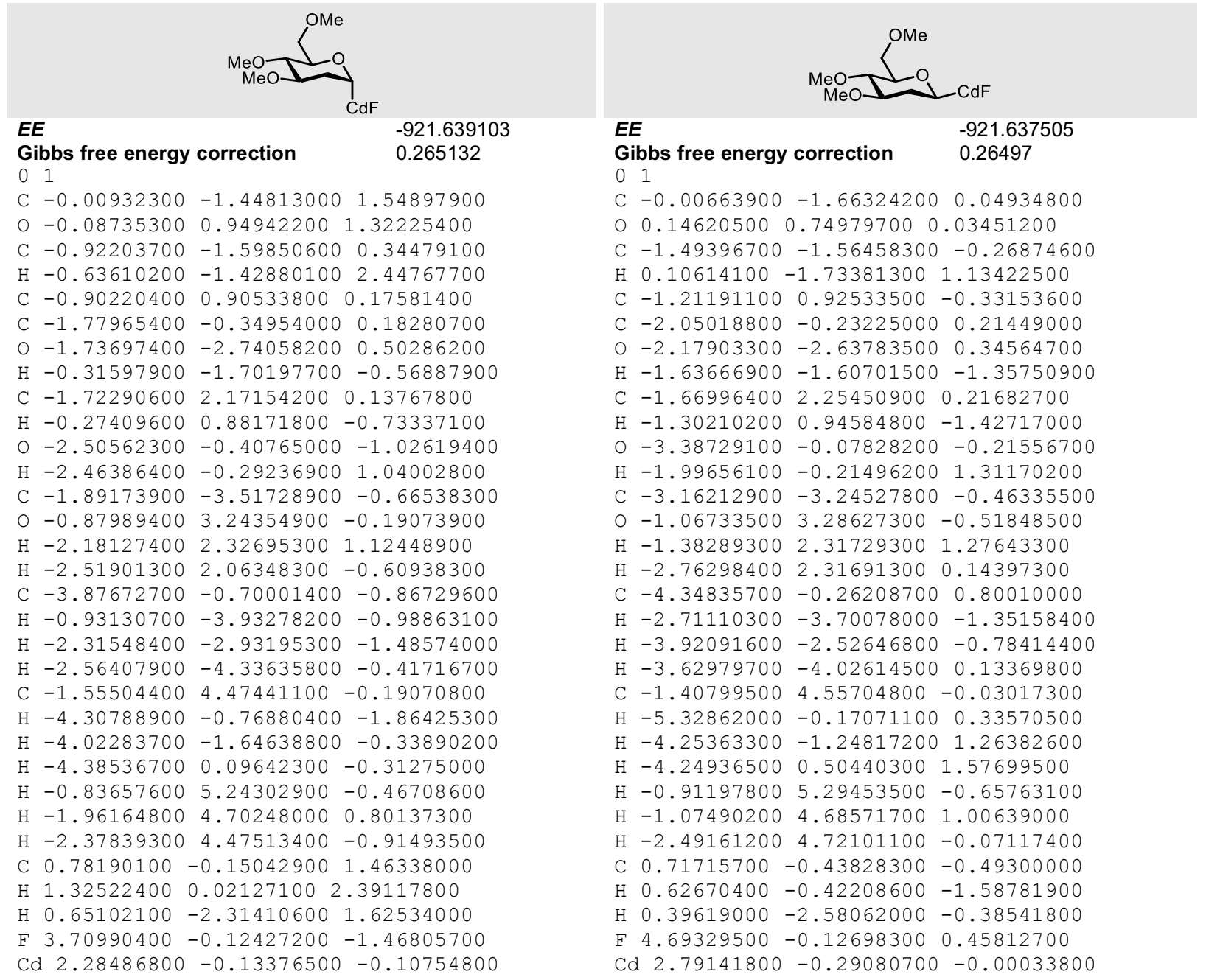

Gibbs free energy correction $\quad 0.26497$

01

C $-0.00663900-1.66324200 \quad 0.04934800$

$\begin{array}{llllll}0 & 0.14620500 & 0.74979700 & 0.03451200\end{array}$

C $-1.49396700-1.56458300-0.26874600$

H $0.10614100-1.73381300 \quad 1.13422500$

C $-1.21191100 \quad 0.92533500-0.33153600$

C $-2.05018800 \quad-0.23225000 \quad 0.21449000$

$\begin{array}{lllll}0 & -2.17903300 & -2.63783500 & 0.34564700\end{array}$

$\mathrm{H}-1.63666900-1.60701500-1.35750900$

C $-1.66996400 \quad 2.25450900 \quad 0.21682700$

$\mathrm{H}-1.30210200 \quad 0.94584800-1.42717000$

$\begin{array}{lllll}0 & -3.38729100 & -0.07828200 & -0.21556700\end{array}$

$\mathrm{H}-1.99656100-0.21496200 \quad 1.31170200$

C $-3.16212900-3.24527800-0.46335500$

$0-1.06733500 \quad 3.28627300-0.51848500$

$\mathrm{H}-1.38289300 \quad 2.31729300 \quad 1.27643300$

$\mathrm{H}-2.76298400 \quad 2.31691300 \quad 0.14397300$

C $-4.34835700 \quad-0.26208700 \quad 0.80010000$

$\mathrm{H}-2.71110300-3.70078000-1.35158400$

$\mathrm{H}-3.92091600 \quad-2.52646800 \quad-0.78414400$

$\mathrm{H}-3.62979700 \quad-4.02614500 \quad 0.13369800$

C $-1.40799500 \quad 4.55704800-0.03017300$

$\mathrm{H}-5.32862000 \quad-0.17071100 \quad 0.33570500$

$\mathrm{H}-4.25363300 \quad-1.24817200 \quad 1.26382600$

$\mathrm{H}-4.24936500 \quad 0.50440300 \quad 1.57699500$

$\mathrm{H}-0.91197800 \quad 5.29453500-0.65763100$

$\mathrm{H}-1.07490200 \quad 4.68571700 \quad 1.00639000$

$\mathrm{H}-2.49161200 \quad 4.72101100-0.07117400$

C $0.71715700-0.43828300-0.49300000$

$\mathrm{H} \quad 0.62670400-0.42208600-1.58781900$

H $0.39619000-2.58062000-0.38541800$

F $4.69329500-0.12698300 \quad 0.45812700$

$\begin{array}{lllll}\mathrm{Cd} 2.79141800 & -0.29080700 & -0.00033800\end{array}$ 
MERCURY

\begin{tabular}{|c|c|c|c|}
\hline \multirow{2}{*}{\multicolumn{2}{|c|}{ EF }} & \multicolumn{2}{|r|}{$\mathrm{HgF}$} \\
\hline & & & -907.31165 \\
\hline \multicolumn{3}{|c|}{ Gibbs free energy correction } & 0.26597 \\
\hline C & -0.27031200 & -1.41335800 & 1.52353000 \\
\hline 0 & -0.48740300 & 0.97642000 & 1.28037100 \\
\hline $\mathrm{C}$ & -1.19005000 & -1.62380400 & 0.33340400 \\
\hline $\mathrm{H}$ & -0.88062900 & -1.42719600 & 2.43236500 \\
\hline C & -1.32693600 & 0.87320400 & 0.14841200 \\
\hline C & -2.12627600 & -0.43145700 & 0.18580800 \\
\hline O & -1.92736400 & -2.81348700 & 0.51418700 \\
\hline $\mathrm{H}$ & -0.59524500 & -1.69329900 & -0.58970900 \\
\hline C & -2.22608400 & 2.08520100 & 0.13319100 \\
\hline $\mathrm{H}$ & -0.71992500 & 0.88488800 & -0.77149500 \\
\hline 0 & -2.87398000 & -0.54112800 & -1.00550500 \\
\hline $\mathrm{H}$ & -2.79312900 & -0.41044200 & 1.05844500 \\
\hline $\mathrm{C}$ & -2.05481500 & -3.60649500 & -0.64715000 \\
\hline 0 & -1.46225900 & 3.21009500 & -0.20820100 \\
\hline $\mathrm{H}$ & -2.67328900 & 2.20770300 & 1.12995100 \\
\hline $\mathrm{H}$ & -3.02928200 & 1.92672400 & -0.59722000 \\
\hline C & -4.22050700 & -0.91763300 & -0.81486700 \\
\hline $\mathrm{H}$ & -1.07633200 & -3.96258300 & -0.98611200 \\
\hline $\mathrm{H}$ & -2.52998400 & -3.05398100 & -1.46191100 \\
\hline $\mathrm{H}$ & -2.66969000 & -4.46414300 & -0.38122800 \\
\hline $\mathrm{C}$ & -2.21747200 & 4.39353700 & -0.19448300 \\
\hline $\mathrm{H}$ & -4.66784600 & -1.01857300 & -1.80184100 \\
\hline $\mathrm{H}$ & -4.29574400 & -1.86840100 & -0.27962400 \\
\hline $\mathrm{H}$ & -4.76547400 & -0.15114500 & -0.25271800 \\
\hline $\mathrm{H}$ & -1.55596600 & 5.20813600 & -0.48086800 \\
\hline $\mathrm{H}$ & -2.62191200 & 4.59314600 & 0.80463100 \\
\hline $\mathrm{H}$ & -3.05079600 & 4.34033000 & -0.90519400 \\
\hline C & $0.43451400-$ & -0.06681400 & 1.43282600 \\
\hline $\mathrm{H}$ & 0.96854700 & 0.143969002 & .35766500 \\
\hline $\mathrm{H}$ & 0.44646100 & -2.23326000 & 1.59289000 \\
\hline & 3.32731600 & 0.03042000 & -1.44498700 \\
\hline & & & \\
\hline
\end{tabular}

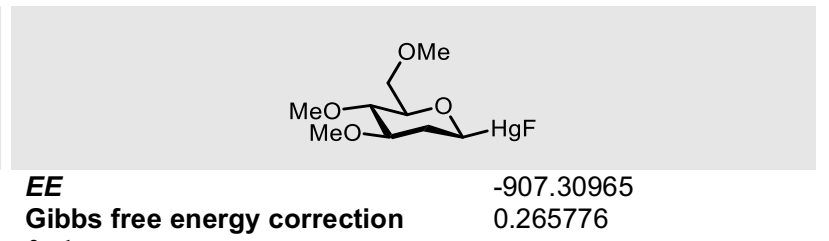

$\begin{array}{ll}0 & 1\end{array}$

$\begin{array}{llll}C & -0.35095300 & -1.59442900 & 0.06553300\end{array}$

$\begin{array}{lllll}0 & -0.33474600 & 0.82031800 & 0.05347200\end{array}$

C $-1.83845000-1.58033900-0.26411600$

$\mathrm{H}-0.23759900-1.65172300 \quad 1.15062700$

C $-1.69893300 \quad 0.92062500-0.32241400$

C $-2.47454900-0.28291700 \quad 0.21675600$

$\begin{array}{lllll}0 & -2.46118100 & -2.69289700 & 0.34330800\end{array}$

$\mathrm{H}-1.97117800-1.62843500-1.35403000$

C $-2.23069400 \quad 2.22263900 \quad 0.22399900$

$\mathrm{H}-1.78267300 \quad 0.93835300-1.41847400$

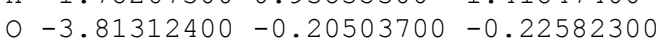

$\mathrm{H}-2.43087500-0.26412000 \quad 1.31428200$

C $-3.40241400-3.35622600-0.47256500$

$\begin{array}{lllll}0 & -1.68947600 & 3.28365100 & -0.51615400\end{array}$

$\mathrm{H}-1.94544500 \quad 2.30394800 \quad 1.28269100$

$\mathrm{H}-3.32567800 \quad 2.22130000 \quad 0.15323000$

C $-4.77242400 \quad-0.44413600 \quad 0.78070500$

$\mathrm{H}-2.91983900-3.78313200-1.35823100$

$\mathrm{H}-4.20014700 \quad-2.68268400 \quad-0.79668400$

$\mathrm{H}-3.82628500 \quad-4.16382000 \quad 0.12138800$

C $-2.09166900 \quad 4.53581200 \quad-0.02546900$

$\mathrm{H}-5.75135700 \quad-0.40944500 \quad 0.30615500$

$\mathrm{H}-4.62550100-1.42319700 \quad 1.24556000$

$\mathrm{H}-4.72527700 \quad 0.32679200 \quad 1.55787900$

$\mathrm{H}-1.64080300 \quad 5.29697400-0.65851500$

$\mathrm{H}-1.75548800 \quad 4.68284000 \quad 1.00748500$

$\mathrm{H}-3.18268500 \quad 4.64254800-0.05644400$

C $0.29779300-0.32663200-0.47379100$

$\mathrm{H} \quad 0.21813400-0.31250200-1.56889300$

$\mathrm{H} \quad 0.11000500-2.48499000 \quad-0.36625700$

F $4.21452300 \quad 0.03330000 \quad 0.45410700$

Hg $2.29780000-0.15153800-0.00323700$ 


\section{NICKEL}

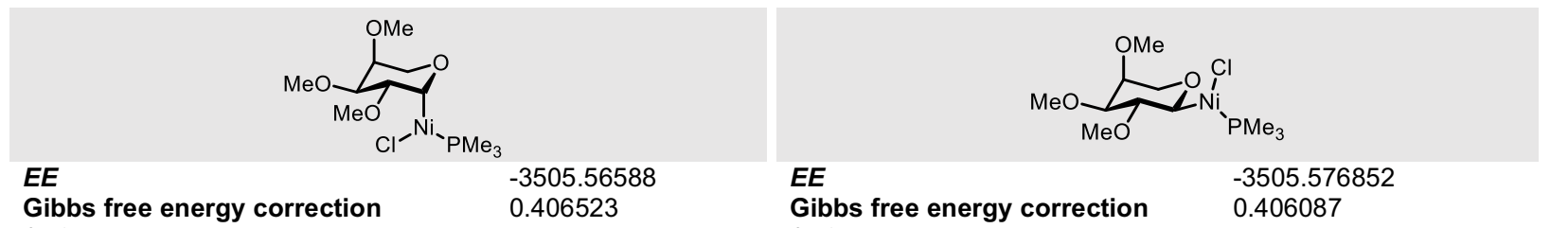

$\begin{array}{ll}0 & 1\end{array}$

C $2.23060100-1.40733700-1.02274700$

C $0.77437200-1.86227100-1.09994500$

C $0.16409100-0.82039900 \quad 0.93021200$

C $1.60314700-0.368035001 .15171800$

C $2.29732900-0.12638800-0.18625800$

$\mathrm{H} \quad 0.20754100-1.10905100-1.67260100$

$\mathrm{H} \quad 0.69709400-2.80972200-1.63589600$

H 2.57051000 -1.20814900 -2.05157400

$\mathrm{H}-0.20885800-1.11585300 \quad 1.91453500$

$\mathrm{H} 2.15900800-1.16985300 \quad 1.65697400$

$\mathrm{H} 1.74622300 \quad 0.64050300-0.74942200$

○ $0.21504100-2.05170400 \quad 0.17026400$

Cl $-2.83988500 \quad 1.22018600-1.28203300$

$\mathrm{P}-0.14560300 \quad 2.49017600-0.20058000$

$\mathrm{P}-2.69695400-1.43797000 \quad 0.39240200$

C $-2.37915000-2.70034800 \quad 1.67136400$

$\mathrm{H}-3.16019500-3.46173600 \quad 1.64955100$

$\mathrm{H}-1.40917500-3.16013700 \quad 1.49243900$

$\mathrm{H}-2.37443900-2.22927600 \quad 2.65473400$

C $-2.88908900-2.42443900-1.12531700$

$\mathrm{H}-1.93533900-2.89807100-1.35654400$

$\mathrm{H}-3.65493800-3.19131700-1.00010800$

$\mathrm{H}-3.16610900-1.75296700-1.93771100$

C $-4.40854600-0.94456500 \quad 0.78124800$

$\mathrm{H}-5.05043100-1.82350100 \quad 0.85845400$

$\mathrm{H}-4.42329700-0.40602400 \quad 1.72894600$

$\mathrm{H}-4.77026700-0.27931400 \quad 0.00020000$

C $-1.32041100 \quad 3.78726000 \quad 0.32615400$

$\mathrm{H}-0.93115900 \quad 4.77462400 \quad 0.07275000$

$\mathrm{H}-2.27799500 \quad 3.62291900-0.16242500$

$\mathrm{H}-1.45764500 \quad 3.72559700 \quad 1.40599600$

C $0.08406400 \quad 2.86022900-1.97756200$

$\mathrm{H}-0.83342700 \quad 2.61961400-2.51086800$

$\mathrm{H} \quad 0.33545900 \quad 3.91269600-2.11780800$

$\mathrm{H} \quad 0.89646200 \quad 2.24923400 \quad-2.37512500$

C $1.405398003 .18056700 \quad 0.48675100$

$\mathrm{H} \quad 1.32459500 \quad 3.23237800 \quad 1.57031400$

H 2.26668700 2.55747500 0.25016100

$\mathrm{H} 1.54902600 \quad 4.18317900 \quad 0.07920700$

$\mathrm{Ni}-1.20389400 \quad 0.33195000 \quad 0.07210700$

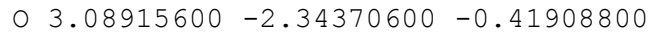

$\begin{array}{lllll}0 & 3.60646200 & 0.32979400 & 0.05401300\end{array}$

O $1.56143800 \quad 0.77087100 \quad 1.97975700$

C $4.35785400 \quad 0.57179200-1.10785100$

$\mathrm{H} 3.80012600 \quad 1.19684800-1.81795900$

$\mathrm{H} 5.259630001 .10063600-0.80541800$

$\mathrm{H} \quad 4.64381400-0.36131900-1.60241300$

C $3.18724800-3.57269200-1.09179800$

$\mathrm{H} \quad 4.04969300-4.09458700-0.68171200$

H 2.29840100 -4.19241100 -0.94205200

$\mathrm{H} \quad 3.33967700 \quad-3.42484000 \quad-2.16847300$

C $2.65435800 \quad 0.91462800 \quad 2.85869300$

$\mathrm{H} \quad 2.76724700 \quad 0.02449300 \quad 3.48706500$

H $3.58665100 \quad 1.08753400 \quad 2.31876000$

$\mathrm{H} 2.43648700 \quad 1.76950200 \quad 3.49870700$

\section{Gibbs free energy correction $\quad 0.406087$}

$0 \quad 1$

C $-3.25242800-0.33627600-0.89168000$

C $-2.31683500 \quad 0.19017000-1.97441100$

C $-0.35299000-0.30017400-0.76122100$

C $-1.17147100-0.86422900 \quad 0.40258500$

C $-2.51932700-1.39860700-0.06762500$

$\mathrm{H}-2.09510400-0.63014800-2.67241400$

$\mathrm{H}-2.79338700 \quad 0.99052700 \quad-2.54297800$

$\mathrm{H}-4.13180700-0.77845600-1.38514100$

$\mathrm{H}-1.36740900-0.07326800 \quad 1.13567300$

$\mathrm{H}-2.32868900-2.26080800-0.72719000$

$\begin{array}{lllll}0 & -1.12979600 & 0.70660400 & -1.43024200\end{array}$

$0-3.67733400 \quad 0.66370300 \quad 0.00714800$

o $-3.24136300-1.82169300 \quad 1.06291400$

o $-0.43373600-1.91402100 \quad 1.00850100$

C $-4.51239200-2.34729200 \quad 0.78146500$

$\mathrm{H}-4.46319900-3.10653800-0.00892700$

$\mathrm{H}-4.87839300-2.81306900 \quad 1.69440600$

$\mathrm{H}-5.21438800-1.56297700 \quad 0.48044200$

C $-4.47431700 \quad 1.66948600 \quad-0.56490900$

$\mathrm{H}-4.92623500 \quad 2.22589200 \quad 0.25419600$

$\mathrm{H}-3.88675200 \quad 2.36197500 \quad-1.17647700$

$\mathrm{H}-5.26955300 \quad 1.23888200-1.18559400$

C $-0.43276600-1.87266300 \quad 2.41859000$

H $0.12121000 \quad-0.99681500 \quad 2.77880100$

$\mathrm{H}-1.45093200-1.84885100 \quad 2.81143000$

$\mathrm{H} \quad 0.06560300 \quad-2.77504400 \quad 2.77147000$

$\mathrm{H}-0.21732300-1.11694000-1.48734300$

$\mathrm{Ni} 1.39214300 \quad 0.35245300-0.13408300$

Cl $3.44554000 \quad 1.02207400 \quad 0.59550200$

P 2.40417100 -1.71244700 -0.52686600

P $0.52437400 \quad 2.48297400 \quad 0.31422400$

C $3.90966600-1.62440900-1.55246300$

$\mathrm{H} \quad 4.58887300-0.89986500-1.10693000$

$\mathrm{H} \quad 4.38974300-2.60201000-1.61837300$

H $3.64688700-1.28576500-2.55480100$

C $1.49855900-3.09254600-1.30701000$

$\mathrm{H} 0.60127100 \quad-3.30189500-0.72592400$

$\mathrm{H} 1.20900000-2.81811600-2.32186900$

H 2.12846000 $-3.98241400-1.34853900$

C $3.00687300-2.46050100 \quad 1.01904700$

$\mathrm{H} 2.15130900-2.68833400 \quad 1.65350100$

H $3.56449000-3.37624300 \quad 0.81703300$

H $3.64499700 \quad-1.73636500 \quad 1.52373900$

C $1.54360300 \quad 3.54615300 \quad 1.38701400$

$\mathrm{H} 2.52787300 \quad 3.66884000 \quad 0.94015200$

H $1.06276500 \quad 4.51614400 \quad 1.52442900$

$\mathrm{H} 1.68165300 \quad 3.06081500 \quad 2.35261200$

C $-1.12061600 \quad 2.67288400 \quad 1.07901300$

$\mathrm{H}-1.86686400 \quad 2.13199100 \quad 0.50070000$

$\mathrm{H}-1.09787600 \quad 2.26042700 \quad 2.08882800$

$\mathrm{H}-1.38356100 \quad 3.73058800 \quad 1.13896700$

C $0.39793000 \quad 3.48059400-1.20652600$

$\mathrm{H}-0.27892000 \quad 2.97976400-1.89592300$

$\mathrm{H} \quad 0.03491600 \quad 4.48510400 \quad-0.98282200$

$\mathrm{H} 1.38589200 \quad 3.54883000-1.66249300$ 
PALLADIUM

\begin{tabular}{|c|c|c|c|c|c|}
\hline \multirow[b]{2}{*}{$E E$} & \multicolumn{2}{|c|}{$\overbrace{\mathrm{Cl}^{-\mathrm{Pd}_{\mathrm{PMe}_{3}}}}^{\mathrm{OMe}}$} & & \multicolumn{2}{|c|}{$\mathrm{MeO} \underbrace{\mathrm{OMe}}_{\mathrm{MeO}}{ }_{\mathrm{Pd}_{\mathrm{PMe}_{3}}^{\prime}}^{\mathrm{Cl}}$} \\
\hline & & -2125.09885 & & & -2125.107893 \\
\hline $\begin{array}{l}\text { Gibbs free energy } \\
0 \quad 1\end{array}$ & y correction & 0.405263 & $\begin{array}{l}\text { Gibbs free energy } \\
0 \quad 1\end{array}$ & y correction & 0.40528 \\
\hline C 2.46098100 & -1.32919600 & -1.02540600 & C -3.40875900 & -0.30278400 & -0.87309300 \\
\hline C 1.05215600 & -1.90608500 & -1.11892900 & C -2.45989200 & 0.18147000 & -1.96288400 \\
\hline C 0.33451300 & -0.92932600 & 0.91538000 & C -0.51443300 & -0.35342200 & -0.75561400 \\
\hline C 1.71779800 & -0.31844800 & 1.12531700 & C -1.33448200 & -0.90346300 & 0.41238600 \\
\hline C 2.40137800 & -0.03498800 & -0.21022900 & C -2.70567100 & -1.38662300 & -0.05211400 \\
\hline $\mathrm{H} 0.42709600$ & -1.21597600 & -1.70536600 & $\mathrm{H}-2.25955400$ & -0.65092500 & -2.65220500 \\
\hline $\mathrm{H} \quad 1.06190500$ & -2.86924300 & -1.63004200 & $\mathrm{H}-2.90553300$ & 0.99237400 & -2.54014600 \\
\hline $\mathrm{H} 2.80093500$ & -1.11547600 & -2.05082100 & $\mathrm{H}-4.30705700$ & -0.71489500 & -1.35812000 \\
\hline $\mathrm{H}-0.01981300$ & -1.25147900 & 01.89521700 & $\mathrm{H}-1.49977700$ & -0.11326200 & 1.15178000 \\
\hline $\mathrm{H} \quad 2.33275700$ & -1.07126900 & 1.63810000 & $\mathrm{H}-2.55388300$ & -2.25866700 & -0.70890700 \\
\hline $\mathrm{H} \quad 1.79412000$ & 0.67282900 & -0.79150500 & $0-1.25816000$ & 0.66971400 & -1.41690900 \\
\hline O 0.48914800 & -2.12363700 & 0.15081400 & $0-3.78688800$ & 0.71634900 & 0.02336500 \\
\hline $\mathrm{Cl}-3.0417240$ & 01.20043600 & $0-1.22914300$ & $0-3.42210000$ & -1.77470200 & 1.09179500 \\
\hline$P-0.16244300$ & 2.46812200 & -0.20532900 & $0-0.64721300$ & -1.99222800 & 0.99878600 \\
\hline$P-2.51287700$ & -1.62463400 & 00.34902000 & $C-4.71162500$ & -2.26698100 & 0.83361800 \\
\hline$C-2.10230300$ & -2.92388800 & 01.56041500 & $\mathrm{H}-4.69389900$ & -3.04027500 & 0.05576300 \\
\hline $\mathrm{H}-2.85492800$ & -3.71279700 & 01.52057500 & $\mathrm{H}-5.07961700$ & -2.70635300 & 1.75858100 \\
\hline $\mathrm{H}-1.12062400$ & -3.33876600 & 01.34249200 & $\mathrm{H}-5.39366200$ & -1.46712000 & 0.52773800 \\
\hline $\mathrm{H}-2.09654400$ & -2.49486800 & 02.56288900 & C -4.52732300 & 1.76332500 & -0.55213300 \\
\hline$C-2.69364600$ & -2.53199200 & $0-1.21469800$ & $\mathrm{H}-4.95787600$ & 2.33865200 & 0.26526700 \\
\hline $\mathrm{H}-1.72194400$ & -2.93107400 & $0-1.50519700$ & $\mathrm{H}-3.89894500$ & 2.42713700 & -1.15498600 \\
\hline $\mathrm{H}-3.40828400$ & -3.35034400 & $0-1.11330900$ & $\mathrm{H}-5.33747600$ & 1.37573400 & -1.18157300 \\
\hline $\mathrm{H}-3.03703200$ & -1.82754200 & $0-1.97242200$ & C -0.49440600 & -1.88005000 & 2.39639900 \\
\hline$C-4.22369600$ & -1.21538400 & $0 \quad 0.81159000$ & H 0.17121500 & -1.04419400 & 2.64627300 \\
\hline $\mathrm{H}-4.82610900$ & -2.12402200 & 00.86269800 & $\mathrm{H}-1.46228600$ & -1.74065100 & 2.88353400 \\
\hline $\mathrm{H}-4.22419400$ & -0.72601400 & 01.78543500 & $\mathrm{H}-0.04882500$ & -2.80844700 & 2.75156800 \\
\hline $\mathrm{H}-4.63050100$ & -0.52470400 & 00.07612900 & $\mathrm{H}-0.37092200$ & -1.16550000 & -1.48174400 \\
\hline$C-1.36374700$ & 3.69234800 & 0.41956300 & $\mathrm{Pd} 1.29723500$ & 0.30356800 & -0.12774500 \\
\hline $\mathrm{H}-1.04078100$ & 4.70307300 & 0.16360100 & $\mathrm{Cl} 3.53829400$ & 0.96107600 & 0.67375500 \\
\hline $\mathrm{H}-2.33870200$ & 3.48365400 & -0.01600300 & P 2.27660800 & -1.79150900 & -0.52849300 \\
\hline $\mathrm{H}-1.43091400$ & 3.60085800 & 1.50377600 & P 0.44690100 & $2.46318700 \quad 0$ & .30210100 \\
\hline$C-0.06903900$ & 2.86941600 & -1.98611500 & C 3.76805700 & -1.68973300 & -1.56736300 \\
\hline $\mathrm{H}-1.01010000$ & 2.58434200 & -2.45412000 & $\mathrm{H} \quad 4.44564700$ & -0.96556100 & -1.11766400 \\
\hline $\mathrm{H} \quad 0.11660000$ & 3.93500600 & -2.13154800 & $\mathrm{H} 4.25002000$ & -2.66577200 & -1.64540900 \\
\hline $\mathrm{H} \quad 0.74307600$ & 2.30279900 & -2.44520800 & $\mathrm{H} 3.49288800$ & -1.34142500 & -2.56277700 \\
\hline C 1.39448400 & 3.217295000 & 0.40405600 & C 1.34303600 & -3.14753200 & -1.31326300 \\
\hline $\mathrm{H} \quad 1.35183200$ & 3.287247001 & 1.48902900 & $\mathrm{H} 0.44845300$ & -3.35272000 & -0.72630100 \\
\hline $\mathrm{H} 2.26766000$ & 2.619835000 & 0.14623500 & $\mathrm{H} 1.05005600$ & -2.85956700 & -2.32346900 \\
\hline $\mathrm{H} \quad 1.49029300$ & 4.21739800 & -0.02346900 & $\mathrm{H} 1.96242900$ & -4.04404800 & -1.36894400 \\
\hline $\mathrm{Pd}-1.1504750$ & 00.22314000 & 00.09271800 & C 2.88512600 & -2.54754300 & 1.00796300 \\
\hline 03.38336600 & -2.18088400 & -0.39201200 & $\mathrm{H} 2.03443000$ & -2.77862500 & 1.64759500 \\
\hline 03.66269200 & 0.531790000 & 0.04590500 & $\mathrm{H} \quad 3.44274500$ & -3.46150600 & 0.79689300 \\
\hline O 1.57293400 & 0.811303001 & 1.94732900 & $\mathrm{H} 3.52508600$ & -1.82145300 & 1.50850000 \\
\hline C 4.40851800 & 0.82833900 & -1.10769300 & C 1.53077700 & 3.506142001 & L. 32491900 \\
\hline $\mathrm{H} \quad 3.80981800$ & 1.39699700 & -1.83138200 & $\mathrm{H} 2.50373000$ & 3.58796500 & .84413500 \\
\hline $\mathrm{H} \quad 5.25644900$ & 1.43511400 & -0.79648800 & $\mathrm{H} 1.08511000$ & 4.49254900 & L. 46540000 \\
\hline $\mathrm{H} \quad 4.78137100$ & -0.08088400 & -1.58860500 & $\mathrm{H} 1.68804500$ & 3.025344002 & .28969900 \\
\hline C 3.62880700 & -3.38854000 & -1.06682600 & C -1.15861800 & 2.63875800 & 1.14563700 \\
\hline $\mathrm{H} \quad 4.51600900$ & -3.83144100 & -0.61871600 & $\mathrm{H}-1.93249600$ & 2.11489200 & 0.58692800 \\
\hline H 2.79678400 & -4.09125600 & -0.96469400 & $\mathrm{H}-1.09057400$ & 2.19982600 & 2.14204100 \\
\hline H 3.81756600 & -3.21454900 & -2.13358400 & $\mathrm{H}-1.41659300$ & 3.69503900 & 1.24330600 \\
\hline C 2.64548700 & 1.056543002 & 2.83069300 & C 0.24182300 & $3.46395100-$ & -1.20579500 \\
\hline H 2.85740100 & 0.171830003 & 3.44047200 & $\mathrm{H}-0.45459400$ & 2.95678700 & -1.87050500 \\
\hline H 3.55129700 & 1.343406002 & 2.29467800 & $\mathrm{H}-0.12795200$ & 4.46104200 & -0.96028000 \\
\hline H 2.33316400 & 1.868025003 & 3.48720300 & $\mathrm{H} 1.20847900$ & $3.54986500-$ & -1.70192900 \\
\hline
\end{tabular}




\section{PLATINUM}

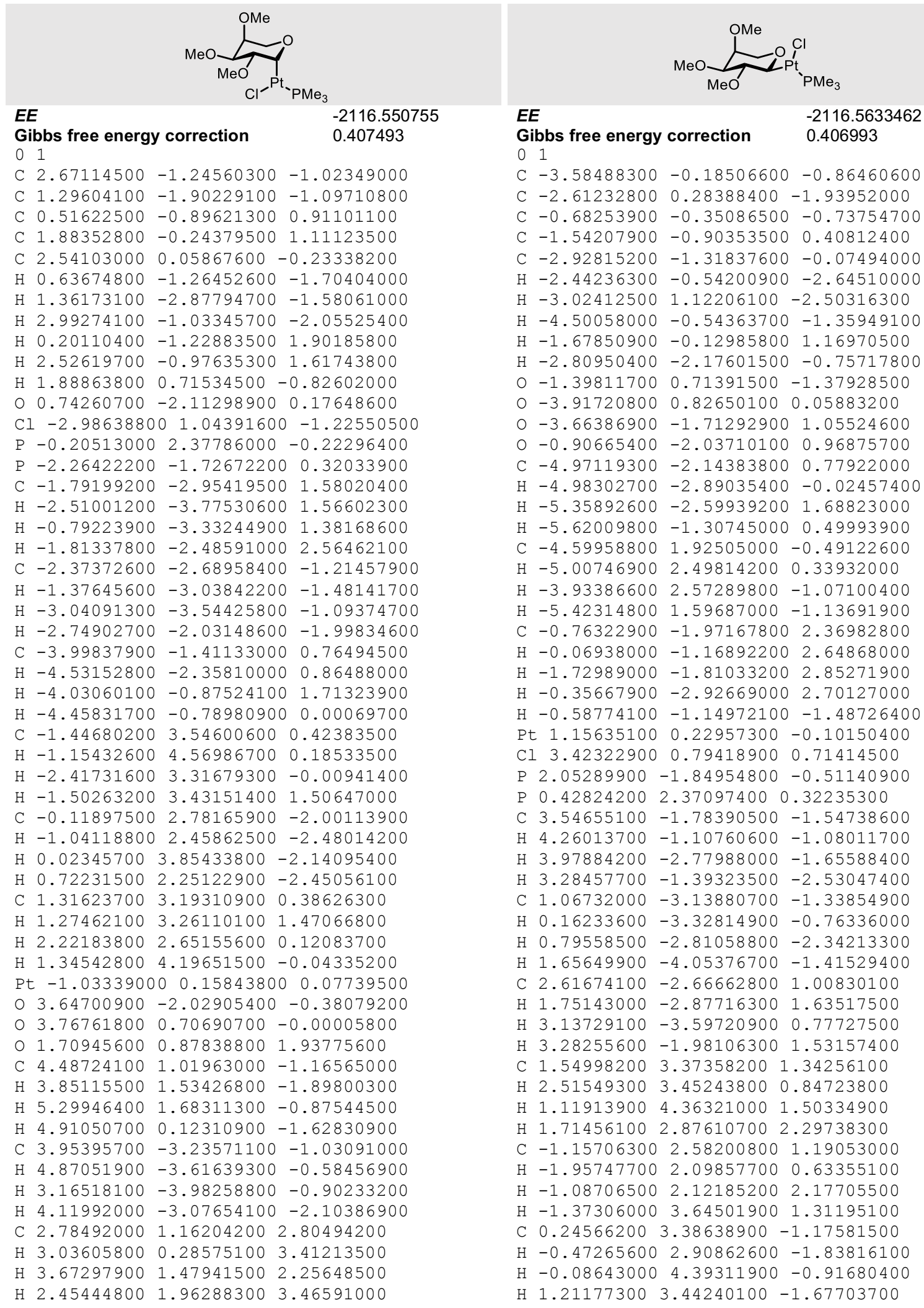


COPPER

\begin{tabular}{|c|c|c|}
\hline \multicolumn{2}{|r|}{ EE } & -2255.2505 \\
\hline \multicolumn{2}{|r|}{ Gibbs free energy correction } & 0.192009 \\
\hline $\mathrm{C}$ & $0.29630600 \quad 0.59009300 \quad-$ & -0.71855200 \\
\hline 0 & $0.18227300-1.84313800$ & -0.79249300 \\
\hline $\mathrm{C}$ & $-0.64232000 \quad 0.59498000$ & 0.48189800 \\
\hline 0 & $1.272464001 .60718700-$ & -0.60948000 \\
\hline $\mathrm{H}$ & $-0.31371800 \quad 0.75175700$ & -1.61785800 \\
\hline $\mathrm{C}$ & -0.60389800 & $0 \quad 0.36074500$ \\
\hline $\mathrm{C}$ & -1.50957800 & $0 \quad 0.47980000$ \\
\hline 0 & $-1.38607400 \quad 1.78849400$ & 0.44955400 \\
\hline $\mathrm{H}$ & $-0.02273800 \quad 0.57291400$ & 1.39336000 \\
\hline $\mathrm{C}$ & $0.942749002 .82419800-$ & -1.24554700 \\
\hline $\mathrm{H}$ & $0.03801200-1.93306100$ & 1.25725900 \\
\hline $\mathrm{C}$ & $-2.31703700 \quad 1.92481500$ & 1.49180600 \\
\hline $\mathrm{H}$ & $0.052610003 .27634500-$ & -0.80661200 \\
\hline $\mathrm{H}$ & $0.77511800 \quad 2.66855700-$ & -2.31662600 \\
\hline $\mathrm{H}$ & $1.795909003 .48815800-$ & -1.11493800 \\
\hline $\mathrm{H}$ & $-1.85521800 \quad 1.72886300$ & 2.46752300 \\
\hline $\mathrm{H}$ & $-3.16978300 \quad 1.25210200$ & 1.35764000 \\
\hline $\mathrm{H}$ & $-2.67436600 \quad 2.95260200$ & 1.47169600 \\
\hline $\mathrm{C}$ & $1.04753900-0.73469600$ & -0.82433400 \\
\hline $\mathrm{H}$ & $1.57024000-0.77561600$ & -1.77951900 \\
\hline $\mathrm{H}$ & $-1.19061300-2.80569500$ & 00.31698400 \\
\hline 0 & $-2.41964600-0.53378300$ & $0-0.58509100$ \\
\hline $\mathrm{H}$ & $-2.06192700-0.74958200$ & 01.42907700 \\
\hline $\mathrm{C}$ & $-3.31873300-1.60365800$ & $0-0.72837900$ \\
\hline $\mathrm{H}$ & $-4.10719000-1.27128300$ & -1.40085600 \\
\hline $\mathrm{H}$ & $-2.83707800-2.48565000$ & -1.15996800 \\
\hline $\mathrm{H}$ & $-3.76580500-1.87780500$ & 0.23567300 \\
\hline $\mathrm{Cu}$ & $2.52428400-0.66189600$ & 00.57871100 \\
\hline
\end{tabular}

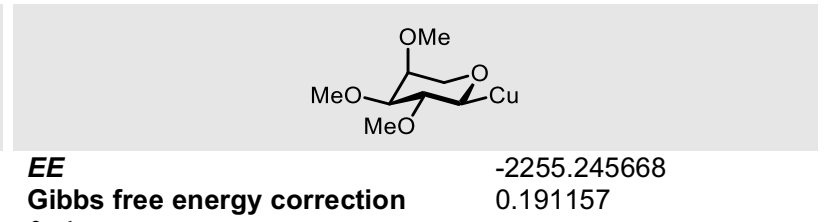

Gibbs

C $0.26110700 \quad 0.63317300 \quad-0.01487000$

$\begin{array}{lllll}0 & 0.45245200 & -1.75308800 & 0.38720400\end{array}$

$\begin{array}{llll}\text { C } & -1.13969100 & 0.63433700 & 0.59323800\end{array}$

$\begin{array}{lllll}0 & 0.93311300 & 1.84114600 & 0.27519300\end{array}$

H $0.14926500 \quad 0.49867200 \quad-1.09632900$

C $-0.80188600-1.79504100 \quad 1.02356300$

C $-1.76305600-0.75772200 \quad 0.45224000$

$\begin{array}{llll}0 & -1.89160500 & 1.64730800 & -0.03134900\end{array}$

$\mathrm{H}-1.04415300 \quad 0.85626900 \quad 1.66774000$

$\begin{array}{llll}\text { C } 0.85796200 & 2.81236900 & -0.74354800\end{array}$

$\mathrm{H}-0.69267200 \quad-1.60854400 \quad 2.10205000$

C $-3.21998800 \quad 1.74649600 \quad 0.41250000$

$\mathrm{H}-0.17471100 \quad 3.10328600-0.94190200$

$\mathrm{H} \quad 1.30750500 \quad 2.43771600-1.67148300$

$\mathrm{H} 1.42397000 \quad 3.67791700 \quad-0.40226300$

$\mathrm{H}-3.27306300 \quad 1.77682700 \quad 1.50789800$

$\mathrm{H}-3.82816500 \quad 0.91280600 \quad 0.04766200$

$\mathrm{H}-3.62408900 \quad 2.67572400 \quad 0.01510200$

C $1.09105300 \quad-0.50270300 \quad 0.57771400$

$\mathrm{H}-1.19554800 \quad-2.80417500 \quad 0.89710700$

$\begin{array}{lllll}0 & -2.04605200 & -0.94212700 & -0.91449700\end{array}$

$\mathrm{H}-2.69881900-0.81560800 \quad 1.03096000$

C $-2.64596300-2.17162100-1.23073400$

$\mathrm{H}-3.03814100-2.08626500-2.24246800$

$\mathrm{H}-1.92741400-2.99579100-1.20046800$

$\mathrm{H}-3.47424100-2.39696900-0.54674300$

$\mathrm{Cu} 2.94719200-0.44353100-0.17163000$

$\mathrm{H} 1.20119600 \quad-0.31111500 \quad 1.65736700$ 


\section{SILVER}

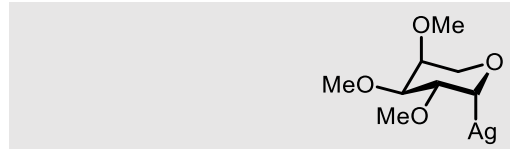

$E E$

Gibbs free energy correction

$0 \quad 1$

C $0.20702300 \quad 0.71372000 \quad 0.78700900$

$\begin{array}{lllll}0 & 0.03111900 & -1.70266800 & 0.98581300\end{array}$

$\begin{array}{llll}\text { C } 1.03596200 & 0.55017700 & -0.48131900\end{array}$

$\begin{array}{lllll}0 & -0.62999900 & 1.84739300 & 0.71511700\end{array}$

$\mathrm{H} \quad 0.90801300 \quad 0.81856800 \quad 1.62722300$

C $0.71804500-1.90302000-0.21544200$

$\begin{array}{llll}\text { C } 1.74596900 & -0.80590600 & -0.47458300\end{array}$

O $1.917104001 .64261800-0.56951900$

$\mathrm{H} \quad 0.34512600 \quad 0.56370700 \quad-1.33967200$

C $-0.09141800 \quad 3.02353200 \quad 1.28166700$

$\mathrm{H} \quad 0.00566800-1.92110800-1.05777600$

C $2.76338400 \quad 1.61999100-1.68967400$

$\mathrm{H} \quad 0.81532800 \quad 3.33804500 \quad 0.76362300$

$\begin{array}{lllll}\mathrm{H} & 0.13475500 & 2.87462300 & 2.34297400\end{array}$

$\mathrm{H}-0.85492100 \quad 3.79425600 \quad 1.18866200$

H $2.19823900 \quad 1.44187700-2.61295300$

$\mathrm{H} \quad 3.53864800 \quad 0.85374600-1.59083500$

$\mathrm{H} \quad 3.24088200 \quad 2.59594300-1.75199500$

C $-0.68301000-0.50058700 \quad 1.02369500$

$\mathrm{H}-1.14775700-0.434980002 .00497600$

$\mathrm{H} \quad 1.19633300-2.88010200-0.15210000$

$\begin{array}{llll}0 & 2.75206400 & -0.73711100 & 0.50655700\end{array}$

$\mathrm{H} 2.20209700-1.00086000-1.45791600$

C $3.54738500-1.88984400 \quad 0.61716900$

H $4.41962200-1.62136900 \quad 1.20997600$

$\mathrm{H} \quad 3.01846600-2.70453800 \quad 1.11993000$

$\begin{array}{llll}\mathrm{H} \quad 3.88070800 & -2.23712700 & -0.36904600\end{array}$

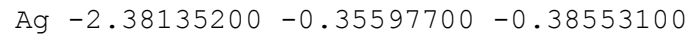

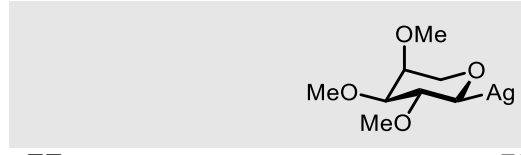

\section{EE $\quad-761645074$ \\ Gibbs free energy correction $\quad 0.190436$}

01

$\begin{array}{lllll}\text { C } & 0.20505300 & 0.69217800 & -0.02570500\end{array}$

$0-0.11867300-1.67359000-0.44974500$

C $1.62226200 \quad 0.60904300-0.59106700$

$\begin{array}{llll}0 & -0.37026800 & 1.94391300 & -0.33073500\end{array}$

H $\quad 0.27631800 \quad 0.54517700 \quad 1.05713000$

C $1.14929500-1.78985100-1.05297500$

C $2.15567300-0.81893900-0.44431600$

O $2.410361001 .57042100 \quad 0.06788500$

H $1.57456500 \quad 0.84497500-1.66579100$

C $-0.31924700 \quad 2.87881700 \quad 0.72208500$

$\mathrm{H} 1.08119800-1.58896400-2.13202700$

C $3.754213001 .60054700-0.33776400$

$\mathrm{H} 0.71057100 \quad 3.09409100 \quad 1.01301000$

$\mathrm{H}-0.86891800 \quad 2.50914700 \quad 1.59668900$

$\mathrm{H}-0.79714900 \quad 3.78985500 \quad 0.36513500$

H $3.839699001 .64378900-1.43065600$

$\mathrm{H} \quad 4.30420300 \quad 0.72856700 \quad 0.03001700$

$\begin{array}{lllll}\mathrm{H} & 4.19851100 & 2.49943800 & 0.08557300\end{array}$

C $-0.67069800-0.38818000-0.64909200$

$\mathrm{H} 1.47353600-2.82288700-0.92431400$

$\begin{array}{llll}0 & 2.38581300 & -1.03145200 & 0.92782300\end{array}$

H $3.10273900-0.92970900-0.99641500$

C $2.90784500-2.29503300 \quad 1.24823700$

H $3.27208700 \quad-2.24122500 \quad 2.27246900$

H $2.14702800-3.078525001 .18640200$

$\mathrm{H} \quad 3.74369700 \quad-2.55828300 \quad 0.58743400$

$\mathrm{H}-0.75843500-0.18556000 \quad-1.72656800$

Ag $-2.69327400 \quad-0.24799400 \quad 0.12220600$ 
GOLD

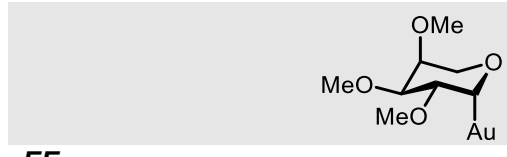

EE

Gibbs free energy correction

01

$\begin{array}{llll}\text { C } & 0.68251400 & 0.80292100 & 0.77100400\end{array}$

$\begin{array}{lllll}0 & 0.25399300 & -1.56002800 & 1.08208700\end{array}$

$\begin{array}{llll}\text { C } 1.46232100 & 0.50585300 & -0.50322700\end{array}$

$\begin{array}{lllll}0 & -0.00274400 & 2.02557400 & 0.70599900\end{array}$

$\mathrm{H} \quad 1.40148700 \quad 0.81028200 \quad 1.60224300$

C $0.93232000-1.89660800-0.10572400$

C $2.04601500-0.90902100-0.43060400$

o $2.441187001 .50285500-0.64743300$

$\begin{array}{lllll}\mathrm{H} & 0.76215000 & 0.54400500 & -1.35179700\end{array}$

C $0.69065300 \quad 3.12518400 \quad 1.25913600$

$\mathrm{H} \quad 0.21972200-1.92223600-0.94260900$

C $3.26177900 \quad 1.35317400-1.77760300$

$\begin{array}{lllll}\mathrm{H} & 1.61925900 & 3.32193000 & 0.72228600\end{array}$

H $0.91595700 \quad 2.94956600 \quad 2.31666900$

$\mathrm{H} \quad 0.02796100 \quad 3.98462100 \quad 1.17720200$

H $2.665416001 .19184600-2.68375800$

H $3.96284900 \quad 0.52122900-1.65636000$

H $3.82840900 \quad 2.27555200-1.88780800$

C $-0.32913700-0.30318800 \quad 1.06730400$

$\mathrm{H}-0.78695500-0.14308000 \quad 2.04170000$

$\mathrm{H} \quad 1.32816000 \quad-2.90040000 \quad 0.04027100$

$\begin{array}{lllll}0 & 3.06719200 & -0.88443700 & 0.53642000\end{array}$

$\mathrm{H} 2.46725800-1.19303000-1.40714200$

C $3.83332600-2.05990000 \quad 0.61288500$

H $4.70567300-1.83430300 \quad 1.22277400$

H $3.28277400 \quad-2.88112000 \quad 1.08084000$

$\mathrm{H} \quad 4.16690300-2.37805400-0.38273300$

$\mathrm{Au}-1.92560500-0.18644300-0.23629600$

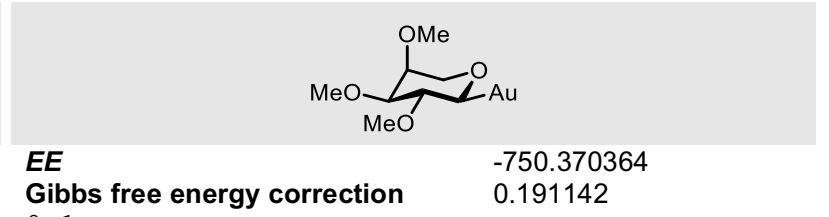

Gibbs

C $0.68157700 \quad 0.76275400-0.04176800$

0
$0.24394700-1.58177900-0.49781900$

C $2.10295500 \quad 0.59632500-0.58111300$

$00.19928300 \quad 2.04009900-0.37695200$

H $0.72173600 \quad 0.61791300 \quad 1.04166800$

C $1.51080600-1.75324600-1.09725900$

C $2.55855400-0.85763100-0.44668900$

$\begin{array}{llll}0 & 2.91230000 & 1.50912300 & 0.11324700\end{array}$

H $2.09254300 \quad 0.85043400-1.65293000$

C $0.14875100 \quad 2.94143400 \quad 0.70577800$

$\mathrm{H} 1.46378200-1.52068300-2.17039900$

C $4.263912001 .49624800-0.26946000$

H $1.14238700 \quad 3.09927400 \quad 1.13127100$

$\mathrm{H}-0.52748500 \quad 2.57236600 \quad 1.48559100$

$\mathrm{H}-0.23729400 \quad 3.88296100 \quad 0.31953000$

H $4.36955800 \quad 1.56847500-1.35882900$

$\mathrm{H} \quad 4.77097000 \quad 0.59191700 \quad 0.08126200$

$\begin{array}{lllll}\mathrm{H} & 4.73557000 & 2.36330600 & 0.18849700\end{array}$

C $-0.23874200-0.27606800-0.67652100$

H $1.76599700 \quad-2.80691100 \quad-0.99247300$

$\begin{array}{llll}0 & 2.74061800 & -1.10762700 & 0.92395800\end{array}$

H $3.50923400-1.01032600-0.98181900$

C $3.17357200-2.408212001 .23400800$

H $3.51317700-2.39432200 \quad 2.26766000$

H $2.36565800-3.13935500 \quad 1.13933000$

$\mathrm{H} \quad 4.00781300 \quad-2.71234300 \quad 0.58940800$

$\mathrm{H}-0.35042800 \quad-0.05067300 \quad-1.74626700$

$\mathrm{Au} \quad-2.11798400 \quad-0.14936900 \quad 0.08097700$ 
ZINC

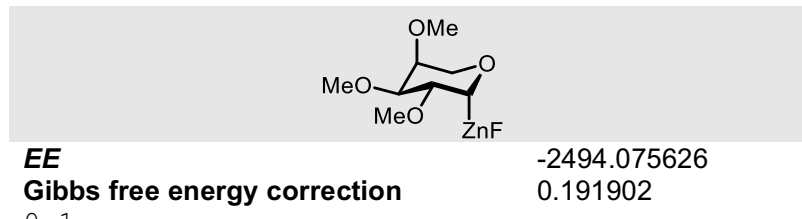

$\begin{array}{ll}0 & 1\end{array}$

C $-0.05064900 \quad 0.49078000 \quad-0.83511700$

$\begin{array}{lllll}0 & -0.31990800 & -1.96253700 & -0.64490800\end{array}$

C $-0.91462200 \quad 0.65948400 \quad 0.40978000$

$\begin{array}{lllll}0 & 1.09885400 & 1.33479200 & -0.78217600\end{array}$

$\mathrm{H}-0.65423900 \quad 0.74077800-1.71357900$

C $-0.98539000-1.81335600 \quad 0.57402000$

C $-1.83621500-0.54595600 \quad 0.58972100$

$\begin{array}{lllll}0 & -1.59341900 & 1.88413000 & 0.29392100\end{array}$

$\mathrm{H}-0.24529000 \quad 0.69207600 \quad 1.28663000$

C $0.93104200 \quad 2.68196400 \quad-1.19297900$

$\mathrm{H}-0.26625500-1.76350600 \quad 1.40878400$

C $-2.46689400 \quad 2.17541500 \quad 1.35803900$

$\mathrm{H} \quad 0.28769400 \quad 3.22581700 \quad-0.50318600$

$\mathrm{H} \quad 0.49661100 \quad 2.71847300 \quad-2.19528500$

$\mathrm{H} \quad 1.92366800 \quad 3.12627600 \quad-1.21175200$

$\mathrm{H}-1.97022600 \quad 2.04780100 \quad 2.32726900$

$\mathrm{H}-3.359365001 .544270001 .32393900$

$\mathrm{H}-2.76658300 \quad 3.21550100 \quad 1.24909300$

C $0.57216300-0.90945000-0.91702300$

$\mathrm{H} \quad 0.91206600-1.06443400-1.94170700$

$\mathrm{H}-1.60060300-2.70125300 \quad 0.71588800$

$\begin{array}{llll}0 & -2.78188400 & -0.49220800 & -0.44729200\end{array}$

$\mathrm{H}-2.34351000-0.49305700 \quad 1.56537800$

C $-3.70546500-1.55344100-0.45883600$

$\mathrm{H}-4.50669900-1.27136700-1.13856000$

$\mathrm{H}-3.24971300-2.48138300-0.81486800$

$\mathrm{H}-4.12784000-1.72095400 \quad 0.53963000$

Zn $2.24401900-0.55614200 \quad 0.16162500$

F $3.73963800-0.261870001 .08128700$

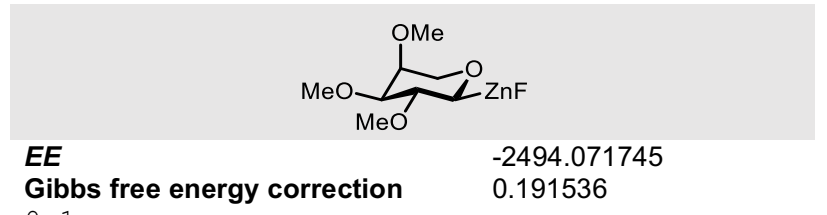

$0 \quad 1$

C $0.03047800 \quad 0.56546000-0.00006900$

O $-0.08395900-1.82591700-0.42832000$

C $1.43394300 \quad 0.64348500-0.58309800$

o $-0.77774800 \quad 1.69791500-0.24909000$

H $0.13257600 \quad 0.39945600 \quad 1.07949700$

C $1.20209800-1.82316100-1.00576800$

C $2.09732400-0.73427500-0.41908200$

o $2.13836400 \quad 1.67366900 \quad 0.06265800$

$\mathrm{H} \quad 1.35706400 \quad 0.86040700 \quad-1.66040600$

C $-0.52673300 \quad 2.83904800 \quad 0.55345900$

H $1.13004100-1.66762400-2.09174300$

C $3.486228001 .79502300-0.31910100$

$\mathrm{H} \quad 0.38062100 \quad 3.35162900 \quad 0.23834200$

$\mathrm{H}-0.42352200 \quad 2.55473300 \quad 1.60488200$

$\mathrm{H}-1.38689300 \quad 3.49591100 \quad 0.43971600$

H $3.59071800 \quad 1.81534200-1.41064300$

$\mathrm{H} \quad 4.09070600 \quad 0.97769100 \quad 0.08534600$

$\mathrm{H} \quad 3.85058200 \quad 2.73614500 \quad 0.08750600$

C $-0.72882900-0.59106700-0.65274600$

$\mathrm{H} \quad 1.63198000-2.81004200-0.83619500$

$\begin{array}{llll}0 & 2.34751500 & -0.89708600 & 0.95518700\end{array}$

$\mathrm{H} \quad 3.04847700-0.75834500-0.97337600$

C $3.05804000-2.06223800 \quad 1.29248900$

H $3.39738100-1.94361200 \quad 2.31943800$

H 2.43099300 $-2.95608800 \quad 1.23130600$

H $3.93131000 \quad-2.19448600 \quad 0.64160200$

$\mathrm{H}-0.74888200-0.38618200-1.73606100$

Zn $-2.58553100-0.37362800-0.00614800$

F $-4.23954200-0.13871100 \quad 0.58280500$ 
CADMIUM
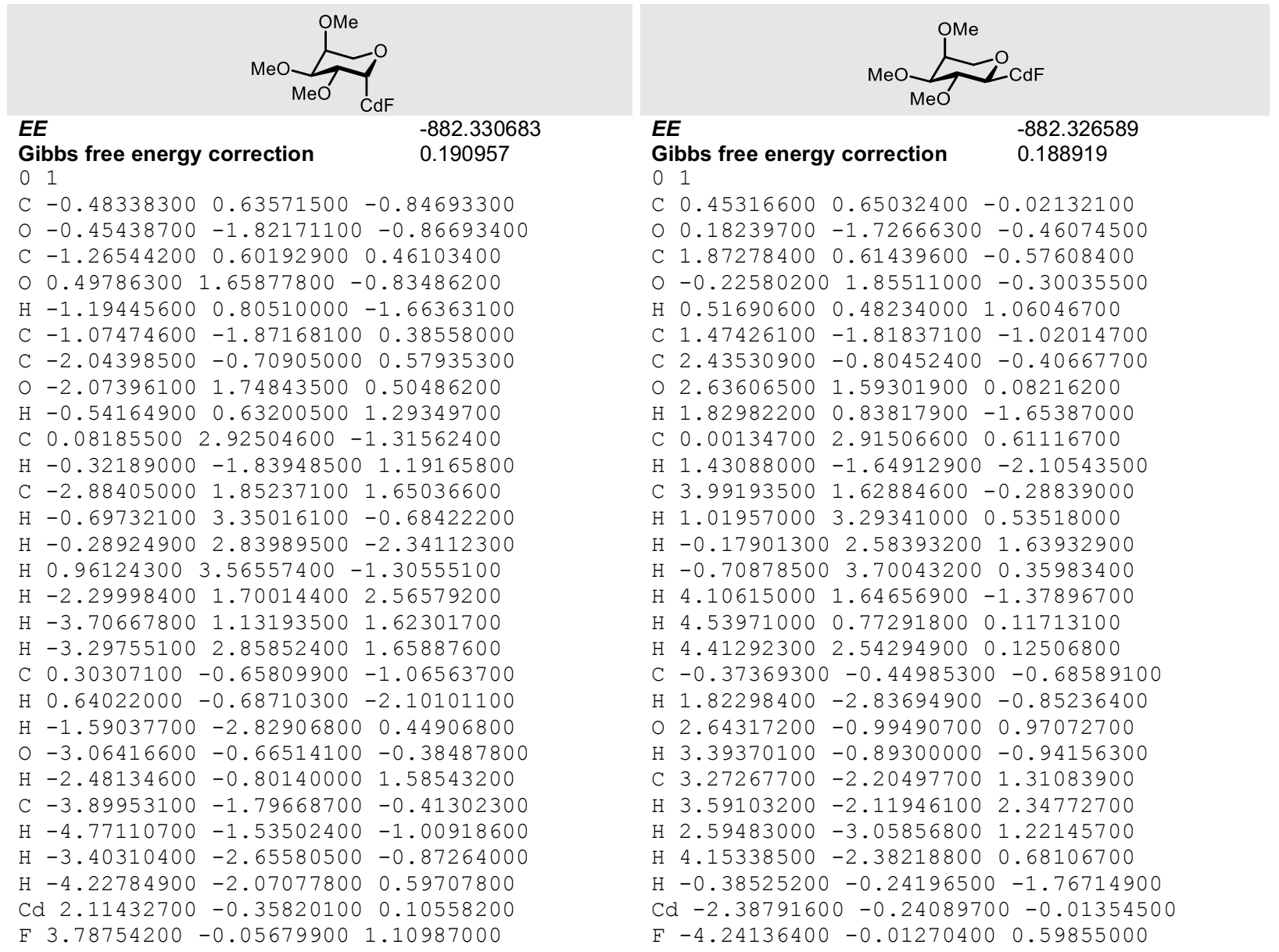
MERCURY

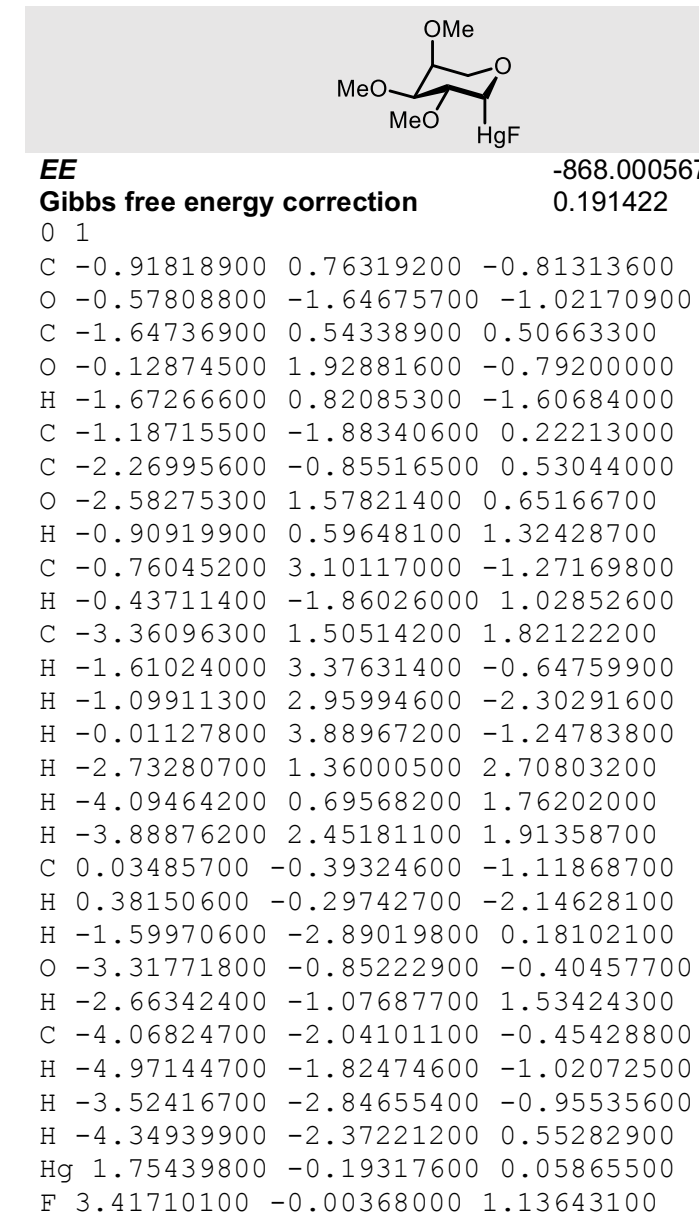

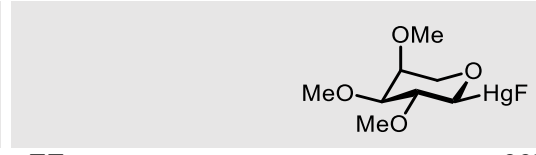

$E E$ Gibbs free energy correction $\quad 0.191287$

C $0.89360000 \quad 0.73994000-0.03737400$

$00.46562000-1.61035500-0.51012900$

C $2.31827400 \quad 0.59035800 \quad-0.56230800$

O $0.35124200 \quad 1.99919100-0.35338700$

H $0.91846500 \quad 0.57907500 \quad 1.04616500$

C $1.75292900-1.78267200-1.06332300$

C $2.77600900-0.86365600-0.40444600$

$\begin{array}{llll}0 & 3.12496400 & 1.50996900 & 0.12507200\end{array}$

H $2.31437900 \quad 0.83027600 \quad-1.63762900$

$\begin{array}{llll}\text { C } & 0.48376800 & 2.97736100 & 0.66032200\end{array}$

$\mathrm{H} 1.73452500-1.57665600-2.14262500$

C $4.485309001 .47539600-0.22926800$

H $1.53244600 \quad 3.18711900 \quad 0.87130400$

$\mathrm{H}-0.01133200 \quad 2.64855500 \quad 1.58073900$

$\mathrm{H}-0.00878500 \quad 3.87620600 \quad 0.29552800$

$\mathrm{H} \quad 4.61361500 \quad 1.51342900-1.31765800$

$\mathrm{H} \quad 4.97674700 \quad 0.57888200 \quad 0.16054400$

H $4.95341200 \quad 2.35266500 \quad 0.21208100$

C $-0.00613100-0.29787800-0.70808800$

H $2.01611500-2.83034100-0.92493100$

$\begin{array}{llll}0 & 2.93124300 & -1.09567400 & 0.97221300\end{array}$

H $3.73775200-1.01400000-0.91905600$

C $3.43577300-2.365910001 .30366500$

$\mathrm{H} \quad 3.73573200-2.32787300 \quad 2.34869300$

$\mathrm{H} 2.68179500-3.14894000 \quad 1.18337100$

H $4.31040600-2.61520700 \quad 0.69034000$

$\mathrm{H}-0.03449800-0.06965300-1.78339000$

$\mathrm{Hg}-1.93881200-0.14230900-0.01388000$

F $-3.79861900 \quad 0.01538500 \quad 0.64685000$ 


\subsection{Tetrahydro-2H-thiopyran Structures}

\section{NICKEL}

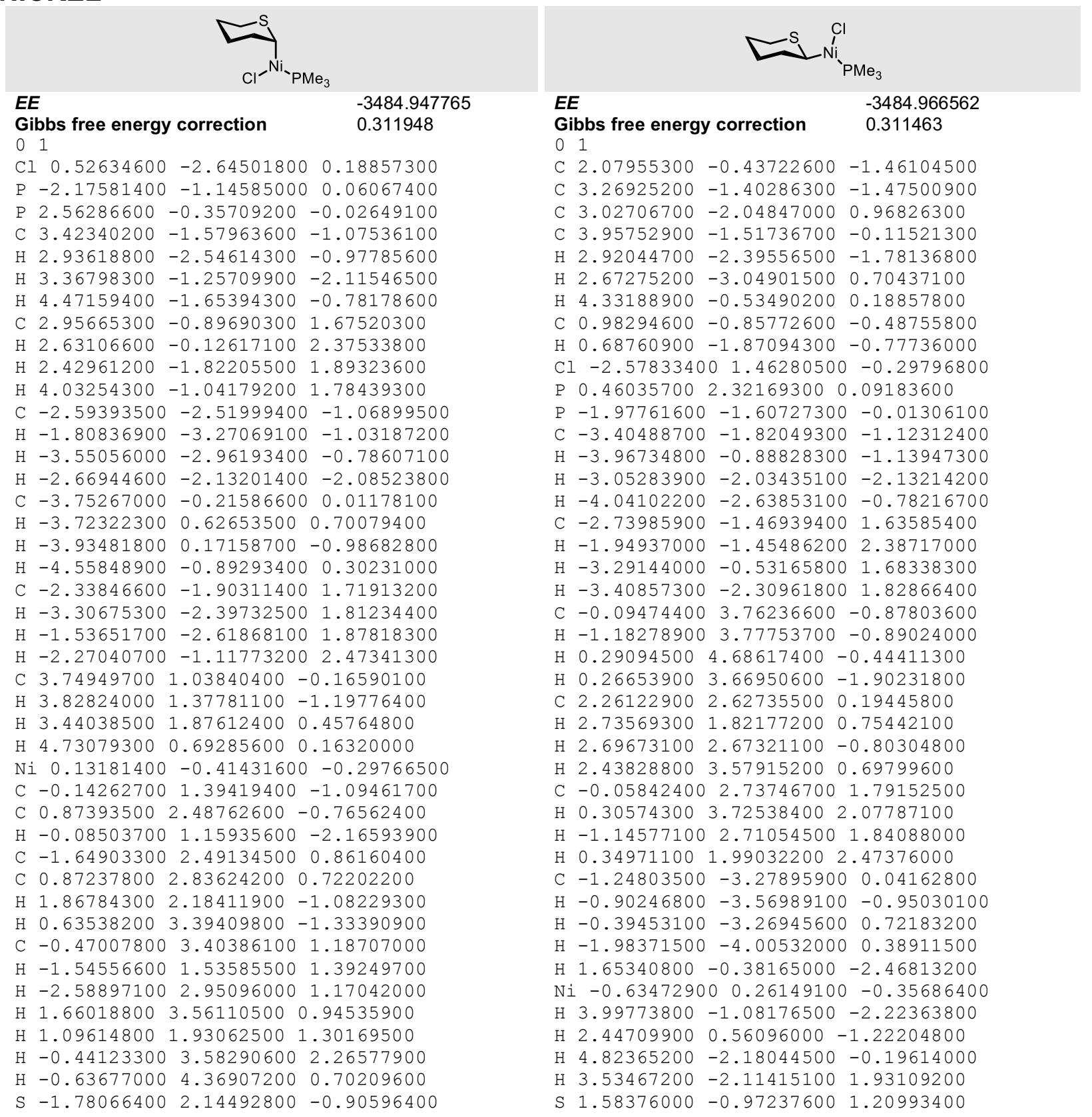


PALLADIUM

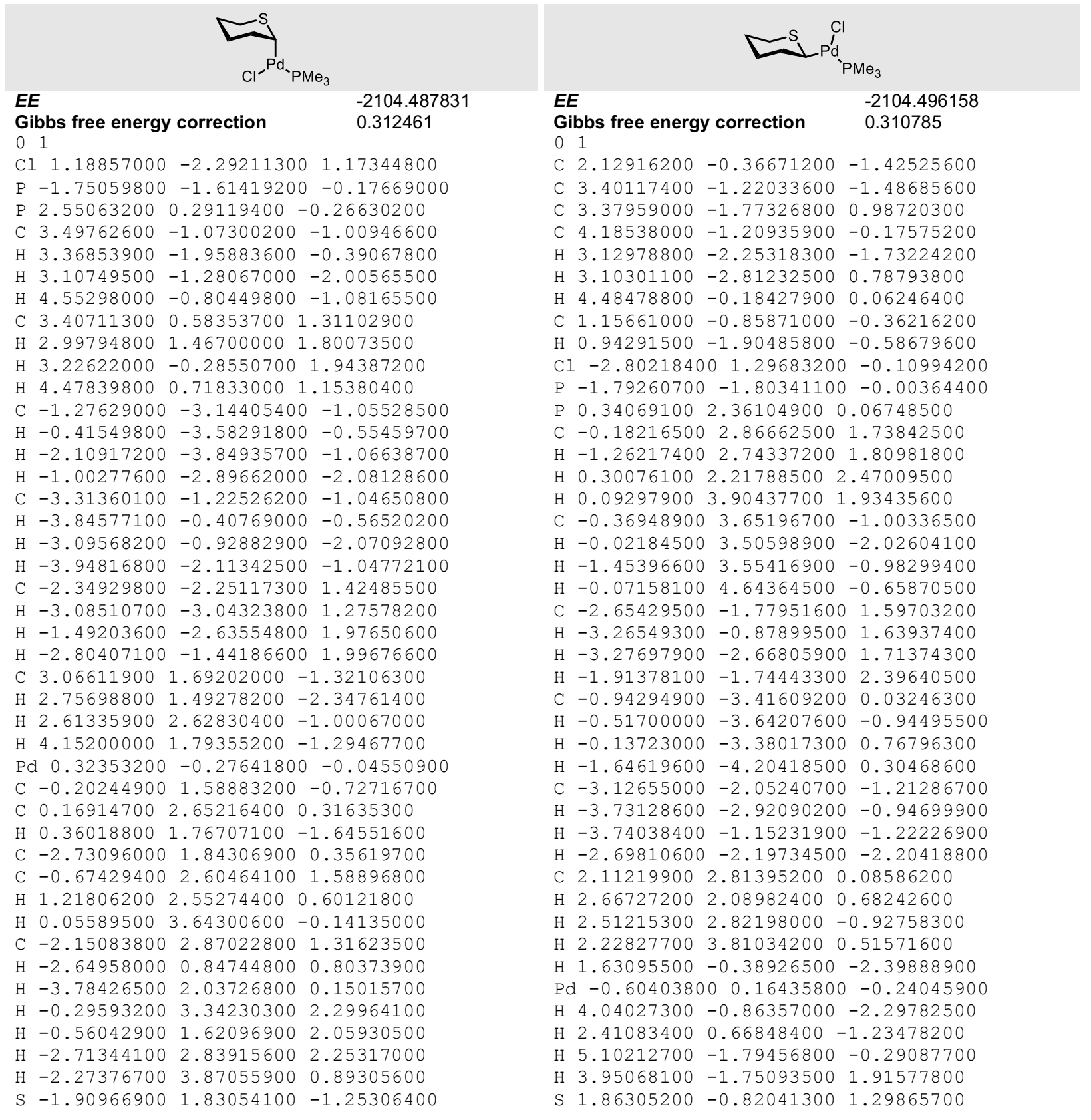




\section{PLATINUM}

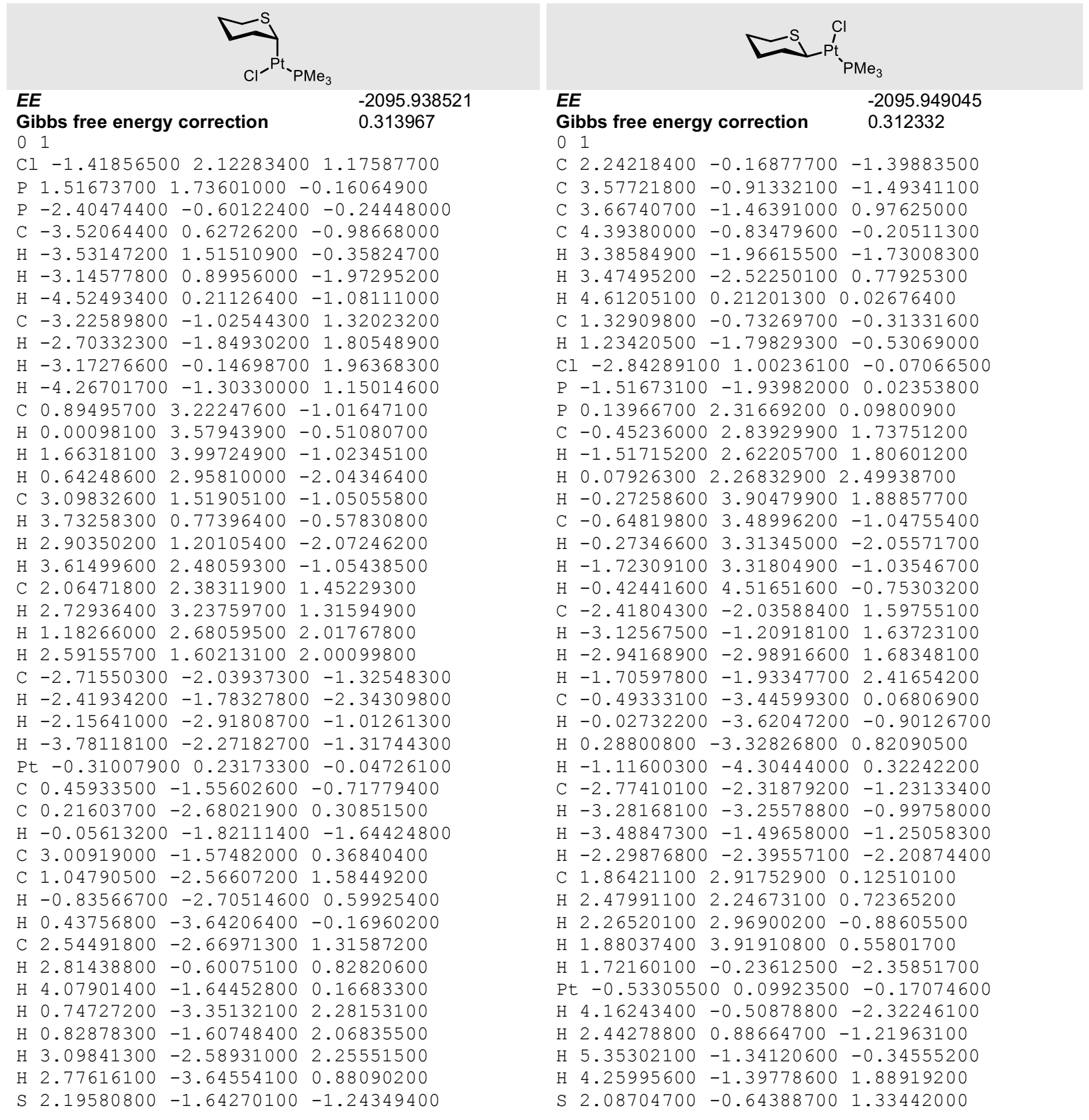


COPPER

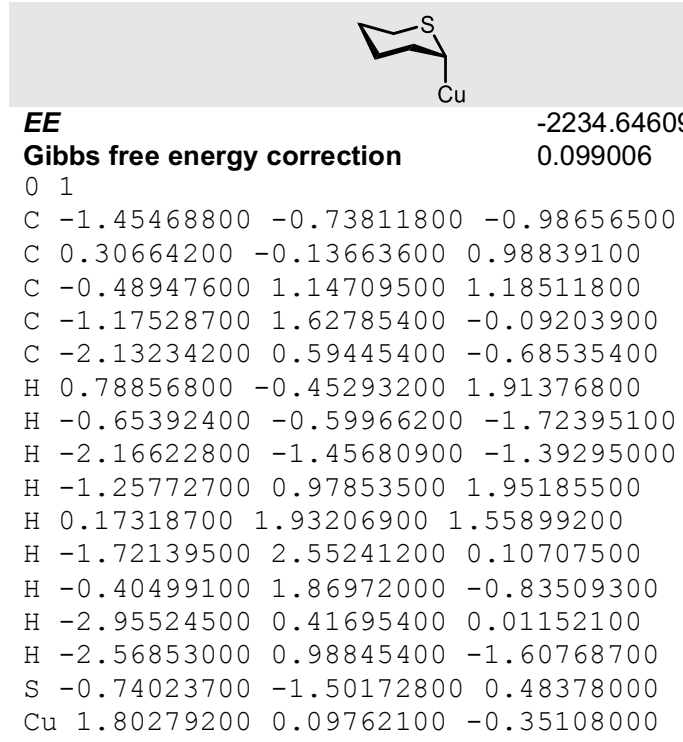

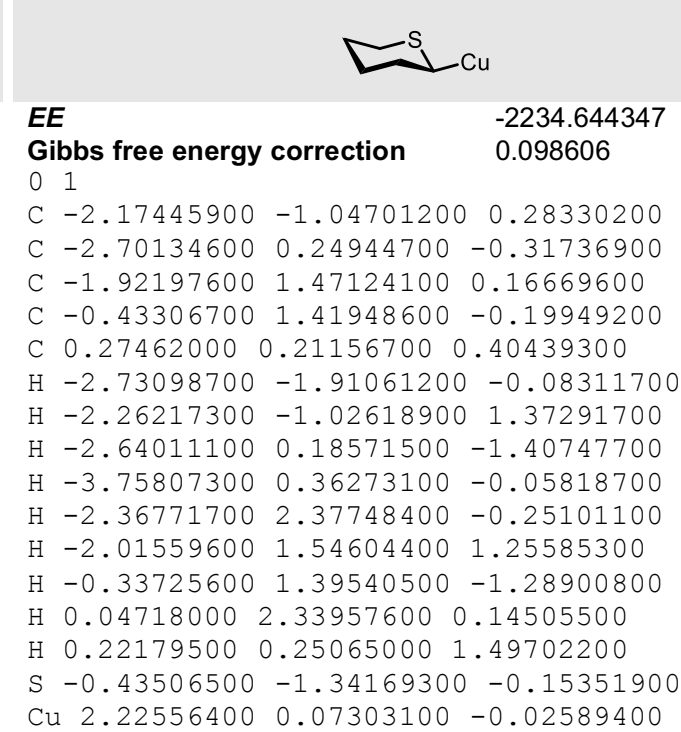




\section{SILVER}

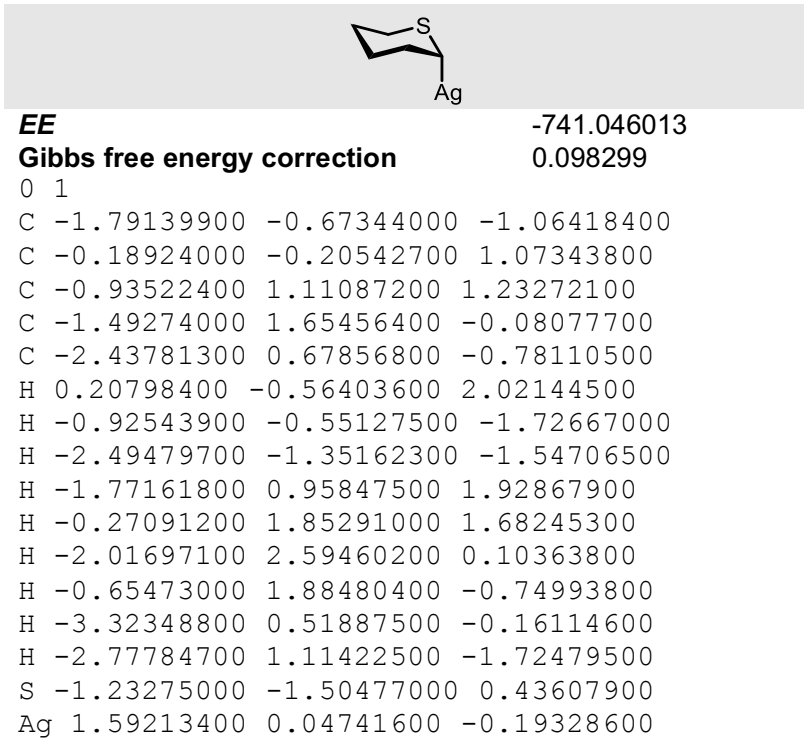

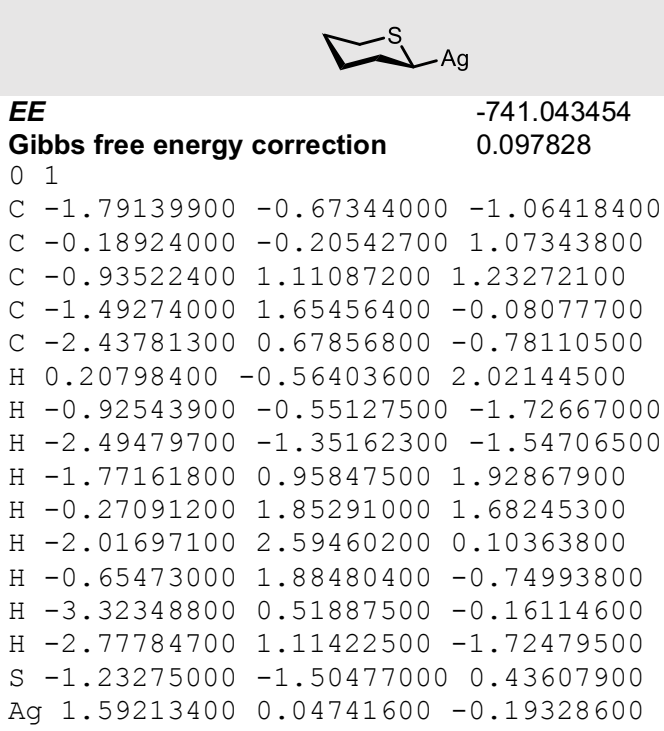


GOLD

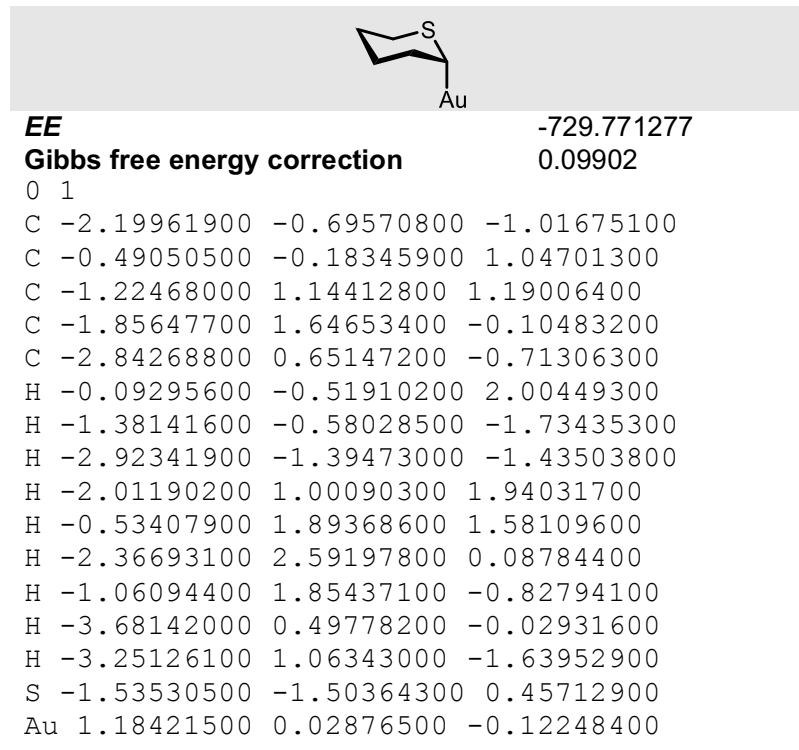

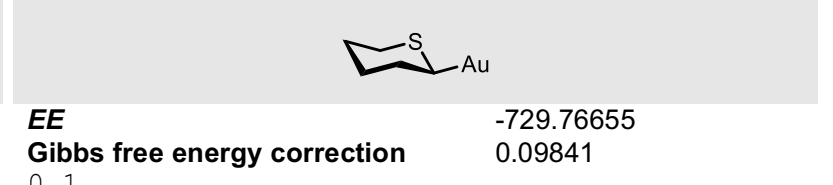

01

C $-3.04638500 \quad-0.99664700 \quad 0.30166200$

C $-3.53801100 \quad 0.31116700-0.30175900$

C $-2.70815600 \quad 1.50502800 \quad 0.16295200$

C $-1.22682200 \quad 1.39534300 \quad-0.22035200$

$\begin{array}{llll}\text { C }-0.58318300 & 0.16290500 & 0.39753200\end{array}$

$\mathrm{H}-3.63901900-1.84424200-0.04383800$

$\mathrm{H}-3.11095500-0.96527300 \quad 1.39240100$

$\mathrm{H}-3.49876400 \quad 0.23777600 \quad-1.39174200$

$\mathrm{H}-4.58457100 \quad 0.46258300 \quad-0.02292700$

$\mathrm{H}-3.11678300 \quad 2.42545500 \quad-0.25933700$

$\mathrm{H}-2.78852700 \quad 1.59592800 \quad 1.25166600$

$\mathrm{H}-1.13361600 \quad 1.34954900-1.30755600$

$\mathrm{H}-0.70182800 \quad 2.29103400 \quad 0.11806100$

$\mathrm{H}-0.63777300 \quad 0.20429100 \quad 1.48814600$

$\mathrm{S}-1.33393600-1.37667900-0.17212100$

$\mathrm{Au} \quad 1.40721700 \quad 0.02535400 \quad-0.00647000$ 
ZINC

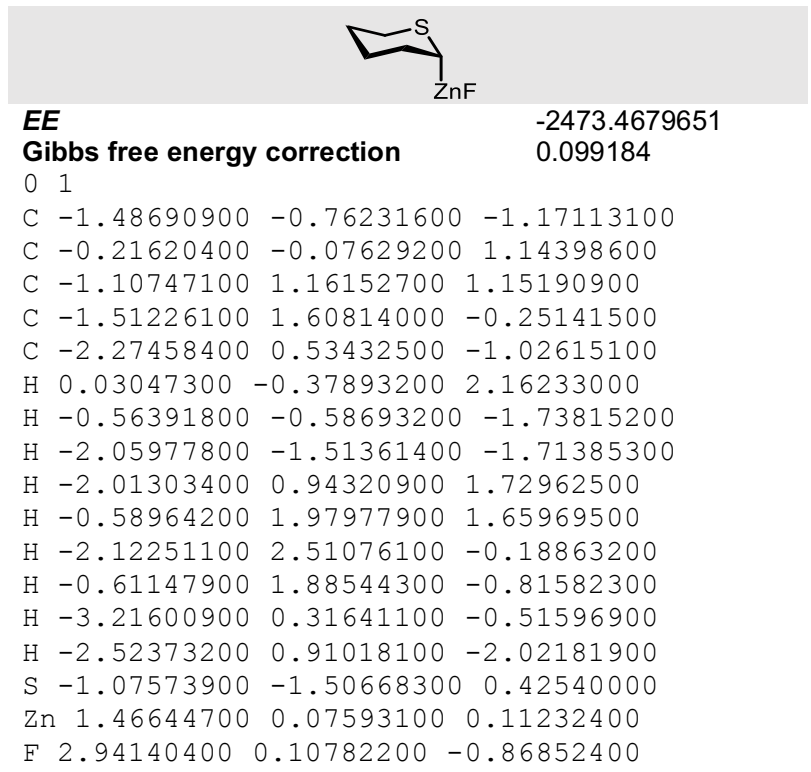

$\begin{array}{ll}E E & -2473.4666323 \\ \text { Gibbs free energy correction } & 0.0991\end{array}$

01

C $-2.58038200-1.03524000 \quad 0.24768400$

C $-3.08049200 \quad 0.26433700-0.36839800$

C $-2.30376100 \quad 1.48089400 \quad 0.13121900$

C $-0.809854001 .41796700-0.19533000$

$\begin{array}{llll}\text { C }-0.12615600 & 0.20006800 & 0.43319200\end{array}$

$\mathrm{H}-3.13542000-1.89432500-0.12961400$

$\mathrm{H}-2.68994800-1.01391100 \quad 1.33492800$

$\mathrm{H}-2.99751500 \quad 0.19717400-1.45648100$

$\mathrm{H}-4.14073500 \quad 0.38530200 \quad-0.13092300$

$\mathrm{H}-2.73015000 \quad 2.38833600-0.30163600$

$\mathrm{H}-2.42533400 \quad 1.56274700 \quad 1.21697800$

$\mathrm{H}-0.68250200 \quad 1.39059500-1.28156100$

$\mathrm{H}-0.33127400 \quad 2.33469000 \quad 0.16099000$

$\mathrm{H}-0.24632100 \quad 0.23976500 \quad 1.52093500$

S $-0.84103200-1.35452200-0.14951500$

F $3.54801400-0.00464700-0.24160600$

$\begin{array}{lllll}\text { Zn } 1.81024800 & 0.07185400 & 0.07142900\end{array}$ 
CADMIUM

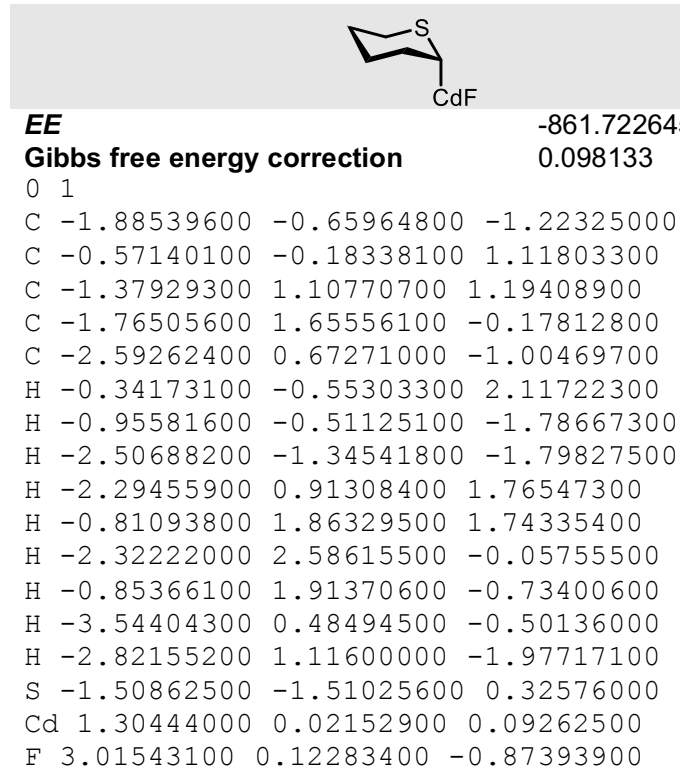

EE
$\begin{array}{lll}\text { EE } \\ \text { Gibbs free energy correction } \\ \text { O } 1 \\ \text { C }-2.0 .0971 .7211359 \\ \text { C }-3.46232700 & 0.27153100 & -0.38183700 \\ \text { C }-2.68155800 & 1.48339000 & 0.12292700 \\ \text { C }-1.18544200 & 1.41193500 & -0.19603400 \\ \text { C }-0.51731600 & 0.19086900 & 0.43689300 \\ \text { H }-3.53004100 & -1.88717300 & -0.14259400 \\ \text { H }-3.08984000 & -1.00838500 & 1.32440100 \\ \text { H }-3.37248900 & 0.20382000 & -1.46932800 \\ \text { H }-4.52336400 & 0.39883300 & -0.15142100 \\ \text { H }-3.09946800 & 2.39389100 & -0.31177000 \\ \text { H }-2.80850800 & 1.56552500 & 1.20799100 \\ \text { H }-1.05288800 & 1.38262300 & -1.28149300 \\ \text { H }-0.70372200 & 2.32578600 & 0.16304800 \\ \text { H }-0.63142500 & 0.23291500 & 1.52384800 \\ \text { S }-1.23243900 & -1.35862000 & -0.14880200 \\ \text { F } 3.51048500 & -0.03955400 & -0.28173700 \\ \text { Cd } 1.58033600 & 0.05256100 & 0.05697000\end{array}$


MERCURY

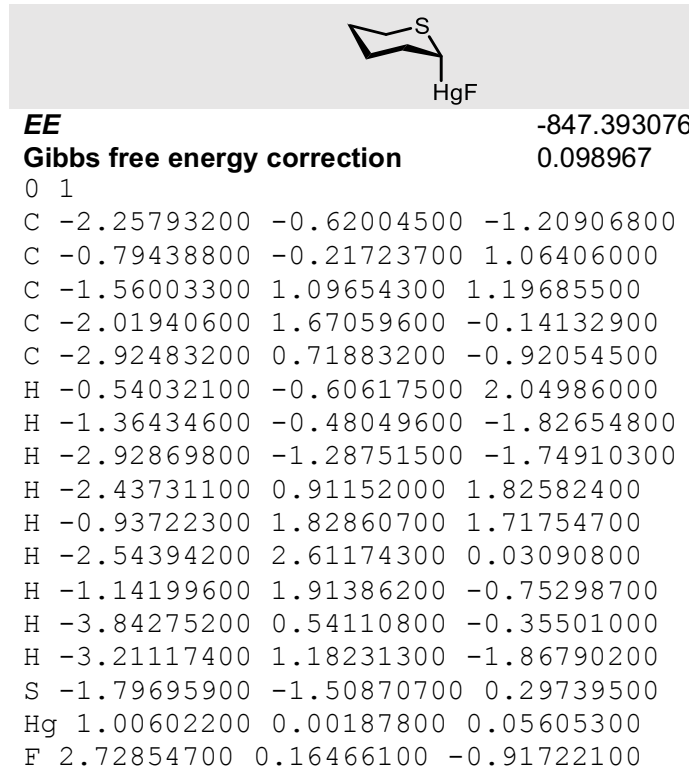

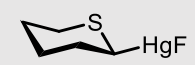

$E E$

Gibbs free energy correction

01

C $-3.29334300-1.00633500 \quad 0.25671200$

C $-3.77246900 \quad 0.29993600-0.36075000$

C $-2.95850900 \quad 1.49843400 \quad 0.12113400$

C $-1.47076900 \quad 1.39426000-0.22289500$

$\begin{array}{llll}\text { C }-0.82429400 & 0.16264400 & 0.41009200\end{array}$

Hg $1.20276800 \quad 0.03569400 \quad 0.04170400$

H $-3.87499000-1.85518200-0.10297800$

$\mathrm{H}-3.38379800 \quad-0.97540900 \quad 1.34545200$

$\mathrm{H}-3.70815900 \quad 0.22453700-1.44932700$

$\mathrm{H}-4.82529800 \quad 0.44783600-0.10683300$

$\mathrm{H}-3.36249700 \quad 2.41472100-0.31345100$

$\mathrm{H}-3.06602600 \quad 1.59215600 \quad 1.20729000$

$\mathrm{H}-1.35136000 \quad 1.35161200-1.30878000$

$\mathrm{H}-0.96112000 \quad 2.29673000 \quad 0.12497200$

$\mathrm{H}-0.93666800 \quad 0.20734100 \quad 1.49656600$

S $-1.57277200-1.37984100-0.17134200$

F $3.14767800-0.06400600-0.30149700$ 
5.2. 1,3-Dioxane Structures

\section{NICKEL}

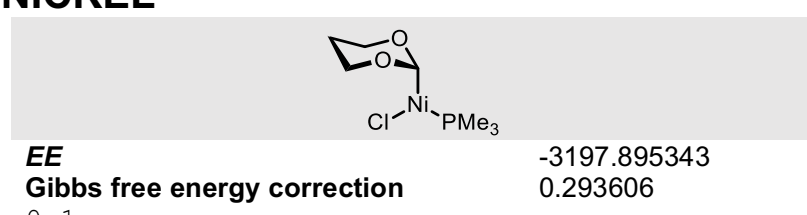

01

Cl $0.00909100 \quad-2.33909600 \quad 1.07572600$

$\mathrm{P}-2.32889900-0.64489200-0.20432200$

P $2.33563800-0.62968200-0.20580500$

C $2.68786500-2.18538200-1.09313100$

H $2.13787300-2.99271700-0.61319400$

H $2.35320900-2.09611600-2.12696700$

H $3.75765100-2.39928100-1.08178900$

C $3.15569400-0.89521800 \quad 1.40120700$

H $3.12846900 \quad 0.02787400 \quad 1.98061400$

H $2.61447700-1.66910300 \quad 1.94298400$

H $4.19492300 \quad-1.19397300 \quad 1.25515200$

C $-2.66833300-2.19833800-1.10058500$

$\mathrm{H}-2.11154700-3.00370200-0.62513000$

$\mathrm{H}-3.73627500-2.42132600-1.09025300$

$\mathrm{H}-2.33459700-2.10048500-2.13392900$

C $-3.48440000 \quad 0.50879200-1.02012200$

$\mathrm{H}-3.55171500 \quad 1.43150900-0.44784700$

$\mathrm{H}-3.11417200 \quad 0.76436600-2.01158400$

$\mathrm{H}-4.47112100 \quad 0.04921400-1.09833600$

C $-3.14658000-0.92696000 \quad 1.40116900$

$\mathrm{H}-4.18318100-1.23370800 \quad 1.25302100$

$\mathrm{H}-2.59891700-1.69930500 \quad 1.93857500$

$\mathrm{H}-3.12746500-0.00715000 \quad 1.98608900$

C $3.48044700 \quad 0.52910100-1.02940800$

H $3.10464800 \quad 0.77896600-2.02035400$

H $3.54285500 \quad 1.45414500-0.46051200$

$\mathrm{H} \quad 4.46992400 \quad 0.07602400-1.11052400$

Ni $0.00252400-0.33054600-0.06024000$

C $0.001125001 .43224000-0.93516100$

H $0.01246800 \quad 1.29284800-2.02046100$

$\begin{array}{llll}\text { C }-1.25053400 & 2.61824400 & 0.65545800\end{array}$

$\begin{array}{llll}\text { C } 1.21392700 & 2.62321600 & 0.68126800\end{array}$

C $-0.02392400 \quad 3.42549100 \quad 1.05646700$

$\mathrm{H}-1.33250200 \quad 1.72887700 \quad 1.30342900$

$\mathrm{H}-2.16656700 \quad 3.20262200 \quad 0.75573700$

$\mathrm{H} 2.12575700 \quad 3.21035200 \quad 0.80165400$

$\mathrm{H} 1.28528200 \quad 1.73280500 \quad 1.32899800$

$\mathrm{H}-0.03597700 \quad 3.64485900 \quad 2.12607800$

$\mathrm{H}-0.01983200 \quad 4.36774000 \quad 0.50560000$

$\begin{array}{llll}0 & -1.15590800 & 2.22169500 & -0.69221200\end{array}$

$\begin{array}{llll}0 & 1.14819600 & 2.22906200 & -0.66888200\end{array}$

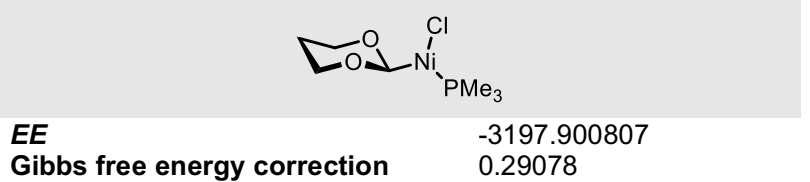

01

C $-2.38858000 \quad 2.48190200-0.98738000$

C $-0.89787200 \quad 3.18243200 \quad 0.84964200$

C $-2.32989100 \quad 3.22530600 \quad 0.33857700$

$\mathrm{H}-1.82572000 \quad 3.03619100-1.75212000$

$\mathrm{H}-0.25387100 \quad 3.78240700 \quad 0.18810600$

$\mathrm{H}-2.98663200 \quad 2.73607700 \quad 1.06125000$

C $-0.491529001 .21492600-0.39013500$

$\mathrm{H} 0.05160400 \quad 1.86372400-1.10444400$

Cl $1.47922100-2.50016400 \quad 0.03633800$

$\begin{array}{llll}\mathrm{P}-1.70593900 & -1.60094800 & 0.08324000\end{array}$

$\begin{array}{lllll}\text { P } 2.43939600 & 0.48567900 & -0.08835100\end{array}$

C $3.73759700-0.18411300-1.17519700$

$\mathrm{H} 3.78109900-1.26226300-1.03049600$

H $3.48103700 \quad 0.01816800-2.21506000$

$\mathrm{H} \quad 4.70396500 \quad 0.26829900-0.94786800$

C $3.12254800 \quad 0.25087800 \quad 1.58274500$

$\begin{array}{llll}\mathrm{H} & 2.45791400 & 0.72857800 & 2.30306600\end{array}$

$\mathrm{H} \quad 3.15665200 \quad-0.81826400 \quad 1.78849500$

H $4.12121600 \quad 0.68235700 \quad 1.66486400$

C $-2.77796000-1.74096700-1.38463300$

$\mathrm{H}-2.25656800-2.31856500-2.14804500$

$\mathrm{H}-3.71091500-2.24641700-1.13004200$

$\mathrm{H}-2.98209500-0.74457600-1.76909700$

C $-2.79417400-0.85514200 \quad 1.34240300$

$\mathrm{H}-2.27240800 \quad-0.84358000 \quad 2.29933900$

$\mathrm{H}-3.01623100 \quad 0.16919400 \quad 1.05396300$

$\mathrm{H}-3.71692200-1.42901600 \quad 1.44188800$

C $-1.61138500 \quad-3.33028200 \quad 0.64676600$

$\mathrm{H}-2.61275500 \quad-3.73736300 \quad 0.79667600$

$\mathrm{H}-1.06956700-3.91861800-0.09163500$

$\mathrm{H}-1.04471500-3.37688600 \quad 1.57563000$

C $2.60944600 \quad 2.28746700-0.32994500$

H $2.348497002 .55369400-1.35436400$

H $1.92688700 \quad 2.79879900 \quad 0.34838000$

H $3.63270000 \quad 2.60799500-0.12985400$

$\mathrm{Ni} \quad 0.35135400-0.53535700-0.19168500$

$\mathrm{H}-3.41341300 \quad 2.35772300-1.33934000$

H $-2.66405600 \quad 4.25798600 \quad 0.21696100$

$\mathrm{H}-0.81548300 \quad 3.57833800 \quad 1.86214500$

$\begin{array}{lllll}0 & -0.42364100 & 1.84882100 & 0.88181700\end{array}$

$0-1.83585400 \quad 1.18660600-0.84673600$ 
PALLADIUM

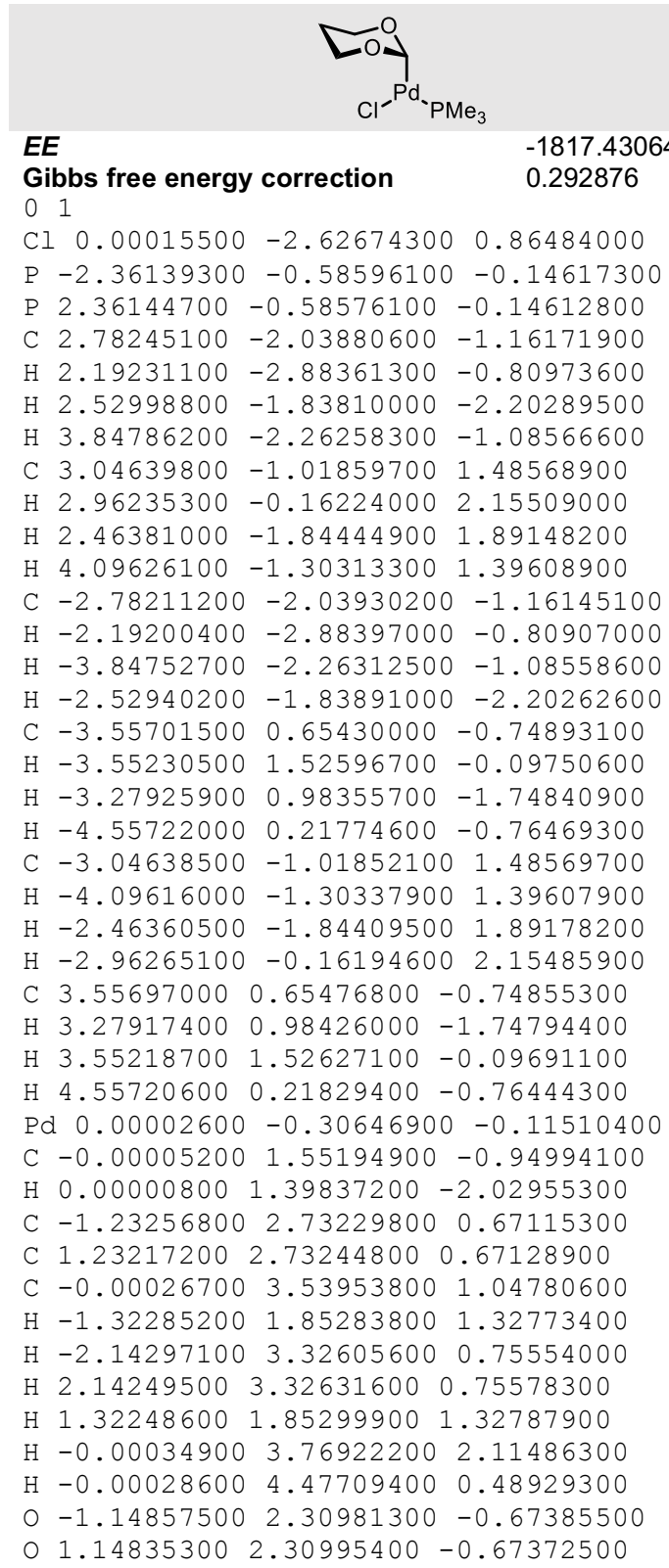

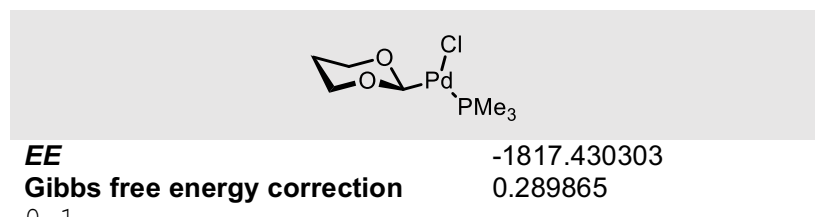

01

C $-2.65018200 \quad 2.42506300-0.96390200$

C $-1.23231000 \quad 3.29480300 \quad 0.85354900$

C $-2.66231400 \quad 3.19704500 \quad 0.34613100$

$\mathrm{H}-2.13944700 \quad 3.01013900-1.74191600$

$\mathrm{H}-0.64195600 \quad 3.93400300 \quad 0.17933400$

$\mathrm{H}-3.26940400 \quad 2.66012300 \quad 1.07775700$

C $-0.66179500 \quad 1.35378000-0.33788000$

$\mathrm{H}-0.15409000 \quad 2.00830300-1.06875100$

Cl $1.66727900 \quad-2.44918400 \quad 0.10841600$

P 2.33811300 $0.76642000-0.07968000$

P $-1.59152900-1.78902600 \quad 0.07930800$

C $-1.49044800-2.70323500 \quad 1.65226500$

$\mathrm{H}-0.51304100-3.18090300 \quad 1.70663400$

$\mathrm{H}-1.58758300-2.00319100 \quad 2.48207700$

$\mathrm{H}-2.28188600-3.45206400 \quad 1.71523400$

C $-1.60397800-3.12112600-1.16282700$

$\mathrm{H}-1.77435900-2.69291700-2.15037300$

$\mathrm{H}-0.62700600-3.60345700-1.15048700$

$\mathrm{H}-2.38695200-3.84896800-0.94304700$

C $3.09019700 \quad 0.55203600 \quad 1.56092100$

$\mathrm{H} \quad 3.18195100-0.51773000 \quad 1.74793200$

H 4.069821001 .030236001 .60962100

$\mathrm{H} \quad 2.43047500 \quad 0.98728800 \quad 2.31152500$

C $2.37847700 \quad 2.57305700-0.32136600$

H 2.08103500 2.81974500 -1.34083000

$\mathrm{H} \quad 1.67703900 \quad 3.03823600 \quad 0.37128000$

$\mathrm{H} 3.38238100 \quad 2.96018700-0.14200200$

C $3.61581700 \quad 0.16269100-1.22331900$

H $4.56990600 \quad 0.65708000-1.03349200$

$\mathrm{H} 3.71045100-0.91337600-1.08187700$

H $3.30486200 \quad 0.35485600-2.24990200$

C $-3.32352500-1.22126300 \quad 0.07426200$

$\mathrm{H}-3.45167300-0.44614700 \quad 0.82846300$

$\mathrm{H}-3.56136500-0.78966500-0.89550400$

$\mathrm{H}-3.98834000-2.06070900 \quad 0.28545600$

$\mathrm{Pd} 0.31699400-0.38461800-0.15120200$

$\mathrm{H}-3.65650900 \quad 2.19370400-1.31299300$

$\mathrm{H}-3.08949900 \quad 4.19208200 \quad 0.20510200$

$\mathrm{H}-1.18123400 \quad 3.71335800 \quad 1.85818600$

o $-0.64137000 \quad 2.00615300 \quad 0.91431200$

$\begin{array}{lllll}0 & -1.98205700 & 1.18638900 & -0.79138900\end{array}$ 


\section{PLATINUM}

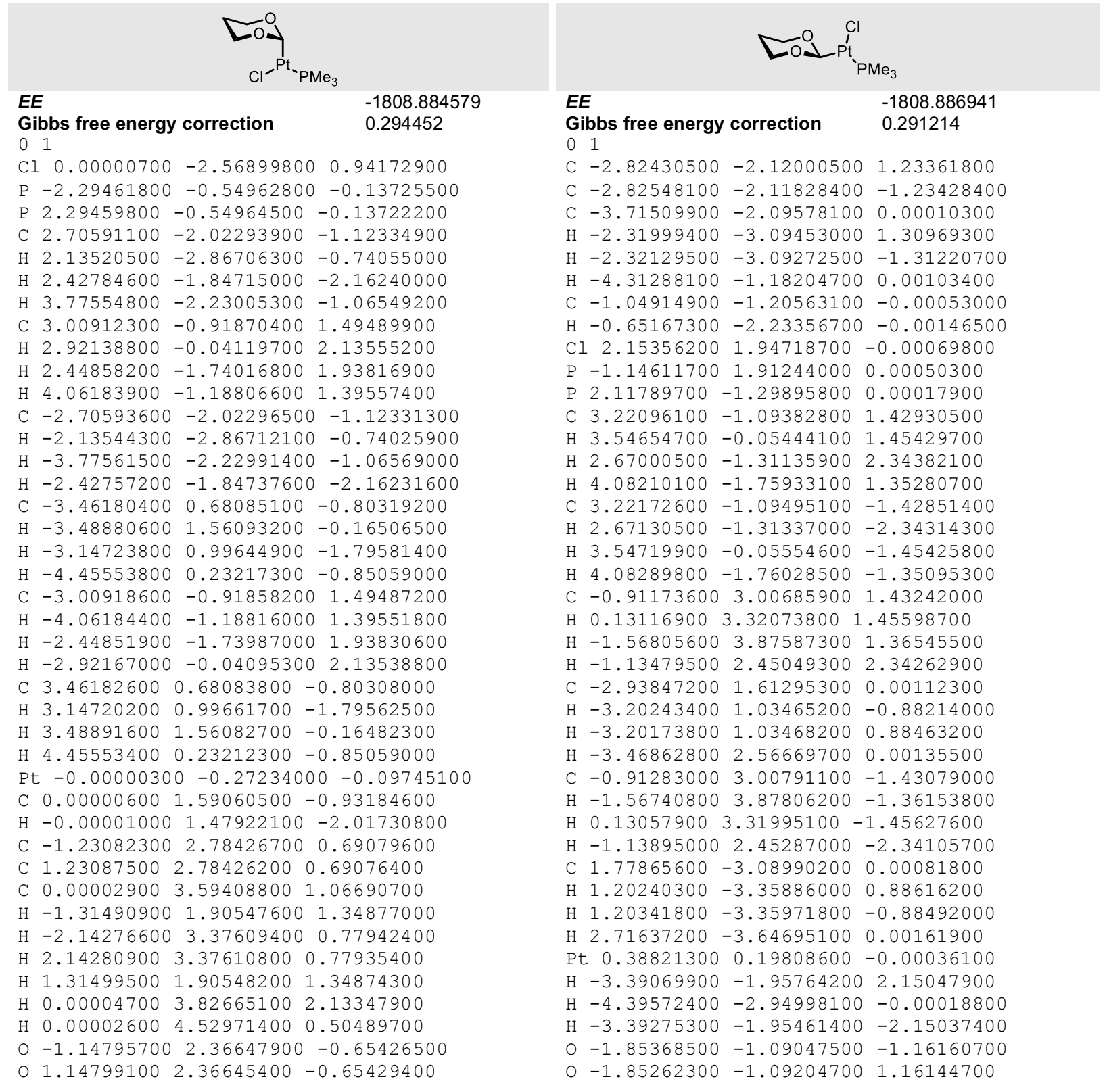


COPPER

\begin{tabular}{|c|c|c|}
\hline \multicolumn{2}{|r|}{ EE } & -1947.57525 \\
\hline \multicolumn{2}{|r|}{ Gibbs free energy correction } & 0.080373 \\
\hline $\mathrm{C}$ & $-1.31603800-1.23678400$ & -0.40204600 \\
\hline $\mathrm{C}$ & $0.08785100-0.00004700$ & 1.00105000 \\
\hline $\mathrm{C}$ & $-1.31597400 \quad 1.23682600$ & -0.40198700 \\
\hline $\mathrm{C}$ & $-2.17059600 \quad 0.00004800$ & -0.64337700 \\
\hline $\mathrm{H}$ & $0.45852700-0.00007900$ & 2.02302900 \\
\hline $\mathrm{H}$ & $-0.54853300-1.32101900$ & $0-1.19008500$ \\
\hline $\mathrm{H}$ & $-1.91027500-2.14941400$ & $0-0.39798400$ \\
\hline $\mathrm{H}$ & -1.910164002 .14948600 & -0.39788100 \\
\hline $\mathrm{H}$ & $-0.54846100 \quad 1.32106100$ & -1.19001900 \\
\hline $\mathrm{H}$ & $-3.00956400 \quad 0.00005400$ & 0.05426200 \\
\hline $\mathrm{H}$ & -2.56606200 & -1.66116000 \\
\hline 0 & -0.67746500 & 0.85150800 \\
\hline 0 & -0.67741300 & 0.85156600 \\
\hline $\mathrm{Cu}$ & 1.69524500 & $0-0.28229600$ \\
\hline
\end{tabular}

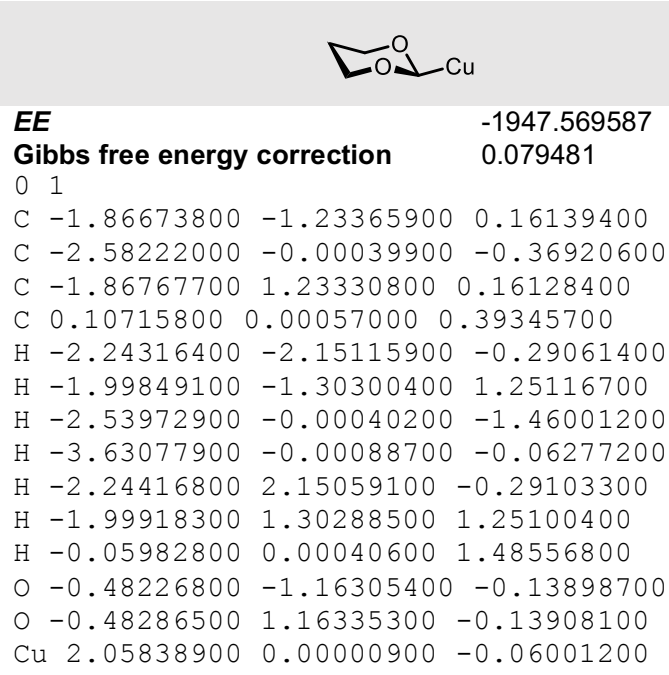




\section{SILVER}

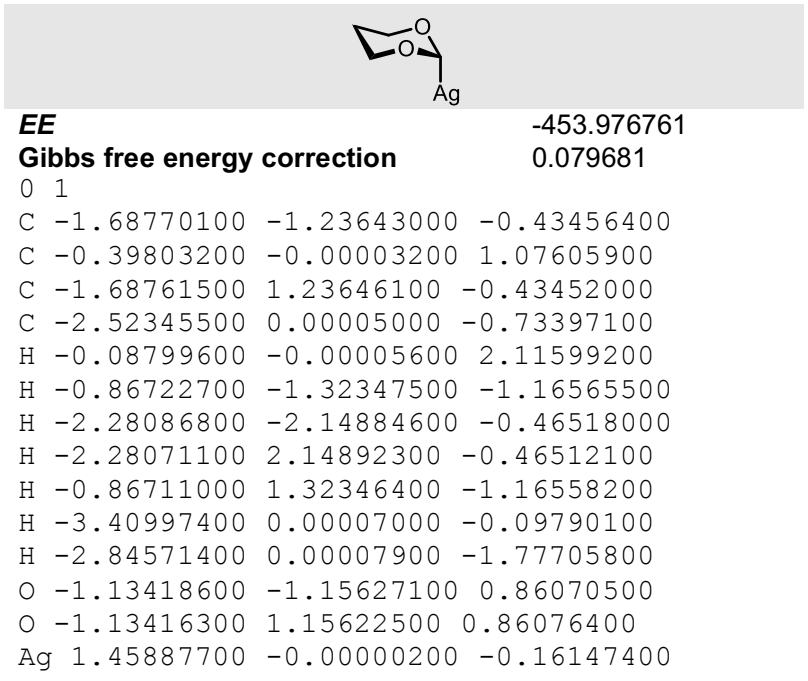

$\begin{array}{ll}\text { EE } & -453.968984 \\ \text { Gibbs free energy correction } & 0.078607\end{array}$

01

C $-2.34101000-1.23350600 \quad 0.15388500$

C $-3.04805700-0.00075800-0.38842500$

C $-2.34267200 \quad 1.23288400 \quad 0.15381200$

C $-0.37578300 \quad 0.00102200 \quad 0.41710500$

$\mathrm{H}-2.70677200-2.15129600-0.30570800$

$\mathrm{H}-2.49015600-1.30431400 \quad 1.24105300$

$\mathrm{H}-2.98818600-0.00058500-1.47832000$

$\mathrm{H}-4.10123200-0.00167900-0.09842000$

$\mathrm{H}-2.70899800 \quad 2.15031000-0.30588800$

$\mathrm{H}-2.49134600 \quad 1.30367700 \quad 1.24096900$

$\mathrm{H}-0.54361900 \quad 0.00079100 \quad 1.50705500$

$\begin{array}{lllll}0 & -0.95015700 & -1.16099400 & -0.12367500\end{array}$

$\begin{array}{llll}0 & -0.95151600 & 1.16148800 & -0.12409500\end{array}$

Ag $1.74231600 \quad 0.00002800-0.03908200$ 


\section{GOLD}

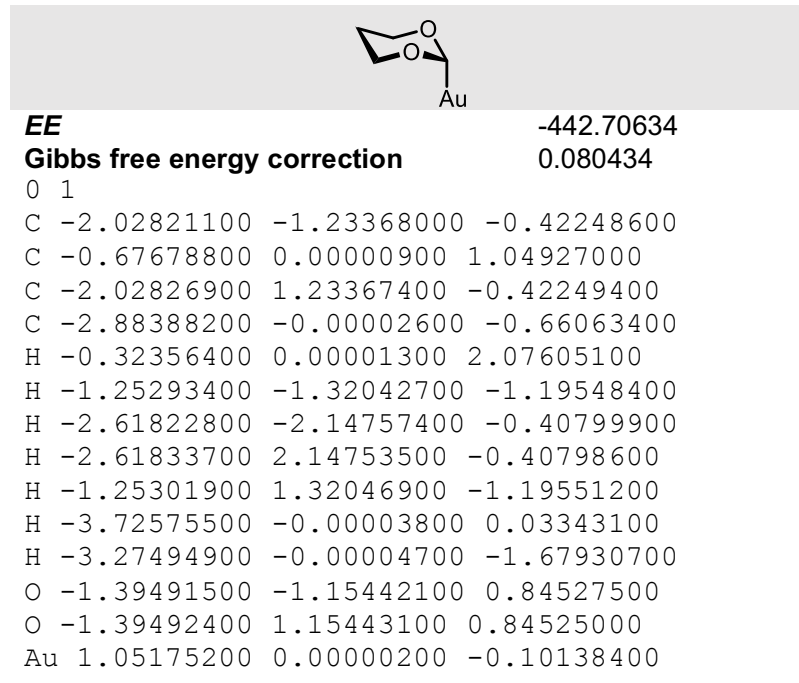

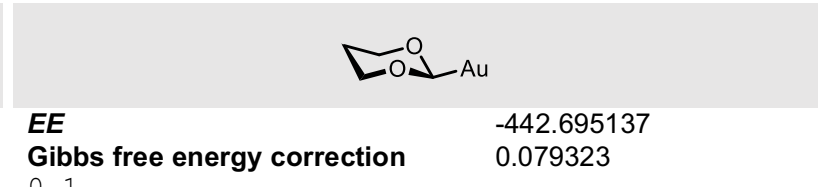

01

C $-2.69135000 \quad-1.23154800 \quad 0.16329400$

C $-3.40789900 \quad 0.00031800-0.36545100$

C $-2.69044000 \quad 1.23188900 \quad 0.16331700$

C $-0.73383400-0.00047200 \quad 0.38530200$

$\mathrm{H}-3.05572900-2.15083100 \quad-0.29136900$

$\mathrm{H}-2.81666900-1.30621400 \quad 1.25238400$

$\mathrm{H}-3.37144100 \quad 0.00025400-1.45594300$

$\mathrm{H}-4.45367900 \quad 0.00081400-0.05156100$

$\mathrm{H}-3.05479800 \quad 2.15132600-0.29108400$

$\mathrm{H}-2.81608600 \quad 1.30631800 \quad 1.25245300$

$\mathrm{H}-0.84826700-0.00036600 \quad 1.48089800$

$0-1.30260500-1.15574800-0.14334400$

$0-1.301974001 .15557100-0.14320100$

Au $1.24549900-0.00001300-0.02129300$ 
ZINC

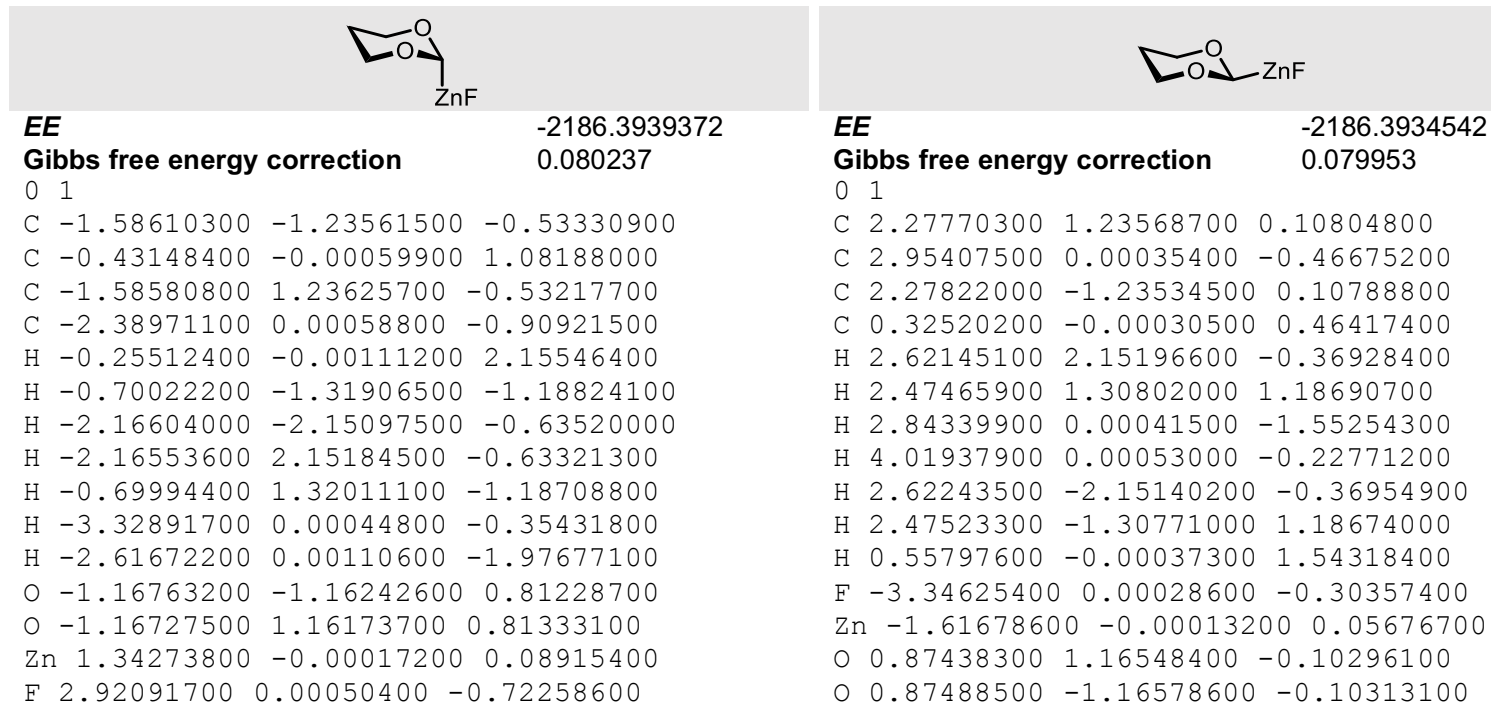


CADMIUM

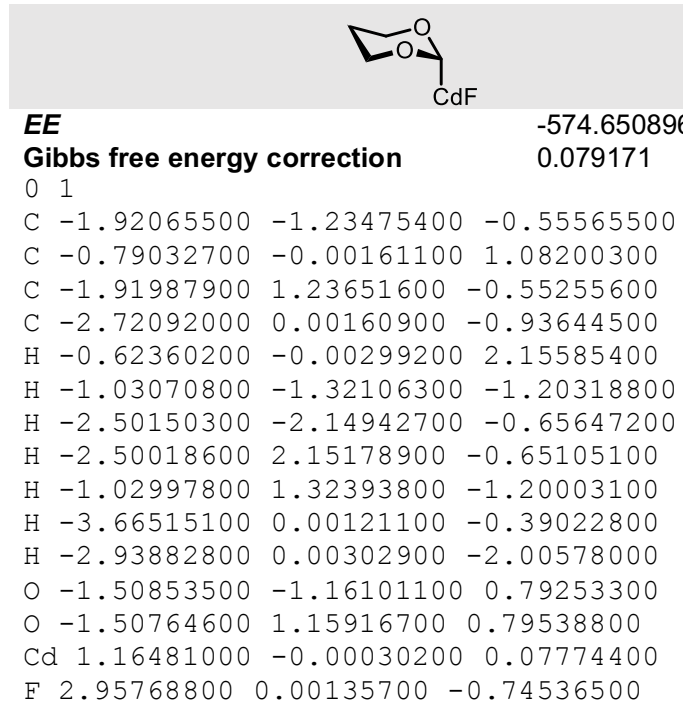

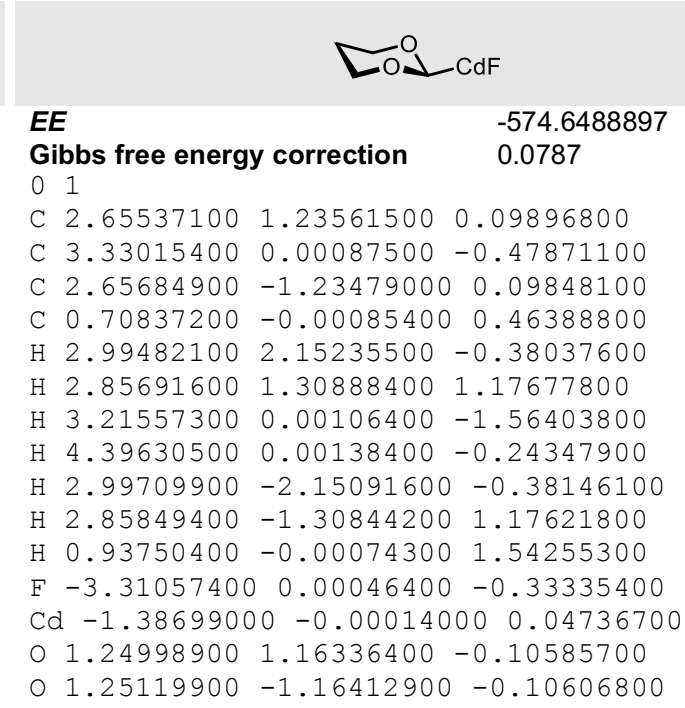


MERCURY

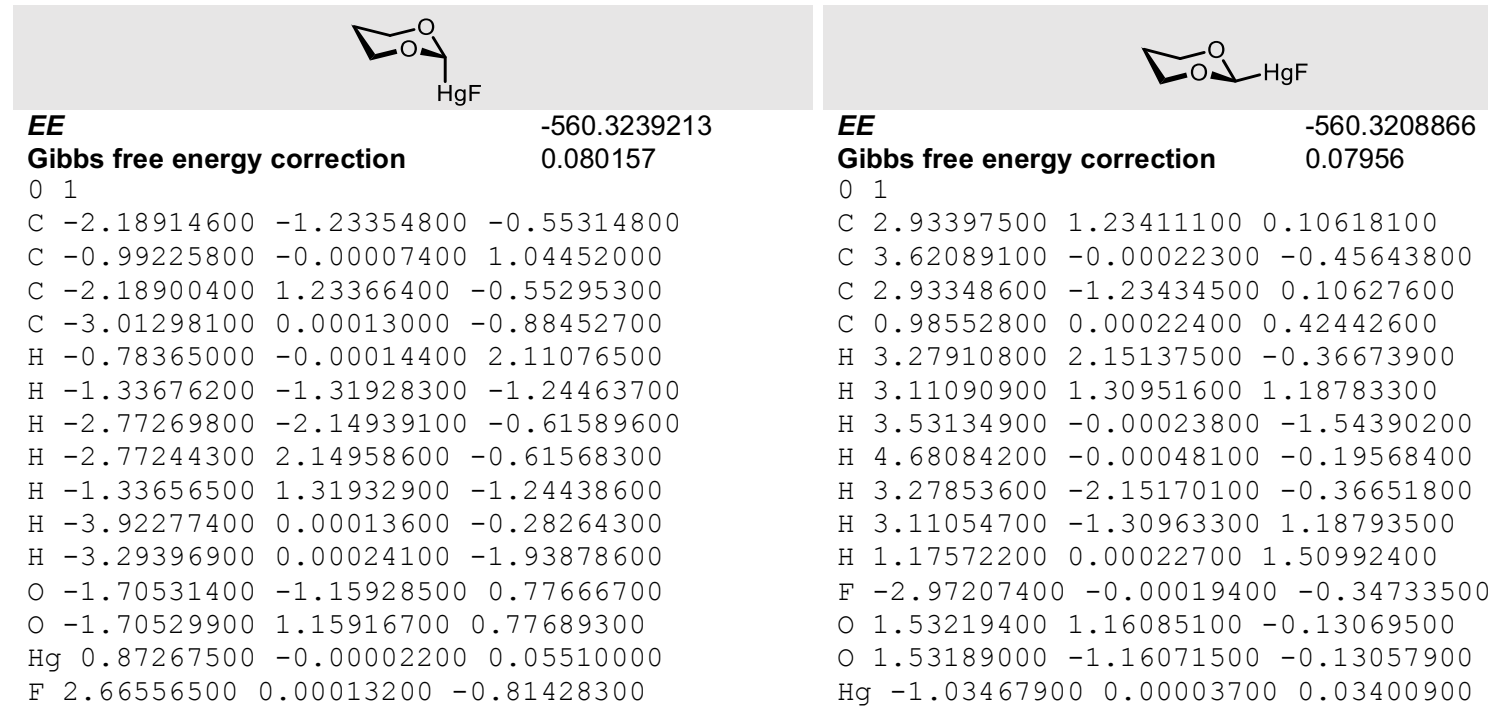


5.3. 1,3-Dithiane Structures

\section{NICKEL}

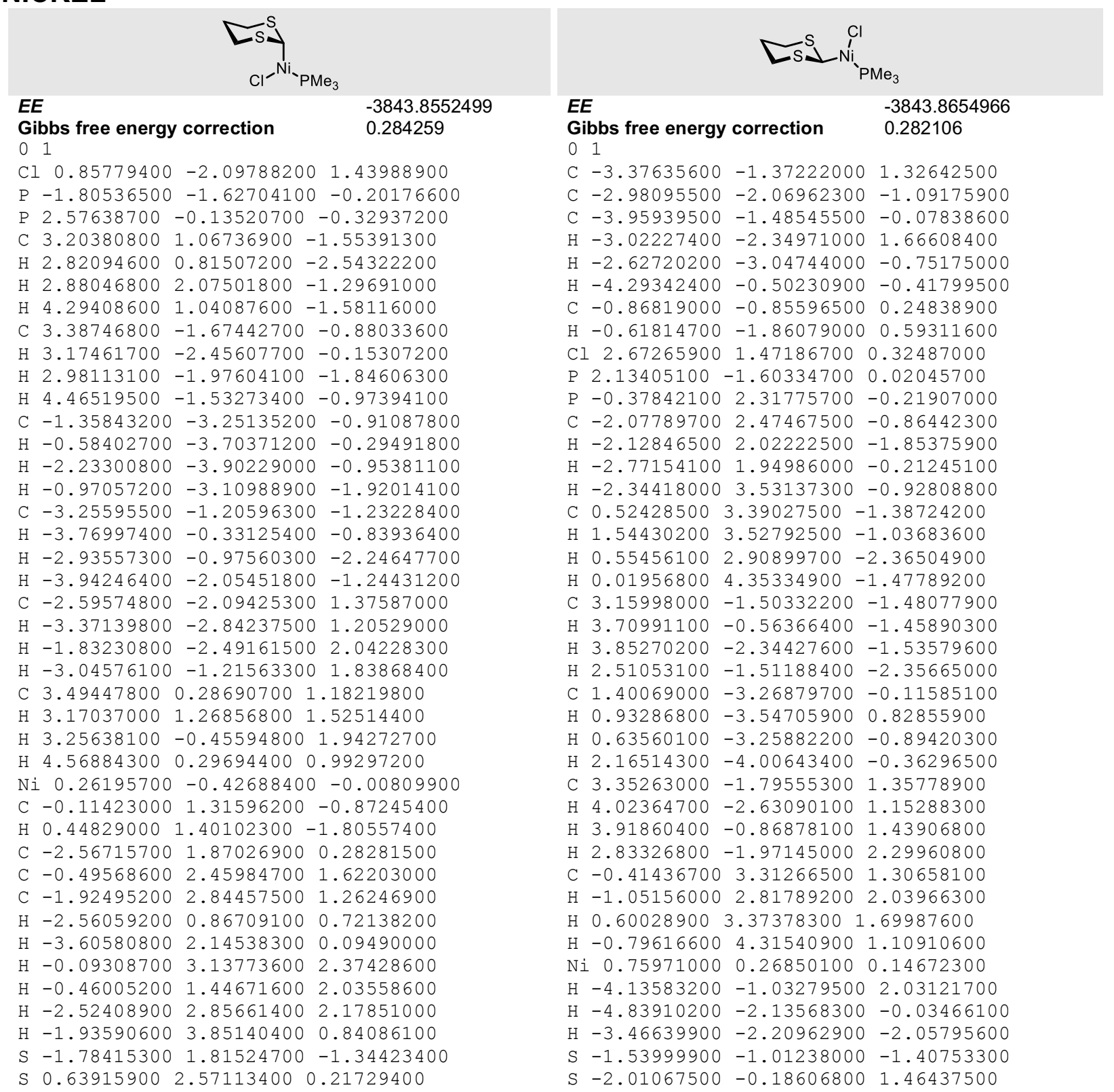


PALLADIUM

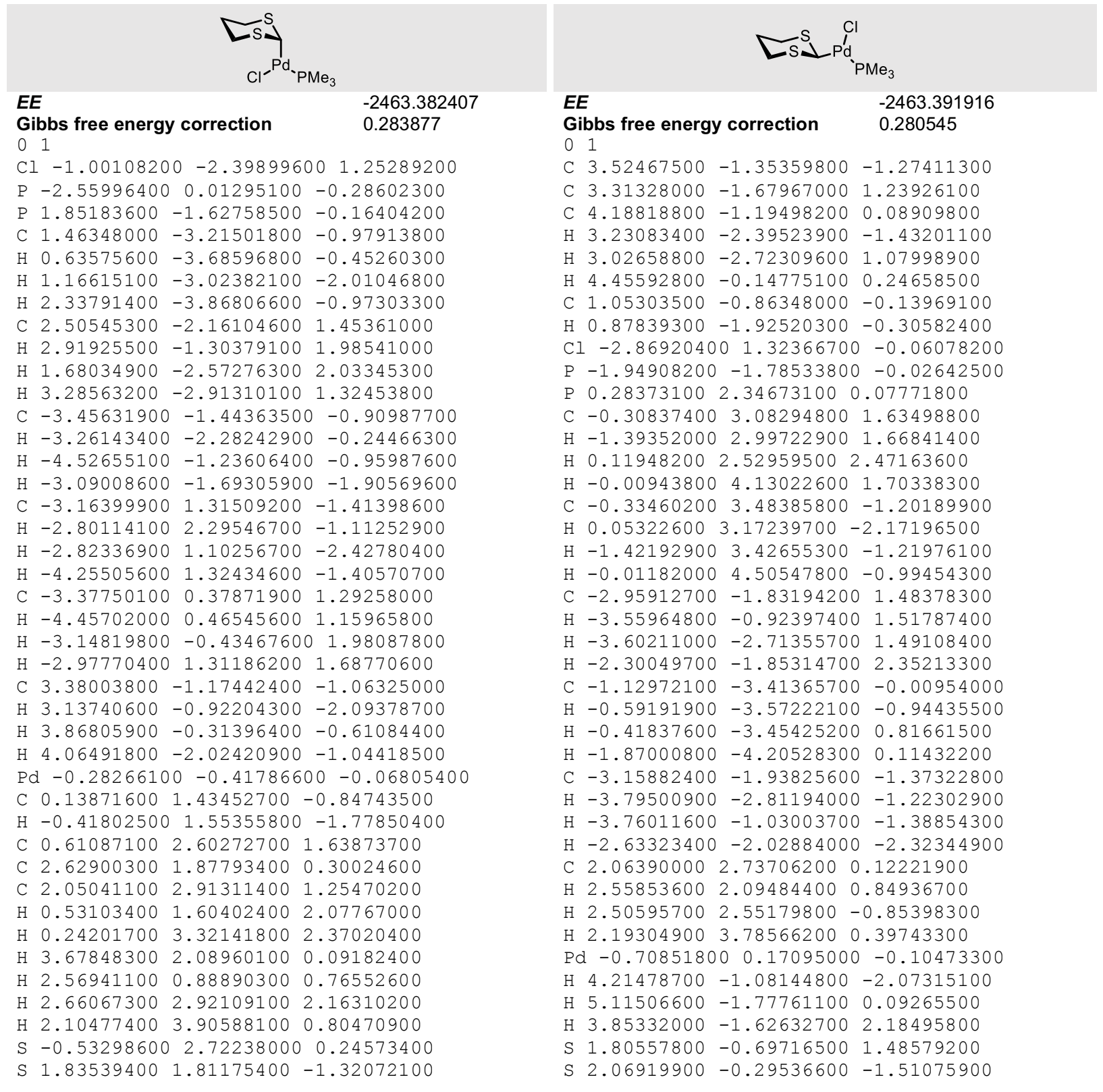




\section{PLATINUM}

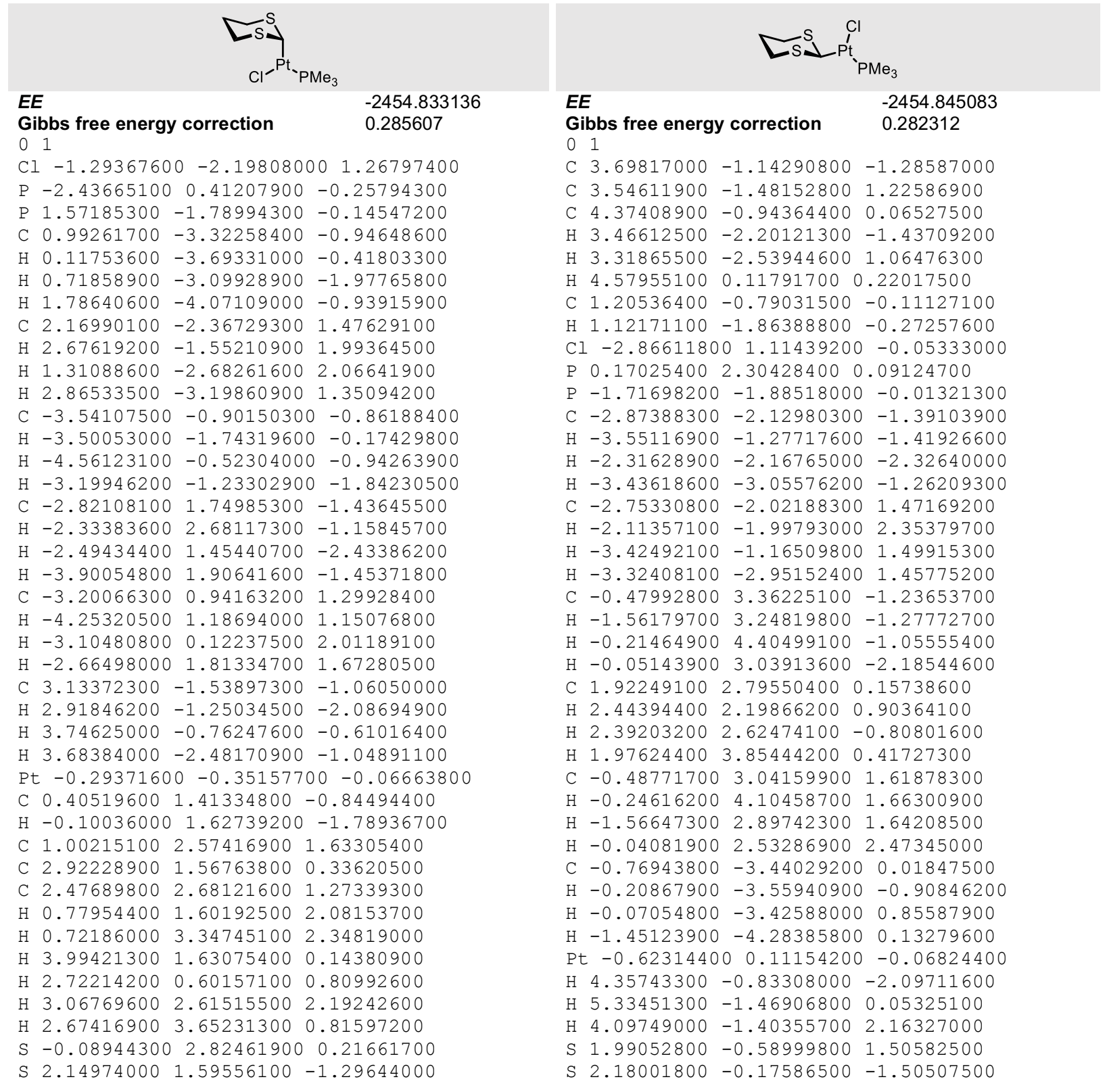


COPPER

\begin{tabular}{|c|c|c|}
\hline \multicolumn{2}{|l|}{$E E$} & -1088.660257 \\
\hline \multicolumn{2}{|r|}{ Gibbs free energy correction } & 0.070587 \\
\hline $\mathrm{C}-$ & $-1.25760800-1.27805900$ & -0.80461700 \\
\hline $\mathrm{CO}$ & $0.37259200-0.00003200$ & 0.96209500 \\
\hline $\mathrm{C}-$ & $-1.25756100 \quad 1.27810500$ & -0.80459200 \\
\hline$C-$ & $-2.07008100 \quad 0.00004000$ & -0.98906600 \\
\hline $\mathrm{H} \mathrm{O}$ & $0.86093000-0.00004700$ & 1.93601800 \\
\hline $\mathrm{H}-$ & $-0.41823000-1.29637500$ & -1.51176600 \\
\hline $\mathrm{H}-$ & $-1.87679200-2.15335700$ & -1.00049900 \\
\hline $\mathrm{H}-$ & $-1.87671400 \quad 2.15342900$ & -1.00045700 \\
\hline $\mathrm{H}-$ & $-0.41818600 \quad 1.29640500$ & -1.51174600 \\
\hline $\mathrm{H}-$ & -2.90747200 & -0.28950500 \\
\hline $\mathrm{H}-$ & 0.00005800 & -2.00275800 \\
\hline$S-$ & $-0.59756600-1.50336200$ & 0.85865600 \\
\hline$S-$ & 1.50335500 & 0.85868200 \\
\hline $\mathrm{Cu}$ & -0.00001300 & -0.42343500 \\
\hline
\end{tabular}

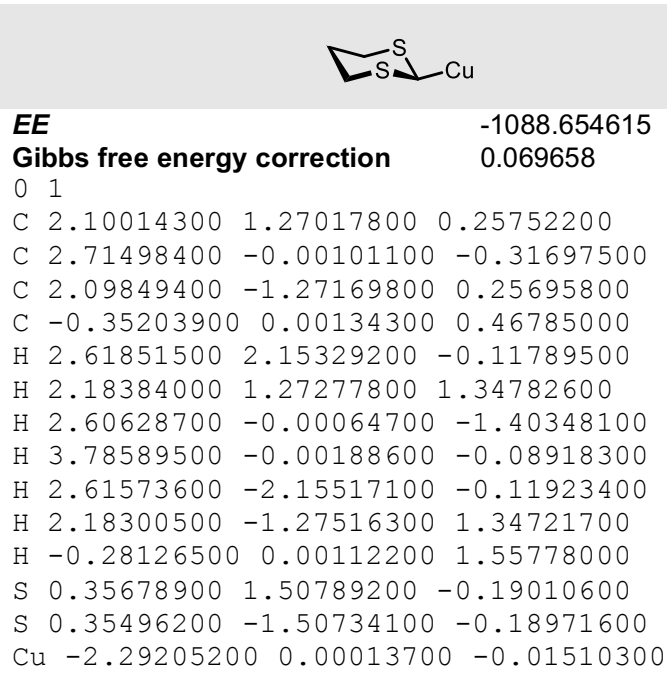




\section{SILVER}

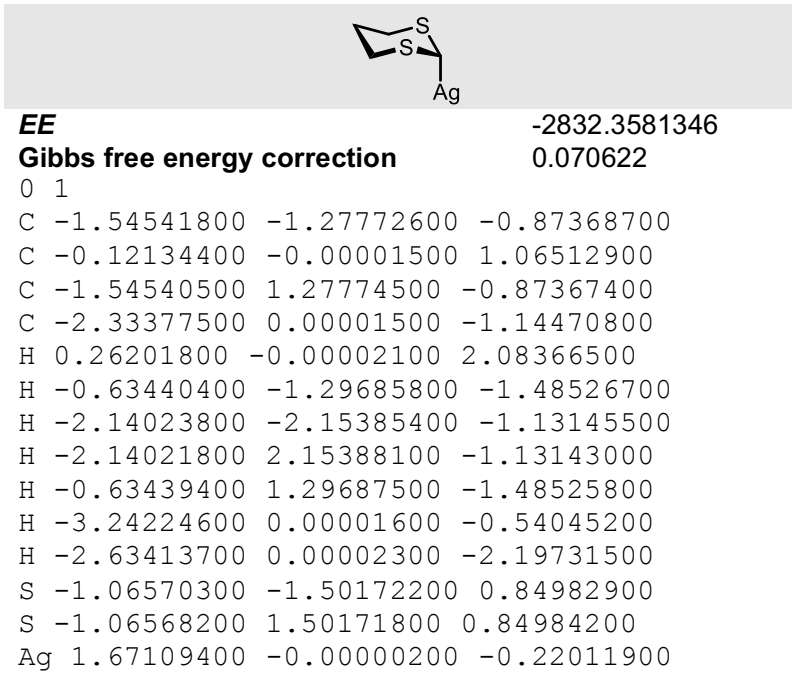

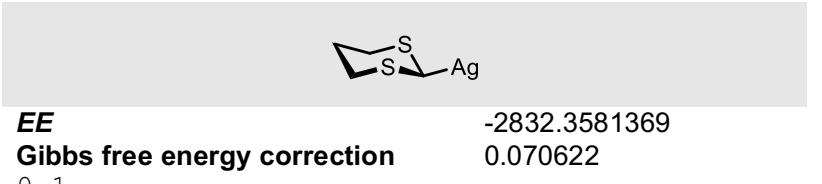

Gibbs free energy correction $\quad 0.070622$

01

$\begin{array}{llll}\text { C } 2.55116200 & 1.27000900 & 0.25051200\end{array}$

C $3.16066400-0.00070400-0.33052200$

$\begin{array}{llll}\text { C } 2.54979400 & -1.27140000 & 0.24931500\end{array}$

C $0.10520800 \quad 0.00141800 \quad 0.48284000$

H $3.06489300 \quad 2.15391700-0.12956900$

H $2.64521000 \quad 1.27245100 \quad 1.33993500$

$\mathrm{H} 3.04100100-0.00005300-1.41585700$

$\mathrm{H} 4.23387600-0.00158400-0.11357500$

$\mathrm{H} \quad 3.06234100-2.15520100-0.13272100$

H $2.64566200-1.27573000 \quad 1.33860400$

H $0.17247500 \quad 0.00128700 \quad 1.57149600$

S $0.802628001 .50714300-0.18109500$

S $0.80113800-1.50670300-0.18035700$

Ag $-2.01546100 \quad 0.00004100 \quad-0.01251000$ 
GOLD

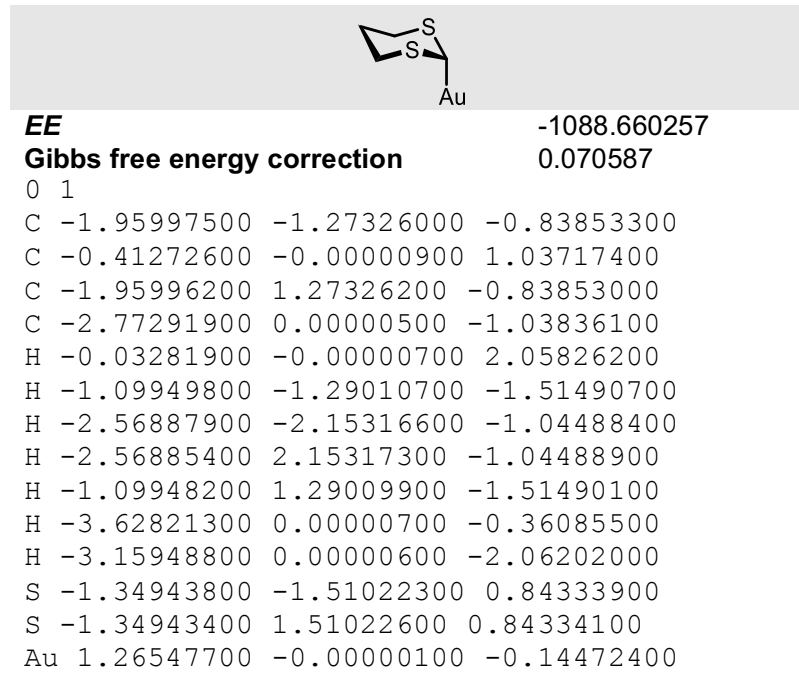

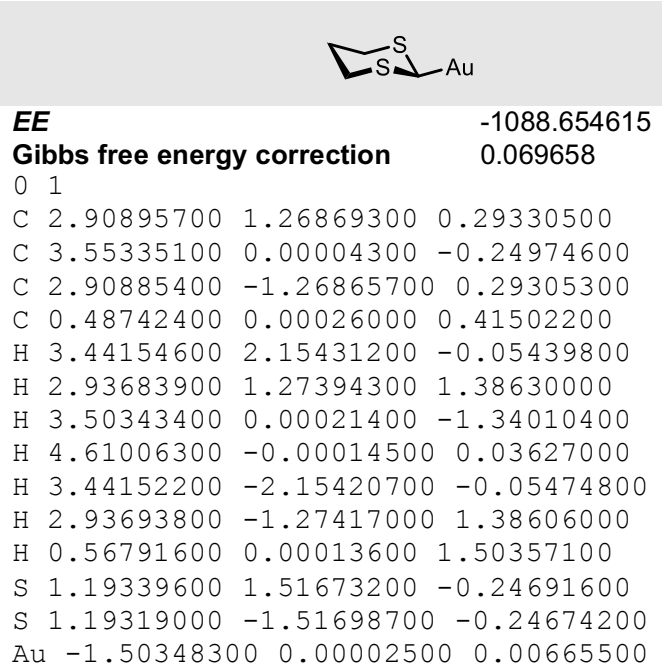


ZINC

$\begin{array}{ll}\text { EE } & -2832.3581346 \\ \text { Gibbs free energy correction } & 0.070622\end{array}$

01

C $1.34608500-1.27587400 \quad 0.99007800$

C $0.14637700-0.00024000-1.10578200$

C $1.34578400 \quad 1.27638500 \quad 0.98965000$

C 2.101242000 .000405001 .34448100

$\mathrm{H}-0.07242100-0.00043700-2.17339300$

$\mathrm{H} \quad 0.37748000-1.29893800 \quad 1.50587600$

H $1.90276900 \quad-2.15296900 \quad 1.31847400$

$\mathrm{H} \quad 1.90225300 \quad 2.15371900 \quad 1.31776800$

$\mathrm{H} \quad 0.37717300 \quad 1.29938200 \quad 1.50544400$

H $3.06932400 \quad 0.00043900 \quad 0.84118400$

$\mathrm{H} 2.28524300 \quad 0.00060700 \quad 2.42299300$

S $1.07991300-1.51096100-0.78208600$

S $1.079570001 .51082700-0.78258800$

Zn $-1.55593700-0.00023200-0.09463700$

F $-3.03915300 \quad 0.00035900 \quad 0.86944300$

\begin{tabular}{|c|c|c|}
\hline 28323581360 & & -2832.3581369 \\
\hline & bs free energy correction & 0.070622 \\
\hline $\mathrm{C}$ & $2.47037200 \quad 1.27076400$ & 0.23050700 \\
\hline $\mathrm{C}$ & $3.07693700-0.00079100$ & -0.35034300 \\
\hline & $2.46947500-1.27181000$ & 0.23063800 \\
\hline $\mathrm{C}$ & $0.02862900 \quad 0.00064900$ & 0.47954100 \\
\hline & 2.983334002 .15297000 & -0.15270900 \\
\hline & $2.56460500 \quad 1.27772500$ & 1.31960100 \\
\hline & $2.96068200-0.00079000$ & -1.43569300 \\
\hline & $4.14881000-0.00118500$ & -0.13033000 \\
\hline & $2.98170600-2.15451200$ & -0.15238800 \\
\hline & $2.56344200-1.27868100$ & 1.31974600 \\
\hline $\mathrm{H}$ & 0.158618000 .00051000 & 1.56489700 \\
\hline & $-3.63710600-0.0003970$ & $0-0.23773000$ \\
\hline & -1.904902000 .0003260 & 00.08652900 \\
\hline & 0.727190001 .51595200 & -0.19789900 \\
\hline & $0.72577000-1.51564700$ & -0.19781700 \\
\hline
\end{tabular}


CADMIUM

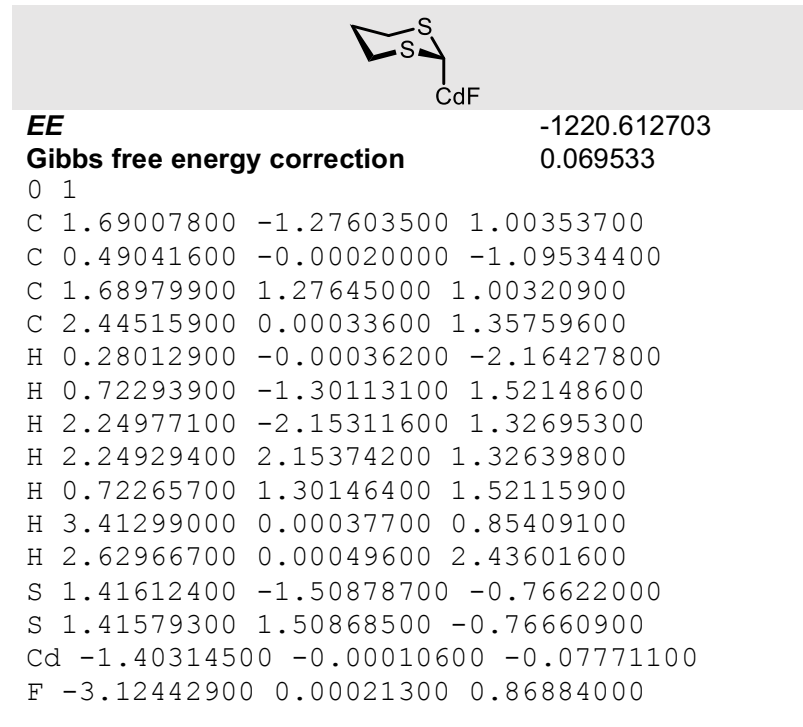

\begin{tabular}{|c|c|c|}
\hline \multicolumn{2}{|c|}{$E E$} & -1220.6125449 \\
\hline \multicolumn{2}{|r|}{$\begin{array}{l}\text { Gibbs free energy correction } \\
01\end{array}$} & 0.06926 \\
\hline & $2.84664300 \quad 1.27073400$ & 0.22350600 \\
\hline & -0.00056500 & -0.35972500 \\
\hline & $2.84602200-1.27153300$ & 0.22356400 \\
\hline $\mathrm{C}$ & $0.40836100 \quad 0.00055600$ & 0.47927400 \\
\hline & $3.35758700 \quad 2.15355900$ & -0.16116400 \\
\hline & 1.27753100 & 1.31226100 \\
\hline & $3.33128400-0.00055800$ & -1.44462000 \\
\hline & -0.00077000 & -0.14375000 \\
\hline & $3.35639400-2.15468400$ & -0.16110600 \\
\hline & -1.27831000 & 1.31231400 \\
\hline & $0.53145100 \quad 0.00045600$ & 1.56440400 \\
\hline & $-3.61247900-0.0004290$ & $0-0.27871800$ \\
\hline & 1.100955001 .51447900 & -0.19936500 \\
\hline & $1.10024100-1.51413700$ & -0.19927100 \\
\hline & $-1.68775700 \quad 0.0001250$ & 00.06684600 \\
\hline
\end{tabular}


MERCURY

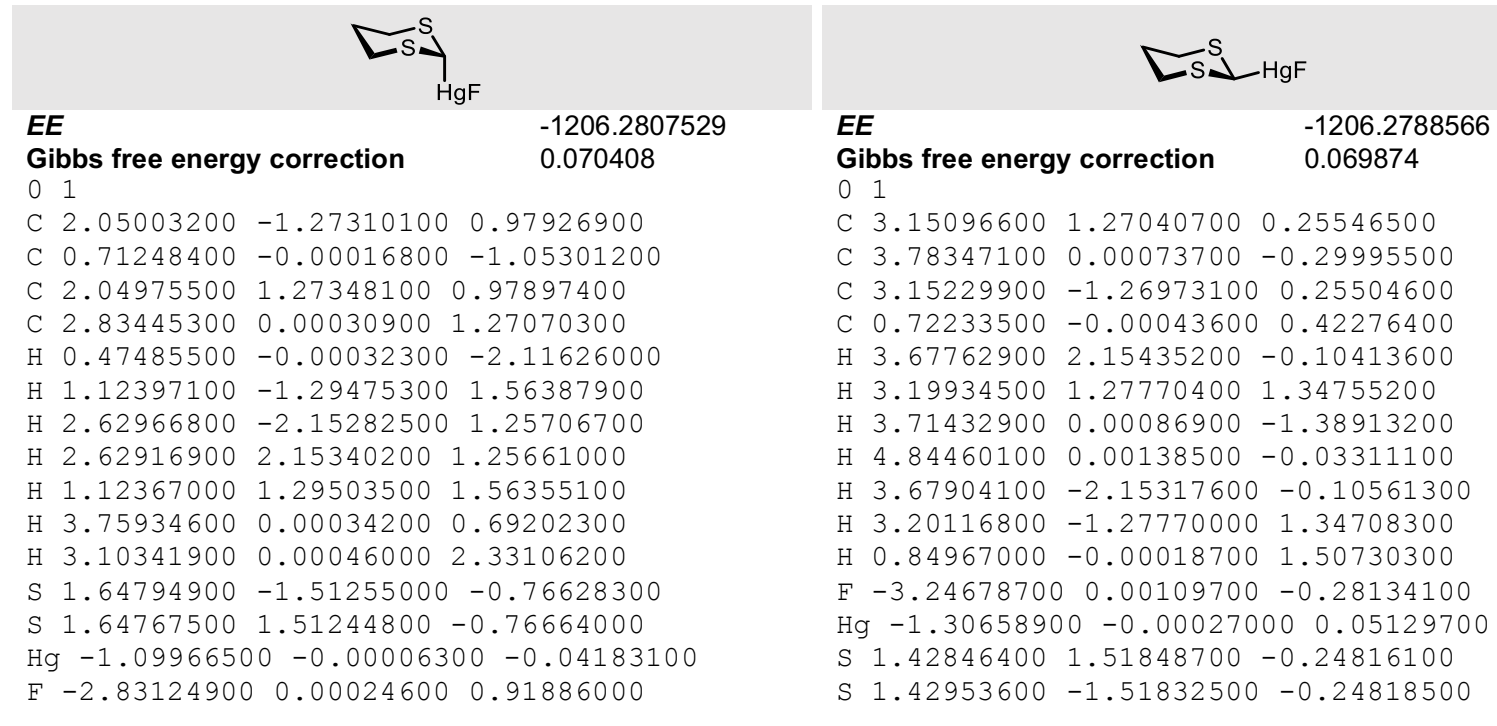




\section{Cartesian Coordinates for Figure 5A}

\subsection{Synclinal and Antiperiplanar Group 10 Structures}

NICKEL

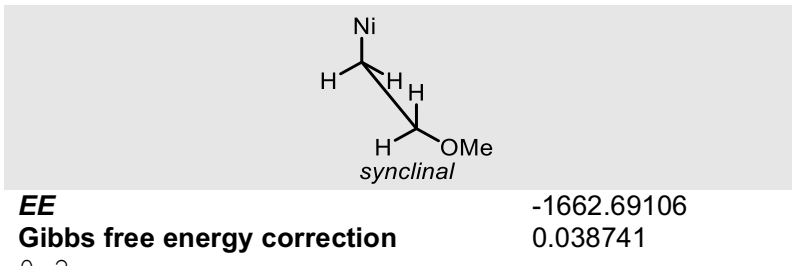

02

$\mathrm{Ni} 1.20823200-0.17035200-0.02493400$

C $-0.44601500 \quad 1.01853200 \quad 0.21214500$

$\mathrm{H}-0.61514500 \quad 1.16503300 \quad 1.28571700$

$\mathrm{H}-0.28344000 \quad 1.98791600-0.25301100$

$\begin{array}{llll}0 & -1.58882700 & 0.45799700 & -0.39034500\end{array}$

C $-1.91973000-0.79188200 \quad 0.12389200$

$\mathrm{H}-2.07773700-0.75590800 \quad 1.20735100$

$\mathrm{H}-1.11659900-1.52143600-0.07498000$

$\mathrm{H}-2.83249200-1.12964100-0.36038500$

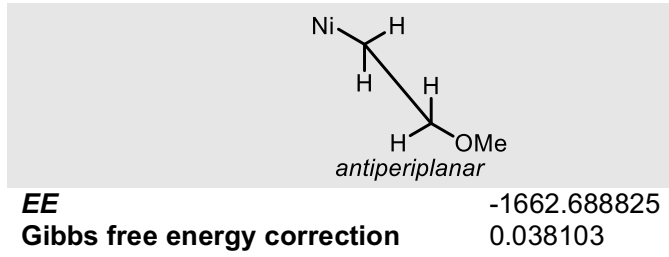

Gibbs
02

$\mathrm{Ni} 1.12074100-0.88409800 \quad 0.00000000$

C $0.00000000 \quad 0.79129800 \quad 0.00000000$

H $0.20699900 \quad 1.40652600 \quad 0.88490700$

$\mathrm{H} \quad 0.20699900 \quad 1.40652600 \quad-0.88490700$

$\begin{array}{lllll}0 & -1.35962900 & 0.38415400 & 0.00000000\end{array}$

C $-2.24349100 \quad 1.47310600 \quad 0.00000000$

$\mathrm{H}-2.09841000 \quad 2.09875000-0.88739700$

$\mathrm{H}-2.09841000 \quad 2.09875000 \quad 0.88739700$

$\mathrm{H}-3.25993900 \quad 1.08453800 \quad 0.00000000$ 
PALLADIUM

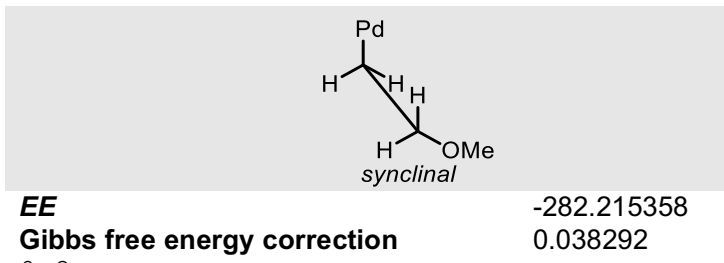

Gibbs
0

Pd $0.91883900-0.10795300-0.01438400$

C $-0.74242300 \quad 0.96747900 \quad 0.24316800$

$\mathrm{H}-0.92693100 \quad 1.00516100 \quad 1.32360000$

$\mathrm{H}-0.53222200 \quad 1.95693900-0.16036300$

$\begin{array}{lllll}0 & -1.82473000 & 0.44512900 & -0.44650500\end{array}$

C $-2.36718400-0.71620800 \quad 0.13039500$

$\mathrm{H}-2.65035400-0.53834900 \quad 1.17178600$

$\mathrm{H}-1.65101300-1.54313300 \quad 0.09902800$

$\mathrm{H}-3.25057500-0.98343400-0.44172200$

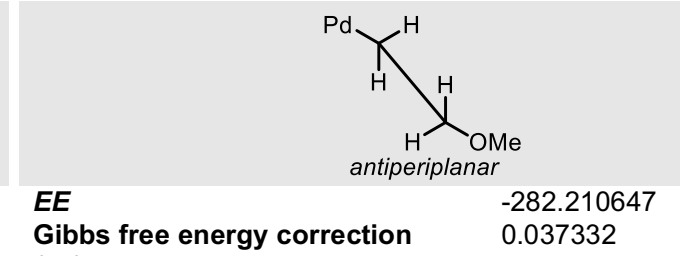

$\begin{array}{ll}\text { Gibbs } \\ 0 & 2\end{array}$

Pd $0.62039900-0.85156300 \quad 0.00000000$

C $0.000000001 .03560500 \quad 0.00000000$

$\mathrm{H} \quad 0.39135900 \quad 1.52689700 \quad 0.89981300$

H $0.39135900 \quad 1.52689700-0.89981300$

O $-1.39877600 \quad 1.01634100 \quad 0.00000000$

C $-1.96234200 \quad 2.30709000 \quad 0.00000000$

$\mathrm{H}-1.65677600 \quad 2.86782100 \quad-0.88847400$

$\mathrm{H}-1.65677600 \quad 2.86782100 \quad 0.88847400$

$\mathrm{H}-3.04328100 \quad 2.19559000 \quad 0.00000000$ 
NICKEL

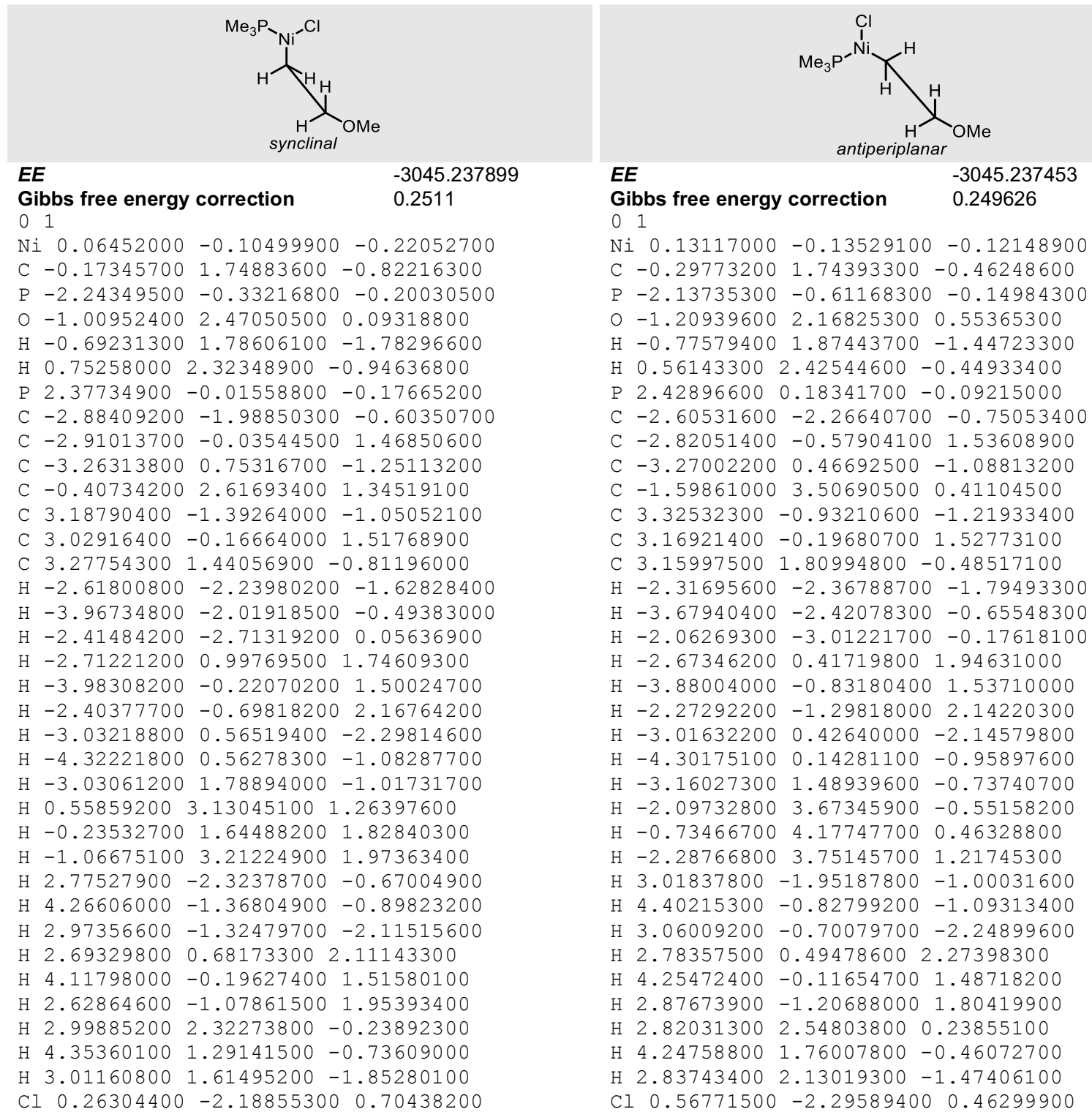


PALLADIUM
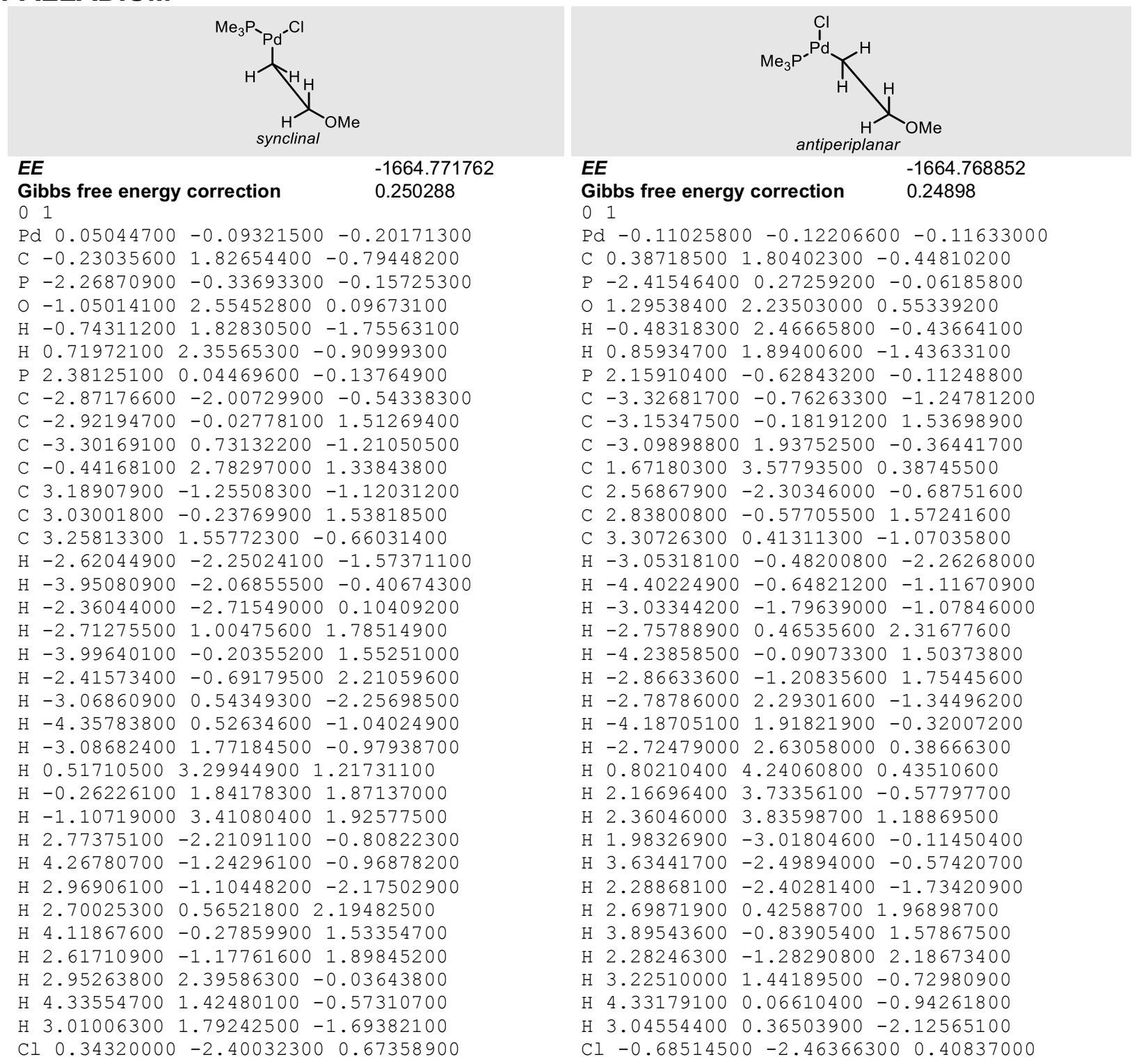

Gibb
01

$\mathrm{Pd}-0.11025800-0.12206600-0.11633000$

C $0.38718500 \quad 1.80402300-0.44810200$

P $-2.41546400 \quad 0.27259200-0.06185800$

$\begin{array}{llllll}0 & 1.29538400 & 2.23503000 & 0.55339200\end{array}$

$\mathrm{H}-0.48318300 \quad 2.46665800-0.43664100$

$\mathrm{H} \quad 0.85934700 \quad 1.89400600-1.43633100$

P 2.15910400-0.62843200-0.11248800

C $-3.32681700-0.76263300-1.24781200$

C $-3.15347500-0.18191200 \quad 1.53698900$

C $-3.09898800 \quad 1.93752500-0.36441700$

C $1.67180300 \quad 3.57793500 \quad 0.38745500$

C $2.56867900-2.30346000-0.68751600$

C $2.83800800-0.57705500 \quad 1.57241600$

C $3.30726300 \quad 0.41311300-1.07035800$

$\mathrm{H}-3.05318100-0.48200800-2.26268000$

$\mathrm{H}-4.40224900-0.64821200-1.11670900$

$\mathrm{H}-3.03344200-1.79639000-1.07846000$

$\mathrm{H}-2.75788900 \quad 0.46535600 \quad 2.31677600$

$\mathrm{H}-4.23858500-0.09073300 \quad 1.50373800$

$\mathrm{H}-2.86633600-1.20835600 \quad 1.75445600$

$\mathrm{H}-2.78786000 \quad 2.29301600-1.34496200$

$\mathrm{H}-4.18705100 \quad 1.91821900-0.32007200$

$\mathrm{H}-2.72479000 \quad 2.63058000 \quad 0.38666300$

$\mathrm{H} \quad 0.80210400 \quad 4.24060800 \quad 0.43510600$

$\mathrm{H} \quad 2.16696400 \quad 3.73356100-0.57797700$

$\mathrm{H} 2.36046000 \quad 3.83598700 \quad 1.18869500$

$\mathrm{H} 1.98326900-3.01804600-0.11450400$

H $3.63441700-2.49894000-0.57420700$

$\mathrm{H} 2.28868100-2.40281400-1.73420900$

H 2.69871900 $0.42588700 \quad 1.96898700$

$\mathrm{H} \quad 3.89543600-0.83905400 \quad 1.57867500$

H 2.28246300 -1.28290800 2.18673400

H $3.22510000 \quad 1.44189500-0.72980900$

$\mathrm{H} \quad 4.33179100 \quad 0.06610400 \quad-0.94261800$

$\mathrm{H} \quad 3.04554400 \quad 0.36503900 \quad-2.12565100$

$\mathrm{Cl}-0.68514500-2.46366300 \quad 0.40837000$ 


\section{PLATINUM}

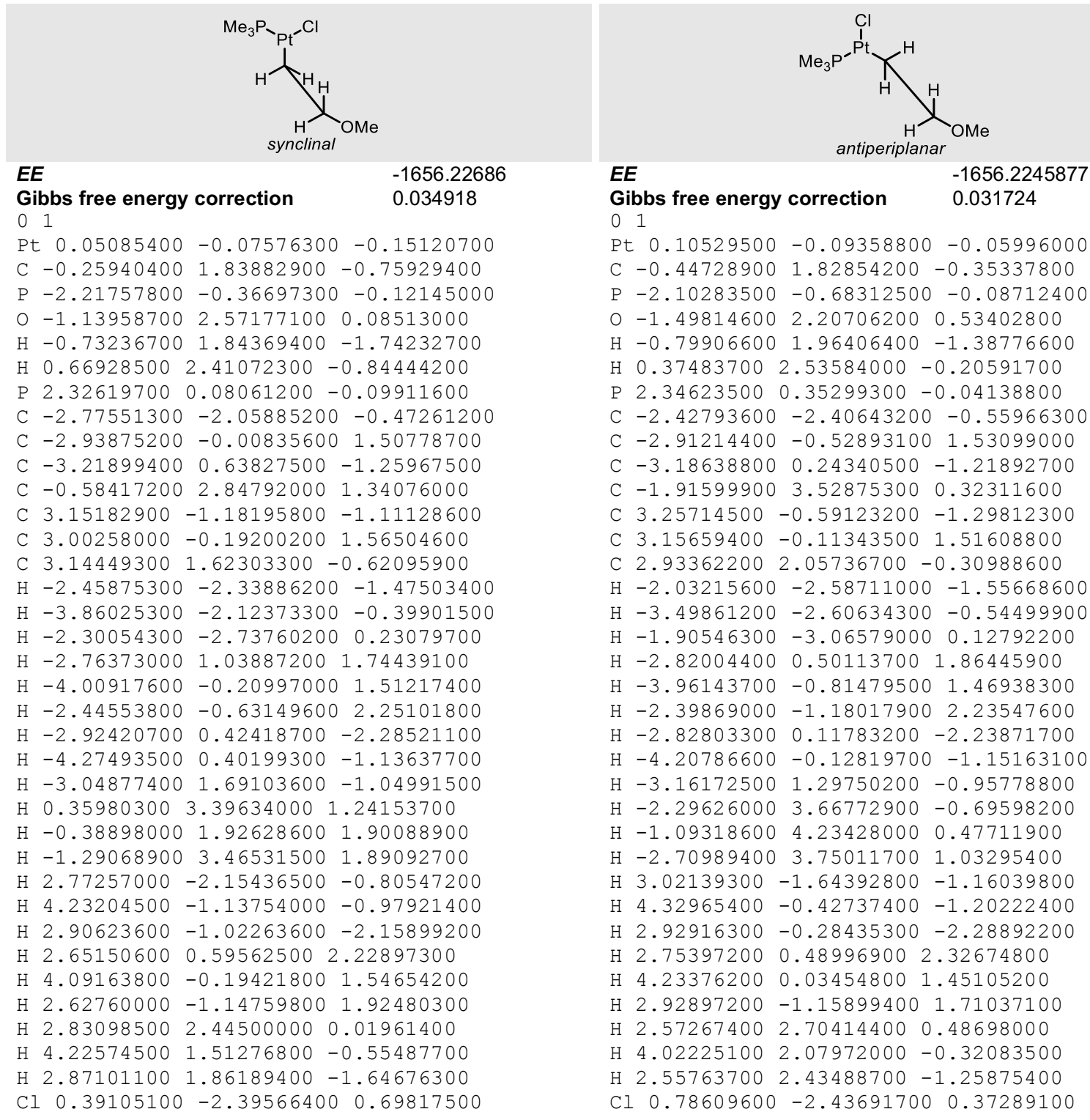




\section{PLATINUM}

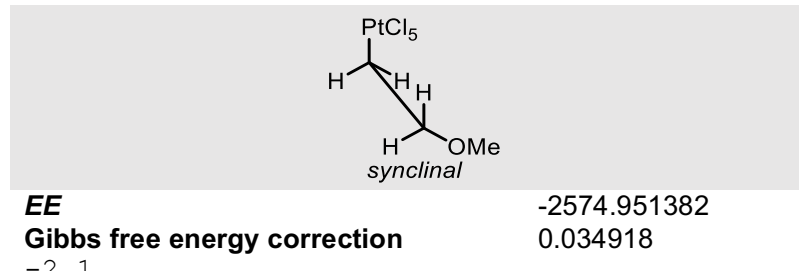

$-21$

Pt $0.17223800 \quad 0.00630300 \quad 0.03638300$

Cl $1.17461300-0.91061100 \quad 1.96697000$

Cl $2.37958400-0.31527600-1.34494600$

Cl $-0.59090100-2.14579300-0.57878700$

$\mathrm{Cl}-0.84648900 \quad 0.94220100-1.86528000$

Cl $0.83075600 \quad 2.15432200 \quad 0.71517500$

C $-1.46052900 \quad 0.19156300 \quad 1.25554600$

$\mathrm{H}-1.61001300-0.81823500 \quad 1.64247300$

$\mathrm{H}-1.13032600 \quad 0.87634700 \quad 2.02870800$

$\begin{array}{lllll}0 & -2.63786500 & 0.70131100 & 0.72477900\end{array}$

C $-3.36984100-0.21460100-0.04785900$

$\mathrm{H}-4.30378900 \quad 0.27720000 \quad-0.32203700$

$\mathrm{H}-2.82528500-0.50119300-0.94599800$

$\mathrm{H}-3.58860000-1.12033100 \quad 0.53135200$

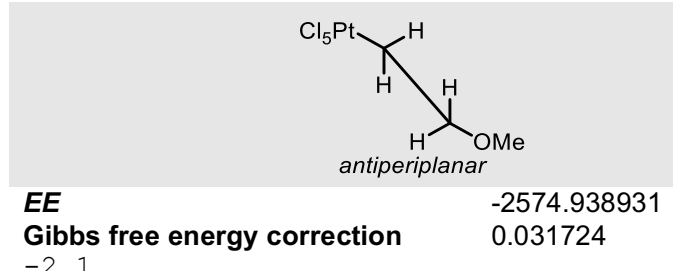

$-21$

Pt $0.19831100 \quad 0.01812400 \quad 0.00007700$

Cl $0.72249300 \quad 2.33157900 \quad 0.00245300$

Cl $2.70143200-0.61179500-0.00270900$

$\begin{array}{lllll}\text { Cl } & 0.13204700 & 0.02777400 & 2.35233700\end{array}$

Cl $-0.35291700-2.25163900-0.00207800$

Cl $0.12835200 \quad 0.03328300 \quad-2.35208100$

C $-1.70576000 \quad 0.68189900 \quad 0.00217400$

$\mathrm{H}-1.80349500 \quad 1.29494500 \quad 0.90156100$

$\mathrm{H}-1.80404400 \quad 1.29852300-0.89469500$

$\begin{array}{llll}0 & -2.69064500 & -0.32564500 & 0.00043600\end{array}$

C $-3.96325100 \quad 0.23052500 \quad 0.00064600$

$\mathrm{H}-4.68691000-0.58530200-0.00096900$

$\mathrm{H}-4.13471500 \quad 0.85484100 \quad 0.88907900$

$\mathrm{H}-4.13379500 \quad 0.85754000-0.88604800$ 


\subsection{Synclinal and Antiperiplanar Group 11 Structures}

\section{COPPER}

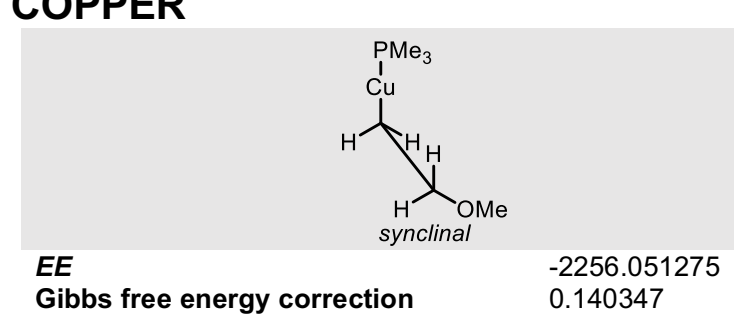

01

C $-3.305540001 .28028900-0.15967100$

C $-2.52933500-0.93411600-0.10208000$

$\mathrm{H}-2.28866600 \quad 1.70271400-0.10958000$

$\mathrm{H}-3.99838400 \quad 1.96936900 \quad 0.32079800$

$\mathrm{H}-2.71841600-1.84483600 \quad 0.46846600$

$\begin{array}{lllll}0 & -3.37914900 & 0.05830600 & 0.49202800\end{array}$

$\mathrm{Cu}-0.56363600-0.48160900-0.07789800$

P $1.70676600 \quad 0.08003600 \quad 0.01230100$

C $2.037387001 .87452200 \quad 0.08444200$

$\mathrm{H} \quad 1.55445000 \quad 2.29603900 \quad 0.96345100$

$\mathrm{H} \quad 3.10721900 \quad 2.07495000 \quad 0.12926200$

$\mathrm{H} \quad 1.61695500 \quad 2.35560600-0.79611300$

C $2.64643300-0.54218000 \quad 1.44919400$

$\mathrm{H} 2.62635800-1.62978600 \quad 1.45279400$

$\mathrm{H} 3.68050700-0.20096600 \quad 1.41758700$

$\mathrm{H} 2.17922100-0.191113002 .36666100$

C $2.75318400-0.44739500-1.38843600$

H $2.35667000-0.03400100-2.31318800$

$\mathrm{H} \quad 3.78120700-0.11230900-1.25573900$

H 2.73602300 $-1.53225700-1.46643600$

$\mathrm{H}-2.89640900-1.13433100-1.11937000$

$\mathrm{H}-3.57235700 \quad 1.19387000-1.22097600$

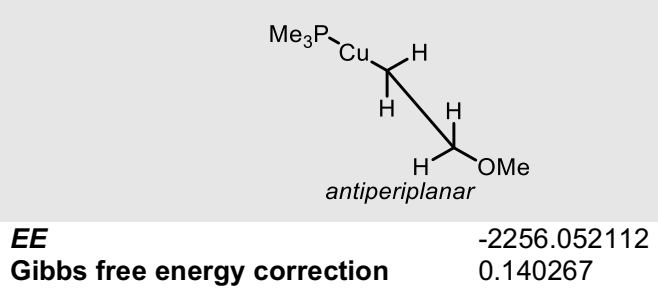

01

C $-4.48729500-0.47128500 \quad 0.00027400$

C $-2.42979100 \quad 0.66983800 \quad 0.00008200$

$\mathrm{H}-4.83617000 \quad 0.07114500 \quad 0.88736600$

$\mathrm{H}-4.92625300-1.46851800-0.00049900$

$\mathrm{H}-2.76904300 \quad 1.24342200-0.87522800$

o $-3.09589200-0.59905800-0.00038800$

$\mathrm{Cu}-0.45224300 \quad 0.36653100-0.00014500$

P $1.83288800-0.08156800-0.00002800$

C $2.45382600-1.020858001 .43734600$

H $1.93051700 \quad-1.97233200 \quad 1.50115000$

H $3.52460700-1.20243500 \quad 1.35261800$

H 2.25412400 $-0.46222500 \quad 2.34912000$

C $2.44198900-1.07833400-1.40321800$

H 2.23374200 $-0.55763900-2.33529200$

H $3.51363700-1.25571900-1.32059400$

$\mathrm{H} \quad 1.91899400-2.03190500-1.42342600$

C $2.95731400 \quad 1.35692800 \quad-0.03375300$

H 2.77104700 $1.98384100 \quad 0.83547400$

H $3.99893800 \quad 1.03798700-0.03122900$

H $2.764238001 .94775500-0.92645200$

$\mathrm{H}-2.76887500 \quad 1.24273300 \quad 0.87593400$

$\mathrm{H}-4.83688900 \quad 0.07275400-0.88559700$ 
COPPER

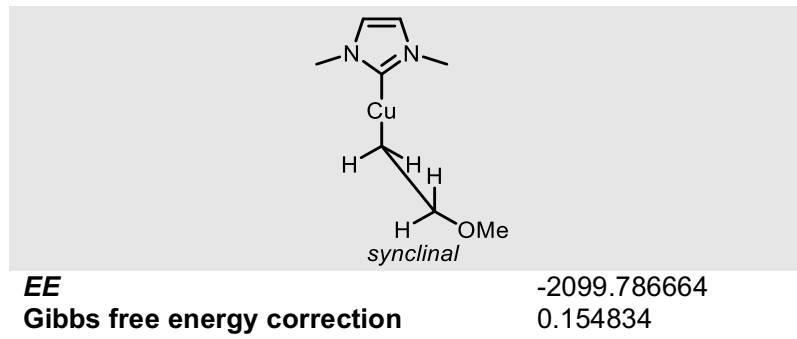

01

$\begin{array}{llll}\text { C } 3.56449700 & 0.85425000 & 0.95062600\end{array}$

C $2.77671500-0.89560700-0.40514700$

H $2.54724600 \quad 1.21374500 \quad 1.17699600$

$\mathrm{H} \quad 4.25141300 \quad 1.69962100 \quad 0.97003300$

H $2.98542200-1.29997800-1.39794900$

C $-3.04666000 \quad 1.20548200 \quad 0.05524800$

C $-3.39098600-0.09028700 \quad 0.20667000$

$\mathrm{H}-3.64614400 \quad 2.09652500 \quad 0.03915700$

$\mathrm{H}-4.34985600-0.55282700 \quad 0.34879300$

$\begin{array}{llll}\text { C }-1.14701600 & -0.01231600 & -0.03700100\end{array}$

C $-0.89994400 \quad 2.44088700-0.27985700$

$\mathrm{H}-1.02325000 \quad 3.10120700 \quad 0.57509900$

$\mathrm{H} \quad 0.14564700 \quad 2.16391500-0.37336800$

$\mathrm{H}-1.21923300 \quad 2.95321000-1.18409800$

C $-2.14681900-2.25670000 \quad 0.26797500$

$\mathrm{H}-1.10514500 \quad-2.55015300 \quad 0.18791200$

$\mathrm{H}-2.53804800 \quad-2.57271400 \quad 1.23199500$

$\mathrm{H}-2.71753200-2.72897700-0.52795500$

$\mathrm{N}-1.67752700 \quad 1.22898300-0.09142500$

$\mathrm{N}-2.21955500-0.81241300 \quad 0.14737800$

$\begin{array}{lllll}0 & 3.62984100 & 0.26289300 & -0.30098100\end{array}$

H $3.15090600-1.64971400 \quad 0.30384000$

H $3.84499700 \quad 0.15443400 \quad 1.74900400$

$\mathrm{Cu} 0.81571400-0.50074700-0.19428800$

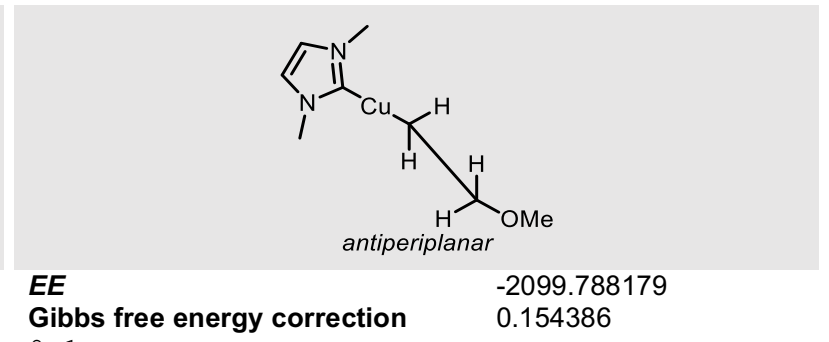

\section{1}

C $4.66268300-0.47349300 \quad 0.00160600$

C $2.68024000 \quad 0.80609200-0.00066500$

H $5.04732600 \quad 0.04526500-0.88508600$

H $5.03797900-1.49693800 \quad 0.00293400$

H $3.06332700 \quad 1.35277800 \quad 0.87394900$

C $-3.50178200-0.04442900 \quad 0.00122000$

C $-3.06319700-1.32061300 \quad 0.00088900$

$\mathrm{H}-4.49718600 \quad 0.35892500 \quad 0.00198800$

$\mathrm{H}-3.60112500-2.25024500 \quad 0.00130100$

C $-1.24501000 \quad 0.01709700-0.00037900$

C $-2.40607900 \quad 2.20244000 \quad 0.00049100$

$\mathrm{H}-2.91473000 \quad 2.56808600 \quad 0.88926600$

$\mathrm{H}-1.38133400 \quad 2.55915900-0.00115300$

$\mathrm{H}-2.91760300 \quad 2.56810000-0.88662300$

C $-0.81837600-2.42406700-0.00116000$

$\mathrm{H} 0.21341400 \quad-2.08409400 \quad 0.00141500$

$\mathrm{H}-1.00162300 \quad-3.02630800 \quad 0.88526400$

$\mathrm{H}-0.99842200-3.02240000-0.89089000$

$\mathrm{N}-2.37778200 \quad 0.75198700 \quad 0.00038400$

$\mathrm{N}-1.68766400-1.25930100-0.00001700$

$03.26672800-0.50939100 \quad 0.00100500$

H $3.063973001 .35086700-0.87620500$

H $5.04649800 \quad 0.04726600 \quad 0.88749500$

$\mathrm{Cu} \quad 0.70044600 \quad 0.55440300-0.00090600$ 
COPPER

$\begin{array}{ll}\text { EE synclinal } & \\ \text { Gibbs free energy correction } & -2255.178879 \\ & 0.033877\end{array}$

02

C $2.54531700 \quad 1.17475000 \quad 0.16563300$

H $3.11583000 \quad 0.94798700 \quad 1.06527600$

H $1.63036800 \quad 1.70547000 \quad 0.42667800$

$\mathrm{H} \quad 3.13920500 \quad 1.77286900-0.51463800$

C $1.57933100 \quad-0.96388100 \quad 0.18678500$

H $1.72346400 \quad-0.94710000 \quad 1.26591800$

$\mathrm{H} \quad 1.54676500-1.92284300-0.31829700$

$02.22650600-0.03305900-0.52197500$

$\mathrm{Cu}-0.43667000 \quad-0.36757100 \quad 0.02158900$

Cl $-2.41483100 \quad 0.47661400 \quad-0.02880700$

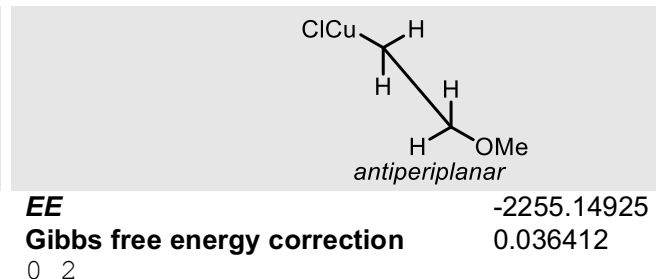

02

$\begin{array}{llll}\text { C } 3.50879200 & -0.31718000 & 0.00007000\end{array}$

H $3.81107100 \quad 0.24600900 \quad 0.88849200$

H $3.81117500 \quad 0.24642400-0.88805200$

H $4.00916000-1.28201100-0.00012700$

C $1.37484000 \quad 0.64948800 \quad 0.00009600$

$\mathrm{H} 1.62788000 \quad 1.26108500-0.87615000$

$\mathrm{H} \quad 1.62781800 \quad 1.26081200 \quad 0.87655100$

O $2.12274400-0.55048600-0.00006600$

$\mathrm{Cu}-0.56741600 \quad 0.19404400-0.00001800$

$\mathrm{Cl}-2.63034000-0.29115000-0.00003800$ 


\section{COPPER}
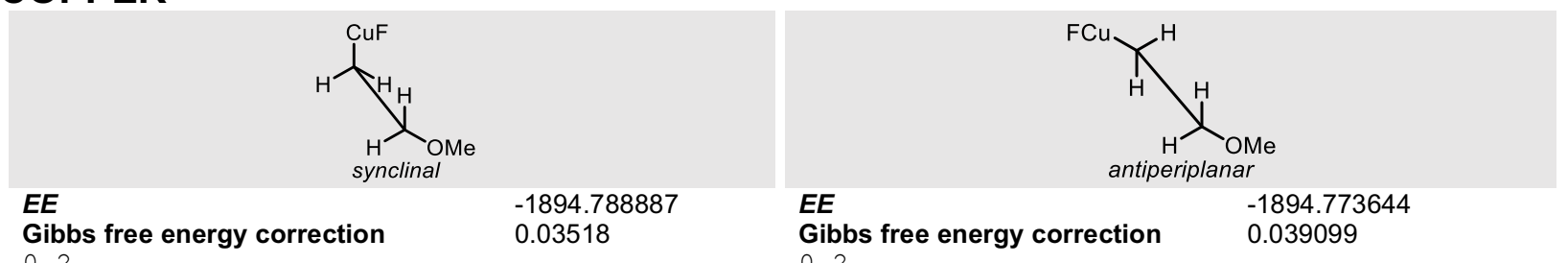
02
C $-2.33527200 \quad-0.94883400 \quad 0.17507700$
$\mathrm{H}-3.02627800 \quad-0.56814600 \quad 0.92642300$
$\mathrm{H}-1.55241200-1.53593900 \quad 0.65410600$
$\mathrm{H}-2.86591800-1.56056600-0.54462100$
C $-1.04791300 \quad 1.01966700 \quad 0.17696500$
$\mathrm{H}-1.29640800 \quad 1.08120800 \quad 1.23571300$
$\mathrm{H}-0.90511800 \quad 1.95587800-0.35512800$
$\begin{array}{lllll}0 & -1.76689400 & 0.13831700 & -0.54847400\end{array}$
$\mathrm{Cu} \quad 0.78337400 \quad 0.17752700 \quad 0.03253600$
F 2.37361700 $-0.67247300-0.06494400$

02

$\begin{array}{llll}\text { C } 3.13111700 & -0.21608700 & 0.00008600\end{array}$

H $3.40597000 \quad 0.36091000 \quad 0.88852700$

H $3.40610000 \quad 0.36148800-0.88793900$

H $3.67728300-1.15565600-0.00018100$

C $0.95327100 \quad 0.64697500 \quad 0.00003600$

$\mathrm{H} 1.17580900 \quad 1.26983200 \quad-0.87623600$

$\mathrm{H} \quad 1.17577400 \quad 1.26959100 \quad 0.87649000$

O $1.75770900-0.51550000-0.00011200$

$\mathrm{Cu}-0.95427500 \quad 0.10069800-0.00001800$

F $-2.63721900-0.38752700 \quad 0.00000200$ 
COPPER

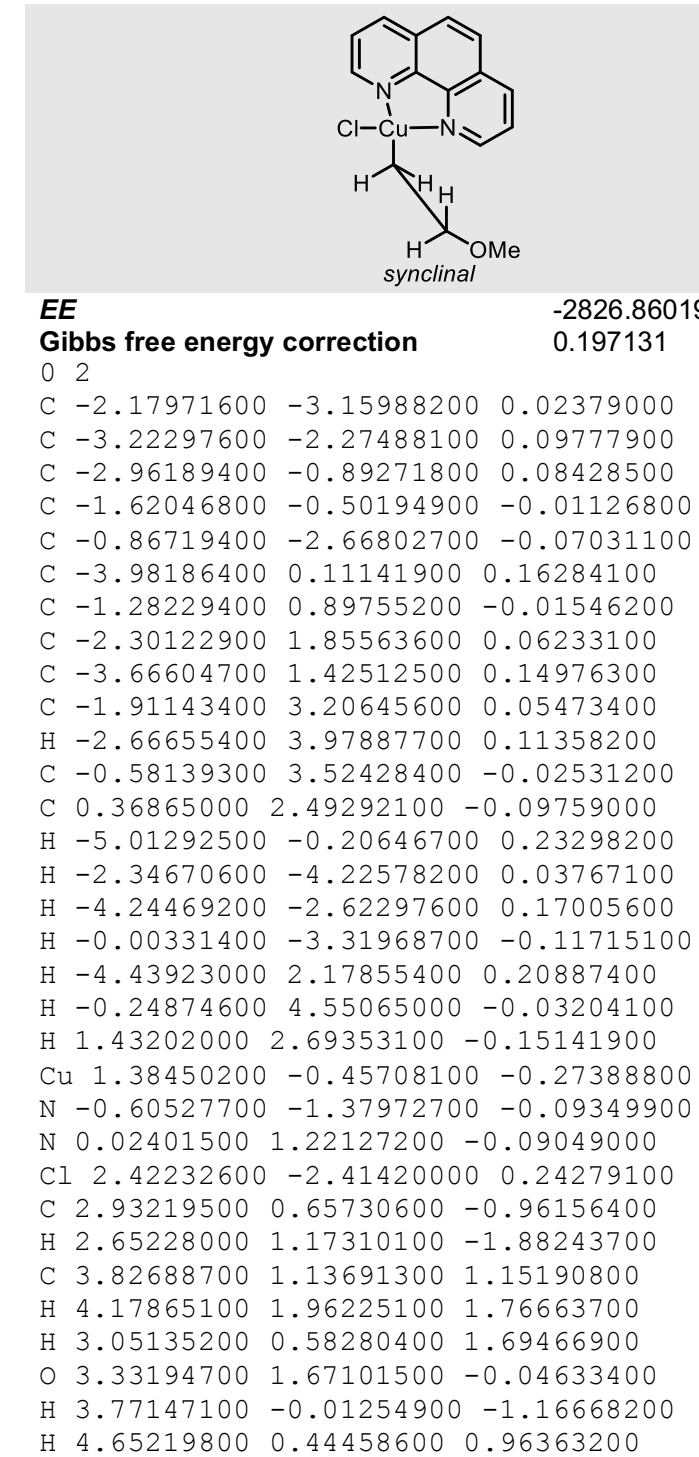

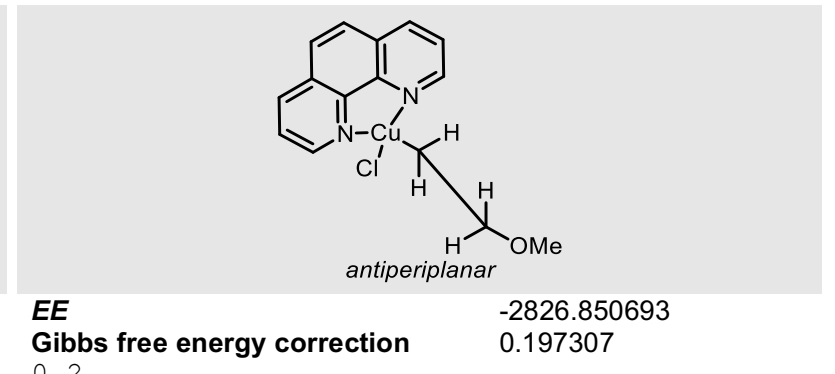

02

C $1.99490300-3.20824300 \quad 0.43673600$

C $3.10463400-2.40557900 \quad 0.48104300$

C $2.96119600-1.01558900 \quad 0.31912800$

C $1.66157800-0.53234500 \quad 0.12911200$

C $0.73185900-2.62602700 \quad 0.23760400$

C $4.05713100-0.09091500 \quad 0.33478000$

C $1.44543600 \quad 0.87705600-0.06387400$

C $2.535421001 .75697900-0.04777600$

C $3.85418300 \quad 1.23395100 \quad 0.16186100$

C $2.258340003 .12011400-0.24947000$

$\mathrm{H} 3.06988000 \quad 3.83526600-0.24562300$

C $0.96390100 \quad 3.52523400-0.44986600$

C $-0.05855800 \quad 2.56520900-0.44780800$

H $5.05487500-0.47868300 \quad 0.48712500$

H 2.07299100 $-4.27850600 \quad 0.54886100$

H $4.08990100 \quad-2.82559600 \quad 0.63279700$

$\mathrm{H}-0.17602700-3.21246100 \quad 0.16984900$

$\mathrm{H} 4.68548500 \quad 1.92501200 \quad 0.17371500$

$\mathrm{H} \quad 0.71840300 \quad 4.56373300-0.60835200$

$\mathrm{H}-1.09321500 \quad 2.84560300-0.59592800$

$\mathrm{Cu}-1.32553800-0.28060700-0.18632900$

$\mathrm{N} \quad 0.57820400-1.32743300 \quad 0.10016900$

N $0.17775100 \quad 1.28457600-0.26320800$

Cl $-2.28727100-2.14154100-1.02776300$

C $-2.80202600 \quad 0.99952800 \quad 0.26885100$

$\mathrm{H}-3.11353200 \quad 1.52843500-0.64753300$

C $-4.99624400 \quad 1.18071800 \quad 1.06392800$

$\mathrm{H}-5.79832500 \quad 0.58750500 \quad 1.49816000$

$\mathrm{H}-4.73612700 \quad 1.99236000 \quad 1.75438600$

$\begin{array}{lllll}0 & -3.90412800 & 0.33589600 & 0.83792000\end{array}$

$\mathrm{H}-2.42986900 \quad 1.76507800 \quad 0.97244300$

$\mathrm{H}-5.34941600 \quad 1.62833100 \quad 0.12804200$ 
COPPER

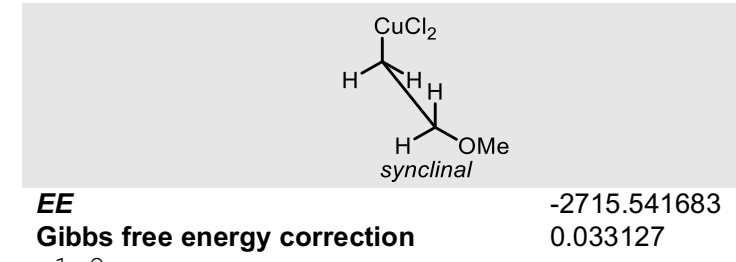

$-12$

C $2.71169900-0.84222900 \quad 0.59032700$

H 2.35728300 $0.15434500 \quad 0.86633400$

$\mathrm{H} \quad 3.76836300-0.79019100 \quad 0.33314000$

$\mathrm{H} 2.58828900-1.51510900 \quad 1.44915900$

C $0.64868900-1.48545800-0.29534600$

$\mathrm{H} \quad 0.46404000 \quad-2.09749900 \quad 0.59722300$

$\mathrm{H} \quad 0.21895800 \quad-1.97606900-1.16713700$

O $2.02526500-1.33094400-0.52659200$

$\mathrm{Cu}-0.47105800 \quad 0.18823900-0.05034000$

Cl $0.67142400 \quad 2.12137300-0.05483700$

Cl $-2.55970000-0.62847600 \quad 0.16213000$

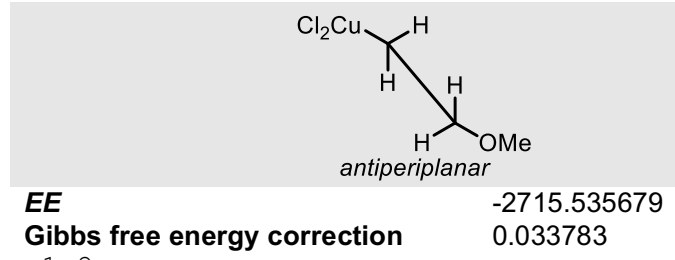

Gibbs
-12

C $-3.55886600-0.00012600-0.15565700$

$\mathrm{H}-3.82636400-0.88759100 \quad 0.43214300$

$\mathrm{H}-4.13854300 \quad 0.00026200-1.07903900$

$\mathrm{H}-3.82633600 \quad 0.88686500 \quad 0.43287300$

C $-1.36308400-0.00010200 \quad 0.65971200$

$\mathrm{H}-1.56493000-0.89396900 \quad 1.26424200$

$\mathrm{H}-1.56497300 \quad 0.89363800 \quad 1.26441500$

$0-2.20439800-0.00001300-0.48596100$

$\mathrm{Cu} 0.55574300 \quad 0.00001000 \quad 0.07511100$

Cl $1.35222600-2.08984700-0.10675900$

Cl $1.35197900 \quad 2.08996300-0.10674100$ 


\section{SILVER}

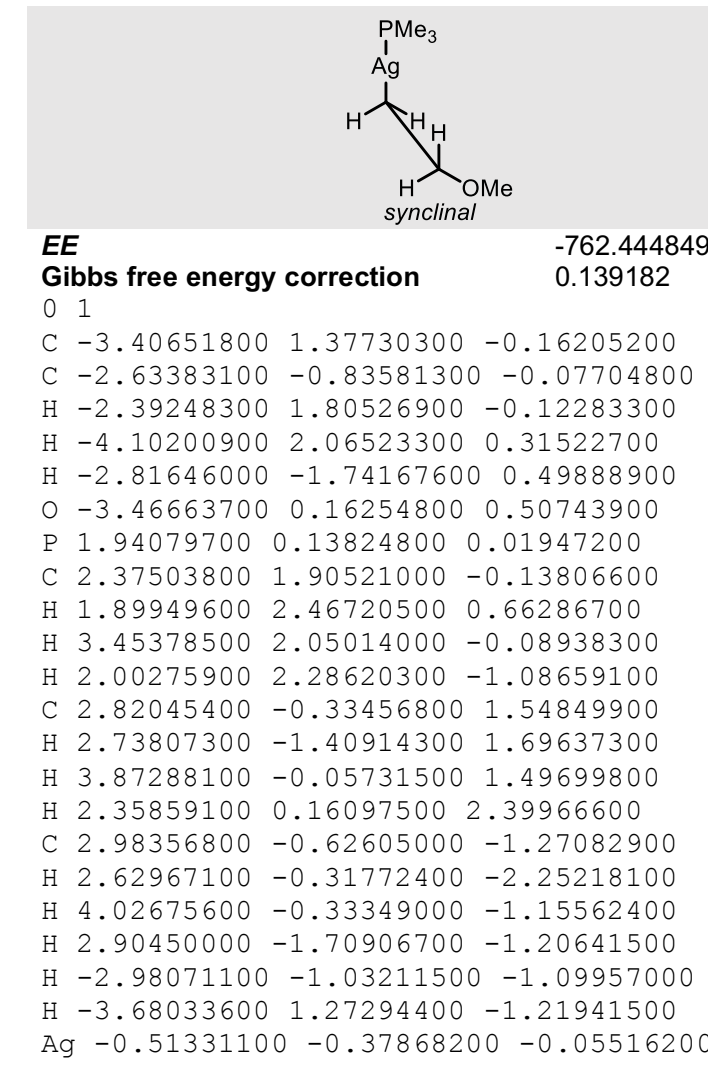

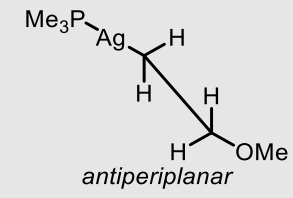

EE

$-762.444566$

Gibbs free energy correction 0.139491

01

C $4.58973700 \quad 0.54630200 \quad 0.00009000$

C $2.54054400-0.59712300 \quad 0.00025800$

$\mathrm{H} \quad 4.93986200 \quad 0.00527600 \quad 0.88722800$

H $5.02398900 \quad 1.54525300-0.00044100$

$\mathrm{H} \quad 2.86743600-1.16945800-0.87787300$

$\begin{array}{llll}0 & 3.19671900 & 0.67063300 & -0.00030500\end{array}$

P $-2.03942800 \quad 0.12696600 \quad 0.00000200$

C $-2.68806200 \quad 1.05503800 \quad 1.43256600$

$\mathrm{H}-2.19304100 \quad 2.02188400 \quad 1.49120500$

$\mathrm{H}-3.76393400 \quad 1.20455800 \quad 1.34768000$

$\mathrm{H}-2.47130600 \quad 0.50730700 \quad 2.34706500$

C $-2.680271001 .09920500-1.40643600$

$\mathrm{H}-2.45798100 \quad 0.58055800 \quad-2.33642700$

$\mathrm{H}-3.75669900 \quad 1.24561200-1.32311200$

$\mathrm{H}-2.18549900 \quad 2.06762500-1.43186800$

C $-3.12427600-1.34177500-0.02587600$

$\mathrm{H}-2.91954600-1.95901700 \quad 0.84613400$

$\mathrm{H}-4.17461600-1.05223100-0.02413500$

$\mathrm{H}-2.91482000-1.93131500-0.91574900$

H 2.86716200 $-1.16851200 \quad 0.87910600$

$\mathrm{H} \quad 4.94028100 \quad 0.00414800-0.88618400$

Ag $0.41256000-0.29236500-0.00008100$ 


\section{SILVER}

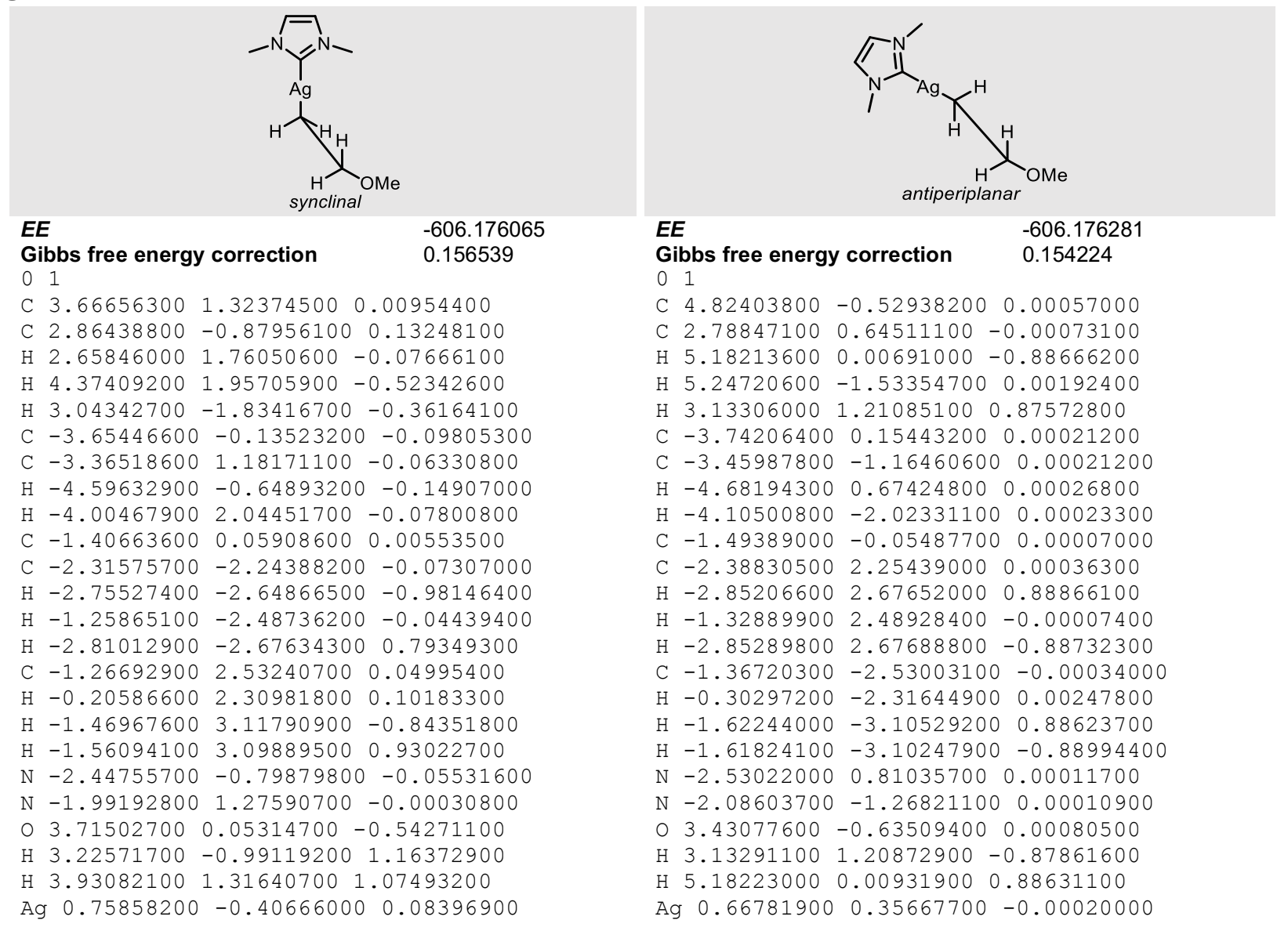


GOLD

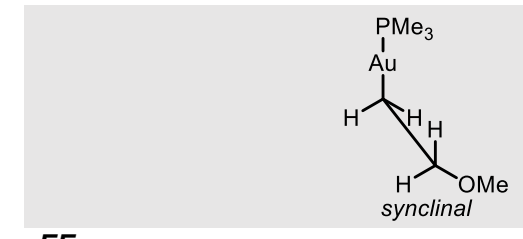

Gibbs free energy correction

$-751.185962$

01

C $-3.25542300 \quad 1.43575700-0.16669700$

C $-2.38921800-0.74271000-0.06528500$

$\mathrm{H}-2.26444700 \quad 1.90955700-0.16087900$

$\mathrm{H}-3.97042000 \quad 2.09683400 \quad 0.31846200$

$\mathrm{H}-2.55828700-1.66019100 \quad 0.49732800$

$\begin{array}{lllll}0 & -3.24430800 & 0.22807600 & 0.52688500\end{array}$

P $1.91092600 \quad 0.20584800 \quad 0.02588600$

C 2.312085001 .950409000 .36661400

$\mathrm{H} 1.88882300 \quad 2.23494300 \quad 1.32737700$

H $3.38965000 \quad 2.10904900 \quad 0.38342700$

$\mathrm{H} \quad 1.86533800 \quad 2.57853200-0.40098000$

C $2.88348100-0.67948900 \quad 1.28708300$

H $2.80465600-1.750764001 .11573300$

$\mathrm{H} \quad 3.93125300 \quad-0.38324700 \quad 1.25440900$

$\begin{array}{llll}\mathrm{H} & 2.47608300 & -0.46222800 & 2.27200900\end{array}$

C $2.84579200-0.12687200-1.50275700$

H $2.41368900 \quad 0.44606000-2.32020400$

$\mathrm{H} \quad 3.89501200 \quad 0.14185300-1.38659300$

H $2.76806300-1.18336200-1.74961800$

$\mathrm{H}-2.72630100-0.93307300-1.09247700$

$\mathrm{H}-3.55406400 \quad 1.29628300-1.21281500$

$\mathrm{Au}-0.34745300-0.28323000 \quad-0.03749700$

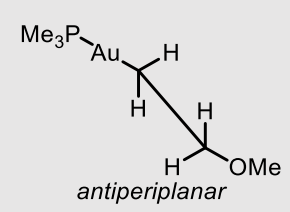

EE

$-751.185726$

Gibbs free energy correction $\quad 0.140638$

01

C $4.41455100 \quad 0.54782900 \quad 0.00005000$

C $2.33106500-0.51918800-0.00006500$

$\mathrm{H} \quad 4.74533300 \quad-0.00461100 \quad 0.88723600$

H $4.881623001 .53093500-0.00009600$

$\mathrm{H} 2.63758200-1.10189100-0.87898300$

$\begin{array}{llll}0 & 3.02656700 & 0.72291300 & -0.00017300\end{array}$

P $-1.99144000 \quad 0.16948800 \quad-0.00001600$

C $-2.63399100 \quad 1.09155300 \quad 1.43387500$

$\mathrm{H}-2.14191800 \quad 2.06011900 \quad 1.48732500$

$\mathrm{H}-3.71107700 \quad 1.23492300 \quad 1.35704300$

$\mathrm{H}-2.40510700 \quad 0.54356700 \quad 2.34516400$

C $-2.62687500 \quad 1.13153100-1.41052500$

$\mathrm{H}-2.39325500 \quad 0.60951200 \quad-2.33574300$

$\mathrm{H}-3.70437600 \quad 1.27255300-1.33513700$

H $-2.13484600 \quad 2.10130800-1.43416200$

C $-3.04452500-1.31719200-0.02355100$

$\mathrm{H}-2.82535300 \quad-1.92696600 \quad 0.85008200$

$\mathrm{H}-4.10023700-1.04872500-0.02302900$

$\mathrm{H}-2.82002900-1.90272100-0.91228200$

$\mathrm{H} 2.63804800-1.10200500 \quad 0.87860600$

H $4.74560900-0.00496600-0.88683800$

Au $0.27349000 \quad-0.20498500 \quad 0.00004700$ 
GOLD
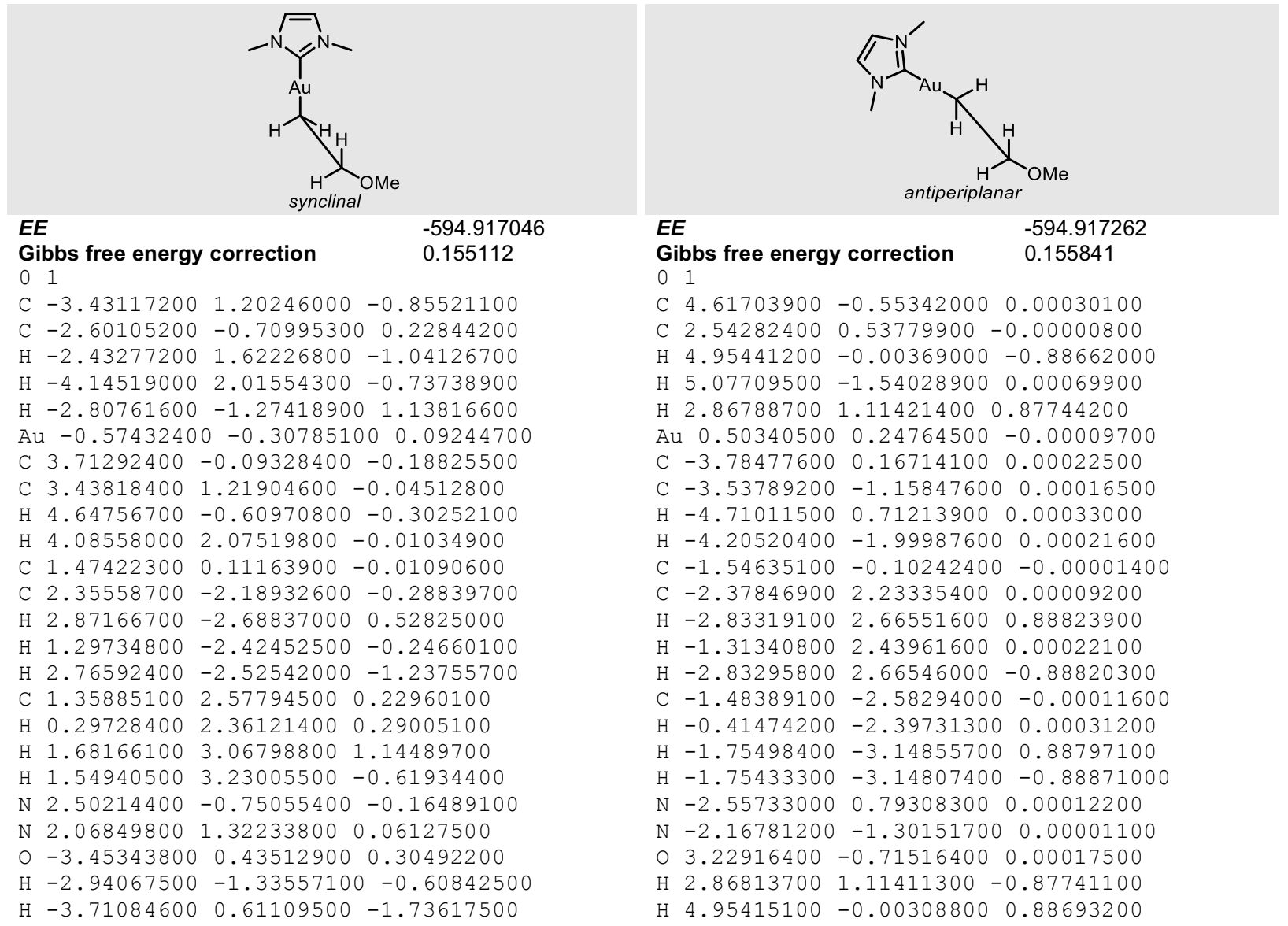

01

$\begin{array}{lllll}\text { C } 4.61703900 & -0.55342000 & 0.00030100\end{array}$

C $2.54282400 \quad 0.53779900-0.00000800$

$\mathrm{H} 4.95441200-0.00369000-0.88662000$

$\mathrm{H} 5.07709500-1.54028900 \quad 0.00069900$

H $2.86788700 \quad 1.11421400 \quad 0.87744200$

$\mathrm{Au} \quad 0.50340500 \quad 0.24764500 \quad-0.00009700$

C $-3.78477600 \quad 0.16714100 \quad 0.00022500$

C $-3.53789200-1.15847600 \quad 0.00016500$

$\mathrm{H}-4.71011500 \quad 0.71213900 \quad 0.00033000$

$\mathrm{H}-4.20520400-1.99987600 \quad 0.00021600$

C $-1.54635100-0.10242400-0.00001400$

C $-2.37846900 \quad 2.23335400 \quad 0.00009200$

$\mathrm{H}-2.83319100 \quad 2.66551600 \quad 0.88823900$

$\mathrm{H}-1.31340800 \quad 2.43961600 \quad 0.00022100$

$\mathrm{H}-2.83295800 \quad 2.66546000-0.88820300$

C $-1.48389100-2.58294000-0.00011600$

H $-0.41474200-2.39731300 \quad 0.00031200$

$\mathrm{H}-1.75498400-3.14855700 \quad 0.88797100$

$\mathrm{H}-1.75433300-3.14807400-0.88871000$

$\mathrm{N}-2.55733000 \quad 0.79308300 \quad 0.00012200$

$\mathrm{N}-2.16781200-1.30151700 \quad 0.00001100$

$\begin{array}{llll}0 & 3.22916400 & -0.71516400 & 0.00017500\end{array}$

$\mathrm{H} 2.868137001 .11411300-0.87741100$

H $4.95415100 \quad-0.00308800 \quad 0.88693200$ 


\section{GOLD}

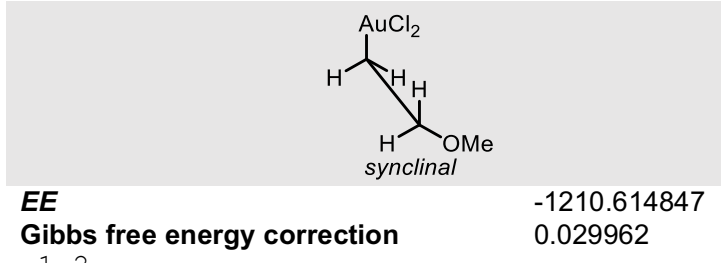

$-12$

C $2.78503000-1.36794100 \quad 0.50002700$

H $2.60655400-0.30809400 \quad 0.69186600$

$\mathrm{H} \quad 3.81164500 \quad-1.51398700 \quad 0.17219600$

H $2.61646300-1.93792200 \quad 1.42074600$

C $0.60879600-1.80636200-0.20457500$

$\mathrm{H} \quad 0.38831200 \quad-2.22387200 \quad 0.78005300$

$\mathrm{H} \quad 0.02083800-2.24142900-1.00353300$

Cl $1.36456600 \quad 1.96530000-0.03961700$

$\mathrm{Cl}-2.52204100 \quad-0.74291500 \quad 0.12267200$

$01.94613100-1.83363200-0.52804400$

$\mathrm{Au}-0.32530100 \quad 0.26784400 \quad-0.01293200$

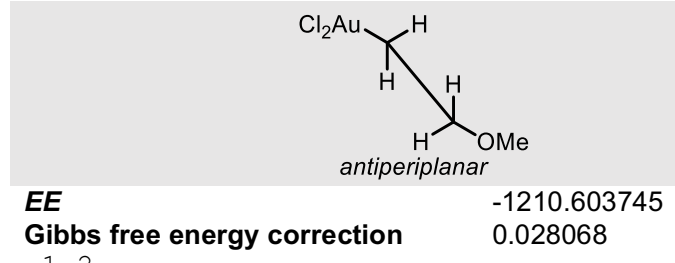

Gibbs
$-1 \quad 2$

C $3.80603000-0.00002600-0.12686500$

$\mathrm{H} \quad 4.07135700 \quad 0.88802000 \quad 0.45840300$

$\mathrm{H} \quad 4.37831000 \quad 0.00019300-1.05333100$

H $4.07145100 \quad-0.88828300 \quad 0.45804000$

C $1.62123000 \quad 0.00001600 \quad 0.66777200$

$\mathrm{H} \quad 1.73651700 \quad 0.91194400 \quad 1.25796300$

H $1.73651400 \quad-0.91186900 \quad 1.25802900$

$\mathrm{Cl}-0.87768800 \quad 2.36000800 \quad-0.07343500$

$\mathrm{Cl}-0.87772400 \quad-2.35999500-0.07343000$

$\mathrm{Au}-0.48462400 \quad 0.00000100 \quad 0.00702700$

$02.44619600-0.00003000-0.46037500$ 
GOLD

\begin{tabular}{|c|c|c|c|}
\hline \multicolumn{3}{|c|}{$E E$} & -1671.6593 \\
\hline \multicolumn{3}{|c|}{ Gibbs free energy correction } & 0.141459 \\
\hline $\mathrm{P}$ & -2.13352300 & -0.30802000 & 0.12019800 \\
\hline C & -2.50556500 & -1.39923700 & 1.52457400 \\
\hline $\mathrm{H}$ & -2.24211500 & -0.89752000 & 2.45319600 \\
\hline $\mathrm{H}$ & -3.56197300 & -1.66343000 & 1.54377400 \\
\hline $\mathrm{H}$ & -1.90041600 & -2.29847200 & 1.43011800 \\
\hline C & -3.25268400 & 1.10668200 & 0.32555100 \\
\hline $\mathrm{H}$ & -3.14108700 & 1.77400600 & -0.52614400 \\
\hline $\mathrm{H}$ & -4.28780500 & 0.77847200 & 0.40805900 \\
\hline H & -2.96609300 & 1.66009200 & 1.21701900 \\
\hline $\mathrm{C}$ & -2.78954200 & -1.21185100 & -1.31295100 \\
\hline $\mathrm{H}$ & -3.83108900 & -1.48855600 & -1.15570600 \\
\hline $\mathrm{H}$ & -2.71155800 & -0.58832200 & -2.20095300 \\
\hline $\mathrm{H}$ & -2.18649900 & -2.10524000 & -1.46102100 \\
\hline $\mathrm{C}$ & 2.33624400 & $0.68220100-c$ & 0.33963100 \\
\hline & 2.49905300 & $1.42226700 \quad 0$ & .44466000 \\
\hline $\mathrm{C}$ & 3.50652600 & -0.82486800 & 0.99692400 \\
\hline & 4.25857900 & -1.59979700 & 0.89315600 \\
\hline & 2.61390600 & -1.24867000 & 1.45985900 \\
\hline & 3.21464200 & -0.35272200 & -0.30021700 \\
\hline & $1-0.0335740$ & 02.48608800 & 0.07338800 \\
\hline & 10.63101800 & -2.09224200 & -0.28862000 \\
\hline & u 0.29845500 & 0.19960100 & -0.09753500 \\
\hline & 3.89527100 & -0.01454700 & 1.61868800 \\
\hline & 2.34319100 & $1.13037500-$ & -1.32855200 \\
\hline
\end{tabular}

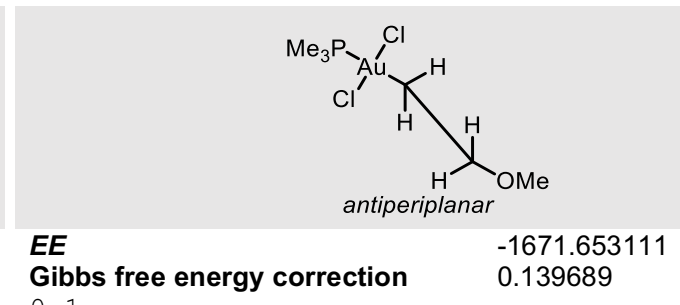

$0 \quad 1$

P $-2.15480400 \quad 0.01916900 \quad 0.23929200$

C $-2.57703100-0.89658000 \quad 1.74802500$

$\mathrm{H}-2.13543700-0.39897100 \quad 2.60863300$

$\mathrm{H}-3.65664000-0.95542800 \quad 1.87718500$

$\mathrm{H}-2.15675600-1.89719900 \quad 1.67058900$

C $-2.95449000 \quad 1.63548700 \quad 0.42379900$

$\mathrm{H}-2.79676900 \quad 2.21556900-0.48257800$

$\mathrm{H}-4.02048900 \quad 1.52157500 \quad 0.61420500$

$\mathrm{H}-2.48398800 \quad 2.17240900 \quad 1.24434500$

C $-3.08686800-0.82632400-1.06860100$

$\mathrm{H}-4.14345200-0.88968500-0.81290000$

$\mathrm{H}-2.97293800-0.28270600-2.00392100$

$\mathrm{H}-2.67437700-1.82547000-1.19268700$

C $2.29525700-0.04313900-0.47693800$

H 2.57847500 $0.86132400-1.01770800$

C $4.26515000-0.12000800 \quad 0.75183600$

$\mathrm{H} \quad 4.61776300 \quad-0.15543500 \quad 1.77815700$

$\mathrm{H} \quad 4.65960300 \quad 0.77470700 \quad 0.26291500$

○ $2.85494500-0.08955900 \quad 0.79349300$

Cl $0.37268600 \quad 2.33186600-0.19172400$

Cl $0.18956900-2.29128100-0.23068100$

Au $0.25735300 \quad 0.02598400 \quad-0.18652000$

$\mathrm{H} \quad 4.62002600-1.00423200 \quad 0.21557700$

$\mathrm{H} 2.50616300-0.94679400-1.05187600$ 


\section{SDM Optimized Structures}

\begin{tabular}{|c|c|c|c|c|}
\hline & \multicolumn{2}{|c|}{ PhH } & \multicolumn{2}{|c|}{ DMSO } \\
\hline & $E E$ & Gibbs energy & $E E$ & Gibbs energy \\
\hline \multicolumn{5}{|c|}{ Tetrahydropyran } \\
\hline NiClPhenAx & -2811.31563 & -2811.04942 & -2811.31918 & -2811.05338 \\
\hline NiClPhenEq & -2811.31559 & -2811.04966 & -2811.32066 & -2811.05576 \\
\hline$\Delta E(\mathrm{kcal} / \mathrm{mol})$ & -0.02447289 & 0.1506024 & 0.92996982 & 1.49284629 \\
\hline PdClPhenAx & -1430.86534 & -1430.60146 & -1430.86534 & -1430.60146 \\
\hline PdCIPhenEq & -1430.85894 & -1430.59518 & -1430.8646 & -1430.60071 \\
\hline$\Delta E(\mathrm{kcal} / \mathrm{mol})$ & -4.01543649 & -3.94452786 & -0.4643574 & -0.47314254 \\
\hline PtClPhenAx & -1422.29194 & -1422.02622 & -1422.2948 & -1422.02947 \\
\hline PtClPhenEq & -1422.29056 & -1422.02552 & -1422.29622 & -1422.03099 \\
\hline$\Delta E(\mathrm{kcal} / \mathrm{mol})$ & -0.86345376 & -0.43800198 & 0.89169171 & 0.95569773 \\
\hline CuAx & -1911.60457 & -1911.50135 & -1911.61655 & -1911.51429 \\
\hline CuEq & -1911.60122 & -1911.49854 & -1911.61431 & -1911.51264 \\
\hline$\Delta E(\mathrm{kcal} / \mathrm{mol})$ & -2.10717858 & -1.76644065 & -1.40750493 & -1.03978407 \\
\hline $\operatorname{AgAx}$ & -418.042821 & -417.94032 & -418.046148 & -417.944276 \\
\hline $\mathrm{AgEq}$ & -418.037457 & -417.935468 & -418.042002 & -417.940685 \\
\hline$\Delta E(\mathrm{kcal} / \mathrm{mol})$ & -3.36596364 & -3.04467852 & -2.60165646 & -2.25338841 \\
\hline AuAx & -406.771774 & -406.668146 & -406.773747 & -406.670677 \\
\hline AuEq & -406.765217 & -406.662426 & -406.768913 & -406.666393 \\
\hline$\Delta E(\mathrm{kcal} / \mathrm{mol})$ & -4.11458307 & -3.5893572 & -3.03338334 & -2.68825284 \\
\hline \multicolumn{5}{|l|}{ Acyclic } \\
\hline CuAnti & -1794.92498 & -1794.88621 & -1794.94031 & -1794.90235 \\
\hline CuSyn & -1794.92639 & -1794.88713 & -1794.94082 & -1794.9025 \\
\hline$\Delta E(\mathrm{kcal} / \mathrm{mol})$ & 0.88227906 & 0.5773092 & 0.32191263 & 0.09977409 \\
\hline AgAnti & -301.317647 & -301.279796 & -301.324024 & -301.28649 \\
\hline AgSyn & -301.320772 & -301.282126 & -301.326116 & -301.288004 \\
\hline$\Delta E(\mathrm{kcal} / \mathrm{mol})$ & 1.96096875 & 1.4620983 & 1.31275092 & 0.95005014 \\
\hline AuAnti & -290.043194 & -290.004731 & -290.048588 & -290.010213 \\
\hline AuSyn & -290.048921 & -290.009207 & -290.052644 & -290.013334 \\
\hline$\Delta E(\mathrm{kcal} / \mathrm{mol})$ & 3.59374977 & 2.80873476 & 2.54518056 & 1.95845871 \\
\hline PdAnti & -282.215432 & -282.178158 & -282.222663 & -282.185101 \\
\hline PdSyn & -282.219049 & -282.181057 & -282.220125 & -282.183074 \\
\hline$\Delta E(\mathrm{kcal} / \mathrm{mol})$ & 2.26970367 & 1.81915149 & -1.59262038 & -1.27196277 \\
\hline
\end{tabular}




\section{Additional Structures}

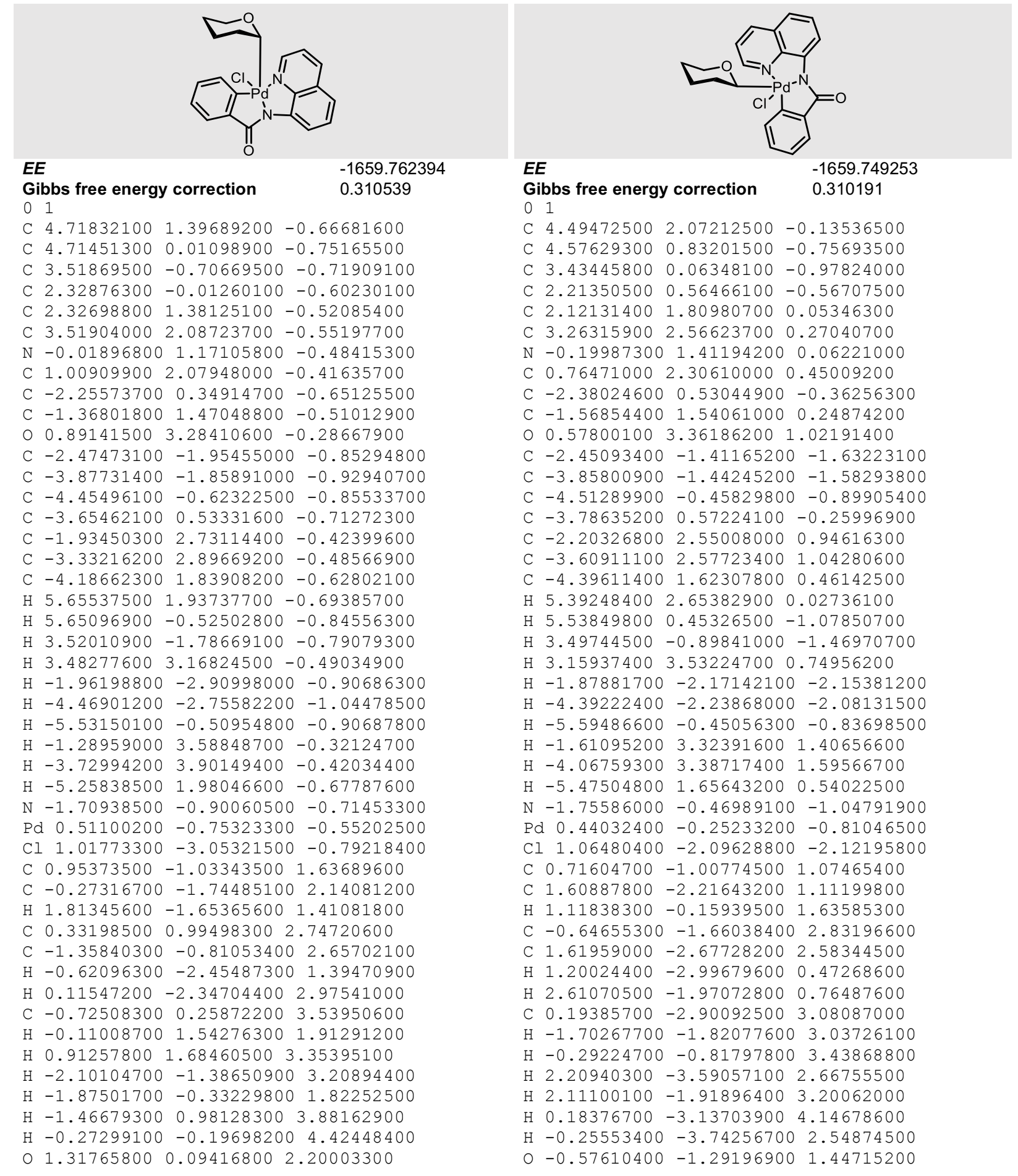




\section{References}

(1) Gaussian 09, R. B., M. J. Frisch, G. W. Trucks, H. B. Schlegel, G. E. Scuseria, M. A. Robb, J. R. Cheeseman, G. Scalmani, V. Barone, B. Mennucci, G. A. Petersson, H. Nakatsuji, M. Caricato, X. Li, H. P. Hratchian, A. F. Izmaylov, J. Bloino, G. Zheng, J. L. Sonnenberg, M. Hada, M. Ehara, K. Toyota, R. Fukuda, J. Hasegawa, M. Ishida, T. Nakajima, Y. Honda, O. Kitao, H. Nakai, T. Vreven, J. A. Montgomery, Jr., J. E. Peralta, F. Ogliaro, M. Bearpark, J. J. Heyd, E. Brothers, K. N. Kudin, V. N. Staroverov, T. Keith, R. Kobayashi, J. Normand, K. Raghavachari, A. Rendell, J. C. Burant, S. S. Iyengar, J. Tomasi, M. Cossi, N. Rega, J. M. Millam, M. Klene, J. E. Knox, J. B. Cross, V. Bakken, C. Adamo, J. Jaramillo, R. Gomperts, R. E. Stratmann, O. Yazyev, A. J. Austin, R. Cammi, C. Pomelli, J. W. Ochterski, R. L. Martin, K. Morokuma, V. G. Zakrzewski, G. A. Voth, P. Salvador, J. J. Dannenberg, S. Dapprich, A. D. Daniels, O. Farkas, J. B. Foresman, J. V. Ortiz, J. Cioslowski, and D. J. Fox, Gaussian, Inc., Wallingford CT, 2010.

(2) Gaussian 16, R. A., M. J. Frisch, G. W. Trucks, H. B. Schlegel, G. E. Scuseria, M. A. Robb, J. R. Cheeseman, G. Scalmani, V. Barone, G. A. Petersson, H. Nakatsuji, X. Li, M. Caricato, A. V. Marenich, J. Bloino, B. G. Janesko, R. Gomperts, B. Mennucci, H. P. Hratchian, J. V. Ortiz, A. F. Izmaylov, J. L. Sonnenberg, D. Williams-Young, F. Ding, F. Lipparini, F. Egidi, J. Goings, B. Peng, A. Petrone, T. Henderson, D. Ranasinghe, V. G. Zakrzewski, J. Gao, N. Rega, G. Zheng, W. Liang, M. Hada, M. Ehara, K. Toyota, R. Fukuda, J. Hasegawa, M. Ishida, T. Nakajima, Y. Honda, O. Kitao, H. Nakai, T. Vreven, K. Throssell, J. A. Montgomery, Jr., J. E. Peralta, F. Ogliaro, M. J. Bearpark, J. J. Heyd, E. N. Brothers, K. N. Kudin, V. N. Staroverov, T. A. Keith, R. Kobayashi, J. Normand, K. Raghavachari, A. P. Rendell, J. C. Burant, S. S. Iyengar, J. Tomasi, M. Cossi, J. M. Millam, M. Klene, C. Adamo, R. Cammi, J. W. Ochterski, R. L. Martin, K. Morokuma, O. Farkas, J. B. Foresman, and D. J. Fox, Gaussian, Inc., Wallingford CT, 2016.

(3) Marenich, A. V.; Cramer, C. J.; Truhlar, D. G. Universal Solvation Model Based on Solute Electron Density and on a Continuum Model of the Solvent Defined by the Bulk Dielectric Constant and Atomic Surface Tensions. J. Phys. Chem. B 2009, 113, 6378-6396.

(4) Foster, J. P.; Weinhold, F. Natural hybrid orbitals. J. Am. Chem. Soc. 1980, 102, 7211-7218.

(5) Reed, A. E.; Curtiss, L. A.; Weinhold, F. Intermolecular interactions from a natural bond orbital, donor-acceptor viewpoint. Chem. Rev. 1988, 88, 899-926.

(6) Reed, A. E.; Weinstock, R. B.; Weinhold, F. Natural population analysis. J. Chem. Phys 1985, 83, 735-746.

(7) Weigend, F.; Ahlrichs, R. Balanced basis sets of split valence, triple zeta valence and quadruple zeta valence quality for $\mathrm{H}$ to Rn: Design and assessment of accuracy. Phys. Chem. Chem. Phys. 2005, 7, 3297-3305.

(8) Weigend, F. Accurate Coulomb-fitting basis sets for H to Rn. Phys. Chem. Chem. Phys. 2006, 8, $1057-1065$. 